\title{
CHARACTERIZATION OF FLOW HOMOGENEITY DOWNSTREAM \\ OF A SLOTTED ORIFICE PLATE IN A TWO-PHASE FLOW USING \\ ELECTRICAL RESISTANCE TOMOGRAPHY
}

\author{
A Thesis \\ by \\ GAUTHAM ANNAMALAI \\ Submitted to the Office of Graduate and Professional Studies of \\ Texas A\&M University \\ in partial fulfillment of the requirements for the degree of \\ MASTER OF SCIENCE
}

Chair of Committee, Gerald L. Morrison

Committee Members, Karen Vierow

Sungyon Lee

Head of Department, Andreas A. Polycarpou

May 2015

Major: Mechanical Engineering

Copyright 2015 Gautham Annamalai 


\begin{abstract}
The accurate measurement of two-phase flow parameters has always been a key issue for many industries. Advancements in flow measurement techniques led to the development of multiphase flow meters which can measure it without separating it to individual phases. This technique is based on the homogeneous flow model which treats the two-phase flow as a single-phase flow at the homogenized location. It has been experimentally proven that a slotted orifice plate is a better flow homogenizer than a standard orifice plate and a V-cone. However, the location downstream of the slotted orifice plate where the two-phase flow becomes most homogeneous is still not known. Knowing this would help in accurate flow metering of the two-phase flow as the sensors (such as the electrical impedance sensor) can be placed at the most homogenized location. Electrical Resistance Tomography (ERT) is a non-invasive measurement technique that determines the conductivity distribution of the flow inside a pipe. The conductivity data can be used to determine concentration data by using the Maxwell's equation. By analyzing the concentration distribution of the components in a two-phase flow at various locations, the optimum location downstream of the slotted orifice plate where the two-phase flow becomes most homogeneous for different flow conditions can be determined.
\end{abstract}




\section{ACKNOWLEDGEMENTS}

I would like to express my heartfelt gratitude to my advisor, Dr. Gerald L. Morrison, for his support and guidance throughout my research. I would also like to express my appreciation to my committee members: Dr. Karen Vierow and Dr. Sungyon Lee for their advice during my research.

I would like to thank all my lab mates in the Turbomachinery Laboratory for being such good friends. Special thanks to Dr. Sahand Pirouzpanah and Sujan Reddy for helping me with my research and giving me good advices.

I would like to thank my family: Dad, Mom, Sister and Dr. Jayakumar for their unconditional support and helping me accomplish my dream of graduating from Texas A\&M University. I thank the Almighty for blessing me with a good life and giving me everything I need. 


\section{TABLE OF CONTENTS}

Page

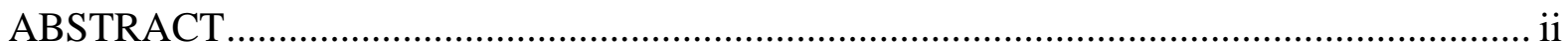

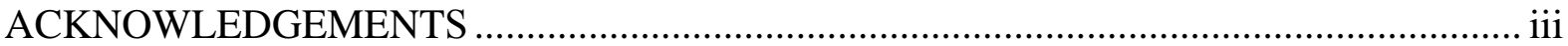

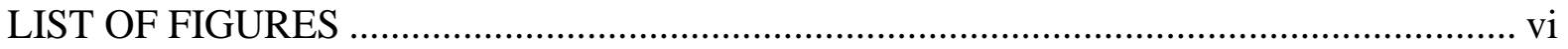

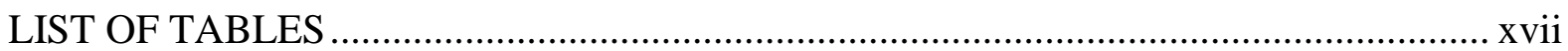

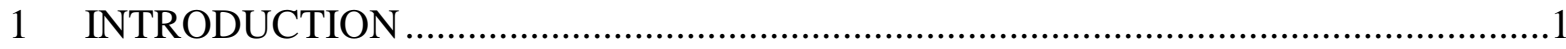

1.1 Orifice flow meter .......................................................................................

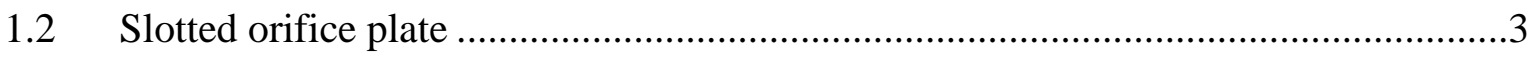

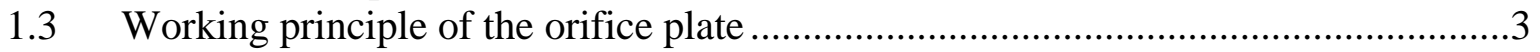

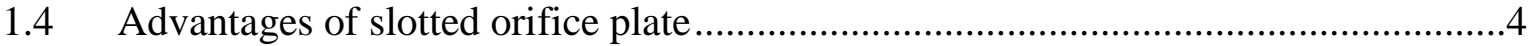

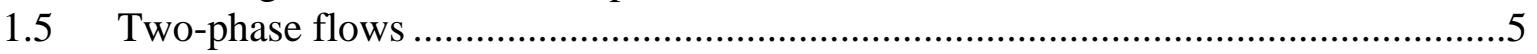

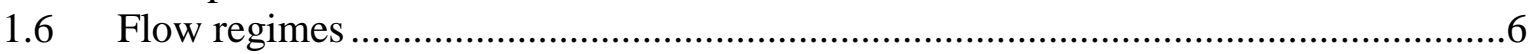

1.7 Process Tomography ...................................................................................

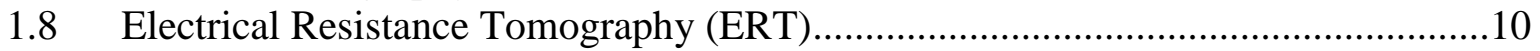

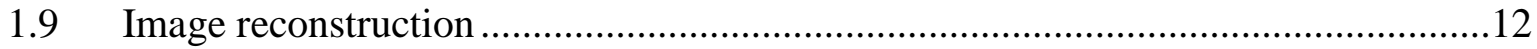

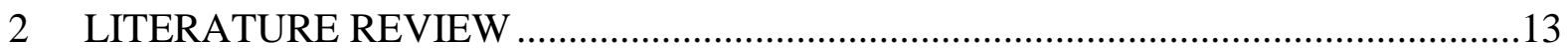

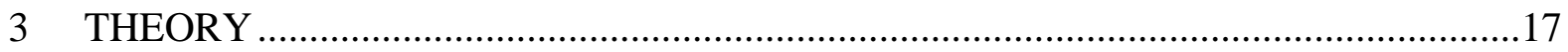

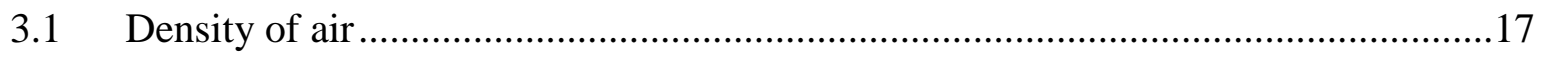

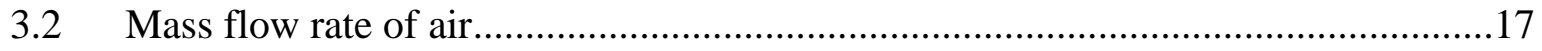

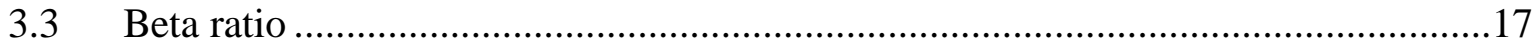

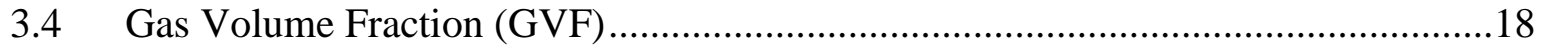

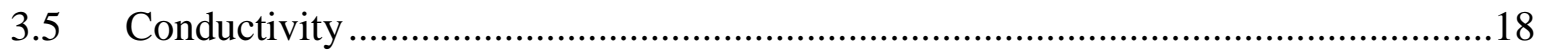

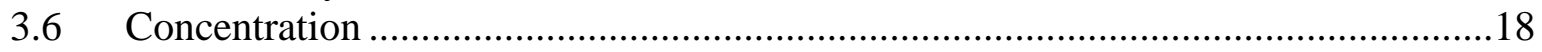

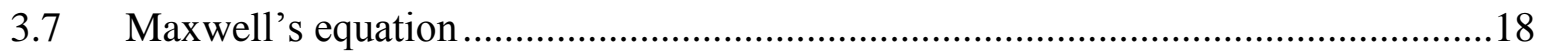

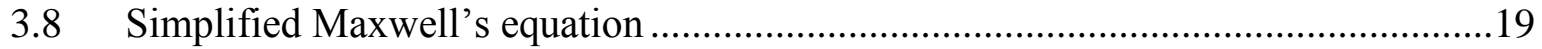

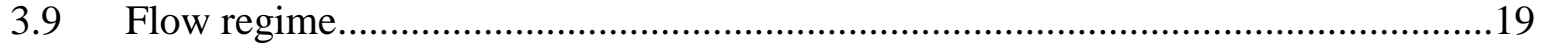

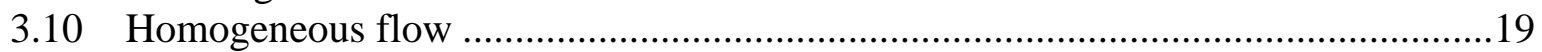

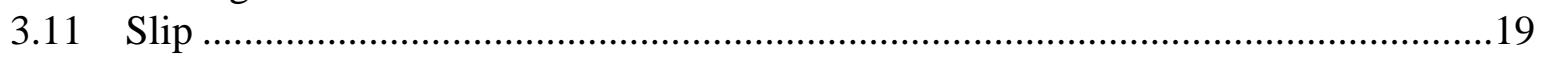

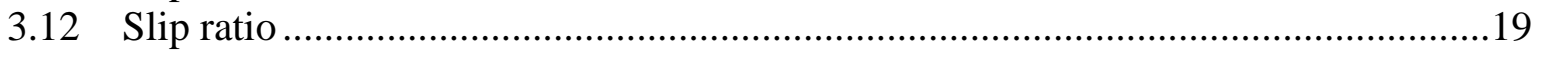

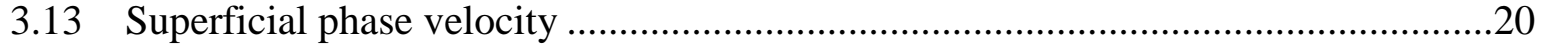

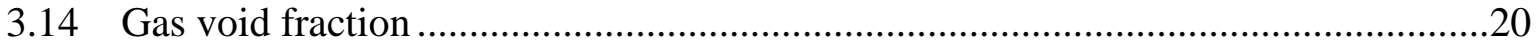

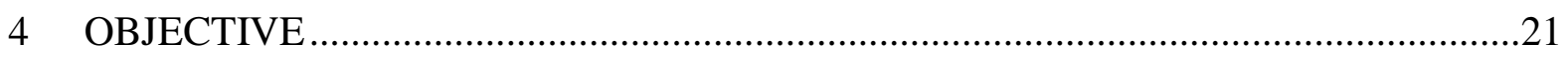

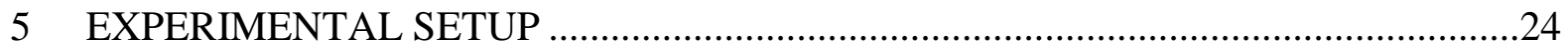




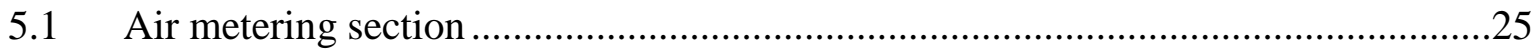

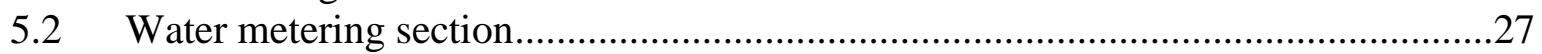

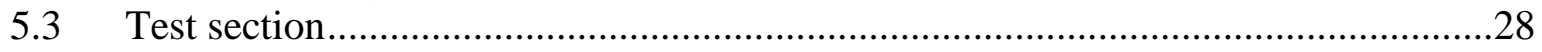

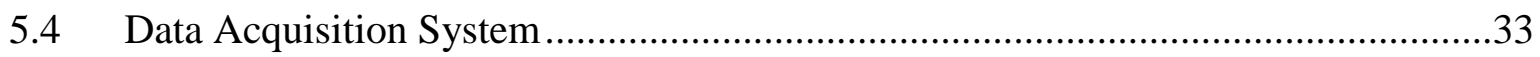

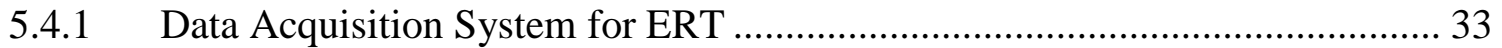

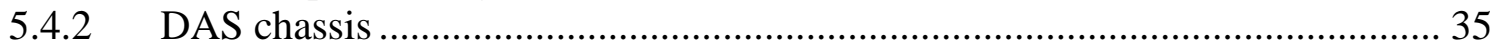

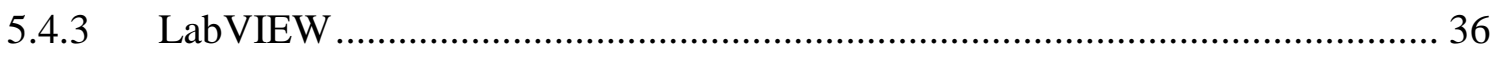

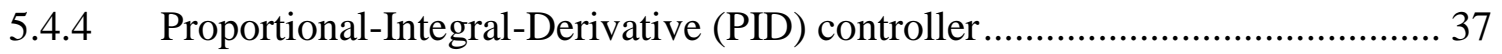

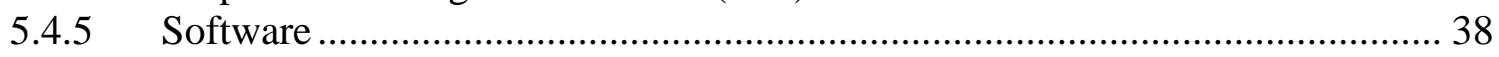

5.4.6 Image reconstruction algorithm ....................................................... 38

6 EXPERIMENTAL PROCEDURE .......................................................................4

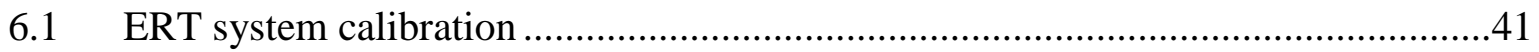

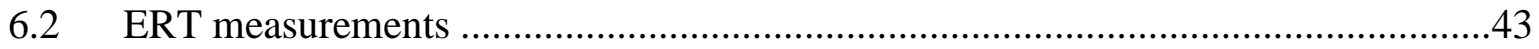

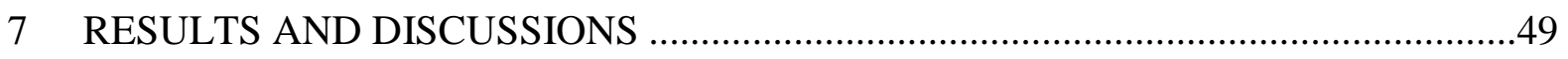

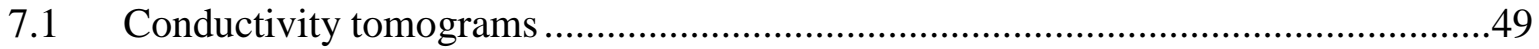

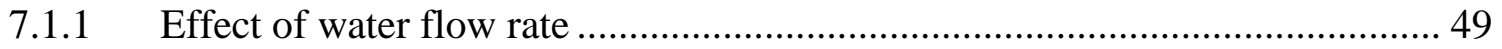

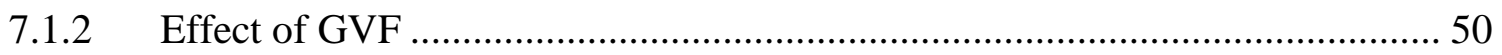

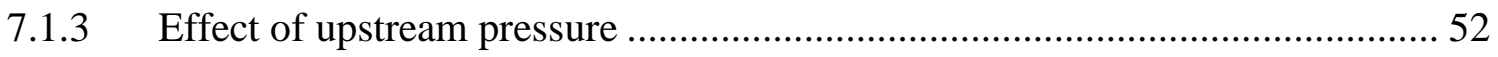

7.1.4 Effect of downstream distance ............................................................... 53

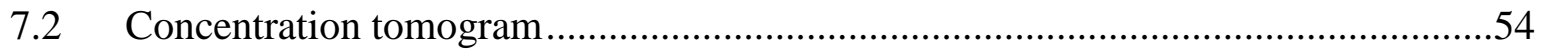

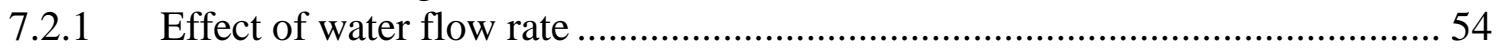

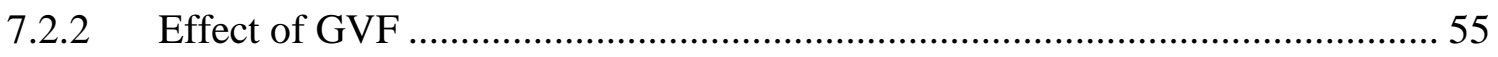

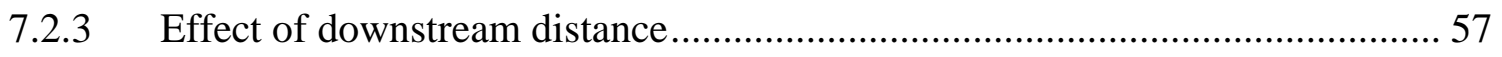

7.3 Determining most homogeneous location ...................................................58

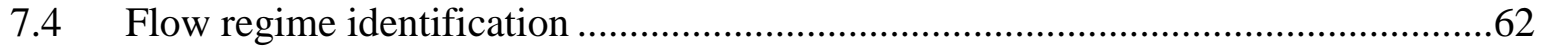

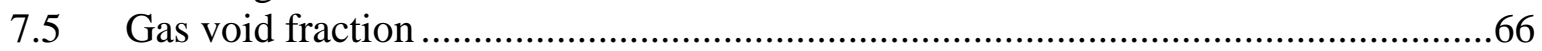

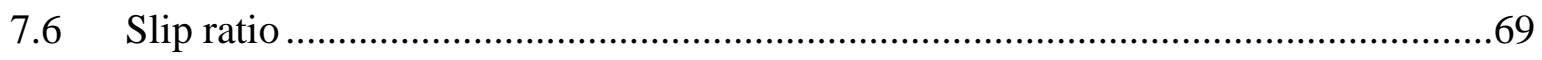

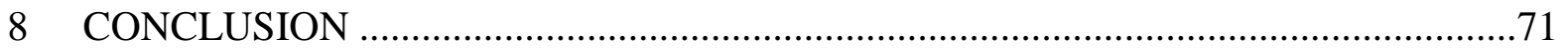

$8.1 \quad$ Recommended Future Work ...................................................................... 72

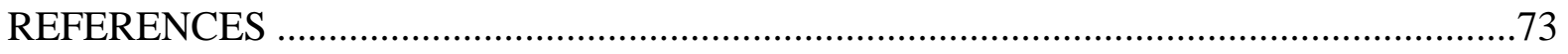

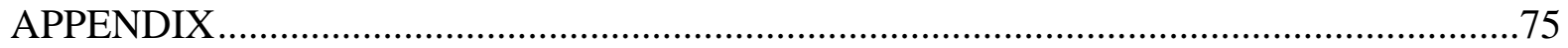




\section{LIST OF FIGURES}

Page

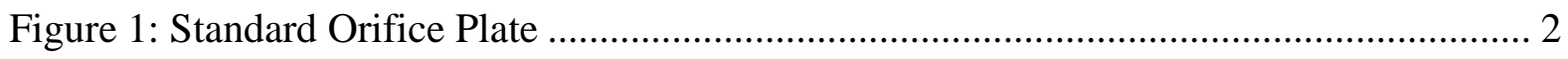

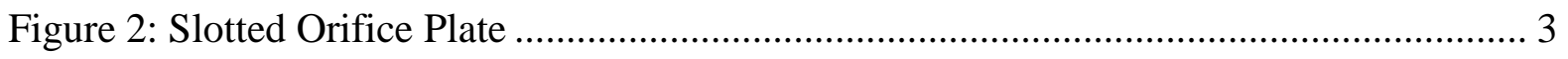

Figure 3: Sketches of flow regimes for horizontal pipe flow ...................................... 6

Figure 4: Horizontal Flow Regime Chart ................................................................... 8

Figure 5: Measurement strategy of ERT system ….................................................... 11

Figure 6: Image reconstruction grid........................................................................ 12

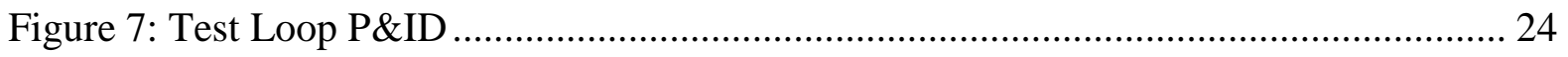

Figure 8: Masoneilan Dresser Varimax 3000 Series Control Valve .................................. 26

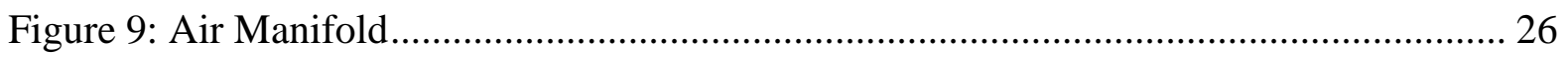

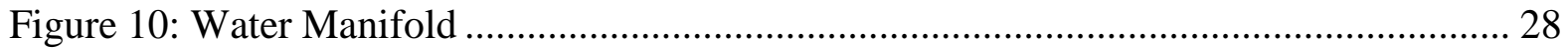

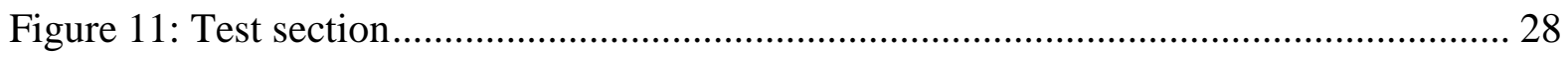

Figure 12: Polycarbonate flanges with slot for placing the slotted orifice plate .................. 29

Figure 13: Cross-sectional view of the pipe showing the electrodes arranged in circular

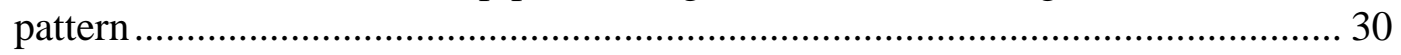

Figure 14: Electrode planes at 1, 3, 5 and 7 inches downstream of the slotted orifice

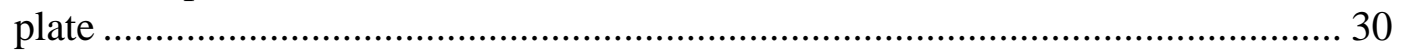

Figure 15: Electrode planes at 8, 10, 12 and 14 inches downstream from the slotted

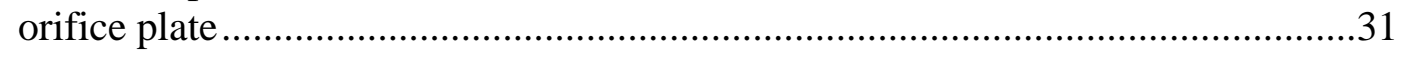

Figure 16: Pressure taps inserted upstream and downstream of the orifice plate................. 32

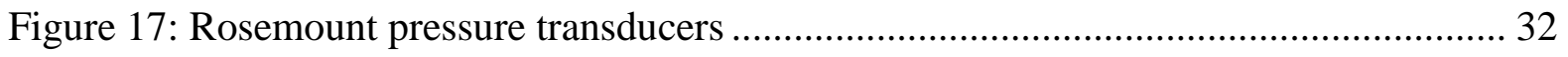

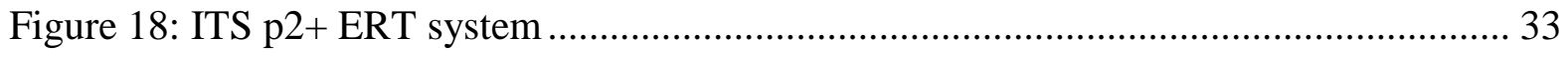

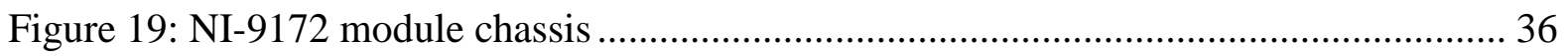

Figure 20: LabVIEW program for two-phase flow meter ............................................... 37 
Figure 21: ERT system configuration window 41

Figure 22: ERT sensor configuration window. 42

Figure 23: Individual voltage measurement at two electrode planes. 44

Figure 24: Graph displaying relative change between actual and reference measurement .... 45

Figure 25: Conductivity tomograms obtained from p2+ ERT system..... 46

Figure 26: Reconstructed conductivity tomograms at different downstream locations for a particular flow condition

Figure 27: Concentration tomograms at different downstream locations for a particular flow condition 48

Figure 28: Effect of increasing water flow rate on conductivity distribution at 20 psig and $40 \% \mathrm{GVF}$ at downstream location $\mathrm{X} / \mathrm{D}=1.5$

Figure 29: Effect of increasing GVF on conductivity distribution at 40 psig and 40 GPM at downstream location $\mathrm{X} / \mathrm{D}=2.5$.

Figure 30: Effect of increasing upstream pressure on conductivity distribution at 40 GPM and $40 \% \mathrm{GVF}$ at downstream location $\mathrm{X} / \mathrm{D}=1.5$. 52

Figure 31: Conductivity tomograms at different downstream locations for $40 \mathrm{psig}$, $20 \mathrm{GPM}$ and $70 \% \mathrm{GVF}$ 53

Figure 32: Effect of increasing water flow rate on concentration distribution for 40 psig and $40 \% \mathrm{GVF}$ at downstream location $\mathrm{X} / \mathrm{D}=1.5$

Figure 33: Effect of increasing GVF on concentration distribution at 40 psig and 20

GPM at downstream location $\mathrm{X} / \mathrm{D}=2.5$ 56

Figure 34: Concentration tomograms at different downstream locations for $40 \mathrm{psig}$, $20 \mathrm{GPM}$ and $70 \% \mathrm{GVF}$

Figure 35: Standard deviation of the concentration tomogram pixel values at different downstream locations for 20 psig \& 20 GPM....

Figure 36: Standard deviation of the concentration tomogram pixel values at different downstream locations for 60 psig \& 20 GPM.

Figure 37: Histogram displaying the number of occurrences of the standard deviation values for each downstream location 
Figure 38: Upstream flow regime map with experimental data points .62

Figure 39: Concentration tomogram for an elongated bubble flow upstream condition of 20 psig, 20 GPM and $40 \%$ GVF. 64

Figure 40: Concentration tomogram for a slugged flow upstream condition of $60 \mathrm{psig}$, $60 \mathrm{GPM}$ and $70 \% \mathrm{GVF}$

Figure 41: Relationship between Gas Void Fraction and GVF in slip and no-slip condition

Figure 42: Graph displaying the standard deviation of Gas Void Fraction and GVF for different GVF values at $40 \mathrm{psig}$ and $40 \mathrm{GPM}$...... 68

Figure 43: Plot of slip ratio V/s downstream locations for 20 psig \& 20 GPM 70

Figure 44: Conductivity Tomogram at 20 psig_20 GPM_5\% GVF...................................... 75

Figure 45: Conductivity Tomogram at 20 psig_20 GPM_10\% GVF................................... 75

Figure 46: Conductivity Tomogram at 20 psig_20 GPM_20\% GVF.................................... 76

Figure 47: Conductivity Tomogram at 20 psig_20 GPM_30\% GVF.................................... 76

Figure 48: Conductivity Tomogram at 20 psig _20 GPM_40\% GVF................................... 77

Figure 49: Conductivity Tomogram at 20 psig_20 GPM_50\% GVF.................................... 77

Figure 50: Conductivity Tomogram at 20 psig _20 GPM_60\% GVF................................... 78

Figure 51: Conductivity Tomogram at 20 psig _20 GPM_70\% GVF................................... 78

Figure 52: Conductivity Tomogram at 20 psig _40 GPM_5\% GVF....................................... 79

Figure 53: Conductivity Tomogram at 20 psig _40 GPM_10\% GVF..................................... 79

Figure 54: Conductivity Tomogram at 20 psig _40 GPM_20\% GVF.................................... 80

Figure 55: Conductivity Tomogram at 20 psig _40 GPM_30\% GVF..................................... 80

Figure 56: Conductivity Tomogram at 20 psig _40 GPM_40\% GVF.................................... 81

Figure 57: Conductivity Tomogram at 20 psig _ 40 GPM_50\% GVF.................................... 81

Figure 58: Conductivity Tomogram at 20 psig _40 GPM_60\% GVF.................................... 82 
Figure 59: Conductivity Tomogram at 20 psig _40 GPM_70\% GVF................................ 82

Figure 60: Conductivity Tomogram at 20 psig _60 GPM_5\% GVF................................. 83

Figure 61: Conductivity Tomogram at 20 psig_60 GPM_10\% GVF................................ 83

Figure 62: Conductivity Tomogram at 20 psig _60 GPM_20\% GVF................................ 84

Figure 63: Conductivity Tomogram at 20 psig _60 GPM_30\% GVF................................. 84

Figure 64: Conductivity Tomogram at 20 psig _60 GPM_40\% GVF............................... 85

Figure 65: Conductivity Tomogram at 20 psig _60 GPM_50\% GVF............................... 85

Figure 66: Conductivity Tomogram at 20 psig _60 GPM_60\% GVF................................. 86

Figure 67: Conductivity Tomogram at 20 psig _60 GPM_70\% GVF................................ 86

Figure 68: Conductivity Tomogram at 40 psig _20 GPM_5\% GVF................................. 87

Figure 69: Conductivity Tomogram at 40 psig _20 GPM_10\%_GVF................................ 87

Figure 70: Conductivity Tomogram at 40 psig _20 GPM_20\% GVF................................ 88

Figure 71: Conductivity Tomogram at 40 psig _20 GPM_30\% GVF................................. 88

Figure 72: Conductivity Tomogram at 40 psig _20 GPM_40\% GVF............................... 89

Figure 73: Conductivity Tomogram at 40 psig _20 GPM_50\% GVF............................... 89

Figure 74: Conductivity Tomogram at 40 psig _20 GPM_60\% GVF............................... 90

Figure 75: Conductivity Tomogram at 40 psig _20 GPM_70\% GVF............................... 90

Figure 76: Conductivity Tomogram at 40 psig _40 GPM_5\% GVF................................ 91

Figure 77: Conductivity Tomogram at 40 psig _40 GPM_10\% GVF............................... 91

Figure 78: Conductivity Tomogram at 40 psig _40 GPM_20\% GVF................................ 92

Figure 79: Conductivity Tomogram at 40 psig _40 GPM_30\% GVF................................ 92

Figure 80: Conductivity Tomogram at 40 psig _40 GPM_40\% GVF............................... 93 
Figure 81: Conductivity Tomogram at 40 psig _40 GPM_50\% GVF................................93

Figure 82: Conductivity Tomogram at 40 psig _40 GPM_60\% GVF................................ 94

Figure 83: Conductivity Tomogram at 40 psig _40 GPM_70\% GVF................................ 94

Figure 84: Conductivity Tomogram at 40 psig _60 GPM_5\% GVF................................. 95

Figure 85: Conductivity Tomogram at 40 psig _60 GPM_10\% GVF................................ 95

Figure 86: Conductivity Tomogram at 40 psig _60 GPM_20\% GVF................................ 96

Figure 87: Conductivity Tomogram at 40 psig _60 GPM_30\% GVF................................ 96

Figure 88: Conductivity Tomogram at 40 psig _60 GPM_40\% GVF................................ 97

Figure 89: Conductivity Tomogram at 40 psig _60 GPM_50\% GVF................................ 97

Figure 90: Conductivity Tomogram at 40 psig_60 GPM_60\% GVF................................ 98

Figure 91: Conductivity Tomogram at 40 psig _ 60 GPM_70\% GVF................................ 98

Figure 92: Conductivity Tomogram at 60 psig _20 GPM_5\% GVF................................. 99

Figure 93: Conductivity Tomogram at 60 psig _20 GPM_10\% GVF............................... 99

Figure 94: Conductivity Tomogram at 60 psig _20 GPM_20\% GVF.............................. 100

Figure 95: Conductivity Tomogram at 60 psig _20 GPM_30\% GVF............................... 100

Figure 96: Conductivity Tomogram at 60 psig _20 GPM_40\% GVF.............................. 101

Figure 97: Conductivity Tomogram at 60 psig _20 GPM_50\% GVF.............................. 101

Figure 98: Conductivity Tomogram at 60 psig _20 GPM_60\% GVF.............................. 102

Figure 99: Conductivity Tomogram at 60 psig _20 GPM_70\% GVF.............................. 102

Figure 100: Conductivity Tomogram at 60 psig _40 GPM_5\% GVF.............................. 103

Figure 101: Conductivity Tomogram at 60 psig _40 GPM_10\% GVF............................. 103

Figure 102: Conductivity Tomogram at 60 psig_40 GPM_20\% GVF............................. 104 
Figure 103: Conductivity Tomogram at 60 psig _40 GPM_30\% GVF...............................104

Figure 104: Conductivity Tomogram at 60 psig _40 GPM_40\% GVF............................. 105

Figure 105: Conductivity Tomogram at 60 psig _40 GPM_50\% GVF............................. 105

Figure 106: Conductivity Tomogram at 60 psig_40 GPM_60\% GVF............................. 106

Figure 107: Conductivity Tomogram at 60 psig _40 GPM_70\% GVF............................. 106

Figure 108: Conductivity Tomogram at 60 psig _60 GPM_5\% GVF............................... 107

Figure 109: Conductivity Tomogram at 60 psig _60 GPM_10\% GVF.............................. 107

Figure 110: Conductivity Tomogram at 60 psig_60 GPM_20\% GVF............................. 108

Figure 111: Conductivity Tomogram at 60 psig _60 GPM_30\% GVF............................. 108

Figure 112: Conductivity Tomogram at 60 psig _60 GPM_40\% GVF............................. 109

Figure 113: Conductivity Tomogram at 60 psig _60 GPM_50\% GVF............................. 109

Figure 114: Conductivity Tomogram at 60 psig _60 GPM_60\% GVF............................. 110

Figure 115: Conductivity Tomogram at 60 psig _60 GPM_70\% GVF............................. 110

Figure 116: Concentration Tomogram at 20 psig _20 GPM_5\% GVF ............................. 111

Figure 117: Concentration Tomogram at 20 psig _20 GPM_10\% GVF............................ 111

Figure 118: Concentration Tomogram at 20 psig _20 GPM_20\% GVF............................ 112

Figure 119: Concentration Tomogram at 20 psig _20 GPM_30\% GVF........................... 112

Figure 120: Concentration Tomogram at 20 psig _20 GPM_40\% GVF........................... 113

Figure 121: Concentration Tomogram at 20 psig _20 GPM_50\% GVF........................... 113

Figure 122: Concentration Tomogram at 20 psig _20 GPM_60\% GVF............................ 114

Figure 123: Concentration Tomogram at 20 psig _20 GPM_70\% GVF........................... 114

Figure 124: Concentration Tomogram at 20 psig _40 GPM_5\% GVF ............................. 115 
Figure 125: Concentration Tomogram at 20 psig _40 GPM_10\% GVF............................ 115

Figure 126: Concentration Tomogram at 20 psig _40 GPM_20\% GVF............................ 116

Figure 127: Concentration Tomogram at 20 psig _40 GPM_30\% GVF ............................ 116

Figure 128: Concentration Tomogram at 20 psig _40 GPM_40\% GVF............................ 117

Figure 129: Concentration Tomogram at 20 psig _40 GPM_50\% GVF............................ 117

Figure 130: Concentration Tomogram at 20 psig _40 GPM_60\% GVF........................... 118

Figure 131: Concentration Tomogram at 20 psig_40 GPM_70\% GVF............................. 118

Figure 132: Concentration Tomogram at 20 psig_60 GPM_5\% GVF............................. 119

Figure 133: Concentration Tomogram at 20 psig _60 GPM_10\% GVF............................ 119

Figure 134: Concentration Tomogram at 20 psig_60 GPM_20\% GVF............................. 120

Figure 135: Concentration Tomogram at 20 psig _60 GPM_30\% GVF............................ 120

Figure 136: Concentration Tomogram at 20 psig_60 GPM_40\% GVF............................ 121

Figure 137: Concentration Tomogram at 20 psig _60 GPM_50\% GVF............................ 121

Figure 138: Concentration Tomogram at 20 psig_60 GPM_60\% GVF............................ 122

Figure 139: Concentration Tomogram at 20 psig _60 GPM_70\% GVF........................... 122

Figure 140: Concentration Tomogram at 40 psig _20 GPM_5\% GVF ............................. 123

Figure 141: Concentration Tomogram at 40 psig _20 GPM_10\% GVF ............................ 123

Figure 142: Concentration Tomogram at 40 psig _20 GPM_20\% GVF ........................... 124

Figure 143: Concentration Tomogram at 40 psig _20 GPM_30\% GVF............................ 124

Figure 144: Concentration Tomogram at 40 psig _20 GPM_40\% GVF ........................... 125

Figure 145: Concentration Tomogram at 40 psig _20 GPM_50\% GVF........................... 125

Figure 146: Concentration Tomogram at 40 psig _20 GPM_60\% GVF........................... 126 
Figure 147: Concentration Tomogram at 40 psig_20 GPM_70\% GVF........................... 126

Figure 148: Concentration Tomogram at 40 psig _40 GPM_5\% GVF............................. 127

Figure 149: Concentration Tomogram at 40 psig _40 GPM_10\% GVF ............................ 127

Figure 150: Concentration Tomogram at 40 psig _40 GPM_20\% GVF........................... 128

Figure 151: Concentration Tomogram at 40 psig _40 GPM_30\% GVF............................ 128

Figure 152: Concentration Tomogram at 40 psig _40 GPM_40\% GVF........................... 129

Figure 153: Concentration Tomogram at 40 psig_40 GPM_50\% GVF........................... 129

Figure 154: Concentration Tomogram at 40 psig _40 GPM_60\% GVF............................ 130

Figure 155: Concentration Tomogram at 40 psig _40 GPM_70\% GVF ........................... 130

Figure 156: Concentration Tomogram at 40 psig _60 GPM_5\% GVF .............................. 131

Figure 157: Concentration Tomogram at 40 psig _60 GPM_10\% GVF............................ 131

Figure 158: Concentration Tomogram at 40 psig _60 GPM_20\% GVF........................... 132

Figure 159: Concentration Tomogram at 40 psig _60 GPM_30\% GVF............................ 132

Figure 160: Concentration Tomogram at 40 psig_60 GPM_40\% GVF........................... 133

Figure 161: Concentration Tomogram at 40 psig _60 GPM_50\% GVF............................ 133

Figure 162: Concentration Tomogram at 40 psig _60 GPM_60\% GVF............................ 134

Figure 163: Concentration Tomogram at 40 psig_60 GPM_70\% GVF........................... 134

Figure 164: Concentration Tomogram at 60 psig _20 GPM_5\% GVF ............................. 135

Figure 165: Concentration Tomogram at 60 psig _20 GPM_10\% GVF........................... 135

Figure 166: Concentration Tomogram at 60 psig_20 GPM_20\% GVF............................ 136

Figure 167: Concentration Tomogram at 60 psig_20 GPM_30\% GVF........................... 136

Figure 168: Concentration Tomogram at 60 psig _20 GPM_40\% GVF........................... 137 
Figure 169: Concentration Tomogram at 60 psig _20 GPM_50\% GVF............................ 137

Figure 170: Concentration Tomogram at 60 psig _20 GPM_60\% GVF ............................ 138

Figure 171: Concentration Tomogram at 60 psig _20 GPM_70\% GVF........................... 138

Figure 172: Concentration Tomogram at 60 psig _40 GPM_5\% GVF.............................. 139

Figure 173: Concentration Tomogram at 60 psig _40 GPM_10\% GVF............................ 139

Figure 174: Concentration Tomogram at 60 psig _40 GPM_20\% GVF............................ 140

Figure 175: Concentration Tomogram at 60 psig _40 GPM_30\% GVF............................. 140

Figure 176: Concentration Tomogram at 60 psig _40 GPM_40\% GVF............................ 141

Figure 177: Concentration Tomogram at 60 psig _40 GPM_50\% GVF ........................... 141

Figure 178: Concentration Tomogram at 60 psig _40 GPM_60\% GVF............................ 142

Figure 179: Concentration Tomogram at 60 psig _40 GPM_70\% GVF............................ 142

Figure 180: Concentration Tomogram at 60 psig _40GPM_5\%GVF ............................... 143

Figure 181: Concentration Tomogram at 60 psig _60 GPM_10\% GVF........................... 143

Figure 182: Concentration Tomogram at 60 psig_60 GPM_20\% GVF............................ 144

Figure 183: Concentration Tomogram at 60 psig _60 GPM_30\% GVF........................... 144

Figure 184: Concentration Tomogram at 60 psig _60 GPM_40\% GVF............................ 145

Figure 185: Concentration Tomogram at 60 psig_60 GPM_50\% GVF........................... 145

Figure 186: Concentration Tomogram at 60 psig _60 GPM_60\% GVF ........................... 146

Figure 187: Concentration Tomogram at 60 psig _60 GPM_70\% GVF........................... 146

Figure 188: Standard deviation of the concentration tomogram pixel values at $20 \mathrm{psig}$ \& 20 GPM

Figure 189: Standard deviation of the concentration tomogram pixel values at $40 \mathrm{psig}$ \& 20 GPM 
Figure 190: Standard deviation of the concentration tomogram pixel values at $60 \mathrm{psig}$ \& 20 GPM

Figure 191: Standard deviation of the concentration tomogram pixel values at 20 psig \& 40 GPM

Figure 192: Standard deviation of the concentration tomogram pixel values at $40 \mathrm{psig}$ \& 40 GPM

Figure 193: Standard deviation of the concentration tomogram pixel values at $60 \mathrm{psig}$ \& 40 GPM

Figure 194: Standard deviation of the concentration tomogram pixel values at $20 \mathrm{psig}$ \& 60 GPM

Figure 195: Standard deviation of the concentration tomogram pixel values at $40 \mathrm{psig}$ \& 60 GPM

Figure 196: Standard deviation of the concentration tomogram pixel values at $60 \mathrm{psig}$ \& 60 GPM 152

Figure 197: Slip Ratio at different downstream location for 20 GPM \& 20 psig..... 152

Figure 198: Slip Ratio at different downstream location for 20 GPM \& 40 psig..... 153

Figure 199: Slip Ratio at different downstream location for 20 GPM \& 60 psig. 153

Figure 200: Slip Ratio at different downstream location for 40 GPM \& 20 psig..... 154

Figure 201: Slip Ratio at different downstream location for 40 GPM \& 40 psig. 154

Figure 202: Slip Ratio at different downstream location for 40 GPM \& 60 psig...... 155

Figure 203: Slip Ratio at different downstream location for 60 GPM \& 20 psig....... 155

Figure 204: Slip Ratio at different downstream location for 60 GPM \& 40 psig. 156

Figure 205: Slip Ratio at different downstream location for 60 GPM \& 60 psig..... 156

Figure 206: Standard deviation of Gas Void Fraction and GVF at 20 psig \& 20 GPM...... 157

Figure 207: Standard deviation of Gas Void Fraction and GVF at 40 psig \& 20 GPM...... 157

Figure 208: Standard deviation of Gas Void Fraction and GVF at 60 psig \& 20 GPM...... 158 
Figure 209: Standard deviation of Gas Void Fraction and GVF at 20 psig \& 40 GPM........158

Figure 210: Standard deviation of Gas Void Fraction and GVF at 40 psig \& 40 GPM...... 159

Figure 211: Standard deviation of Gas Void Fraction and GVF at 60 psig \& 40 GPM...... 159

Figure 212: Standard deviation of Gas Void Fraction and GVF at 20 psig \& 60 GPM...... 160

Figure 213: Standard deviation of Gas Void Fraction and GVF at 40 psig \& 60 GPM...... 160

Figure 214: Standard deviation of Gas Void Fraction and GVF at 60 psig \& 60 GPM....... 161 


\section{LIST OF TABLES}

Page

Table 1: Different sensing methods used for Process Tomography ...................................10

Table 2: Conductivity and resistivity values of air and water at $20{ }^{\circ} \mathrm{C} \ldots \ldots \ldots \ldots \ldots \ldots \ldots \ldots \ldots \ldots \ldots . . . . . . . . .11$

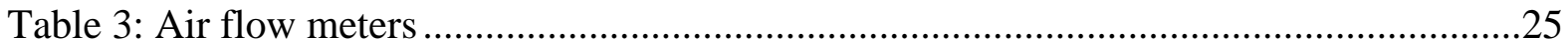

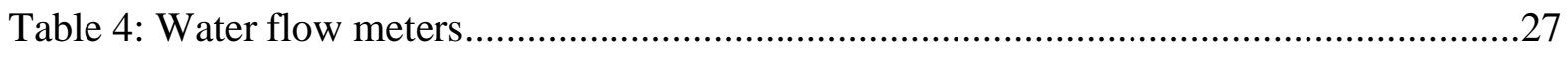

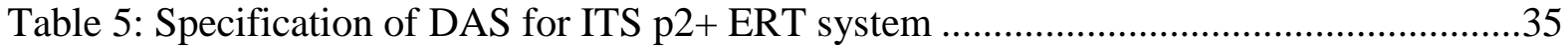

Table 6: Test Matrix for different flow conditions ........................................................40

Table 7: Characterizing elongated bubble flow and slug flow conditions..........................63 


\section{INTRODUCTION}

Two-phase flows are complex and unpredictable phenomena, commonly encountered in oil \& gas, chemical and aerospace industry. In many applications there is a need to accurately quantify the amount of mixture transported over time. On account of the complications of twophase flows, their measurements are much more difficult than that for single phase flows.

Conventionally, separators are used to separate each phase of the multiphase flow and then measure individual phases by single-phase flow metering devices. But this technique has several disadvantages such as high equipment and maintenance cost, large space requirement for separator and inaccurate measurements due to incomplete separation [1].

Advancements in flow measurement techniques and methods led to the development of multiphase flow meters which can measure the multiphase flow as such, without separating it to individual phases. This is done by making the flow homogeneous so that the two-phase flow can be treated as being analogous to a single-phase flow as the two phases are travelling at the same velocity [2]. This homogeneous model approach allows single-phase flow metering devices such as orifice plate, venturi meter, turbine meter, coriolis flow meter, etc. to measure multiphase flows and also eliminates the need of a separator and its associated costs.

Although significant advancements are made in multiphase flow meters, the accuracy and convenience of measurements are still needing to be raised. Industries are looking for multiphase flow measuring systems which are cheaper, compact in design, more accurate, easier to use and time saving [3]. Accuracy and repeatability of the flow meters are of paramount importance for the industries. Accuracy is how close a measured value is to the actual value and repeatability is the closeness of agreement between independent 
measurements taken by a single person on the same item and under the same conditions. Maximum efficiency could be attained with increased measurement accuracy of the multiphase flow meter which will provide a significant amount of cost savings. Therefore, it is necessary to find the location where the two-phase flow becomes most homogeneous in order to take accurate flow measurements.

\subsection{Orifice flow meter}

The orifice flow meter is a type of differential pressure flow meter which is based on Bernoulli's equation. One of the simplest ways to measure the fluid flow rate is by restricting the flow in a pipe by decreasing the cross-sectional area of the pipe which will result in a decrease in pressure and an increase in flow velocity.

A standard orifice plate consists of a plate with a large circular hole in the center as shown in Figure 1. Although the standard orifice plate is relatively inexpensive, the accuracy of the standard orifice plate is quite low and it is very sensitive to upstream flow condition. In order to have better flow measurement accuracy, a new type of orifice plate called the "slotted orifice plate" was designed and fabricated [4].

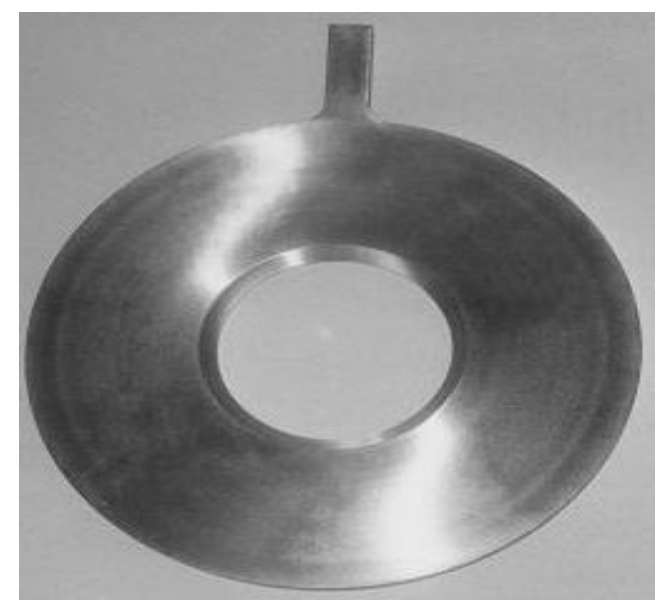

Figure 1: Standard Orifice Plate 


\subsection{Slotted orifice plate}

The slotted orifice plate was designed by Morrison [4] to measure two-phase flow. It has the same working principle as that of a standard orifice plate but its design is completely different. The slotted orifice plate has a circular array of radially aligned slots instead of a large hole in the center as shown in Figure 2. The slots disperse the flow over the entire cross-section of the pipe which results in well-distributed pipe-wall pressure and also eliminates swirl. Slots of square contour is preferred over round or beveled contour as it produces lower differential pressures [4]. The slotted orifice plate has higher accuracy and better consistency in flow measurement than the standard orifice plate [5].

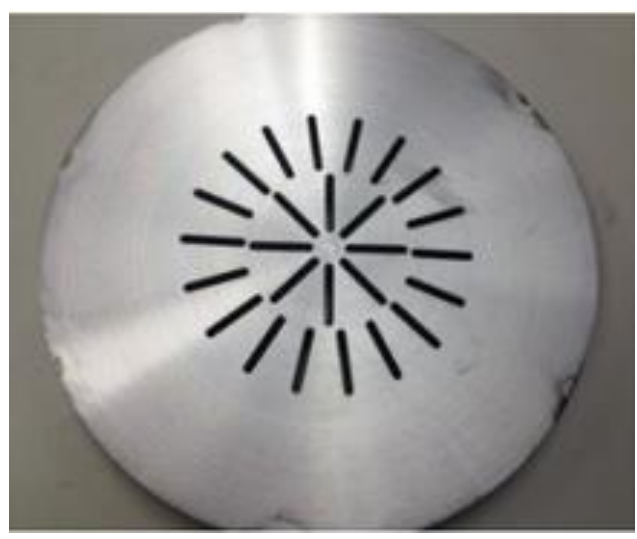

Figure 2: Slotted Orifice Plate

\subsection{Working principle of the orifice plate}

The orifice plate is mounted between two flanges in a pipe such that the plate is concentric to the pipe. The orifice plate acts as an obstruction to the flow in the pipe. The flow is assumed to be steady, uniform, frictionless and incompressible with no body forces. As the cross-section of the plate decreases, the flow is constricted and it attains radial momentum and accelerates. Due to the fluid constriction, there is a difference in the pressure upstream and downstream of the plate. The flow is separated from the downstream of the pipe as it exits the orifice plate and 
a recirculation zone is created on the backside of the orifice plate. At some distance downstream, the cross-sectional area of the flow becomes a minimum and this point of minimum flow diameter is called vena contracta. At this point the pressure is minimum and the velocity is maximum. After this point, the fluid velocity starts decreasing and the fluid reattaches with the pipe wall.

By observing the pressure drop across the orifice plate, the mass flow rate of the flow can be determined from this equation below:

$$
\dot{\mathrm{m}}=\frac{\mathrm{C}_{\mathrm{d}} \pi\left(\mathrm{D}_{\text {pipe }} \beta\right)^{2} \sqrt{2 \rho \Delta \mathrm{P}}}{4 \sqrt{1-\beta^{4}}}
$$

Where $C_{d}$ is the discharge coefficient, $D_{\text {pipe }}$ is the diameter of the pipe, $\beta$ is the ratio of area of slotted region to the cross-sectional area of the pipe, $\rho$ is the density and $\Delta \mathrm{P}$ is the differential pressure.

\subsection{Advantages of slotted orifice plate}

The slotted orifice plate provides the following advantages over the standard orifice plate:

- It has quicker pressure recovery.

- It has lower overall head loss.

- It is independent of the upstream flow condition.

- It has well-distributed pipe-wall pressure

- It eliminates swirl.

- It can be used both as a flow metering device as well as a flow conditioner.

- It has superior homogenizer performance. 


\subsection{Two-phase flows}

The term "two-phase" flow here refers to a flowing mixture of air and water. As mentioned earlier, the measurement of two-phase flows are much more complex than single-phase flows. The accurate measurement of two-phase flows is challenging because of the following reasons:

- The two phases interact with each other and hence their cross-sectional distributions vary in accordance with the flow conditions.

- Two-phase flows have different flow patterns and hence knowing the flow regime is critical for two-phase flow measurement as most of the existing two-phase flow meters are flow-regime dependent.

- Gas and liquid velocities can vary over a wide range which causes difficulties for data interpretation and thus causes significant errors in flow measurement.

In order to overcome these difficulties and facilitate accurate measurement of two-phase flow measurement, the flow has to be homogenized prior to taking measurements. By homogenizing the two-phase flow, the phase velocities become equal and hence the fluid can be treated as a single phase fluid with a mixture density given by the following equation:

$$
\rho_{\text {mix }}=\alpha \rho_{g}+(1-\alpha) \rho_{1}
$$

where $\alpha$ is the volume fraction of the gas phase, $\rho_{\mathrm{g}}$ is the gas phase density and $\rho_{1}$ is the liquid phase density.

The flow density can be measured accurately by placing an electrical impedance sensor at the homogenized location downstream of the slotted orifice plate for both conductive and nonconductive materials [6]. 


\subsection{Flow regimes}

A particular type of geometrical distribution of the components in a flow is called a flow regime or flow pattern. The flow regime depends on the flow rates of each of the phases, the pipe size, the fluid properties and the orientation of the tube. It is different for a horizontal and vertical pipe flow. In this study, we focus only on the flow regimes observed in a horizontal pipe flow. The various flow regimes observed in a horizontal pipe flow are shown below in Figure 3.

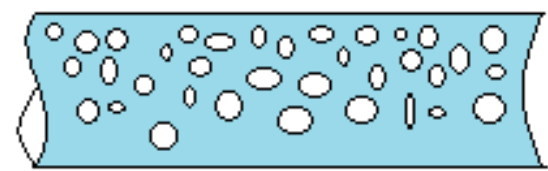

Dispersed Bubble flow

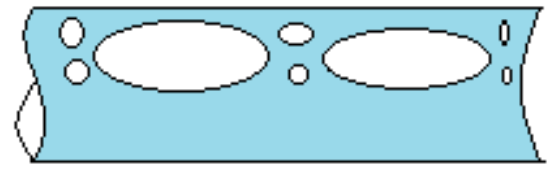

Elongated Bubble Flow

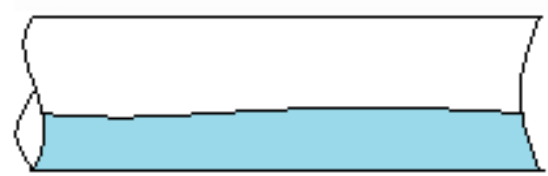

Stratified Flow

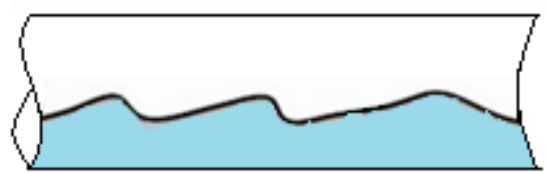

Wavy Flow

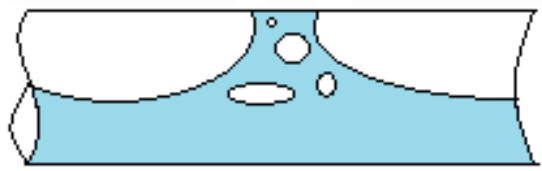

Slug Flow

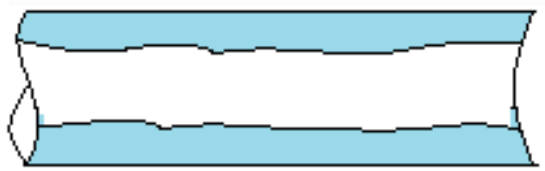

Annular Flow

Figure 3: Sketches of flow regimes for horizontal pipe flow [7] 
The characteristics of each of the flow regime are discussed below:

Dispersed Flow: The liquid flow rate is high enough to break up the gas into bubbles such that the gas phase appears as distinct bubbles in a continuous liquid phase. Due to buoyancy effects, the bubbles tend to rise to the top of the flow in the pipe.

Elongated Bubble Flow: The liquid flow rate decreases such that the size of the bubbles elongates resulting in the formation of plugs, which tend to remain at the top of the flow channel due to buoyancy force. This flow regime is also called Plug Flow.

Stratified Flow: The liquid and gas velocities are relatively slow here. The liquid phase is along the bottom of the tube while the gas phase is at the top of the tube due to gravity. There are no waves at the phase boundary.

Wavy Flow: It is similar to the stratified flow but waves are present at the phase boundary here. This is because the gas velocity increases and the shear forces of the gas flow over the liquid results in the formation of waves.

Slug Flow: The amplitude of the waves increases as the liquid flow rate increases and the gas velocities are much higher. This region is characterized by large pressure fluctuations and liquid flow rate fluctuations. Occasionally the waves are so intense that they occupy the whole cross section of the tube. The liquid and gas phases are separate here.

Annular Flow: The gas velocity is very large here. The liquid forms a continuous annular film around the wall of the pipe and the gas flows in the central core. The interface between the liquid annulus and the vapor core is distributed by small amplitude waves and the core region may contain entrained liquid droplets. 
Mist flow: At very high gas velocities, all the liquid may be stripped from the wall and be entrained as small droplets in the now continuous gas phase.

The horizontal two-phase flow regime chart is plotted with the superficial velocity of gas $\left(\mathrm{V}_{\mathrm{SG}}\right)$ along the $\mathrm{x}$-axis and the superficial velocity of liquid $\left(\mathrm{V}_{\mathrm{SL}}\right)$ along the $\mathrm{y}$-axis as shown below in Figure 4. A chart like this is dimensional so it will be valid only for a specific pipe size, pressure and specific fluids.

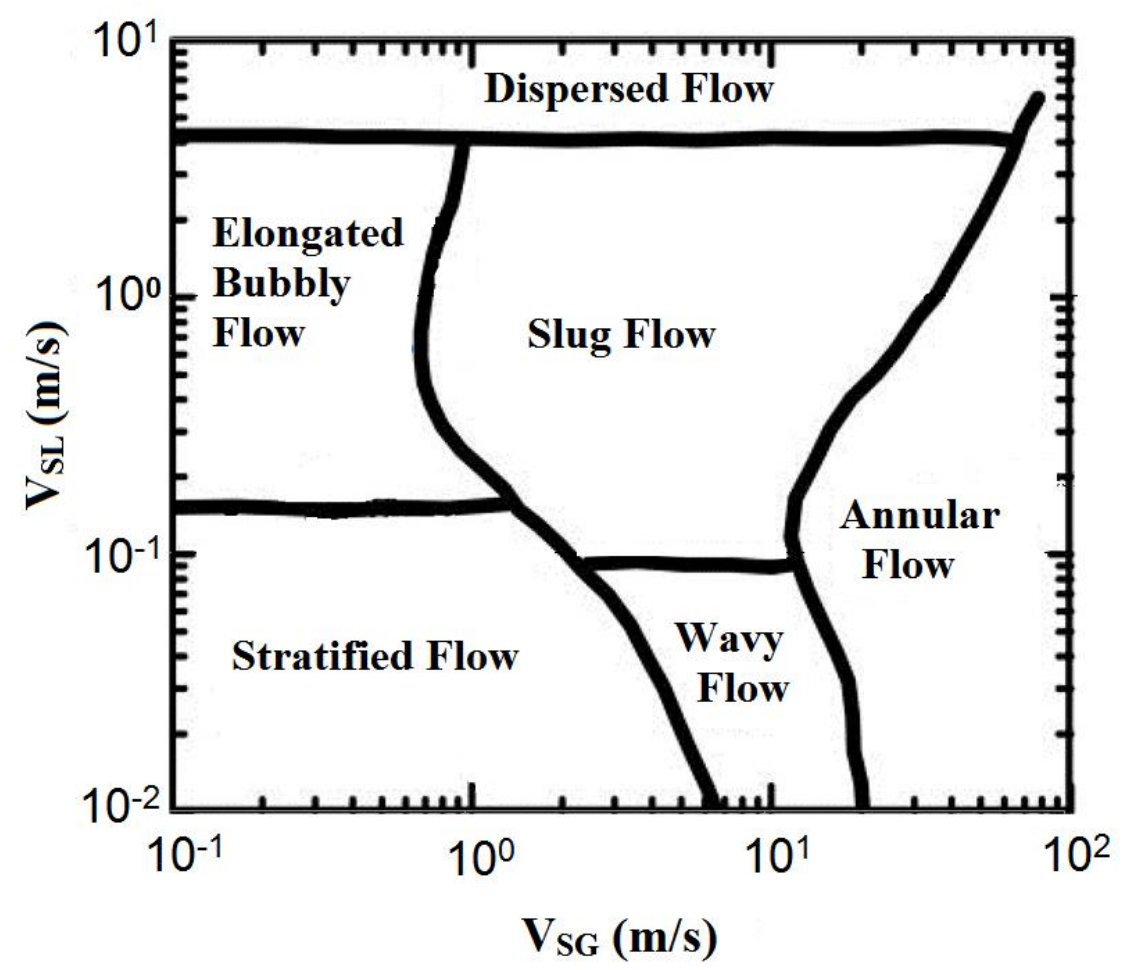

Figure 4: Horizontal Flow Regime Chart [8]

The flow regime boundaries are not distinctive lines but poorly defined transition zones. This is because the two-phase transitions are unpredictable and they may depend on minor features of the flow such as the roughness of the walls or the entrance conditions. Also it is important to realize that there may exist no unique flow pattern for a given fluid for a given flow rate. 
There may be several possible flow patterns whose occurrence may depend on the initial conditions, specifically on the manner in which the multiphase flow is generated.

\subsection{Process Tomography}

Process Tomography (PT) is of great interest in the field of two-phase flow measurement in the past few decades. It involves using a tomographing imaging method to manipulate data from remote sensors in order to obtain precise quantitative information from inaccessible locations. Flow regime, conductivity distribution, concentration distribution, vector velocity and other important information about the two-phase flow in process vessels and pipelines can be determined from tomographic images.

The key requirements for a tomographic technique is that it should be non-invasive (i.e. it should not necessitate rupture of the walls) and non-intrusive (i.e. it should not disturb the nature of the process being examined). Most of the industries rely on non-intrusive tomographic techniques so that flow inside the vessel or pipeline is not disturbed while recording flow measurements.

Sensors are the heart of the tomographic technique. Different sensing methods are available and the choice of sensing system is determined largely by the nature of components contained in the vessel or pipeline, the information to be collected, the process environment and the size of the equipment. Different sensors used for process tomography [9] is shown below in Table 1 .

Among the different types of PT, electrical PT which includes Electrical Resistance Tomography (ERT) and Electrical Capacitance Tomography (ECT) is very popular and is widely used because it is cheap, rapid, convenient and non-intrusive and it is safer compared 
to the other methods based on electromagnetic radiation, ultrasound waves and nuclear magnetic resonance. ERT is used for conductive phase systems (such as water-based systems) whereas ECT is used for non-conductive phase systems (such as oil/gas based systems).

\begin{tabular}{|c|c|c|c|}
\hline Principle & Resolution & Practical Realization & Comments \\
\hline \multirow[t]{4}{*}{$\begin{array}{l}\text { Electromagnetic } \\
\text { Radiation }\end{array}$} & \multirow[t]{4}{*}{1} & Optical & $\begin{array}{l}\text { Fast } \\
\text { Optical access required }\end{array}$ \\
\hline & & X-ray and gamma-ray & $\begin{array}{l}\text { Slow } \\
\text { Radiation Containment }\end{array}$ \\
\hline & & Positron Emission & $\begin{array}{l}\text { Labeled Particle } \\
\text { Not on-line }\end{array}$ \\
\hline & & Magnetic Resonance & $\begin{array}{l}\text { Fast } \\
\text { Expensive for large size }\end{array}$ \\
\hline Acoustics & 3 & Ultrasonic & $\begin{array}{l}\text { Sonic speed limitation } \\
\text { Complex to use }\end{array}$ \\
\hline $\begin{array}{l}\text { Measurement of } \\
\text { Electrical properties }\end{array}$ & 10 & $\begin{array}{l}\text { Capacitive, } \\
\text { Conductive, } \\
\text { Inductive }\end{array}$ & $\begin{array}{l}\text { Fast } \\
\text { Low cost } \\
\text { Suitable for small and large } \\
\text { sizes }\end{array}$ \\
\hline
\end{tabular}

Table 1: Different sensing methods used for Process Tomography [9]

\subsection{Electrical Resistance Tomography (ERT)}

ERT is widely used in various industrial investigations for visualization of the concentration profiles and characteristics of the fluid dynamics in gas-liquid two-phase systems. It is based on the principle that conductivity of different medium varies as shown in Table 2. It is useful in determining the component distribution in a flow by using the conductivity distribution of 
the sensing field. In this technique, multiple electrodes are arranged around the boundary of the pipe and electrical contact is established between the electrodes and the fluid flowing inside the pipe. This technique requires the main continuous phase to be at least slightly conductive and the dispersed phase to be of different conductivity value.

\begin{tabular}{|l|c|c|}
\hline \multicolumn{1}{|c|}{ Medium } & $\begin{array}{c}\text { Resistivity at } 20^{\circ} \mathrm{C} \\
(\Omega . \mathrm{m})\end{array}$ & $\begin{array}{c}\text { Conductivity at } 20^{\circ} \mathrm{C} \\
(\mathrm{S} / \mathrm{m})\end{array}$ \\
\hline Air & $1.3 \times 10^{16}-3.3 \times 10^{16}$ & $3 \times 10^{-15}-8 \times 10^{-15}$ \\
Sea Water & 0.2 & 4.8 \\
Drinking Water & $2 \times 10^{1}-2 \times 10^{3}$ & $5 \times 10^{-4}-5 \times 10^{-2}$ \\
\hline
\end{tabular}

Table 2: Conductivity and resistivity values of air and water at $20^{\circ} \mathrm{C}$ [10]

In operation, an ERT system first injects exciting current between a pair of electrodes and measures the resultant voltage differences between the other pairs of electrodes as shown in Figure 5. Then the current is applied through the next pair of electrodes and the voltage measurements repeated until all the independent measurements have been made. A 16electrode sensor delivers 104 individual voltage measurements in approximately 25 ms [11].

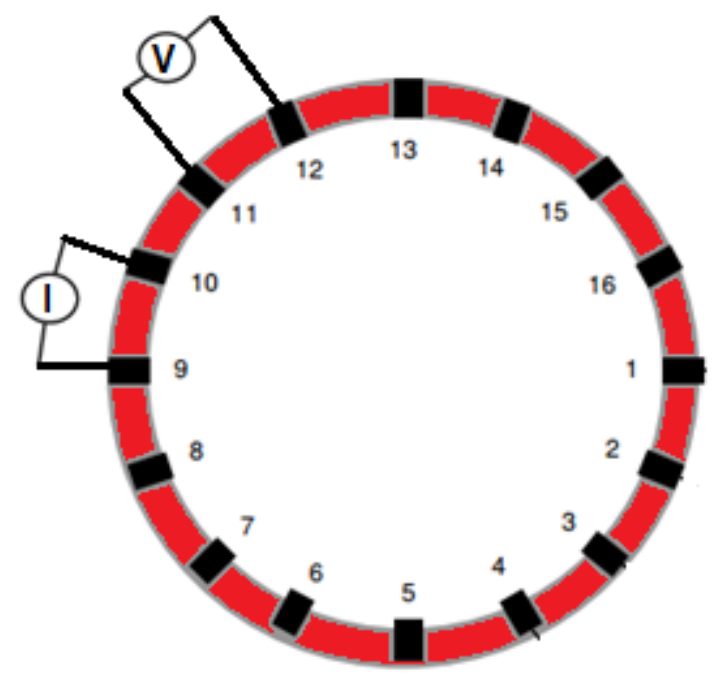

Figure 5: Measurement strategy of ERT system [11] 


\subsection{Image reconstruction}

Following the acquisition of data from the boundary of the pipe, an appropriate image reconstruction algorithm is used to process the data and reconstruct an image containing information on the cross-sectional distribution of electrical conductivity of the components within the measurement plane.

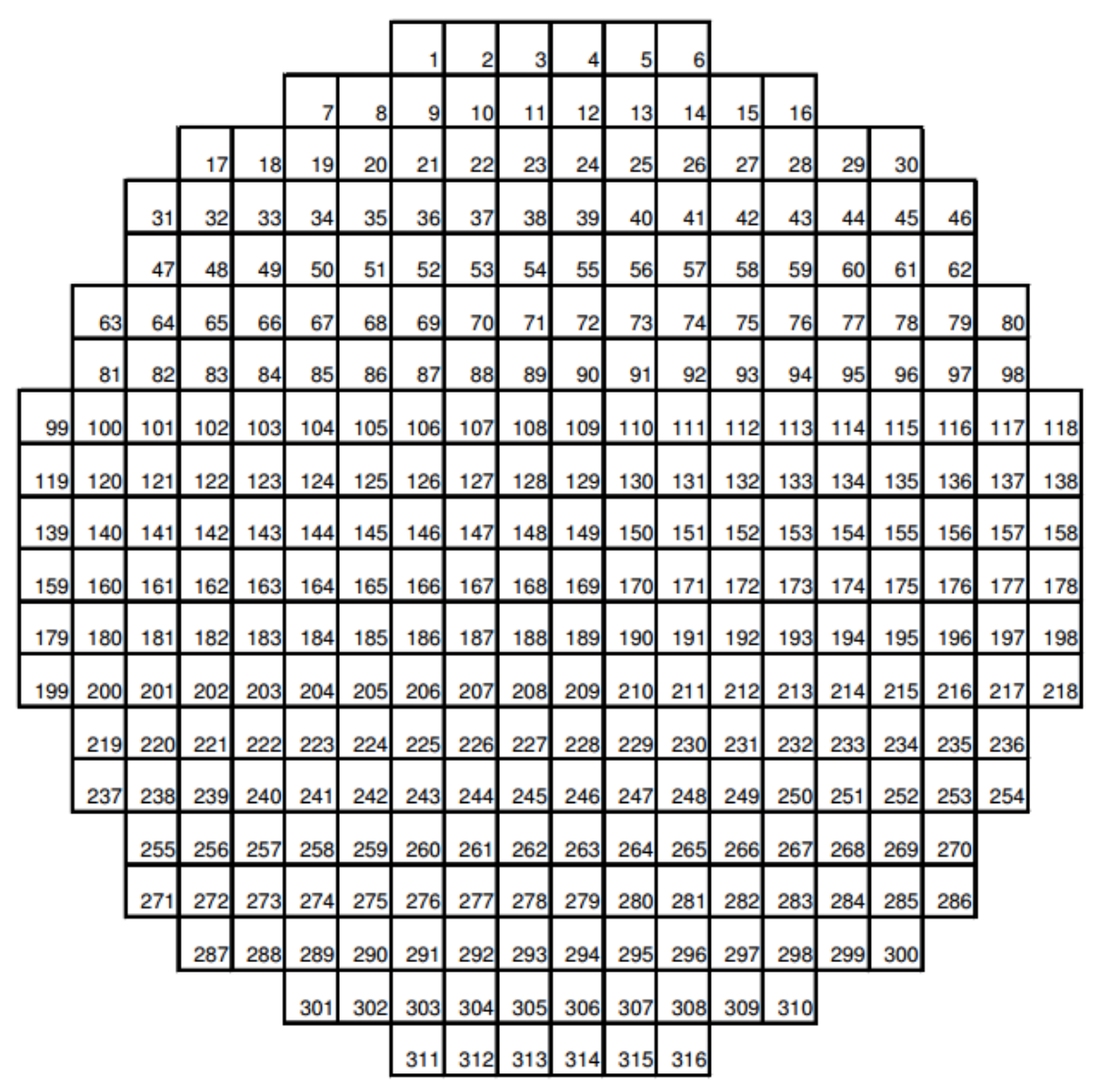

Figure 6: Image reconstruction grid [11]

The circular image is constructed using 316 pixels as shown in Figure 6 . The choice of image reconstruction algorithm is a trade-off between the accuracy of image and the time required for reconstruction. Industries are demanding fast image reconstruction algorithms that can be used for real time imaging of fast-dynamic processes such as multiphase flows. 


\section{LITERATURE REVIEW}

Macek [5] compared the performance of a slotted orifice plate to that of a standard orifice plate. By investigating on the pipe-wall pressure distributions and the velocity profiles associated with the flow through both the plates at different flow conditions, it was determined that the slotted orifice plate was superior to the standard orifice plate in flow measurement. The slotted orifice plate showed better consistency in flow measurement, less sensitivity to upstream velocity profiles and swirl, lower overall head loss and provided quicker pressure recovery downstream of the plate.

Sparks [12] studied the response characteristics of slotted orifice plate to the standard orifice plate and a V-cone for liquid-gas two-phase flows at different flow conditions. It was experimentally verified that the slotted orifice plate mixes the two-phase flow better than the standard orifice plate and a V-cone. The slotted orifice plate was independent of the upstream flow condition and created a uniform, turbulent mixing downstream. The standard orifice plate and V-cone did not exhibit a uniform, turbulent mixing and showed poor homogenizer performance. Hence the slotted orifice plate was a better flow homogenizer than the standard orifice plate and a V-cone.

Muralidharan [13] tested the performance of slotted orifice plate to horizontal two-phase flow. The performance of a slotted orifice plate flow meter was compared to that of a standard orifice plate and a venturi meter. Also the effect of varying the upstream flow quality on the differential pressure and the coefficient of discharge was evaluated. It was observed that with an increase in upstream flow quality there was a decrease in differential pressure and a decrease in coefficient of discharge when the differential pressure was maintained constant. It was also determined that the slotted orifice plate showed better repeatability compared to the standard 
orifice plate and the performance of the slotted orifice plate (with beta ratio 0.43 and 0.467 ) was unaffected by the location of the plates. The response of the venturi was unpredictable even though it showed good repeatability. Because of several advantages of the slotted orifice plate such as lower differential pressures, lower sensitivity to upstream flow conditions, higher coefficient of discharge values, better consistency, higher accuracy and better repeatability than the standard orifice plate, it was concluded that the slotted orifice plate is a better choice for metering horizontal liquid-gas two- phase flows.

Razak [14] used Electrical Resistance Tomography (ERT) as the imaging technique to study flow parameters such as phase holdup and individual phase velocities in a liquid-solid twophase flow. Using ERT, conductivity distribution was obtained which was then converted to concentration distribution based on Maxwell's equation and then the local solid holdup was measured. Local solid holdup was also measured by using optical fiber probe to compare and verify the result obtained from ERT. It was observed that there was a good agreement between the data obtained from the two methods. ERT, being a non-intrusive method, was used to measure time-based average, hence producing a more specific and accurate data compared to optic fiber. Optic fiber, being intrusive in nature, can only be used to measure at a particular location. Hence, unlike the ERT, the phase hold-up cannot be measured at all radial position simultaneously by the optic fiber which can introduce minor measurement errors. Thus it was concluded that ERT is a better measurement technique than optic fiber in the flow measurement of two-phase flow.

Bolton [15] used ERT to visualize the flow pattern and distribution of components inside a radial flow fixed bed reactor for improving the reactor performance with lower pressure drop. The information yielded by ERT was suitable for validation using Computational Fluid 
Dynamics (CFD). The ERT measurement technique was chosen for comparison with the CFD results because data could be collected with sufficient temporal resolution to facilitate the tracking of an injection current and reconstruction of the flow patterns. Thus the tomographic images of conductivity distribution obtained by using the ERT system can quantitatively demonstrate the flow pattern through the reactor and also effectively validate the CFD calculations.

Deng [16] employed fusions of electrical tomography methods to improve the accuracy of twophase flow measurements. The shortcomings of one method was compensated by the other methods so that accurate measurements were obtained. ERT can be used to measure the phase size, void fractions and flow regime parameters but the dynamic fluid parameters such as average velocity measurement cannot be obtained. In order to measure the average velocity measurement, electromagnetic (EM) flow meter is used. Thus by the fusion of ERT with EM flow meter, it was made possible to measure gas-liquid two-phase flows. Similarly Electrical Capacitance Tomography (ECT) can be used to measure non-conductive substances whereas ERT can be used to measure conductive substances. By the fusion of ERT with ECT, it is possible to measure oil-gas-liquid multi-phase flows.

Jin [17] carried out experiments to determine the gas holdup in concurrent gas-liquid twophase flow using ERT system and differential pressure method. The local gas volume fraction can be determined from the conductivity data obtained using ERT system using the Maxwell equation. From the differential pressure method, the axial holdup was measured. Experimental results showed that at the homogeneous flow regime, the results obtained from the differential pressure method and ERT method were similar. But in transition region and heterogeneous flow regime, the ERT method was more accurate. 
Triplett [18] experimentally investigated the void fraction and two-phase frictional pressure drop in micro-channels. Gas and liquid superficial velocities were varied in the range of 0.02$80 \mathrm{~m} / \mathrm{s}$ and $0.02-8 \mathrm{~m} / \mathrm{s}$ respectively and the void fractions were calculated. It was found that the homogeneous flow model provided the best prediction of the experimental void fractions in bubble and slug flow regions and two-phase frictional pressure drop. For annular flow, the homogeneous mixture model significantly over-predicted the void fractions and two-phase frictional pressure drop. 


\section{THEORY}

\subsection{Density of air}

The density of air is dependent on pressure when the temperature is maintained constant and is given by the ideal-gas law as follows:

$$
\rho_{\text {air }}=\frac{\mathrm{P}_{\text {air }}}{\mathrm{R} \mathrm{T}_{\text {air }}}
$$

where $\mathrm{P}_{\text {air }}$ and $\mathrm{T}_{\text {air }}$ are the pressure and temperature of air respectively and $\mathrm{R}$ is the specific gas constant. The density of water can be assumed to be constant at different pressures.

\subsection{Mass flow rate of air}

The air mass flow rate is the mass of air flowing through the cross-section of the conduit in unit time. It is calculated by using the following equation:

$$
\dot{\mathrm{m}}_{\mathrm{air}}=\rho_{\text {air }} \dot{\mathrm{Q}}_{\mathrm{air}}
$$

where $\rho_{\text {air }}$ is the density of air and $\dot{Q}_{\text {air }}$ is the volumetric flow rate of air.

\subsection{Beta ratio}

The beta ratio of the slotted orifice plate is defined as the square root of the ratio of the area of the slotted region in the orifice plate to the cross-sectional area of the pipe.

$$
\beta=\sqrt{\frac{\mathrm{A}_{\text {slots }}}{\mathrm{A}_{\text {pipe }}}}
$$

where $\mathrm{A}_{\text {slots }}$ is the area of the slotted region and $\mathrm{A}_{\mathrm{pipe}}$ is the cross-sectional area of the pipe. 


\subsection{Gas Volume Fraction (GVF)}

GVF is the volumetric flow rate of gas relative to the total volumetric flow rate at the pressure and temperature prevailing at that location. It is usually expressed as a percentage.

$$
\mathrm{GVF}=\frac{\dot{\mathrm{Q}}_{\text {gas }}}{\dot{\mathrm{Q}}_{\text {total }}}
$$

where $\dot{\mathrm{Q}}_{\mathrm{gas}}$ is the volumetric flow rate of gas and $\dot{\mathrm{Q}}_{\text {total }}$ is the total volumetric flow rate.

\subsection{Conductivity}

Conductivity is defined as the ability of the medium to allow electric current to pass through it. It is the reciprocal of resistivity and it is expressed in $\mathrm{mS} / \mathrm{cm}$.

\subsection{Concentration}

Concentration is the ratio of area occupied by the dispersed phase in the two-phase flow to the total cross-sectional area of the pipe and is denoted by $\alpha$.

$$
\alpha=\frac{A_{\text {dispersed }}}{A_{\text {total }}}
$$

\subsection{Maxwell's equation}

The conductivity data can be converted into concentration data by the Maxwell's equation:

$$
\alpha=\frac{2 \sigma_{1}+\sigma_{2}-2 \sigma_{\mathrm{mc}}-\frac{\sigma_{\mathrm{mc}} \sigma_{2}}{\sigma_{1}}}{\sigma_{\mathrm{mc}}-\frac{\sigma_{2}}{\sigma_{1}} \sigma_{\mathrm{mc}}+2\left(\sigma_{1}-\sigma_{2}\right)}
$$


where $\alpha$ is the volume fraction of the dispersed phase, $\sigma_{1}$ is the conductivity of the continuous phase $(\mathrm{mS} / \mathrm{cm}), \sigma_{2}$ is the conductivity of the dispersed phase $(\mathrm{mS} / \mathrm{cm})$ and $\sigma_{\mathrm{mc}}$ is the reconstructed measured conductivity $(\mathrm{mS} / \mathrm{cm})$.

\subsection{Simplified Maxwell's equation}

For a non-conductive dispersed phase $\left(\sigma_{2}=0\right)$, the Maxwell's equation can be simplified to:

$$
\alpha=\frac{2 \sigma_{1}-2 \sigma_{\mathrm{mc}}}{\sigma_{\mathrm{mc}}+2 \sigma_{1}}
$$

\subsection{Flow regime}

A particular type of geometrical distribution of the components in a flow is called a flow regime or flow pattern.

\subsection{Homogeneous flow}

The flow condition where the two-phases are uniformly distributed over the entire crosssection of the pipe; i.e. the composition is the same at all points in the cross-section and the liquid and gas phase velocities are equal.

\subsection{Slip}

Slip is the term used to express a flow condition when there is relative difference between the phase velocities. It is quantitatively expressed by the phase velocity difference between the phases. If there is no relative change in the phase velocities, it is termed as no-slip condition.

\subsection{Slip ratio}

Slip ratio is the ratio between the two phase velocities. It is calculated by dividing the GVF with the Gas Void Fraction: 


$$
\text { Slip Ratio }=\frac{(1-\alpha)}{\alpha} \frac{G V F}{(1-G V F)}
$$

\subsection{Superficial phase velocity}

Superficial phase velocity is defined as the flow velocity of one phase of a multiphase flow, assuming that the phase occupies the whole pipe by itself. The superficial velocity of air $\left(\mathrm{V}_{\mathrm{SG}}\right)$ and water $\left(\mathrm{V}_{\mathrm{SL}}\right)$ is calculated by the following equation:

$$
\begin{gathered}
\mathrm{V}_{\mathrm{SG}}=\frac{4 \dot{\mathrm{m}}_{\text {air }}}{\pi \mathrm{D}^{2} \rho_{\text {air }}} \\
\mathrm{V}_{\mathrm{SL}}=\frac{4 \dot{\mathrm{m}}_{\text {water }}}{\pi \mathrm{D}^{2} \rho_{\text {water }}}
\end{gathered}
$$

where $\dot{\mathrm{m}}_{\text {air }}$ and $\dot{\mathrm{m}}_{\text {water }}$ is the mass flow rate of air and water respectively, $\mathrm{D}$ is the diameter of pipe and $\rho_{\text {air }}$ and $\rho_{\text {water }}$ are the density of air and water respectively.

\subsection{Gas void fraction}

The Gas Void Fraction is defined as the ratio of the cross-sectional area of the pipe occupied by the gas phase to the liquid phase. It is one of the most important parameter to obtain the relative velocity of the two phases.

$$
\text { Void Fraction }=\frac{\mathrm{A}_{\text {gas }}}{\mathrm{A}_{\text {liquid }}}=\frac{\alpha}{1-\alpha}
$$

where $\alpha$ is the concentration measured by using the Maxwell's equation. 


\section{OBJECTIVE}

Gas-liquid two-phase flow is commonly encountered in fluid transportation industry. Measuring accurately the individual flow rate of the gas and liquid on-line is still a great challenge. Conventional method of separating the phases and measuring individual phases by using single-phase flow metering devices has several disadvantages. The separation efficiency significantly affects the metering uncertainty and also the separators are bulky and heavy which require large space and impose high maintenance cost. Hence multiphase flow meters which measures multiphase flows as such, without separating individual phases, is highly desirable.

By using the homogeneous model approach, the two-phase flow can be treated as a single phase flow as the two phases travel at the same velocity at the fully homogenized location. Hence single-phase flow metering devices such as orifice plate, venturi meter, turbine meter, coriolis flow meter, vortex shedding flow meter, etc. can be employed to measure the twophase flows.

It has been experimentally proven that a slotted orifice plate is a better flow homogenizer than a standard orifice plate and a V-cone. The slotted orifice plate has radially aligned slots which disperses the flow over the entire cross-section of the pipe resulting in well-distributed pipewall pressure and eliminates swirl. It also offers quicker pressure recovery downstream and a lower overall head loss compared to a standard orifice plate. Hence the slotted orifice plate is a superior flow homogenizer but the location downstream of the slotted orifice plate where the two-phase flow becomes most homogeneous is still not determined. Knowing the most homogenized location is very important as the sensors (such as the electrical impedance sensor) can be placed at that location in order to take accurate measurements. Hence the objective of 
this study is to determine the optimum location downstream of the slotted orifice plate where the two-phase flow becomes most homogeneous for different flow conditions.

In order to meet this objective, Electrical Resistance Tomography (ERT) measurement technique is employed. In this method, electrical contact is established between the electrodes arranged around the pipe and the flow inside the pipe. Sixteen electrodes are arranged in a circular pattern around the pipe at eight different locations downstream of the slotted orifice plate. By injecting current through the electrodes at different electrode planes and taking the corresponding voltage measurements, the conductivity distribution of the two-phase flow inside the pipe at different downstream locations is obtained. The conductivity data is then converted into concentration data by using the Maxwell's equation. Thus the concentration distribution of components in the two-phase flow for different flow conditions at different downstream locations is obtained.

By analyzing the concentration tomograms at different downstream locations, the location where the two-phase flow becomes most homogenized can be determined. At the most homogeneous location, the concentration distribution of the components in the two-phase flow will be uniformly distributed throughout the entire cross-section of the concentration tomogram and hence the standard deviation of the pixels in the tomogram will be the lowest at the most homogeneous location. Whereas at the heterogeneous or non-homogeneous location, the distribution will be non-uniform with low concentration values dominating in some zones and high concentration values dominating in other zones of the tomogram and hence the standard deviation of the pixels will be high. Thus by calculating the standard deviation of pixels in the concentration tomograms, the location where the two-phase flow becomes most homogeneous can be determined for different flow conditions. 
Also by analyzing the concentration tomograms for different upstream flow conditions, the different flow regimes exhibited by the two-phase flow downstream of the slotted orifice plate can be determined which is useful in studying the flow regime transition downstream of the slotted orifice plate and the dependence of the slotted orifice plate on the upstream flow conditions.

Moreover, possible metering errors in the two-phase flow measurements can be determined by calculating the gas void fraction for each flow condition and comparing it to the gas volume fraction $(\mathrm{GVF})$ measured by the flow meters. At the homogeneous condition, the gas and liquid phases are moving at the same velocity and hence there is no slip. Hence the gas void fraction will be equal to the gas volume fraction at the no-slip condition. But at the slip condition, there is a difference in phase velocities as the gas phase being lighter moves much faster than the liquid phase. Also the liquid phase tends to accumulate in the pipe segments and so the area occupied by the gas phase is much lesser than the area occupied by the liquid phase. Hence the gas void fraction is lesser than the gas volume fraction at the slip condition. The ratio of GVF and gas void fraction gives the slip ratio. Hence if the slip ratio is high, then there will be buildup of gas or liquid in that section and the local gas void fraction measured will be different from the GVF, leading to possible metering errors in the two-phase flow measurements. 


\section{EXPERIMENTAL SETUP}

This section describes the experimental hardware, test section, instrumentation, data acquisition system and software used to carry out the experiment at the Turbomachinery Laboratory, College Station. The Piping \& Instrumentation Diagram (P\&ID) for the test loop is shown in Figure 7.

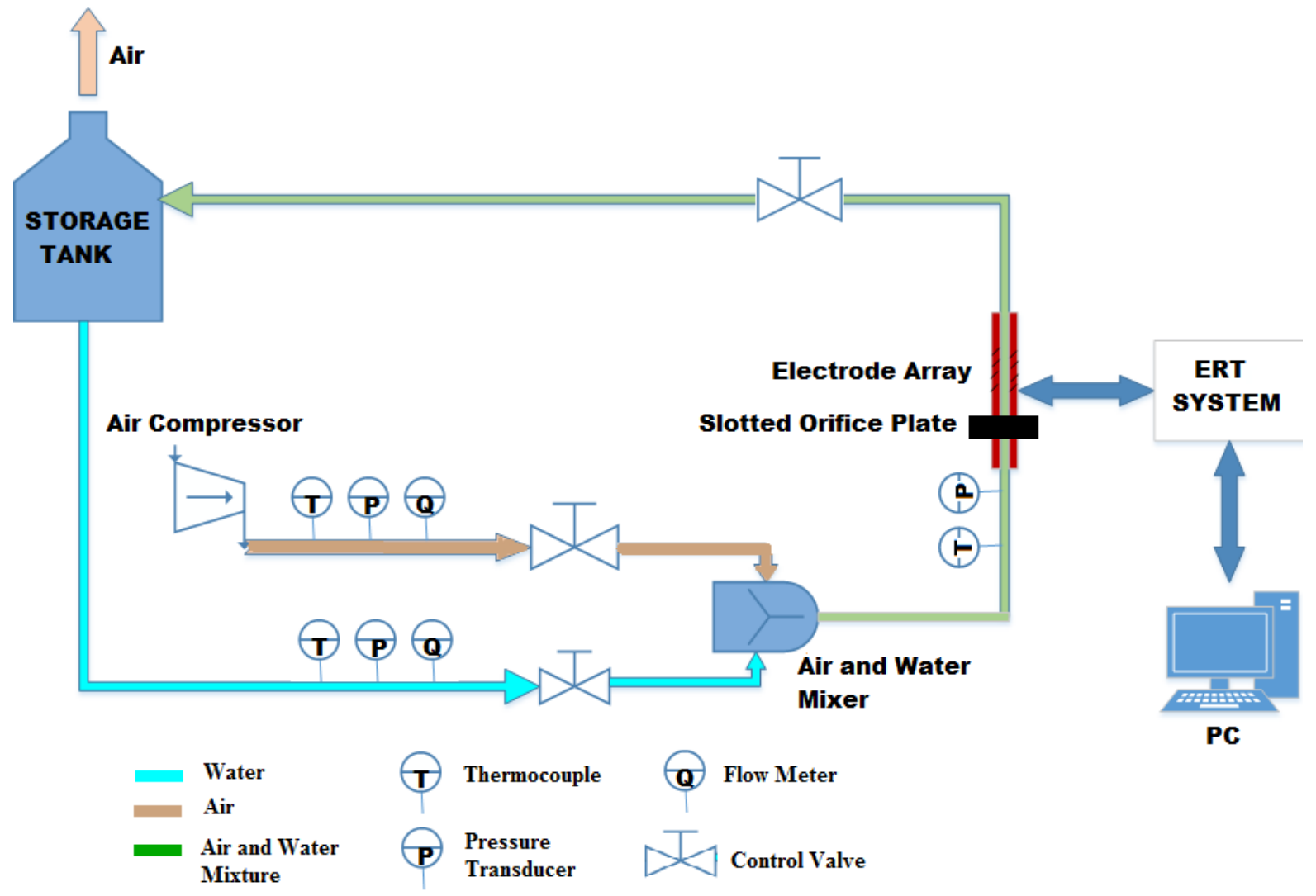

Figure 7: Test Loop P\&ID

As shown in the test loop, water and air are metered separately and are mixed together at a distance of approximately 50 inches before the inlet of the test section. After passing through the test section, water is returned back to the storage tank whereas air is released to the atmosphere. 


\subsection{Air metering section}

Air at about 120 psig is supplied by Ingersoll-Rand air compressors located outside the test cell. A pair of desiccant dryers filters and dries the compressed air to a dew point temperature of $-40^{\circ} \mathrm{F}$. The compressed air is brought into the test cell through a 4-inch stainless steel pipe. Then the volumetric flow rate, pressure and temperature of the air is measured prior to mixing with water. Two turbine flow meters and a coriolis flow meter with different measurement range are used to measure the volumetric flow rate of air accurately. The details of the flow meters with their measurement range and accuracy is listed in the Table 3 below.

\begin{tabular}{|l|l|l|l|l|}
\hline Type & Manufacturer & Accuracy & Repeatability & Range \\
\hline \multirow{3}{*}{ Air flow meters } & $\begin{array}{l}\text { Micro Motion } \\
\text { CMFS015M }\end{array}$ & $0.35 \%$ & $0.2 \%$ & $0-2$ ACFM \\
\cline { 2 - 5 } & Omega FTB-935 & $1 \%$ & $0.25 \%$ & $1-10$ ACFM \\
\cline { 2 - 5 } & Omega FTB-938 & $1 \%$ & $0.25 \%$ & 8 -250ACFM \\
\hline
\end{tabular}

Table 3: Air flow meters

The air pressure is measured with a Rosemount Model 3051C SMART pressure transducer which has a range of 10 to $1035 \mathrm{kPa}$ and an accuracy of $0.075 \%$. Air temperature is measured by an Omega T-type thermocouple which has an accuracy of $1{ }^{\circ} \mathrm{F}$.

Once the flow rate, pressure and temperature measurements are taken, then the air flows through a control valve. Masoneilan Dresser Varimax 3000 Series shown in Figure 8, is an electro-pneumatic control valve, which is used to regulate the air flow rate and the air pressure upstream of the test section. 


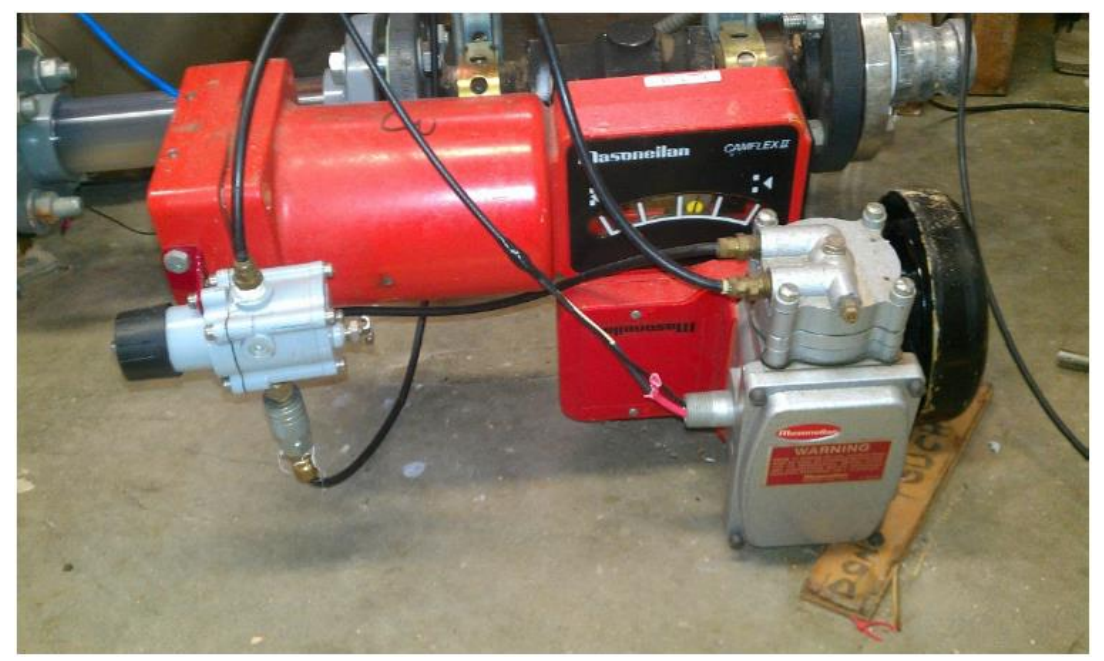

Figure 8: Masoneilan Dresser Varimax 3000 Series Control Valve

A Proportional-Integral-Derivative (PID) controller in Labview is used to control the electropneumatic control valve by sending a current signal in the range of $4-20 \mathrm{~mA}$, where $4 \mathrm{~mA}$ adjusts the valve to the fully closed position and $20 \mathrm{~mA}$ adjusts it to the fully opened position. A check valve is also present in the control valve to prevent the back flow. The air manifold used in the experiment is shown in Figure 9.

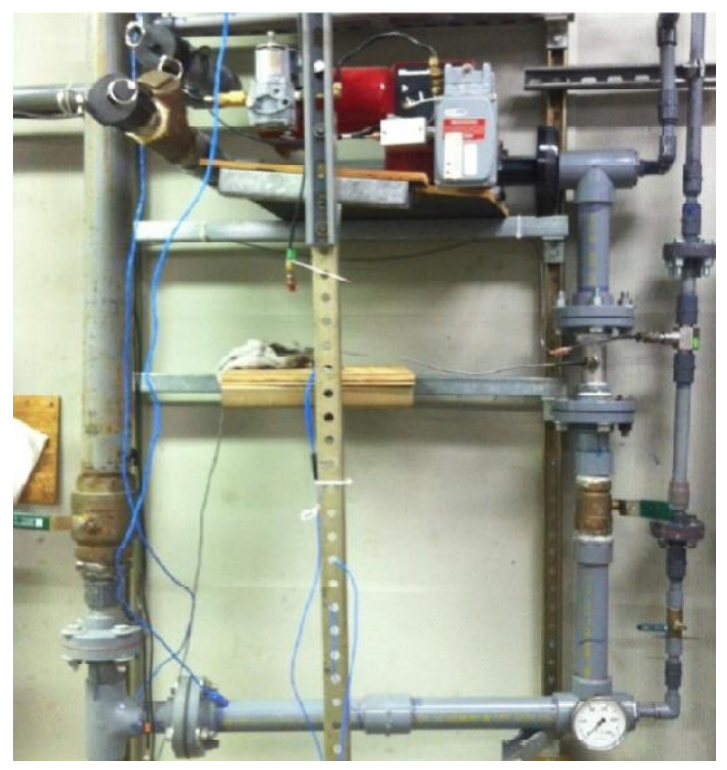

Figure 9: Air Manifold 


\subsection{Water metering section}

Water is supplied to the test section from a 5000 gallon storage tank by using a 100 GPM centrifugal pump with a maximum pressure of 120 psig. A pressure relief valve is used for adjusting the pump pressure and to recirculate the water back to the tank in case of excessive pressure build-up on the downstream side of the pump, thereby preventing pump damage. Similar to the air metering section, three Masoneilan model electro-pneumatic valve are present to control the flow rate of water through the flow meters. Three independent turbine flow meters were used to measure the water flow rate. The details of the flow meter with their measuring range is listed in the Table 4 below.

\begin{tabular}{|l|l|c|c|c|}
\hline Type & Manufacturer & Accuracy & Repeatability & Range \\
\hline \multirow{3}{*}{$\begin{array}{l}\text { Water } \\
\text { meters }\end{array}$} & Daniel Industries & $0.25 \%$ & $0.02 \%$ & $25-250 \mathrm{GPM}$ \\
\cline { 2 - 5 } & Omega FTB-1425 & $1 \%$ & $0.1 \%$ & $5-50 \mathrm{GPM}$ \\
\cline { 2 - 5 } & Omega FTB-1422 & $1 \%$ & $0.1 \%$ & $\begin{array}{c}0.75-7.5 \\
\text { GPM }\end{array}$ \\
\hline
\end{tabular}

Table 4: Water flow meters

The temperature of water is measured using an Omega T-type thermocouple. The accuracy of the thermocouple is $1^{\circ} \mathrm{F}$.

The mixing of air and water takes place at a distance of approximately 50 inches before the test section. After passing through the test section, water is returned to the storage tank and air is released into the atmosphere. The water manifold used in the experiment is shown in Figure 10. 


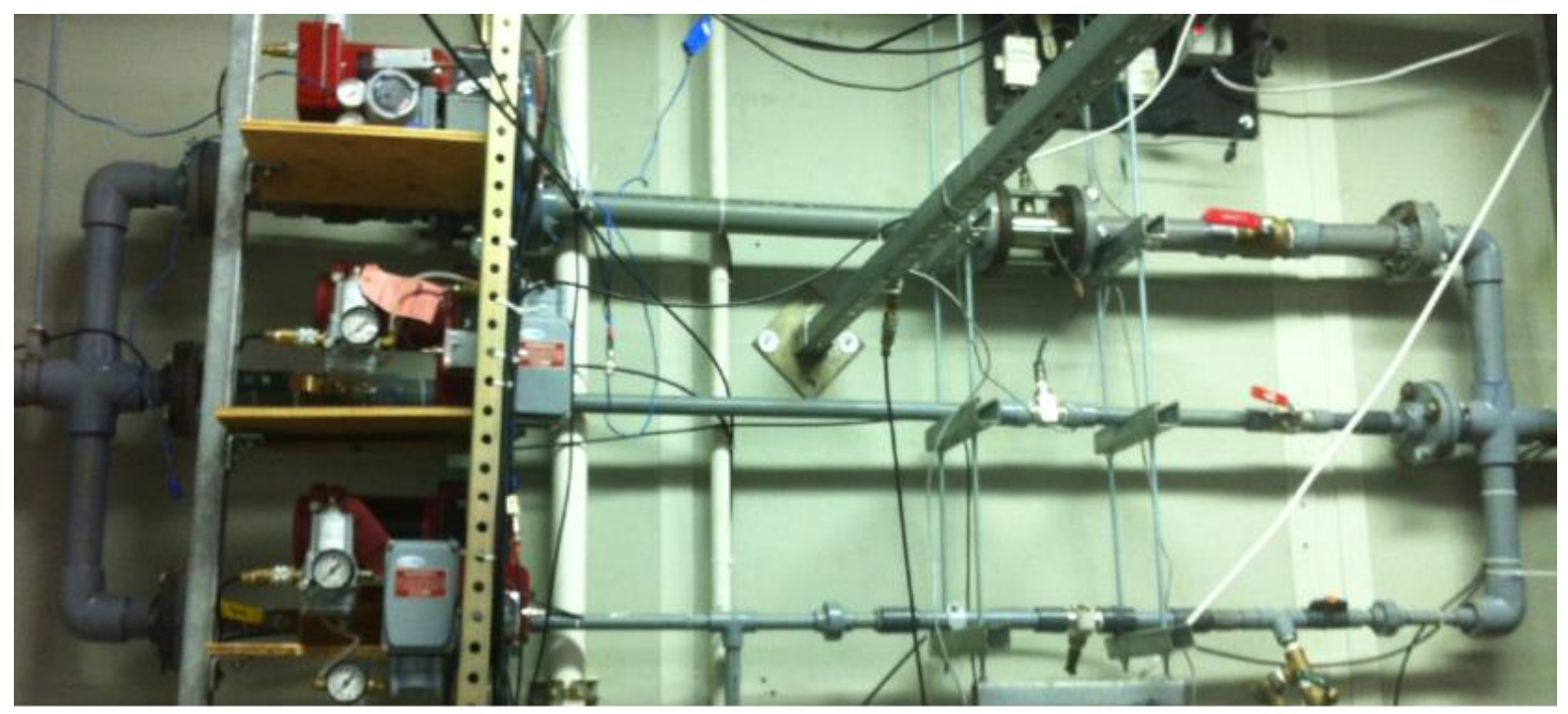

Figure 10: Water Manifold

\subsection{Test section}

The entire test section is made transparent to visualize the flow inside the pipe. Polycarbonate tubes are used upstream and downstream of the slotted orifice plate as they are highly transparent and are more resistant to breakage or cracking than acrylic pipes. The inner diameter of the tubes is 2 inches and the thickness is 0.25 inches. The length of the entire test section is $15 \mathrm{D}$ and is shown in Figure 11.

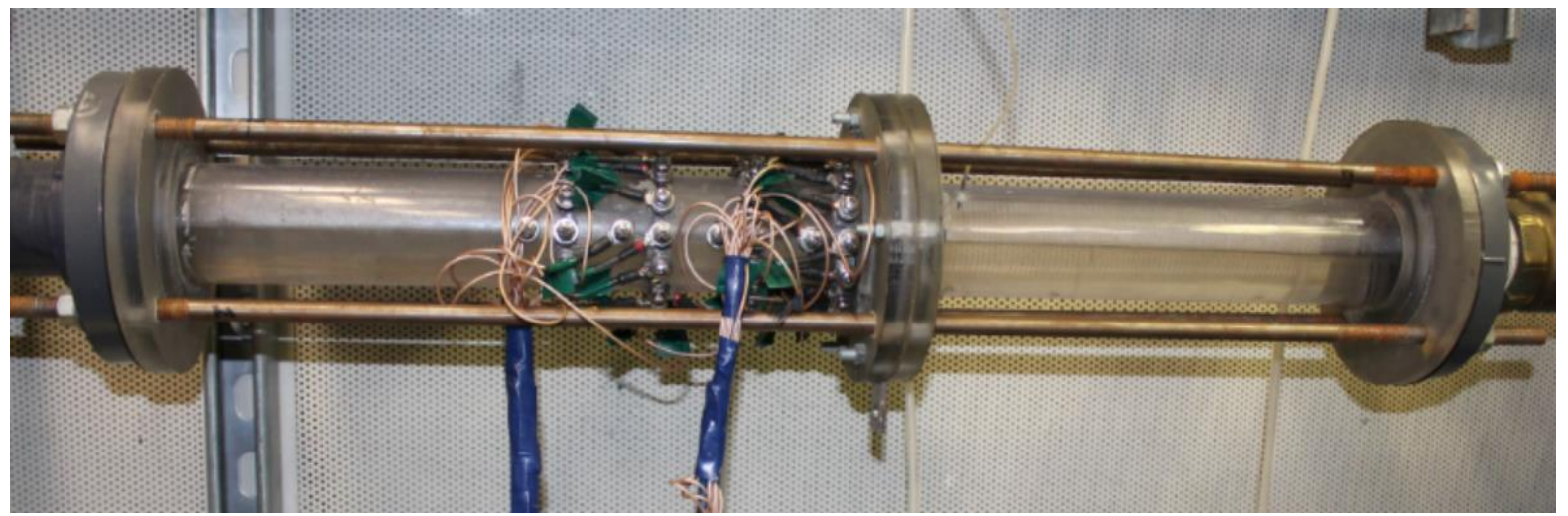

Figure 11: Test section 
Slotted orifice plate with a beta ratio of 0.467 is used in this experiment. Flanges are machined from polycarbonate discs as shown in Figure 12 and a slot is provided in the flanges for placing the slotted orifice plate. Grooves are provided in the flanges for placing the O-rings which are used to tightly seal the gap between the pipe and the flange during assembly. The slotted orifice plate is sandwiched between the two flanges and the flanges are screwed with hexagonal nuts. Also holes are provided for placing the tie-rods to tightly assemble the test section.
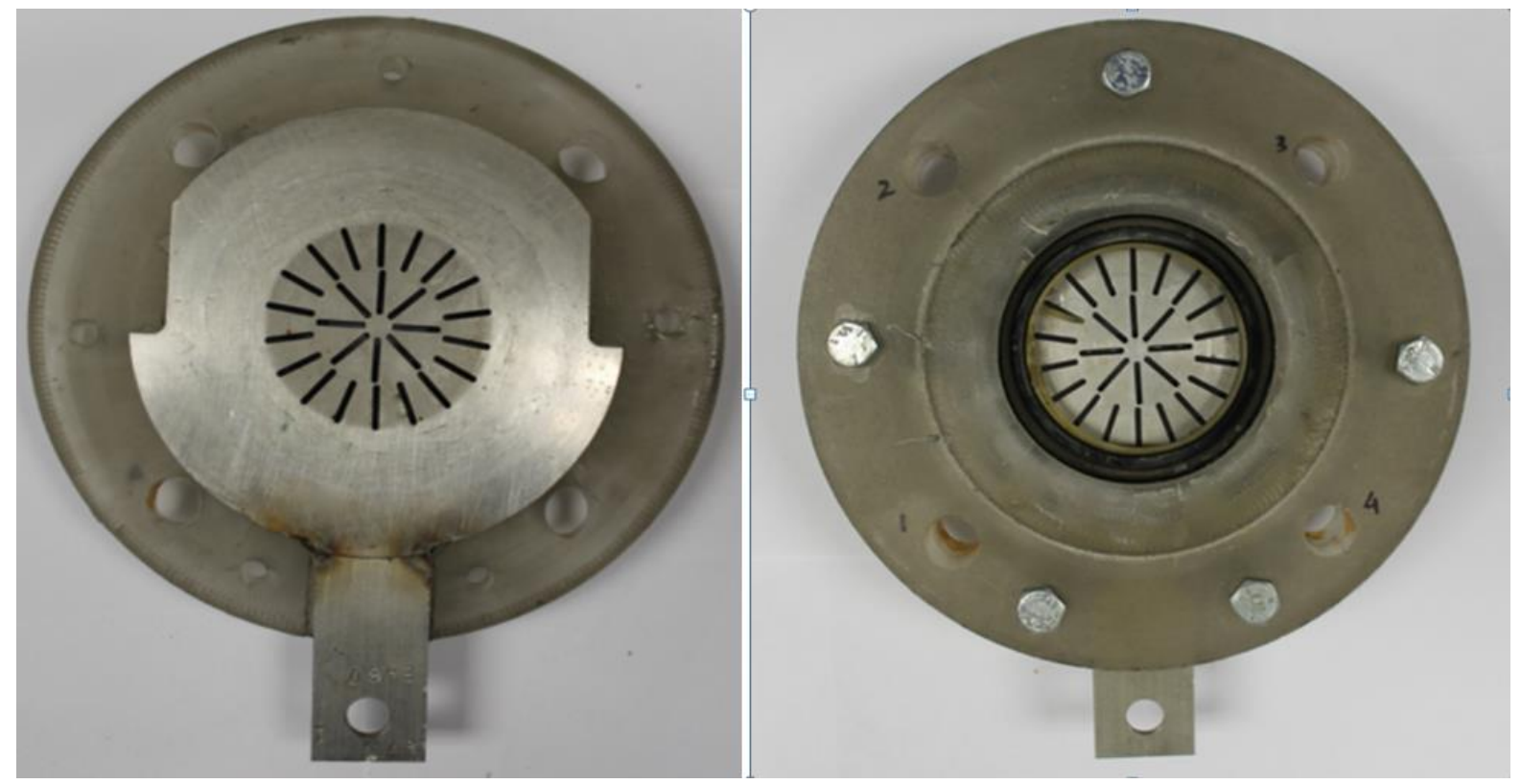

Figure 12: Polycarbonate flanges with slot for placing the slotted orifice plate

Stainless steel machine screws (size number 10) are used as electrodes in this experiment. Stainless steel was chosen as the electrode material in this experiment because it is more conductive than the fluid being imaged and thus prevents problems due to contact impedance.

Seventeen holes (including one for ground electrode) are drilled around the circumference of the downstream pipe at four different planes for inserting the electrodes. The ground electrode is positioned away from the measurement electrode but it is also in contact with the internal 
fluid so as to ensure all the voltage requirements are fixed against a common ground source. The electrodes are made to flush with the pipe-wall as shown in Figure 13 so as not to disturb the flow inside the pipe.

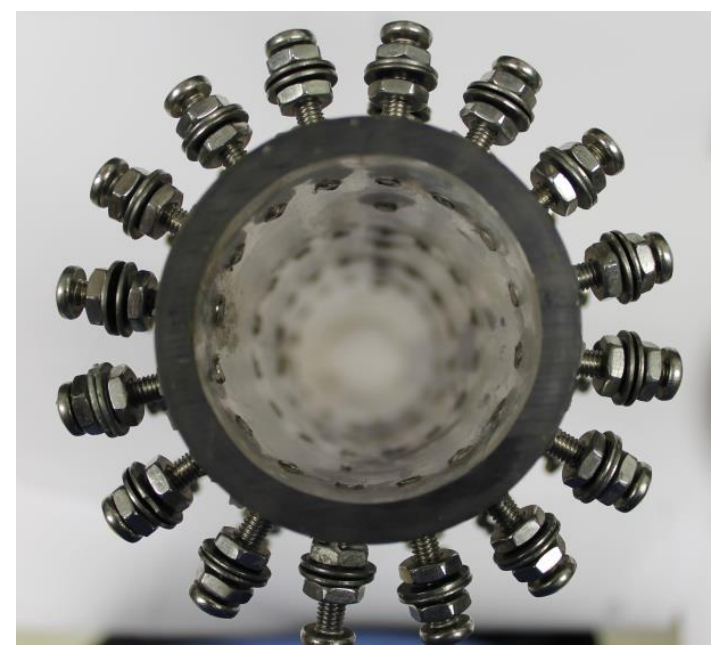

Figure 13: Cross-sectional view of the pipe showing the electrodes arranged in circular pattern

The downstream pipe has four electrode planes which are located at a distance of 1, 3, 5 and 7 inches from the slotted orifice plate as shown in Figure 14.

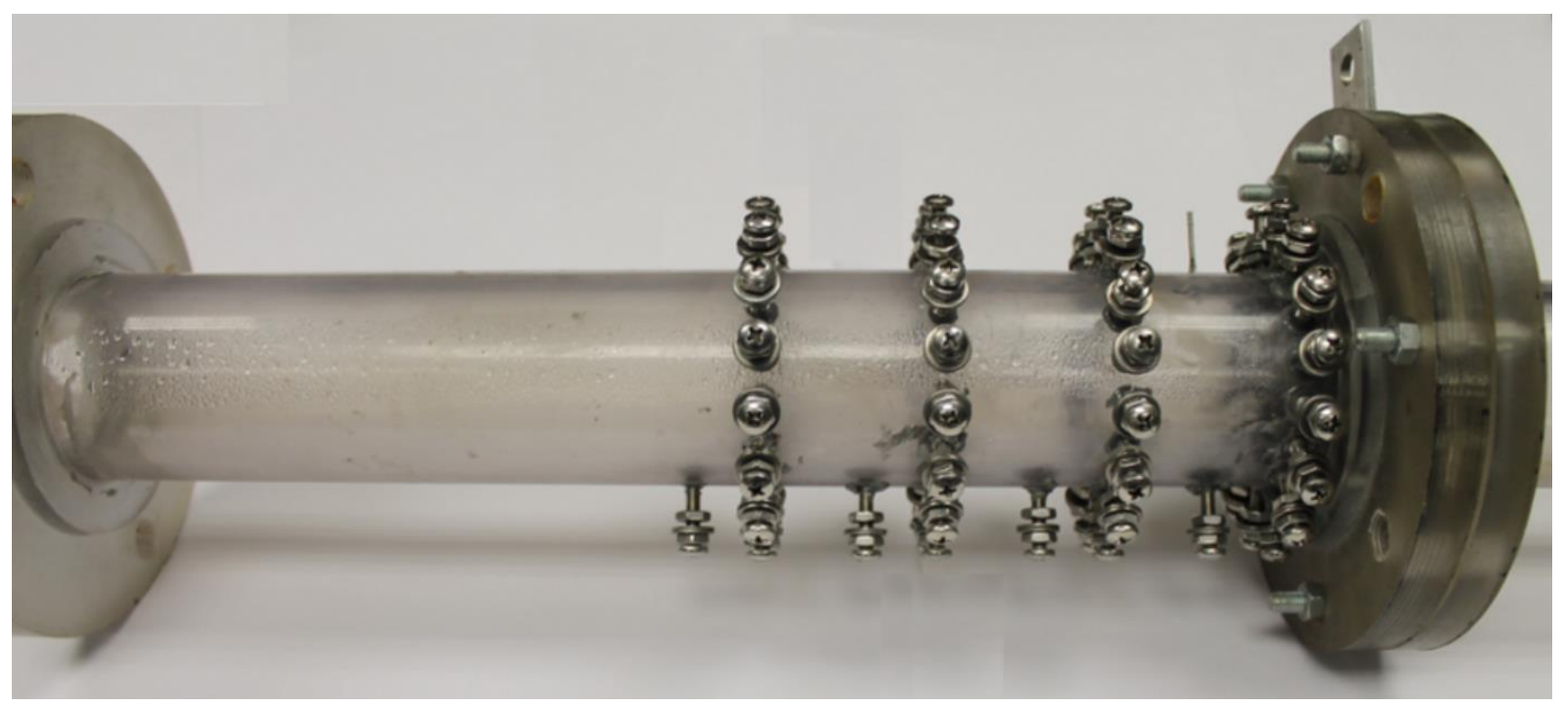

Figure 14: Electrode planes at 1, 3, 5 and 7 inches downstream of the slotted orifice plate 
By orienting the downstream pipe in the opposite direction, measurements can be taken at another 4 planes which are at a distance of 8,10,12 and 14 inches from the slotted orifice plate as shown in Figure 15.

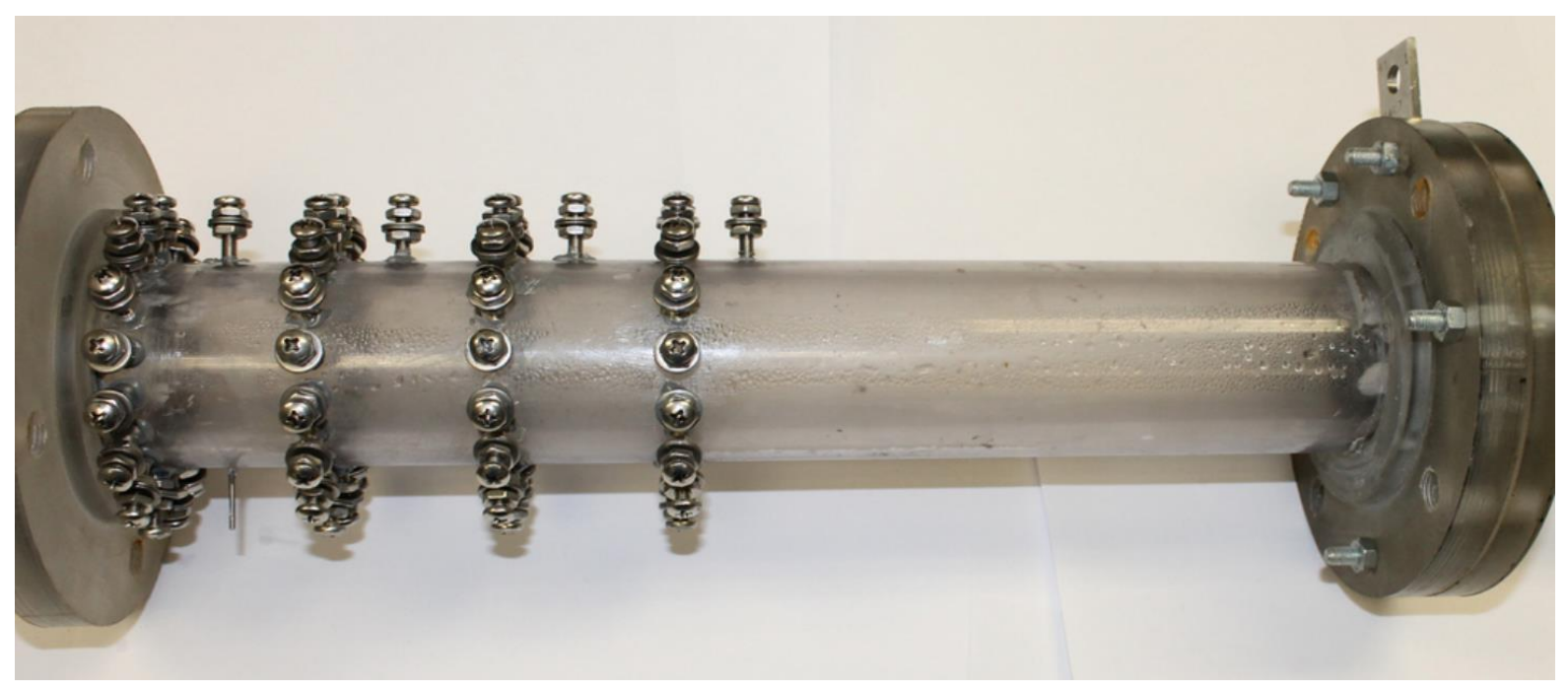

Figure 15: Electrode planes at 8, 10, 12 and 14 inches downstream from the slotted orifice plate

Thus measurements can be taken at eight different planes which are located at a distance of 1 , $3,5,7,8,10,12$ and 14 inches downstream of the slotted orifice plate. An omega T-type thermocouple is used to measure the temperature of the flow downstream of the orifice plate. Pressure taps of dimension 1/16 inch are inserted at locations 0.5D upstream and 0.75D downstream of the slotted orifice plate as shown in Figure $\mathbf{1 6 .}$ 


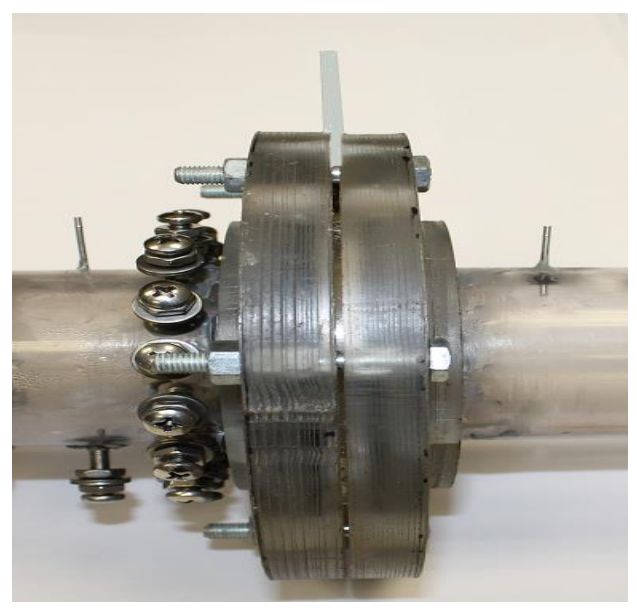

Figure 16: Pressure taps inserted upstream and downstream of the orifice plate

Two Rosemount Model 3051 pressure transducers are connected to the pressure taps as shown in Figure 17 such that one measures the pressure upstream of the plate and the other measures the differential pressure across the plate.

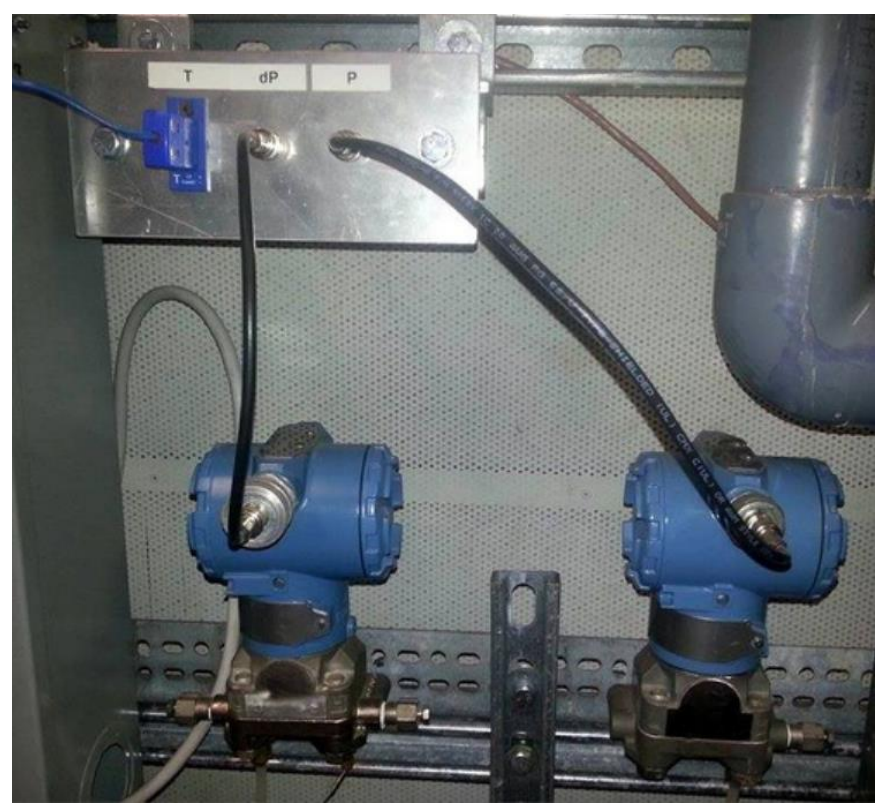

Figure 17: Rosemount pressure transducers 


\subsection{Data Acquisition System}

\subsubsection{Data Acquisition System for ERT}

The Data Acquisition System (DAS) for ERT is responsible for obtaining the quantitative data which describes the conductivity distribution of the components inside the pipe. It has the ability to capture the data very quickly and accurately thereby allowing small changes in conductivity to be tracked in real-time. This feature of the DAS is very important so that the reconstruction algorithm is able to provide an accurate measurement of the true conductivity distribution in a two-phase flow.

The ITS p2+ ERT system data acquisition system (DAS) is connected to the personal computer (PC) by a USB 2.0 cable. Electrical power is supplied to the system via a power adapter (100$240 \mathrm{~V}$ a.c., $2.5 \mathrm{~A}, 50 / 60 \mathrm{~Hz}$ ) at the rear of the instrument.

Each plane of electrodes on the ERT sensor is connected to the p2+ ERT system by connectors on the front of the instrument as shown in Figure 18. The sensor cables are color coded in order to match the front panel connectors.

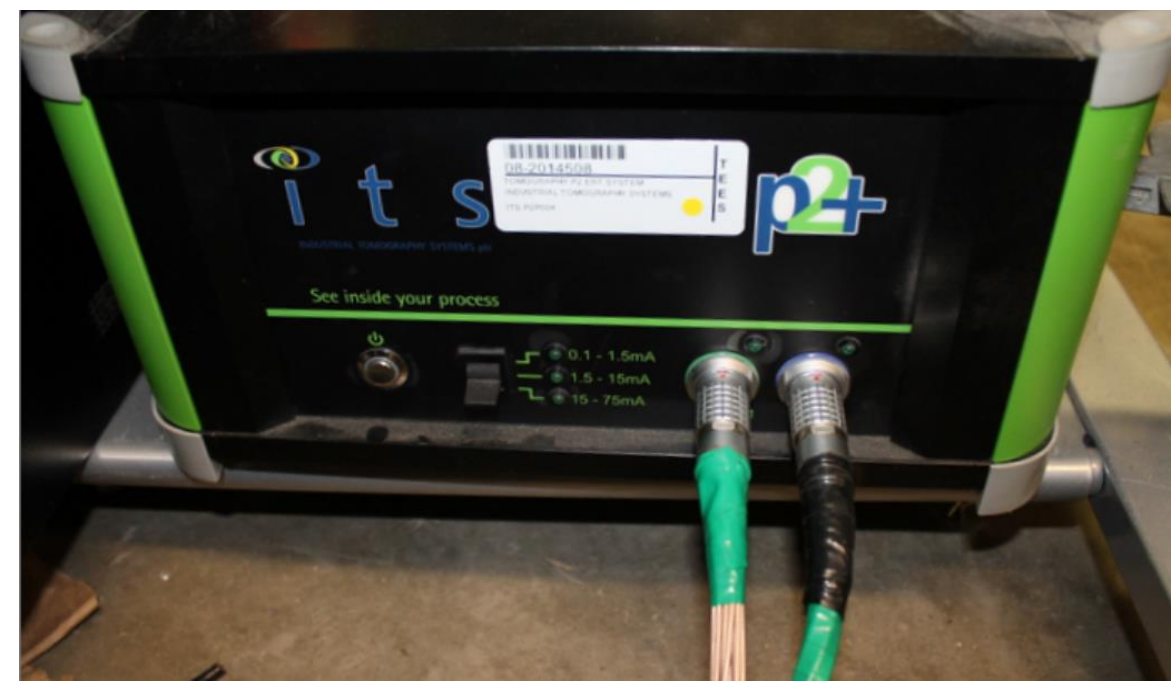

Figure 18: ITS p2+ERT system 
The data capture rate for a given sensor is primarily affected by the following:

- Samples per frame

- Sampling time interval

- Frames per download

- Frequency

- Delay cycle

There is a balance between the data capture rate and the data quality. With an increase in the data capture rate, there is a decrease in data quality. In this application, the data capture rates are increased as it is a fast moving process, which leads to a decrease in the quality of data.

The complete specification of the DAS for ITS p2+ ERT system [11] is shown in Table 5. An accuracy of $\pm 1 \%$ should be achieved for a single plane system and $\pm 3 \%$ should be achieved for a multi-plane system with background conductivities of order $0.1 \mathrm{mS} / \mathrm{cm}$. 


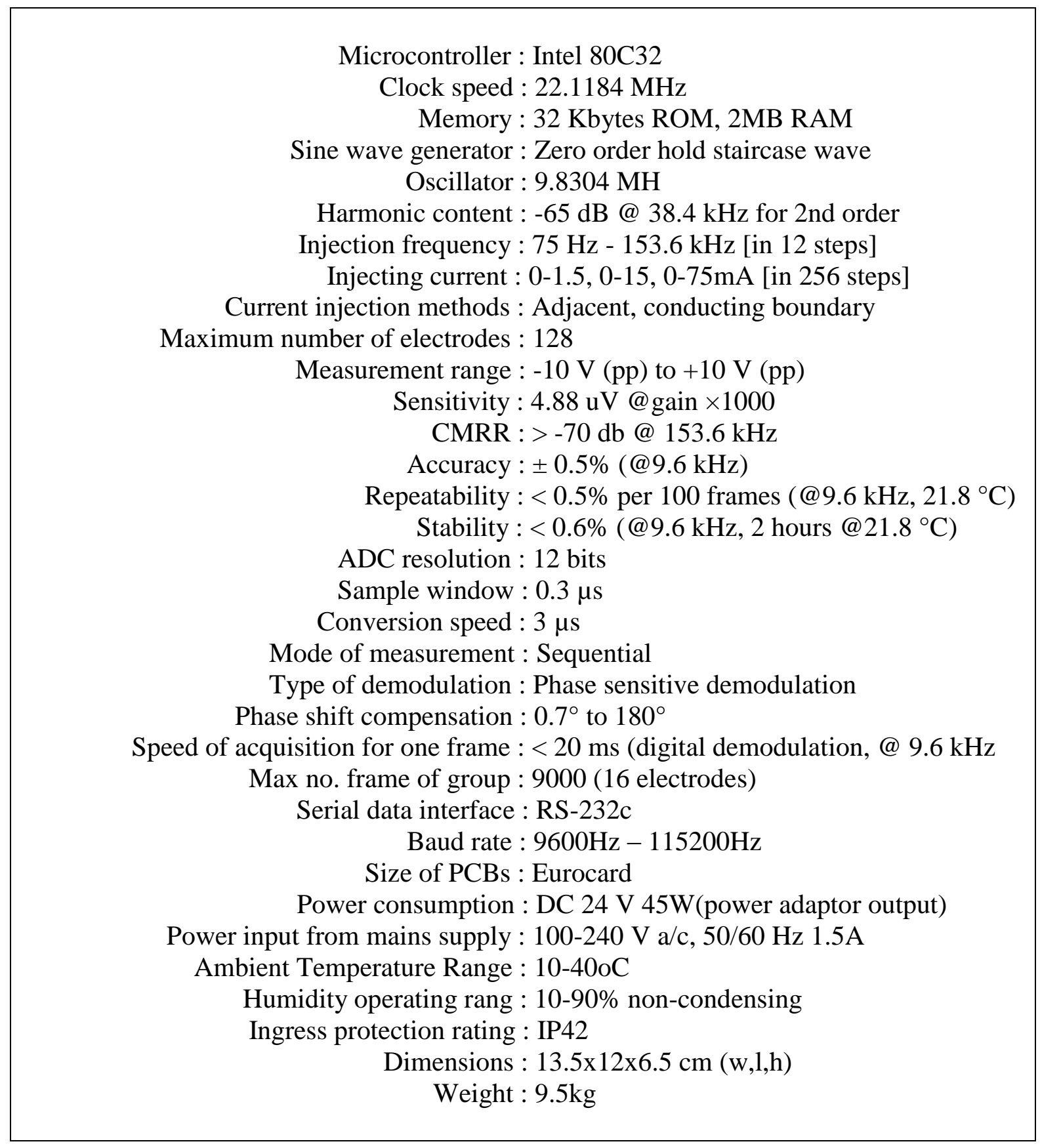

Table 5: Specification of DAS for ITS p2+ ERT system

\subsubsection{DAS chassis}

To control, monitor and record the pressures, temperatures and volumetric flow rates, a NIbased data acquisition system is used. The sensors, flow meters and control valves are all integrated into an NI-9172 module chassis with NI data acquisition cards. NI-9205 and NI- 
9215 modules provide to measure the pressure and the differential pressure from the pressure transducers respectively. NI-9213 module provides to measure the temperatures as ${ }^{\circ} \mathrm{F}$ from the thermocouple data and NI-9265 module provides to regulate the control valve positions to reach the desired flow condition. Figure 19 shows the NI-9172 module chassis which stores the four NI modules connected to LabVIEW.

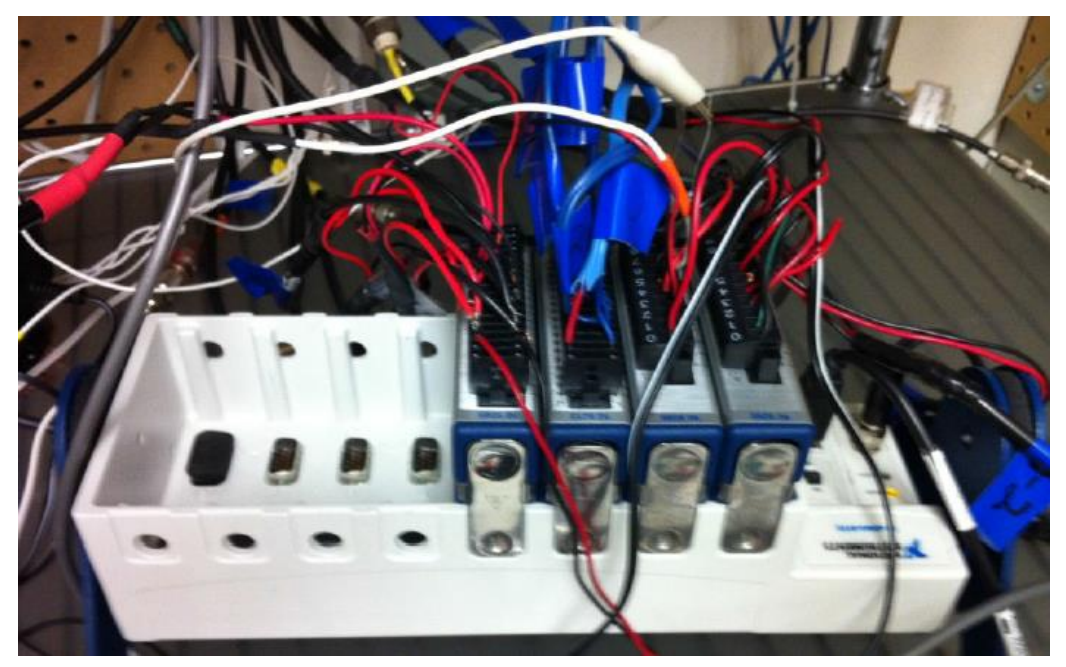

Figure 19: NI-9172 module chassis

\subsubsection{LabVIEW}

LabVIEW has a comprehensive tool-set for acquiring, analyzing, displaying and storing data. A variety of controls (such as dials, buttons, switches, knobs, etc.) and indicators (such as graphs, LEDs, etc.) can be used to build a user-friendly interface. The LabVIEW program for a two-phase flow meter is shown in Figure 20.

The LabVIEW program shows the temperatures, air and water flow rates, GVF, air supply pressure, pressure upstream, differential pressure, and valve positions. The ProportionalIntegral-Derivative (PID) controller is used to control and regulate the pressure upstream of the test section, water flow rate and GVF. 


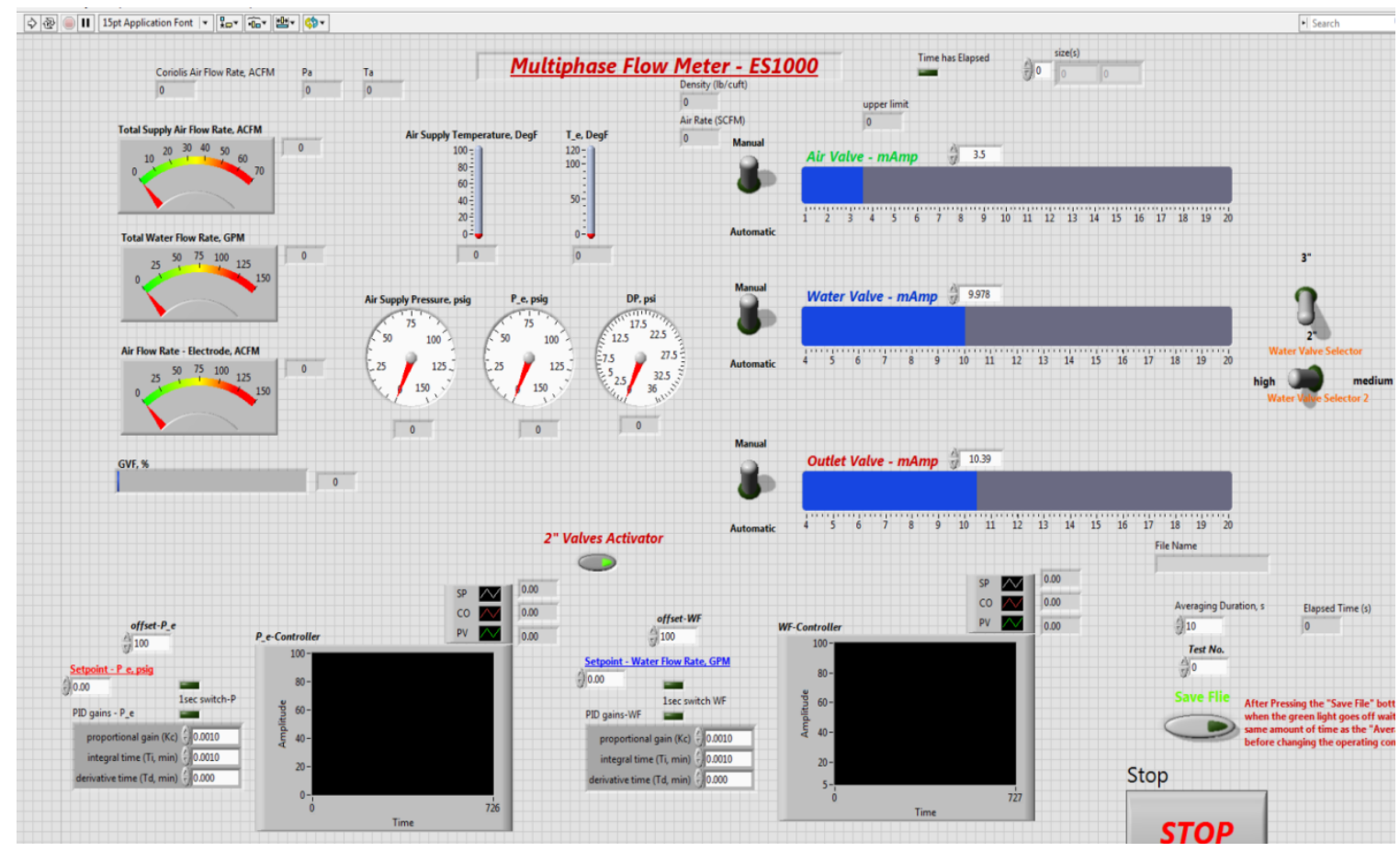

Figure 20: LabVIEW program for two-phase flow meter

\subsubsection{Proportional-Integral-Derivative (PID) controller}

A Proportional-Integral-Derivative (PID) controller in LabVIEW is used to control and regulate the pressure upstream of the test section, water flow rate and GVF. The PID controller first reads a sensor and then computes the desired actuator output by calculating proportional, integral and derivative responses and summing those three components to compute the output. The value of the sensor is known as the process variable.

The characteristics of P, I and D controllers are listed below:

- A proportional controller $(\mathrm{Kp})$ will reduce the rise time but will not eliminate the steady-state error.

- An integral controller (Ki) will eliminate the steady-state error but will make the transient response worse. 
- A derivative controller (Kd) will increase the stability of the system, reduce overshoot and improve transient response.

After performing the PID algorithm on the process variable, the controller output is set to a desired value. Adjustments could be made using the PID controller such that the error falls within a specified limit.

The mean, standard deviation and percentage error of each process variable is recorded and saved as '.tdms' file. The rate at which the data is being recorded can also be adjusted.

\subsubsection{Software}

The software used by the ITS p2+ ERT system is p2000 - p2+ version 7.0. It is compatible with Windows XP and 7. This software is very user-friendly and has a one-click calibration.

\subsubsection{Image reconstruction algorithm}

An image reconstruction algorithm processes the data collected by DAS and constructs a tomographic image containing information about the conductivity distribution of the components inside the pipe.

The main requirement for an image reconstruction algorithm used in ERT system is its processing speed which has to be very fast so that it can be used for real time imaging of the flow inside the pipe. Accuracy also plays a crucial role as the reconstructed image has to provide true conductivity distribution of the components for determining flow properties.

The p2+ ERT system uses Linear Back-Projection (LBP) image reconstruction algorithm which is the simplest and fastest among non-iterative algorithms. It is well-suited for fastdynamic processes like multi-phase flows and is widely used for on-line image reconstruction 
because of its fast processing times in comparison to other algorithms. The only limitation of LBP is that it produces qualitative images rather than quantitative images.

The p2+ ERT system contains two versions of the LBP image reconstruction algorithm: Sensitivity Back Projection (SBP) and Modified Sensitivity Back Projection (MSBP) algorithm. The two versions of the LBP image reconstruction algorithm can be toggled be selecting the reconstruction scheme in control menu.

MSBP is the default image reconstruction algorithm used in p2+ ERT system. There is little or no difference to the reconstructed conductivity tomogram based on the choice of the algorithm in most applications. But in cases where the contrast is extremely high, SBP can be used as it produces more representative images. 


\section{EXPERIMENTAL PROCEDURE}

The air-water two-phase flow was passed through the slotted orifice plate at different flow conditions and the ITS p2+ ERT system is used to obtain the conductivity distribution at eight different locations downstream of the slotted orifice plate.

Different flow conditions of the two-phase flow are obtained by changing the pressure upstream of the orifice plate, water volumetric flow rate and Gas Volume Fraction (GVF). The PID controller in LabVIEW adjusts the control valves in order to obtain the desired set values of the process variables. The upstream pressure values are set to $20 \mathrm{psi}, 40 \mathrm{psi}$ and $60 \mathrm{psi}$, the water volumetric flow rate values are set to 20 GPM, 40 GPM and 60 GPM and the GVF set values ranges from $0 \%$ to $70 \%$. Thus a total combination of eighty-one flow conditions is available and the test matrix for the experiment is shown in Table 6.

\begin{tabular}{|c|c|c|}
\hline $\begin{array}{c}\text { Water Flow Rate } \\
(\mathrm{GPM})\end{array}$ & $\begin{array}{c}\text { Pressure Upstream } \\
(\mathrm{psi})\end{array}$ & $\begin{array}{c}\text { GVF } \\
(\%)\end{array}$ \\
\hline 20 & 20 & 0 \\
\hline 40 & 40 & 5 \\
\hline 60 & 60 & 10 \\
\hline & & 30 \\
\hline & & 40 \\
\hline & & 50 \\
\hline & & 70 \\
\hline & & \\
\hline
\end{tabular}

Table 6: Test Matrix for different flow conditions 


\subsection{ERT system calibration}

Prior to taking measurements, the ERT system has to be first configured. The ERT configuration window is shown in Figure 21.

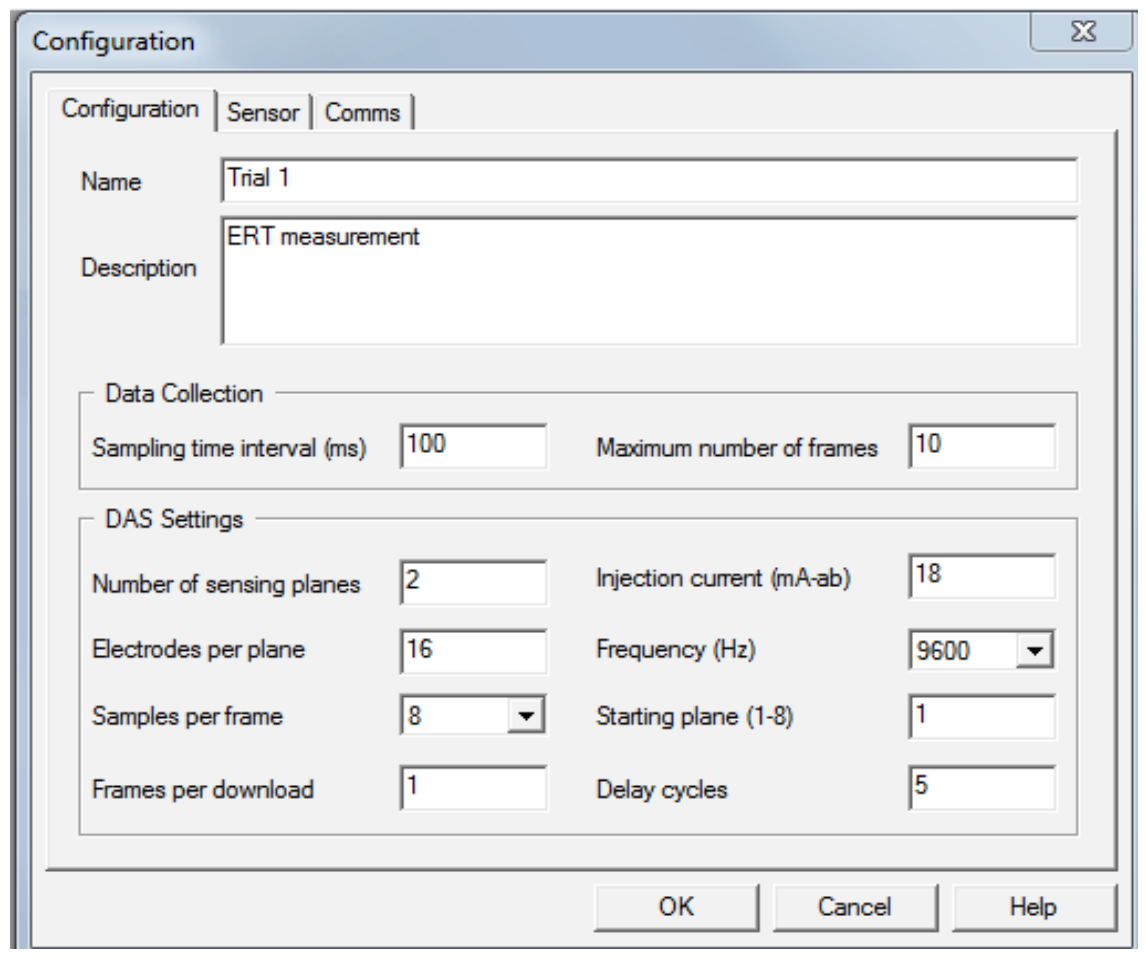

Figure 21: ERT system configuration window

The optimum injection current for the experiment is determined after several iterations. The injection current is mostly influenced by the electrode geometry and the conductivity of the media within the sensor. For this experiment, the optimum value of injection current and frequency is found out to be $18 \mathrm{~mA}$ and $9600 \mathrm{~Hz}$ respectively.

The DAS needs to be configured for fast data capture so the sampling time interval is set to $100 \mathrm{~ms}$, maximum number of frames is set to 10 and delay cycle is set to 5. If the sampling time interval and delay cycle is further reduced then the data quality becomes very poor. 
'Frames per download' has a significant effect on the data collection rates. When this parameter is set to a value of 1 , then a single measurement frame is transferred from the DAS to the PC in a single transmission. But if it is set to a value larger than 1, then that number of frames of data are collected by the DAS and stored in its internal buffer and then transferred to the PC. Hence for this experiment, the frames per download is set to a value of 1 so that the data obtained can be viewed on-line. The number of sensing planes is set to 2 and electrodes per plane is set to 16 in this experiment.

After DAS configuration, sensors need to be configured. Sensor configuration includes the type of sensor used, electrical properties and the type of conversion. For this experiment, the sensor type is Circular non-conducting vessel, the continuous phase is water with a conductivity value of $1 \mathrm{mS} / \mathrm{cm}$ and the dispersed phase is air with a conductivity value of 0.1 $\mathrm{mS} / \mathrm{cm}$ and the conversion from conductivity to concentration is for non-conductive phase.

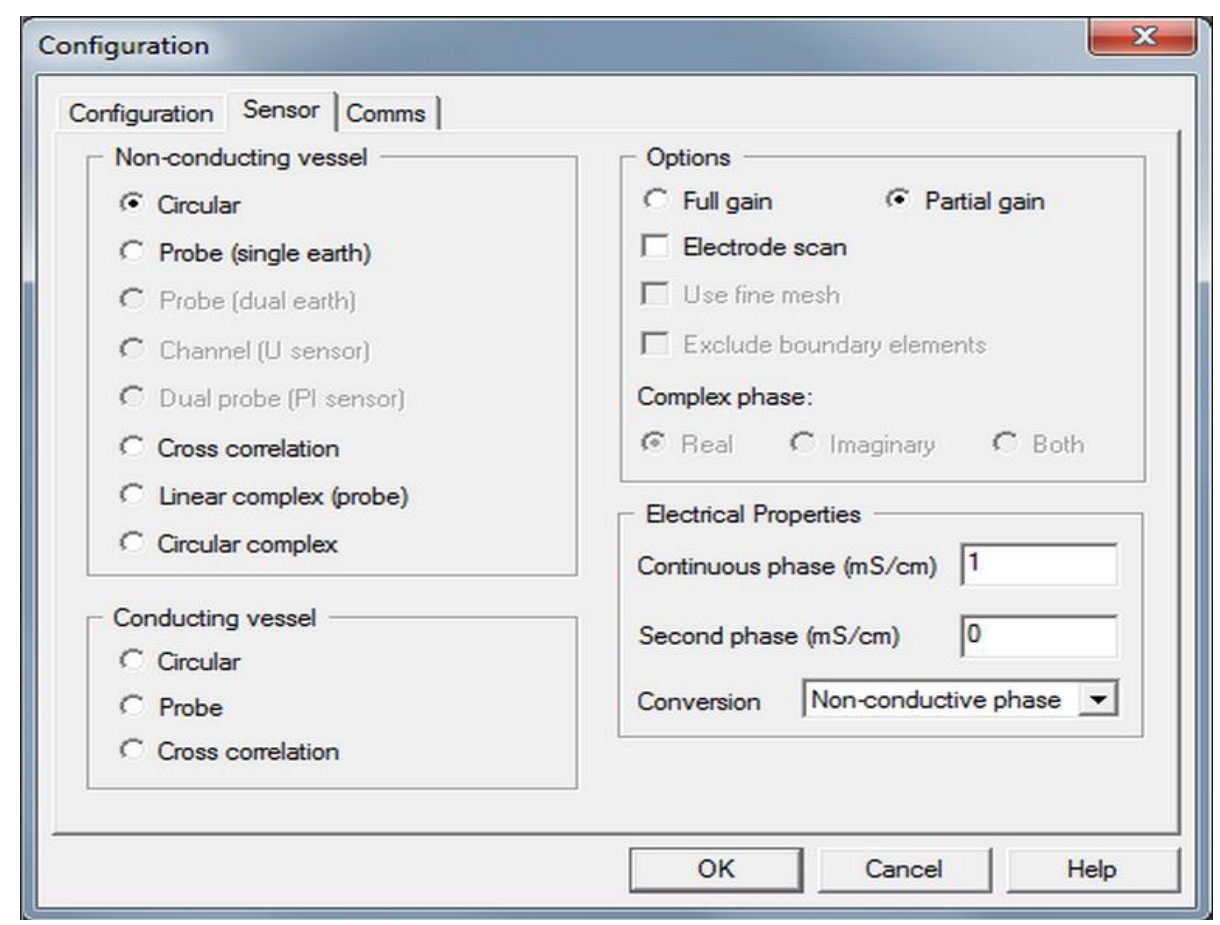

Figure 22: ERT sensor configuration window 
Before measurements can be taken it is necessary to calibrate the current source by selecting DAS/Calibrate Current. A window confirming the magnitude and frequency of injection current that was entered in the configuration window will appear. It is also necessary to calibrate the gain map by selecting DAS/Calibrate gain prior to data collection. Once this calibration is complete, measurements can be taken.

A reference measurement is taken initially since the ERT system works on the relative change in measurements between the measured and the reference measurement. Thus the reference measurement for an air-water two-phase flow is taken with the sensor full of water only. All subsequent measurements are based on changes in voltage measurements in relation to the reference measurement.

\subsection{ERT measurements}

Once the calibration is done and the reference measurement is taken, the ERT system is operated by injecting current through the electrodes in the sensing plane and obtaining all the voltage measurements in the sensing plane. For each flow conditions the corresponding measurements are taken at eight different electrode planes located downstream of the slotted orifice plate. 


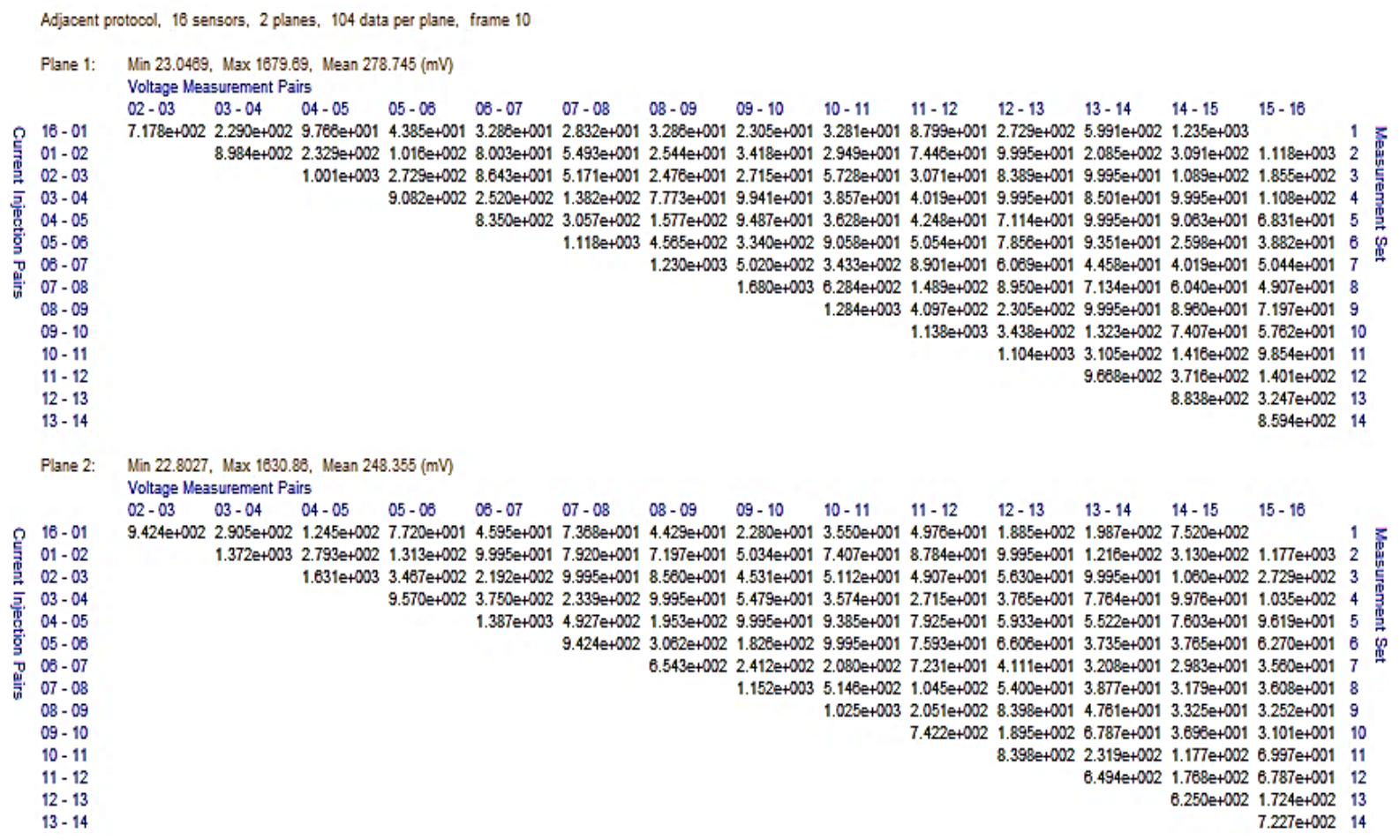

Figure 23: Individual voltage measurement at two electrode planes

The ERT system obtains the individual voltage measurement for each frame of data as shown in Figure 23. The electrode pair through which current is injected is shown in left hand side and the electrode pairs at which the voltage drop is measured is shown across the top of the window. The data is grouped into 14 measurement sets associated with each of the 14 current injection electrode pairs as indicated by the number on the right. Measurements sets 1 and 2 have 13 data points with each subsequent measurement set having one less data point. 
The relative change between the actual voltage measurement and the reference voltage measurement is shown in a graphical form in Figure 24. The measurement set numbers 1 to 14 are labelled in the horizontal axis.
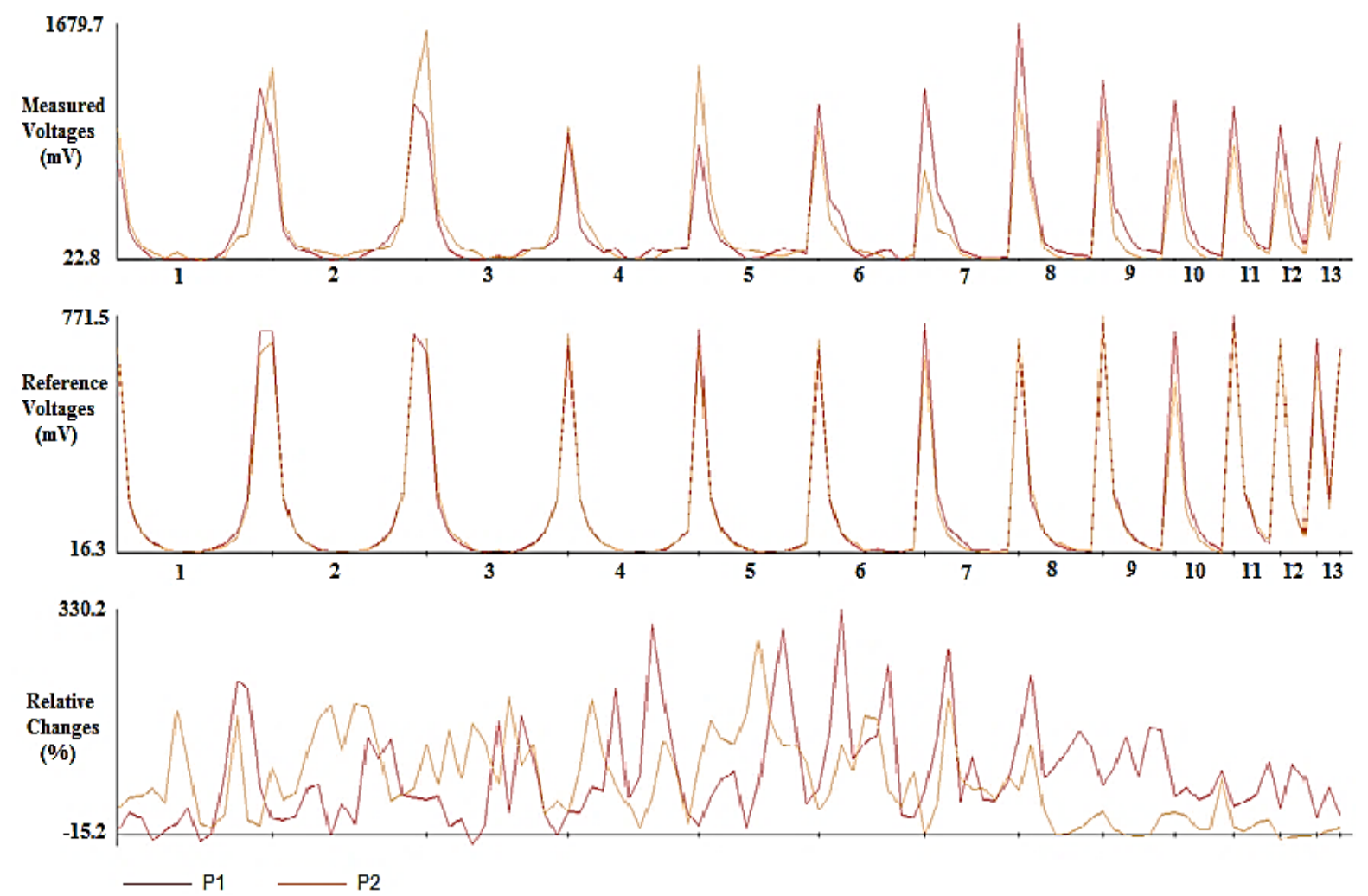

Figure 24: Graph displaying relative change between actual and reference measurement

The relative change between the actual and the reference measurement represents the noise. If the noise is too high, then the injection current has to be modified. 
A maximum of ten frames are taken at a sampling time interval of $100 \mathrm{~ms}$ by the ERT system for each flow condition at each electrode plane. Figure 25 shows the ten conductivity tomograms obtained by the ERT system at a particular downstream location for a particular flow condition.
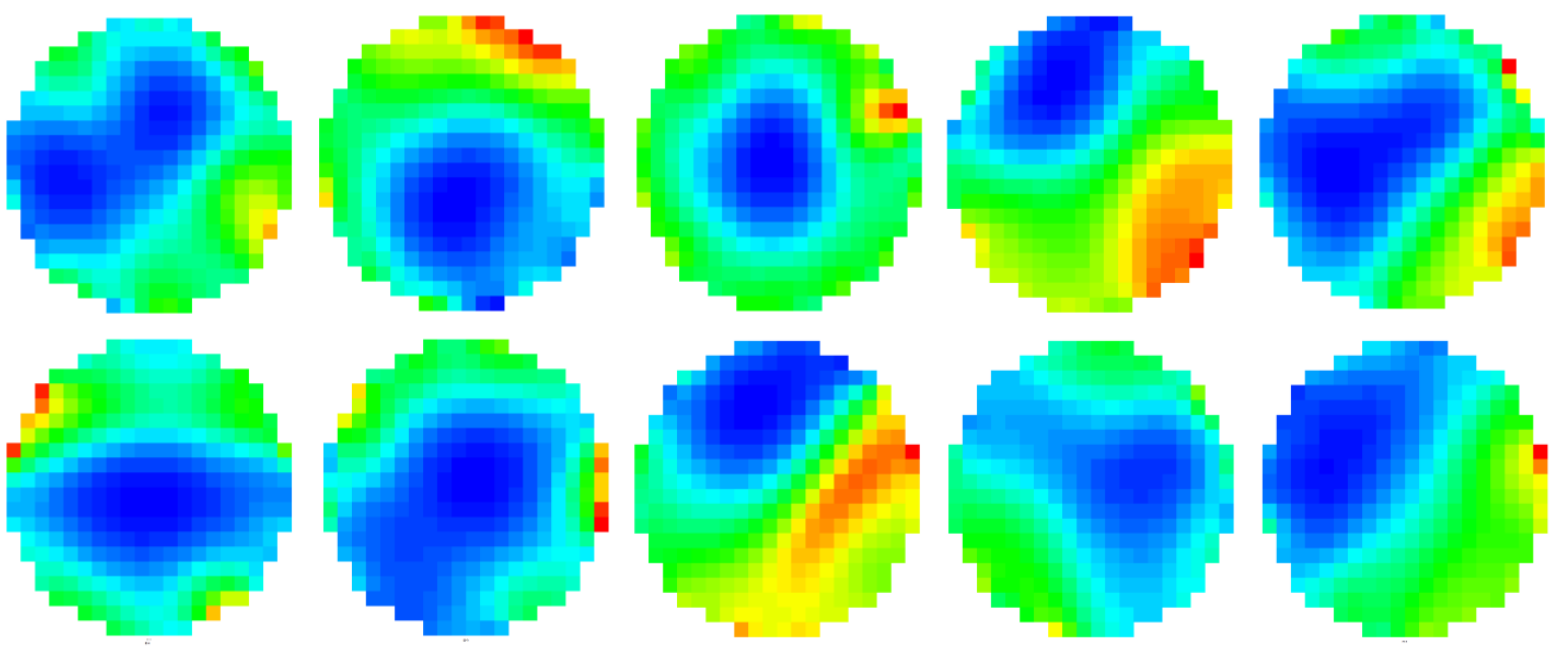

thete

Figure 25: Conductivity tomograms obtained from p2+ ERT system

Using Matlab software, the average of the ten frames is taken and the tomographic image is reconstructed from the average measurement. The reconstructed conductivity tomograms at eight different downstream locations for a particular flow condition are stacked together as shown in Figure 26. A color-scale is used to display the variation in conductivity. 

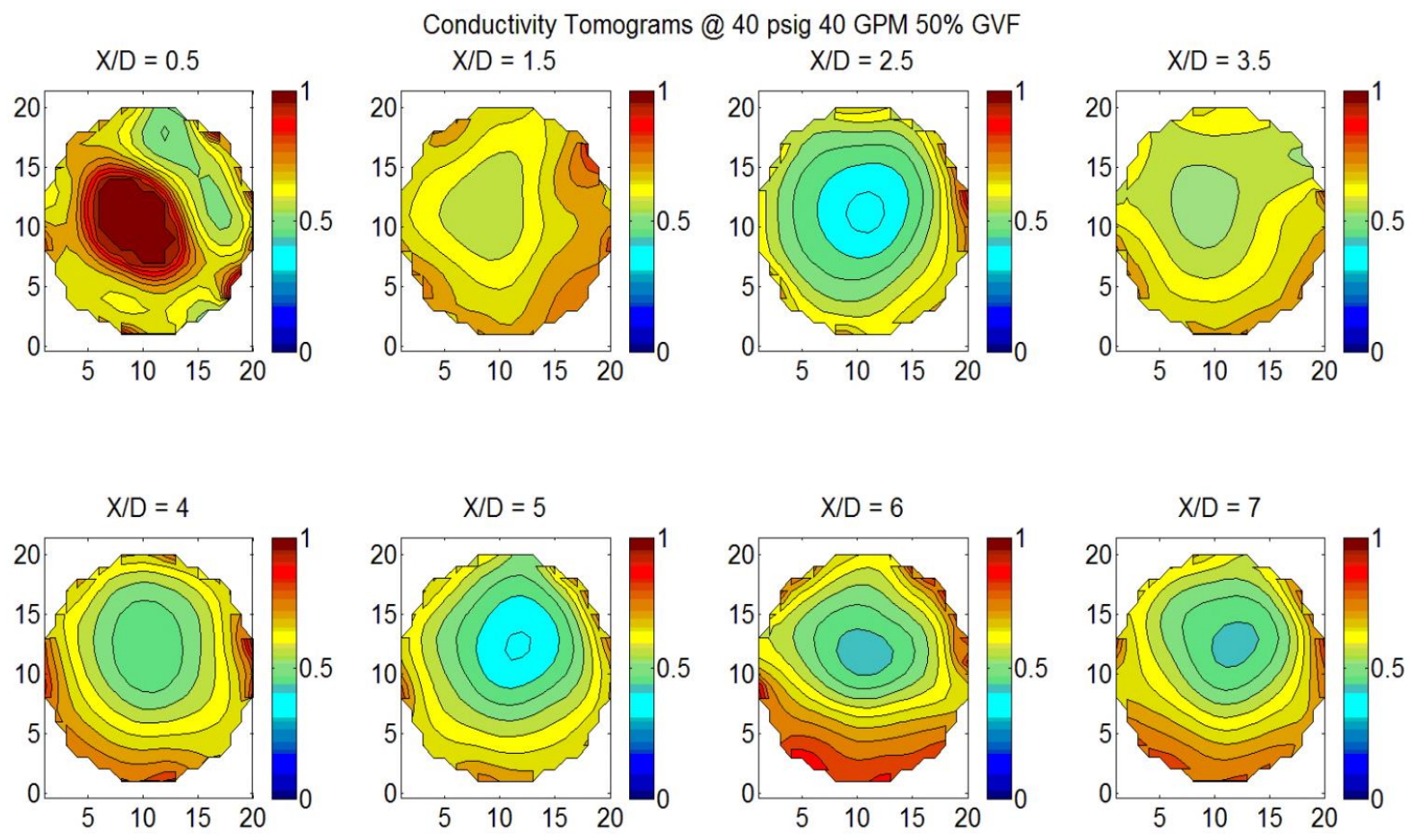

\section{Figure 26: Reconstructed conductivity tomograms at different downstream locations for a particular flow condition}

The conductivity value of each pixel in the conductivity tomogram can be obtained. The conductivity values is converted into concentration values by using the appropriate Maxwell's equation shown in equation 3-7. From the concentration data, tomograms displaying the concentration of each component in the two-phase flow can be constructed.

A sample concentration tomogram for a particular flow condition at different locations downstream of the slotted orifice plate is shown in Figure 27. Similar to the conductivity tomogram, a color-scale is present to display the variation in concentration. 


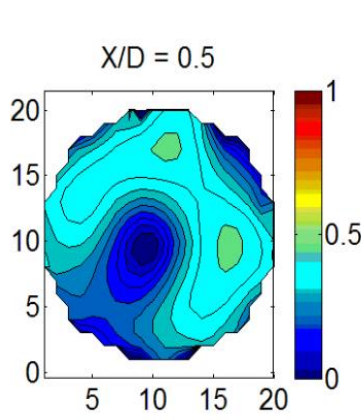

Concentration Tomograms @ 40 psig 20 GPM 70\% GVF
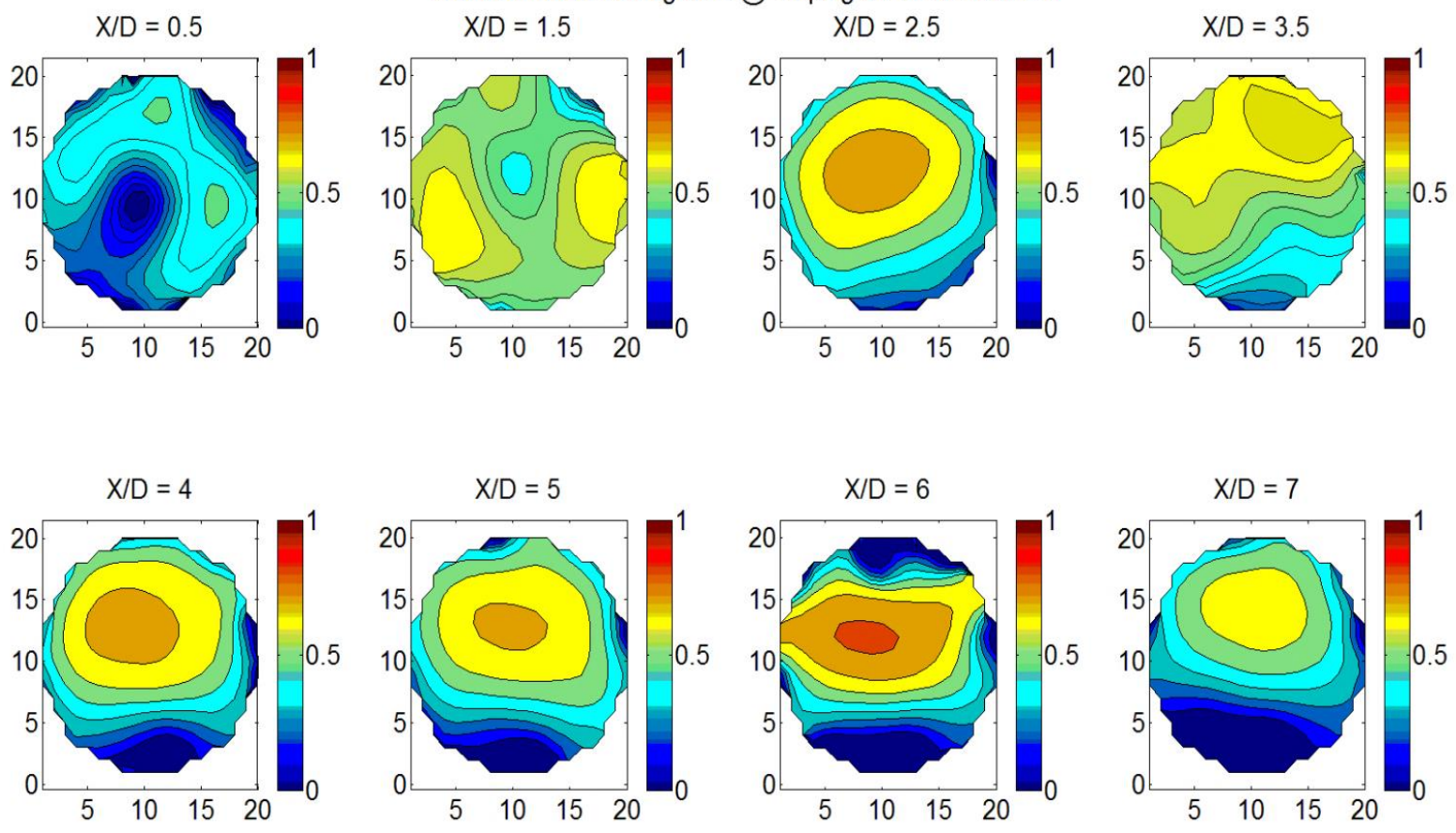

Figure 27: Concentration tomograms at different downstream locations for a particular flow condition 


\section{RESULTS AND DISCUSSIONS}

This section presents the results of the experiments. The ERT system measures the conductivity distribution of the two-phase flow for different flow condition at eight different locations downstream of the slotted orifice plate. The results are discussed in detail below.

\subsection{Conductivity tomograms}

\subsubsection{Effect of water flow rate}

The conductivity increases with increasing water flow rate when the pressure upstream of the slotted orifice plate and the GVF are maintained constant. The effect of increasing water flow rate from 20 GPM to 60 GPM on the conductivity distribution when the pressure upstream is maintained constant at 20 psig and the GVF at $40 \%$ is shown in Figure 28.
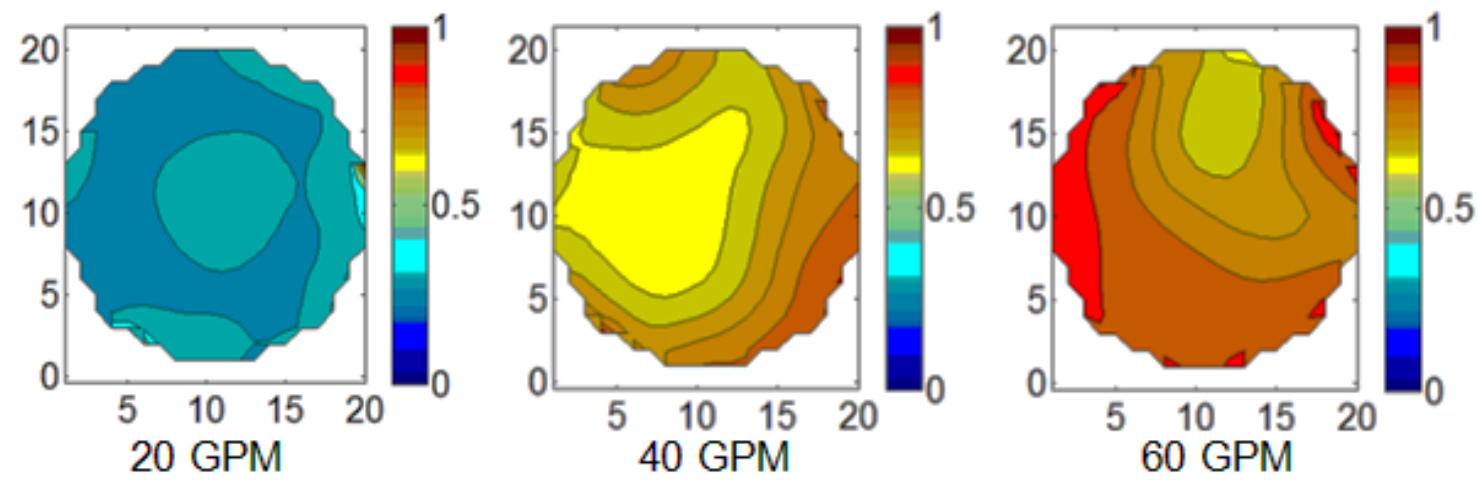

Figure 28: Effect of increasing water flow rate on conductivity distribution at 20 psig and 40\% GVF at downstream location $X / D=1.5$

It can be seen that at low water flow rate (20 GPM), the conductivity tomogram displays more blue zones indicating the presence of more air in the mixture and hence lower conductivity value. On increasing water flow rates to 40 GPM and to 60 GPM, while maintaining the pressure upstream and GVF constant, more red zones are displayed in the tomograms indicating the presence of more water in the mixture and hence higher conductivity value. 
It can be also be seen from the conductivity tomograms that at low water flow rate of 20 GPM, the two-phase flow exists as dispersed flow regime with uniform distribution of the gas and liquid phase. As the water flow rate is increased to 40 GPM, the flow transits to annular flow with the water forming a continuous annular film around the pipe-wall and air flowing in the central core. As the water flow rate is further increased to 60 GPM, the flow transits to stratified flow with the water and gas phase completely separated by a horizontal interface. Water flows along the bottom of the pipe and air flows at the top due to gravity.

Thus increasing water flow rate increases the mean conductivity value and also changes the flow regime of the two-phase flow from mist flow to stratified flow regime.

\subsubsection{Effect of GVF}

The conductivity decreases with increasing GVFs when the pressure upstream of the slotted orifice plate and water flow rate is maintained constant. The effect of increasing GVFs from $5 \%$ to $70 \%$ on the conductivity distribution when the water flow rate is maintained constant at 40 GPM and the pressure upstream at 40 psig is shown in Figure 29.

It can be seen from Figure 29 that on increasing the GVFs from 5\% to 70\%, the red zones in the tomograms are replaced by more blue zones indicating the presence of more air in the mixture. Therefore there is a decrease in the mean conductivity value of the mixture. 


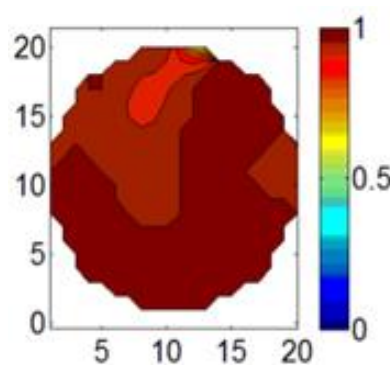

$5 \%$ GVF

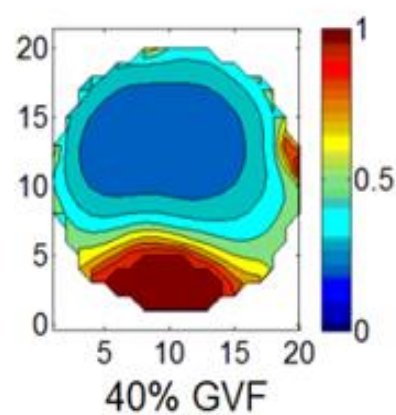

$40 \%$ GVF

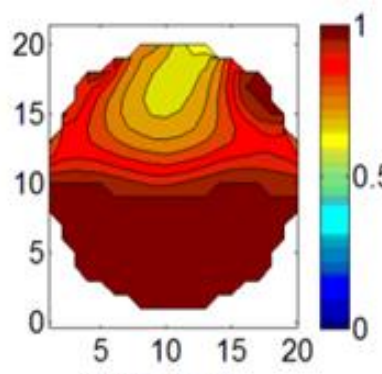

$10 \%$ GVF

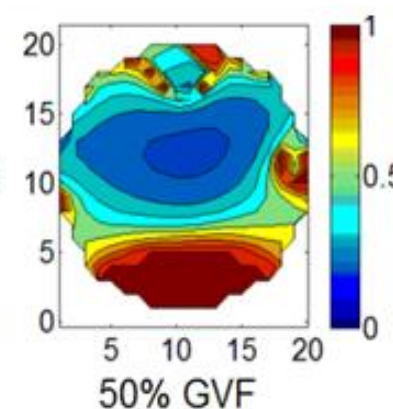

$50 \%$ GVF

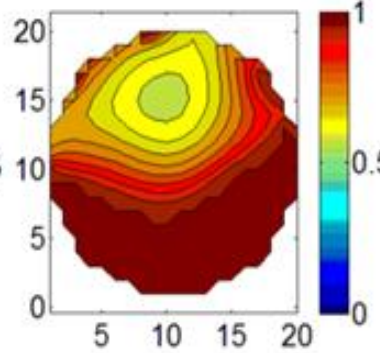

$20 \%$ GVF

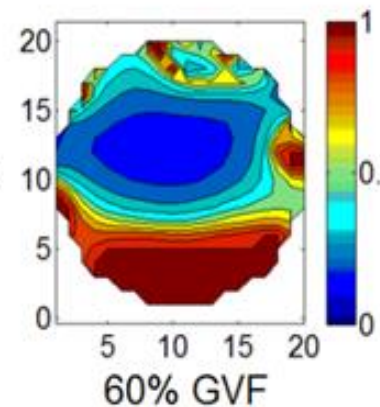

$60 \%$ GVF

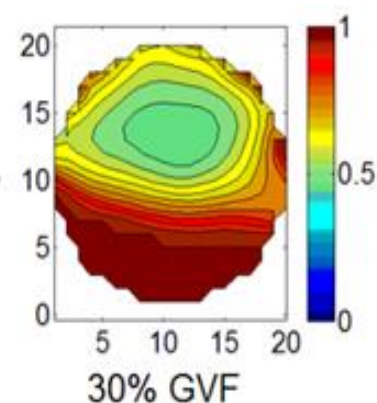

$30 \%$ GVF

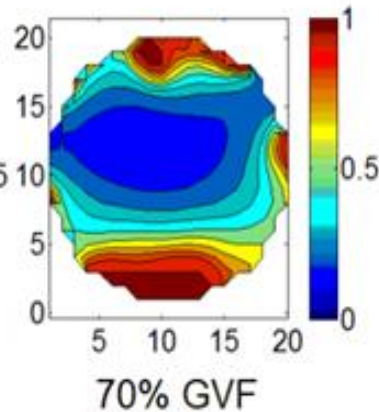

Figure 29: Effect of increasing GVF on conductivity distribution at 40 psig and 40 GPM at downstream location $X / D=2.5$

It can also be seen from the tomograms that at low GVF values $(\mathrm{GVF}=5 \%)$, the flow exists as dispersed bubble flow with the air phase dispersed as small bubbles in the continuous water phase and flowing in the top due to buoyancy. As the GVF is increased (up to 40\%), the flow becomes stratified with the water flowing along the bottom of the pipe and the air flowing in the top due to gravity. As the GVF is further increased (GVF>40\%), the velocity of the gas phase becomes much larger than the water phase and the flow becomes annular flow, with the water flowing around the pipe-wall and air flowing in the central core. Thus the two-phase flow transits from dispersed bubble flow regime to annular flow regime on increasing the GVF and holding the water flow rate and pressure upstream constant. 


\subsubsection{Effect of upstream pressure}

On increasing the pressure upstream of the slotted orifice plate, when the water flow rate and the GVF are maintained constant, there was no noticeable change in the conductivity distribution at different downstream locations. It can be inferred from Figure 30 that when the upstream pressure is increased from 20 psig to 60 psig while maintaining the water flow rate at $40 \mathrm{GPM}$ and GVF at $40 \%$, there is no significant change in the conductivity distribution.
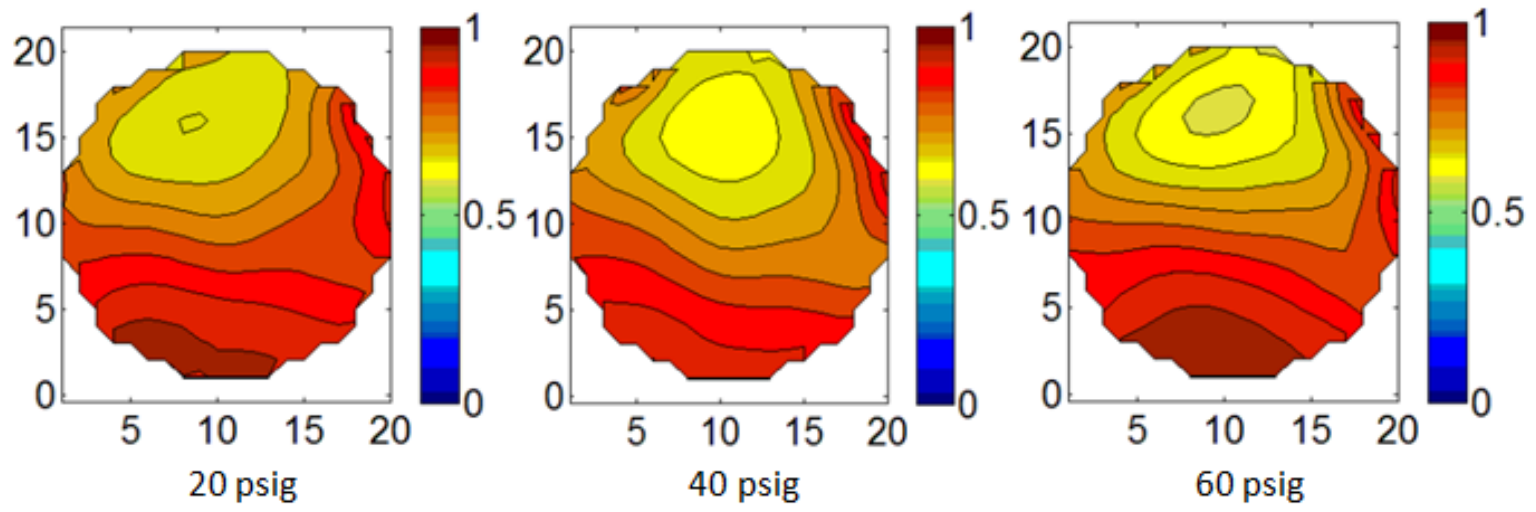

Figure 30: Effect of increasing upstream pressure on conductivity distribution at 40 GPM and $40 \%$ GVF at downstream location $X / D=1.5$

With increasing pressure upstream, there is also no change in the flow regime downstream of the slotted orifice plate. It can be seen from the conductivity tomograms in Figure $\mathbf{3 0}$ that the two-phase flow exists in stratified flow regime in all the three cases. Hence varying the pressure upstream of the slotted orifice plate has no effect on the conductivity as well as the flow regime downstream. 


\subsubsection{Effect of downstream distance}

It is important to determine how the conductivity distribution varies at different locations downstream of the slotted orifice plate for each flow condition. The conductivity tomograms obtained at eight different downstream locations (X/D) when the upstream pressure is $40 \mathrm{psig}$, water flow rate is 20 GPM and GVF is $70 \%$ is shown in Figure 31. Similarly the conductivity tomograms obtained for other flow conditions is shown in the appendix.
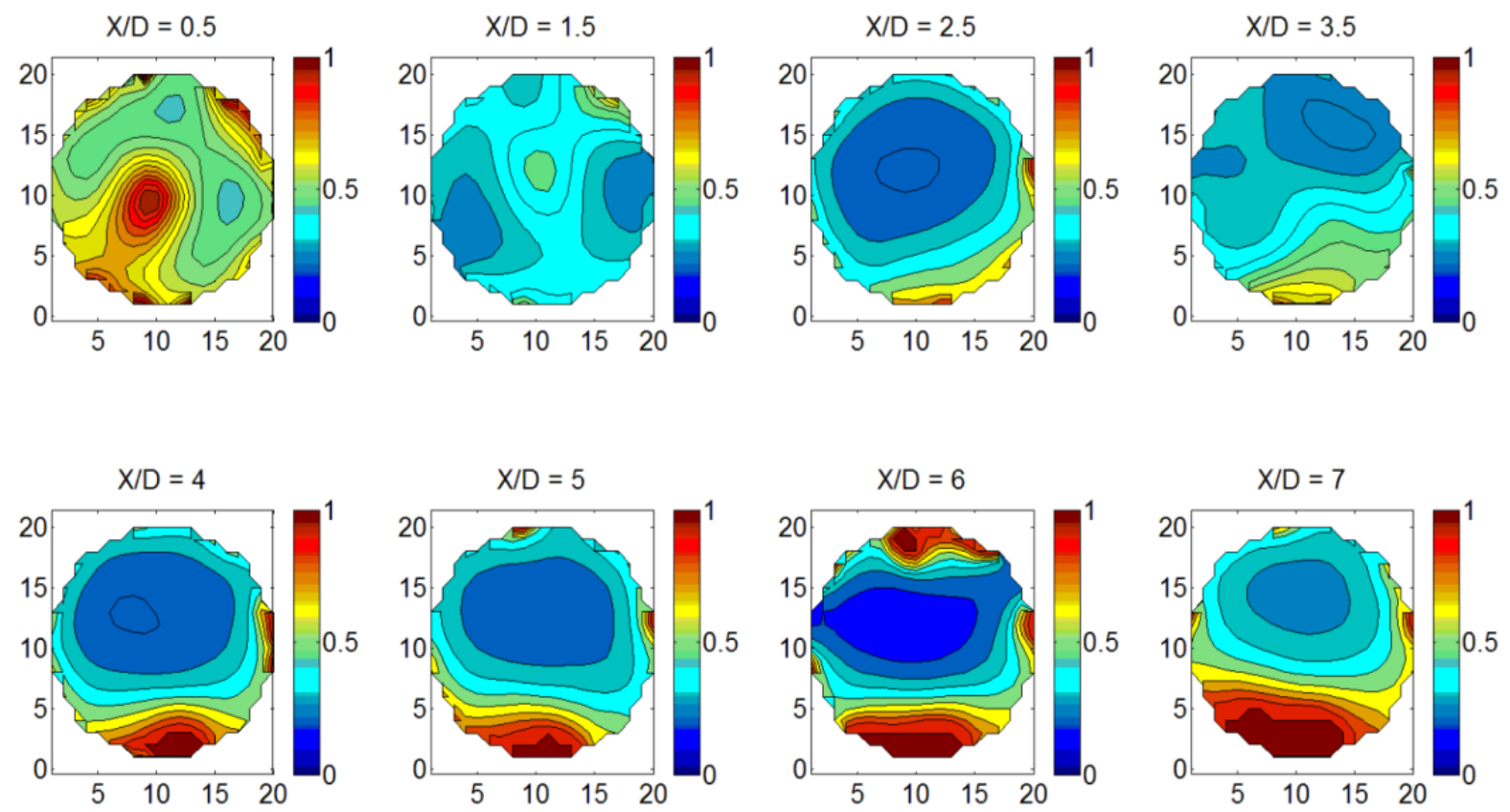

\section{Figure 31: Conductivity tomograms at different downstream locations for 40 psig, 20 GPM and $70 \%$ GVF}

It can be inferred from the conductivity tomograms shown in Figure 31 that the flow immediately downstream of the slotted orifice plate $(\mathrm{X} / \mathrm{D}=1.5)$ is well-mixed and exists in dispersed bubble flow regime with the two phases uniformly distributed over the entire crosssection of the pipe. This location is the best approximation for the location where the twophase flow gets most homogeneous. At the downstream location $\mathrm{X} / \mathrm{D}=2.5$, the two-phases begin to separate and the flow transits to annular flow with the air flowing in the central core 
and the water flowing along the pipe-wall. The liquid film is thicker at the bottom than at the top due to gravity. At farther downstream location (X/D>6), the flow exists in stratified flow regime with the water flowing along the bottom of the pipe and air flowing in the top due to gravity. Hence the two-phase flow becomes most homogeneous at locations closest to the slotted orifice plate.

\subsection{Concentration tomogram}

In order to determine the location where the two-phase flow becomes fully-homogeneous, the concentration tomograms are analyzed. Similar to the conductivity tomograms, the concentration tomograms are also analyzed to study the effect of water flow rate, GVF and downstream distance on the concentration distribution.

\subsubsection{Effect of water flow rate}

Increasing the water flow rate decreases the concentration when the pressure upstream of the slotted orifice plate and GVF are maintained constant. The effect of increasing water flow rate from $20 \mathrm{GPM}$ to $60 \mathrm{GPM}$ on the concentration distribution when maintaining the pressure upstream constant at 40 psig and the GVF at $40 \%$ is shown in Figure 32.
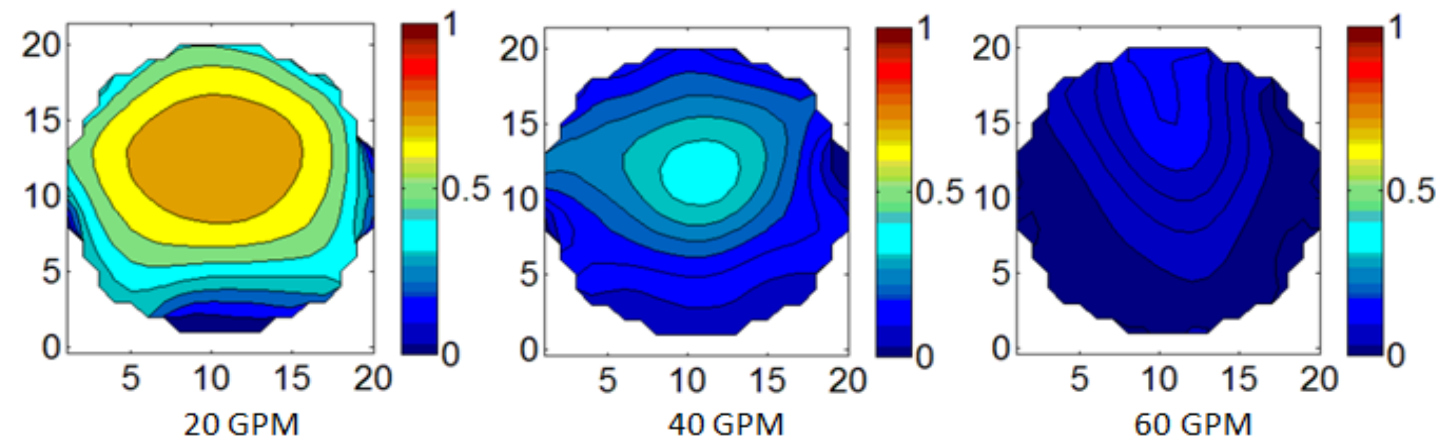

Figure 32: Effect of increasing water flow rate on concentration distribution for 40 psig and $40 \% \mathrm{GVF}$ at downstream location $X / D=1.5$ 
It can be seen from the concentration tomograms in Figure 32 that on increasing the water flow rate from 20 GPM to 60 GPM, while maintaining the upstream pressure and GVF constant, the concentration decreases as shown by the presence of more blue zones in the tomograms.

At low water flow rate of $20 \mathrm{GPM}$, it can be seen that the flow exists in dispersed bubble flow regime with uniform distribution of the components throughout the entire cross-section of the pipe. As the water flow rate increases to $40 \mathrm{GPM}$, the flow transits to annular flow with the air present in the central core and water flowing along the periphery of the pipe. With further increase in the water flow rate to $60 \mathrm{GPM}$, the flow becomes stratified flow with the water flowing along the bottom of the pipe and the air flowing on top due to gravity. Thus the flow transits from bubble flow regime to stratified flow on increasing the water flow rate.

\subsubsection{Effect of GVF}

The concentration increases with increasing GVFs when the pressure upstream and water flow rate are maintained constant. The effect of increasing GVFs from 5\% to $70 \%$ on the concentration distribution when the water flow rate is maintained constant at 20 GPM and the pressure upstream is maintained constant at 40 psig is shown in Figure 33. 

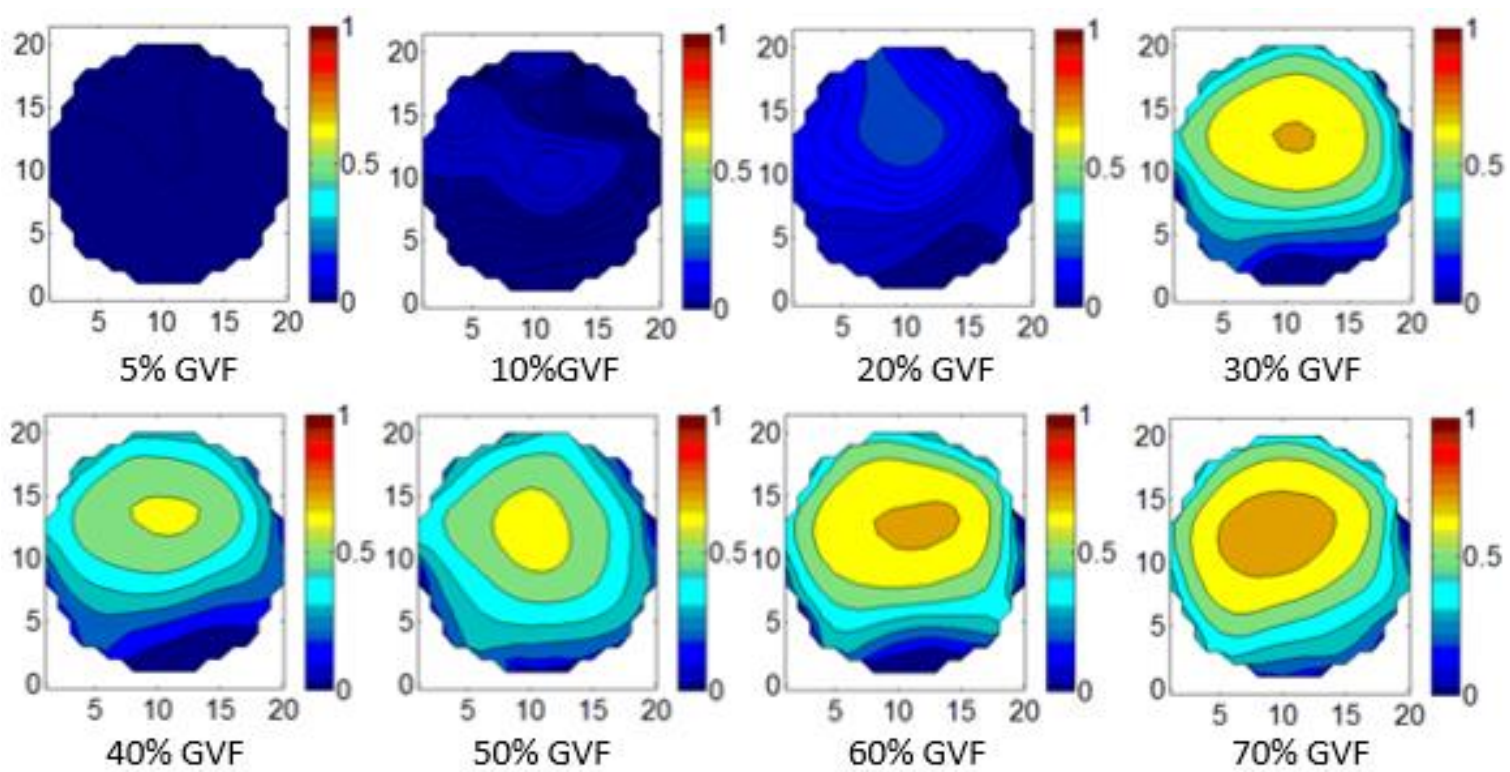

Figure 33: Effect of increasing GVF on concentration distribution at 40 psig and 20 GPM at downstream location $X / D=2.5$

It can be seen that on increasing the GVFs from 5\% to $70 \%$, when the pressure upstream and the water flow rate are maintained constant, the blue zone in the tomograms decreases which indicates the presence of more air in the mixture. Thus the mean concentration value increases on increasing GVF.

At low GVF values (5\%-20\%), the liquid flow rate is high enough to break up the gas into bubbles which rise to the top of the flow due to buoyancy. Thus the flow exists in dispersed bubble flow regime with the gas phase appearing as distinct bubbles in the continuous water phase. When GVF is increased (up to $40 \%$ ), the flow gets separated with the water flowing along the bottom of the pipe and air flowing at the top due to gravity and hence the flow exists in stratified flow regime. At higher GVFs (above 40\%), the flow becomes annular flow as the gas velocity becomes very large. The gas flows in the central core and the liquid forms a continuous annular film around the pipe-wall. The liquid film is thicker at the bottom than at the top due to gravity. 


\subsubsection{Effect of downstream distance}

The distribution of the two phases in the flow at different locations downstream of the slotted orifice plate can be analyzed from the concentration tomograms. The concentration tomograms obtained at eight different downstream locations for a particular flow condition with upstream pressure at 40 psig, water flow rate at 20 GPM and GVF at 70\% are shown in Figure 34.
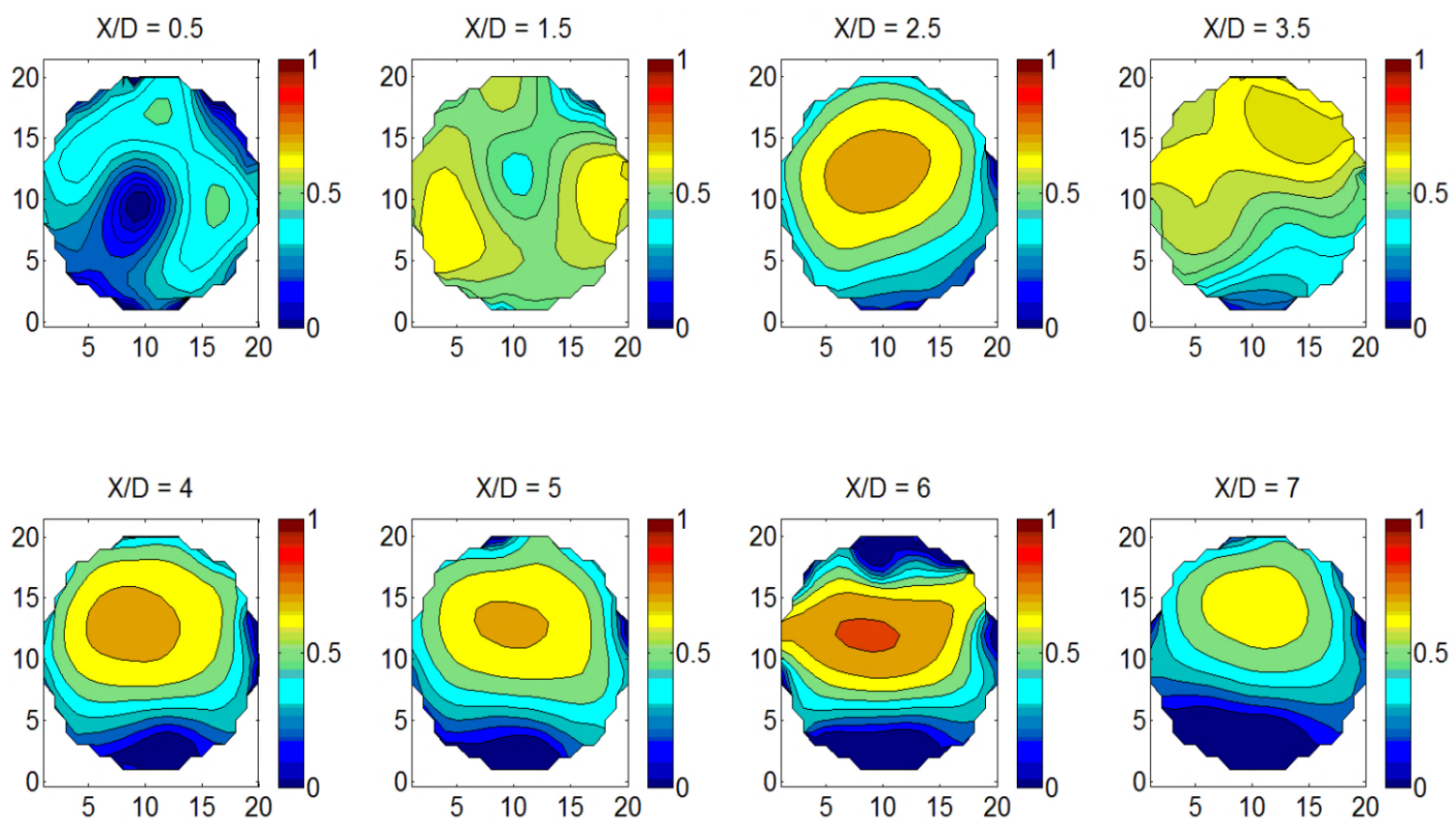

Figure 34: Concentration tomograms at different downstream locations for 40 psig, 20 GPM and $70 \%$ GVF

By analyzing the concentration tomograms, the flow regime downstream of the slotted orifice plate can be determined. From Figure 34, it can be seen that at $\mathrm{X} / \mathrm{D}=1.5$, the two phases are well-mixed with the air bubbles uniformly distributed over the entire cross-section of the tomogram. Hence the two-phase flow exists in dispersed bubble flow regime at this location. At $\mathrm{X} / \mathrm{D}=2.5$, the flow gets separated and transits to annular flow as the gas flows in the central core and the liquid forms a continuous annular film around the pipe-wall. At farther 
downstream location $(\mathrm{X} / \mathrm{D}>6)$, the flow transits to stratified flow as the two phases are completely separated. The liquid phase flows along the bottom of the pipe and the gas phase flows at the top of the pipe due to gravity. The location where the flow exists as dispersed bubble flow is the best approximation for the most homogeneous location. Once the two phases get separated it can never become homogeneous. Similarly, the flow regime downstream of the slotted orifice plate for other flow conditions can be analyzed from the concentration tomograms.

\subsection{Determining most homogeneous location}

In order to determine the location where the two-phase flow becomes most homogeneous, the standard deviation of the pixel values within each concentration tomographic image is calculated. It is based on the fact that at the most homogeneous location, the tomograms contain well-mixed components throughout the entire cross-section whereas at the heterogeneous location, it contains regions of differing component concentration. Hence the location where the standard deviation of the pixel values is the lowest can be concluded to be the location where the two-phase flow is closest to being fully homogeneous.

Figure 35 shows a 3-D graph plotted with the different downstream locations (X/D) on the xaxis, the standard deviation of concentration tomogram pixel values on the $y$-axis and the different GVF values on the z-axis for a particular flow condition with upstream pressure at 20 psig and water flow rate at 20 GPM. Similar graphs for other flow conditions are plotted and are shown in the appendix. 


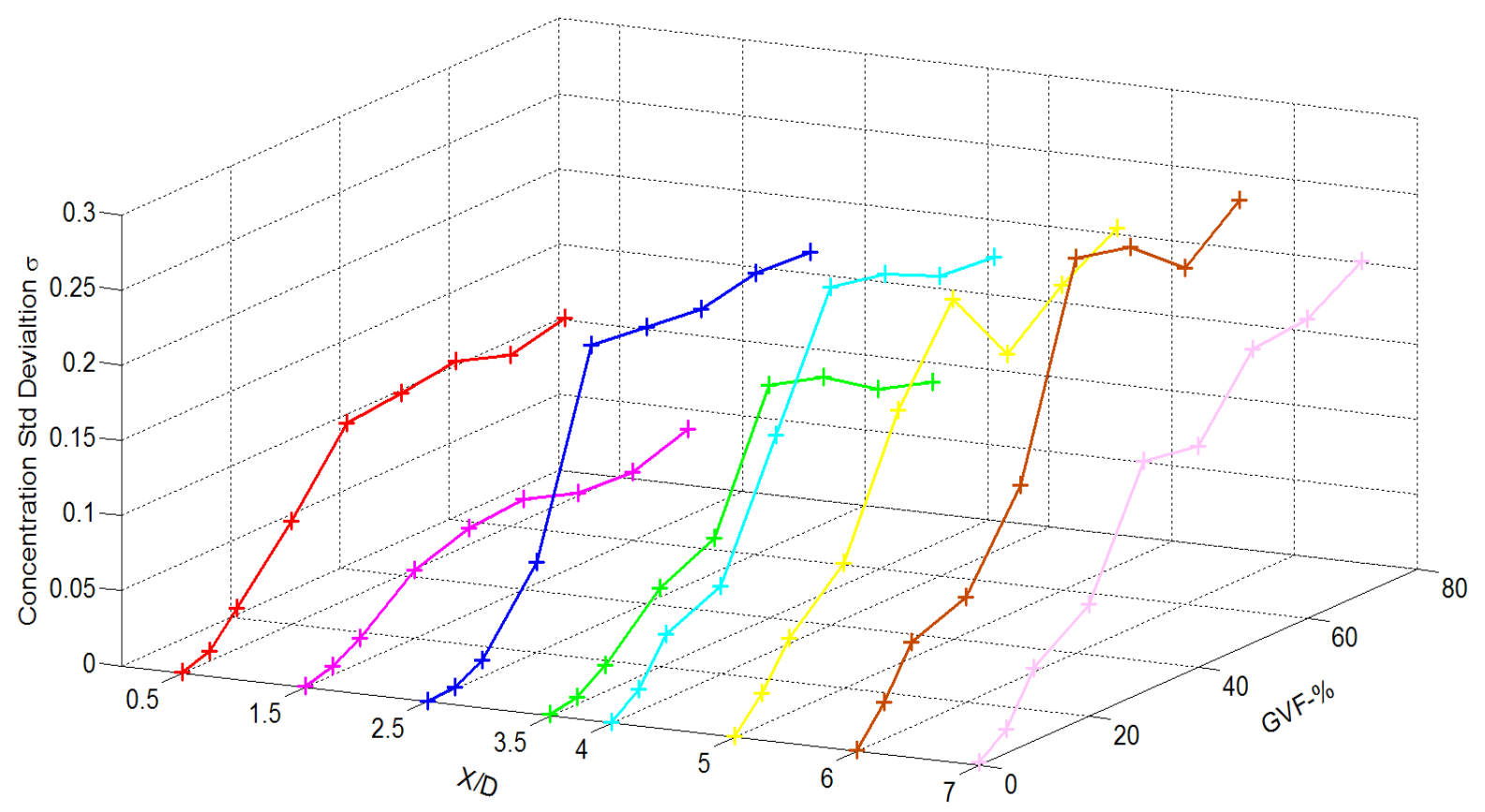

Figure 35: Standard deviation of the concentration tomogram pixel values at different downstream locations for 20 psig \& 20 GPM

It can be inferred from Figure 35 that at downstream location $\mathrm{X} / \mathrm{D}=1.5$, the standard deviation of the pixel values is the lowest for most GVF values. Hence for the given flow condition, the two-phase flow best represents the most homogeneous location at $\mathrm{X} / \mathrm{D}=1.5$.

Similarly, the location where the two-phase flow becomes most homogeneous for other flow conditions can be determined.

It can be found out that the downstream location from the slotted orifice plate where the twophase flow becomes most homogeneous is not the same for all flow conditions. For instance, for a flow condition with upstream pressure of $20 \mathrm{psig}$, water flow rate of 20 GPM and GVF of $50 \%$, the most homogenized location is at $\mathrm{X} / \mathrm{D}=1.5$ (Figure 35) whereas for a flow condition with upstream pressure of $60 \mathrm{psig}$, water flow rate of $20 \mathrm{GPM}$ and GVF of $20 \%$, the most homogenized location is at $\mathrm{X} / \mathrm{D}=2.5$ (Figure 36). 


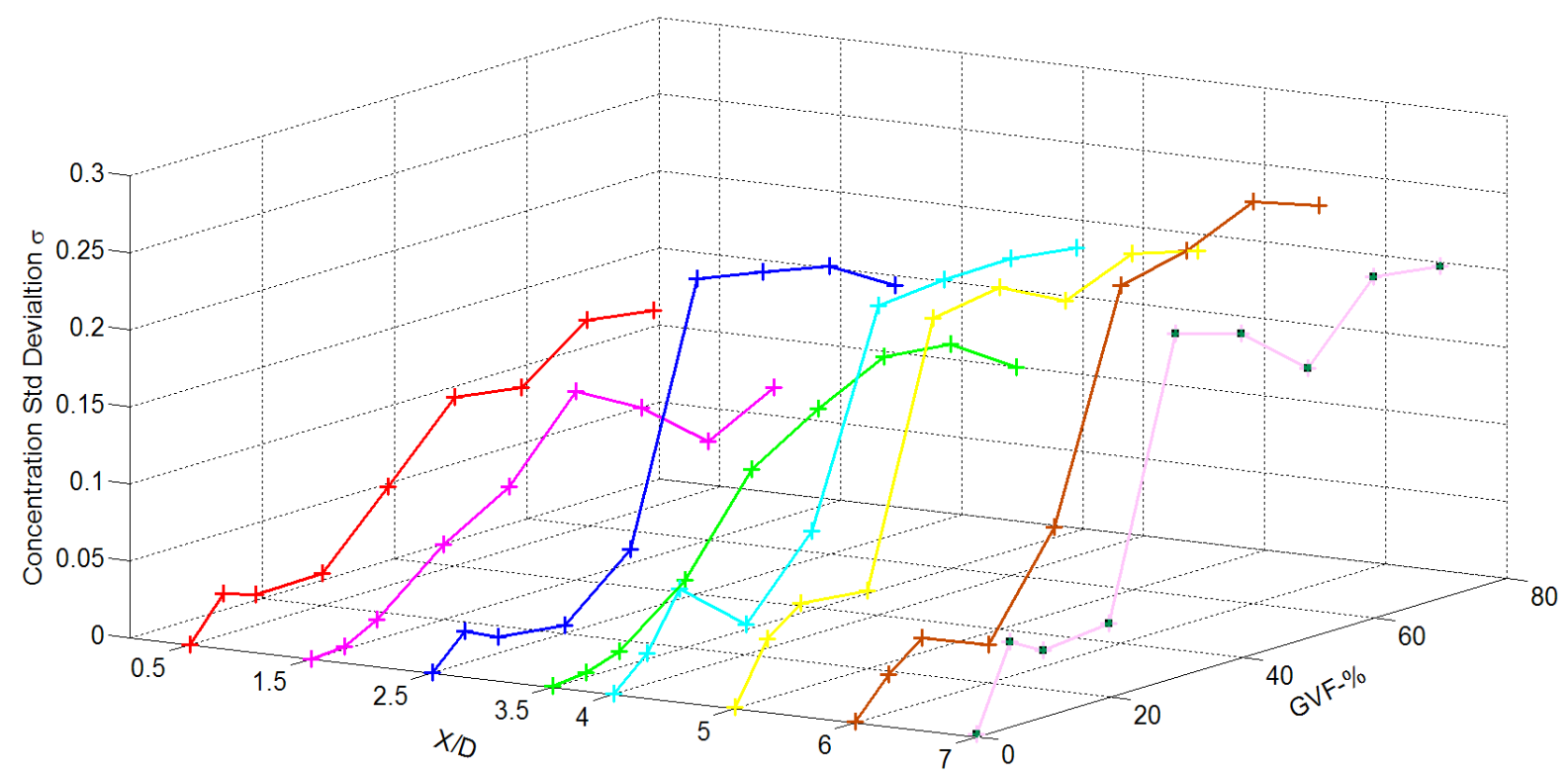

Figure 36: Standard deviation of the concentration tomogram pixel values at different downstream locations for 60 psig \& 20 GPM

In order to determine the optimum location where the two-phase flow becomes most homogeneous for most of the flow conditions, a histogram is plotted for all the flow conditions. The histogram is plotted with the standard deviation of pixel values on the $\mathrm{x}$-axis and the number of occurrences on the y-axis. 


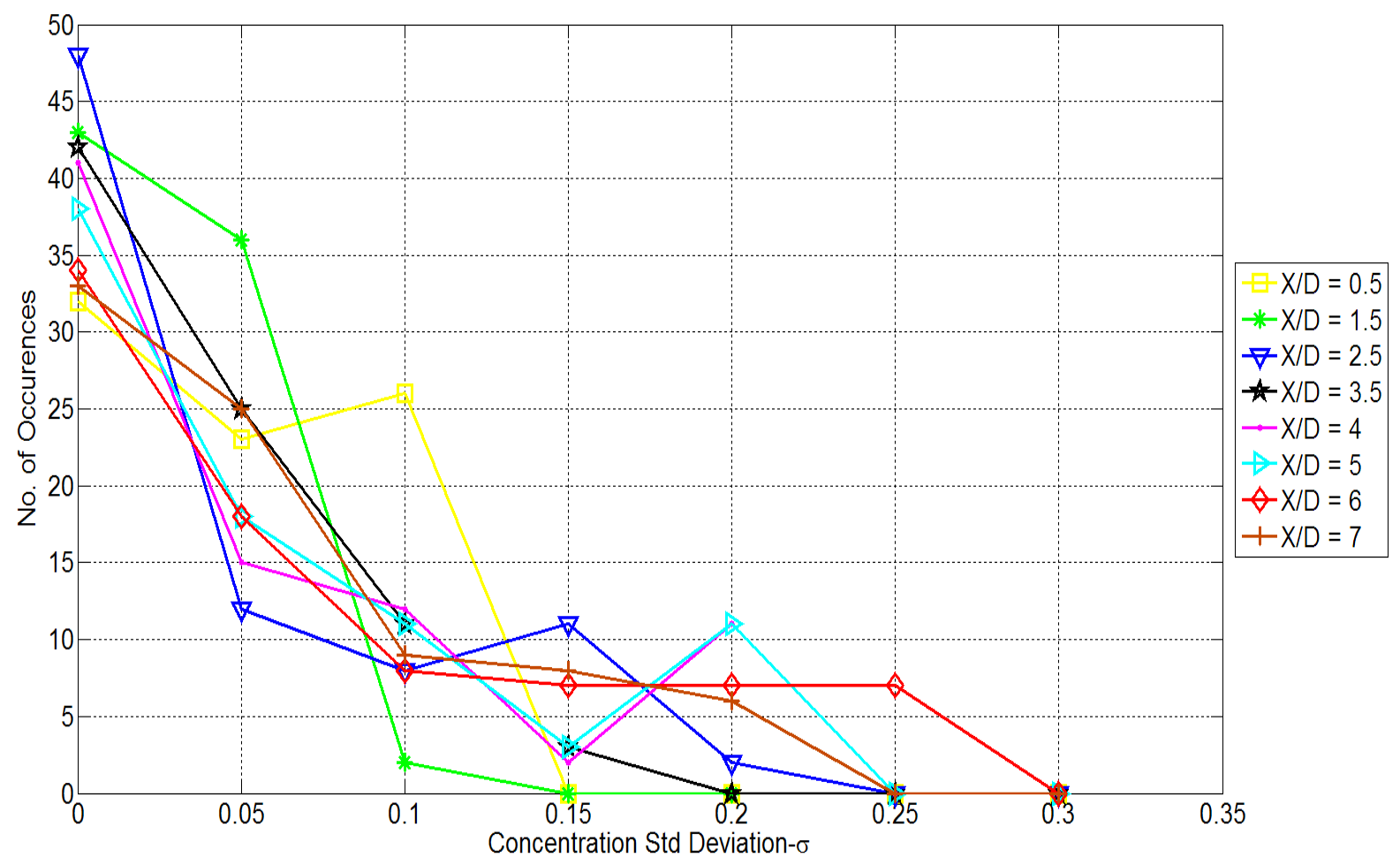

Figure 37: Histogram displaying the number of occurrences of the standard deviation values for each downstream location

From Figure 37, it can be seen that the number of occurrences of lower standard deviation is highest for $\mathrm{X} / \mathrm{D}=1.5$. Therefore, the optimum location where the two-phase flow becomes homogeneous for most flow conditions is at $1.5 \mathrm{D}$ downstream of the slotted orifice plate. 


\subsection{Flow regime identification}

Identifying the flow regime is critical for two-phase flow measurement as most of the existing two-phase flow meters are flow-regime dependent.

From the volumetric flow rate of air and water and the cross-sectional area of the pipe, the superficial velocity of air and water can be calculated from equation 3-9 and 3-10 respectively. By superimposing the experimental test points upstream of the flow meter with the flow regime map shown in Figure 4, the upstream flow regime for each flow condition is identified.

It can be seen from Figure 38 that upstream of the slotted orifice plate, the two-phase flow lies in the elongated bubble flow regime and the slug flow regime.

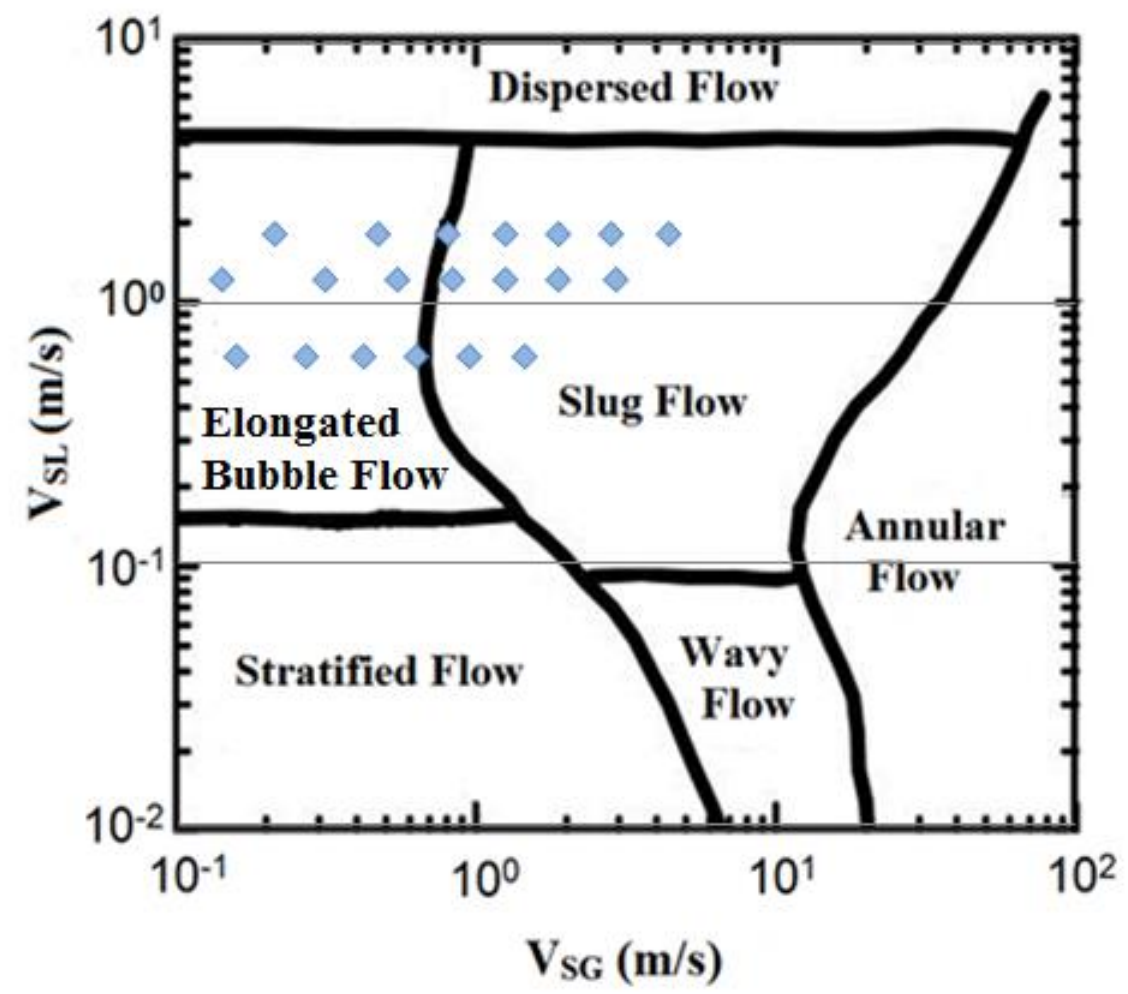

Figure 38: Upstream flow regime map with experimental data points 
The flow conditions for which the upstream flow regime is in elongated bubble flow regime and slug flow regime is shown below.

\begin{tabular}{|c|c|c|c|}
\hline \multicolumn{2}{|c|}{ ELONGATED BUBBLE FLOW } & \multicolumn{2}{|c|}{ SLUG FLOW } \\
\hline $\begin{array}{c}\text { Water Flow Rate } \\
\text { (GPM) }\end{array}$ & $\begin{array}{c}\text { Gas Volume } \\
\text { Fraction } \\
(\%)\end{array}$ & $\begin{array}{c}\text { Water Flow Rate } \\
\text { (GPM) }\end{array}$ & $\begin{array}{c}\text { Gas Volume } \\
\text { Fraction } \\
(\%)\end{array}$ \\
\hline 20 & 0 & 20 & 50 \\
\hline 20 & 5 & 20 & 60 \\
\hline 20 & 10 & 20 & 70 \\
\hline 20 & 20 & 40 & 40 \\
\hline 20 & 30 & 40 & 50 \\
\hline 20 & 40 & 40 & 60 \\
\hline 40 & 0 & 40 & 70 \\
\hline 40 & 5 & 60 & 30 \\
\hline 40 & 10 & 60 & 40 \\
\hline 40 & 20 & 60 & 50 \\
\hline 40 & 30 & 60 & 60 \\
\hline 60 & 0 & 60 & 70 \\
\hline 60 & 5 & & \\
\hline 60 & 10 & & \\
\hline 60 & 20 & & \\
\hline
\end{tabular}

Table 7: Characterizing elongated bubble flow and slug flow conditions 
Determining the flow regime transition as the two-phase flow passes through the slotted orifice plate is very important. The flow regime downstream of the slotted orifice plate can be determined by analyzing the concentration tomograms.

For a flow condition with upstream pressure at 20 psig, water flow rate at 20 GPM and $40 \%$ GVF, the flow exists in elongated bubble flow regime at the upstream of the plate. The concentration tomograms for this flow condition are shown in Figure 39.
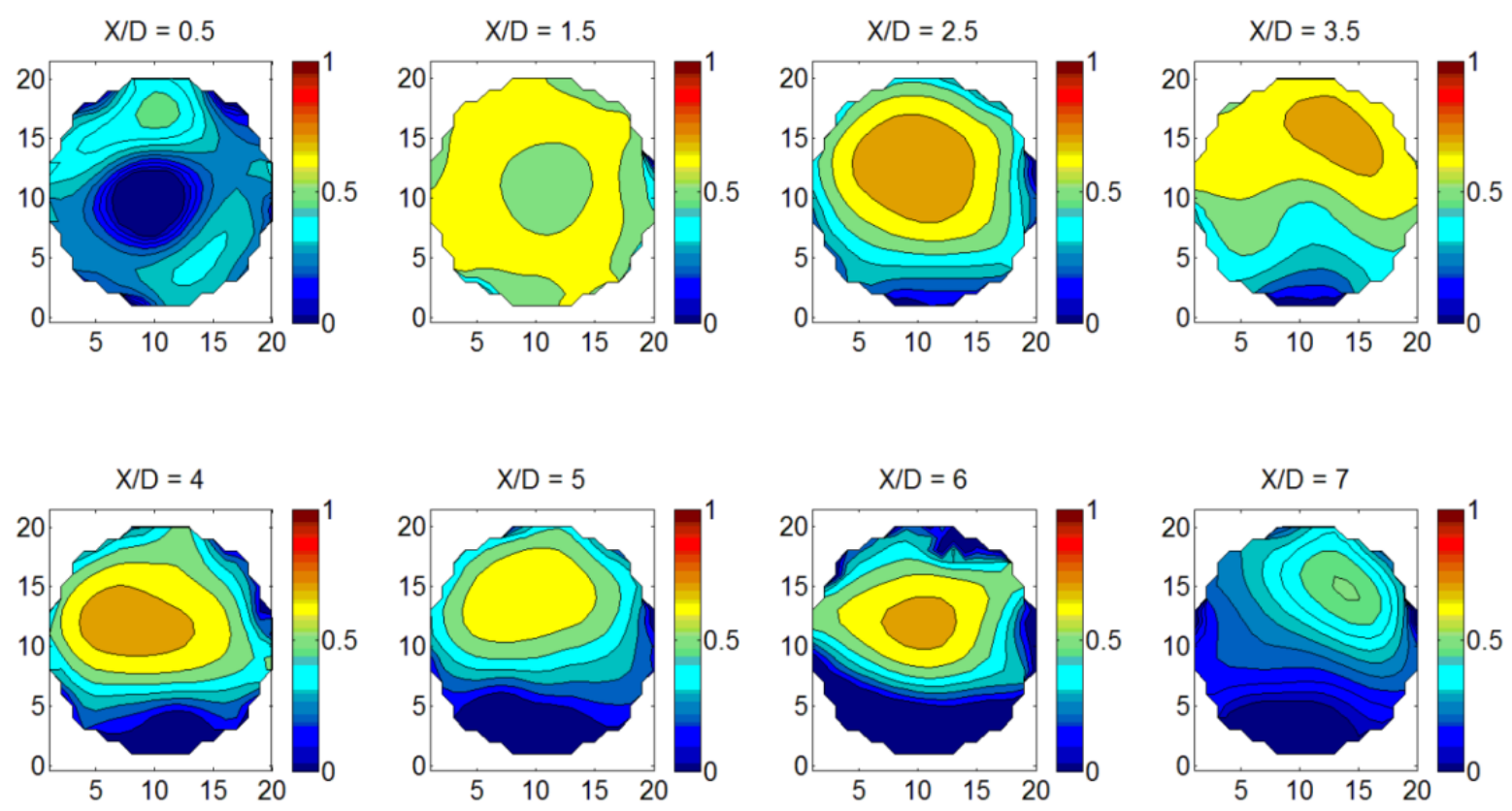

\section{Figure 39: Concentration tomogram for an elongated bubble flow upstream condition of 20 psig, 20 GPM and 40\% GVF}

From the tomograms it can be seen that at location $\mathrm{X} / \mathrm{D}=1.5$, the concentration distribution is most uniformly distributed throughout the entire cross-section of the pipe and hence this is the location where the two-phase flow is most homogeneous. It can also be seen that the flow exists as dispersed bubble flow regime until $\mathrm{X} / \mathrm{D}=3.5$ with uniform distribution of air and water. As the axial distance further increases (X/D>3.5), the flow transits to annular flow with the air flowing in the center and water flowing along the pipe-wall. And at downstream location 
$\mathrm{X} / \mathrm{D}=7$, the two-phase flow becomes stratified with the water flowing along the bottom of the pipe and the air flowing at the top due to gravity. Thus for an elongated bubble flow upstream condition, the two-phase flow becomes most homogeneous closer to the slotted orifice plate and exists as dispersed flow until $\mathrm{X} / \mathrm{D}=3.5$ after which it transits to annular and stratified flow regime.

Now let's consider a slug flow upstream condition. For a flow condition with upstream pressure at 60 psig, water flow rate at $60 \mathrm{GPM}$ and $70 \% \mathrm{GVF}$, the flow exists in slug flow regime upstream of the slotted orifice plate as seen from Table 7. The concentration tomograms for this flow condition are shown in Figure 40.
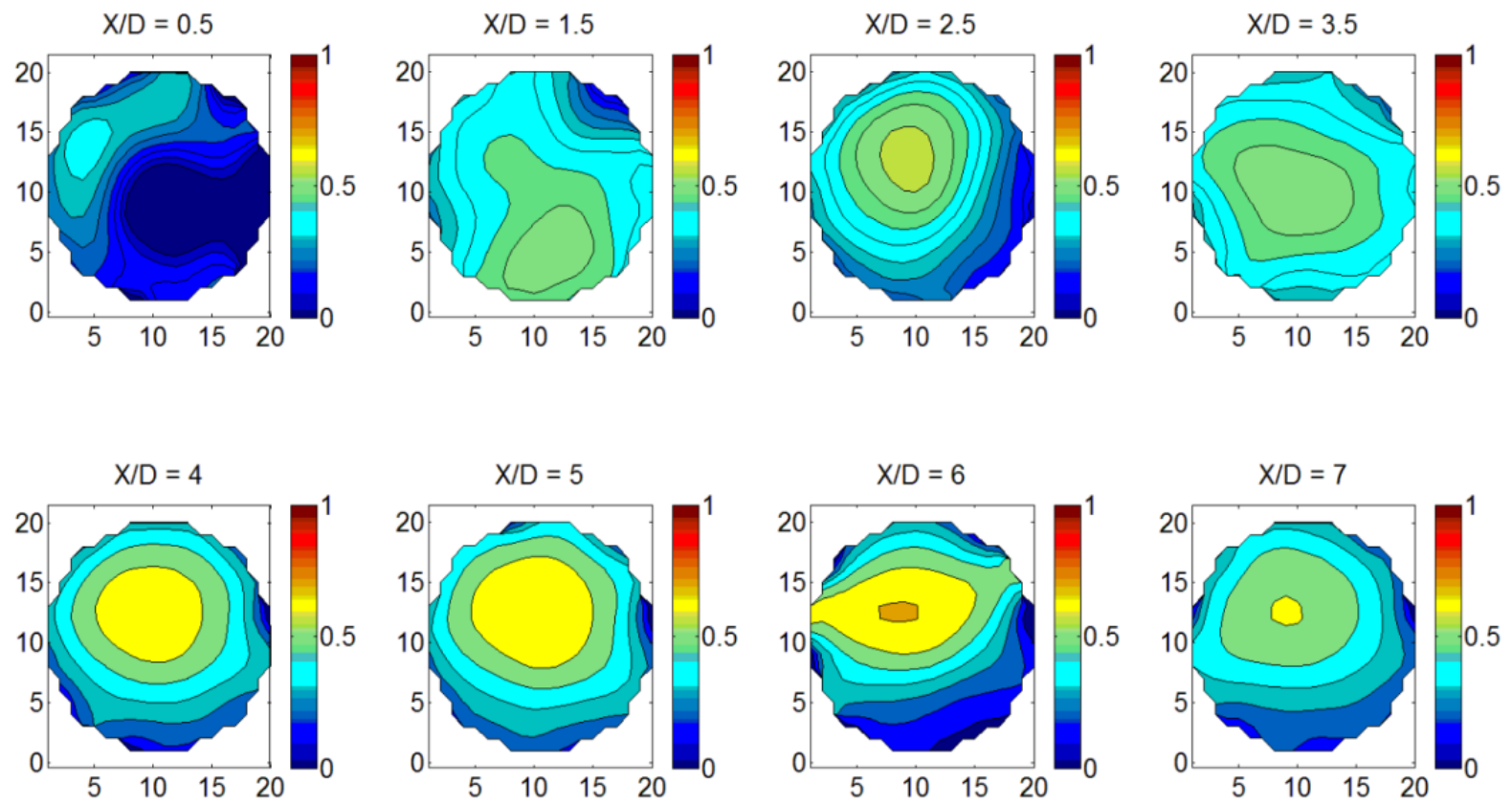

Figure 40: Concentration tomogram for a slugged flow upstream condition of 60 psig, 60 GPM and $70 \%$ GVF

Similarly, it can be seen from the tomograms that the two-phase flow becomes most homogeneous at $\mathrm{X} / \mathrm{D}=1.5$ for this condition and until $\mathrm{X} / \mathrm{D}=3.5$, the flow exists in dispersed flow regime with uniform distribution of air and water. And at axial location X/D>3.5, the flow 
transits to annular flow regime as the gas phase flowing in the center of the pipe and the liquid phase flowing around the pipe-wall. The flow continues to exist as annular flow until $\mathrm{X} / \mathrm{D}=6$. At axial location $\mathrm{X} / \mathrm{D}=7$, the flow transits from annular flow to stratified flow with the water flowing along the bottom of the pipe and air flowing at the top due to gravity. Thus it can be seen that the flow regime transition for an elongated bubble flow upstream condition and for a slug flow upstream condition is very similar. Also for both upstream conditions, the two-phase flow becomes most homogenized closer downstream of the slotted orifice plate.

Therefore it can be concluded that the slotted orifice plate has no dependence on the upstream flow conditions and homogenizes the two-phase flow similarly for different upstream flow conditions.

\subsection{Gas void fraction}

Gas void fraction is one of the most important parameters used to characterize the two-phase flows and to obtain the relative average velocity of the two phases. In the homogeneous condition, the two-phases have the same velocity and hence there is no slip between the two phases. So the gas void fraction will be equal to the gas volume fraction (GVF) at the no-slip condition. But at the non-homogeneous location, the cross-sectional area occupied by liquid phase will be greater than the area occupied by the gas phase. This is due to the effect of slip between the liquid and gas phase as the lighter gas phase moves much faster than the liquid phase and also because the liquid phase tends to accumulate in the pipe segments. Hence the gas void fraction measured will be lesser than the gas volume fraction due to liquid buildup in the section as shown in Figure 41. The gas void fraction is calculated from the mean concentration value using equation 3-11. 
Possible metering errors in the two-phase flow measurements can be determined by calculating the gas void fraction for each flow condition and comparing it to the gas volume fraction (GVF) measured by the flow meters. The ratio of GVF and gas void fraction gives the slip ratio between the two phases. At the no-slip condition (homogeneous condition), the gas void fraction will be equal to the GVF measured and hence the slip ratio will be equal to one. But if there is slip between the two phases, then due to the liquid buildup in the section and the local gas void fraction measured will be lesser than the GVF leading to possible metering errors in the two-phase flow measurement.

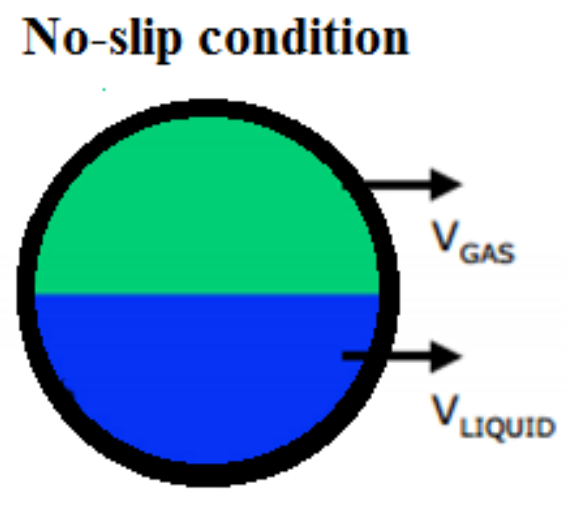

$$
\text { GVF }=\lambda_{\text {gas }}=\mathbf{5 0} \%
$$

\section{Slip Condition}

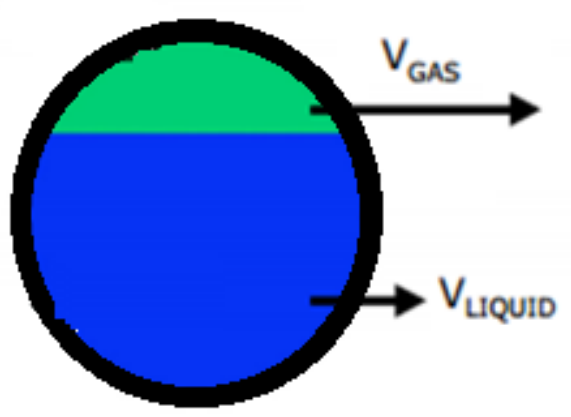

GVF $=\mathbf{5 0} \%, \lambda_{\text {gas }}<\mathbf{5 0} \%$

\section{Figure 41: Relationship between Gas Void Fraction and GVF in slip and no-slip condition}

A 3-D graph is plotted for a flow condition with water flow rate at 40 GPM and pressure upstream at $40 \mathrm{psig}$ with the downstream locations X/D on the $\mathrm{x}$-axis, GVFs on the $\mathrm{y}$-axis and gas void fraction on the z-axis as shown in Figure 42. The standard deviation of GVF measured by the flow meter is shown by horizontal error bars and the standard deviation of gas void fraction is shown by vertical error bars. 


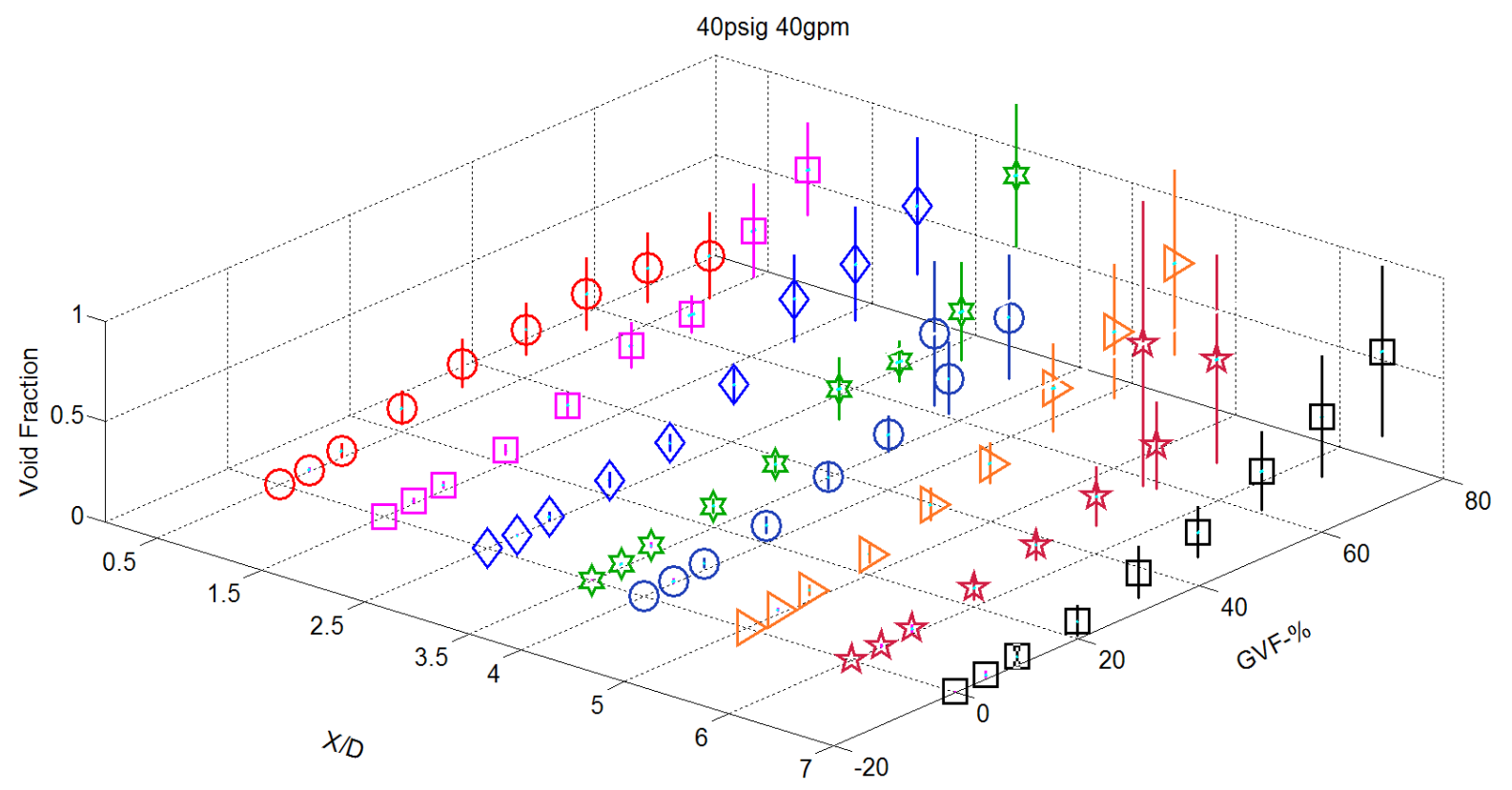

Figure 42: Graph displaying the standard deviation of Gas Void Fraction and GVF for different $G V F$ values at 40 psig and 40 GPM

It can be seen from Figure 42 that at the downstream location $\mathrm{X} / \mathrm{D}=1.5$, the gas void fraction and the GVF are almost equal. This implies that there is no appreciable slip between the two phases and hence this is the location where the two-phase flow becomes most homogeneous. But at the other downstream locations, it can be seen that the gas void fraction measured is much lower than the GVF due to the slip condition. Since the gas void fraction is much lower than the GVF measured, there is excessive liquid build up in the section which leads to possible metering errors in the two-phase flow measurement. 


\subsection{Slip ratio}

Slip ratio is the ratio of velocity of gas phase to the liquid phase. It is calculated by dividing the Gas Volume Fraction (GVF) and the Gas Void Fraction as shown in equation 3-8.

At the most homogeneous location, the velocity of the gas phase and the liquid phase are almost equal and also the gas void fraction is very close to the GVF. Hence the slip ratio will be close to one at the most homogeneous location. But at the non-homogeneous location, the gas void fraction is lesser than the GVF and the velocity of gas phase is much higher than the velocity of the liquid phase. Hence the slip ratio is greater than one at the non-homogeneous location.

For a flow condition with water flow rate at $20 \mathrm{GPM}$, upstream pressure at $20 \mathrm{psig}$ and at different GVF values, the slip ratio at different downstream locations is shown in Figure 43. It can be seen that the slip ratio is closer to 1 at the downstream location $\mathrm{X} / \mathrm{D}=1.5$ but is much greater than 1 at the other downstream locations. This again proves that the downstream location $\mathrm{X} / \mathrm{D}=1.5$ is the most homogenized location for this flow condition.

Also from the concentration tomograms, it could be seen that until $\mathrm{X} / \mathrm{D}=3.5$, the flow exists in dispersed bubble flow regime. At this flow regime, the difference in the phase velocities is not very high and hence the slip ratio is lesser. But after $\mathrm{X} / \mathrm{D}=3.5$, the two-phase flow gets separated into annular and stratified flow where the difference in phase velocities become much higher. The gas phase moves much faster than the liquid phase and hence the slip ratio is higher. 


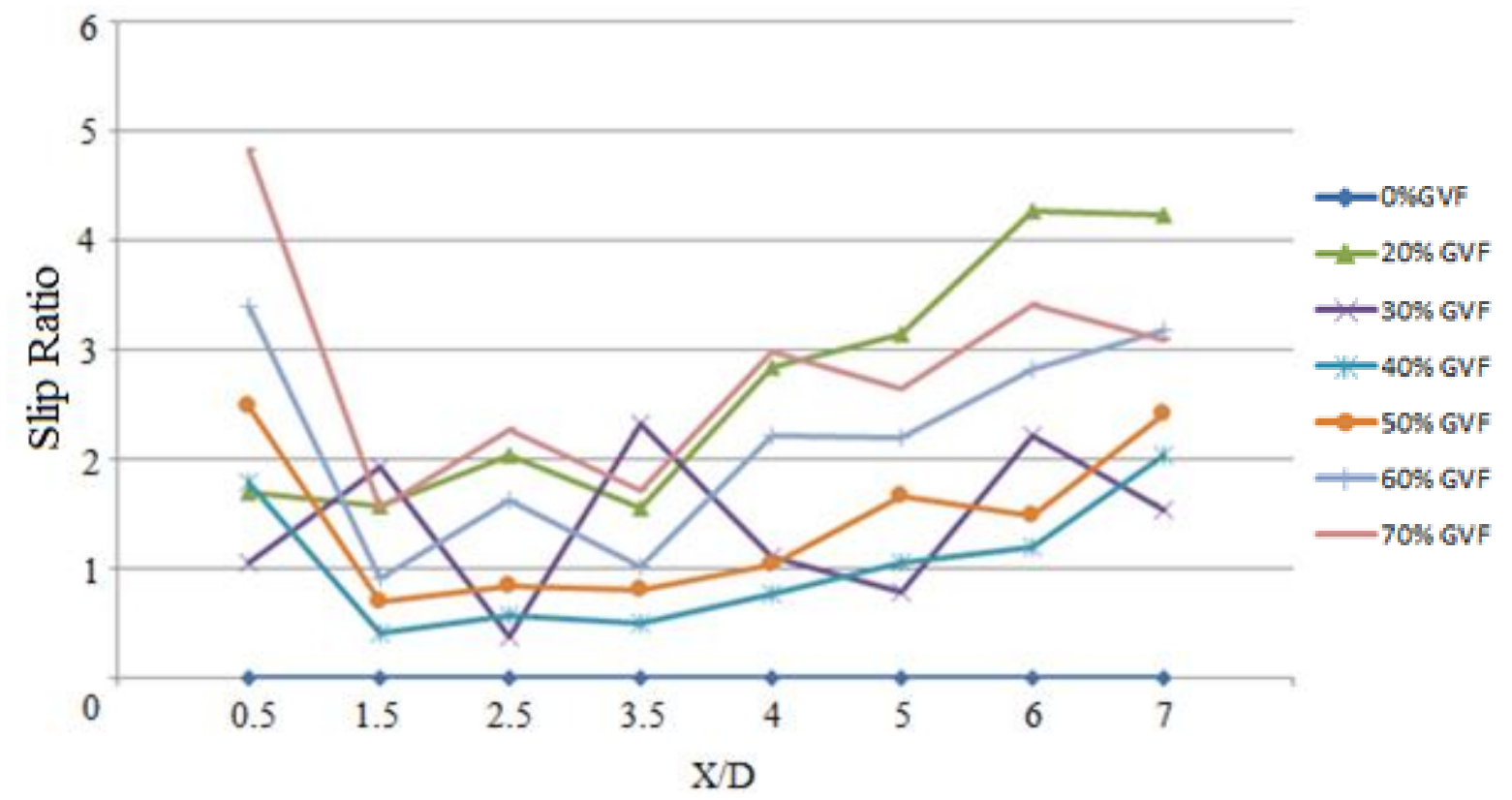

Figure 43: Plot of slip ratio V/s downstream locations for 20 psig \& 20 GPM

Thus it can be seen from the Figure 43 that at the most homogenized location $X / D=1.5$, the slip ratio is closer to one but after $\mathrm{X} / \mathrm{D}=3.5$ the slip ratio increases drastically as the velocity of gas phase is much higher than the liquid phase. 


\section{CONCLUSION}

The following conclusions were derived from this study:

- By analyzing the conductivity and concentration tomograms, it is determined that the two-phase flow becomes most homogenized at closer distances downstream of the slotted orifice plate.

- The location where the two-phase flow becomes most homogeneous varies for different upstream flow conditions. The optimum location where the two-phase flow becomes most homogeneous for most flow conditions is determined to be $\mathrm{X} / \mathrm{D}=1.5$.

- Increasing the water flow rate increases the mean conductivity value whereas increasing the GVF decreases it.

- Increasing the water flow rate decreases the mean concentration value whereas increasing the GVF increases it.

- Varying the pressure upstream of the slotted orifice plate has no significant effect on the conductivity and concentration distribution.

- The transition in flow regimes as the two-phase flow passes through the slotted orifice plate is similar for both elongated as well as slugged flow upstream conditions. Hence the slotted orifice plate has no dependence on the upstream flow conditions and homogenizes the two-phase flow in a similar manner for both flow conditions.

- In the most homogeneous location, the two phases travel at the same velocity and hence there is no slip between the two phases. The Gas Void Fraction measured is equal to the Gas Volume Fraction (GVF) as there is no gas or liquid buildup in the section. Hence accurate measurements can be taken at the most homogeneous location. 
- At the non-homogeneous location, the Gas Void Fraction measured is much lower than the GVF due to slip. This implies liquid build up in the section leading to possible metering errors in the two-phase flow measurement.

- The slip ratio is closer to 1 at the most homogenized location as the Gas Void Fraction measured is very close to the GVF and it is greater than 1 at other locations as the Gas Void Fraction measured is lower than the GVF due to slip condition.

\subsection{Recommended future work}

- Since corrosion of metal parts in pumps is a common phenomenon, the effect of corrosion on the conductivity measurement can be analyzed.

- Effect of pipe size on the homogeneous location can be analyzed by conducting experiment with increasing pipe sizes.

- Experiment can be conducted for higher water flow rates (above $60 \mathrm{GPM}$ ) and the result obtained can be compared to this to analyze the effect of increasing water flow rates.

- ERT has the limitation of measuring the conductivity value of the mixture upto $70 \%$ GVF. Higher GVFs upto $100 \%$ should be studied by using other process tomography methods. 


\section{REFERENCES}

[1] Perdue, J. M., "Five Critical Technologies Will Determine Industry's Future Success", Hart's First Look, SPE 1997:4 (Aug.).

[2] Pirouzpanah, S., Cevik, M., \& Morrison, G. L., "Multiphase Flow Measurements Using Coupled Slotted Orifice Plate and Swirl Flow Meter", Flow Measurement and Instrumentation, Vol. 40, 9 (2014) 157-161.

[3] Geng, Y. F., Zheng, J. W., Shi, T. M., "Study on the Metering Characteristics of a Slotted Orifice for Wet Gas Flow", Flow Measurement and Instrumentation, 17 (2006) 123-128.

[4] Morrison, G. L., Terracina, D., \& Brewer, C., "Response of a Slotted Orifice Flow Meter to an Air/Water Mixture", Flow Measurement and Instrumentation, 12 (2001) 175-180.

[5] Macek, M. L., “A Slotted Orifice Plate Used as a Flow Measurement Device”, M. S. Thesis, Texas A\&M University, College Station, TX, 1993.

[6] Cevik, M., "Evaluation of a Close Coupled Slotted Orifice, Electrical Impedance and Swirl Flow Meter for Multiphase Flow”, M. S. Thesis, Texas A\&M University, TX, 1993.

[7] Hewitt, G. F., "Multiphase Fluid Flow and Pressure Drop", Heat Exchanger Design Handbook, Vol. 2, Begell House, New York, NY.

[8] Mandhane, J. M., Gregory, G. A., Aziz, K., "A Flow Pattern for Gas-Liquid Flow in Horizontal Pipes”, International Journal of Multiphase Flow, 1 (4), 537-553.

[9] Williams, R. A., Beck, M. S., "Process Tomography: Principles, Techniques and Applications", Butterworth-Heinemann Ltd., Chapter 3, $41-45$ (1995).

[10] Helmestine, A., "Flow of Electric Current through Materials", Engineering Materials Science, Upper Saddle River, New Jersey: Prentice Hall, Vol. 1, $3^{\text {rd }}$ Edition, 561 (1995). 
[11] ITS System 2000 Version 7.0 p2+ Electrical Resistance Tomography System- User's Manual; Industrial Tomography Systems, 11 (2009).

[12] Sparks, S., "Two Phase Mixing Comparison, Oil Contamination Comparison and Manufacturing Accuracy Effect On Calibration Of Slotted Orifice Meters", M.S. Thesis, Texas A\&M University, College Station, TX, 2004.

[13] Muralidharan, V., "Response Of A Slotted Plate Flow Meter To Horizontal Two-Phase Flow”, M.S. Thesis, Texas A\&M University, College Station, TX, 2003

[14] Razzak, S. A., Barghi, S., Zhu, J., “Application of Electrical Resistance Tomography on Liquid-Solid Two-Phase Flow Characterization in an LSCFB Riser", Chemical Engineering Science, Vol. 64, Issue 12, 6 (2009) 2851-2858.

[15] Bolton, G. T., Hooper, C., Mann, R., "Flow Distribution and Velocity Measurement in a Radial Flow Fixed Bed Reactor using Electrical Resistance Tomography”, Chemical Engineering Science, Vol. 59, Issue 10, 5 (2004) 1989-1997.

[16] Deng, X., Yang, W. Q, "Fusion Research of Electrical Tomography with Other Sensors for Two-Phase Flow Measurement”, Measurement Science Review, Vol. 12, No. 2, 2012.

[17] Jin, H., Yang, S., Wang, M., "Measurement of Gas Holdup profiles in a Gas-Liquid Concurrent bubble Column Using Electrical Resistance Tomography”, Flow Measurement and Instrumentation, 1 (2007), 200-206.

[18] Triplett, K. A., Ghiaasiaan, S. M., Abdel, S. I., "Gas-Liquid Two-Phase Flow in Microchannels: Part II: Void Fraction and Pressure Drop", International Journal of Multiphase Flow, Vol. 25, Issue 3, 4 (1999) 395-410. 


\section{APPENDIX}
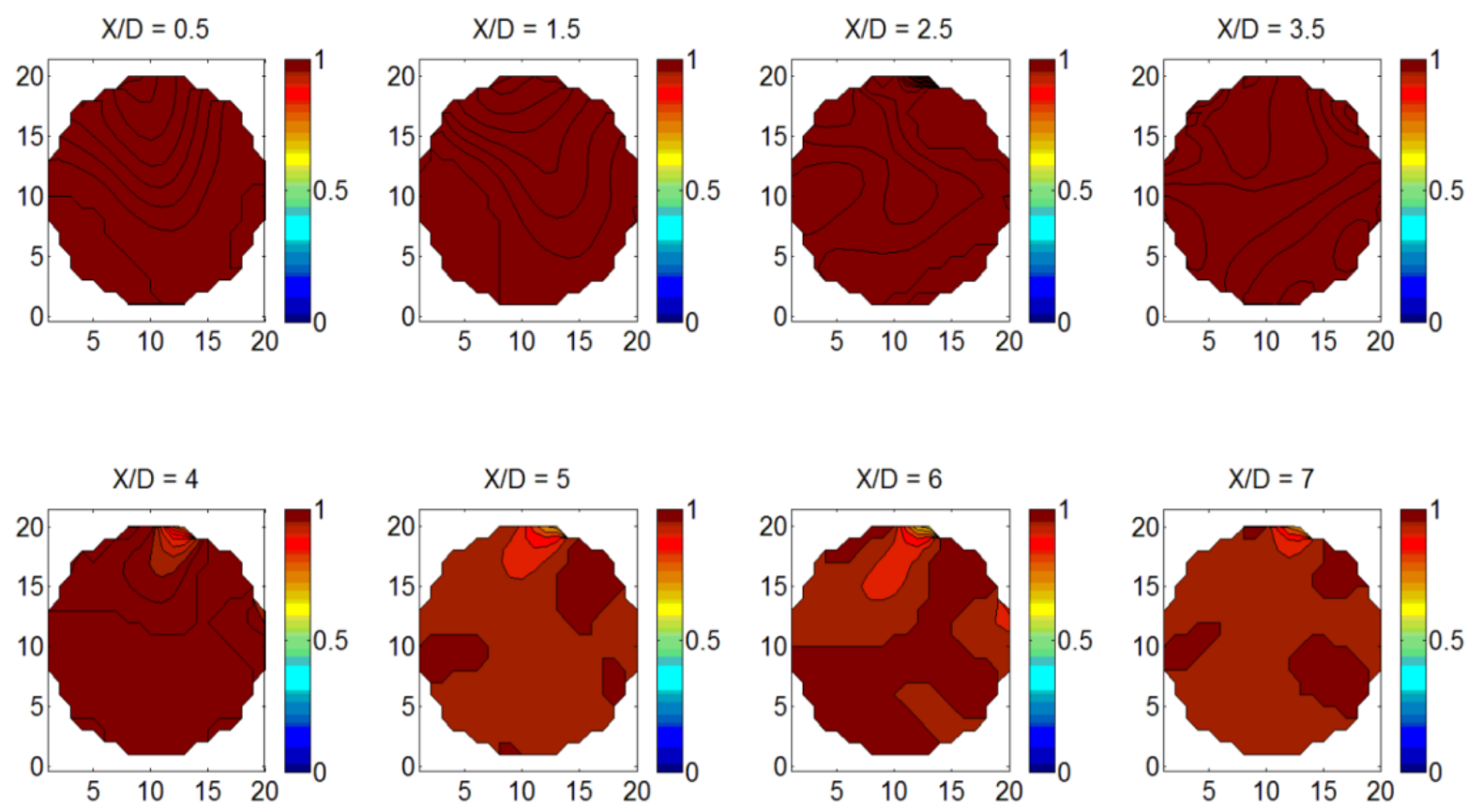

Figure 44: Conductivity Tomogram at 20 psig_20 GPM_5\% GVF
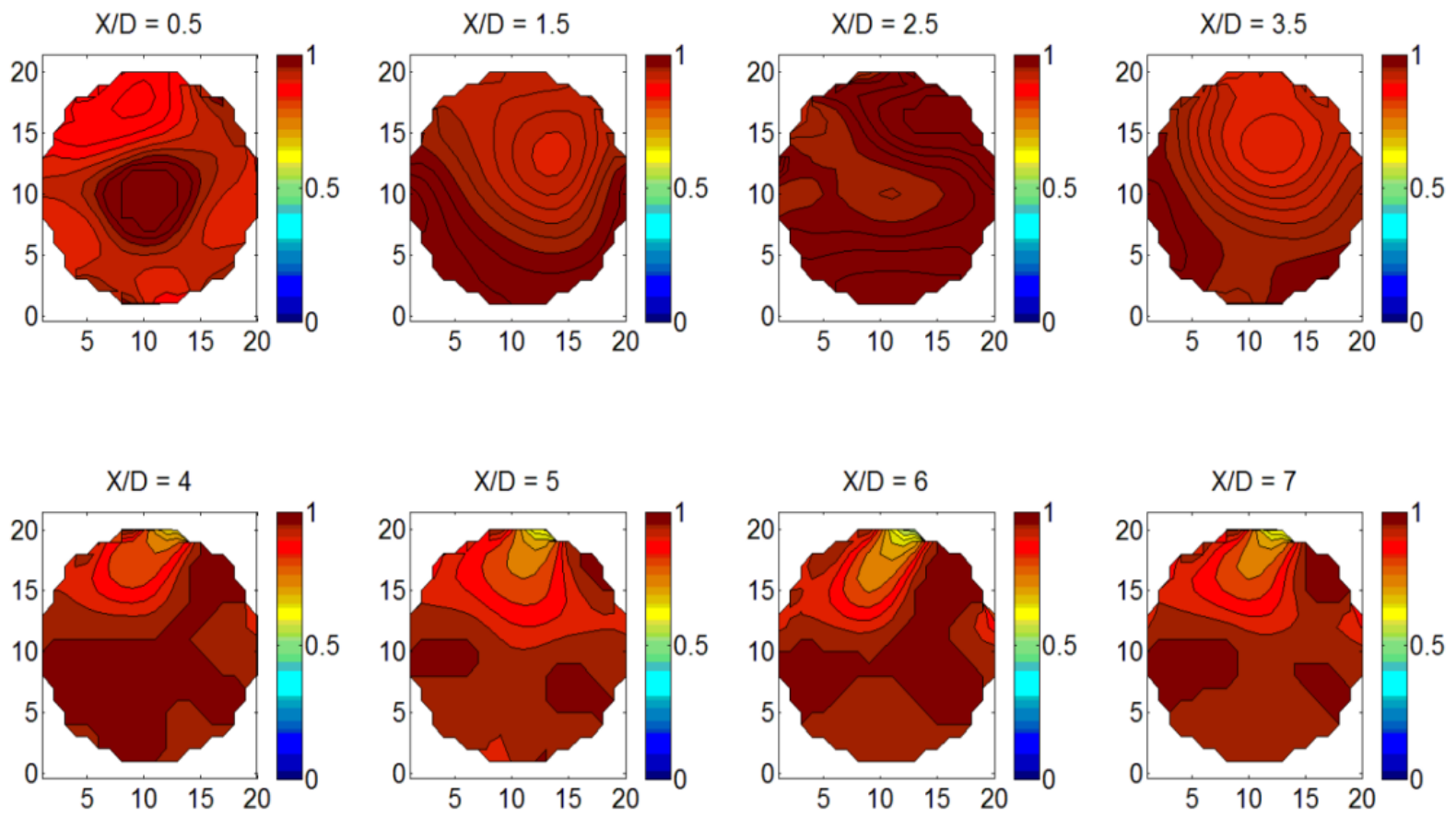

Figure 45: Conductivity Tomogram at 20 psig_20 GPM_10\% GVF 

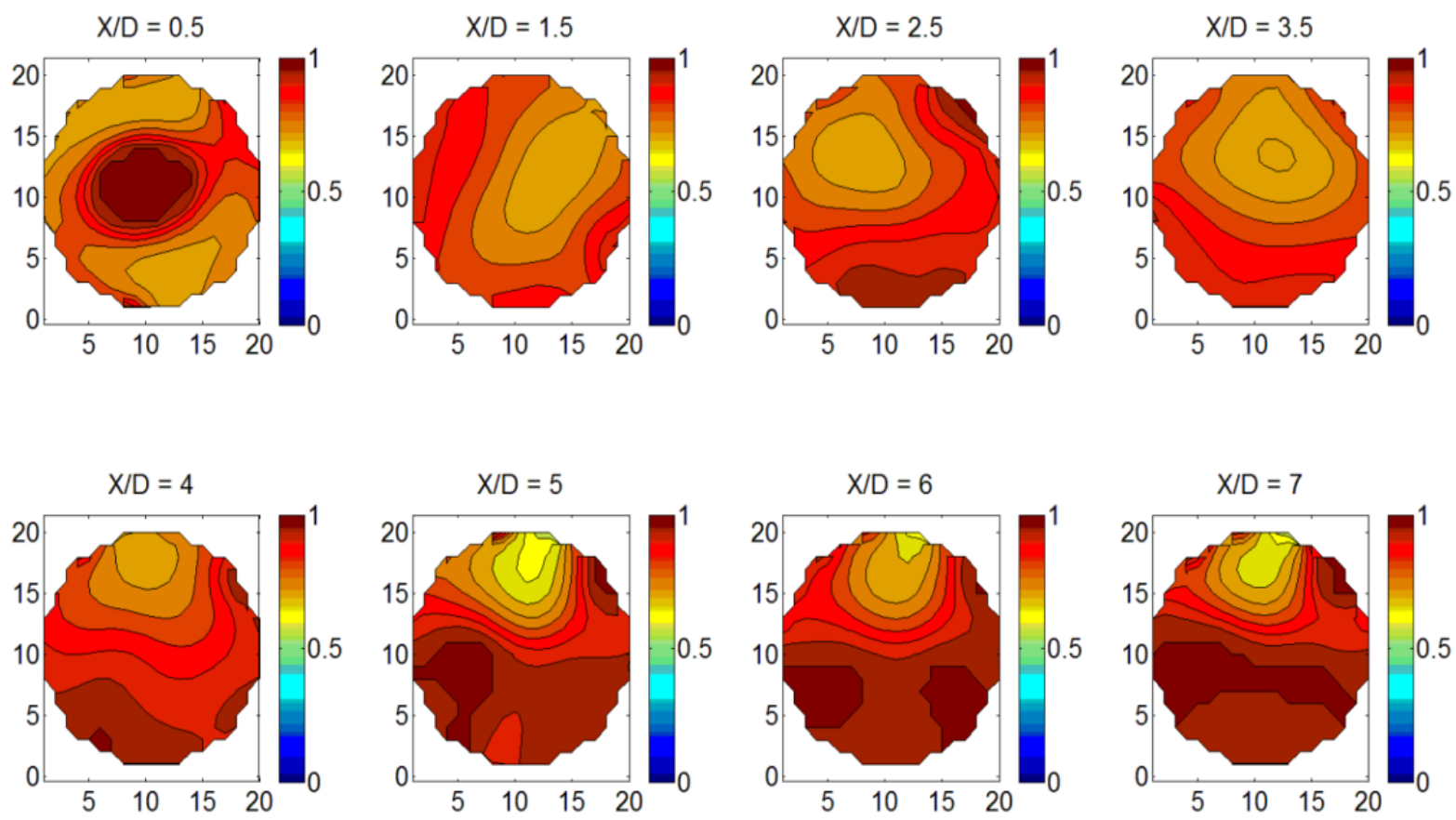

Figure 46: Conductivity Tomogram at 20 psig_20 GPM_20\% GVF
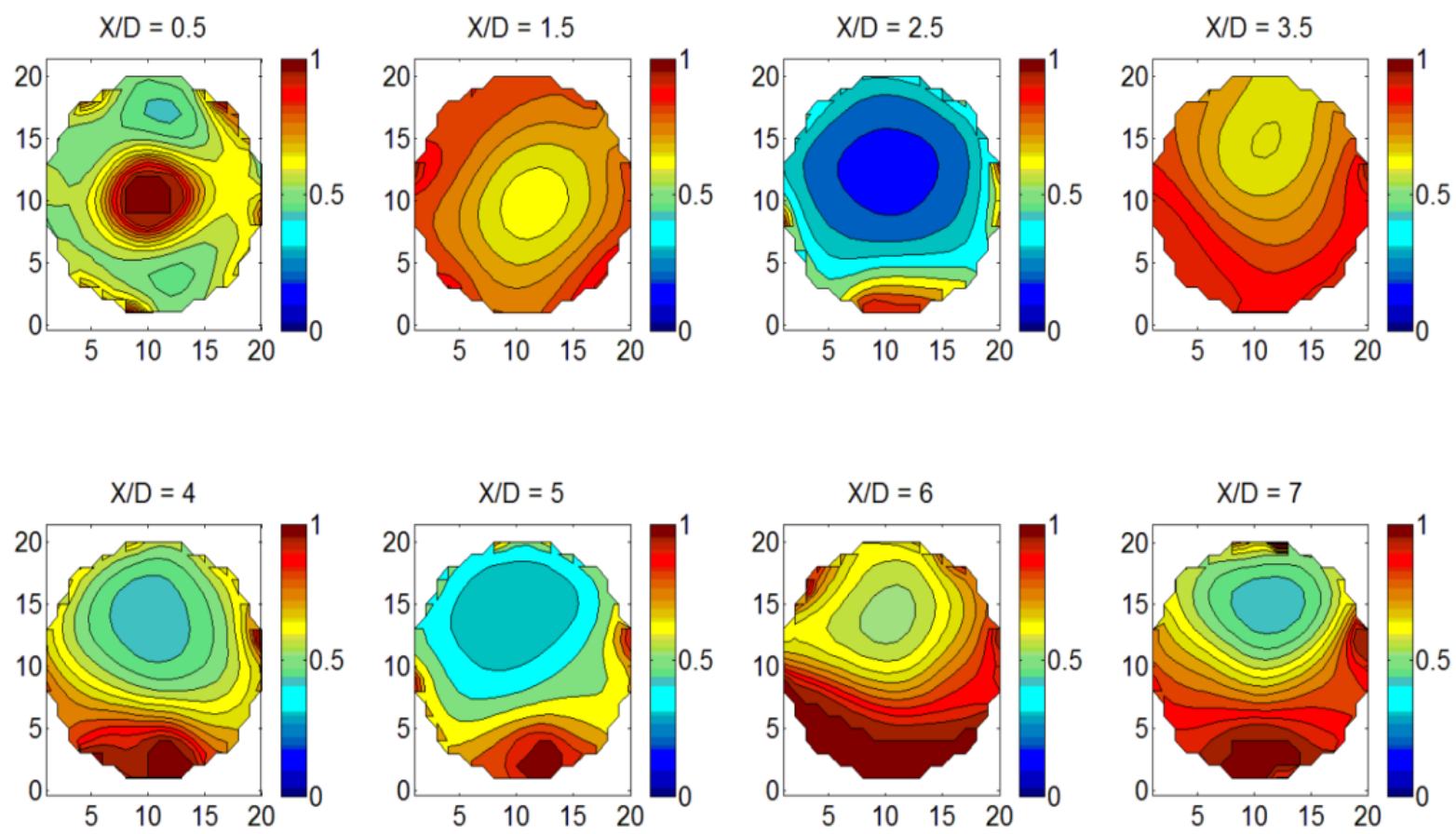

Figure 47: Conductivity Tomogram at 20 psig_20 GPM_30\% GVF 

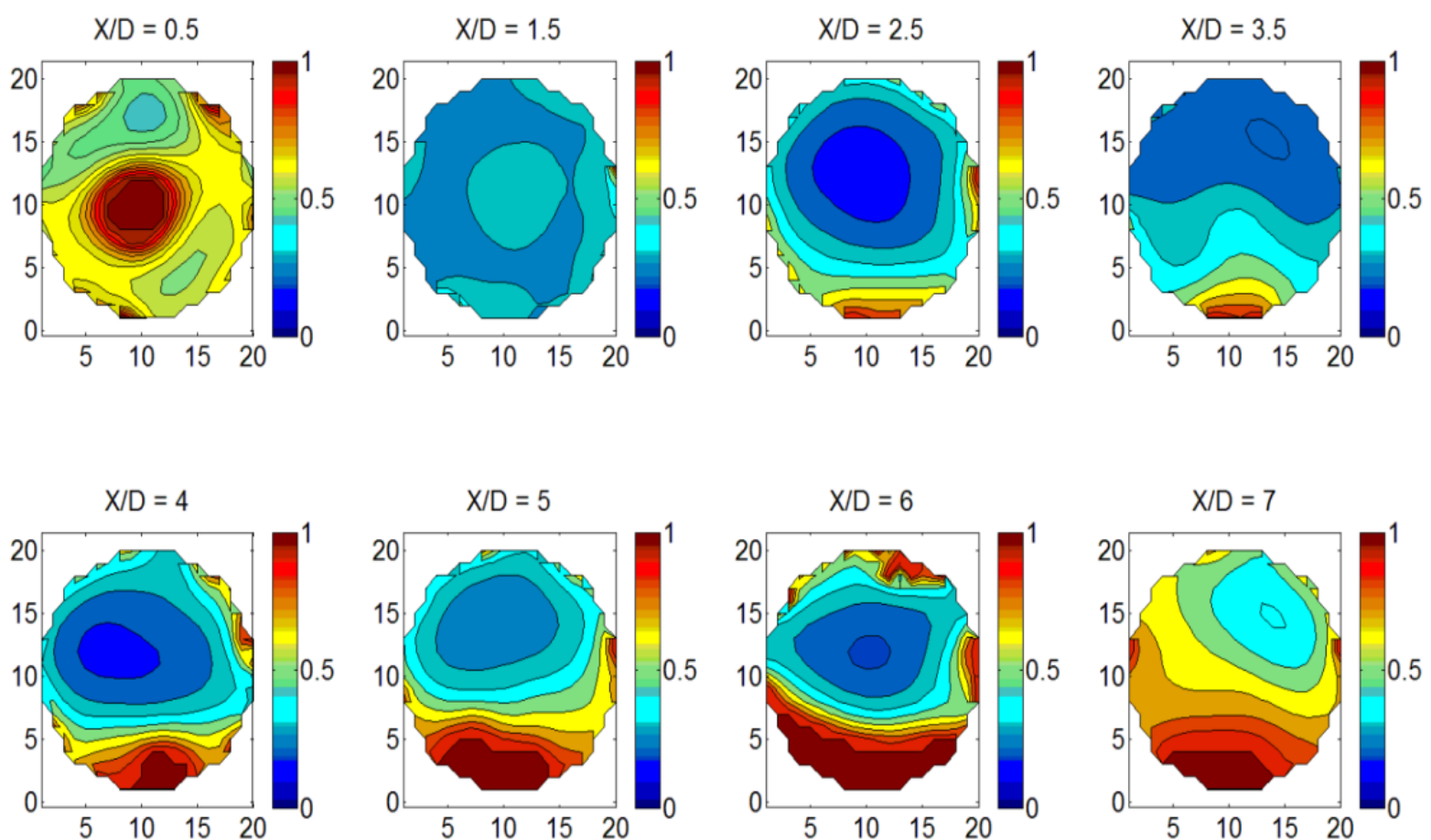

Figure 48: Conductivity Tomogram at 20 psig_20 GPM_40\% GVF
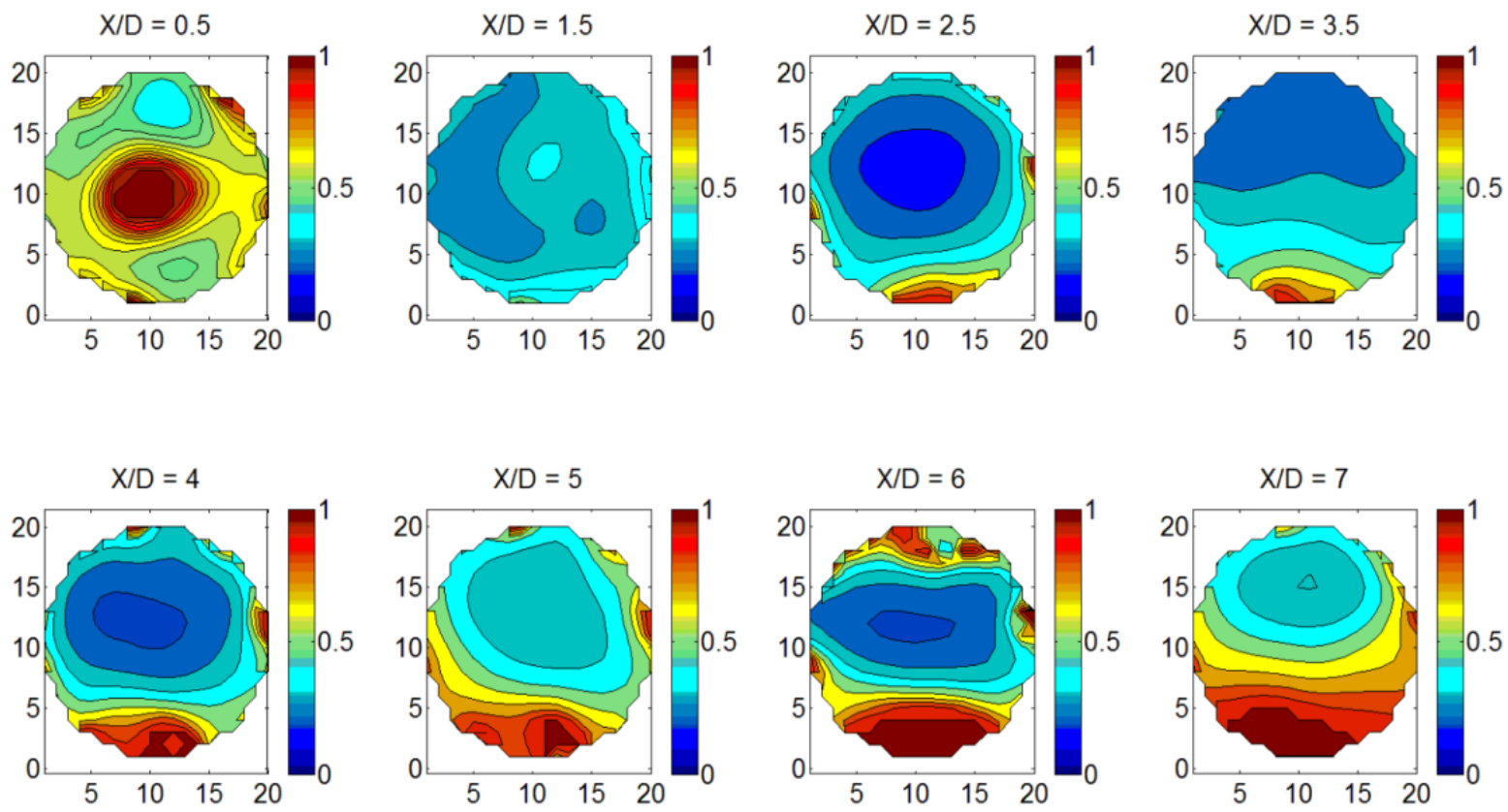

Figure 49: Conductivity Tomogram at 20 psig_20 GPM_50\% GVF 

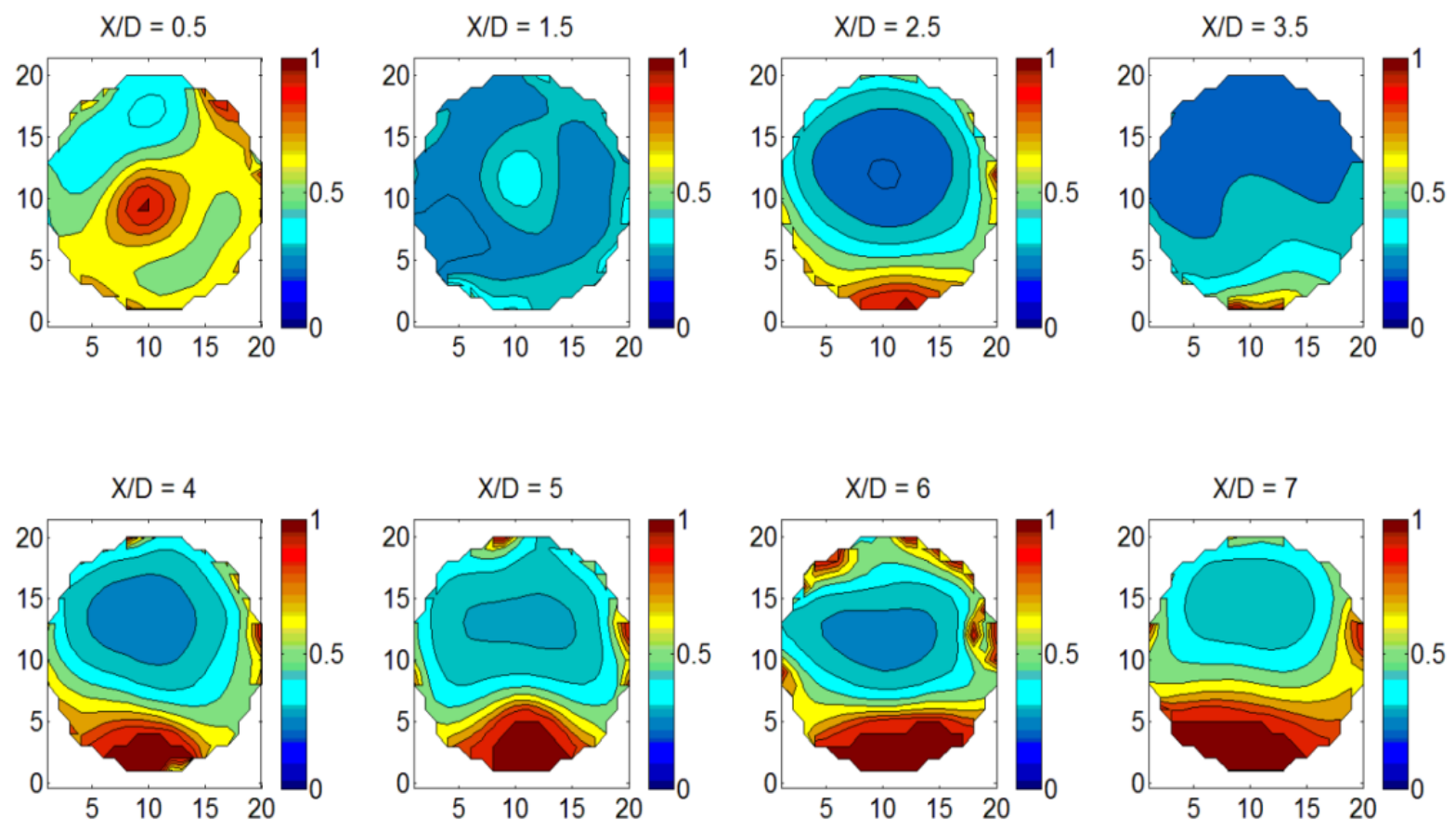

Figure 50: Conductivity Tomogram at 20 psig_20 GPM_60\% GVF
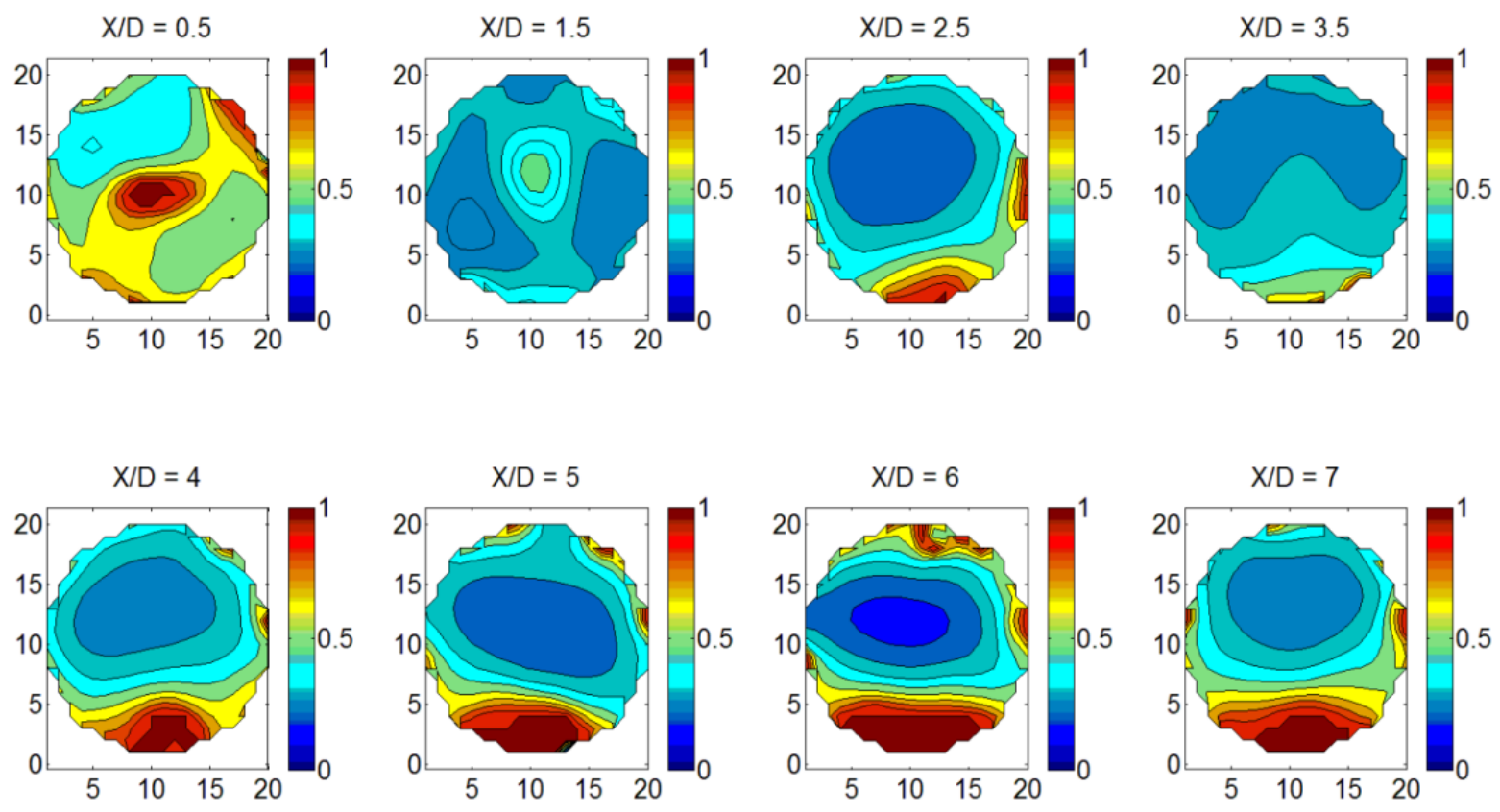

Figure 51: Conductivity Tomogram at 20 psig_20 GPM_70\% GVF 

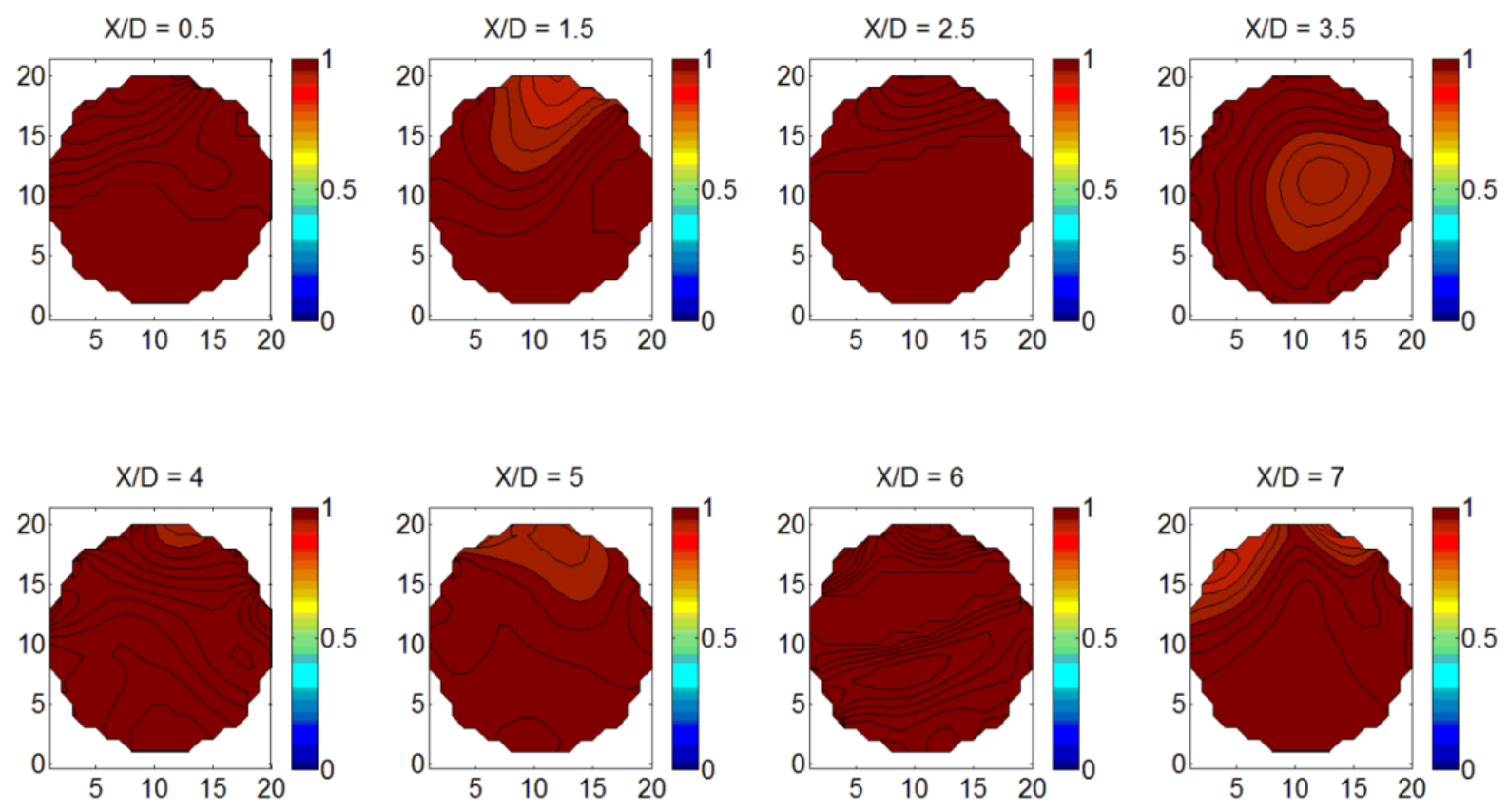

Figure 52: Conductivity Tomogram at 20 psig_40 GPM_5\% GVF
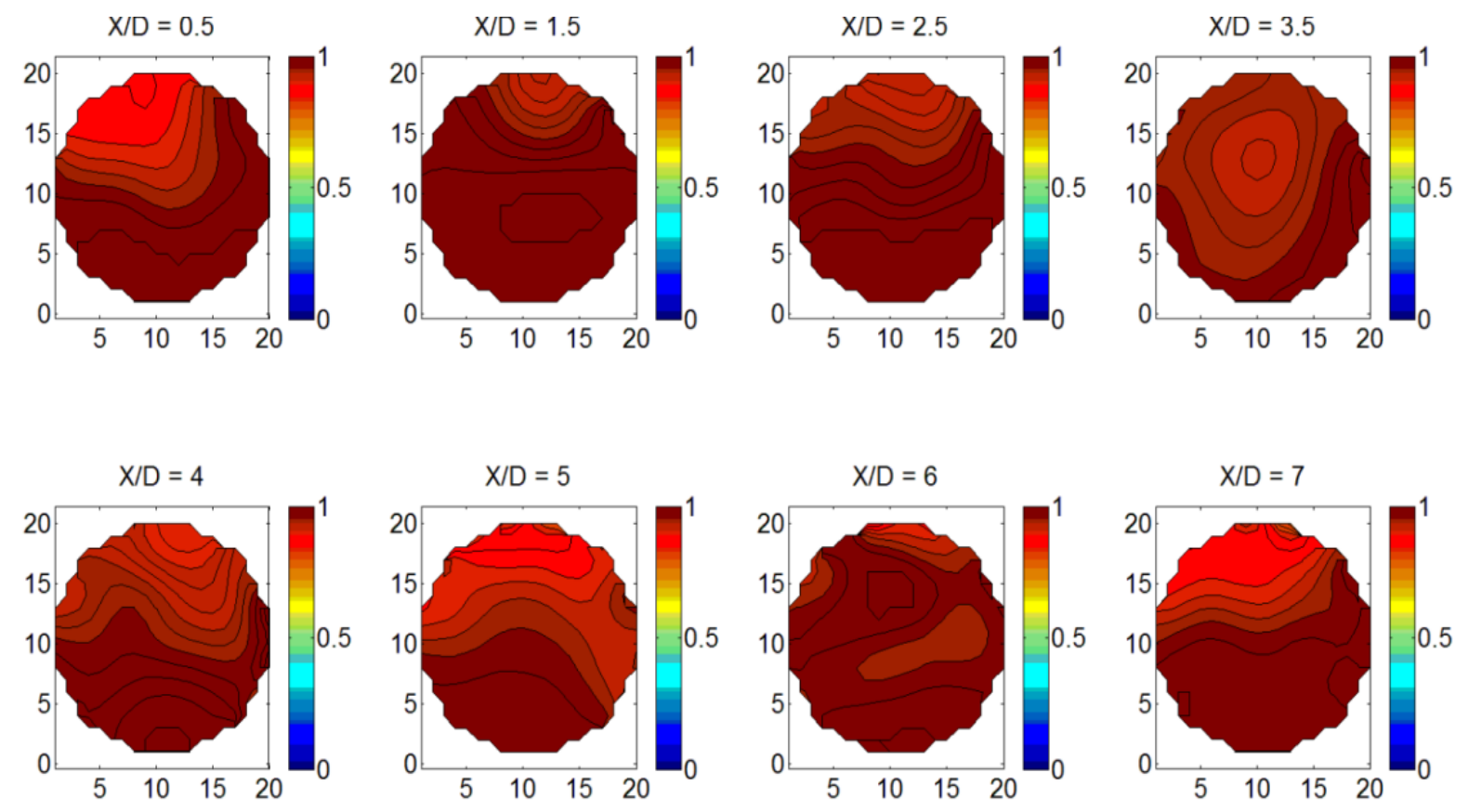

Figure 53: Conductivity Tomogram at 20 psig_40 GPM_10\% GVF 

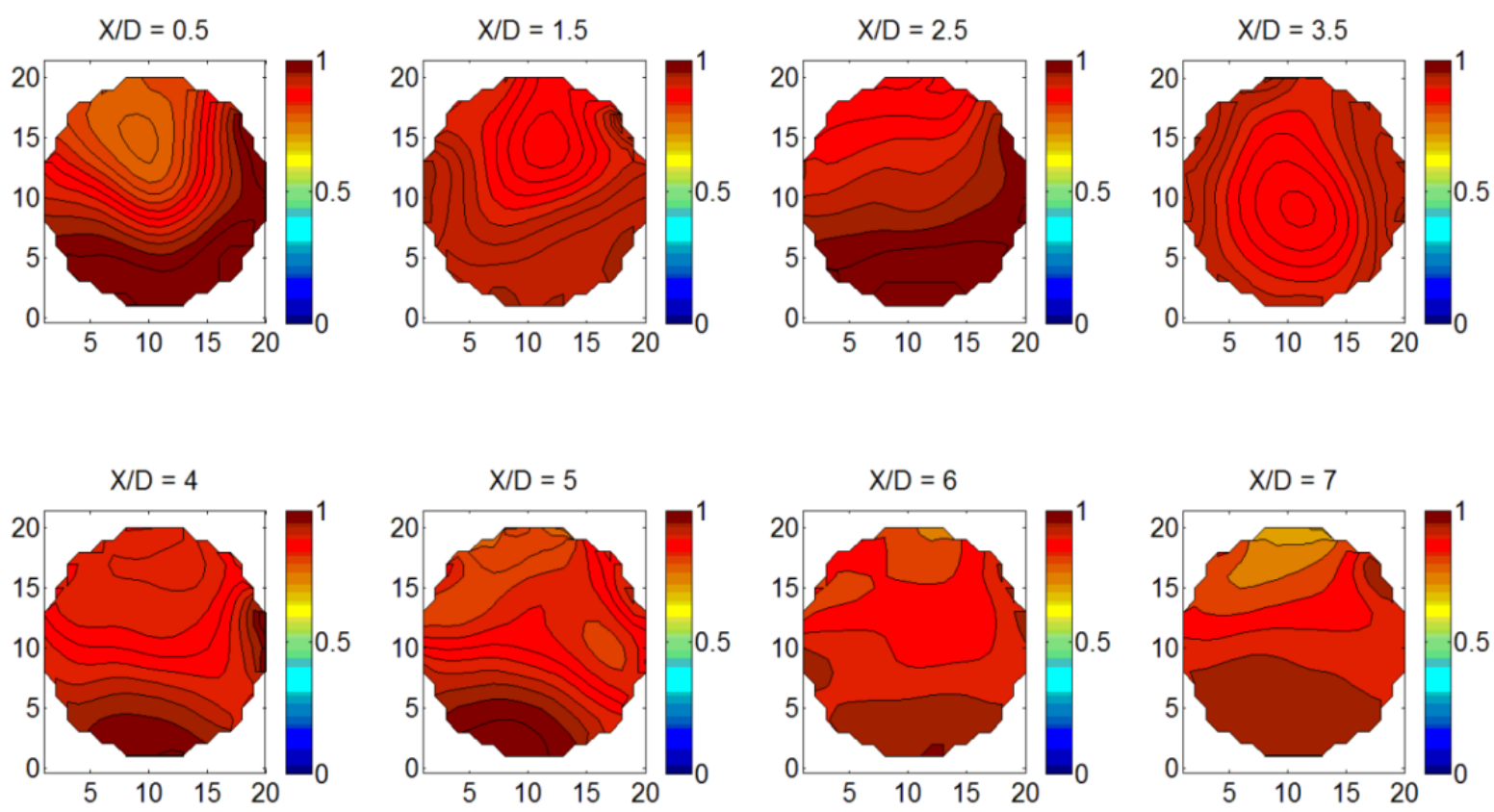

Figure 54: Conductivity Tomogram at 20 psig_40 GPM_20\% GVF
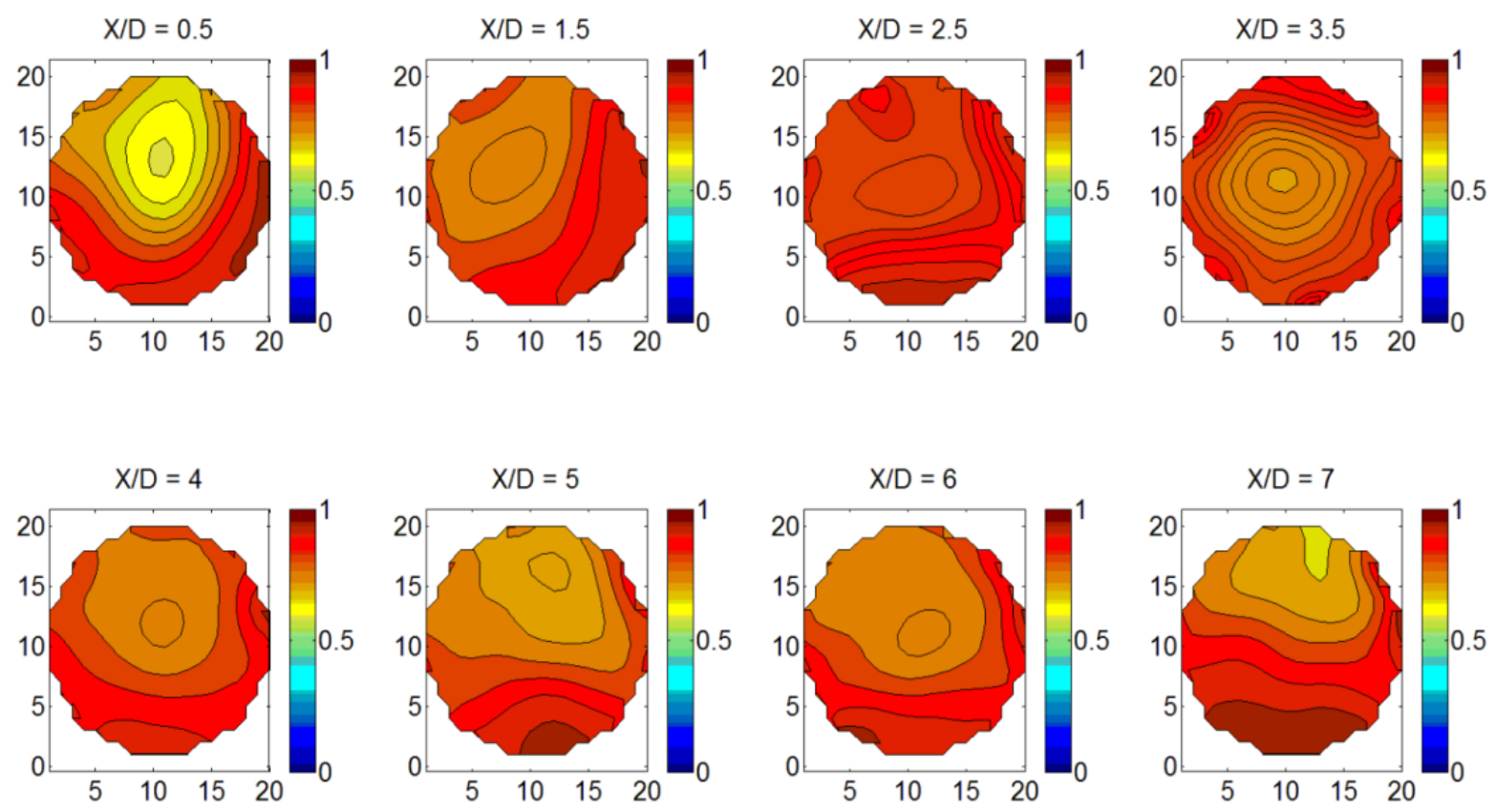

Figure 55: Conductivity Tomogram at 20 psig_40 GPM_30\% GVF 

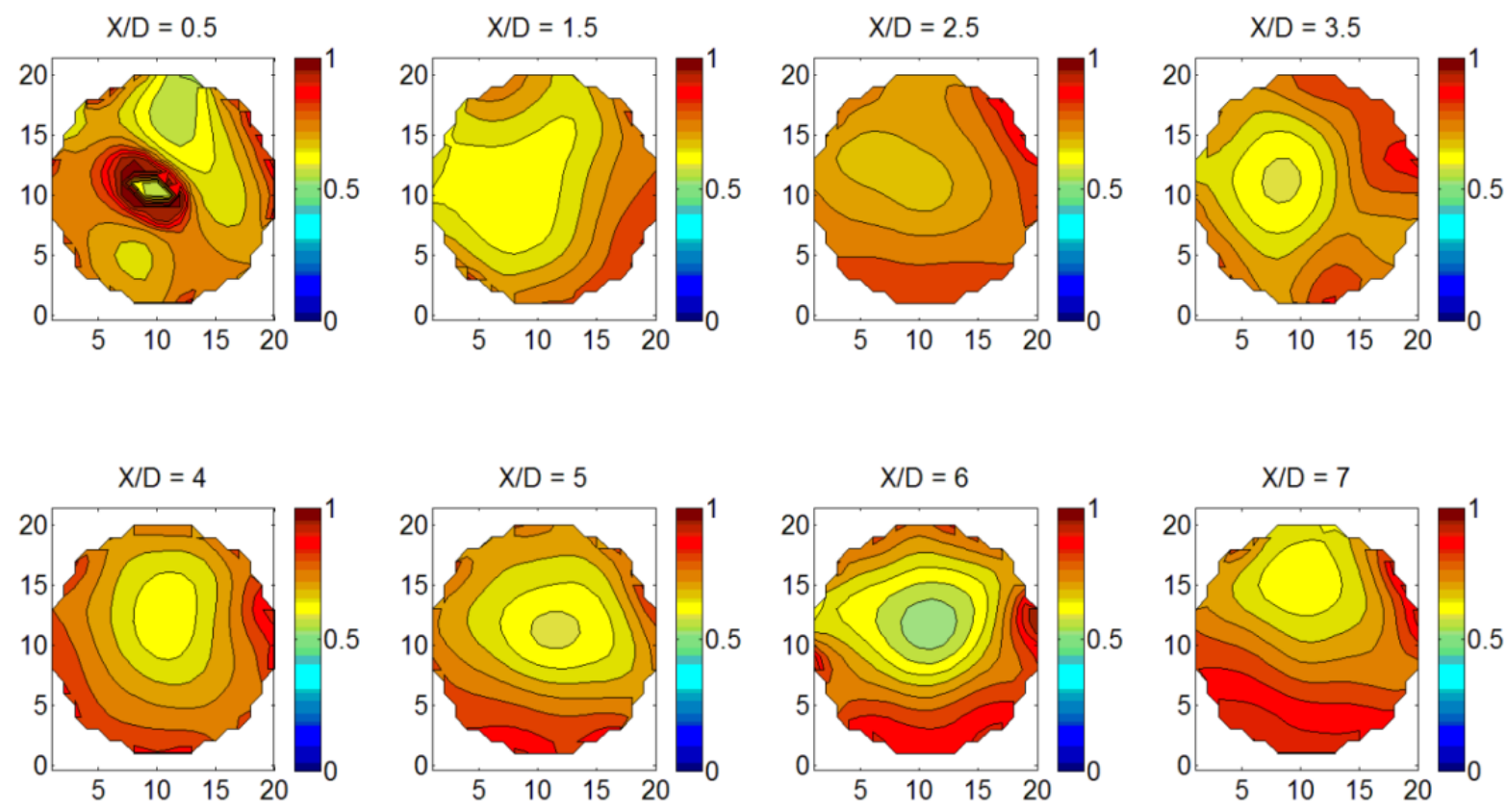

Figure 56: Conductivity Tomogram at 20 psig_40 GPM_40\% GVF
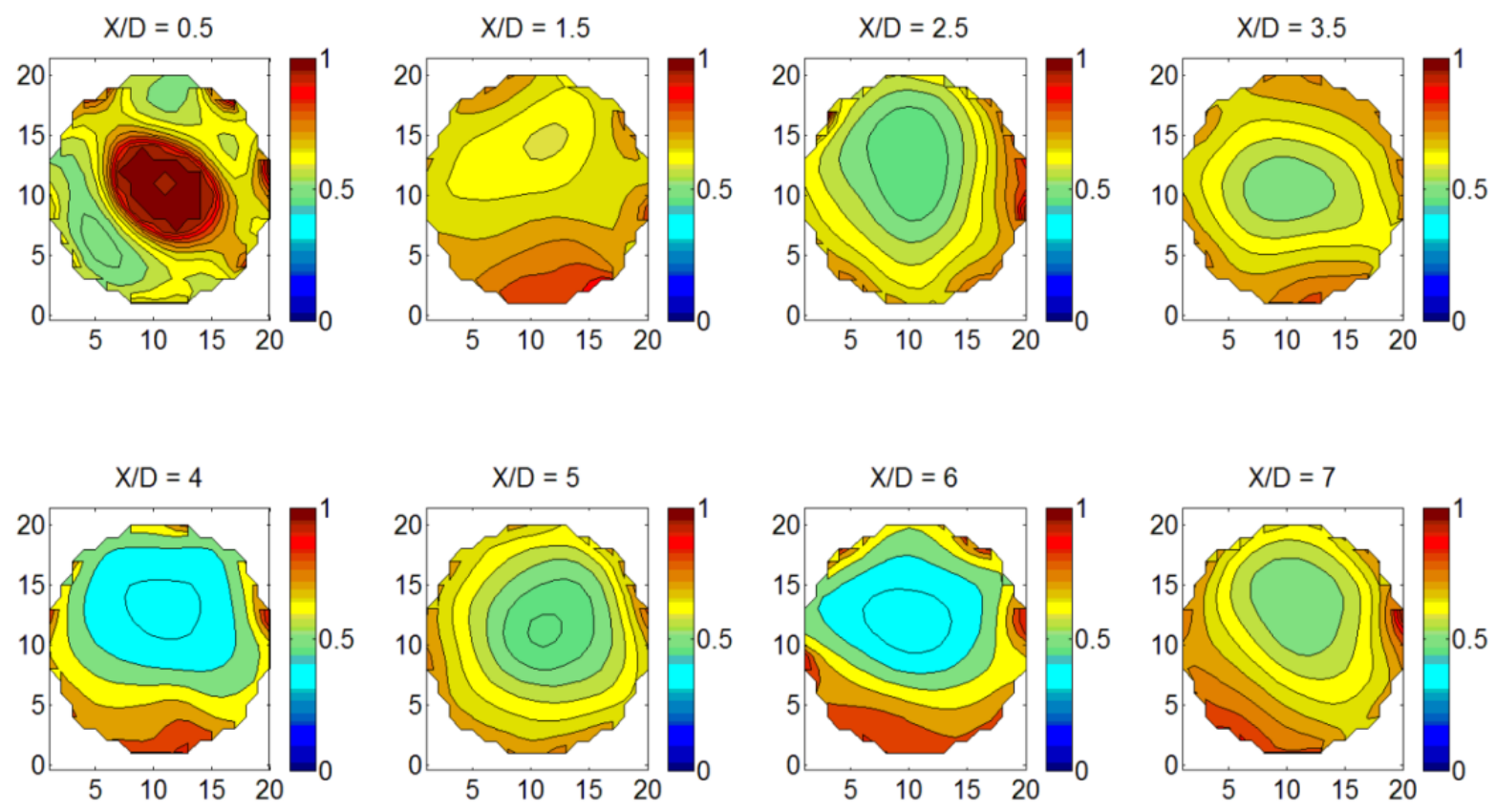

Figure 57: Conductivity Tomogram at 20 psig_40 GPM_50\% GVF 

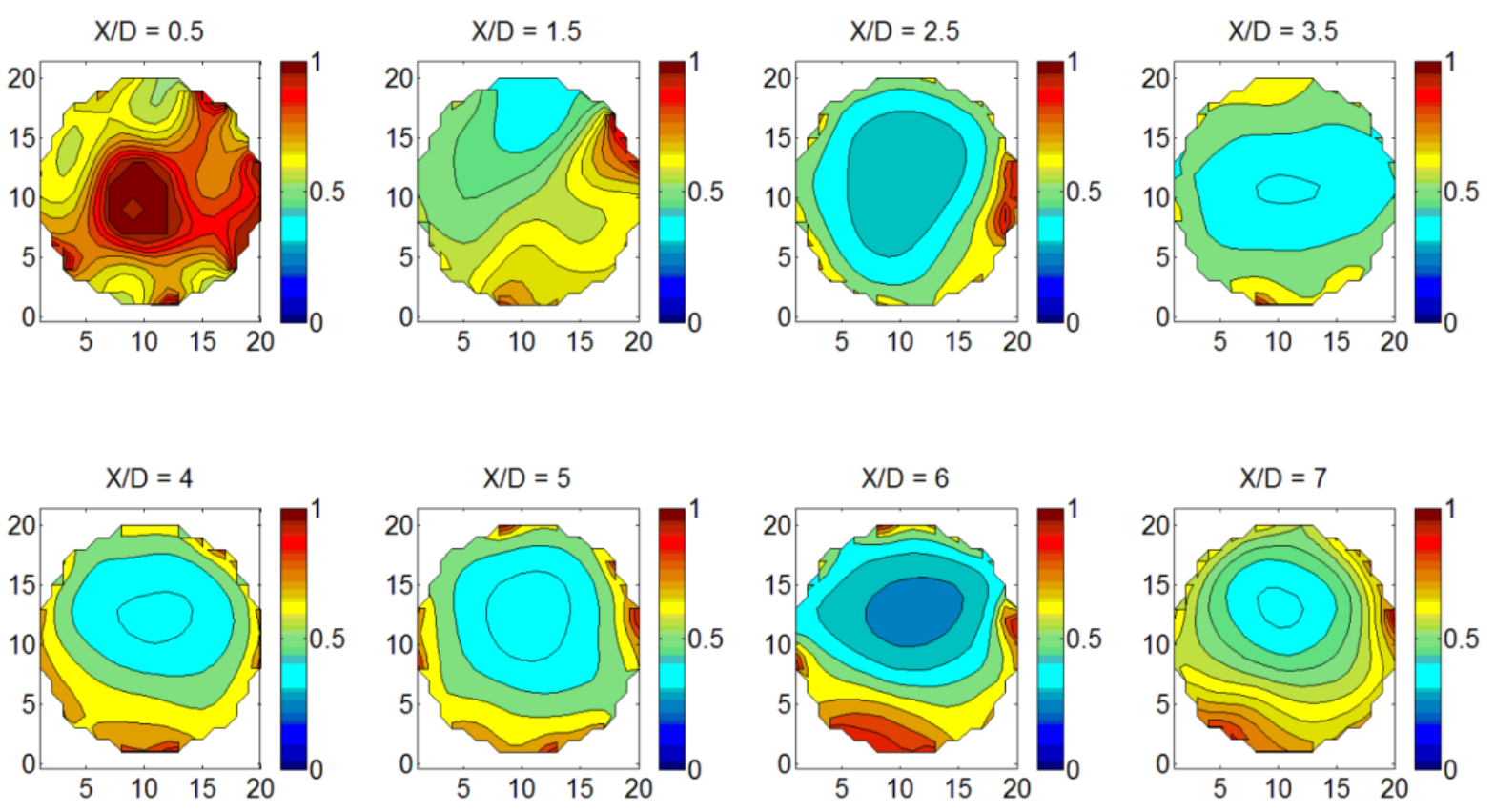

Figure 58: Conductivity Tomogram at 20 psig_40 GPM_60\% GVF
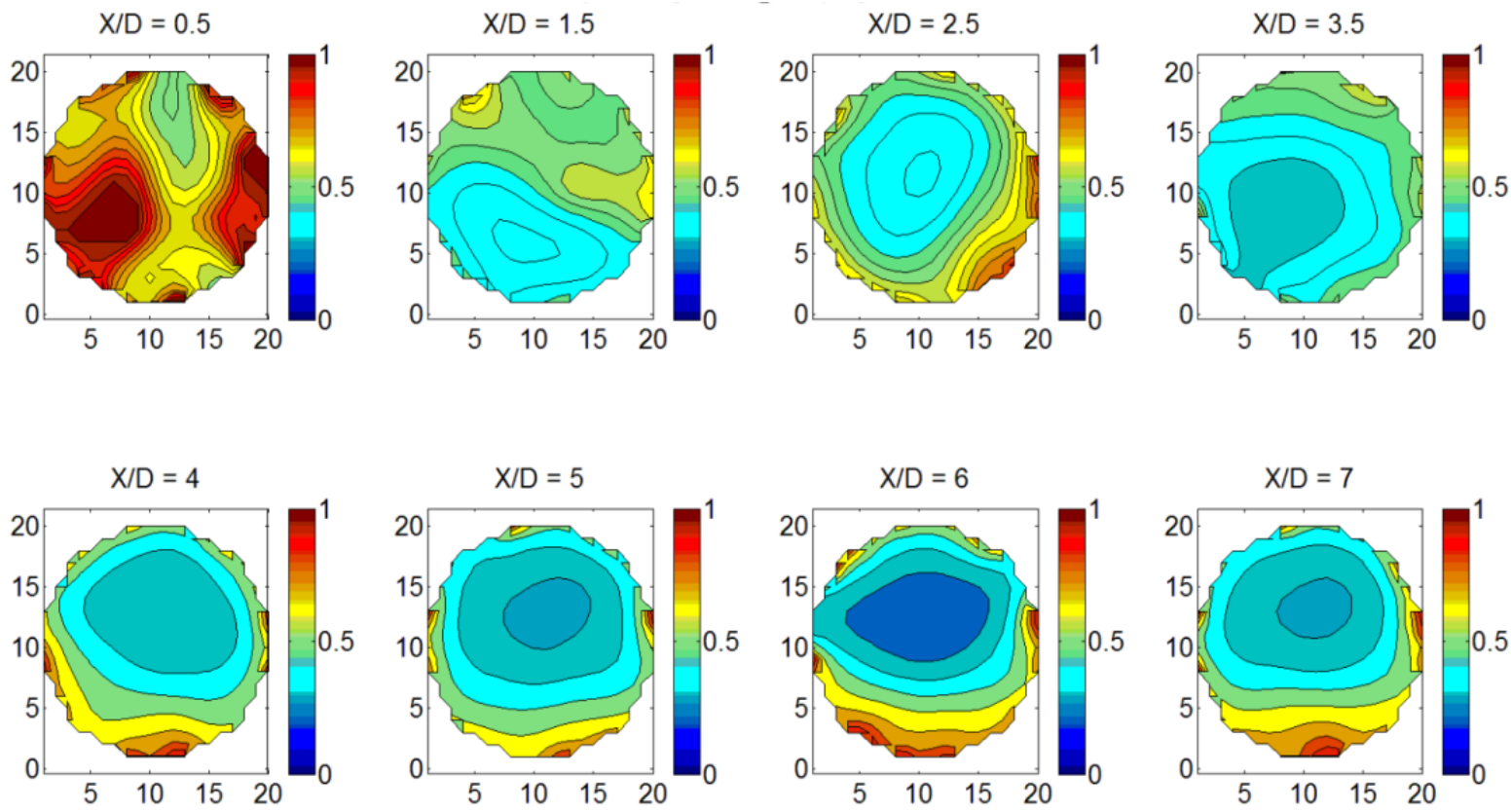

Figure 59: Conductivity Tomogram at 20 psig_40 GPM_70\% GVF 

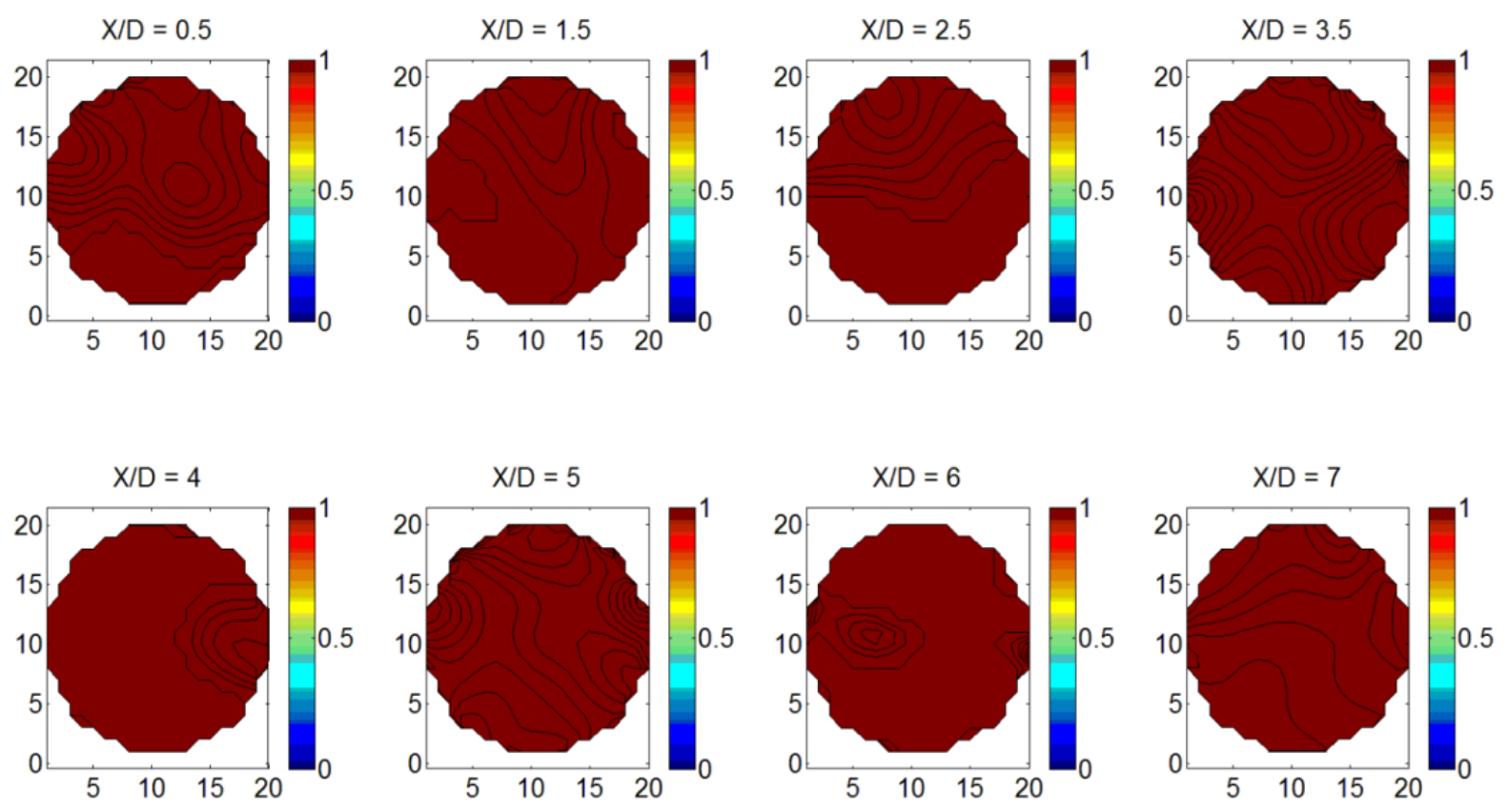

Figure 60: Conductivity Tomogram at 20 psig_60 GPM_5\% GVF
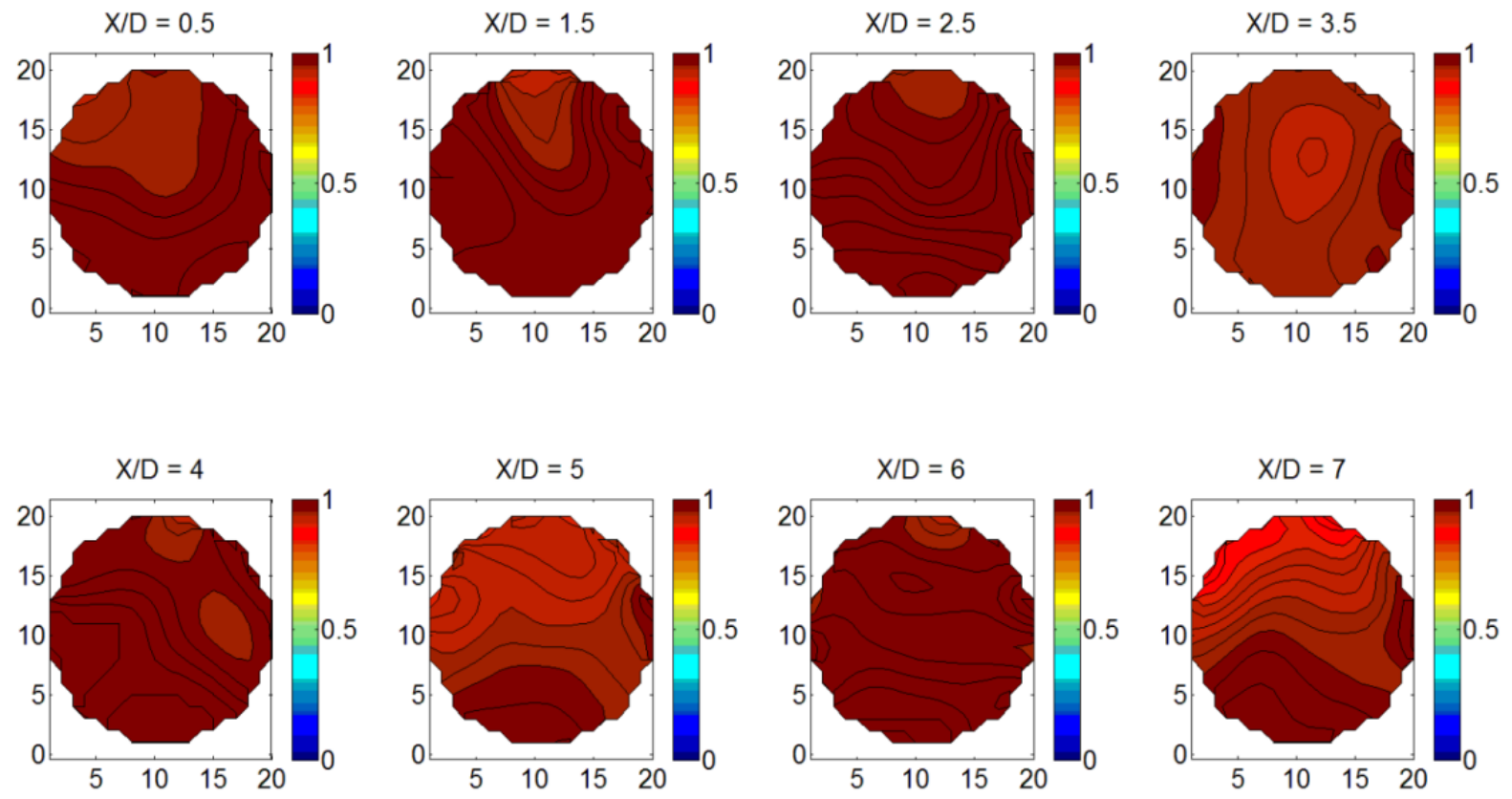

Figure 61: Conductivity Tomogram at 20 psig_60 GPM_10\% GVF 

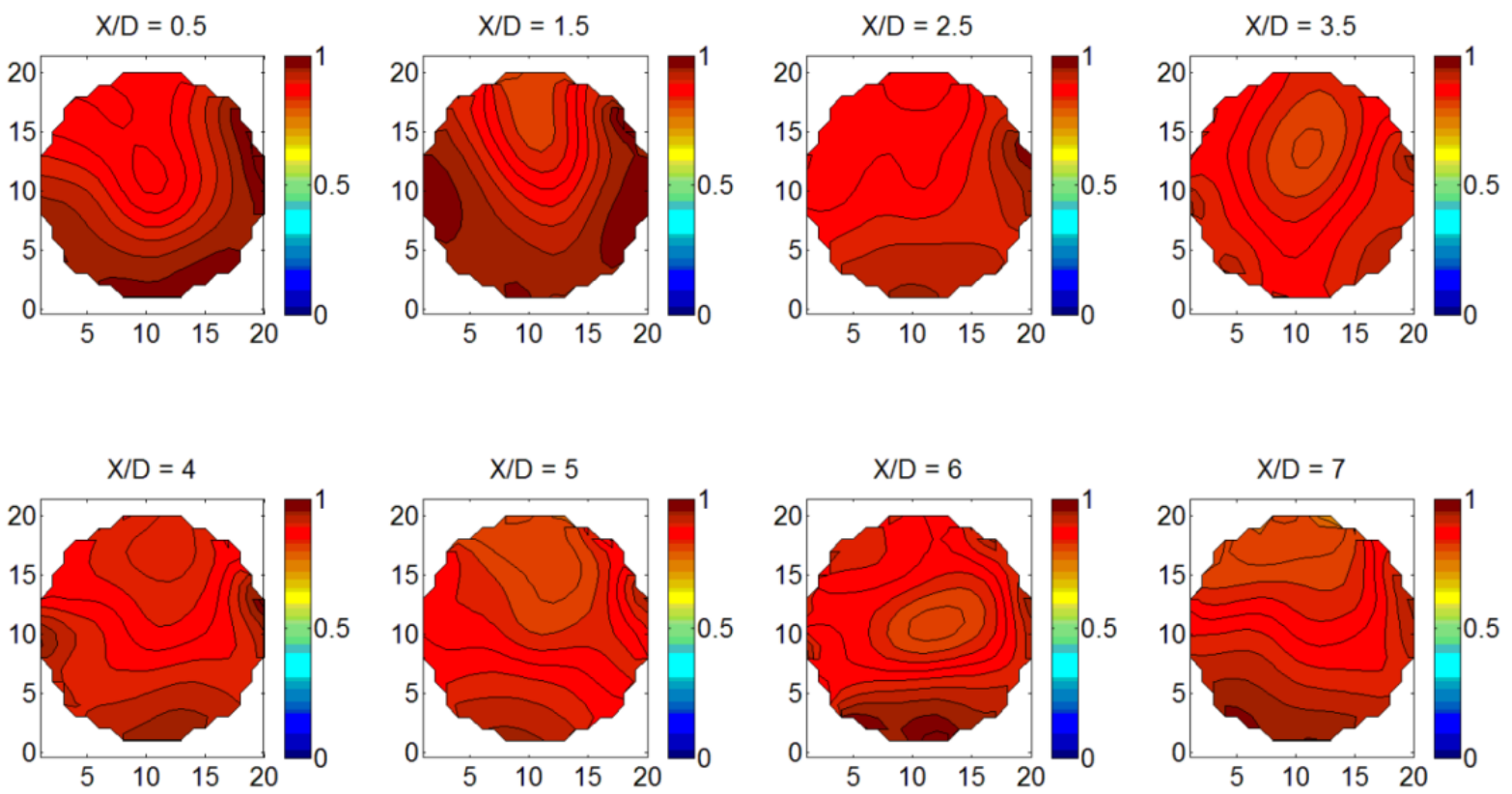

Figure 62: Conductivity Tomogram at 20 psig_60 GPM_20\% GVF
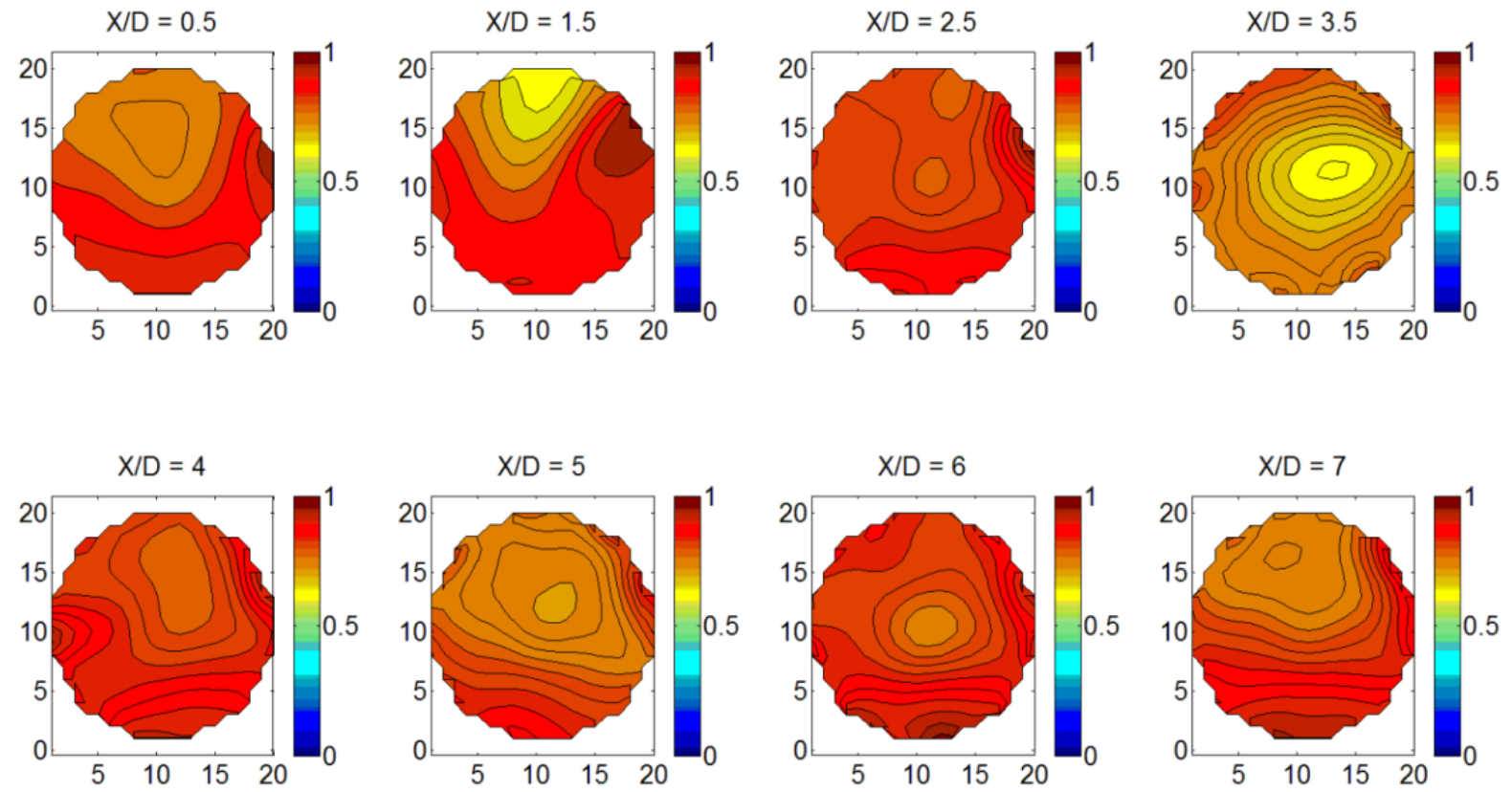

Figure 63: Conductivity Tomogram at 20 psig_60 GPM_30\% GVF 

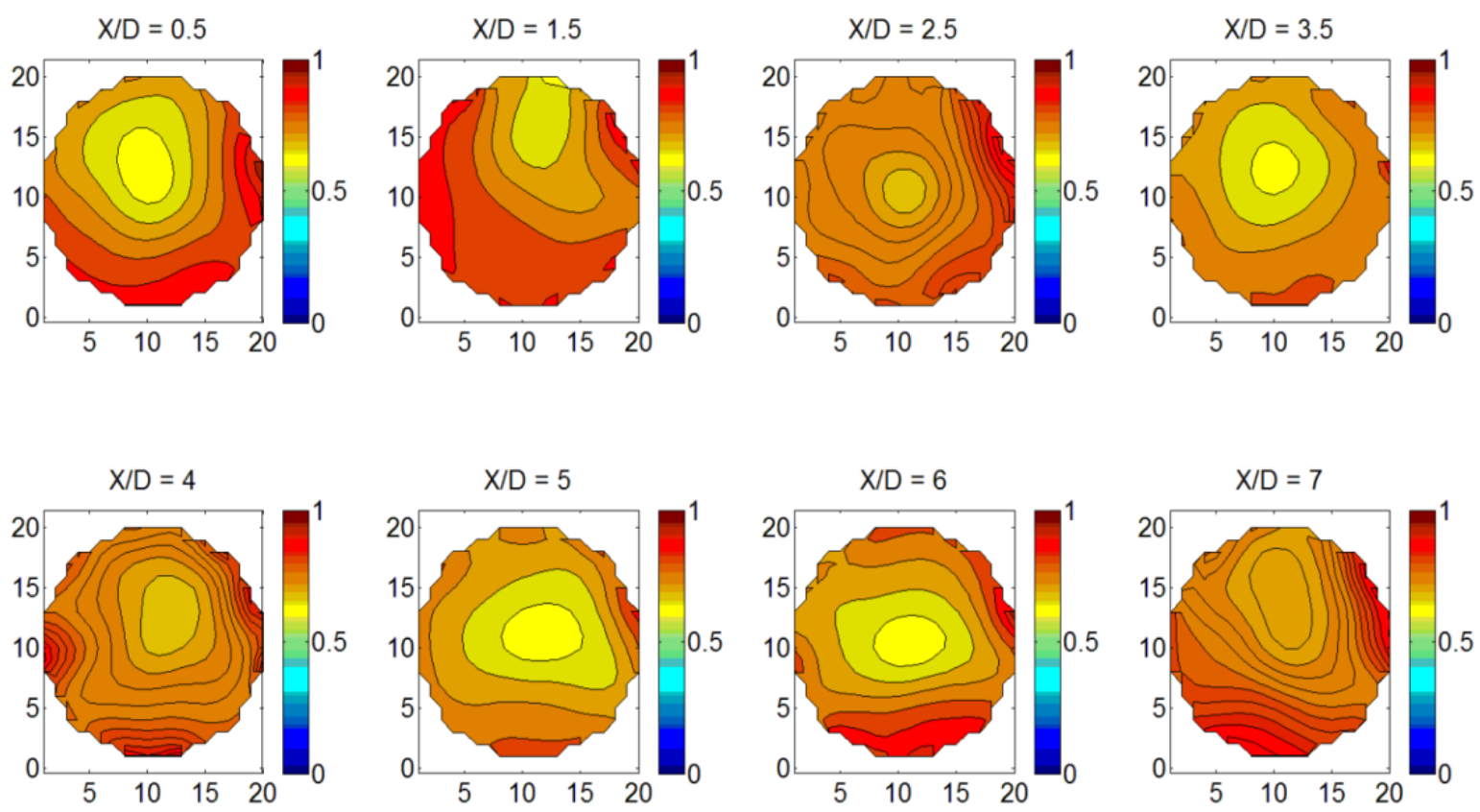

Figure 64: Conductivity Tomogram at 20 psig_60 GPM_40\% GVF
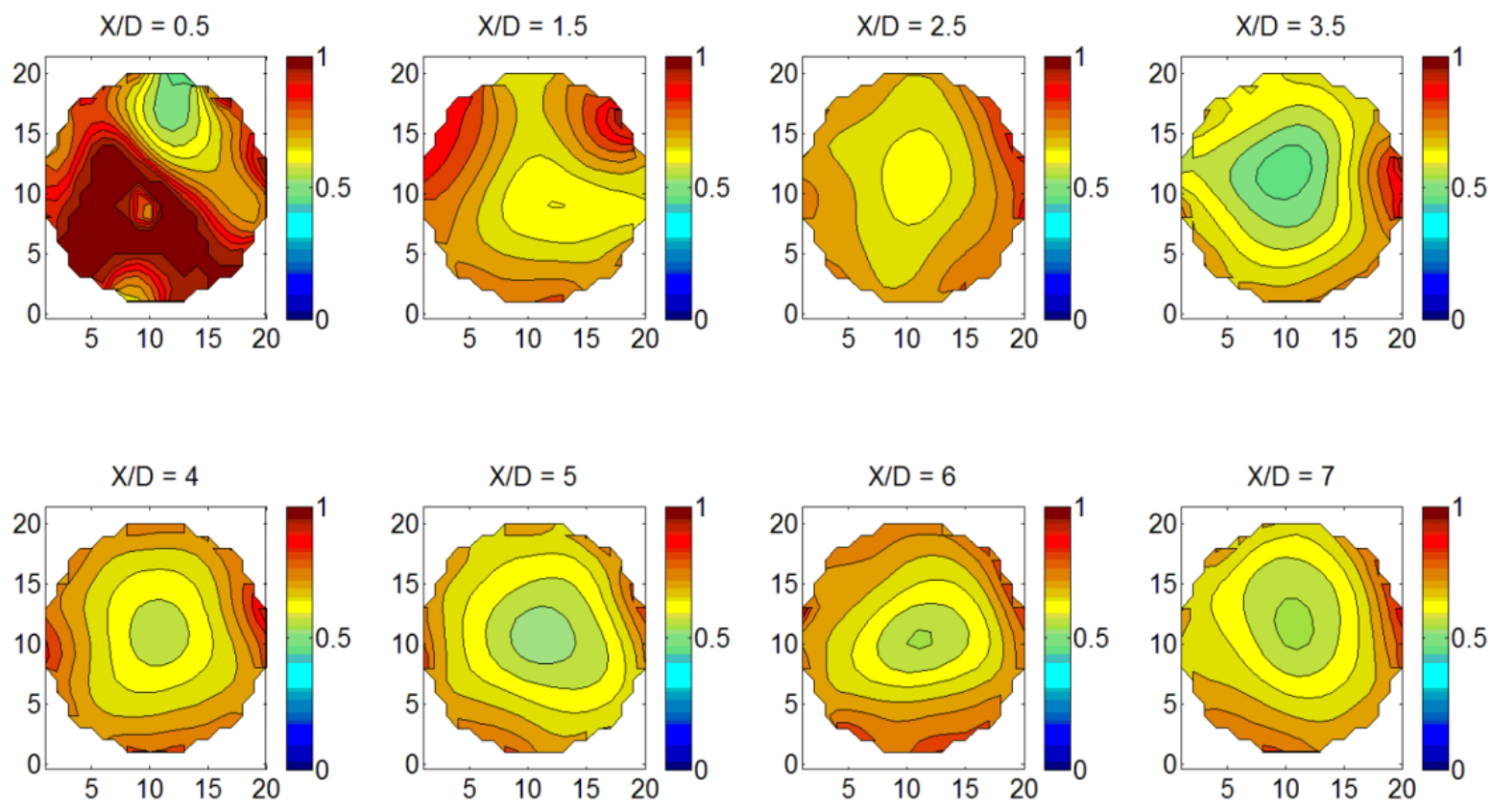

Figure 65: Conductivity Tomogram at 20 psig_60 GPM_50\% GVF 

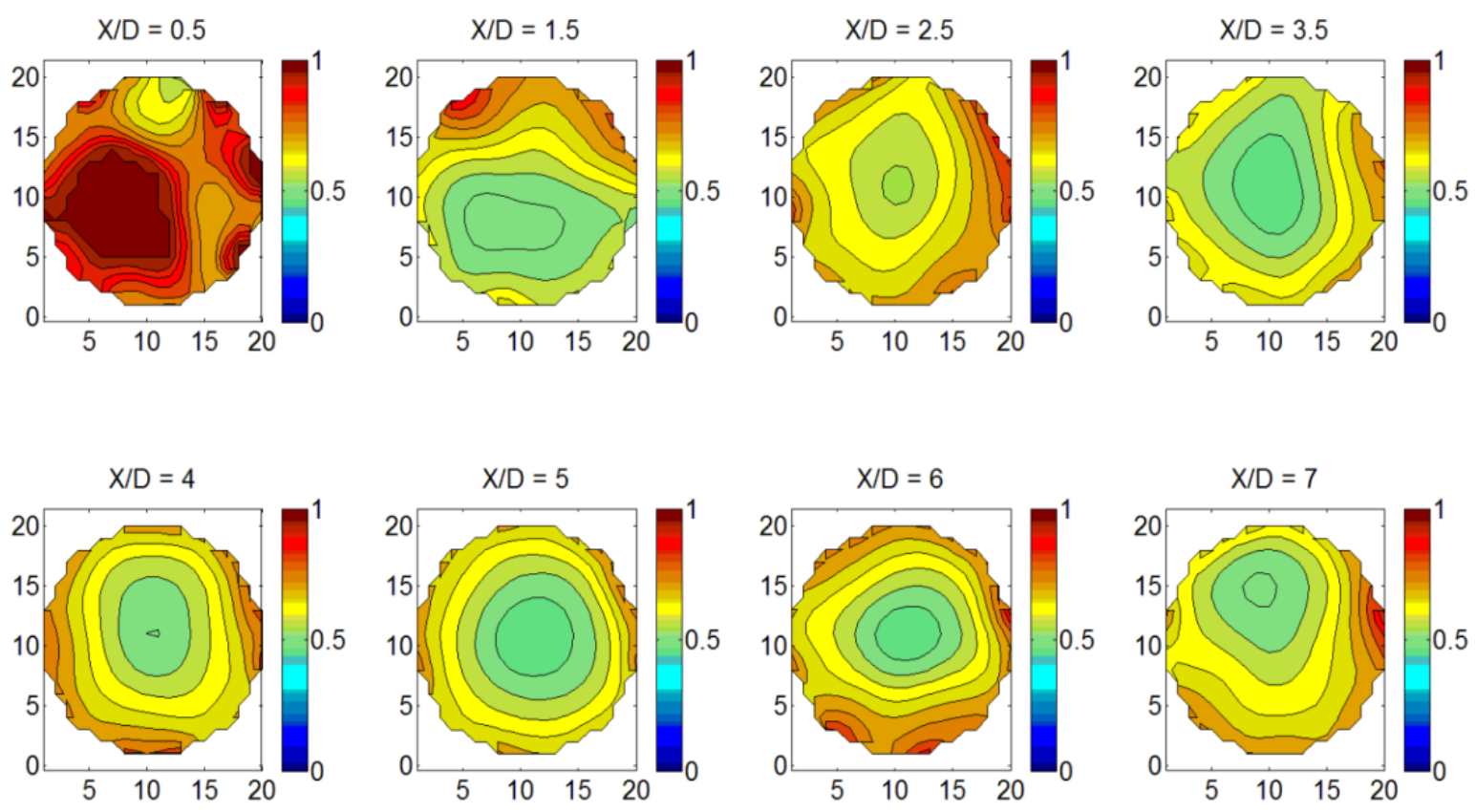

Figure 66: Conductivity Tomogram at 20 psig_60 GPM_60\% GVF
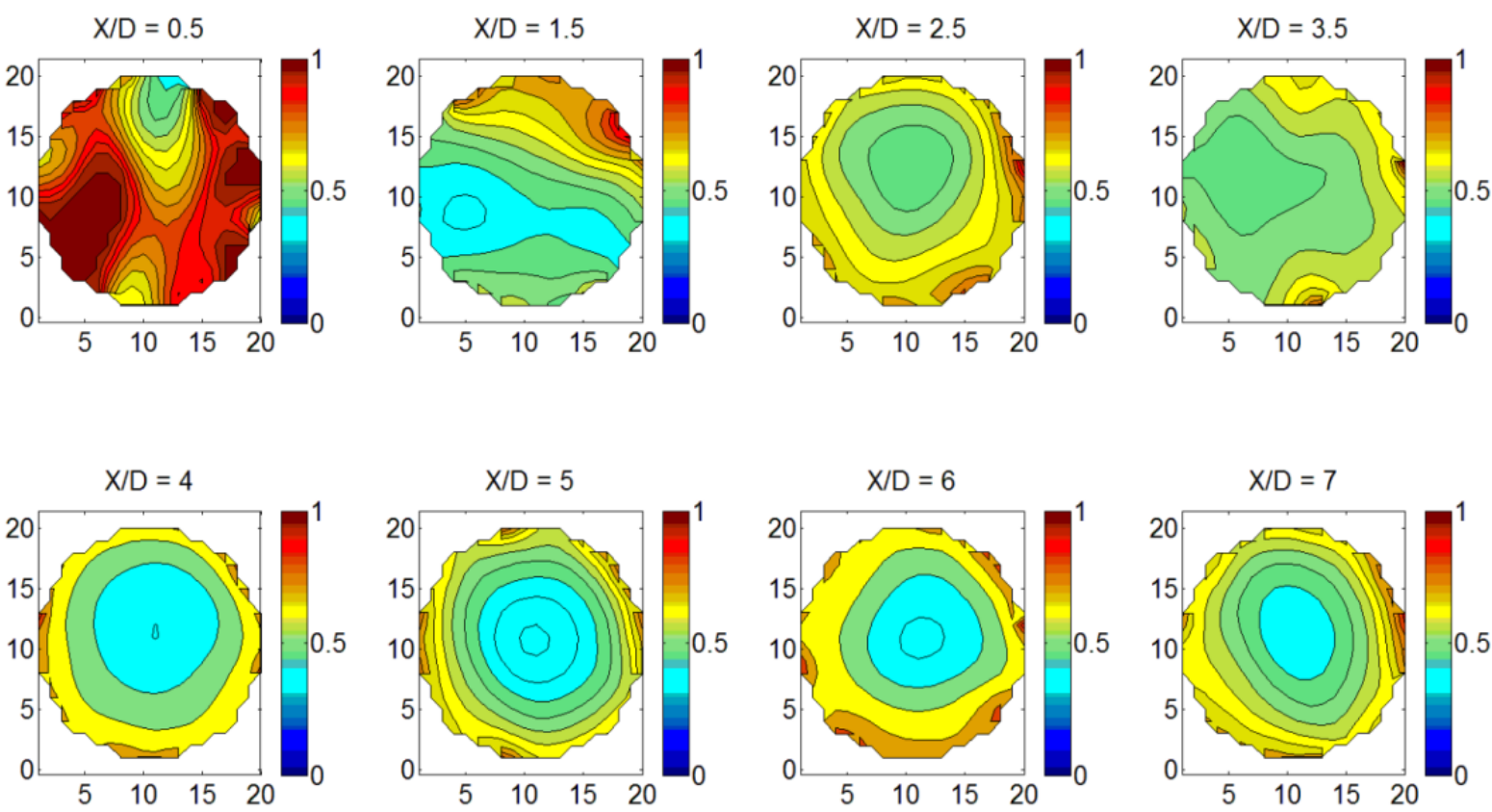

Figure 67: Conductivity Tomogram at 20 psig_60 GPM_70\% GVF 

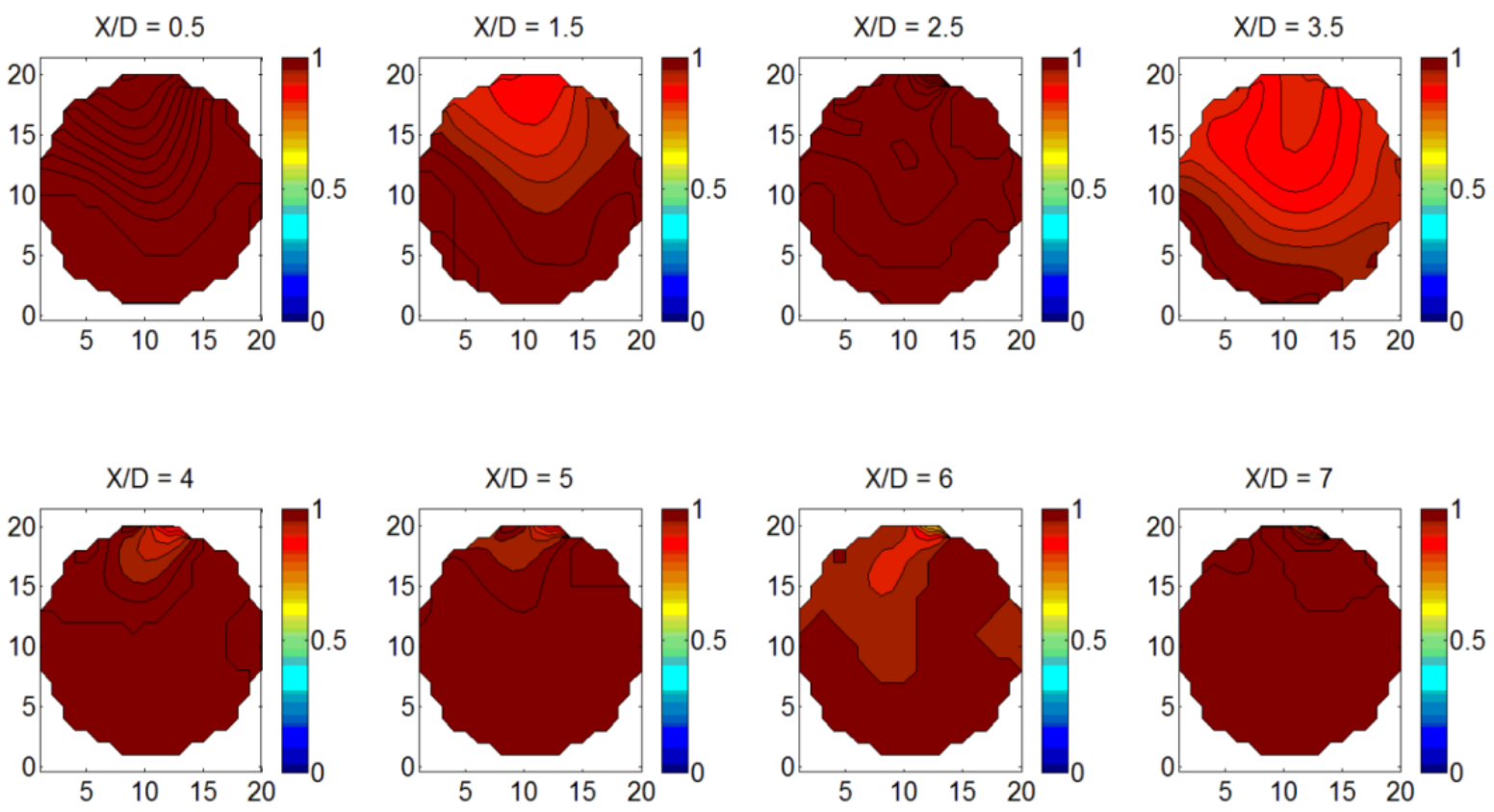

Figure 68: Conductivity Tomogram at 40 psig_20 GPM_5\% GVF
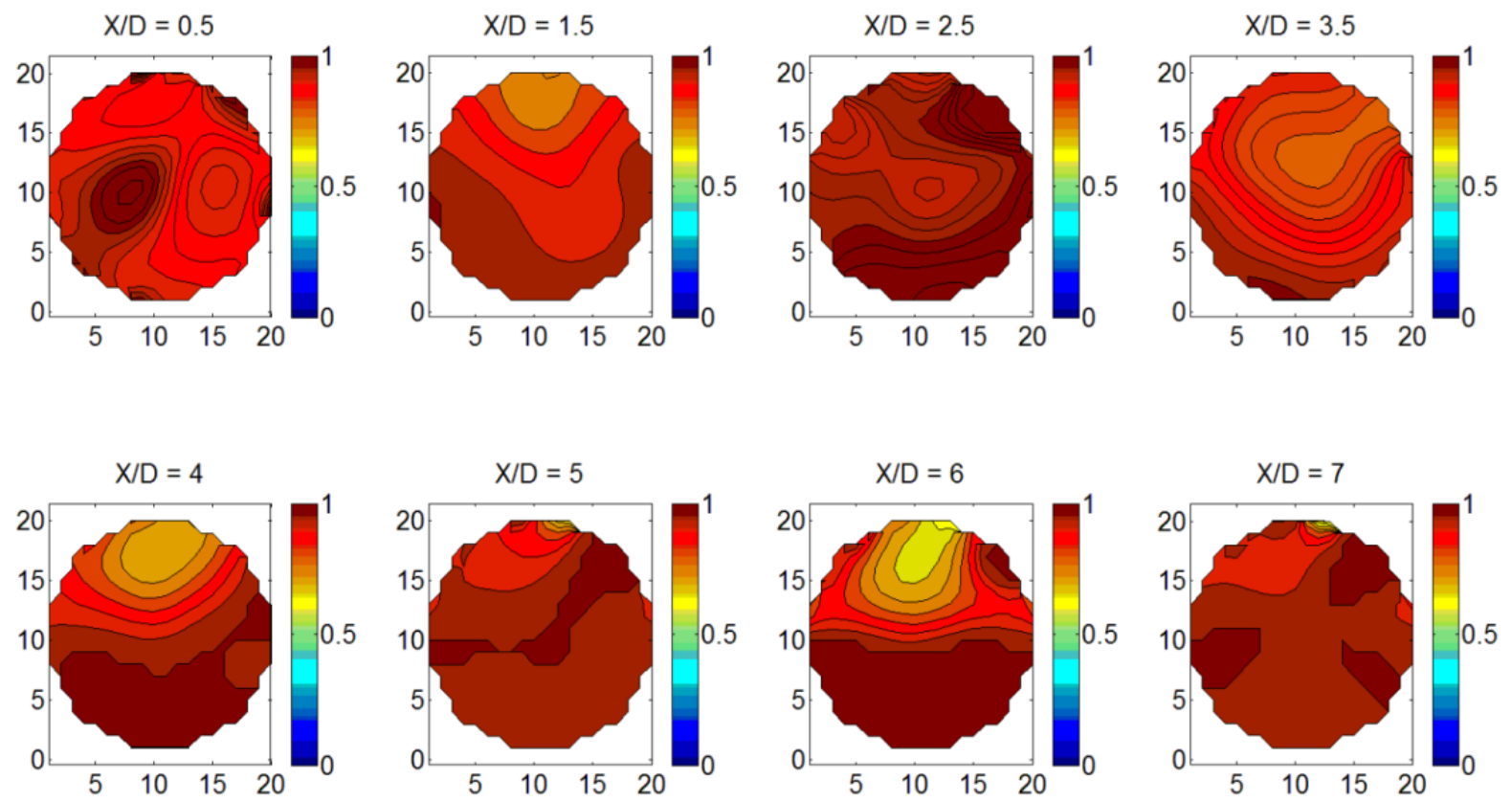

Figure 69: Conductivity Tomogram at 40 psig_20 GPM_10\% GVF 

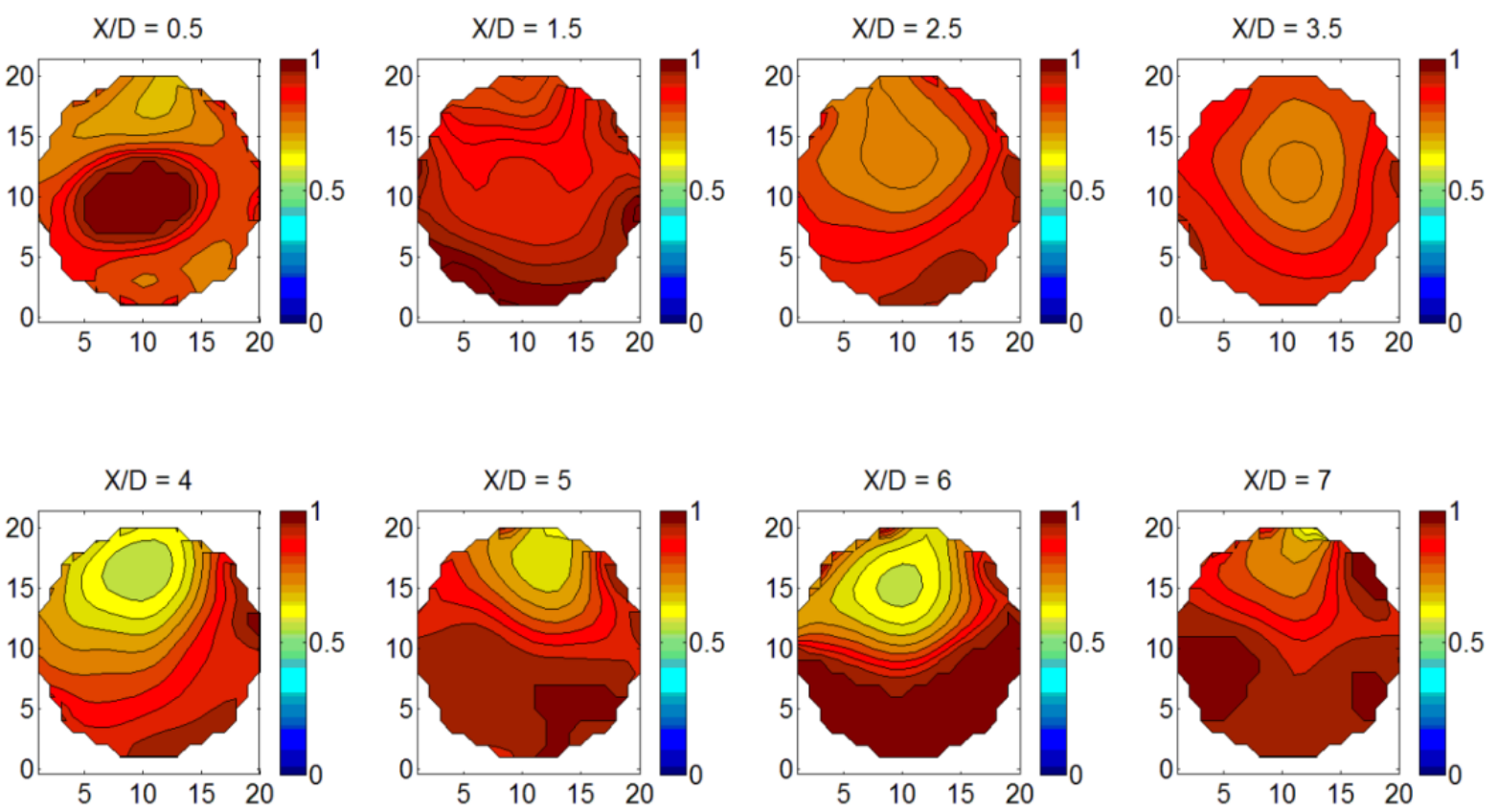

Figure 70: Conductivity Tomogram at 40 psig_20 GPM_20\% GVF
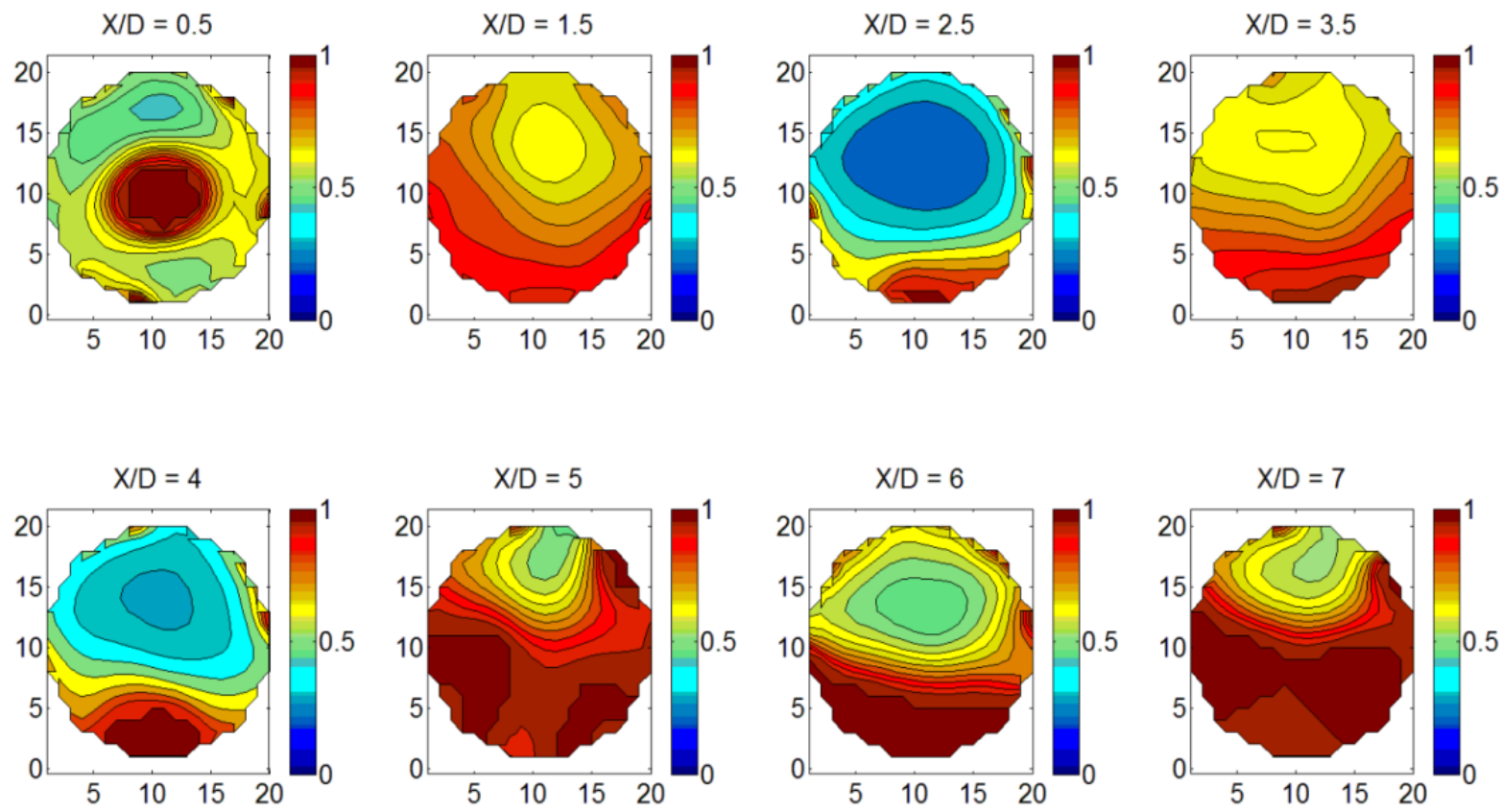

Figure 71: Conductivity Tomogram at 40 psig_20 GPM_30\% GVF 

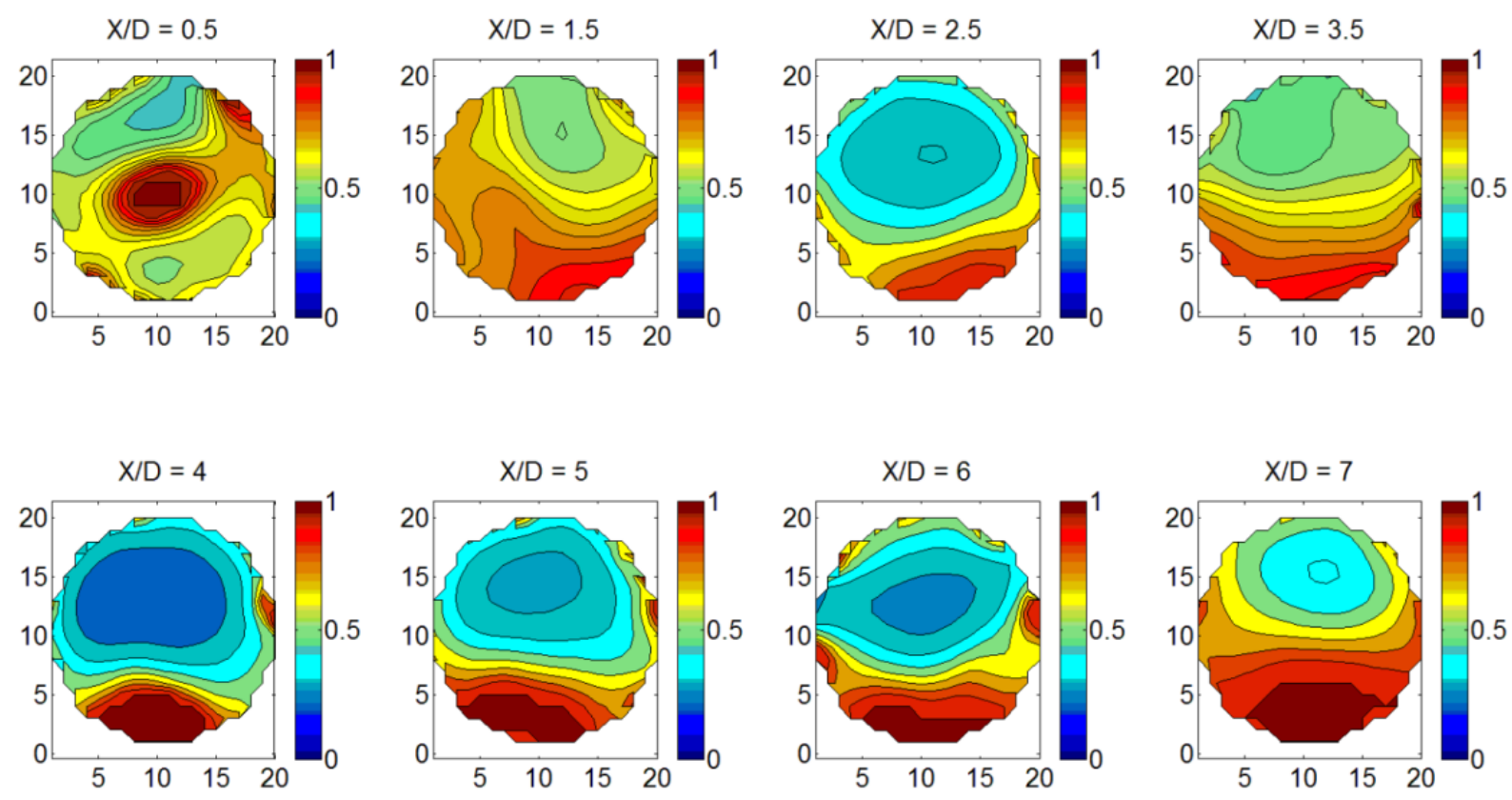

Figure 72: Conductivity Tomogram at 40 psig_20 GPM_40\% GVF
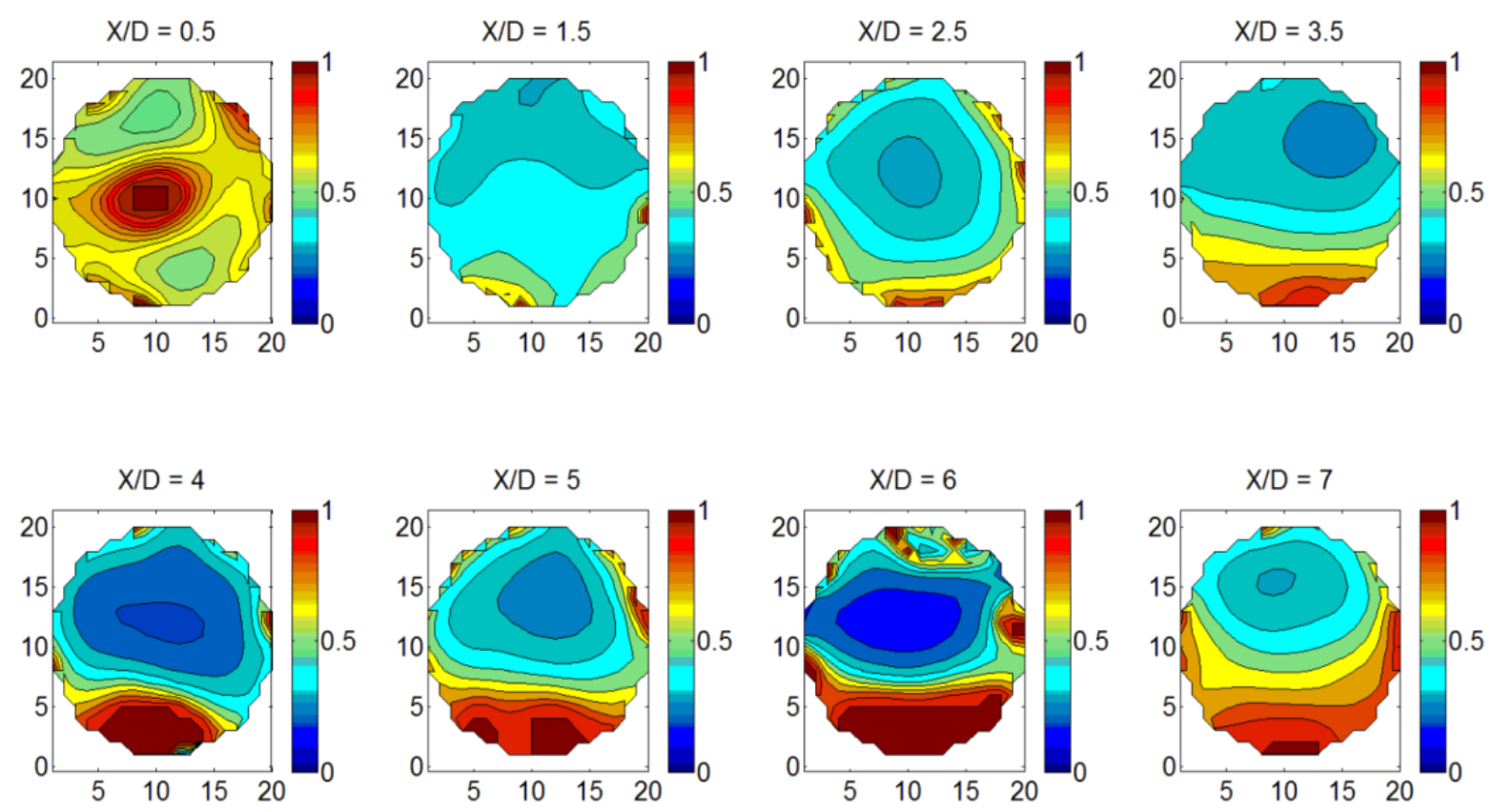

Figure 73: Conductivity Tomogram at 40 psig_20 GPM_50\% GVF 

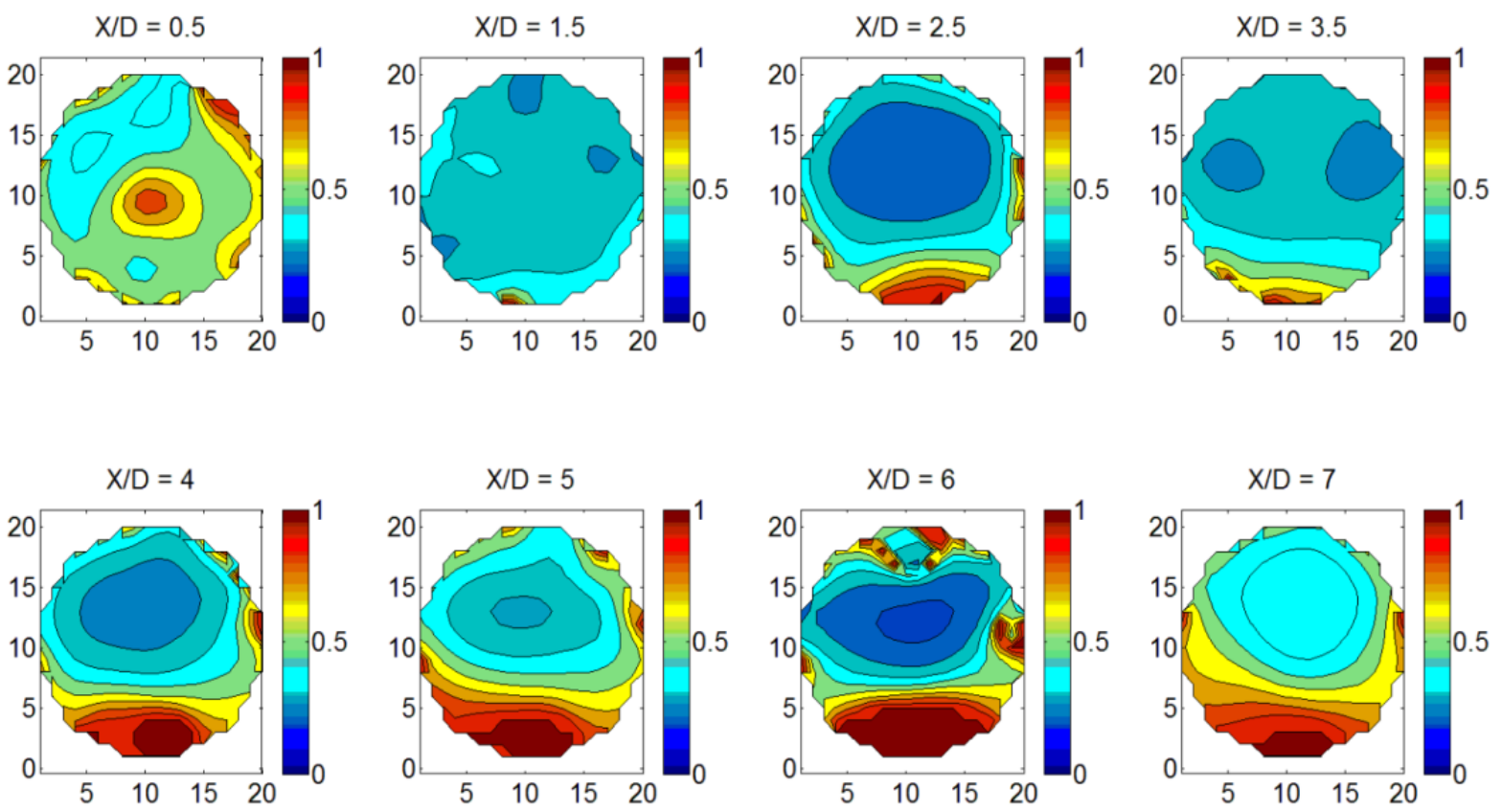

Figure 74: Conductivity Tomogram at 40 psig_20 GPM_60\% GVF
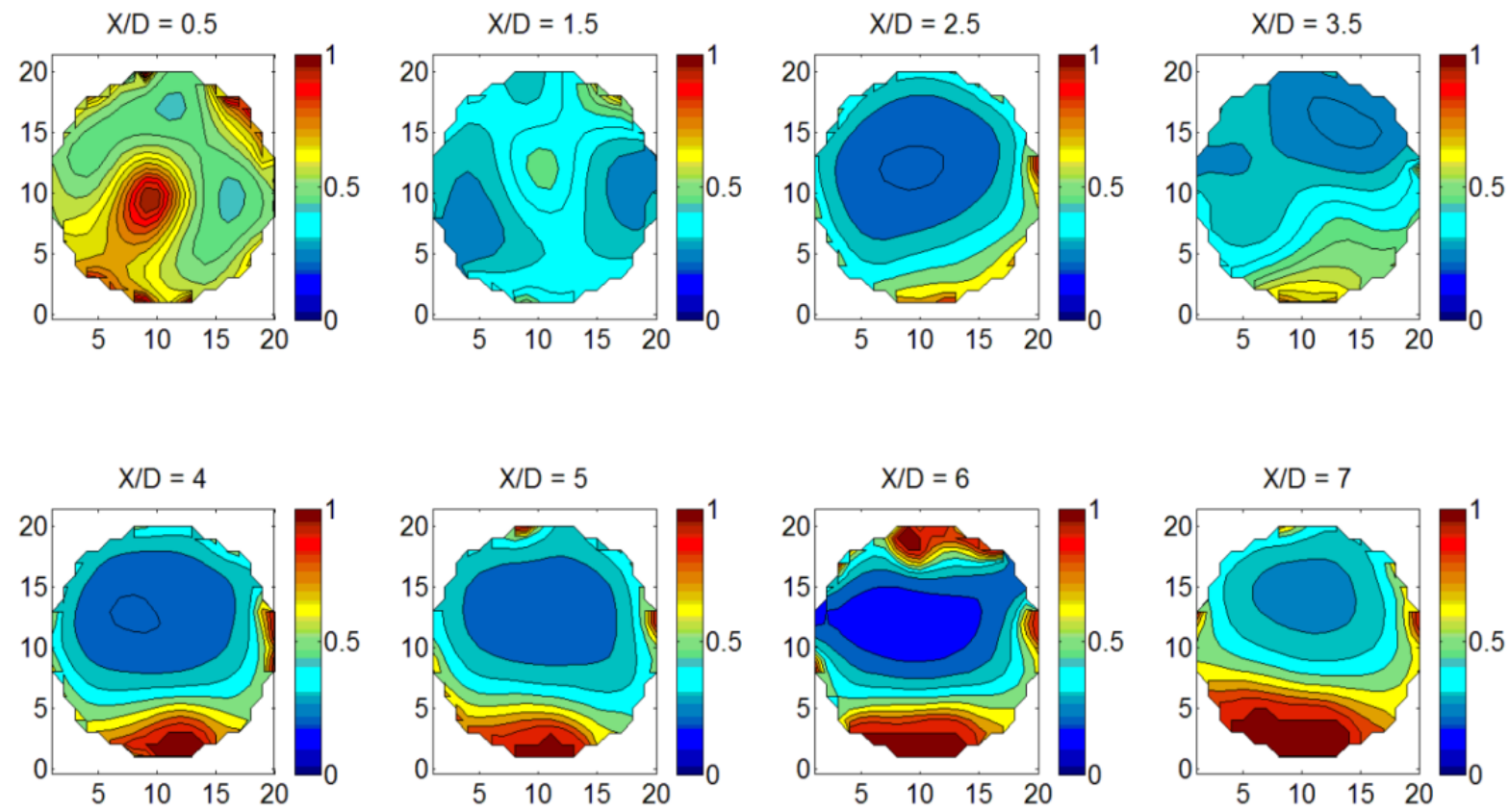

Figure 75: Conductivity Tomogram at 40 psig_20 GPM_70\% GVF 

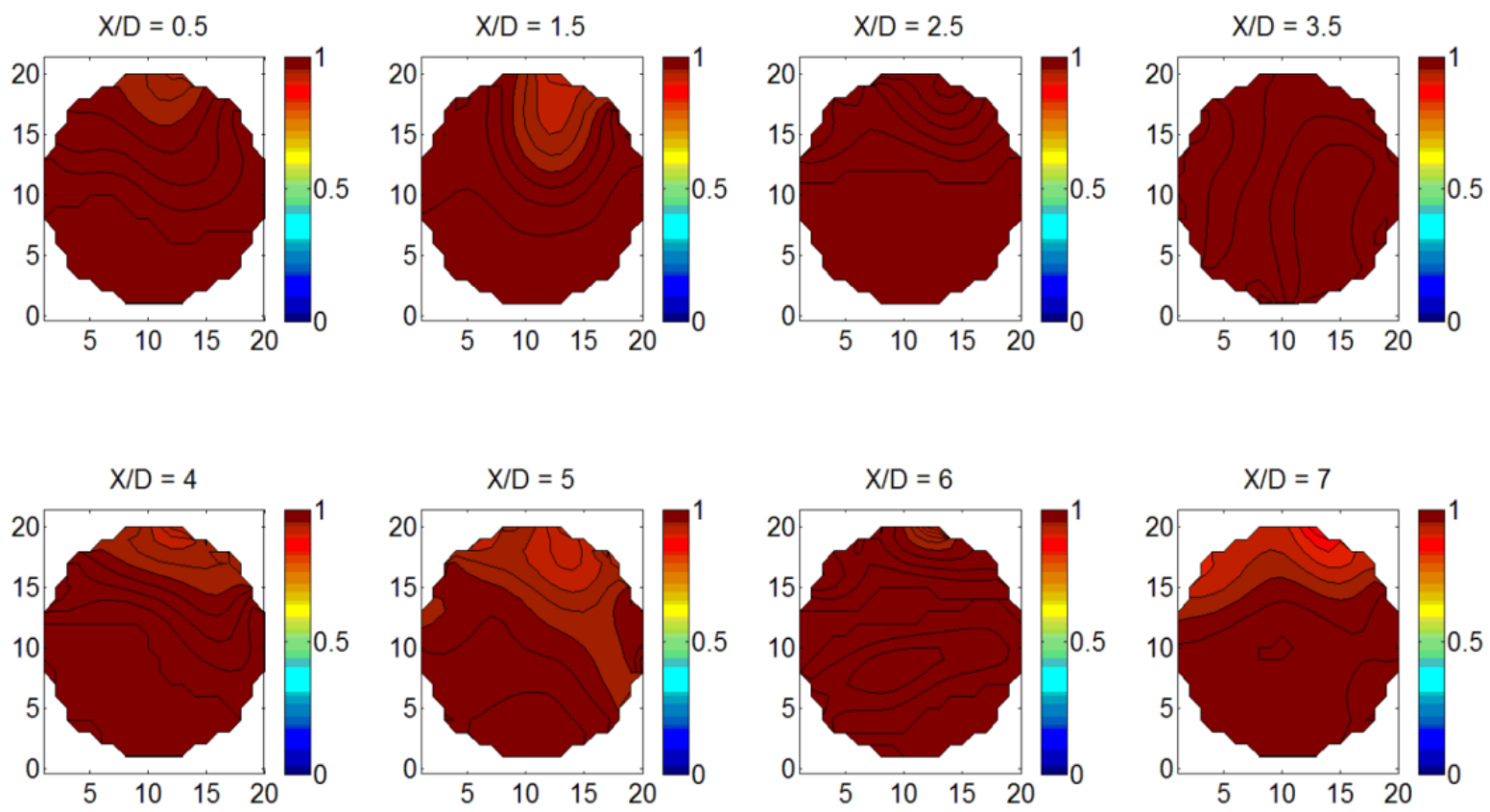

Figure 76: Conductivity Tomogram at 40 psig_40 GPM_5\% GVF
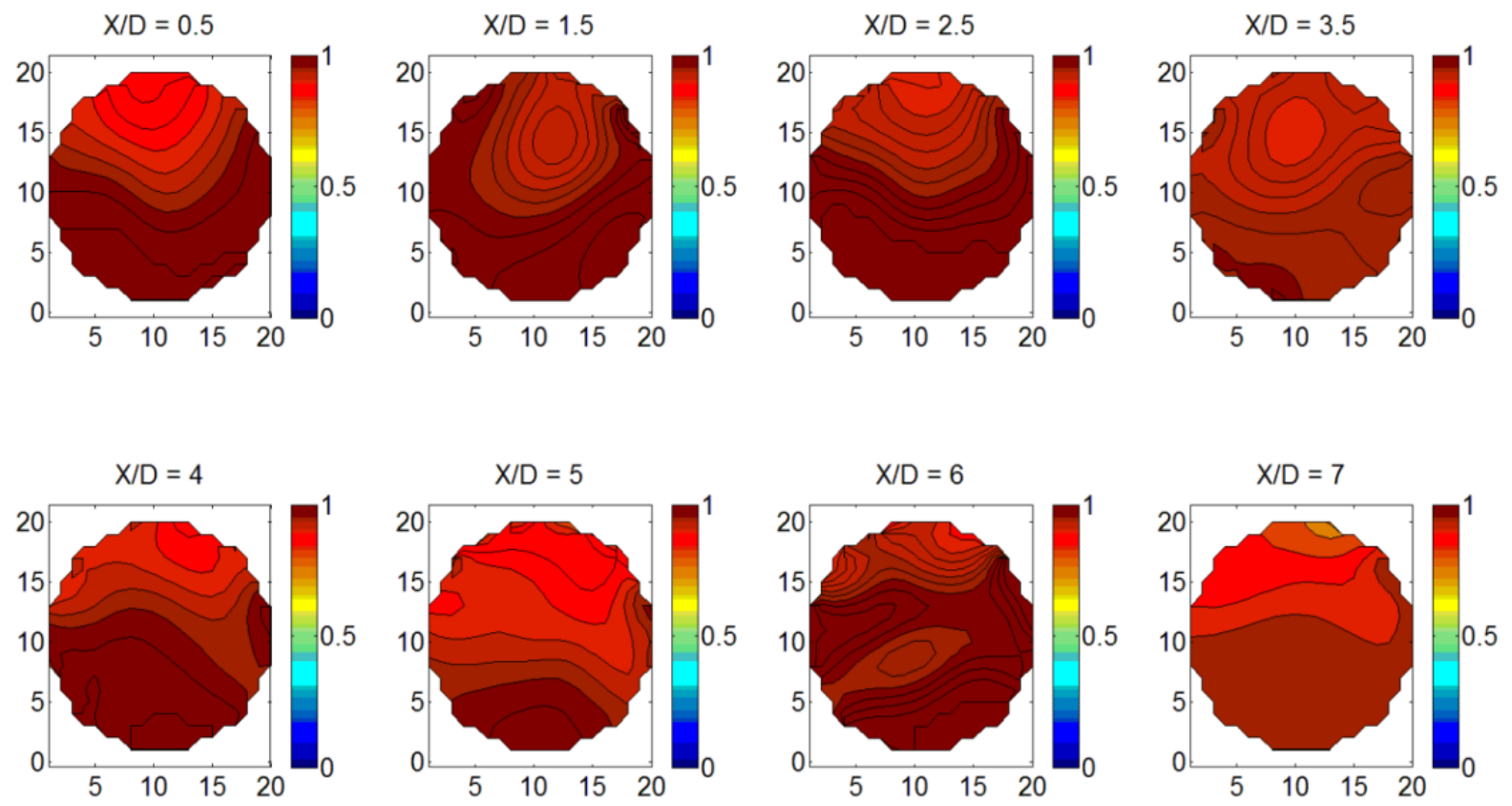

Figure 77: Conductivity Tomogram at 40 psig_40 GPM_10\% GVF 

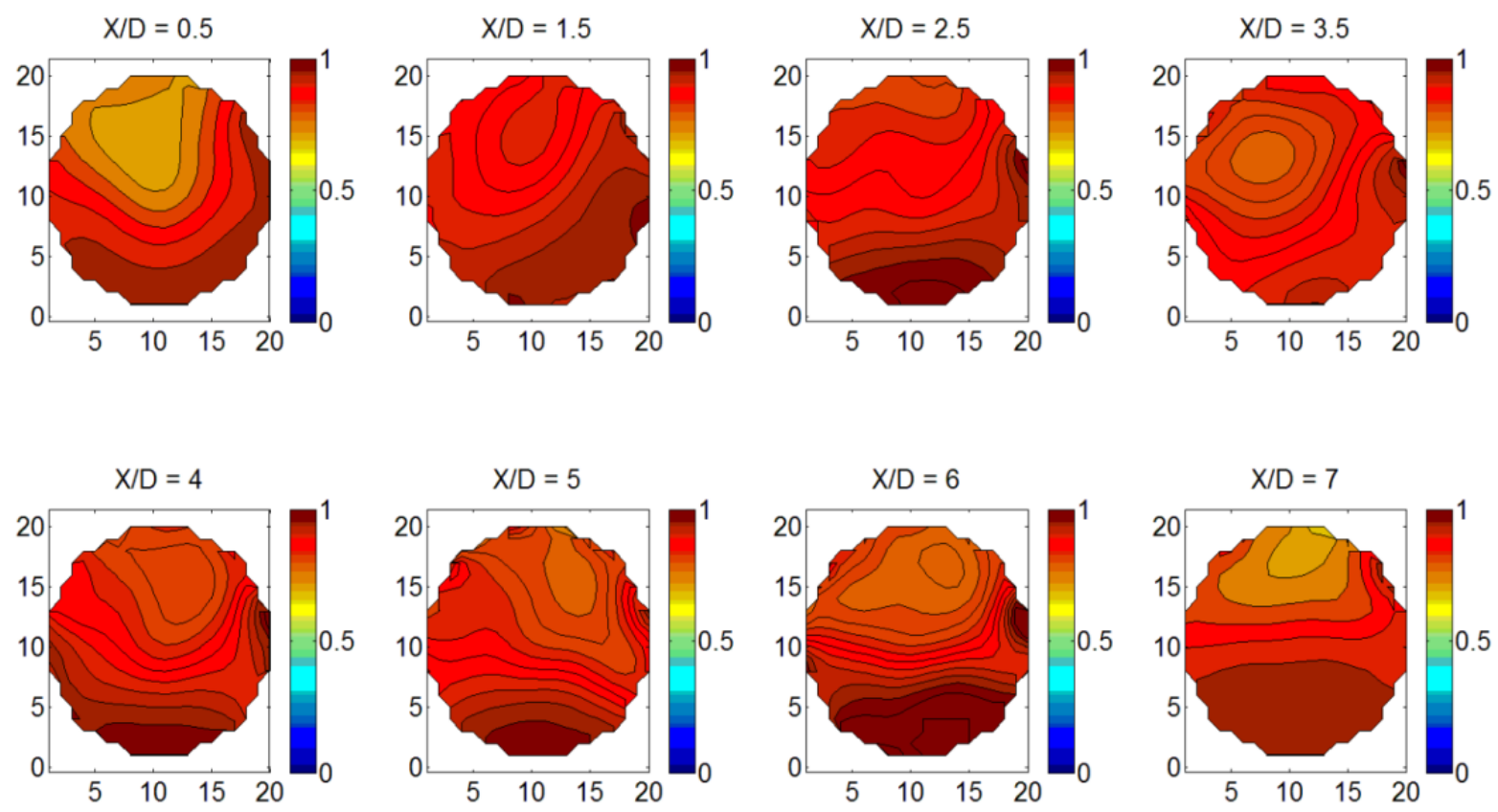

Figure 78: Conductivity Tomogram at 40 psig_40 GPM_20\% GVF
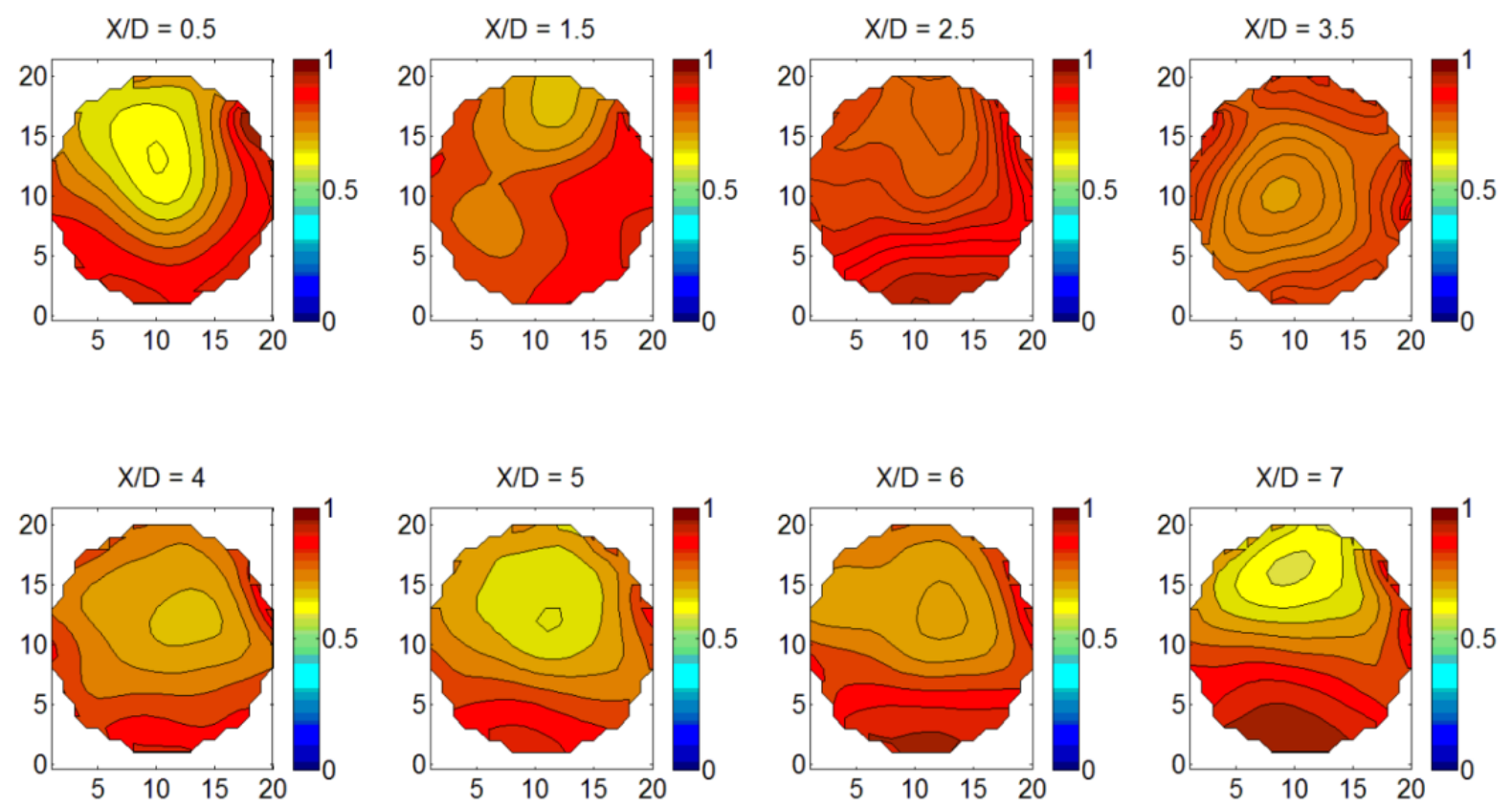

Figure 79: Conductivity Tomogram at 40 psig_40 GPM_30\% GVF 

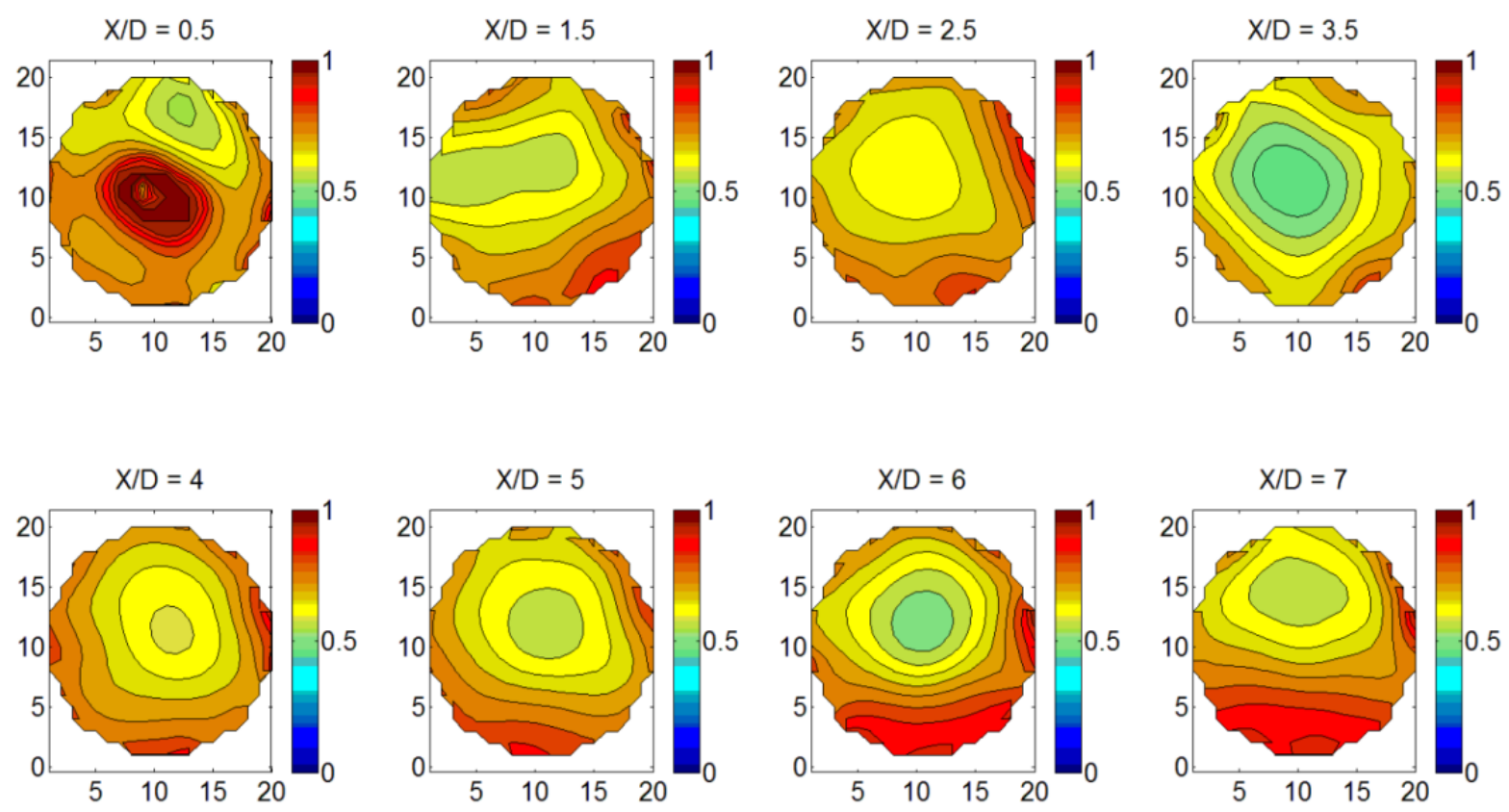

Figure 80: Conductivity Tomogram at 40 psig_40 GPM_40\% GVF
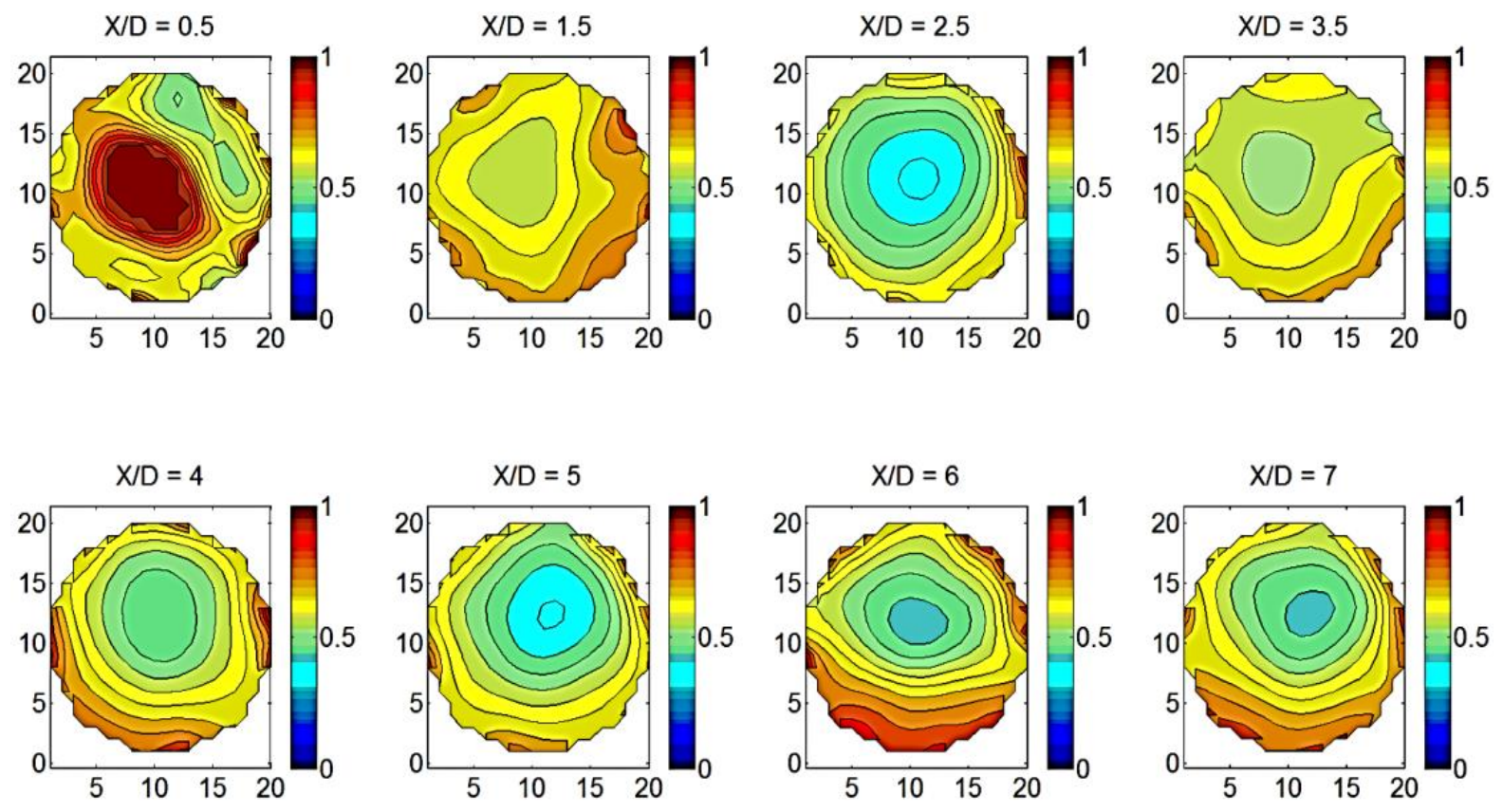

Figure 81: Conductivity Tomogram at 40 psig_40 GPM_50\% GVF 

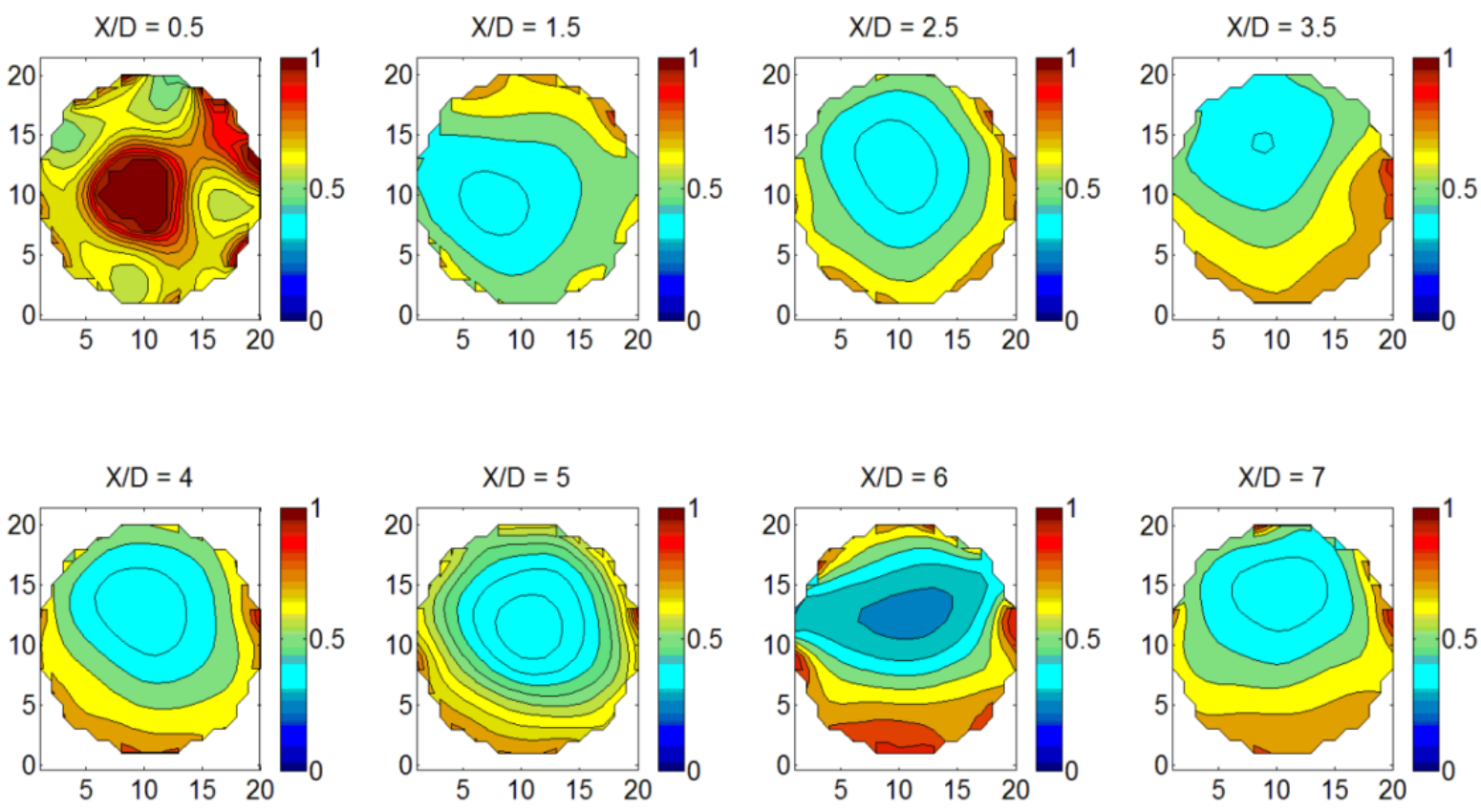

Figure 82: Conductivity Tomogram at 40 psig_40 GPM_60\% GVF
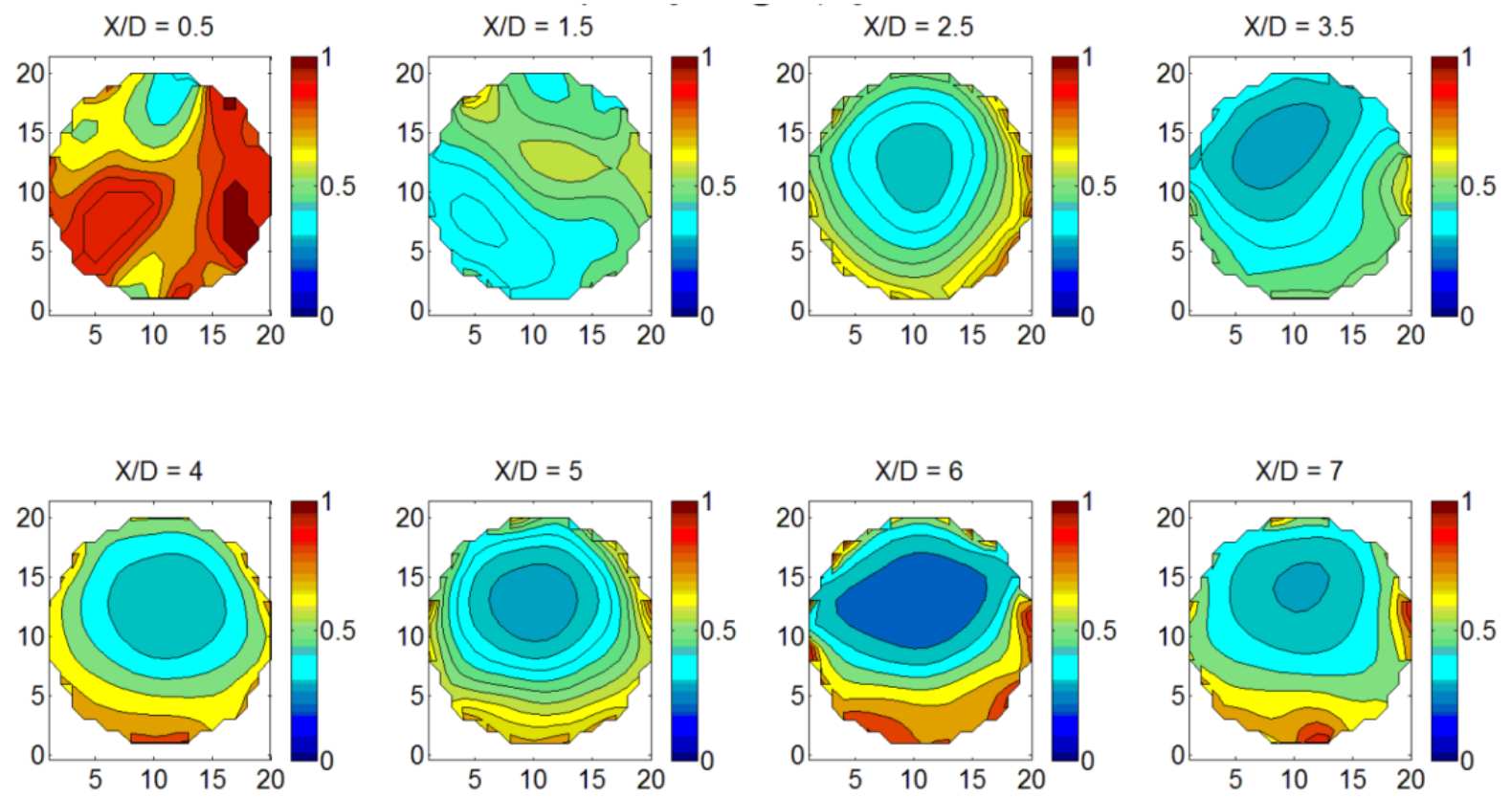

Figure 83: Conductivity Tomogram at 40 psig_40 GPM_70\% GVF 

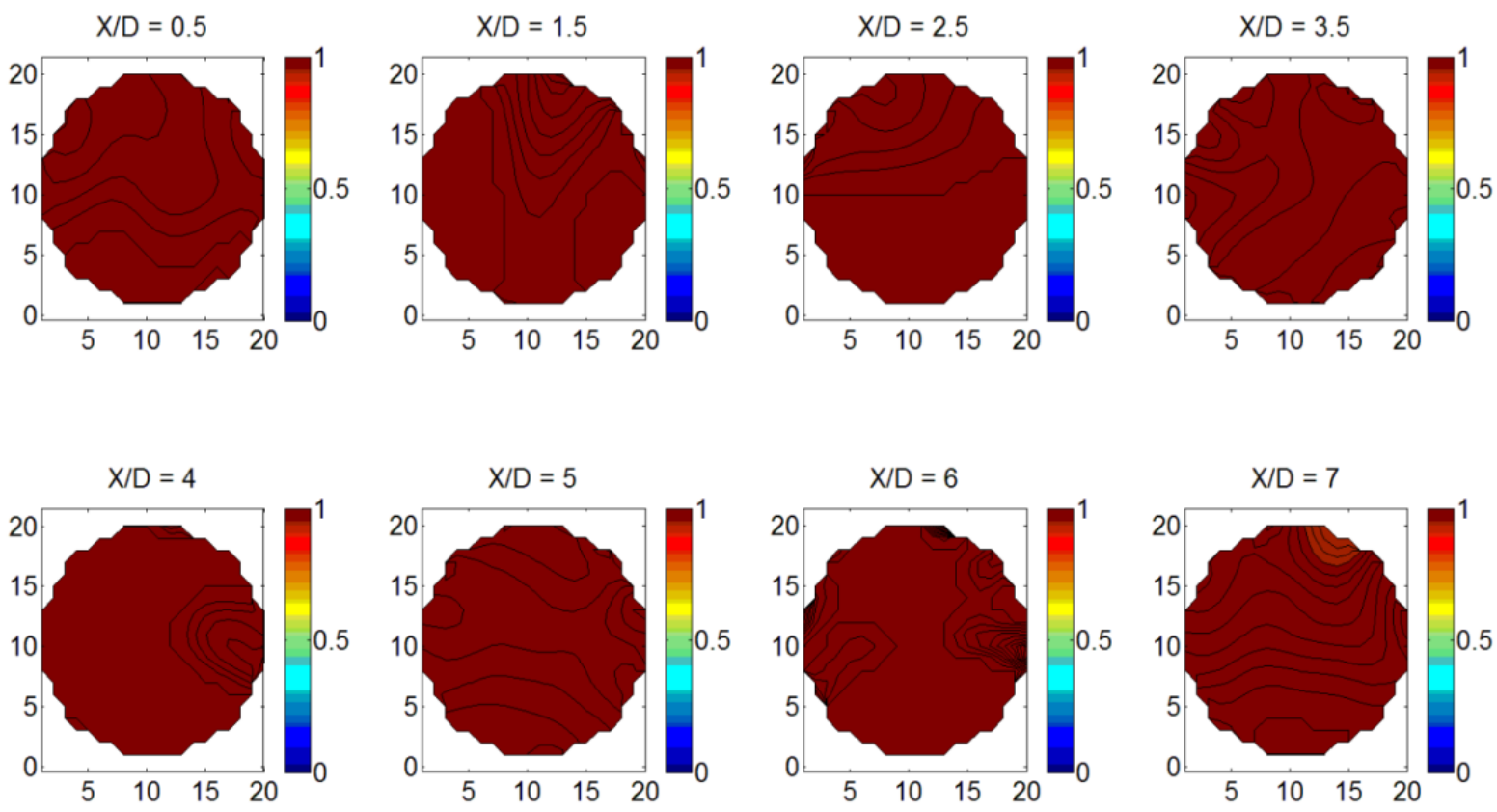

Figure 84: Conductivity Tomogram at 40 psig_60 GPM_5\% GVF
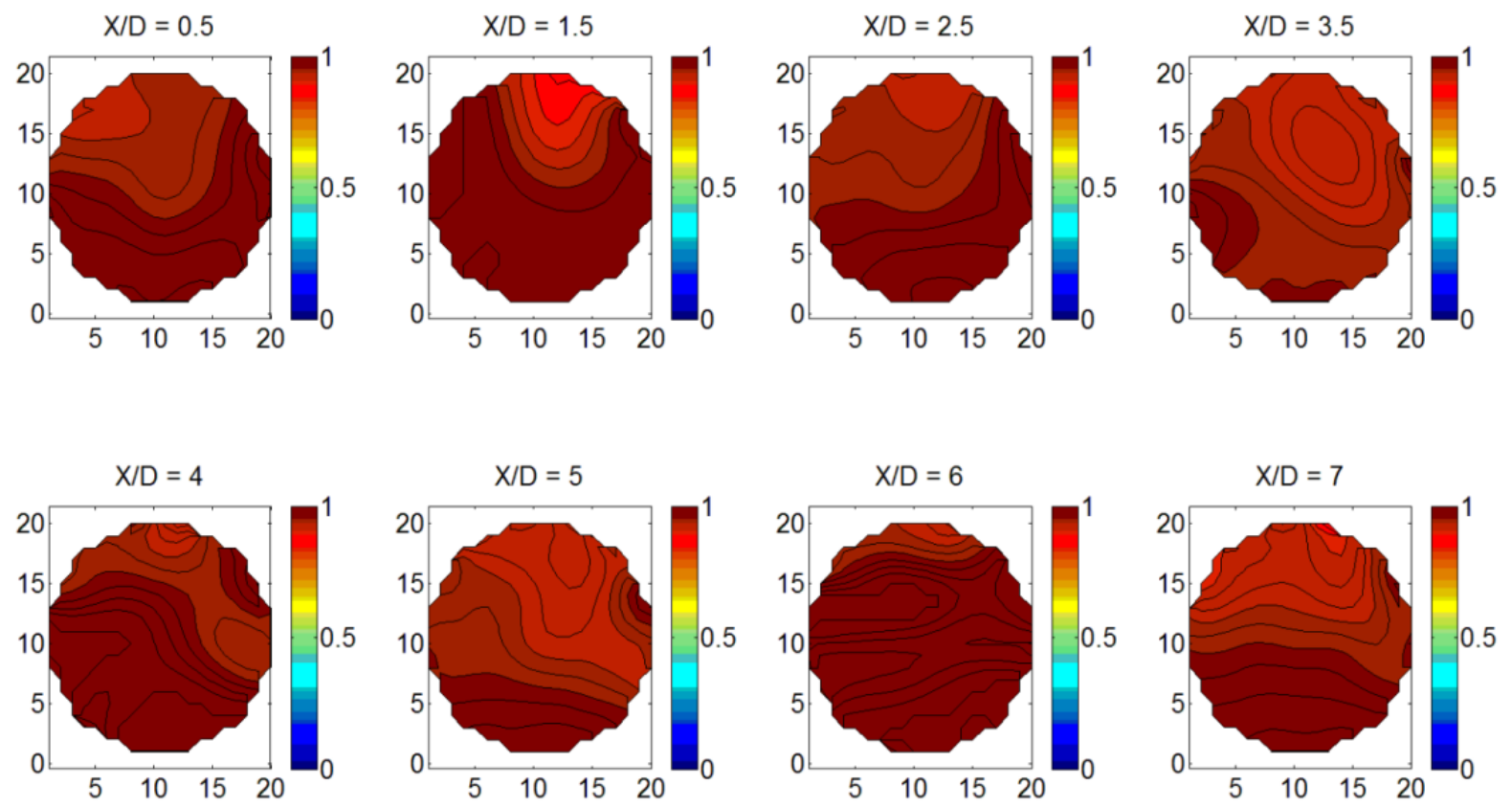

Figure 85: Conductivity Tomogram at 40 psig_60 GPM_10\% GVF 

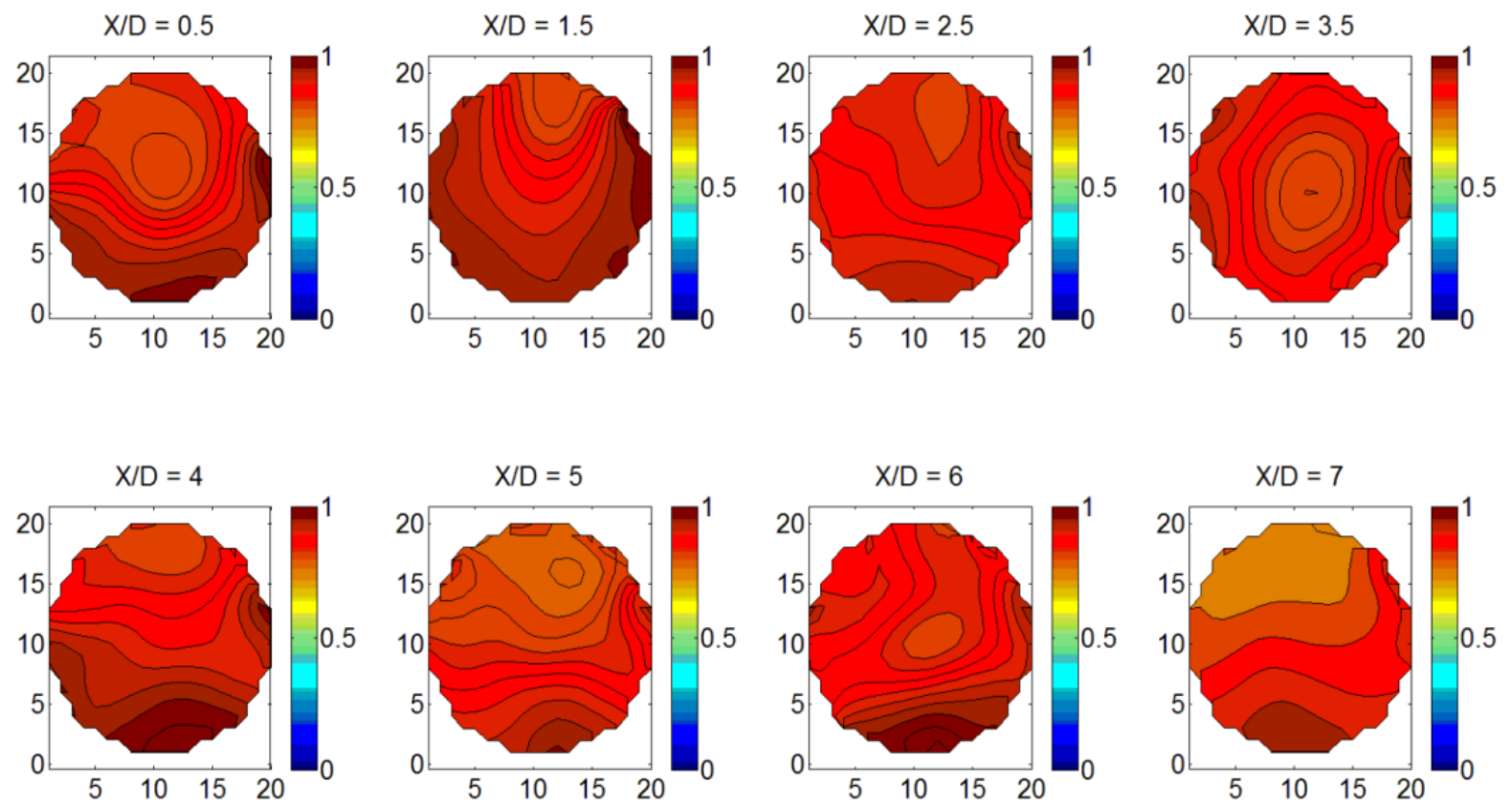

Figure 86: Conductivity Tomogram at 40 psig_60 GPM_20\% GVF
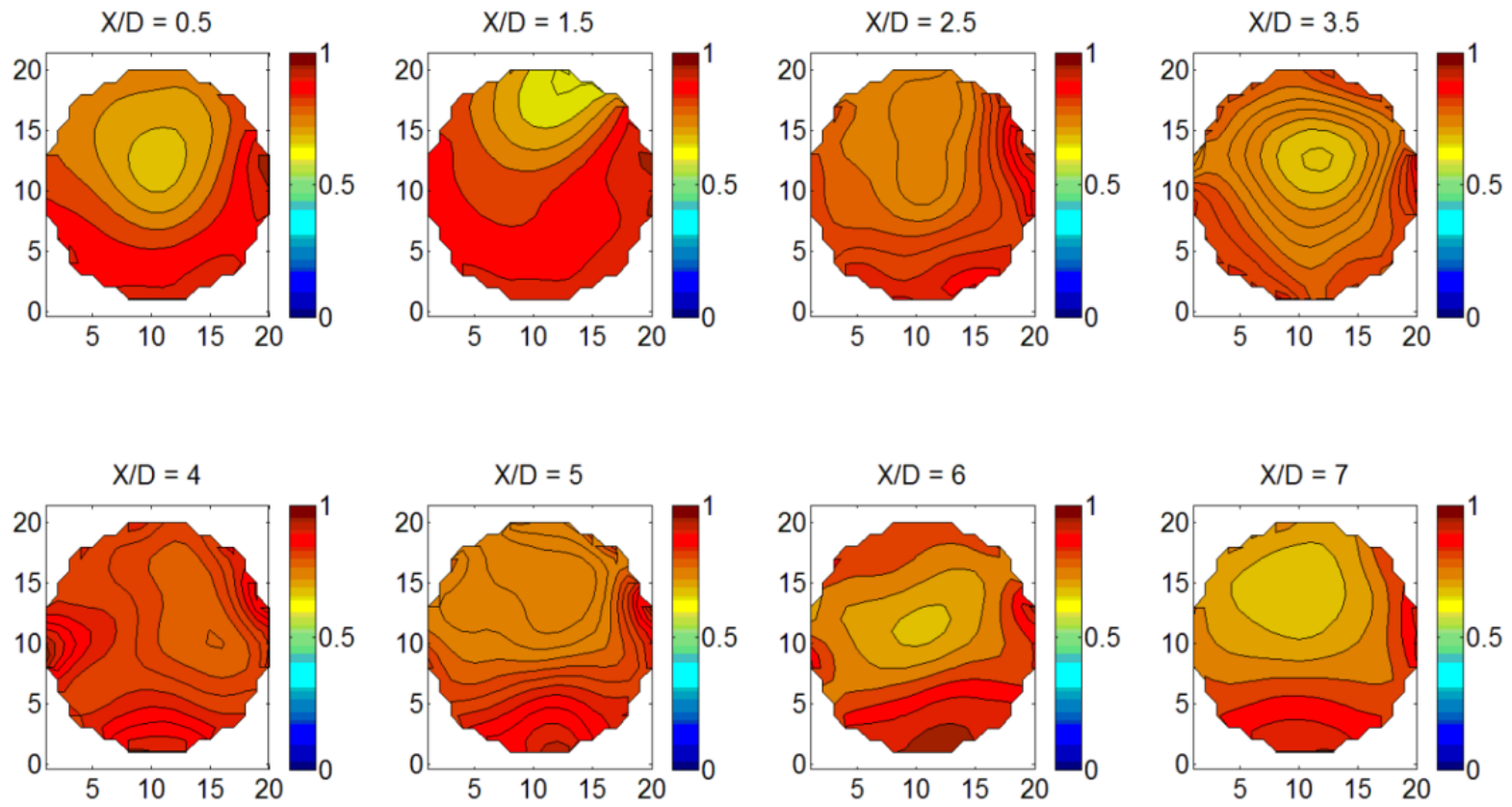

Figure 87: Conductivity Tomogram at 40 psig_60 GPM_30\% GVF 

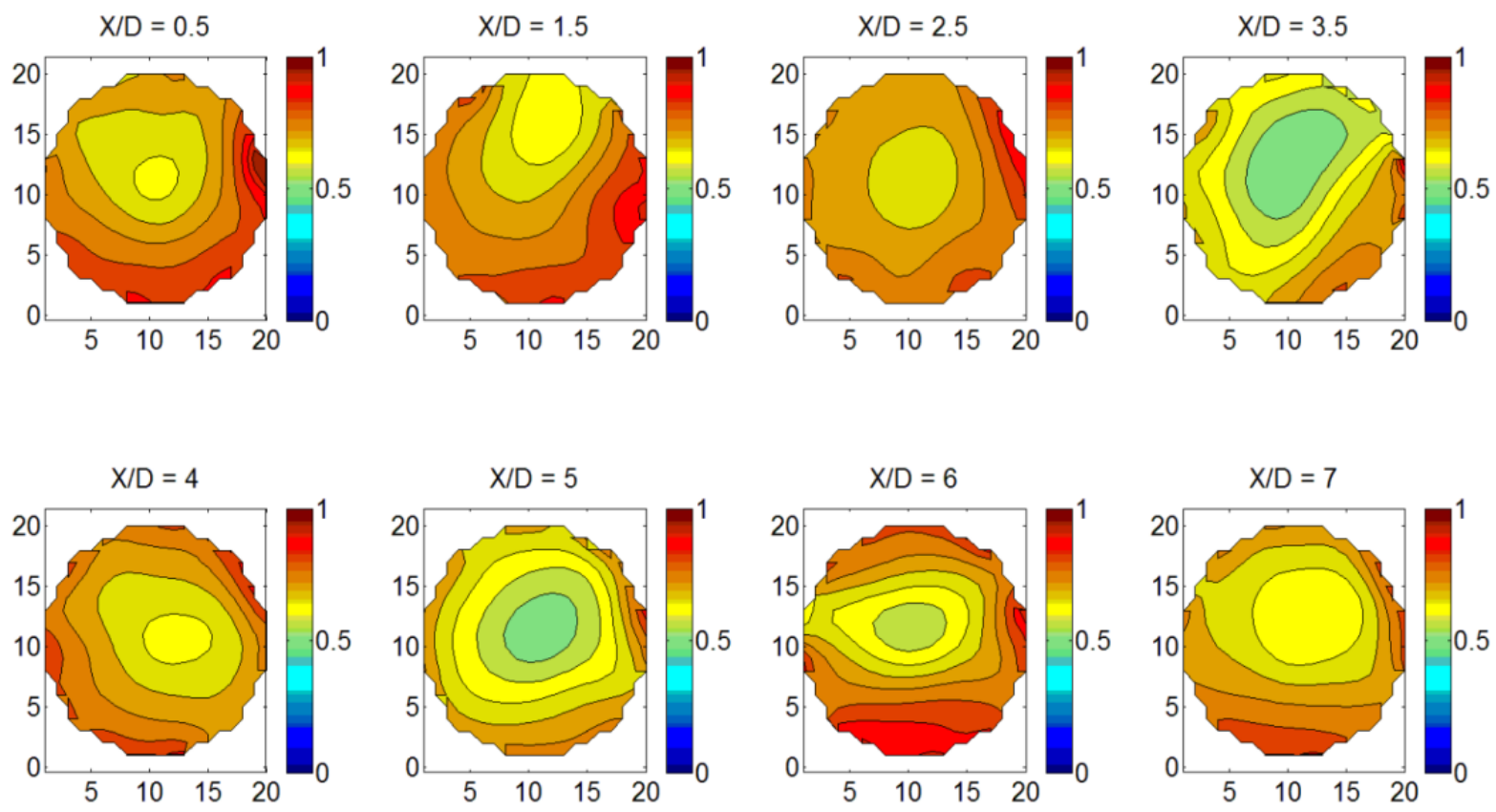

Figure 88: Conductivity Tomogram at 40 psig_60 GPM_40\% GVF
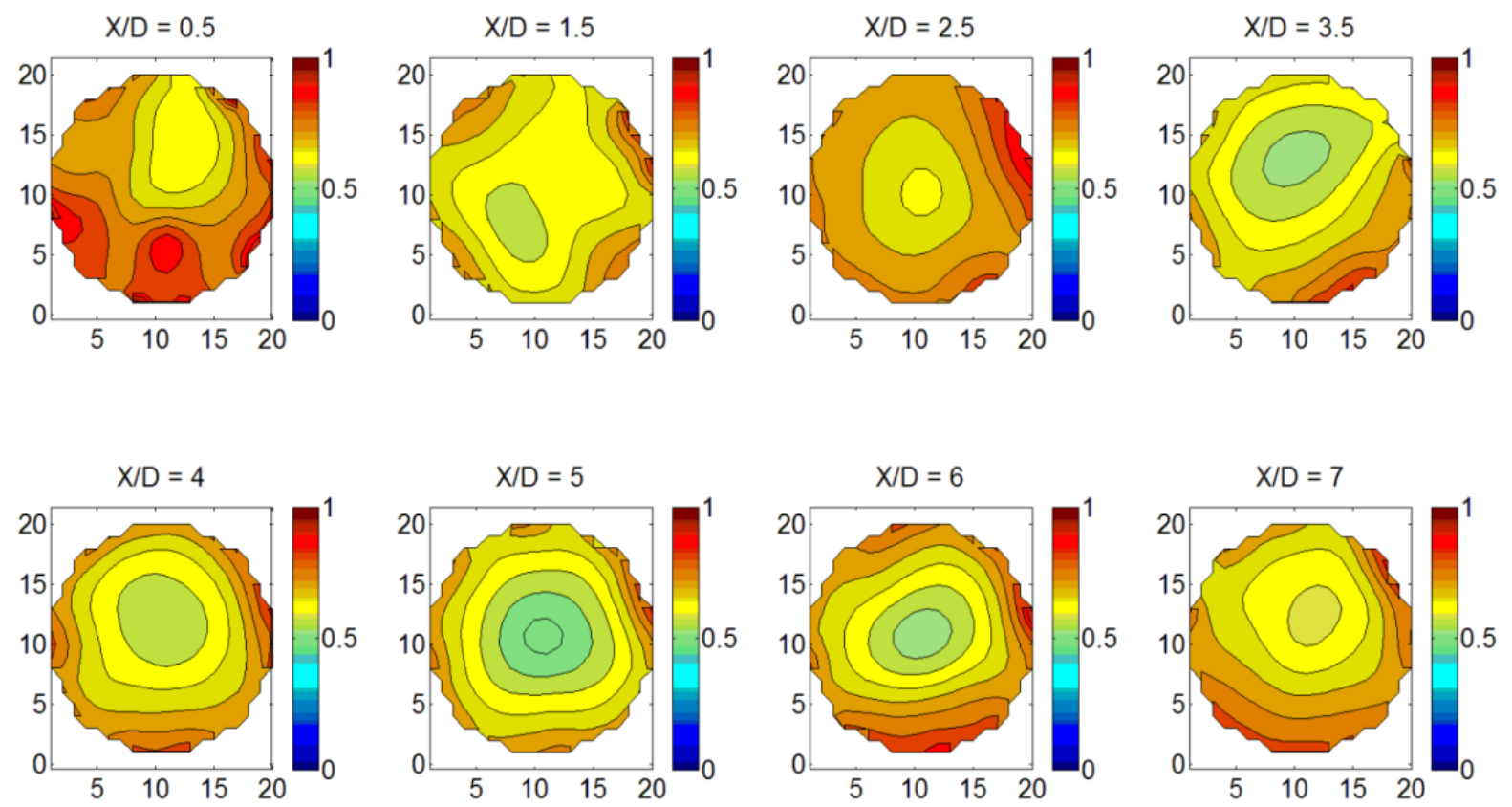

Figure 89: Conductivity Tomogram at 40 psig_60 GPM_50\% GVF 

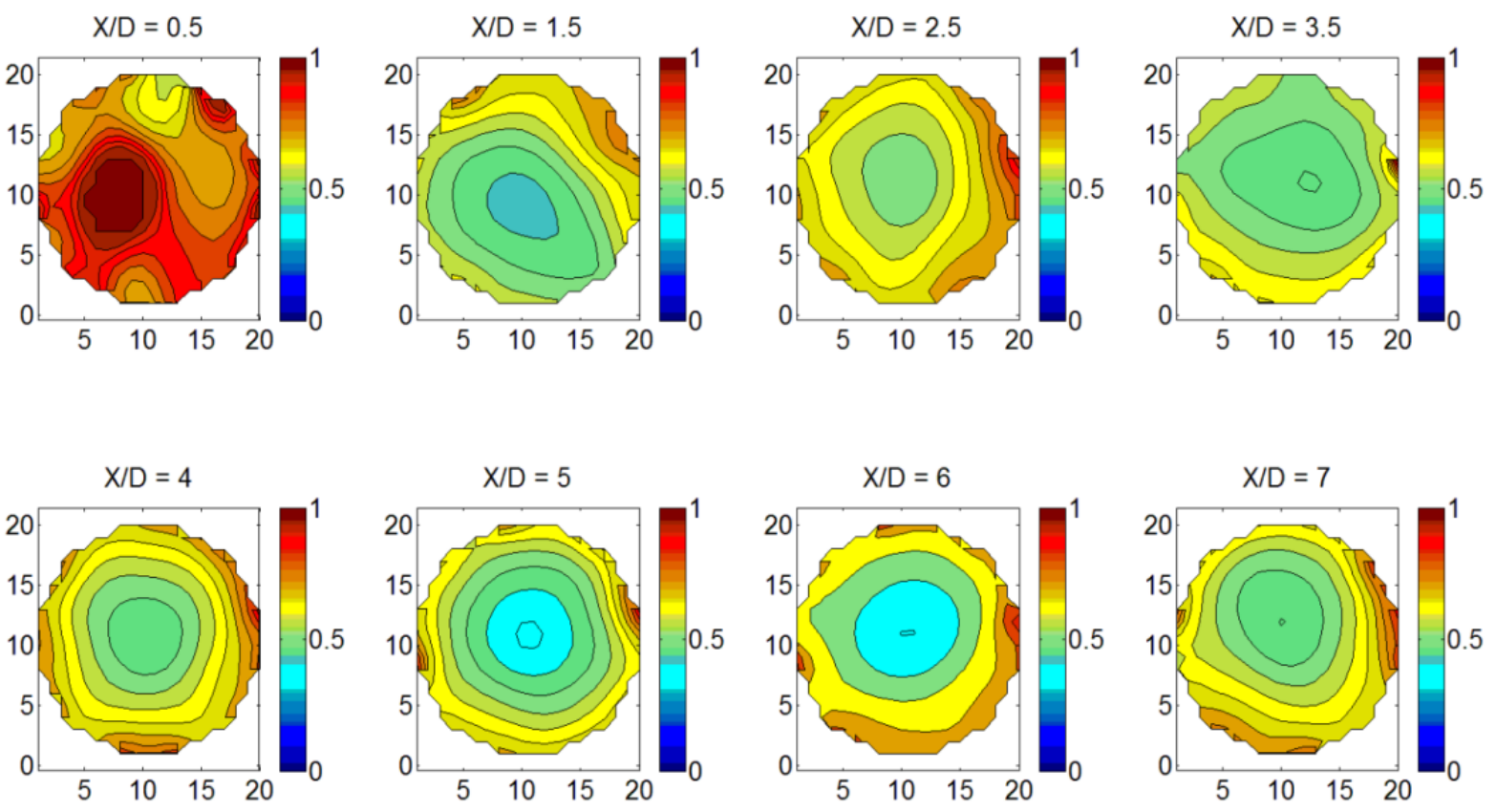

Figure 90: Conductivity Tomogram at 40 psig_60 GPM_60\% GVF
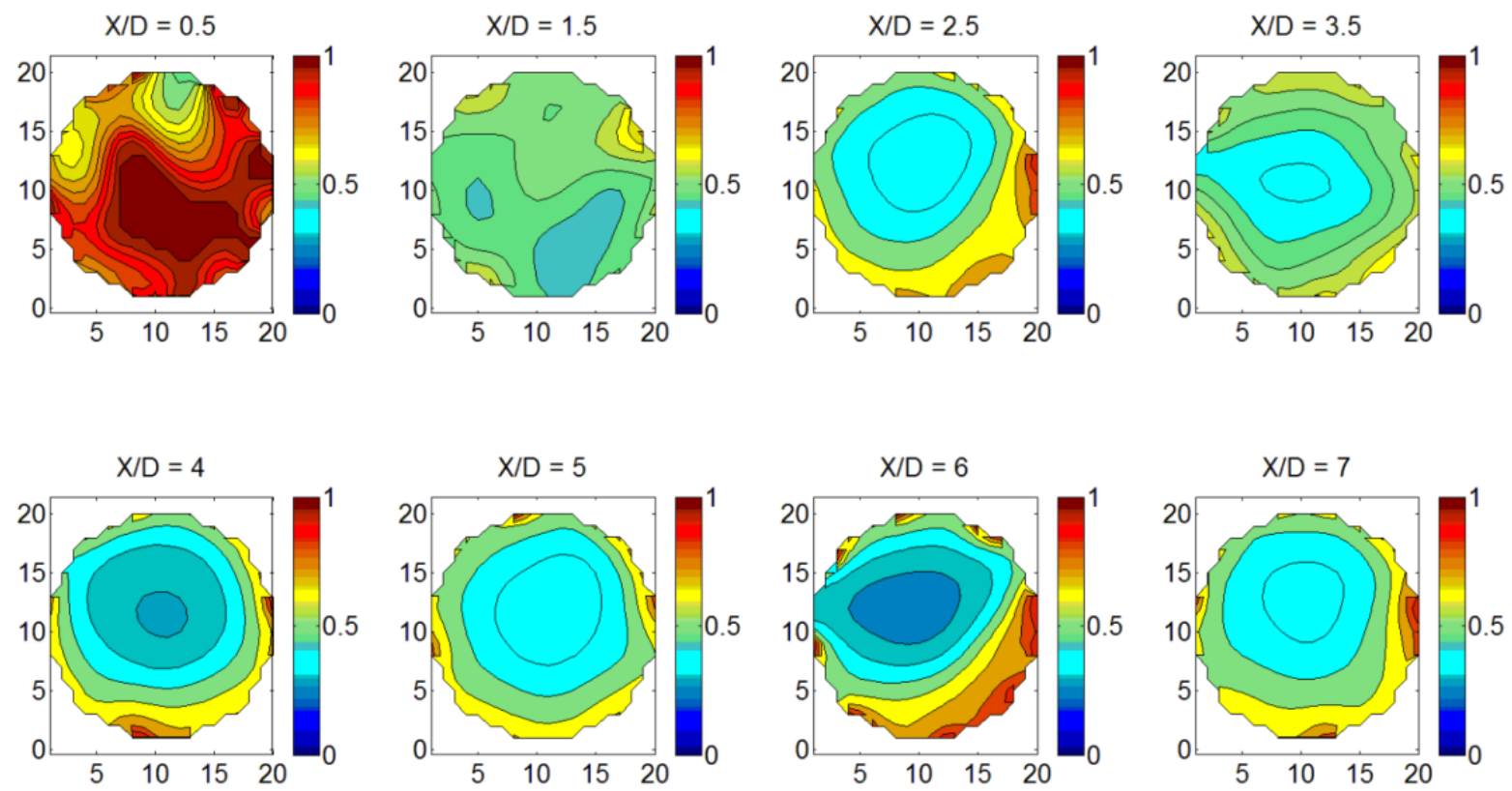

Figure 91: Conductivity Tomogram at 40 psig_60 GPM_70\% GVF 

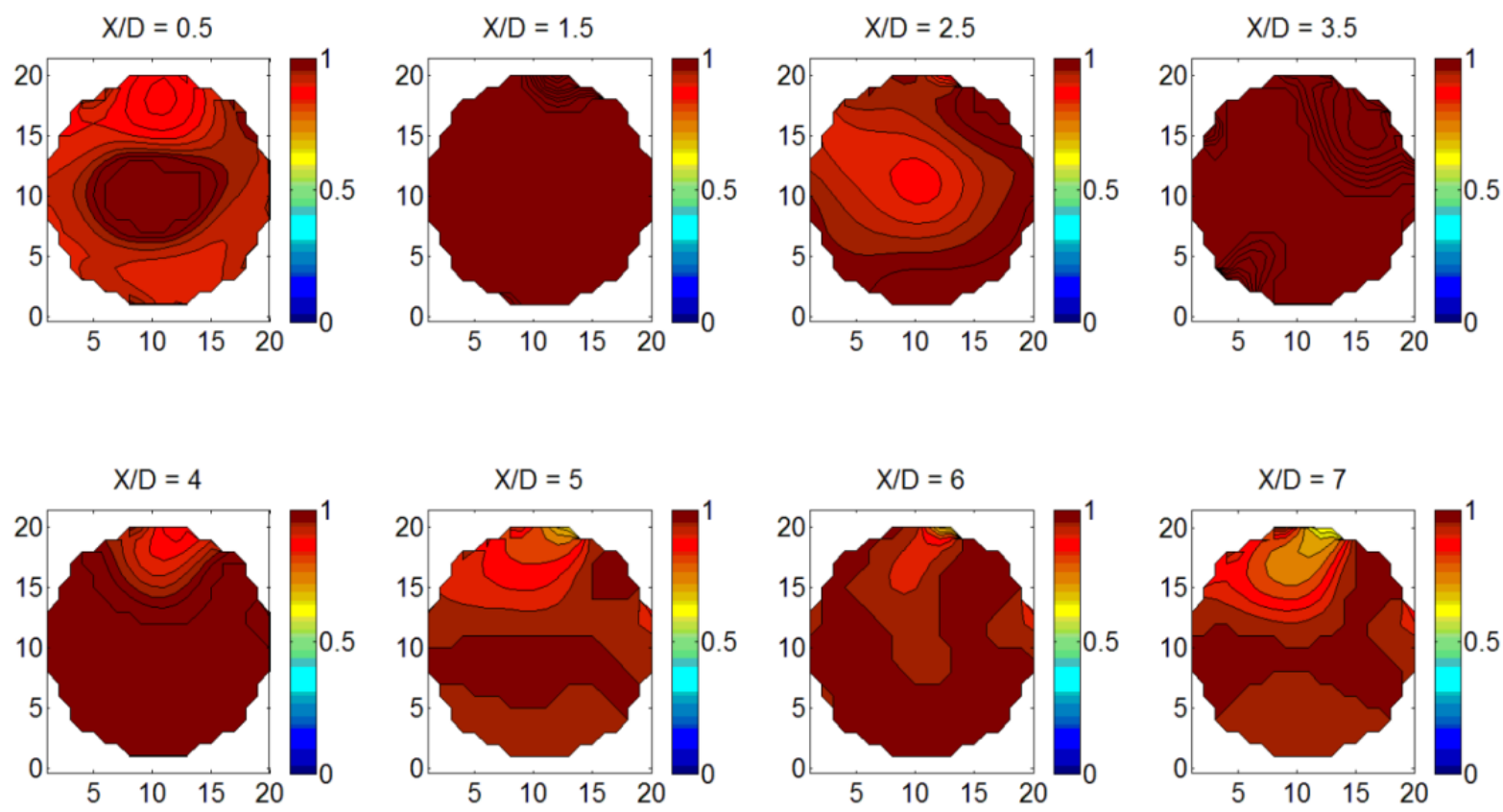

Figure 92: Conductivity Tomogram at 60 psig_20 GPM_5\% GVF
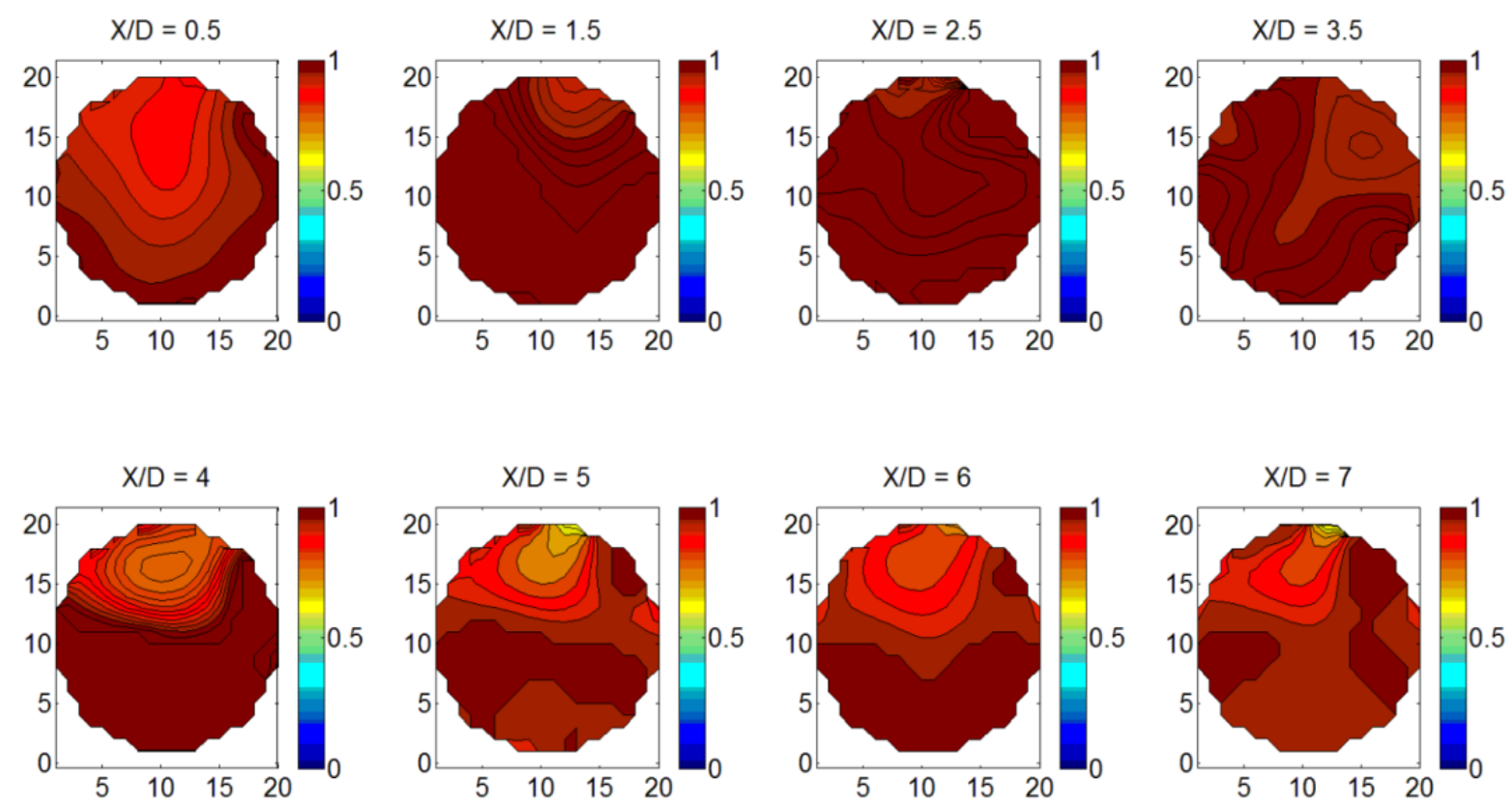

Figure 93: Conductivity Tomogram at 60 psig_20 GPM_10\% GVF 

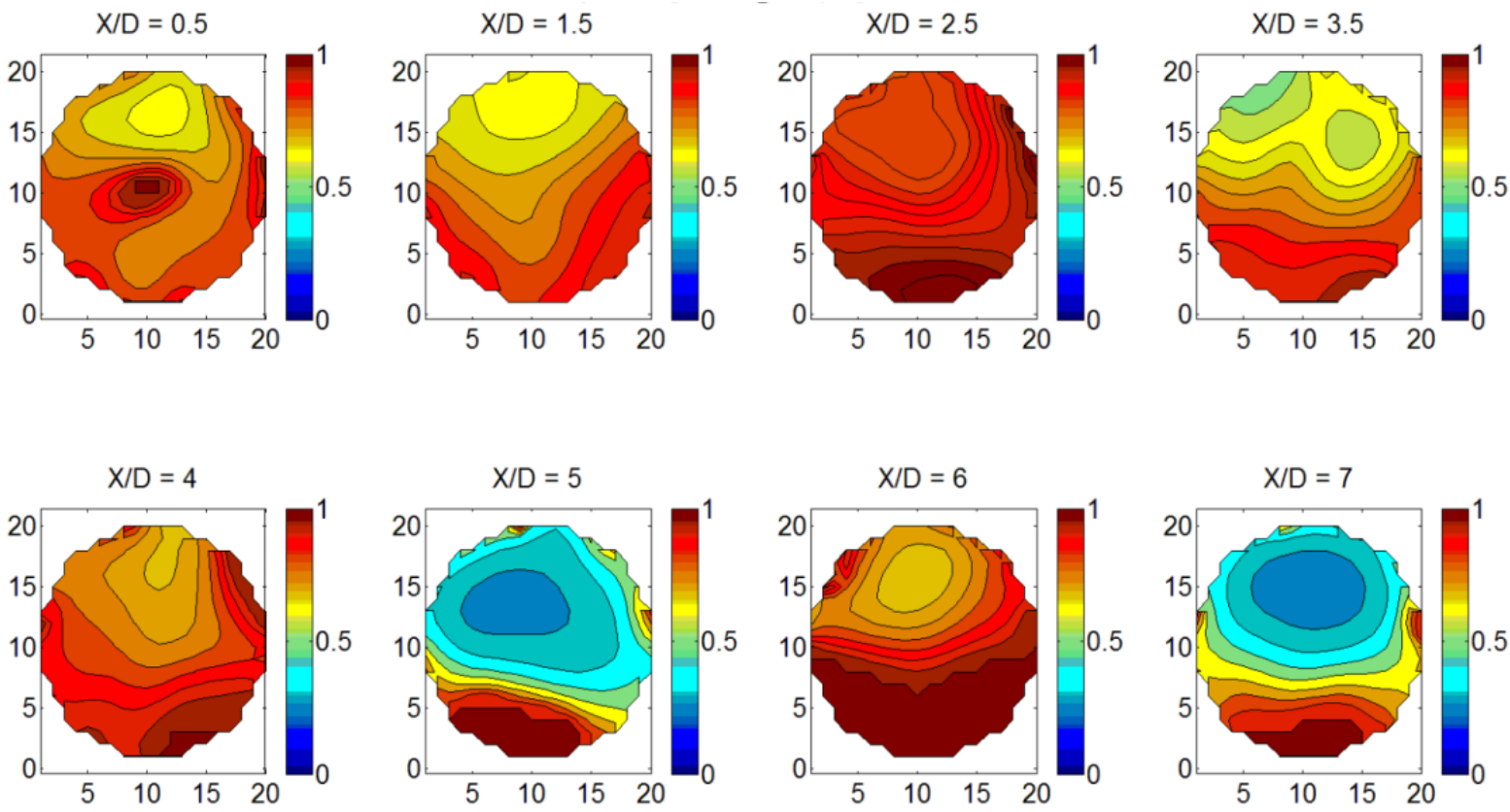

Figure 94: Conductivity Tomogram at 60 psig_20 GPM_20\% GVF
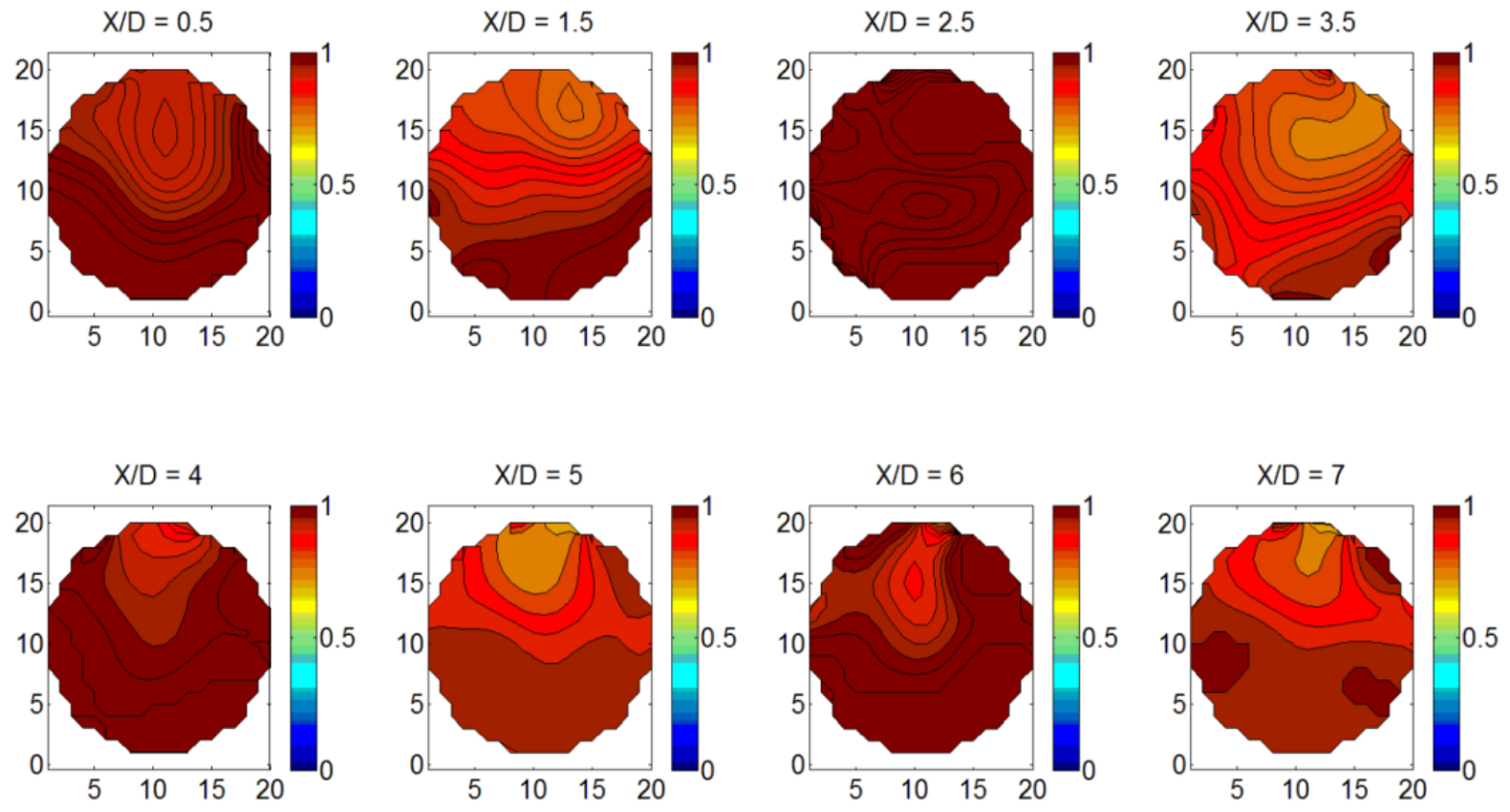

Figure 95: Conductivity Tomogram at 60 psig_20 GPM_30\% GVF 

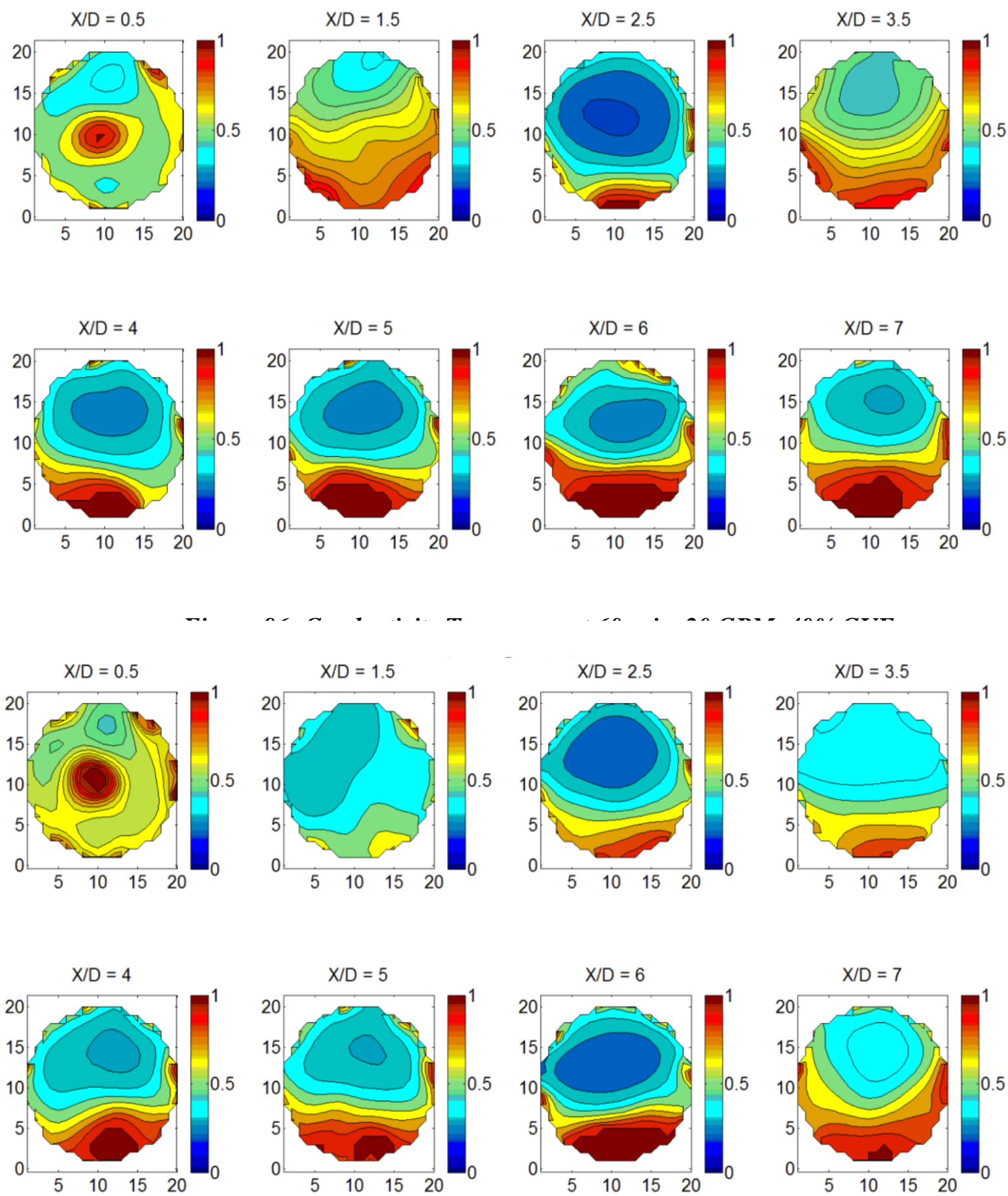

Figure 97: Conductivity Tomogram at 60 psig_20 GPM_50\% GVF 

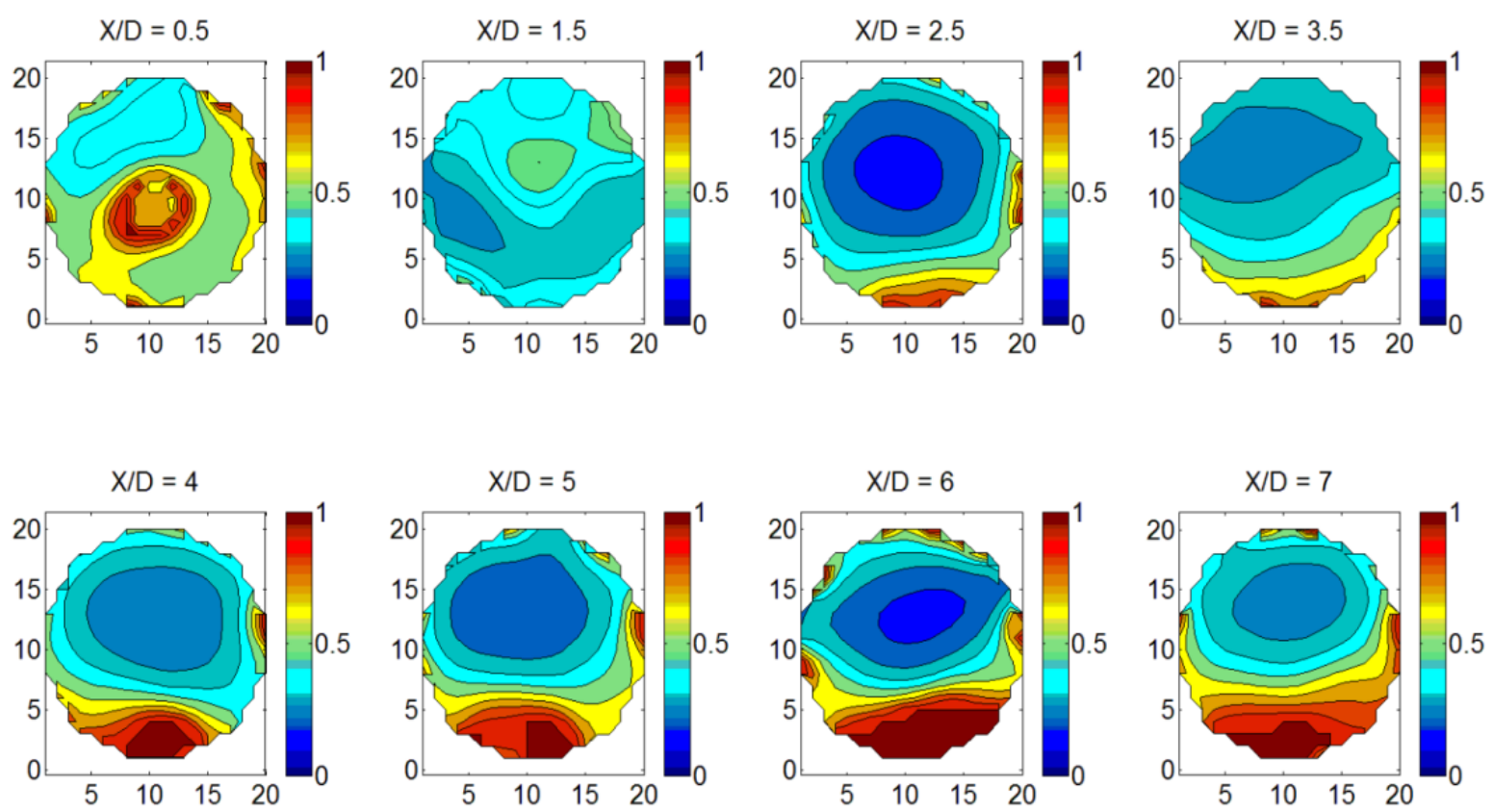

Figure 98: Conductivity Tomogram at 60 psig_20 GPM_60\% GVF
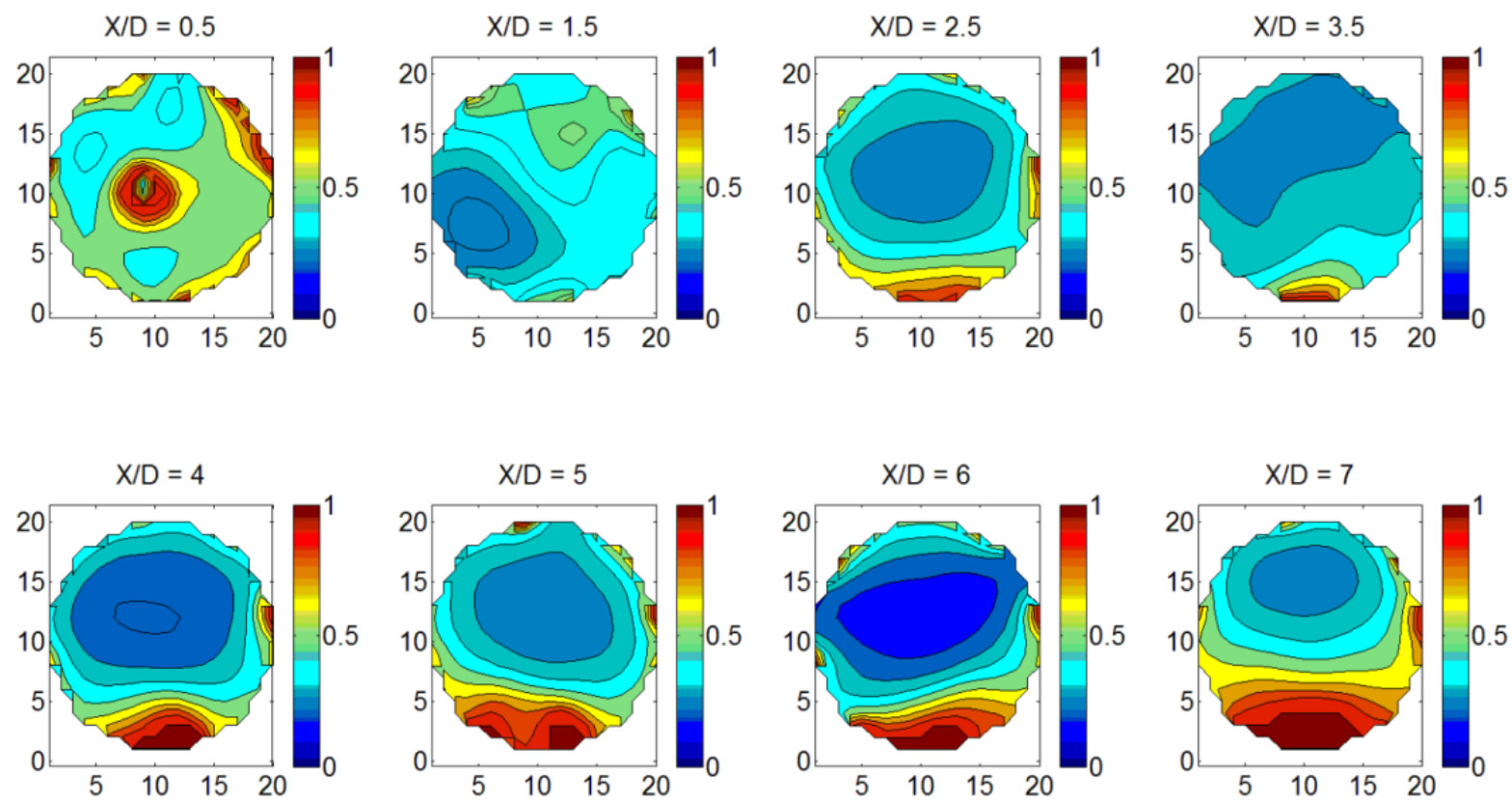

Figure 99: Conductivity Tomogram at 60 psig_20 GPM_70\% GVF 

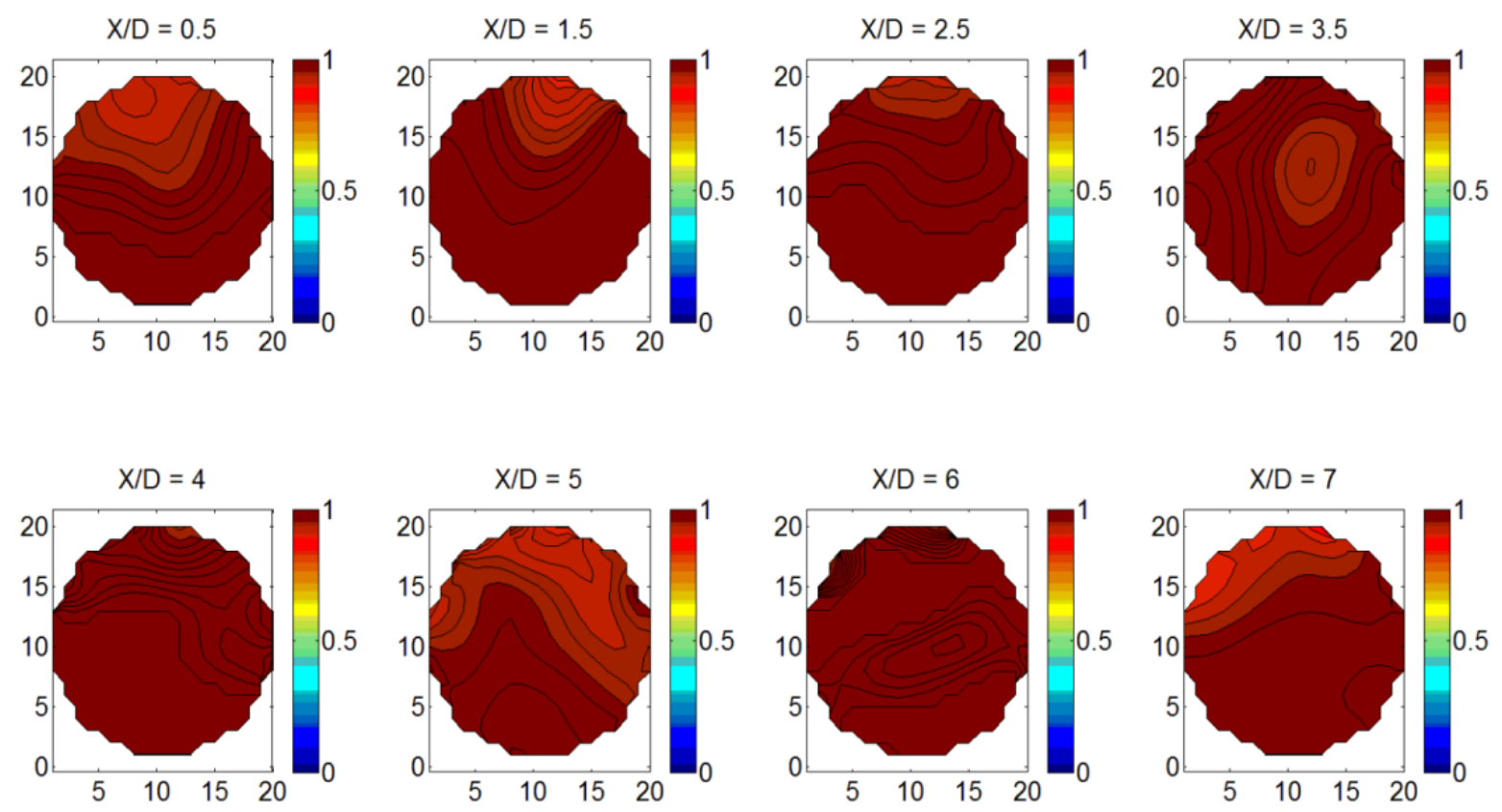

Figure 100: Conductivity Tomogram at 60 psig_40 GPM_5\% GVF
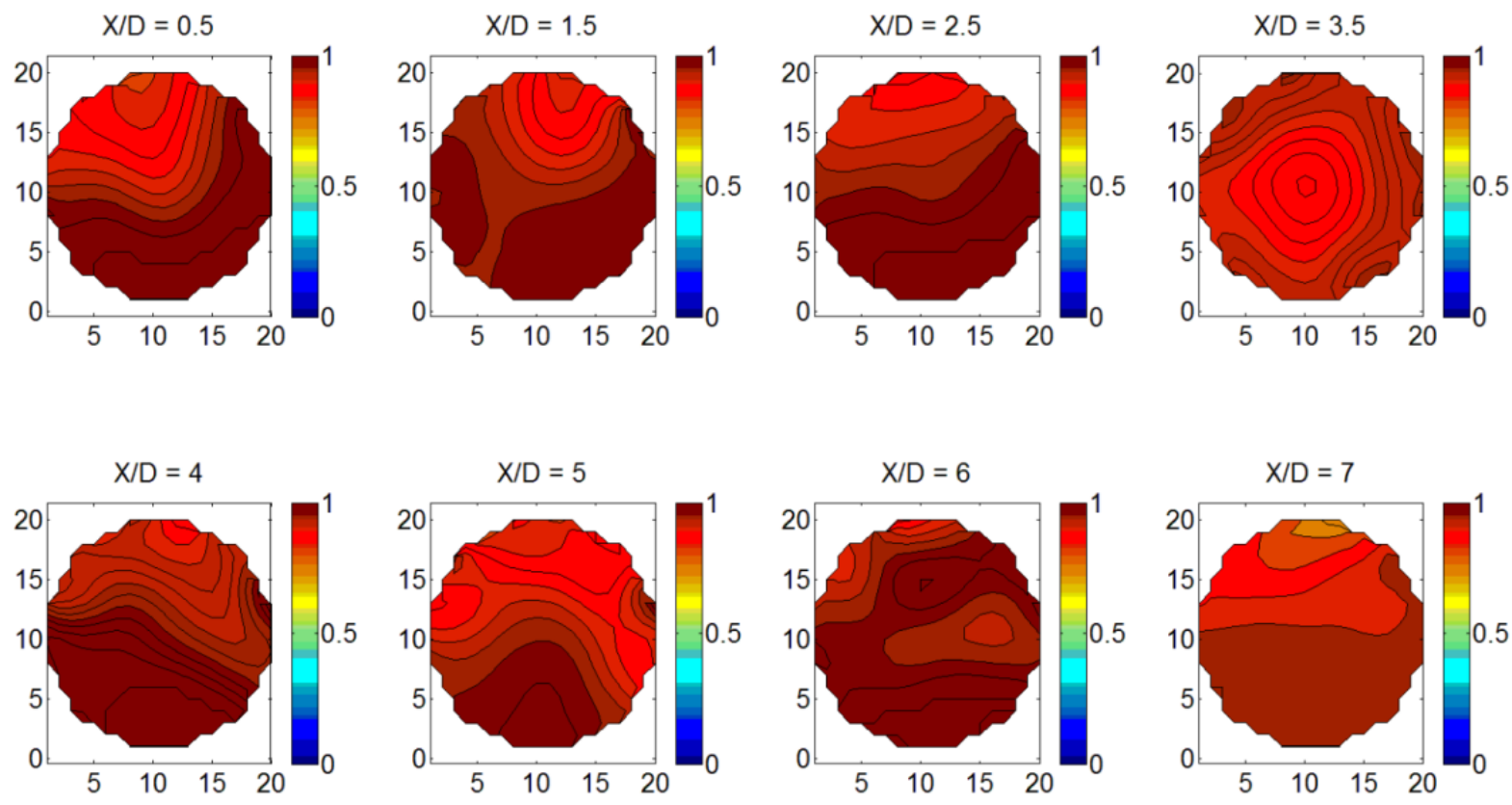

Figure 101: Conductivity Tomogram at 60 psig_40 GPM_10\% GVF 

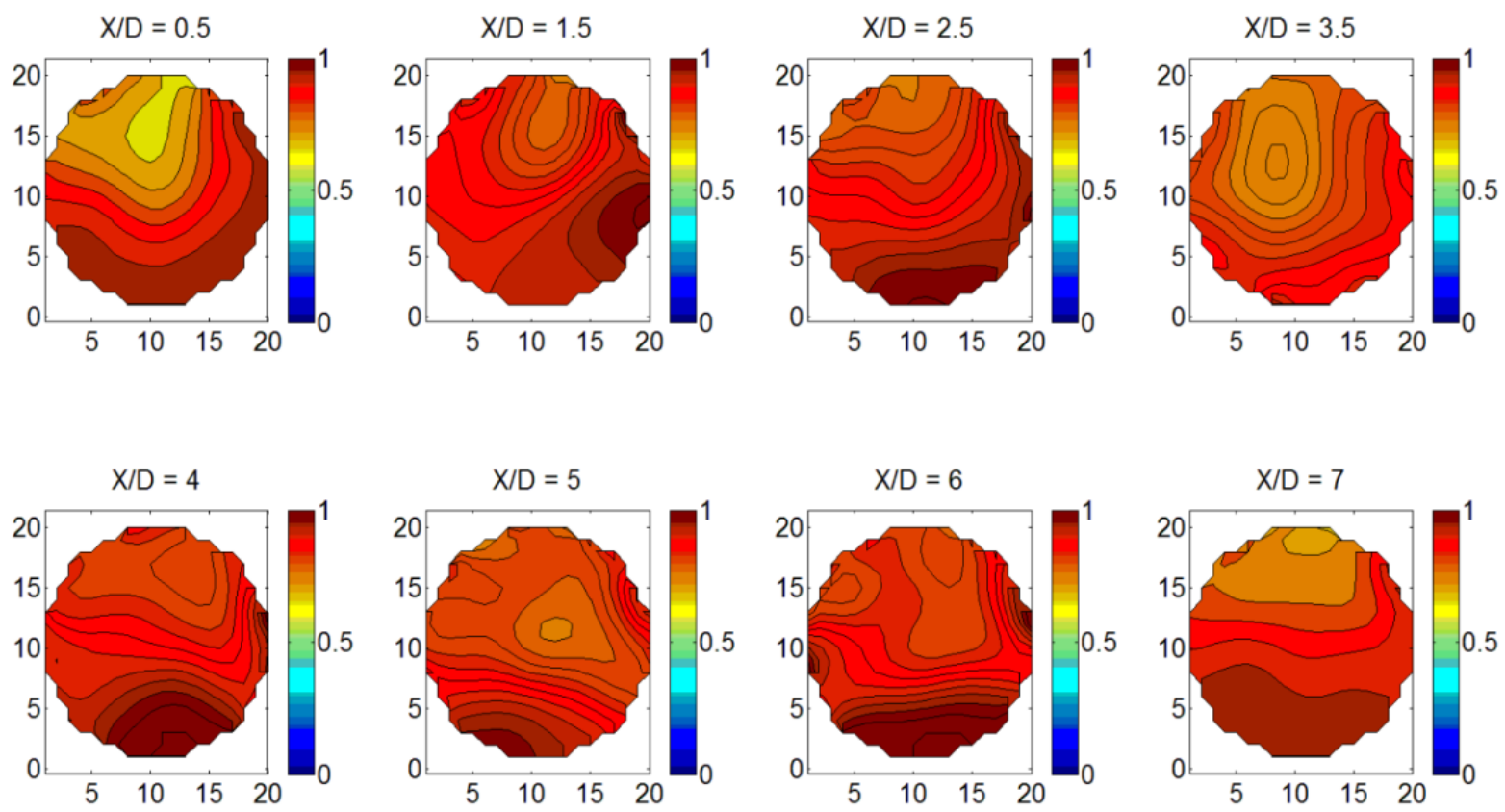

Figure 102: Conductivity Tomogram at 60 psig_40 GPM_20\% GVF
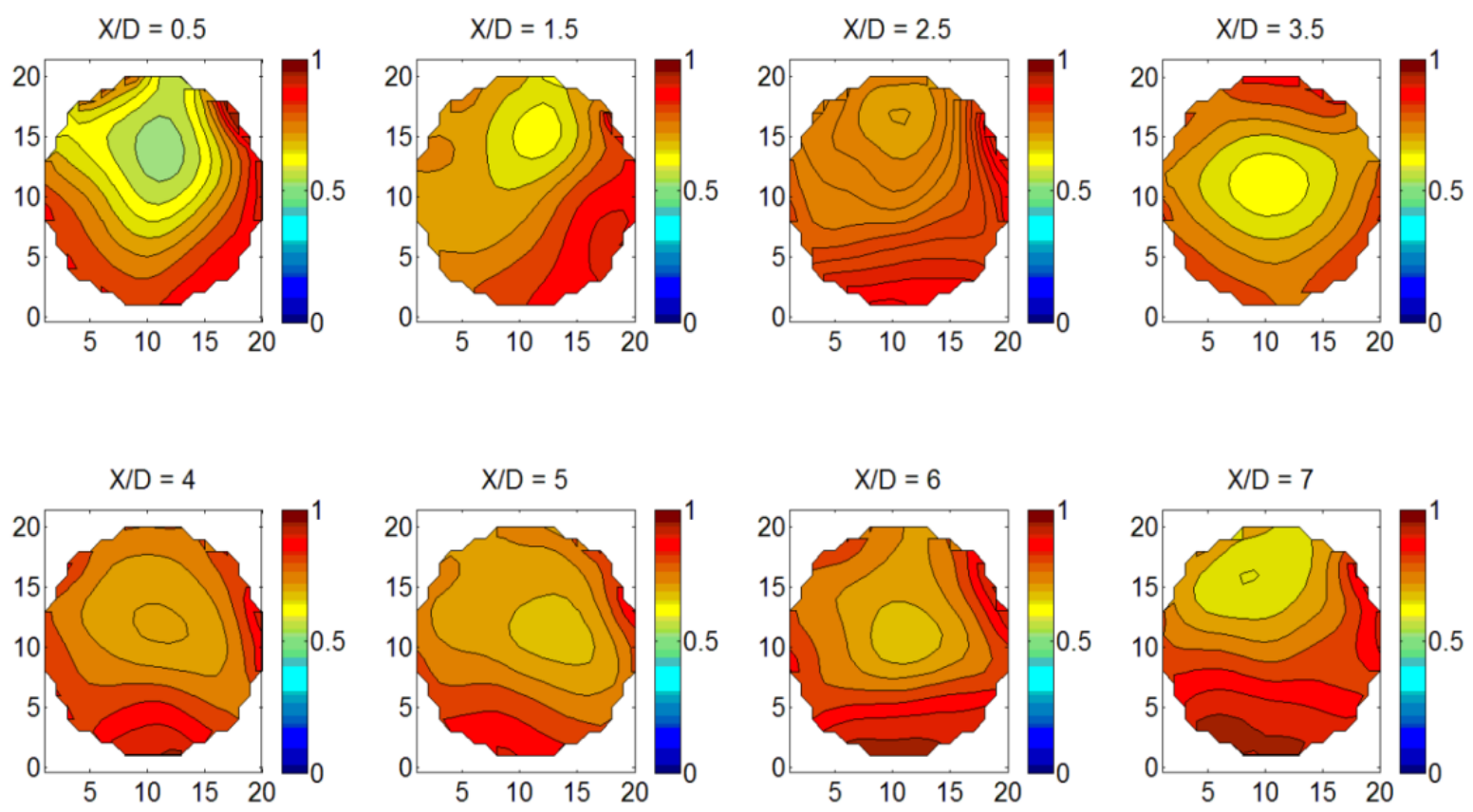

Figure 103: Conductivity Tomogram at 60 psig_40 GPM_30\% GVF 

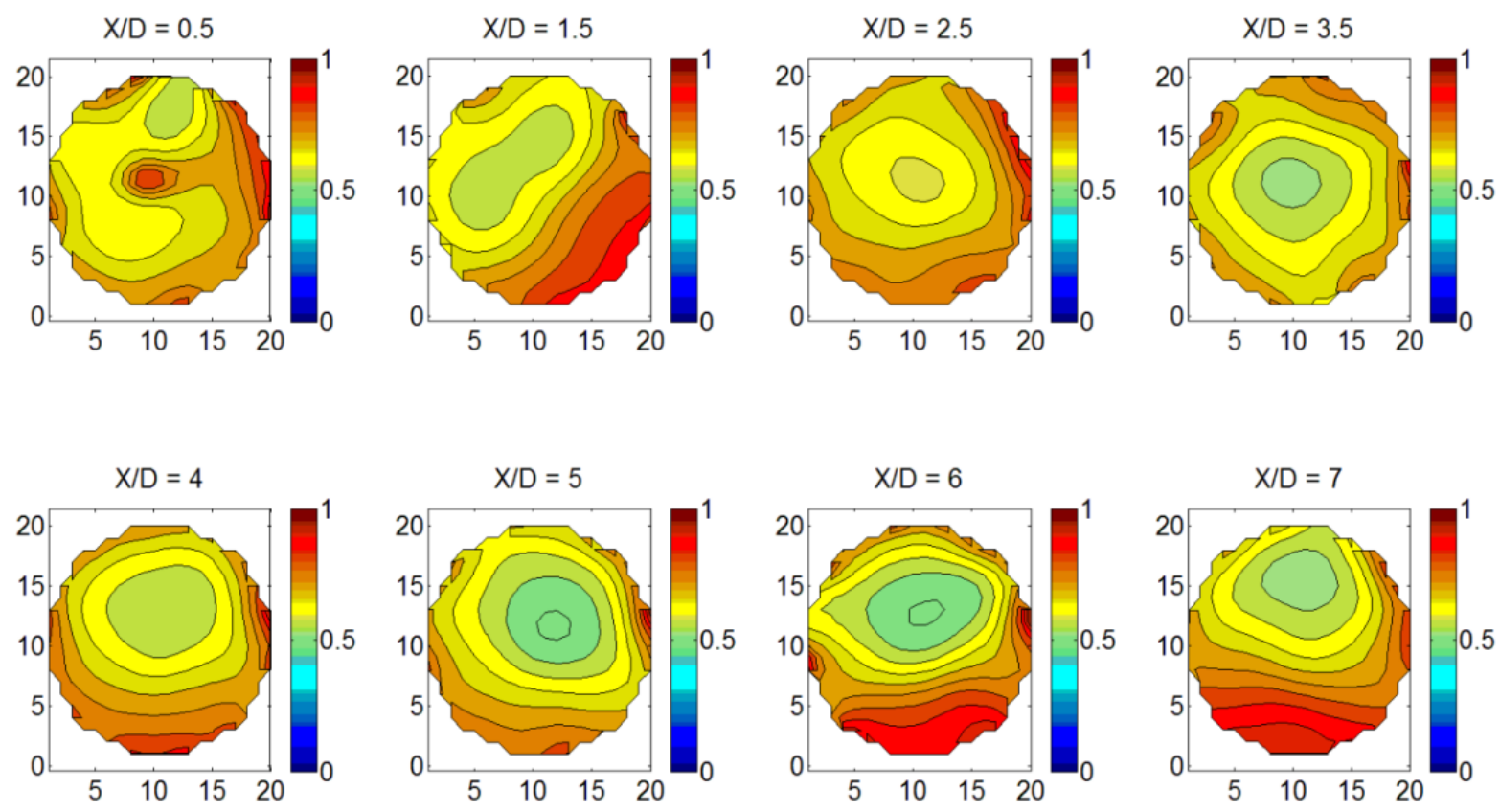

Figure 104: Conductivity Tomogram at 60 psig_40 GPM_40\% GVF
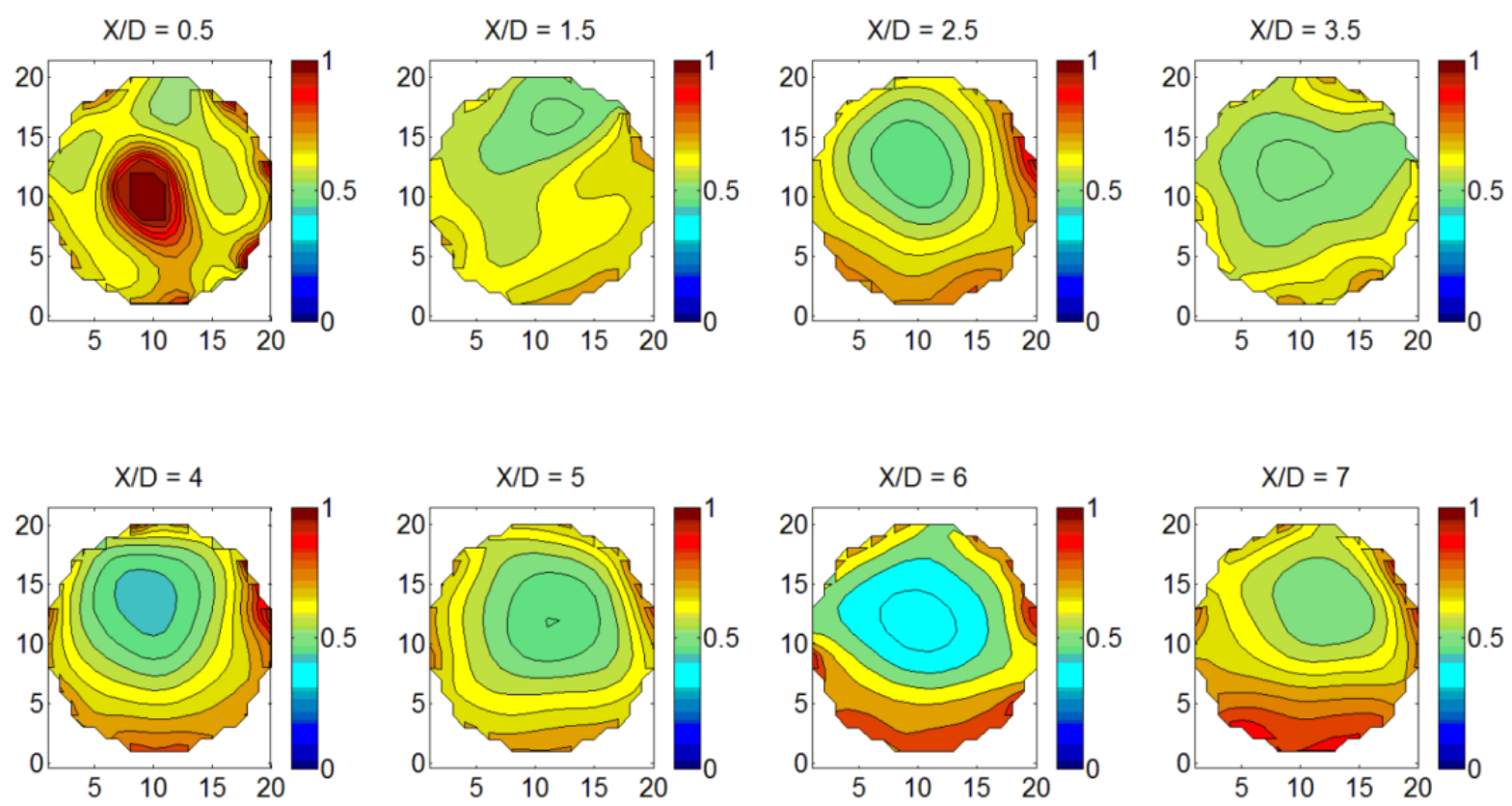

Figure 105: Conductivity Tomogram at 60 psig_40 GPM_50\% GVF 

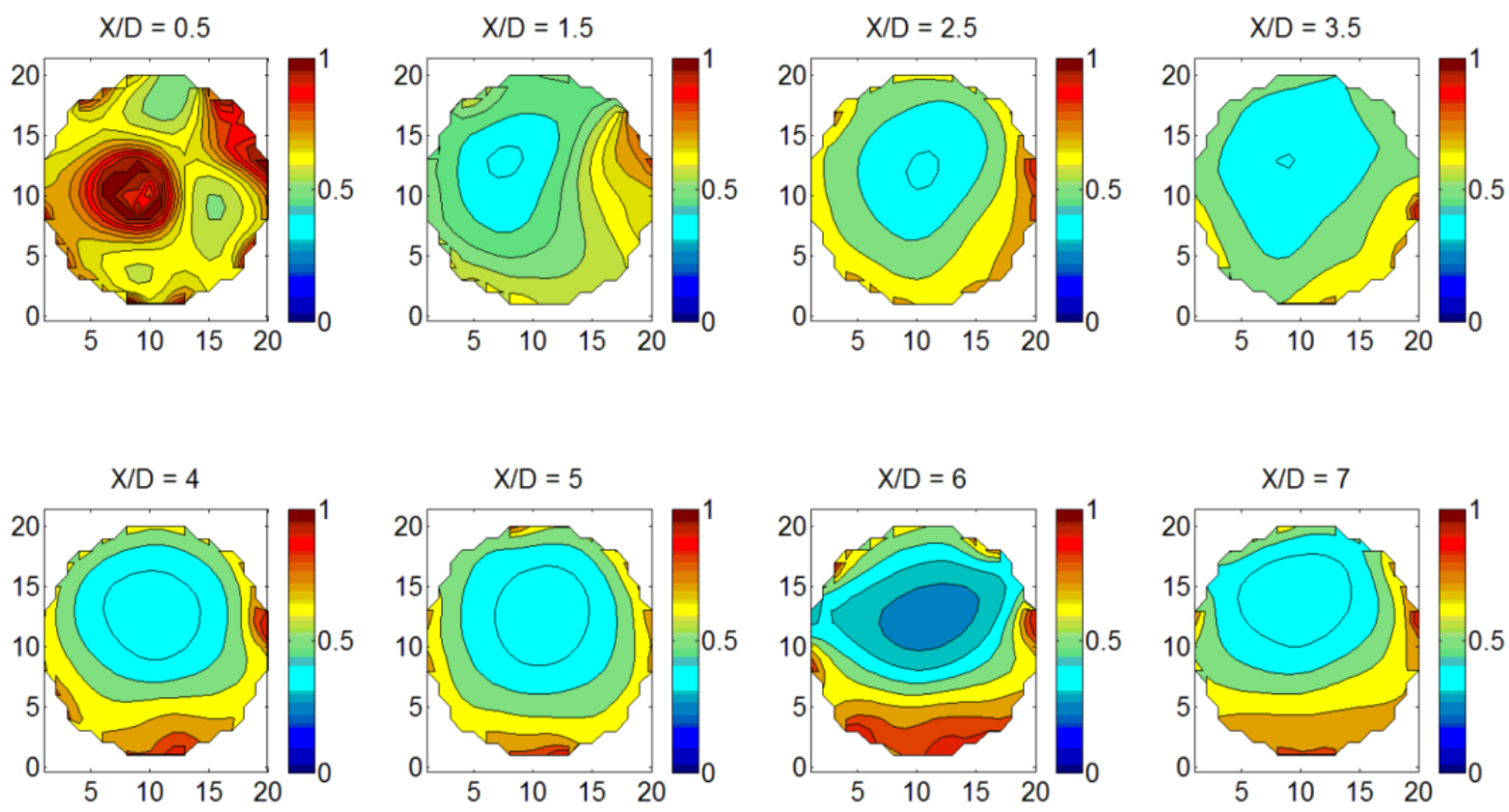

Figure 106: Conductivity Tomogram at 60 psig_40 GPM_60\% GVF
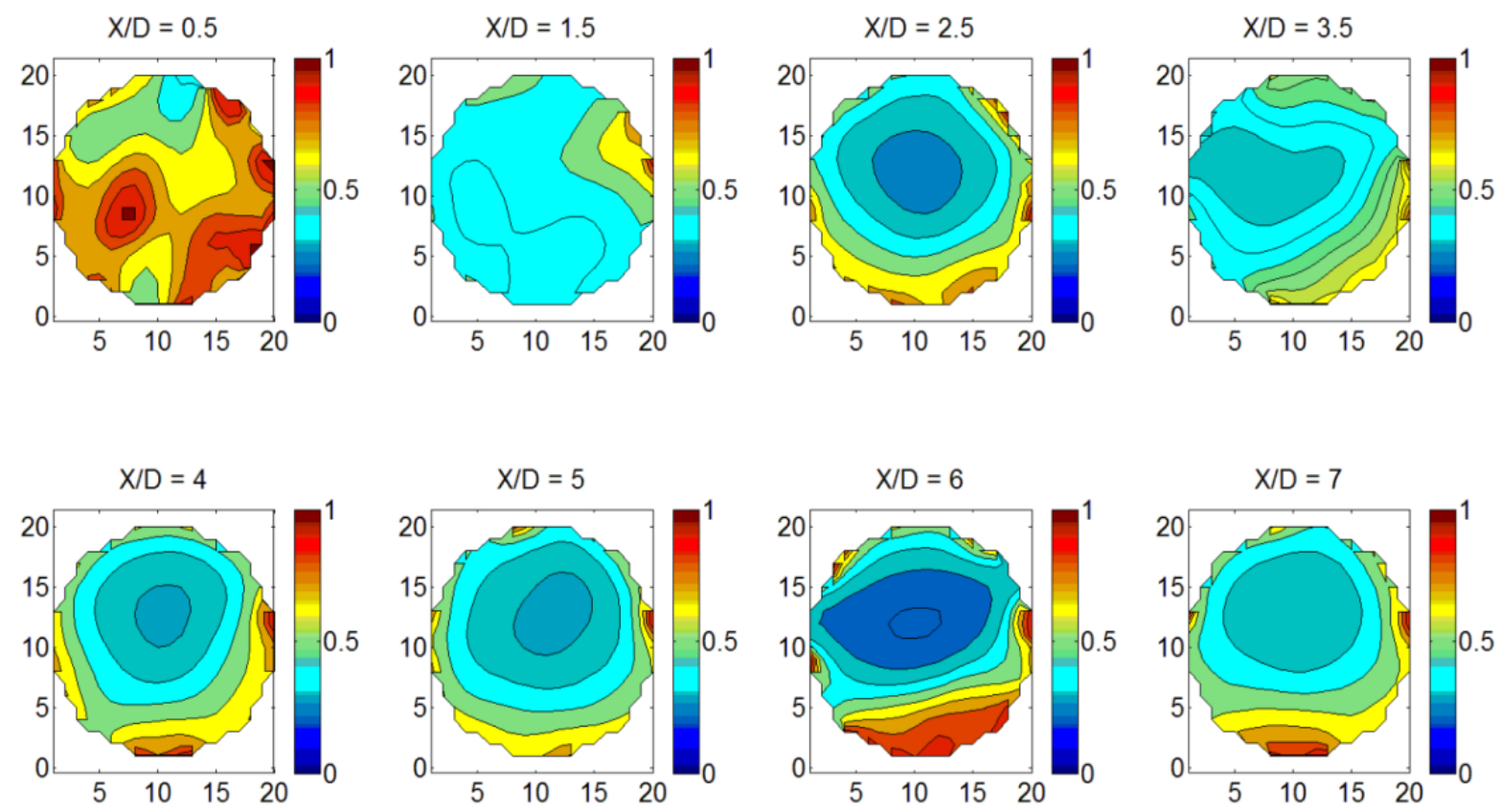

Figure 107: Conductivity Tomogram at 60 psig_40 GPM_70\% GVF 

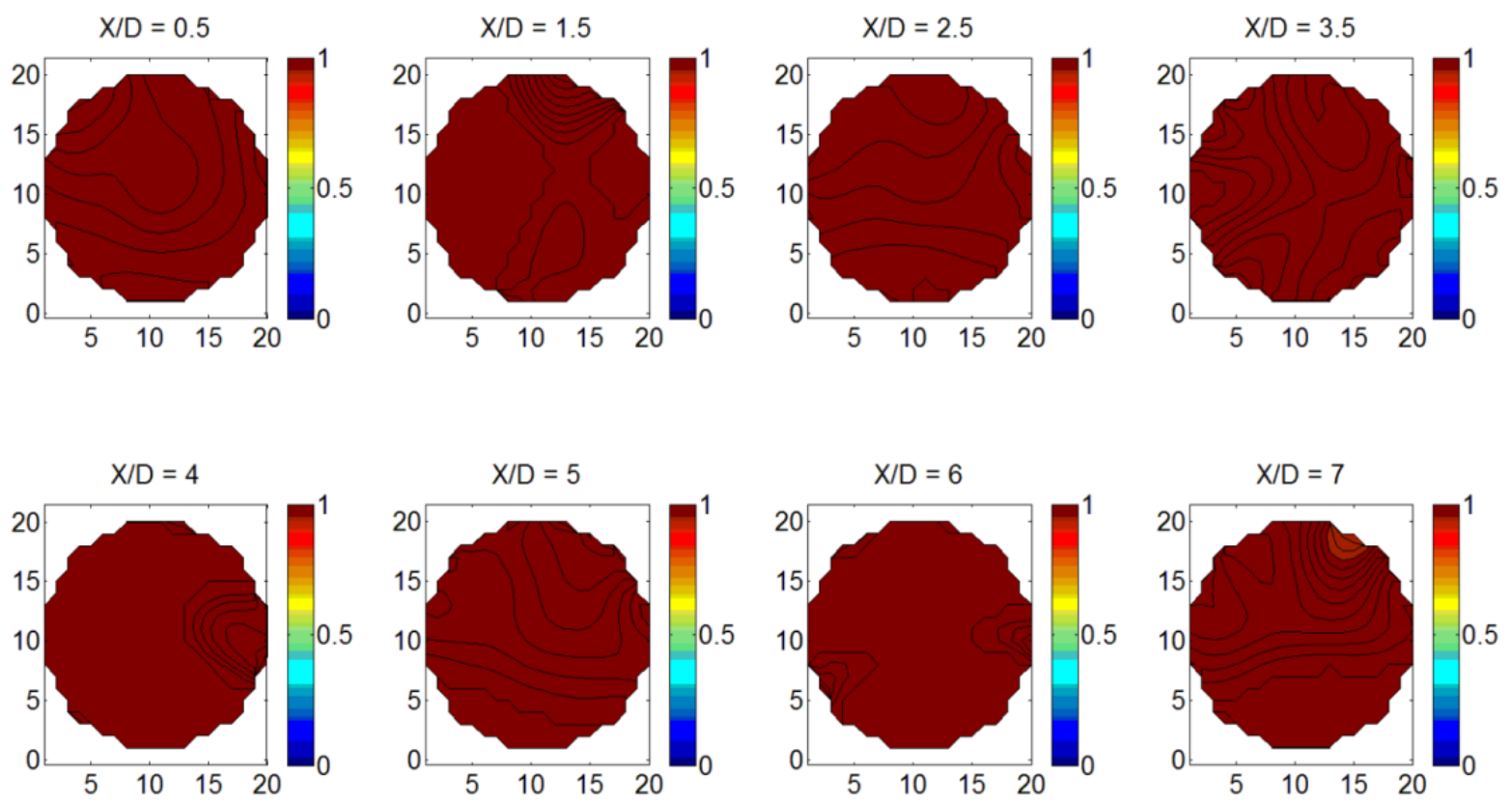

Figure 108: Conductivity Tomogram at 60 psig_60 GPM_5\% GVF
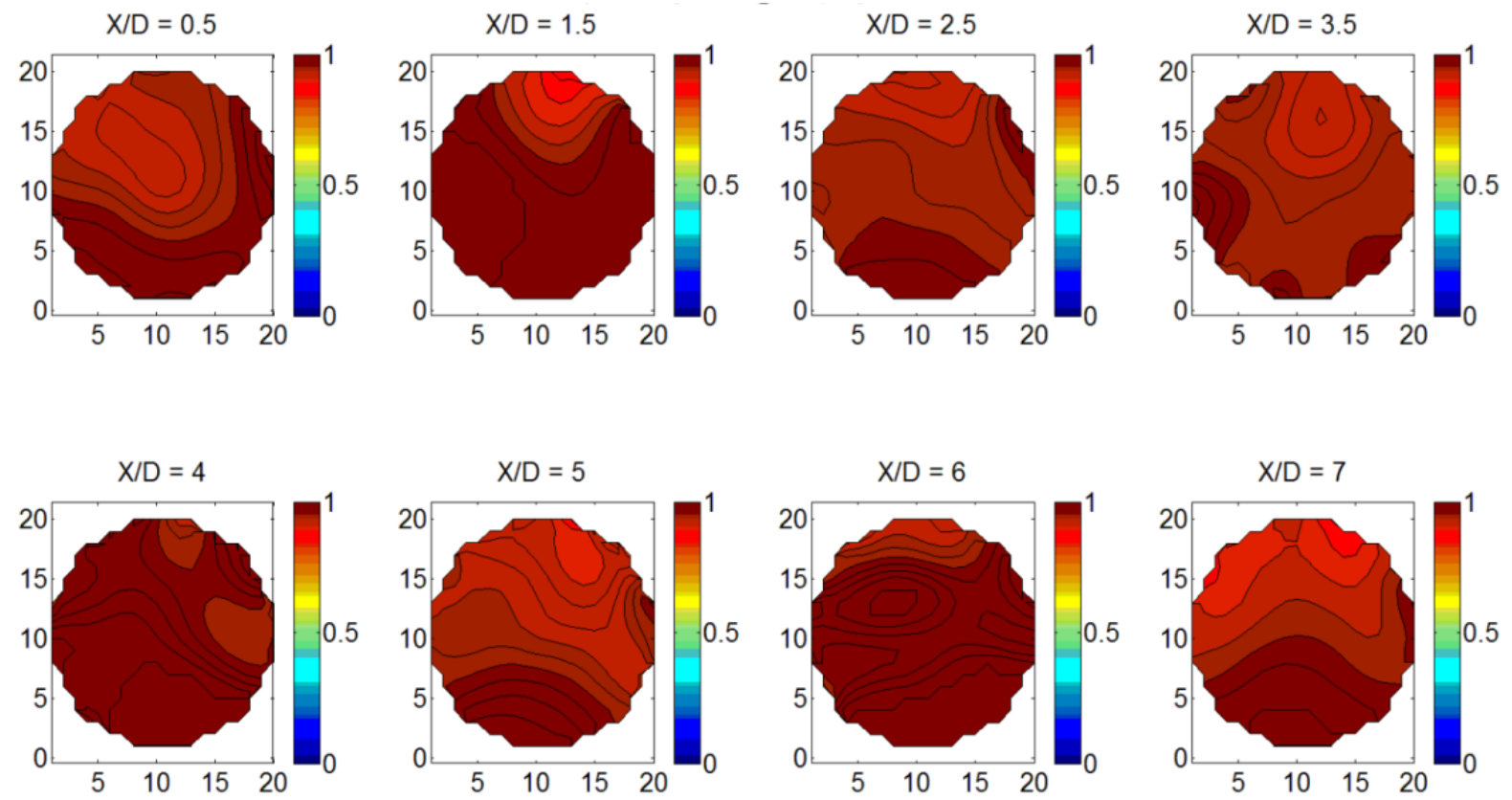

Figure 109: Conductivity Tomogram at 60 psig_60 GPM_10\% GVF 

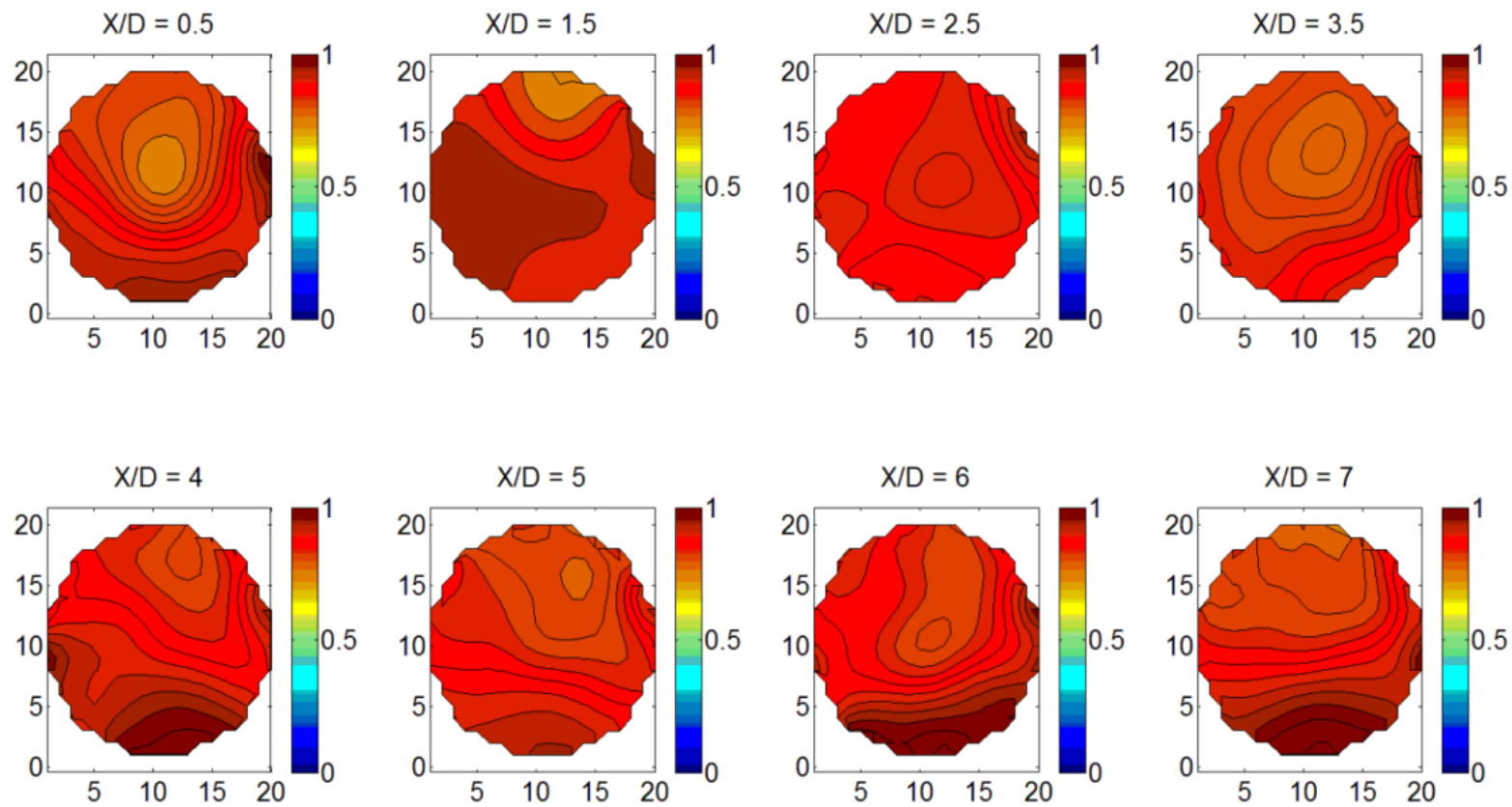

Figure 110: Conductivity Tomogram at 60 psig_60 GPM_20\% GVF
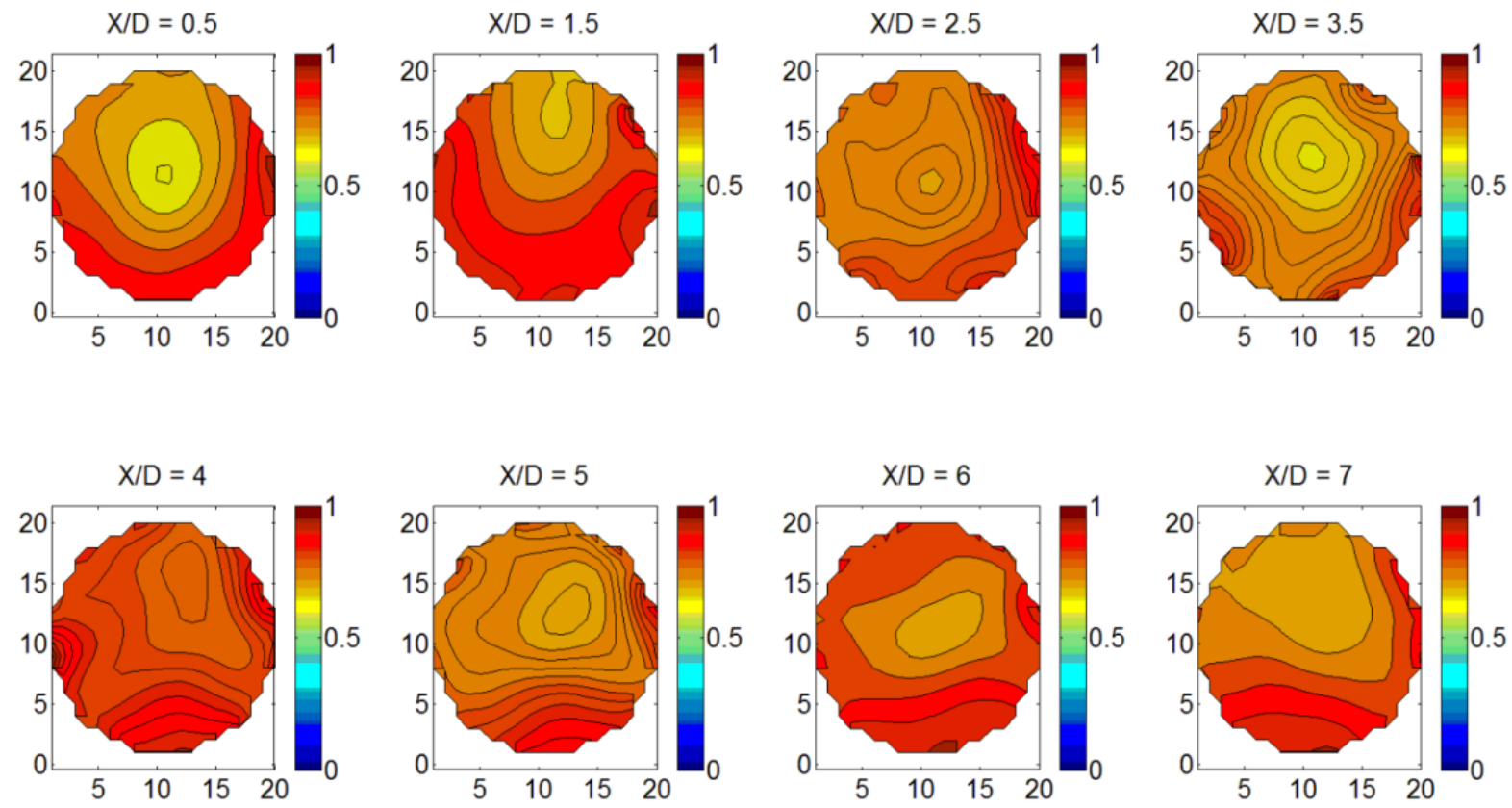

Figure 111: Conductivity Tomogram at 60 psig_60 GPM_30\% GVF 

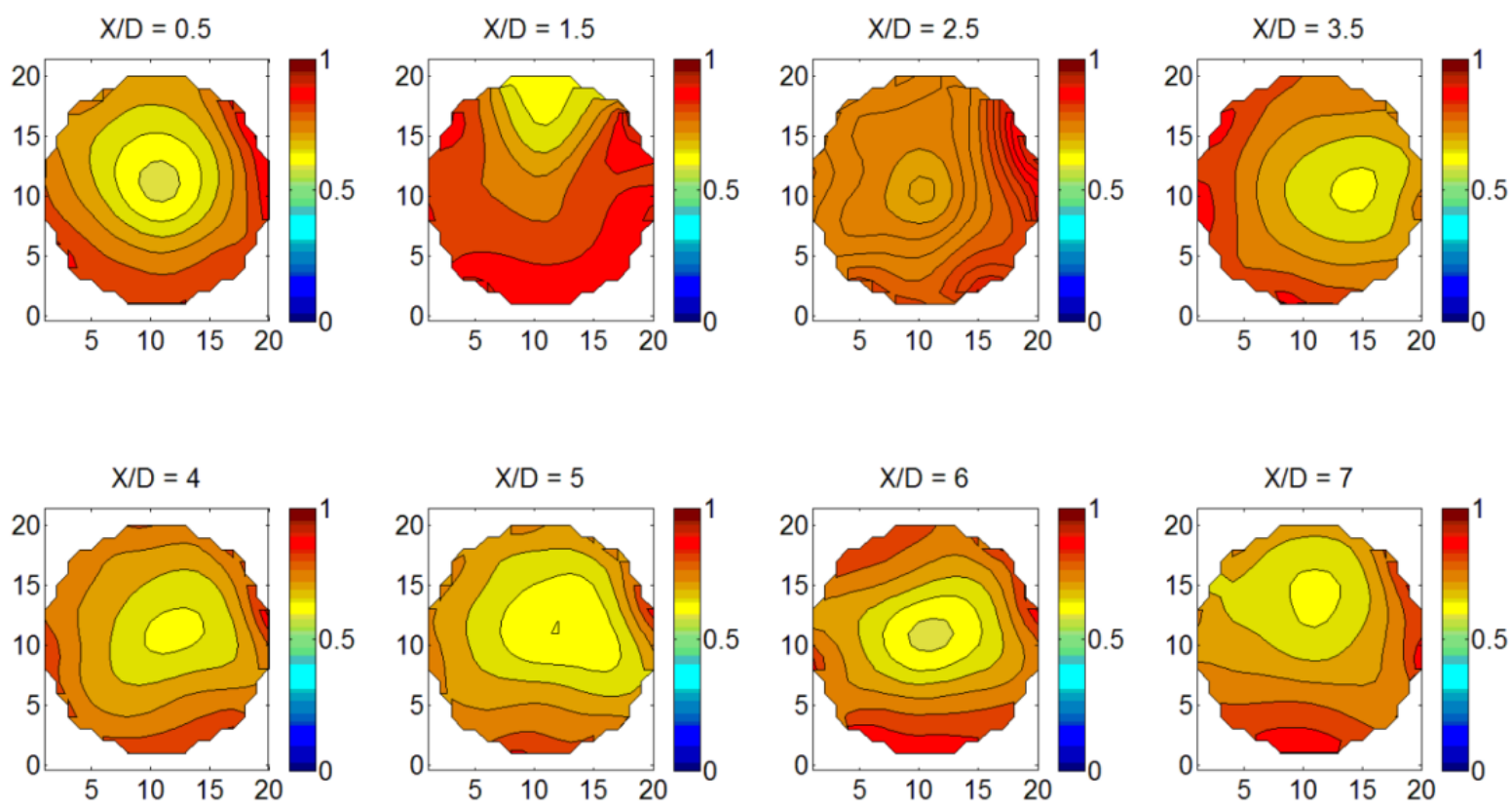

Figure 112: Conductivity Tomogram at 60 psig_60 GPM_40\% GVF
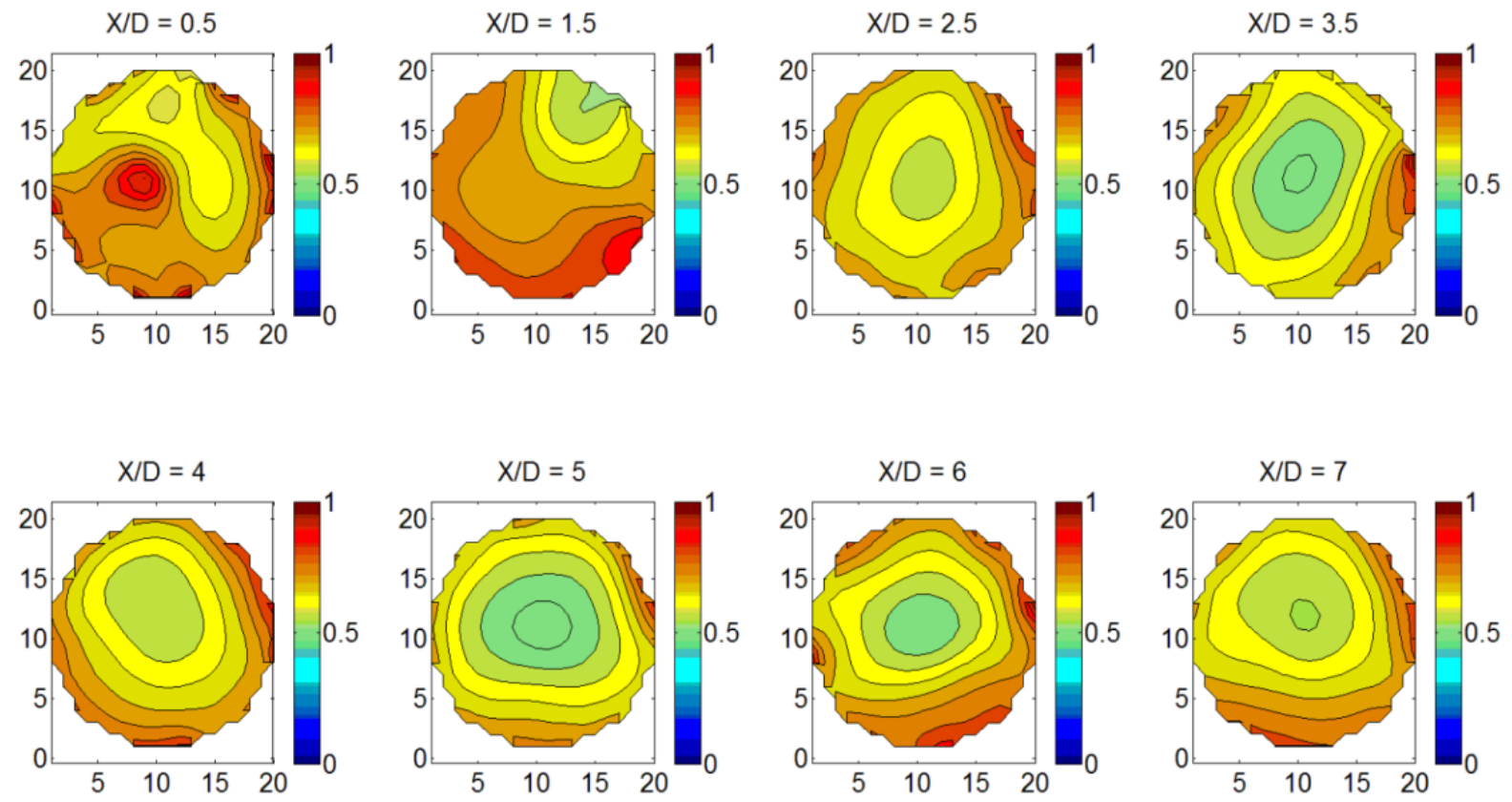

Figure 113: Conductivity Tomogram at 60 psig_60 GPM_50\% GVF 

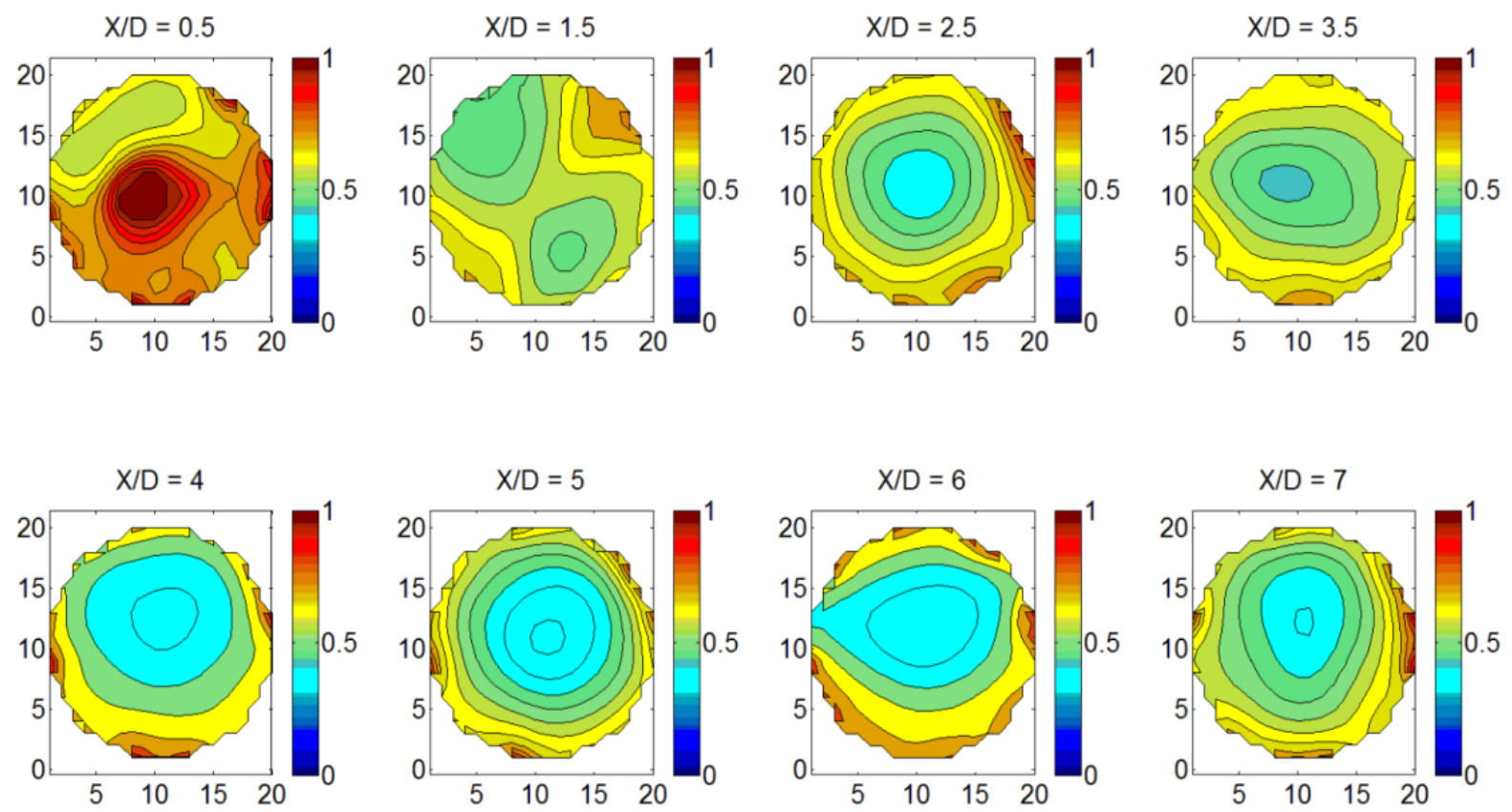

Figure 114: Conductivity Tomogram at 60 psig_60 GPM_60\% GVF
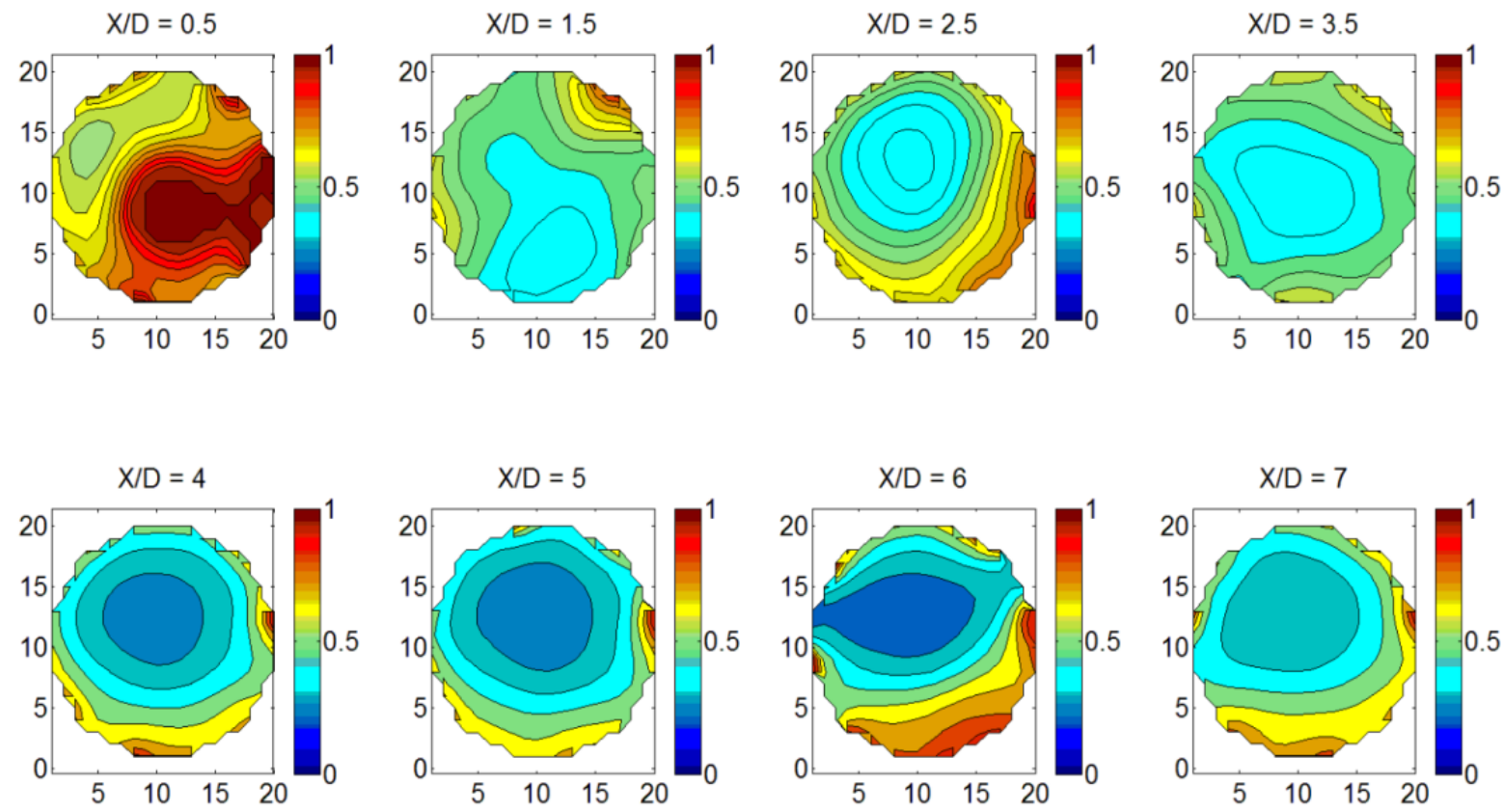

Figure 115: Conductivity Tomogram at 60 psig_60 GPM_70\% GVF 


\section{Concentration Tomograms}
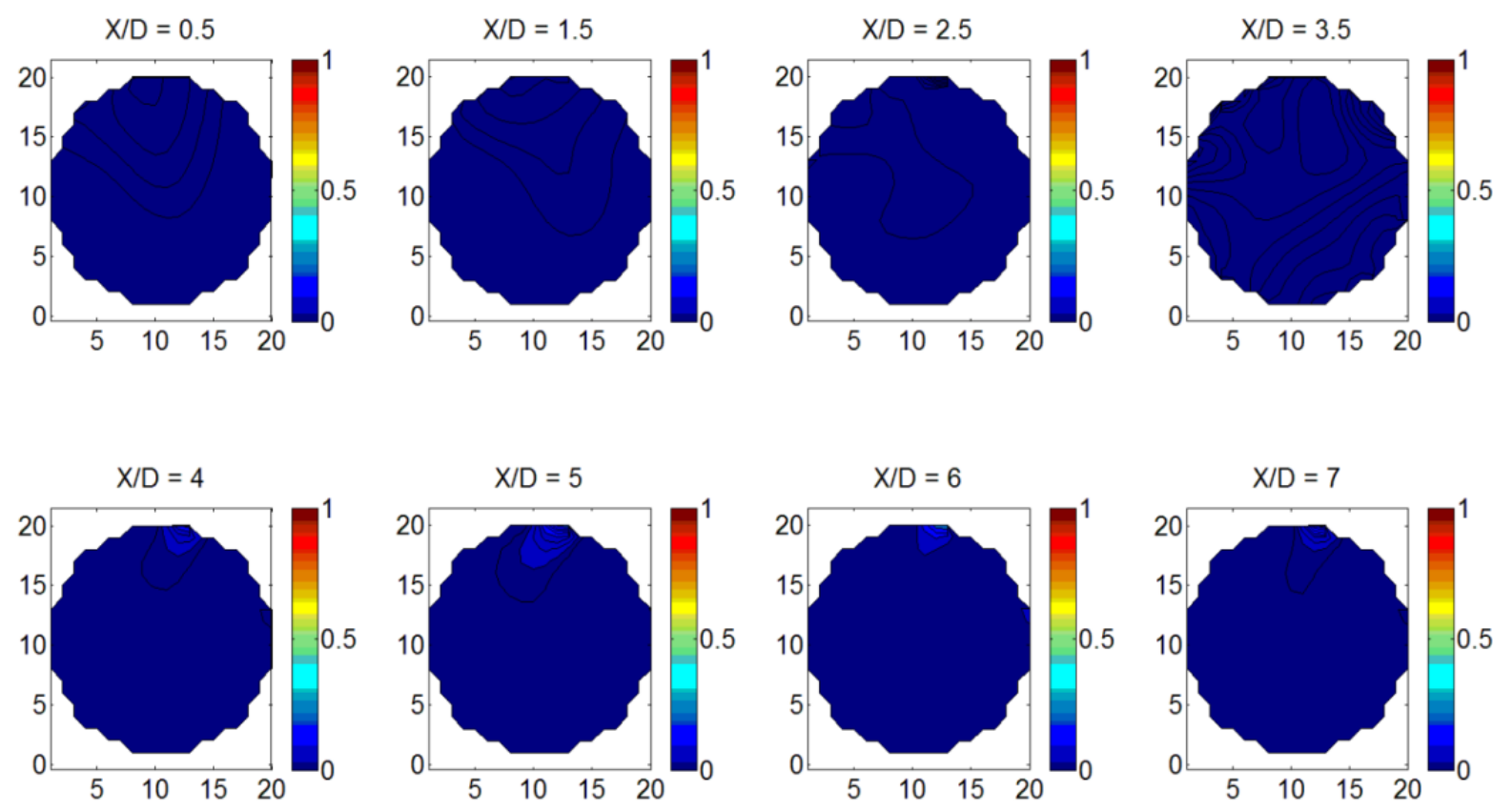

Figure 116: Concentration Tomogram at 20 psig_20 GPM_5\% GVF
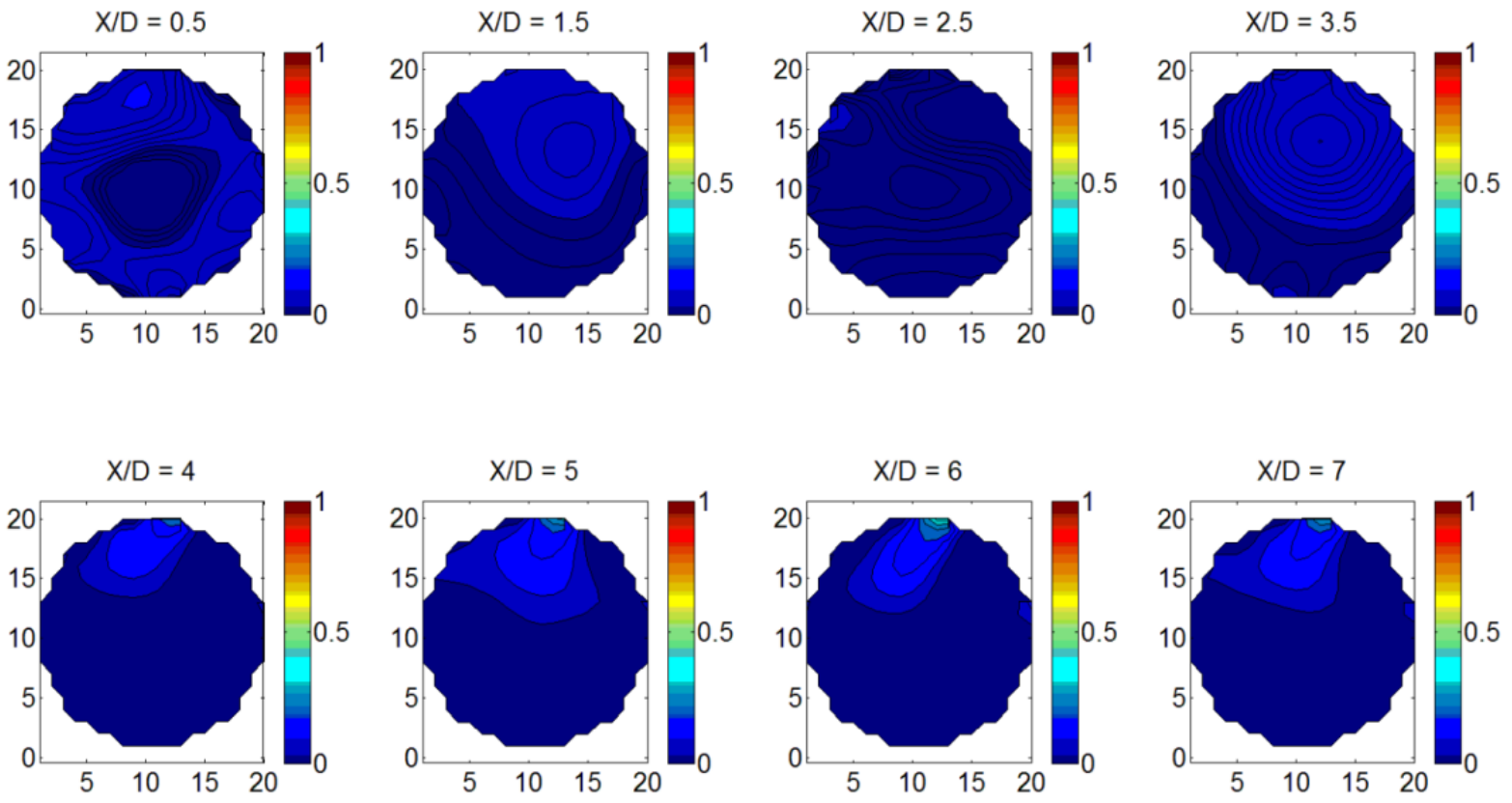

Figure 117: Concentration Tomogram at 20 psig_20 GPM_10\% GVF 

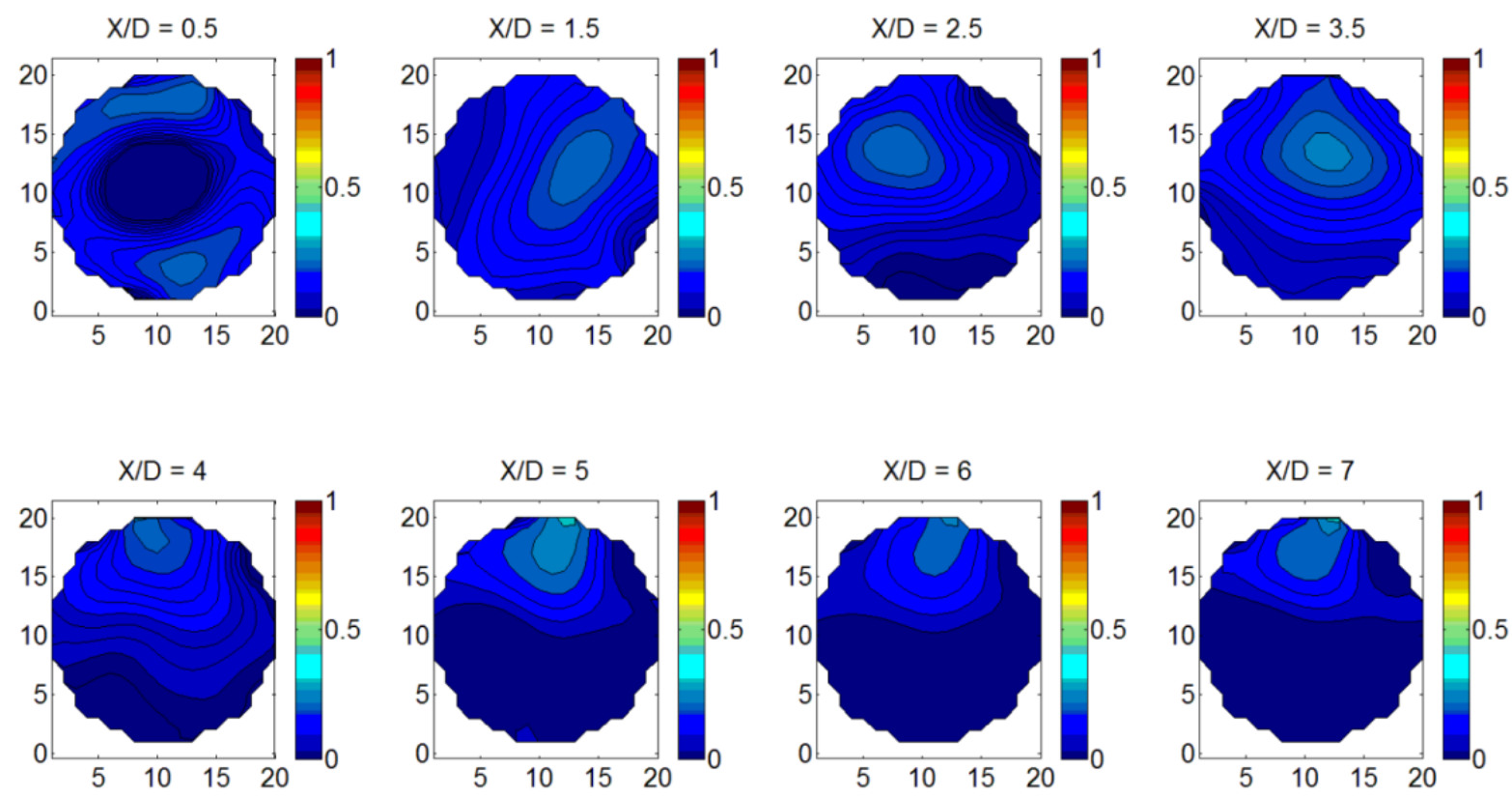

Figure 118: Concentration Tomogram at 20 psig_20 GPM_20\% GVF
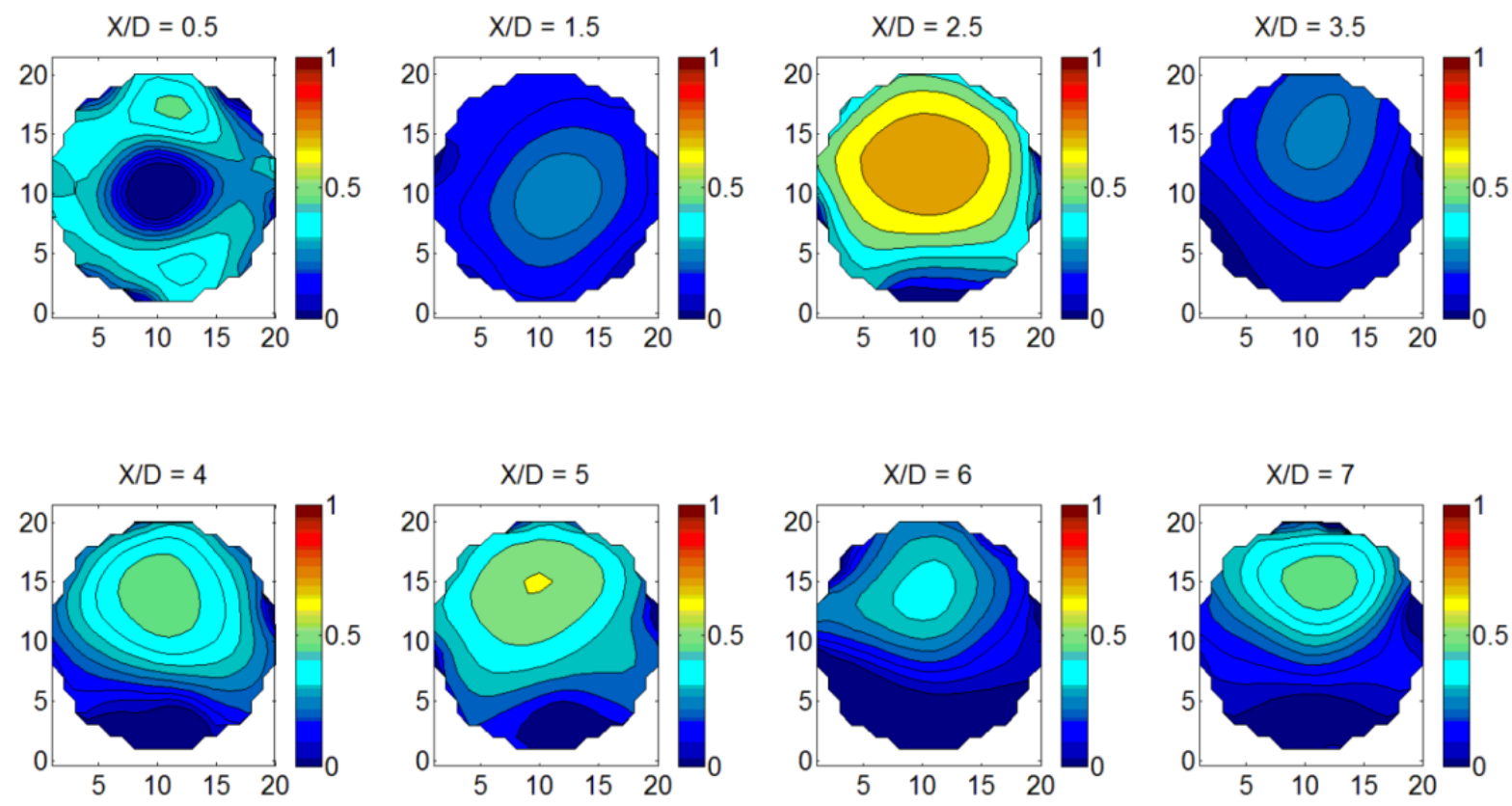

Figure 119: Concentration Tomogram at 20 psig_20 GPM_30\% GVF 

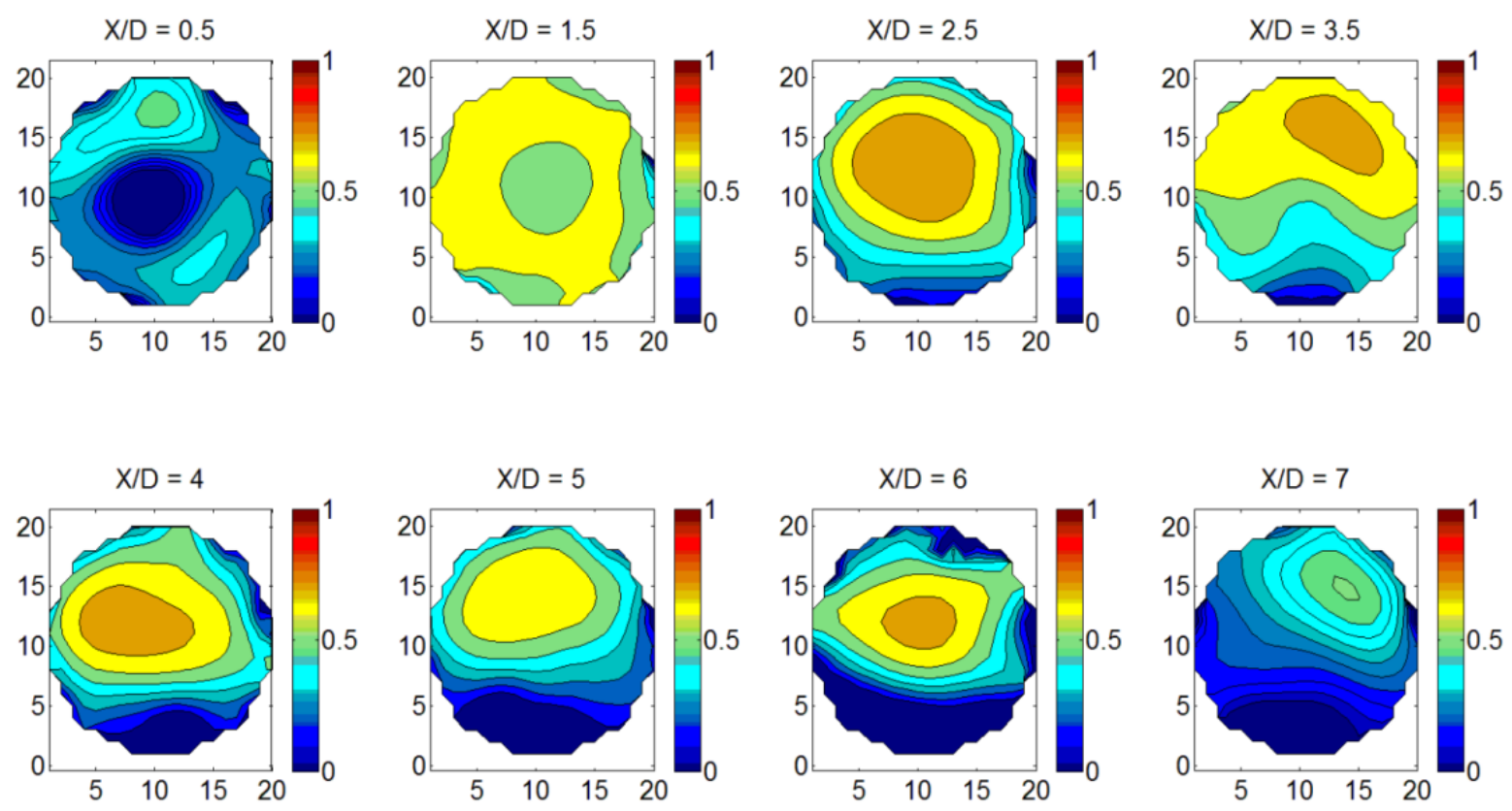

Figure 120: Concentration Tomogram at 20 psig_20 GPM_40\% GVF
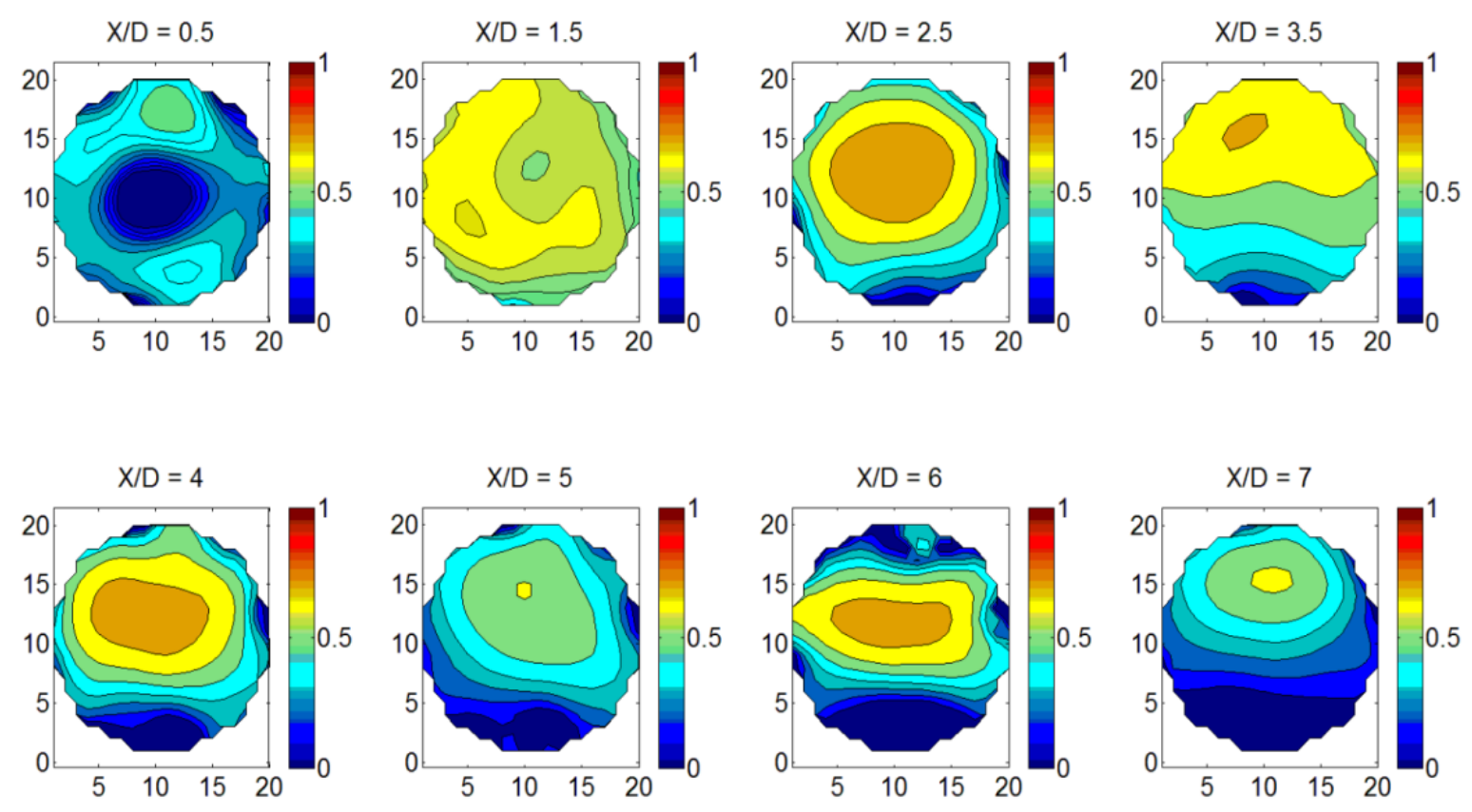

Figure 121: Concentration Tomogram at 20 psig_20 GPM_50\% GVF 

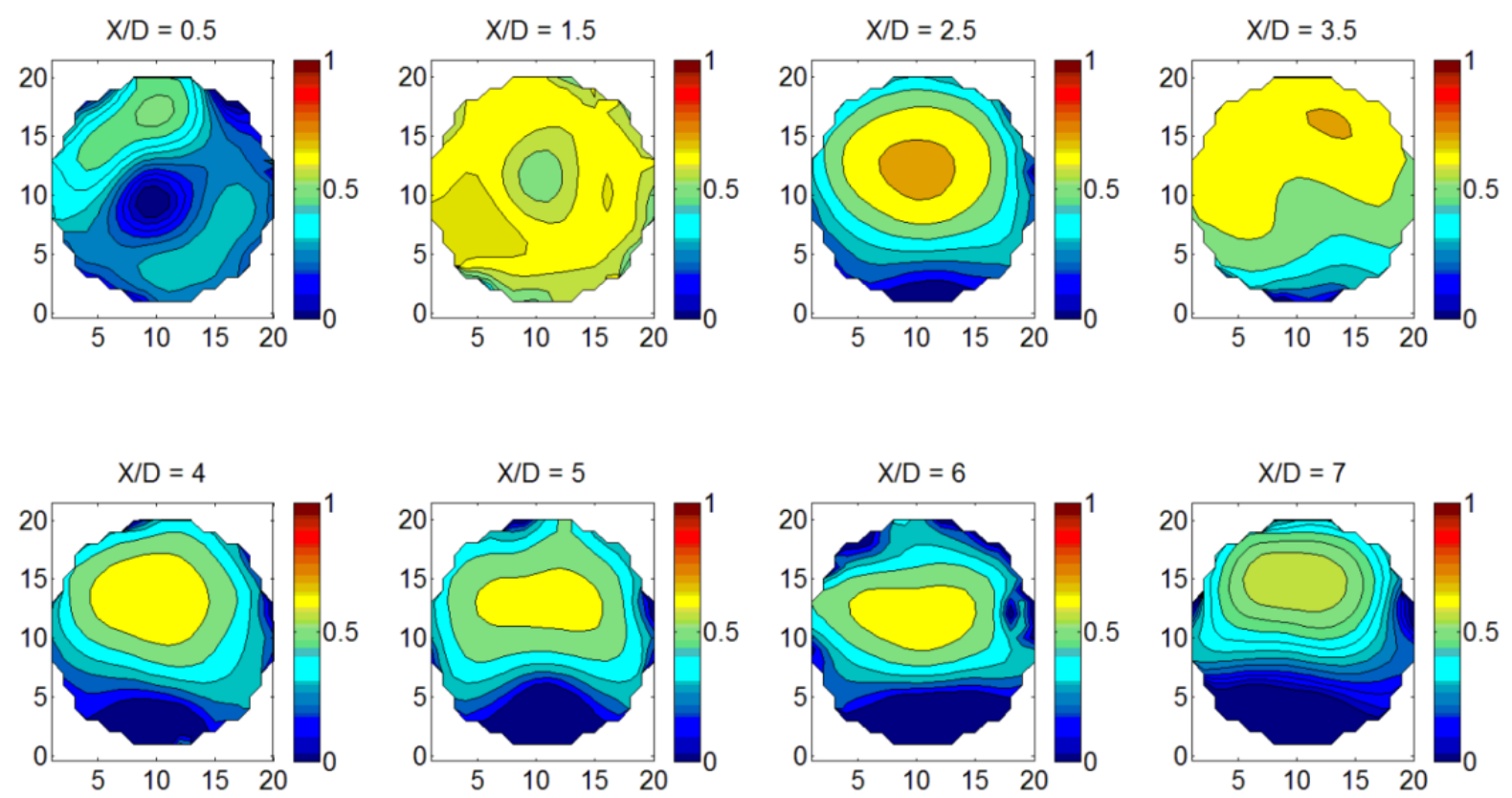

Figure 122: Concentration Tomogram at 20 psig_20 GPM_60\% GVF
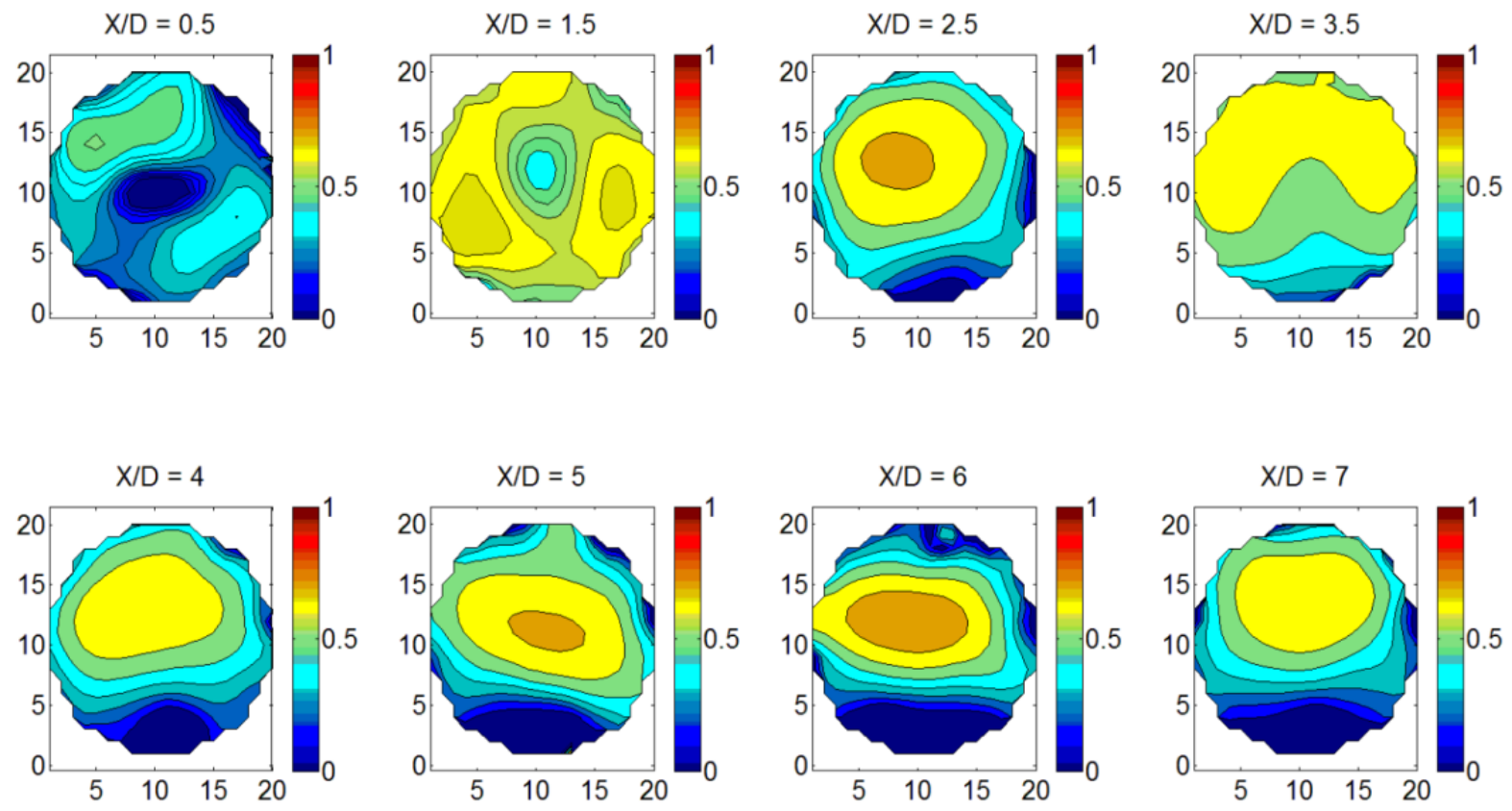

Figure 123: Concentration Tomogram at 20 psig_20 GPM_70\% GVF 

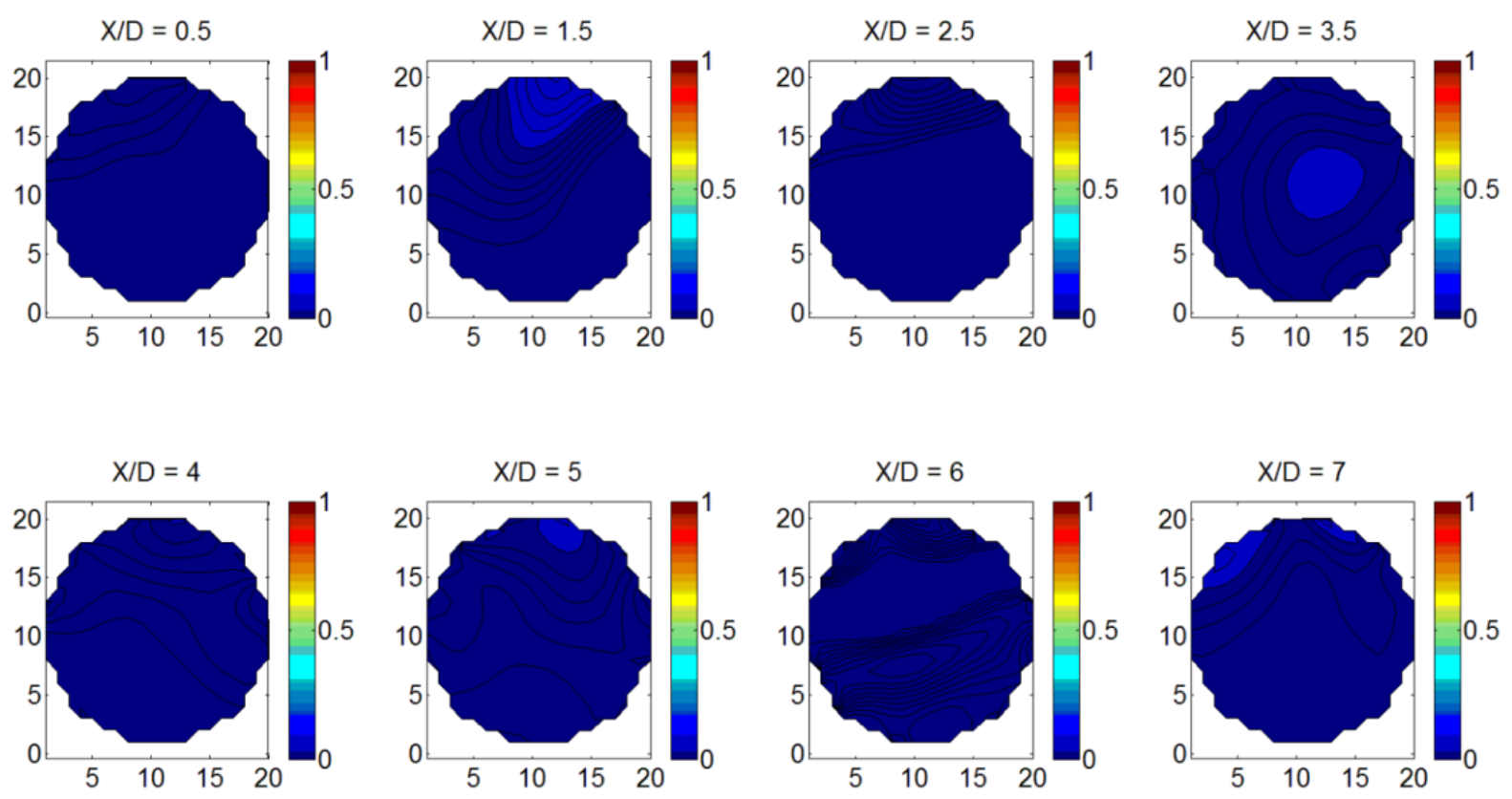

Figure 124: Concentration Tomogram at 20 psig_40 GPM_5\% GVF
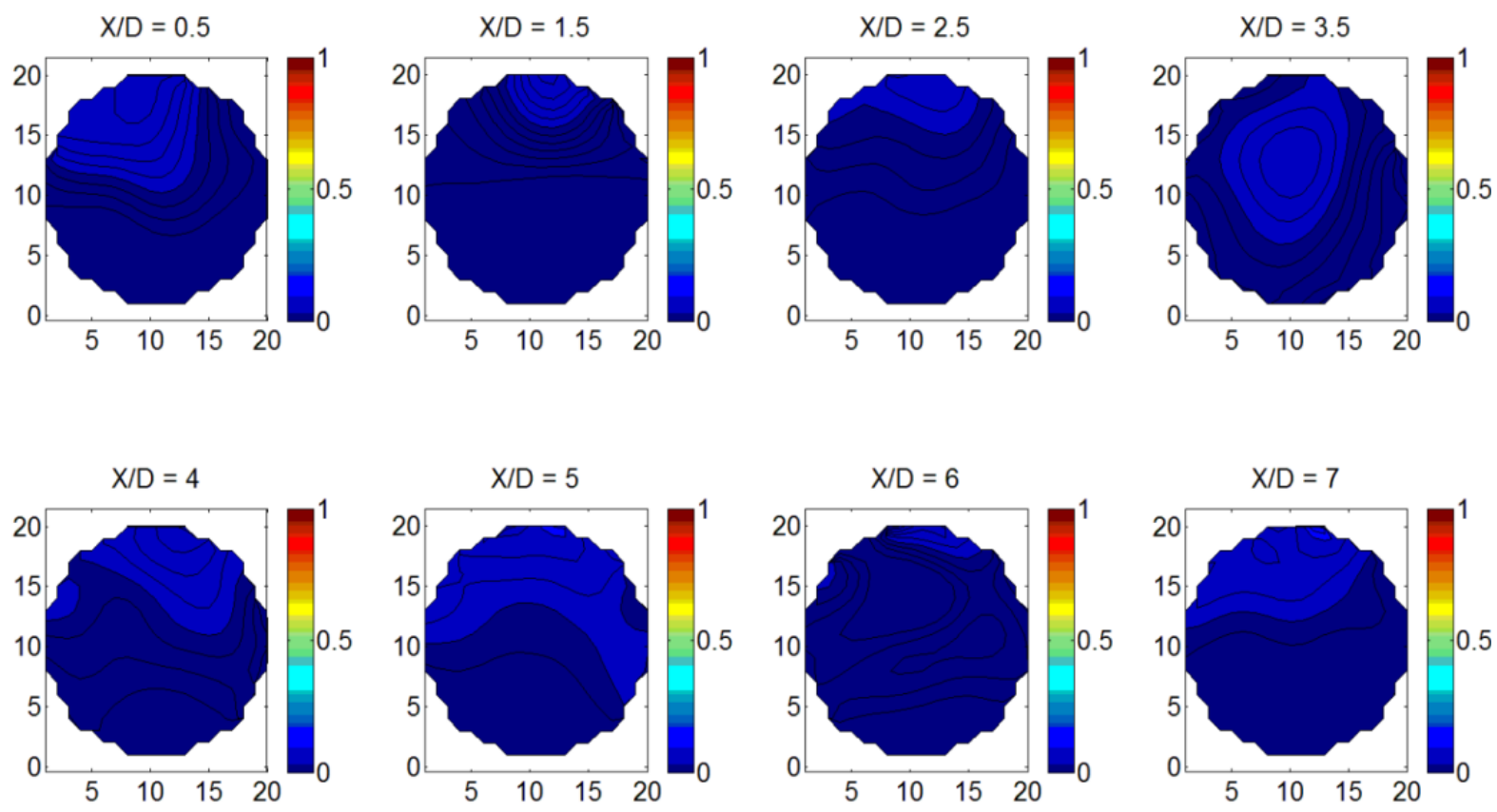

Figure 125: Concentration Tomogram at 20 psig_40 GPM_10\% GVF 

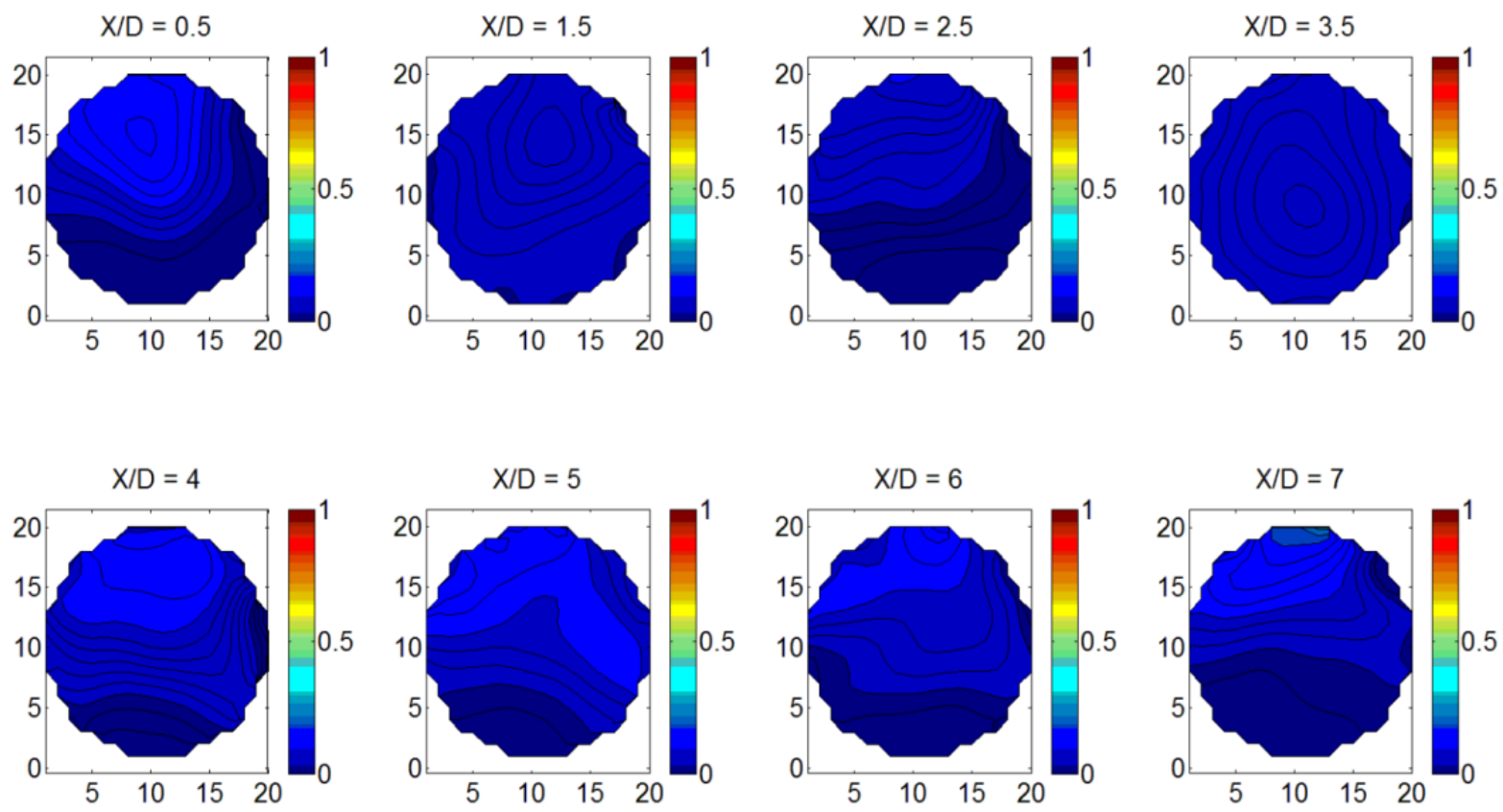

Figure 126: Concentration Tomogram at 20 psig_40 GPM_20\% GVF
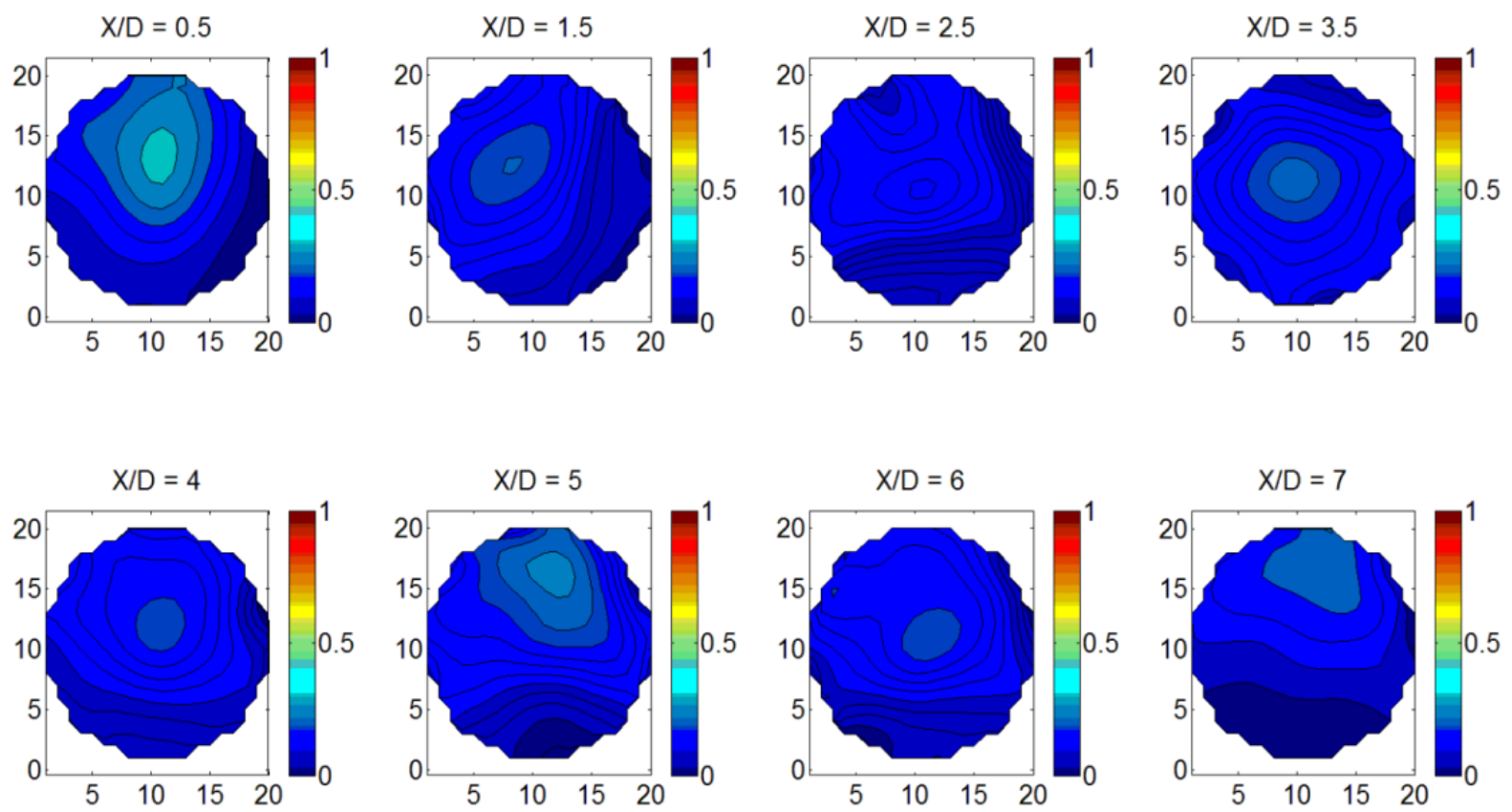

Figure 127: Concentration Tomogram at 20 psig_40 GPM_30\% GVF 

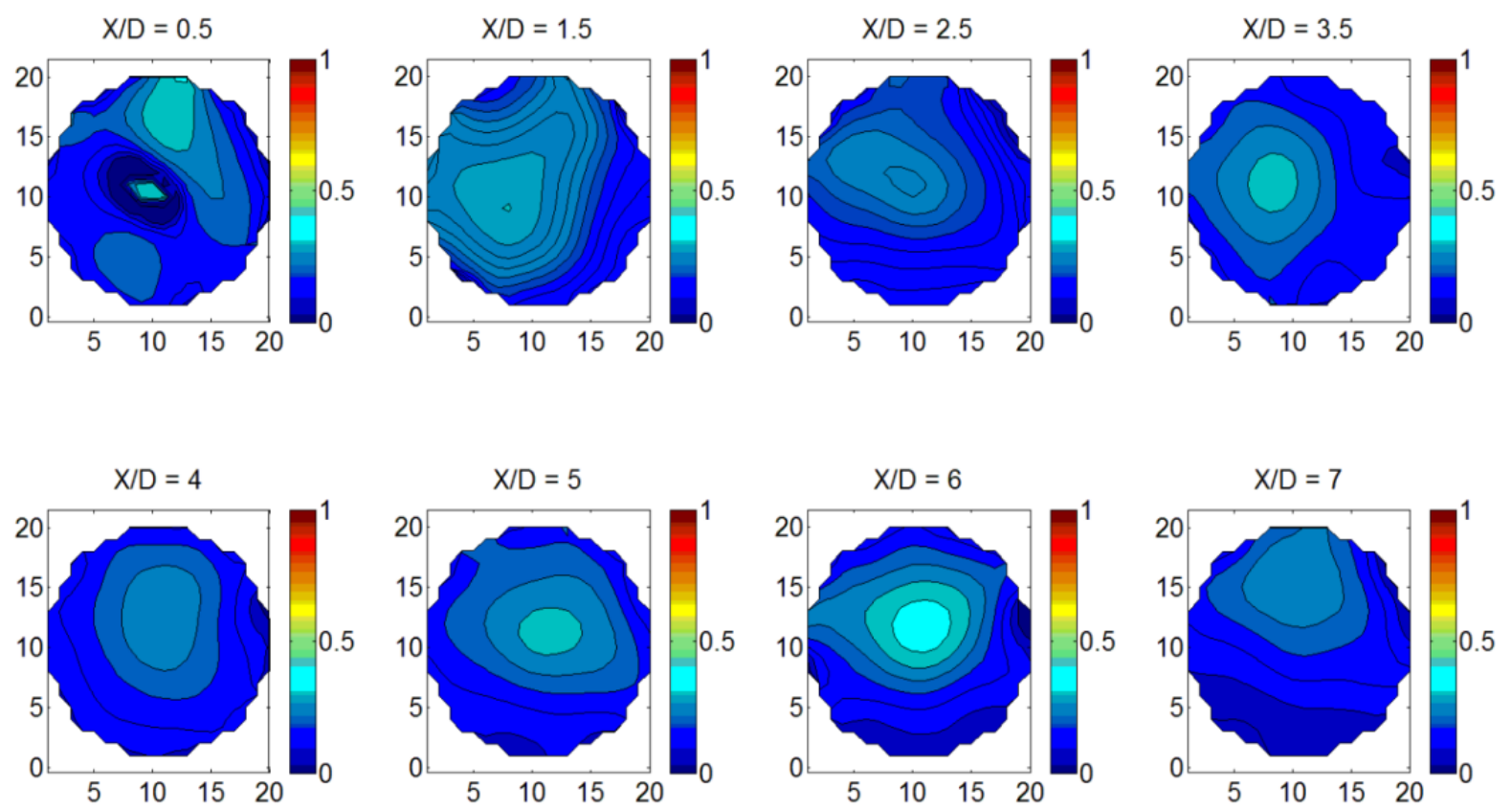

Figure 128: Concentration Tomogram at 20 psig_40 GPM_40\% GVF
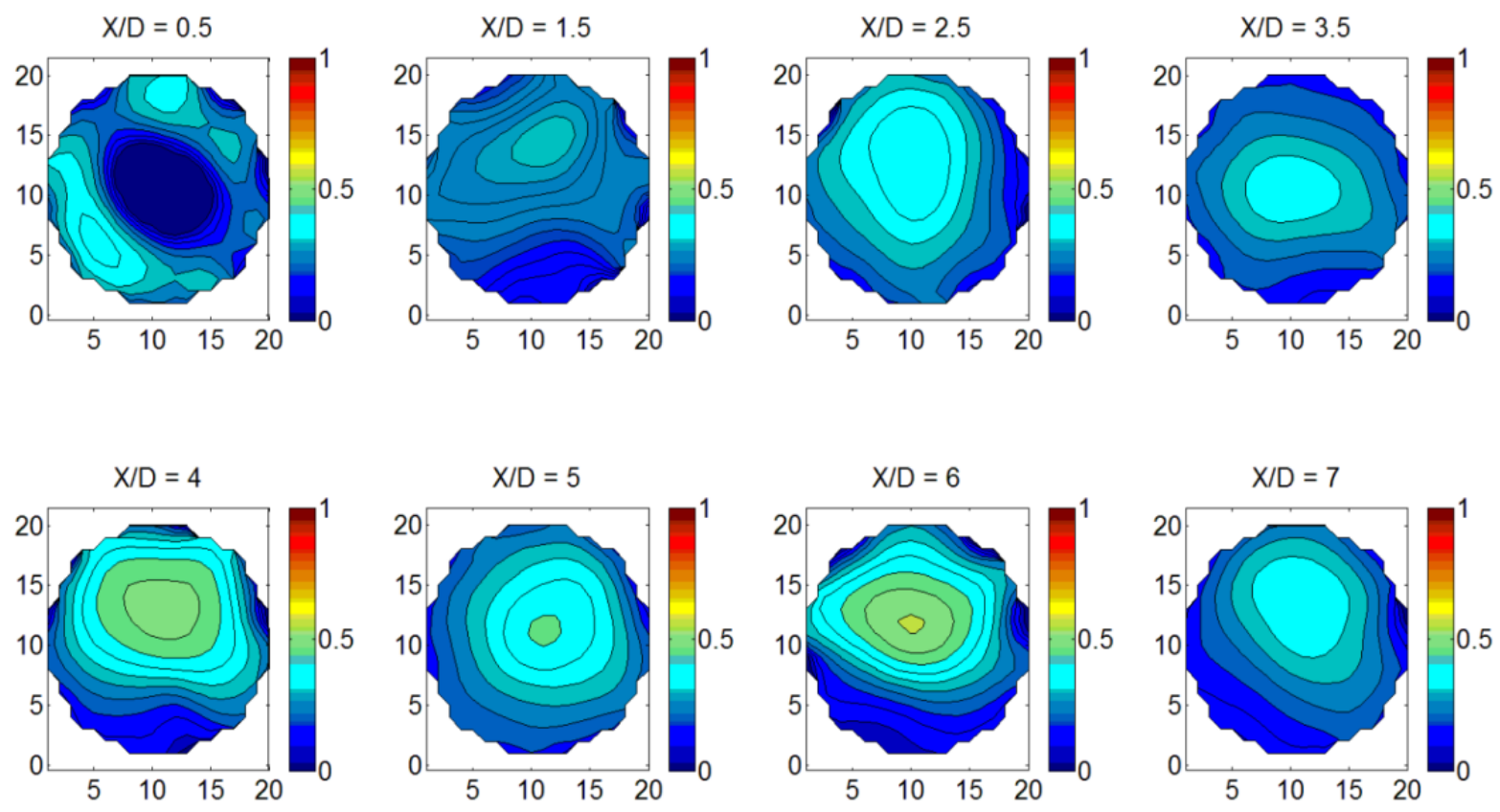

Figure 129: Concentration Tomogram at 20 psig_40 GPM_50\% GVF 

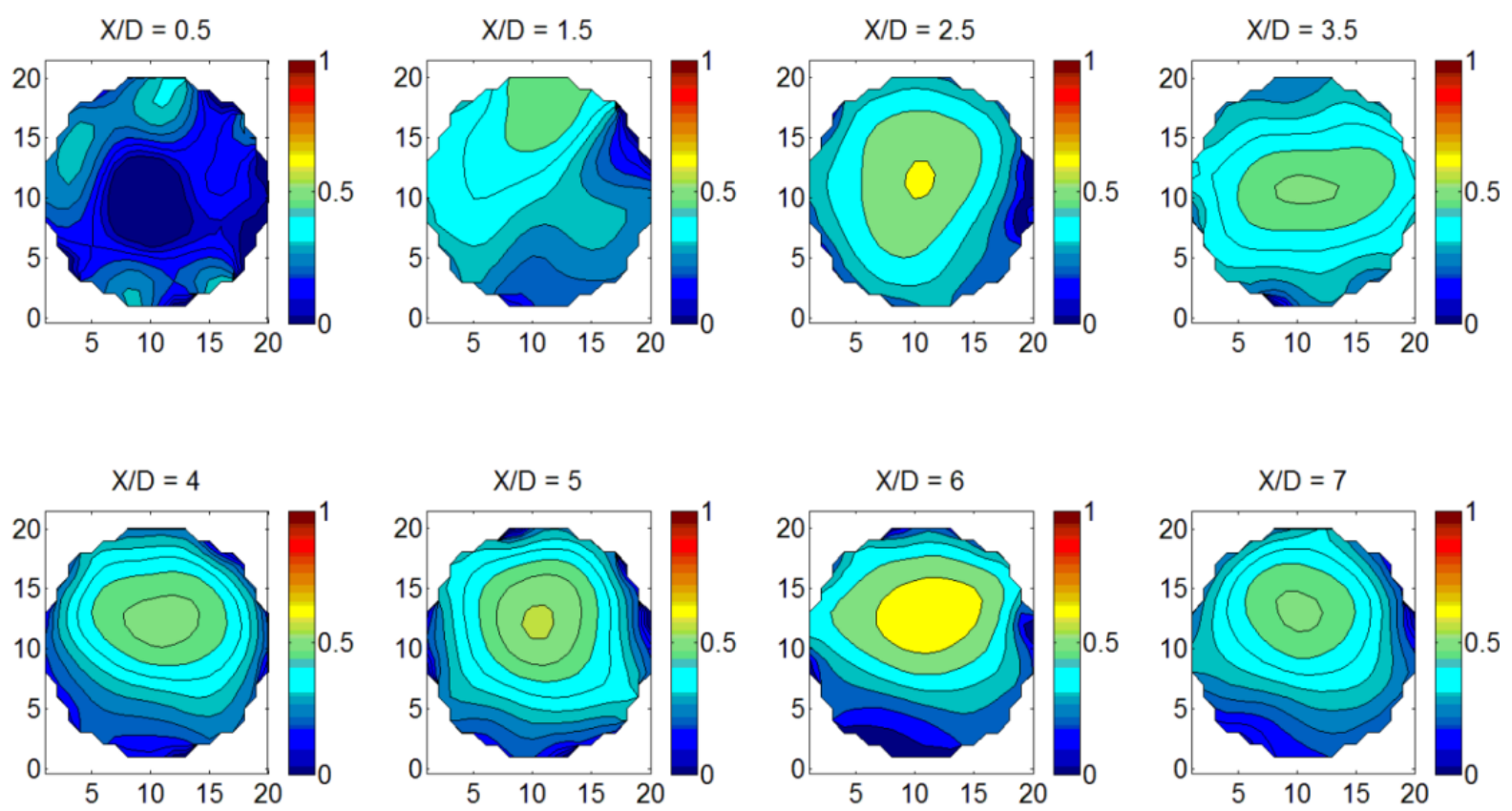

Figure 130: Concentration Tomogram at 20 psig_40 GPM_60\% GVF
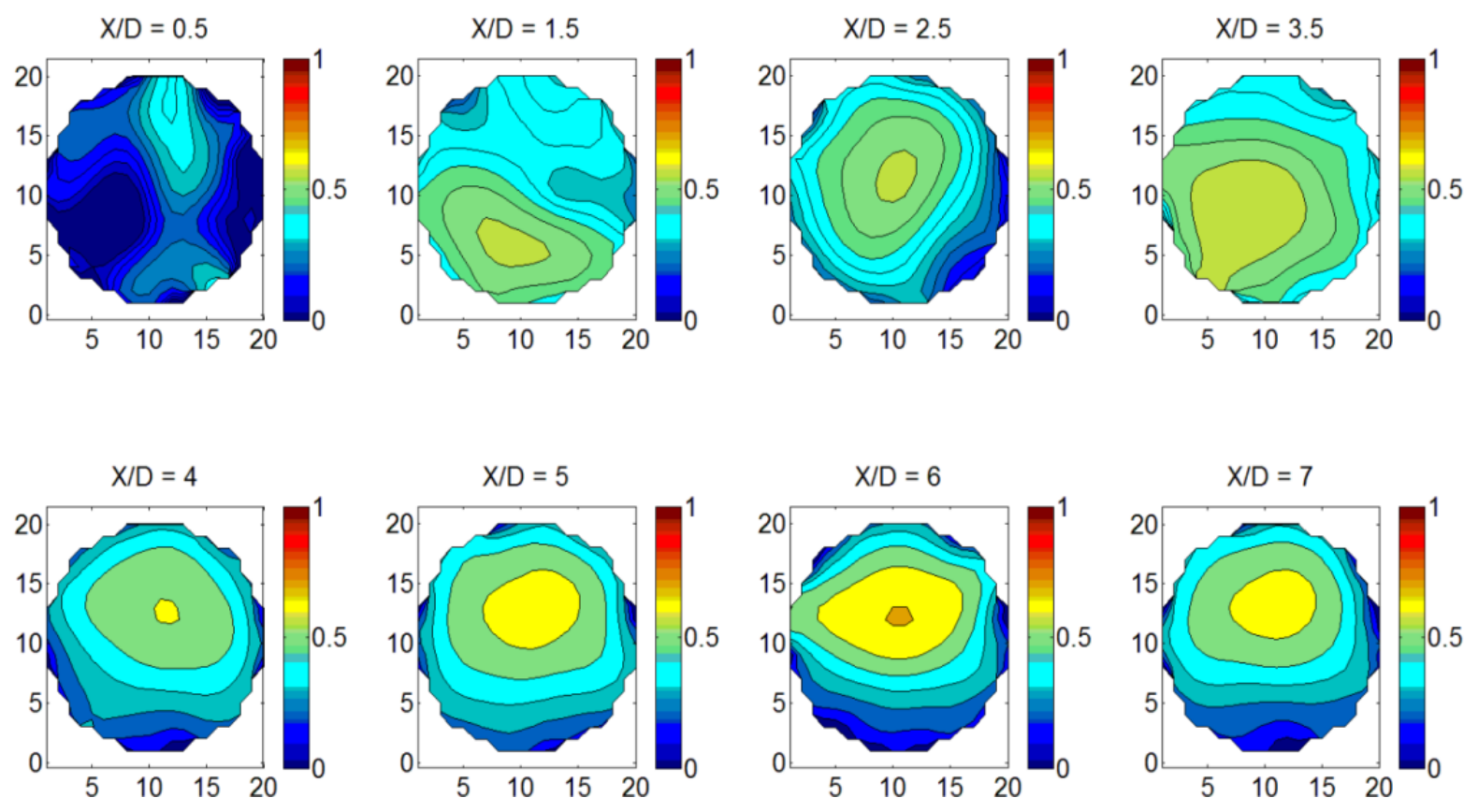

Figure 131: Concentration Tomogram at 20 psig_40 GPM_70\% GVF 

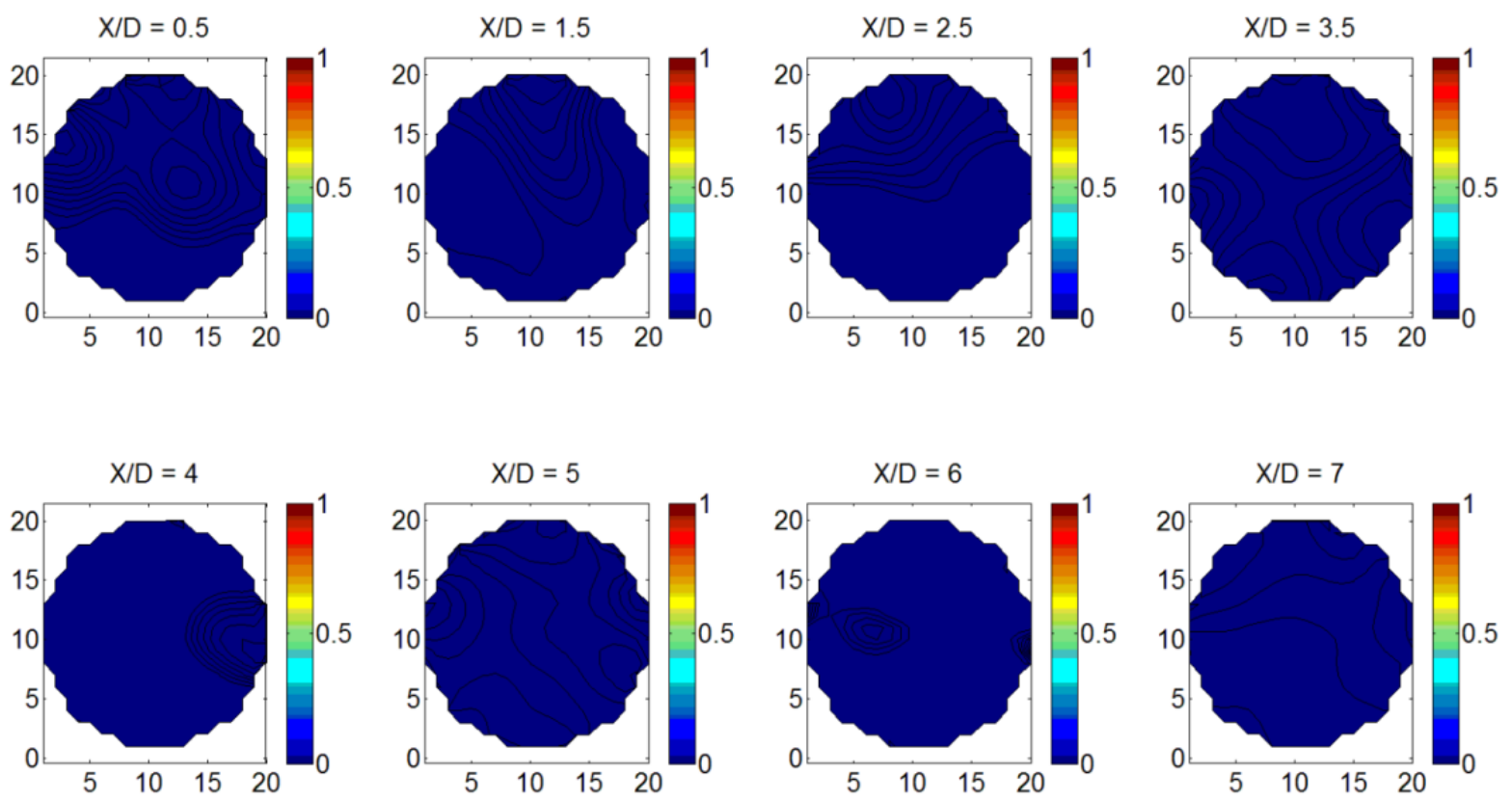

Figure 132: Concentration Tomogram at 20 psig_60 GPM_5\% GVF
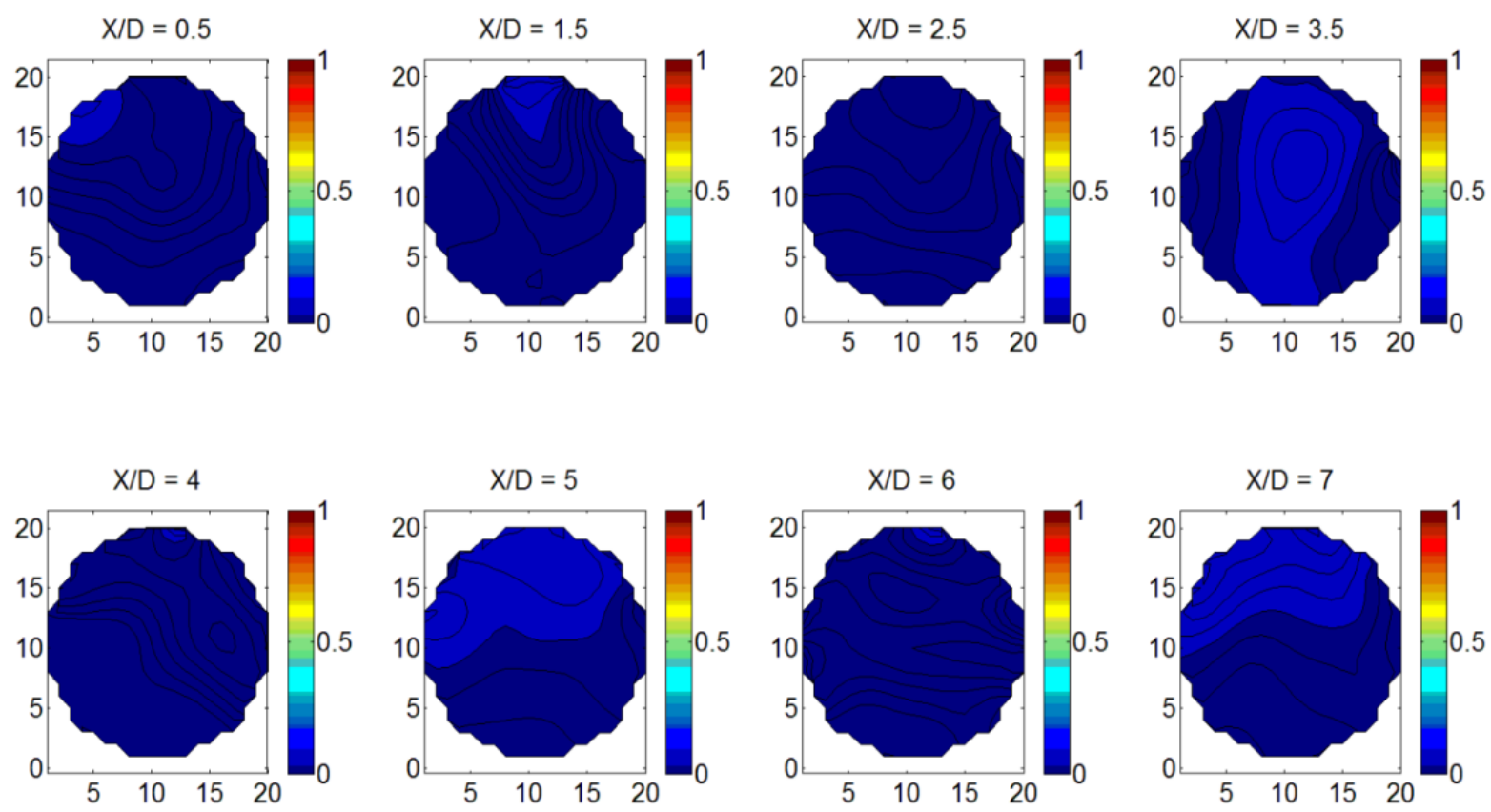

Figure 133: Concentration Tomogram at 20 psig_60 GPM_10\% GVF 

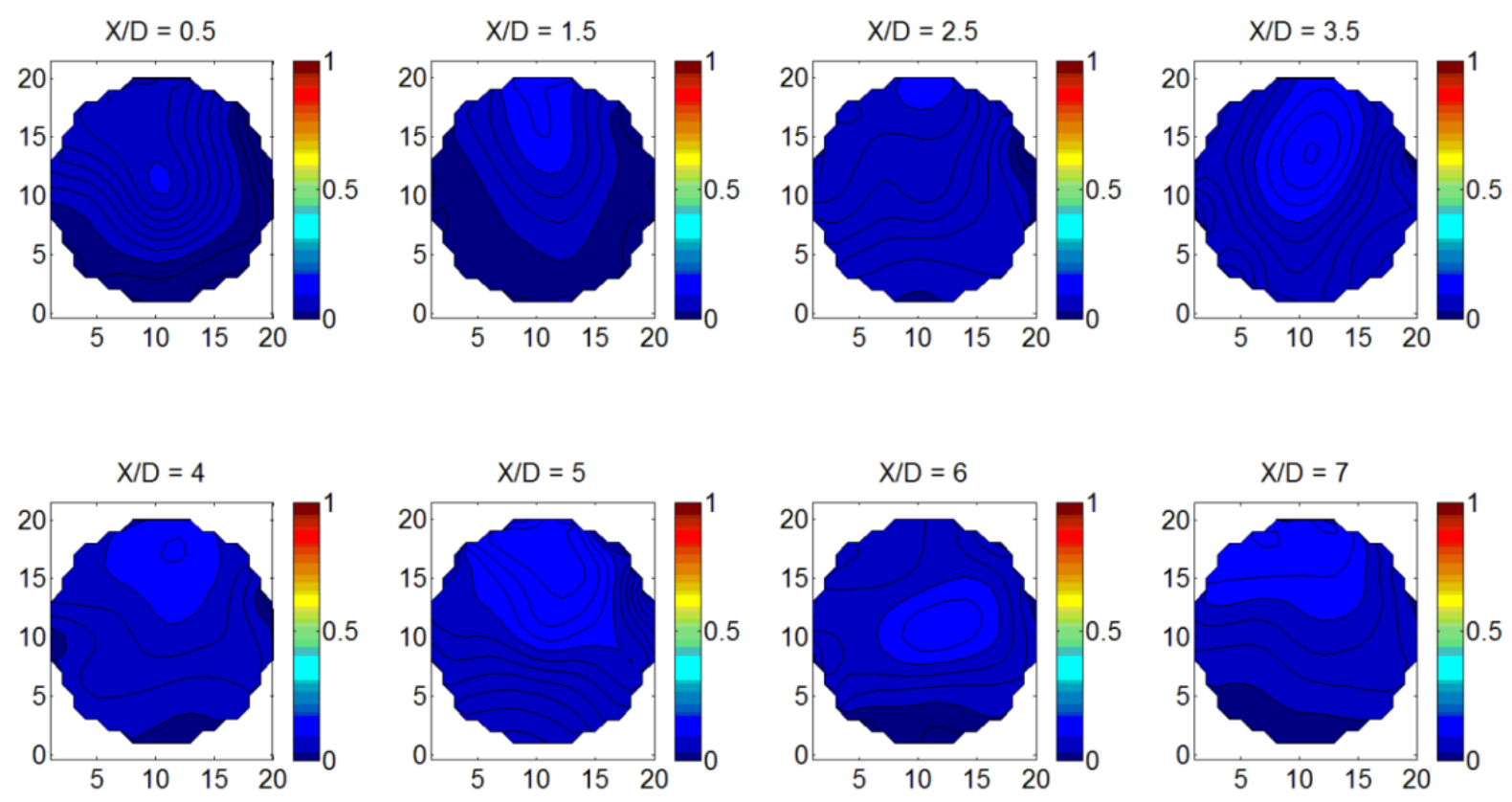

Figure 134: Concentration Tomogram at 20 psig_60 GPM_20\% GVF
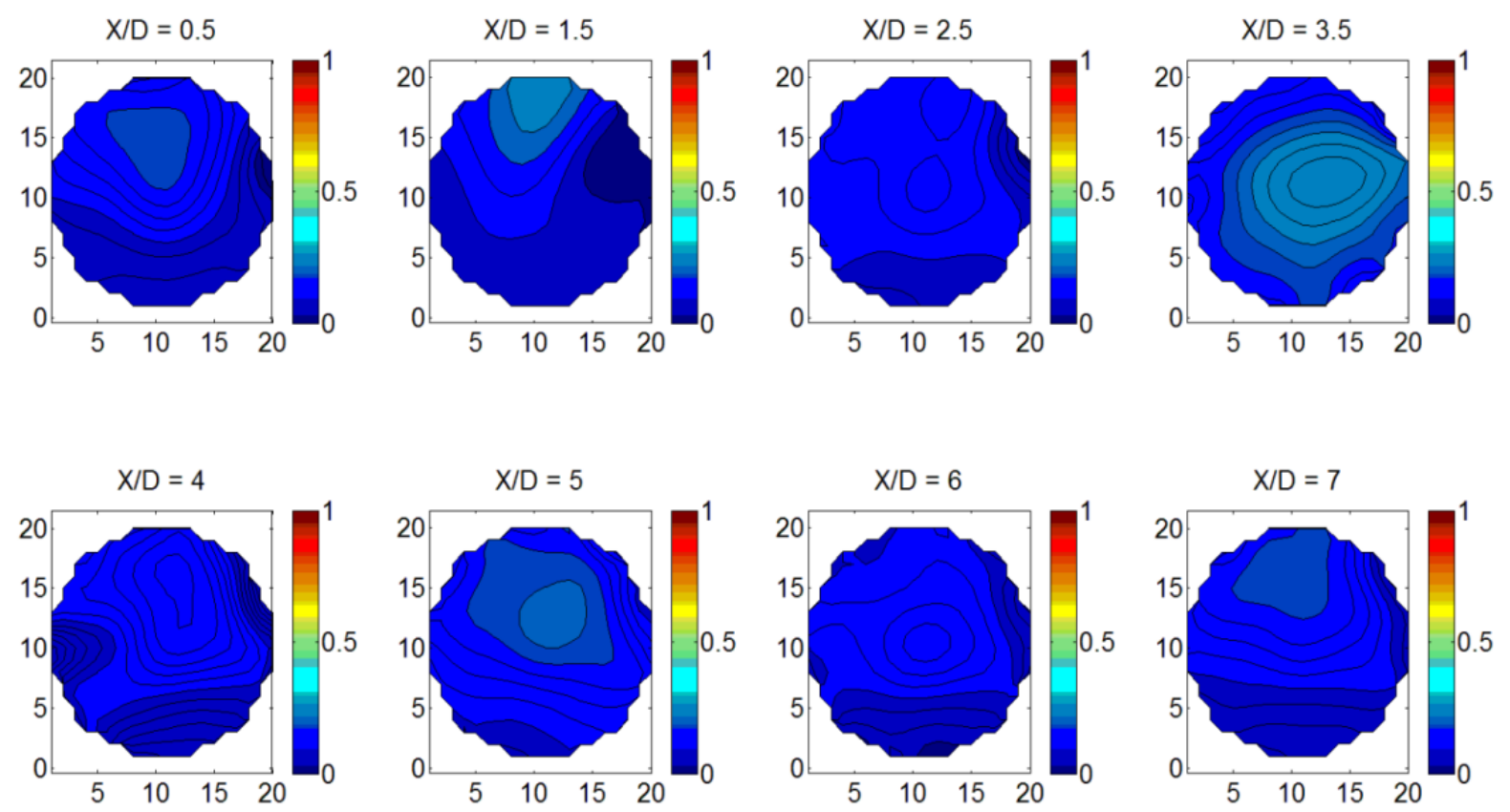

Figure 135: Concentration Tomogram at 20 psig_60 GPM_30\% GVF 

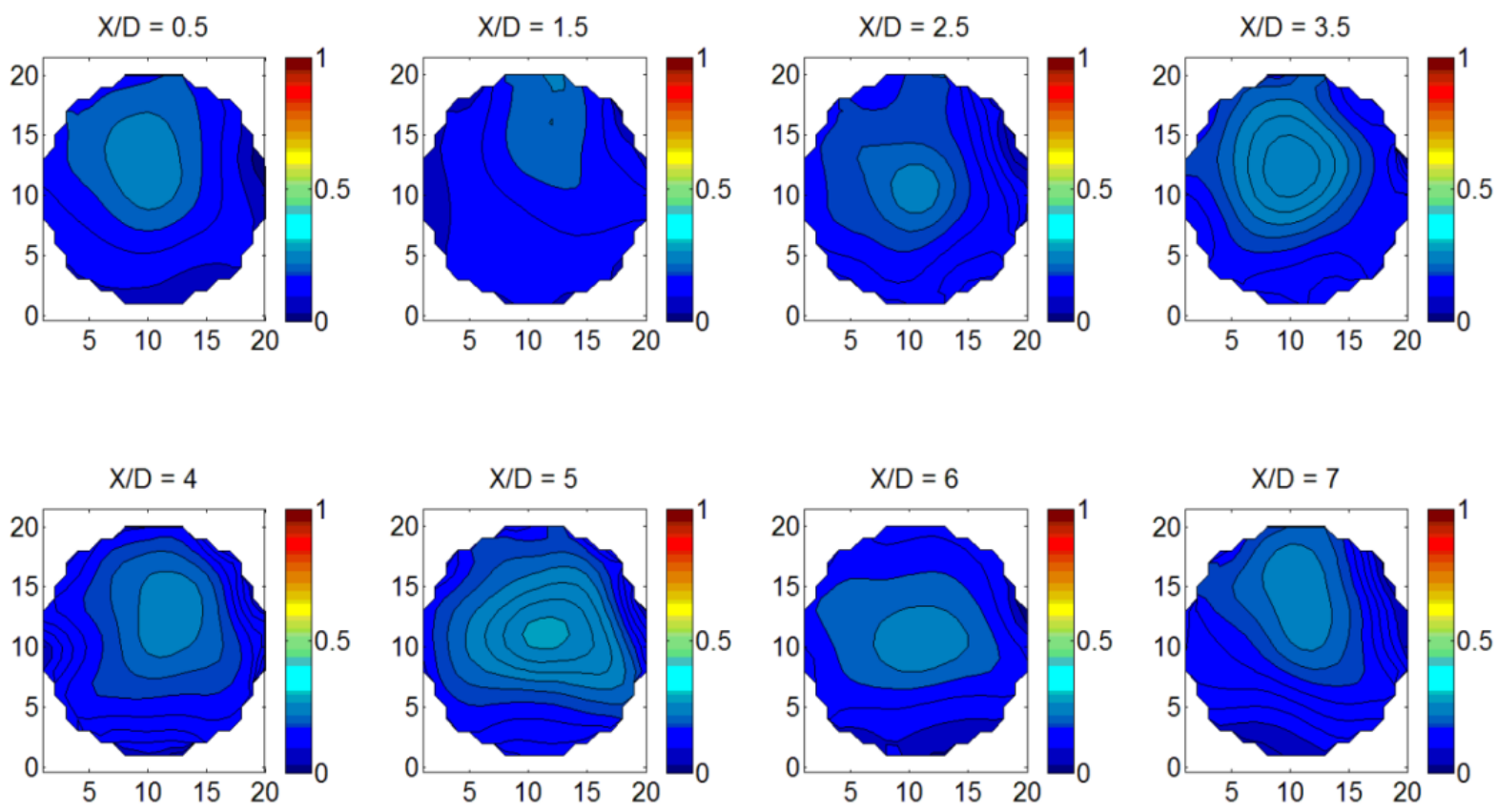

Figure 136: Concentration Tomogram at 20 psig_60 GPM_40\% GVF
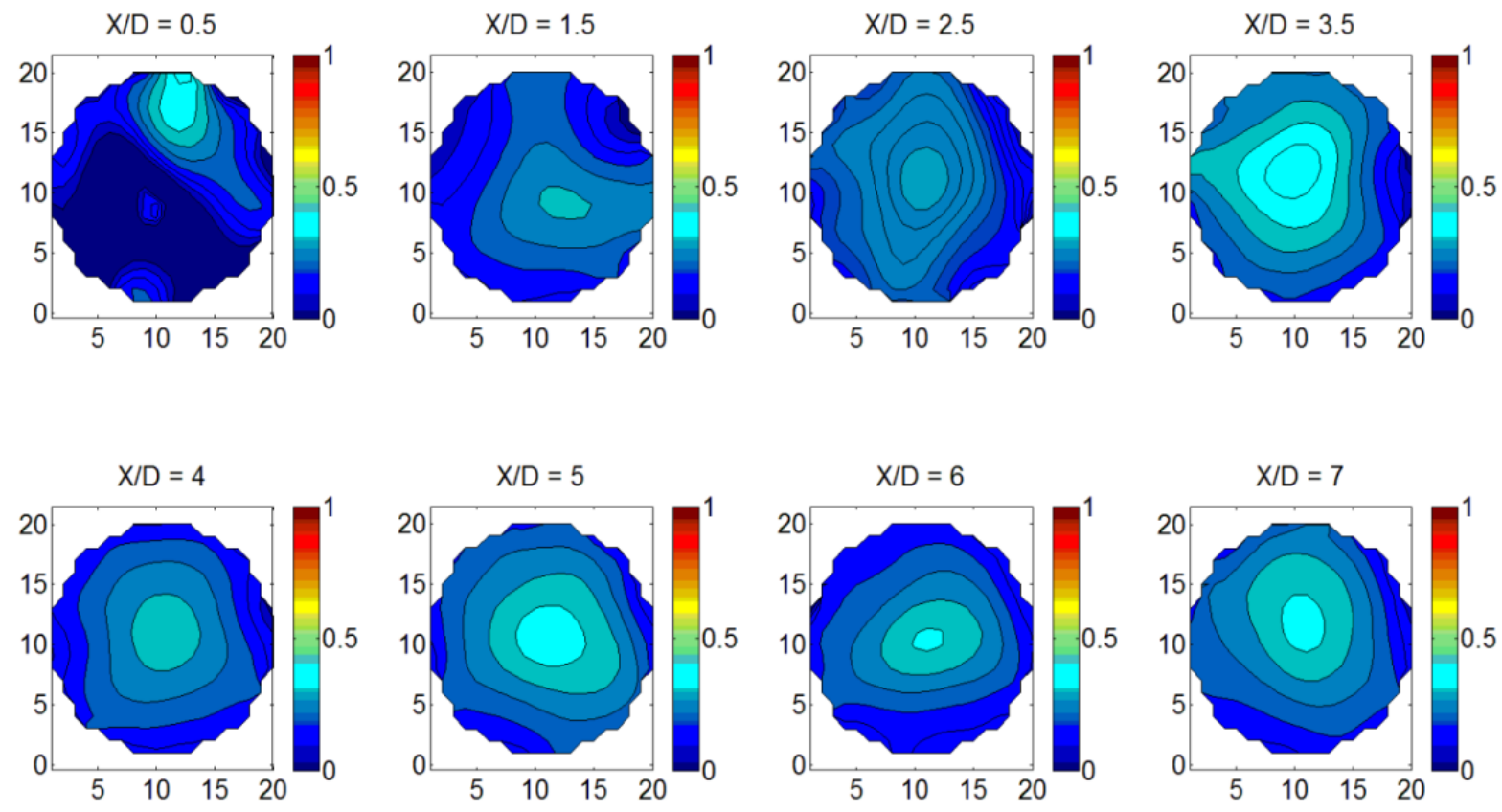

Figure 137: Concentration Tomogram at 20 psig_60 GPM_50\% GVF 

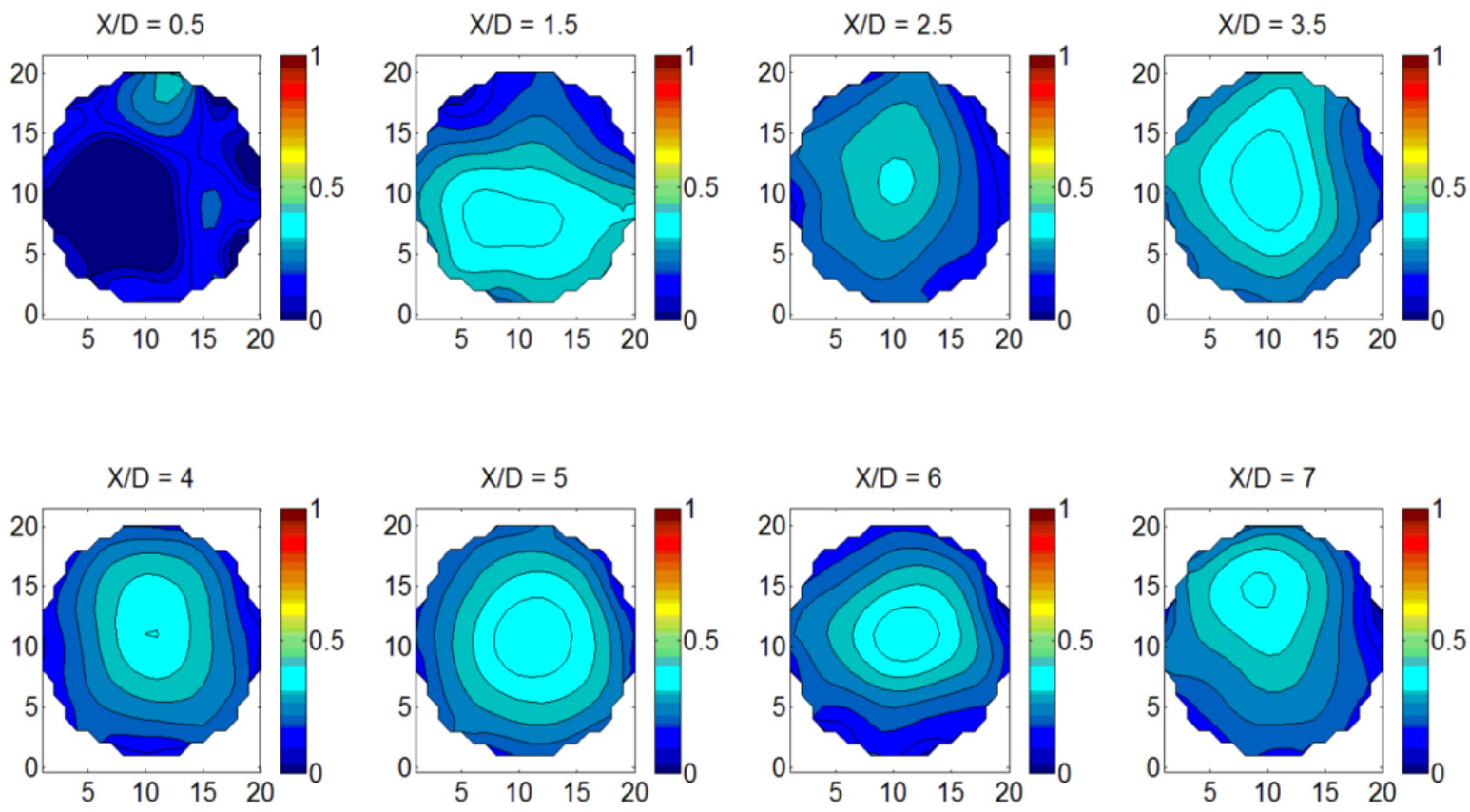

Figure 138: Concentration Tomogram at 20 psig_60 GPM_60\% GVF
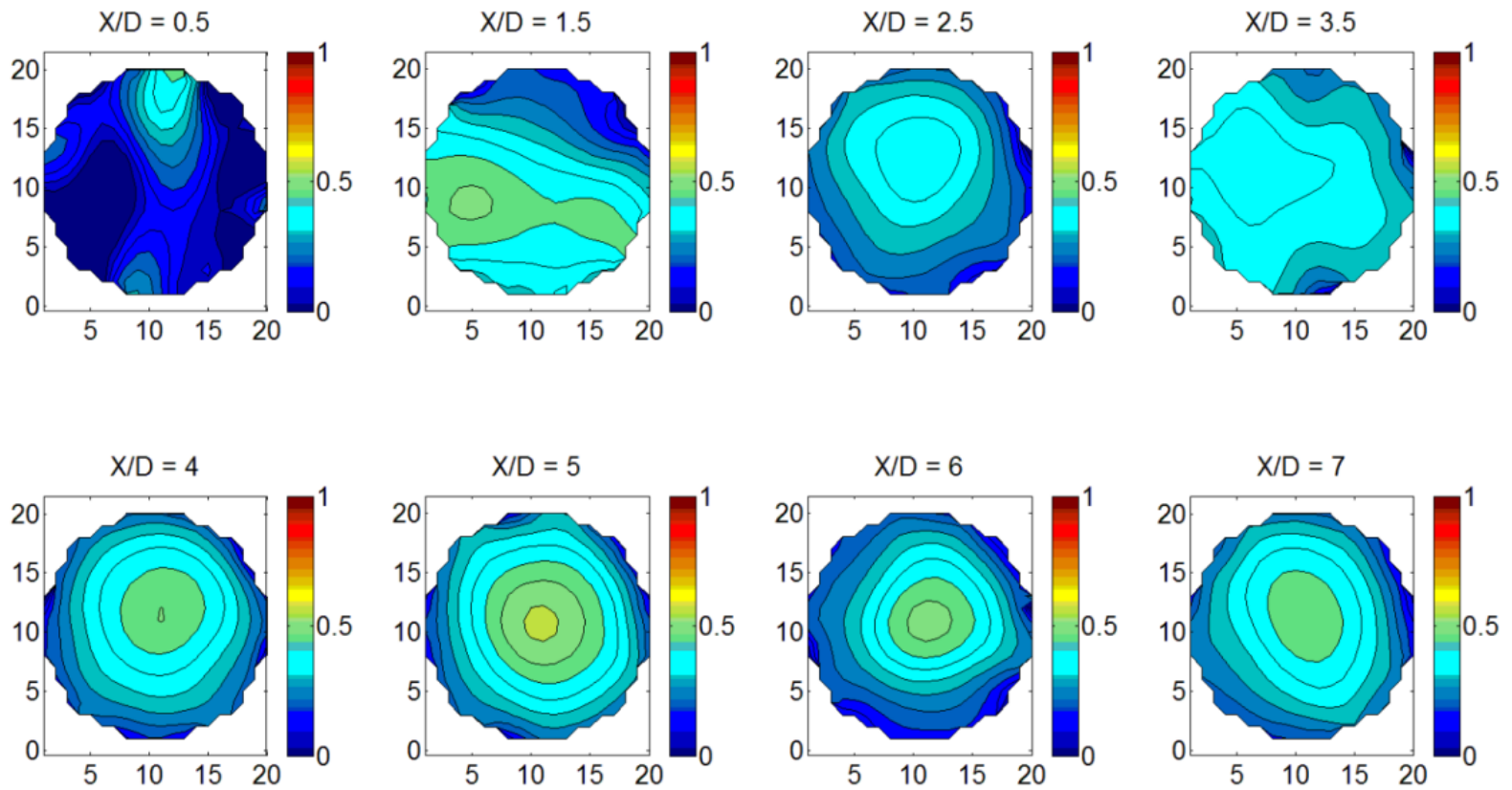

Figure 139: Concentration Tomogram at 20 psig_60 GPM_70\% GVF 

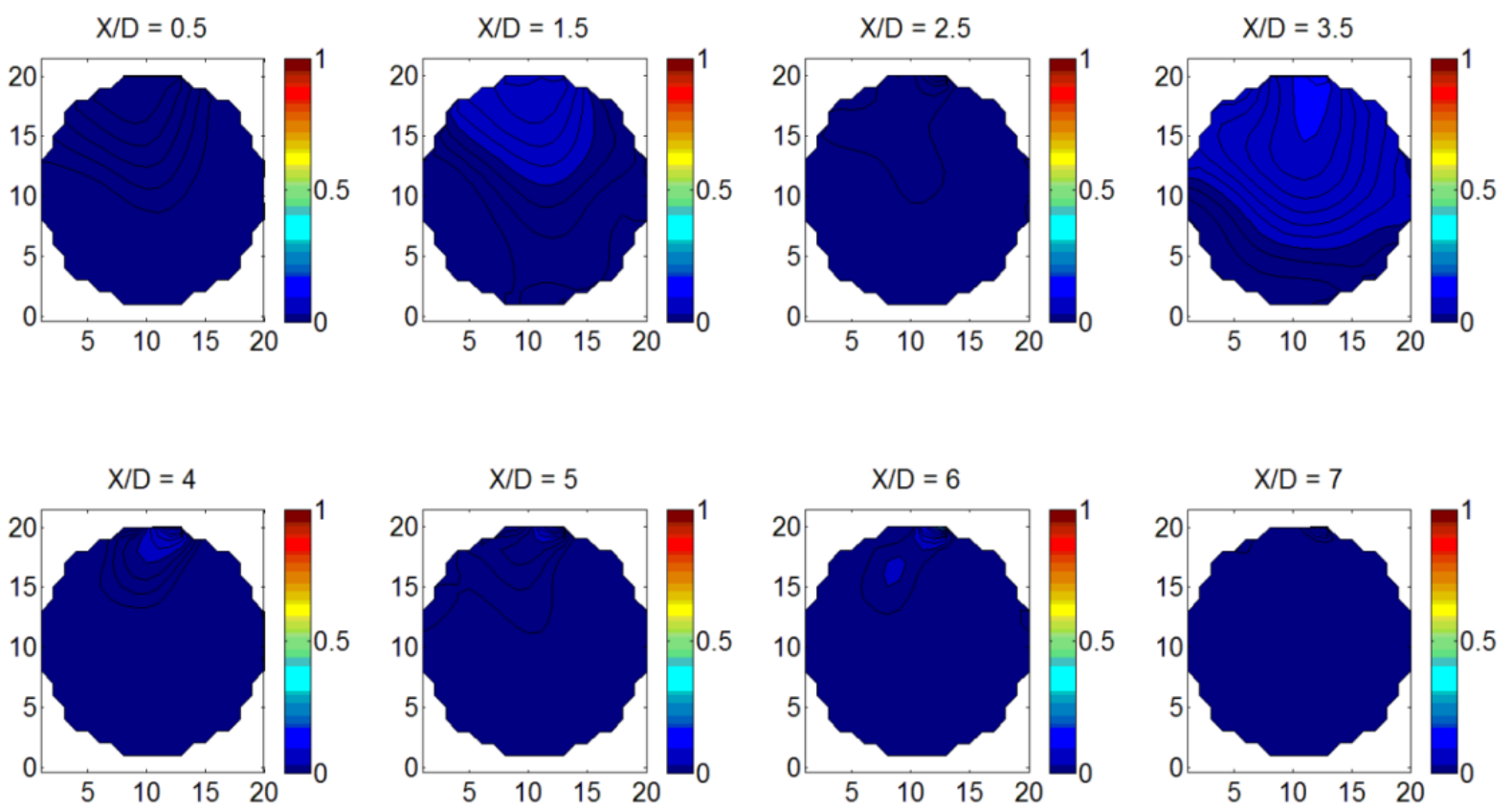

Figure 140: Concentration Tomogram at 40 psig_20 GPM_5\% GVF
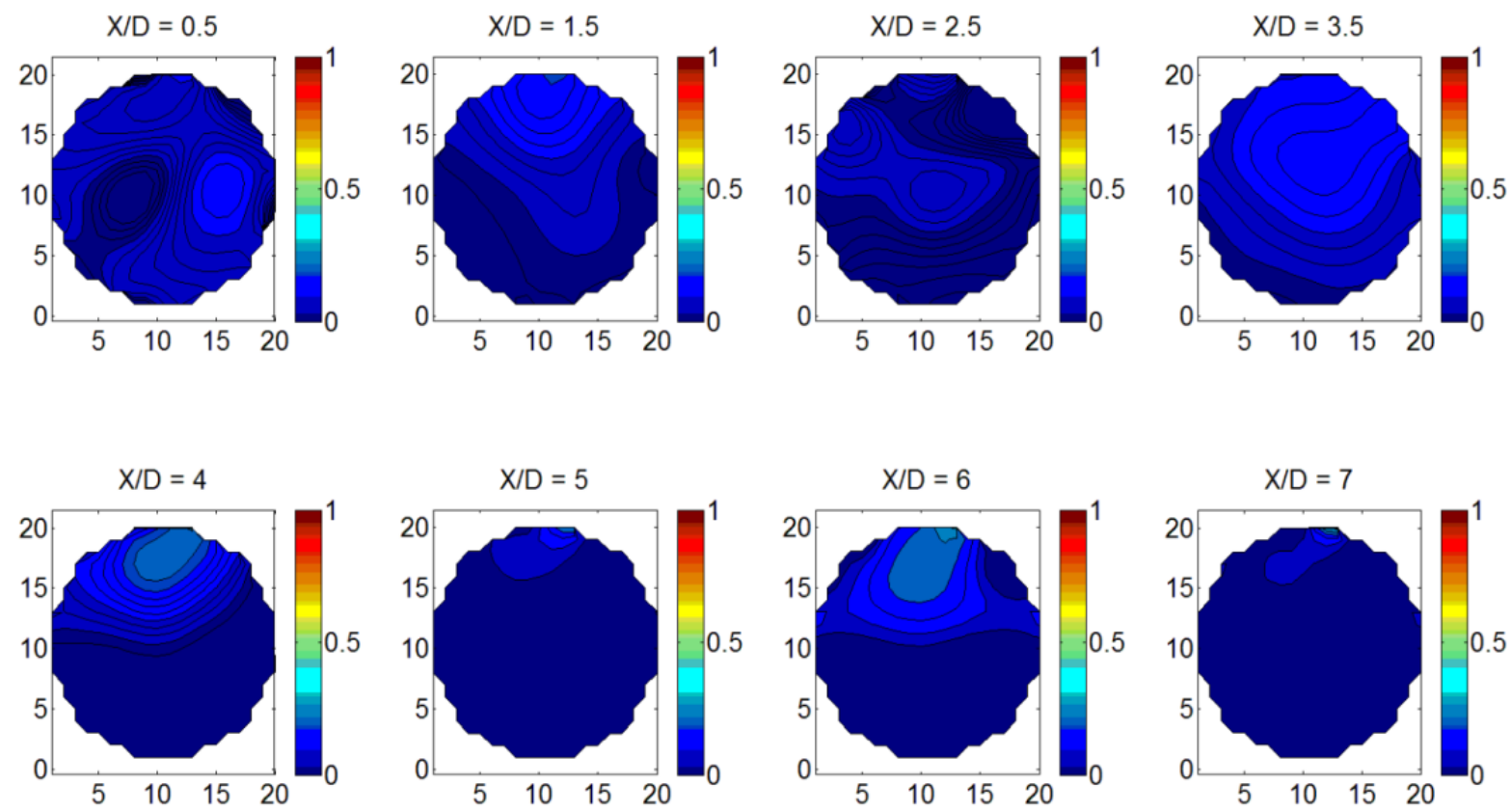

Figure 141: Concentration Tomogram at 40 psig_20 GPM_10\% GVF 

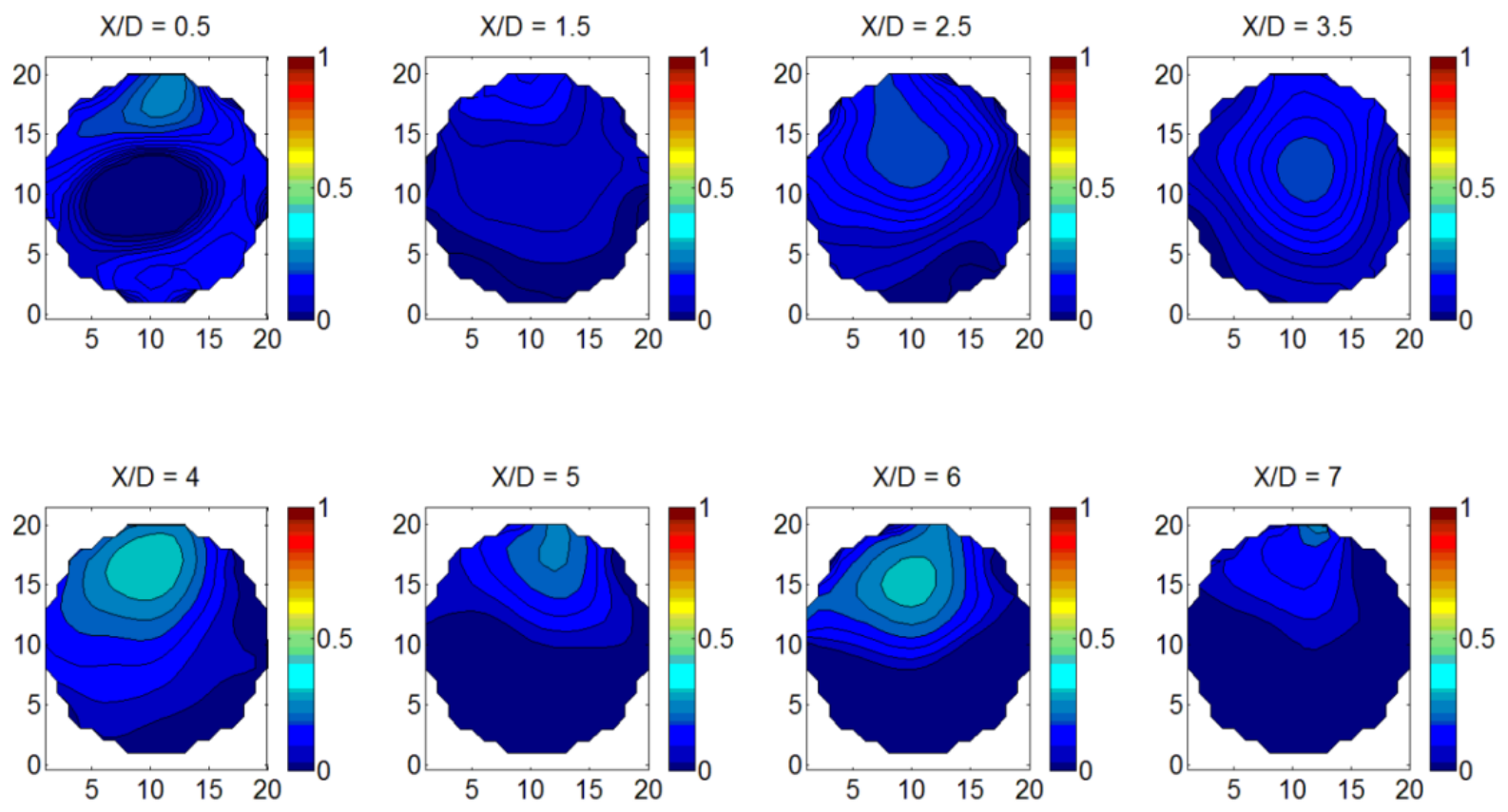

Figure 142: Concentration Tomogram at 40 psig_20 GPM_20\% GVF
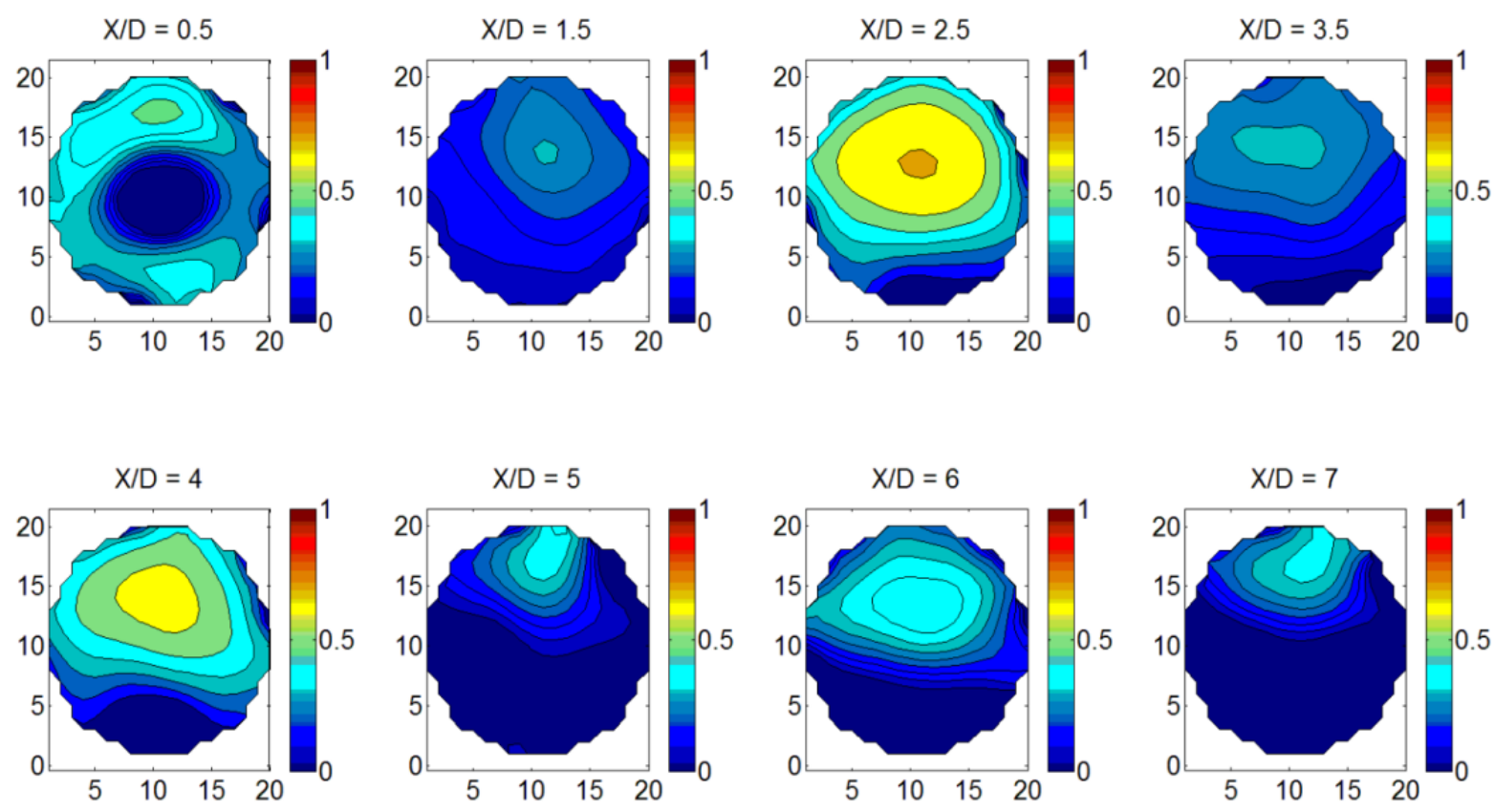

Figure 143: Concentration Tomogram at 40 psig_20 GPM_30\% GVF 

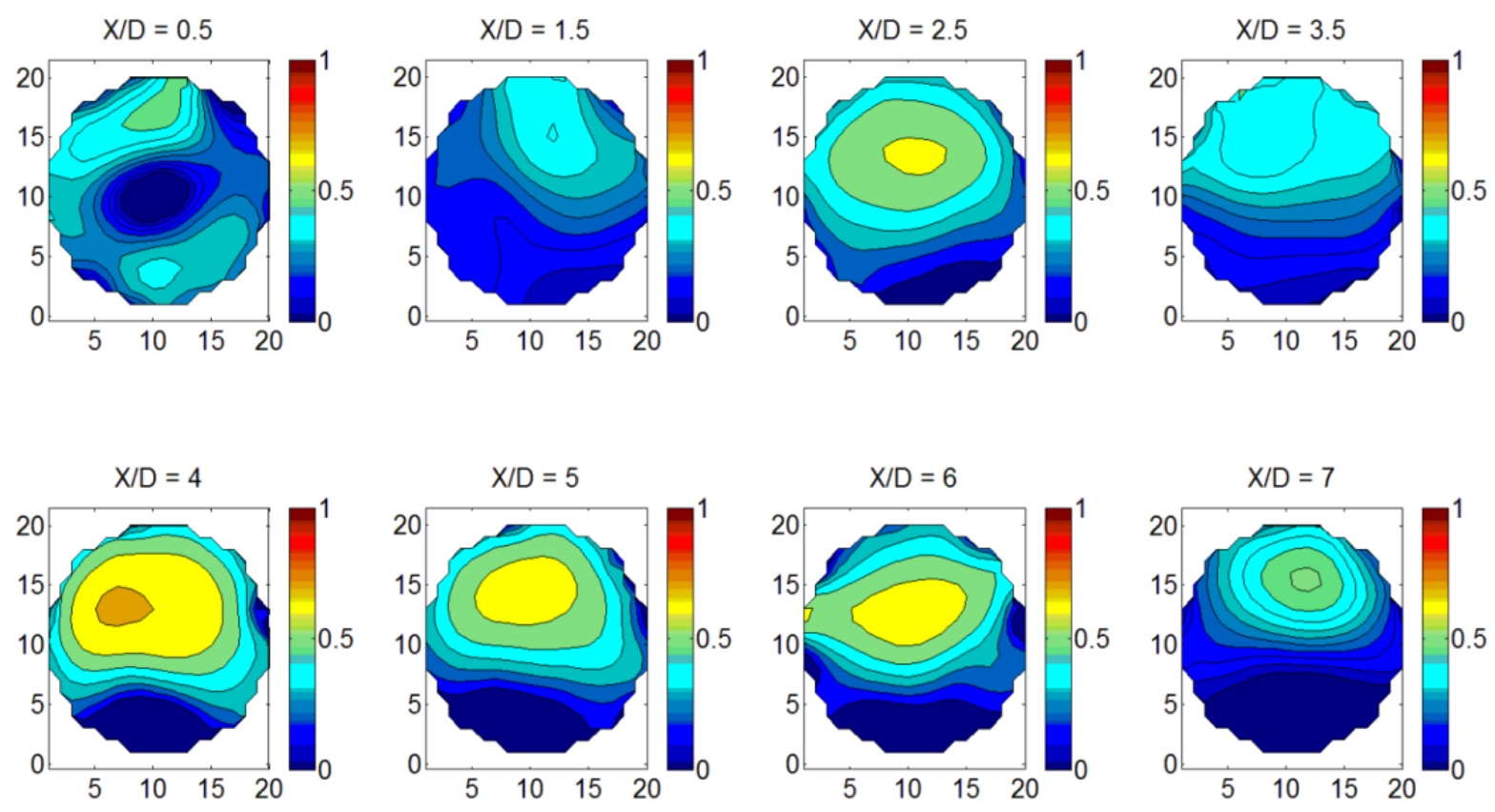

Figure 144: Concentration Tomogram at 40 psig_20 GPM_40\% GVF
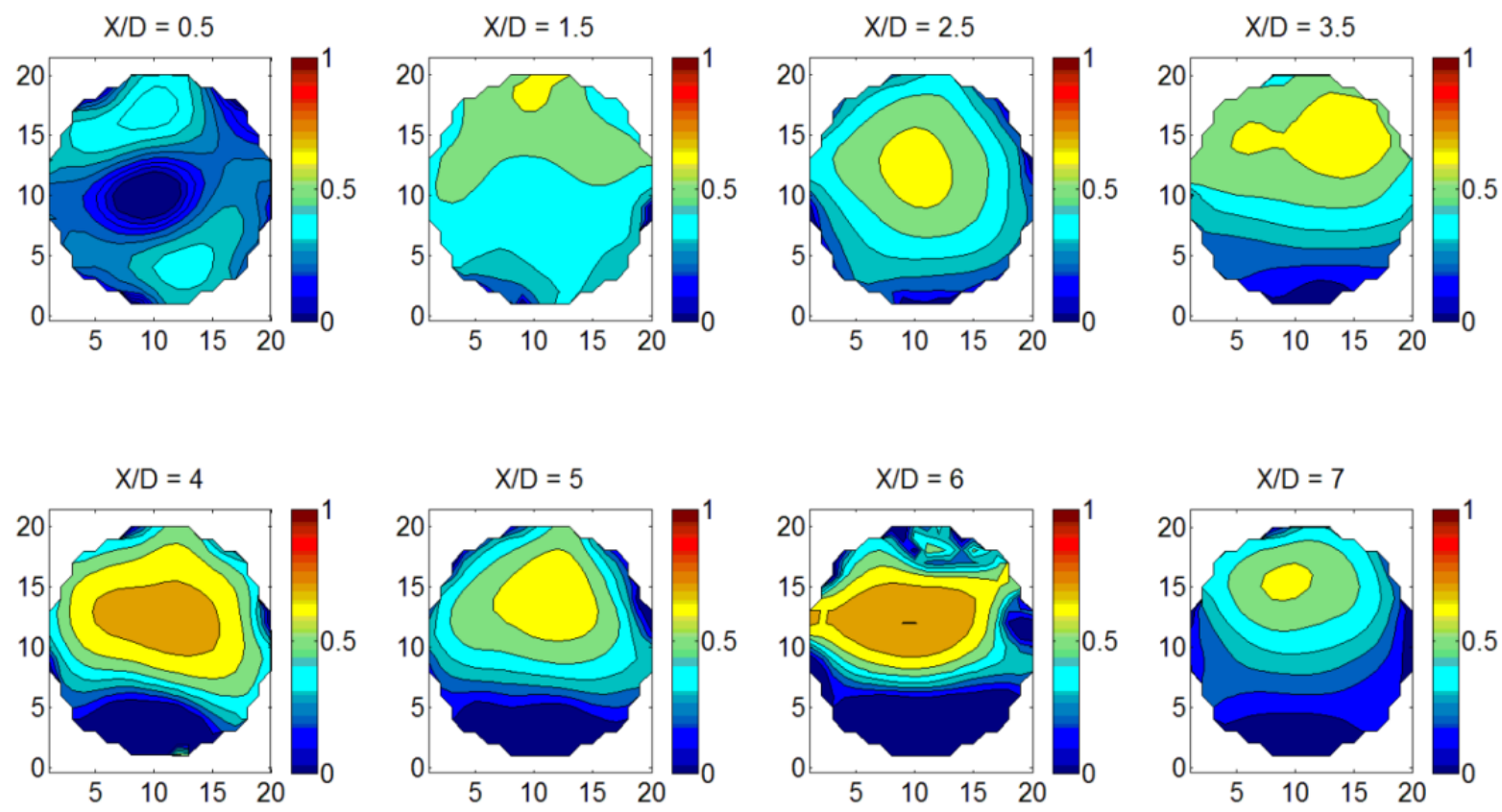

Figure 145: Concentration Tomogram at 40 psig_20 GPM_50\% GVF 

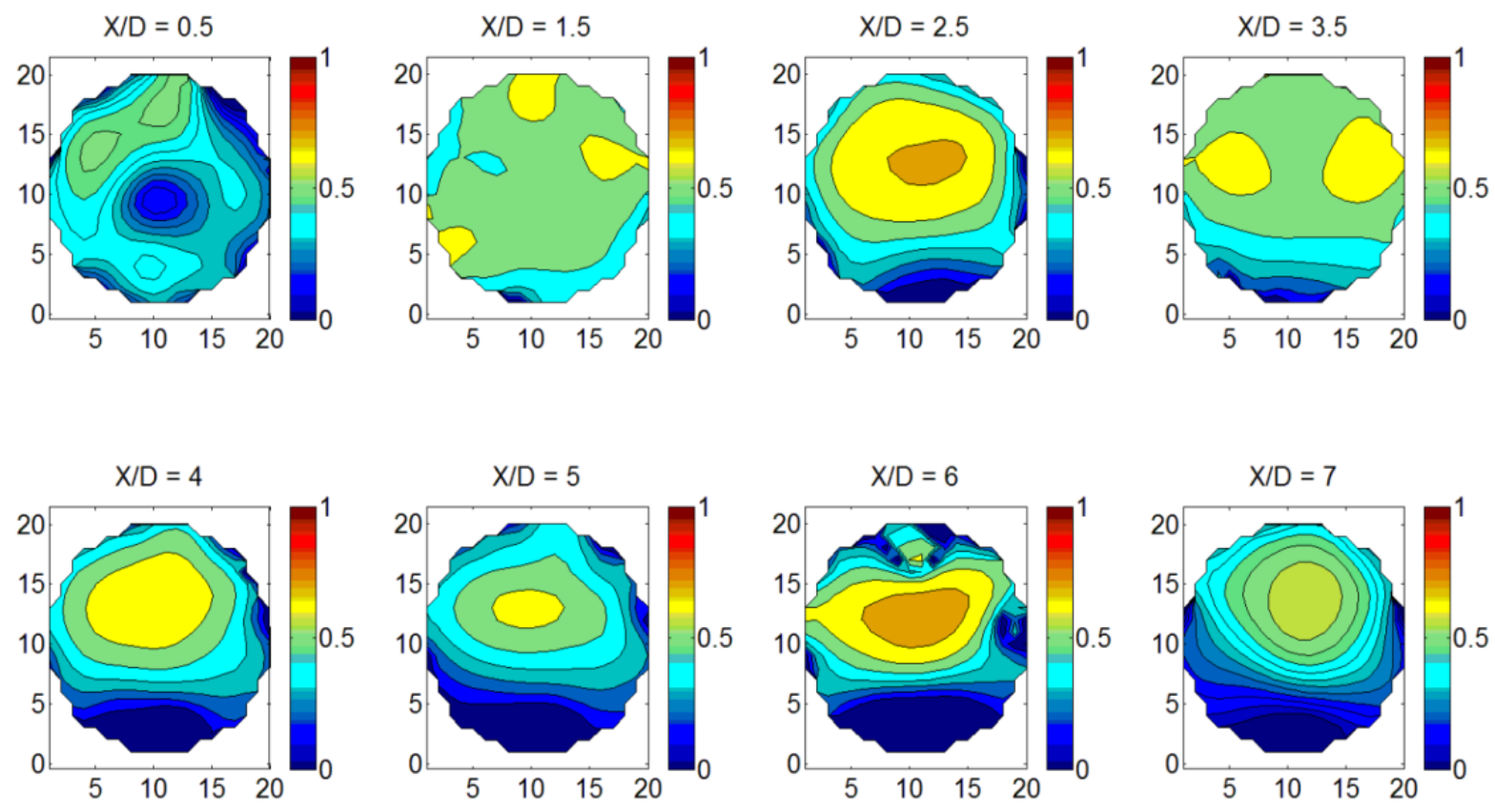

Figure 146: Concentration Tomogram at 40 psig_20 GPM_60\% GVF
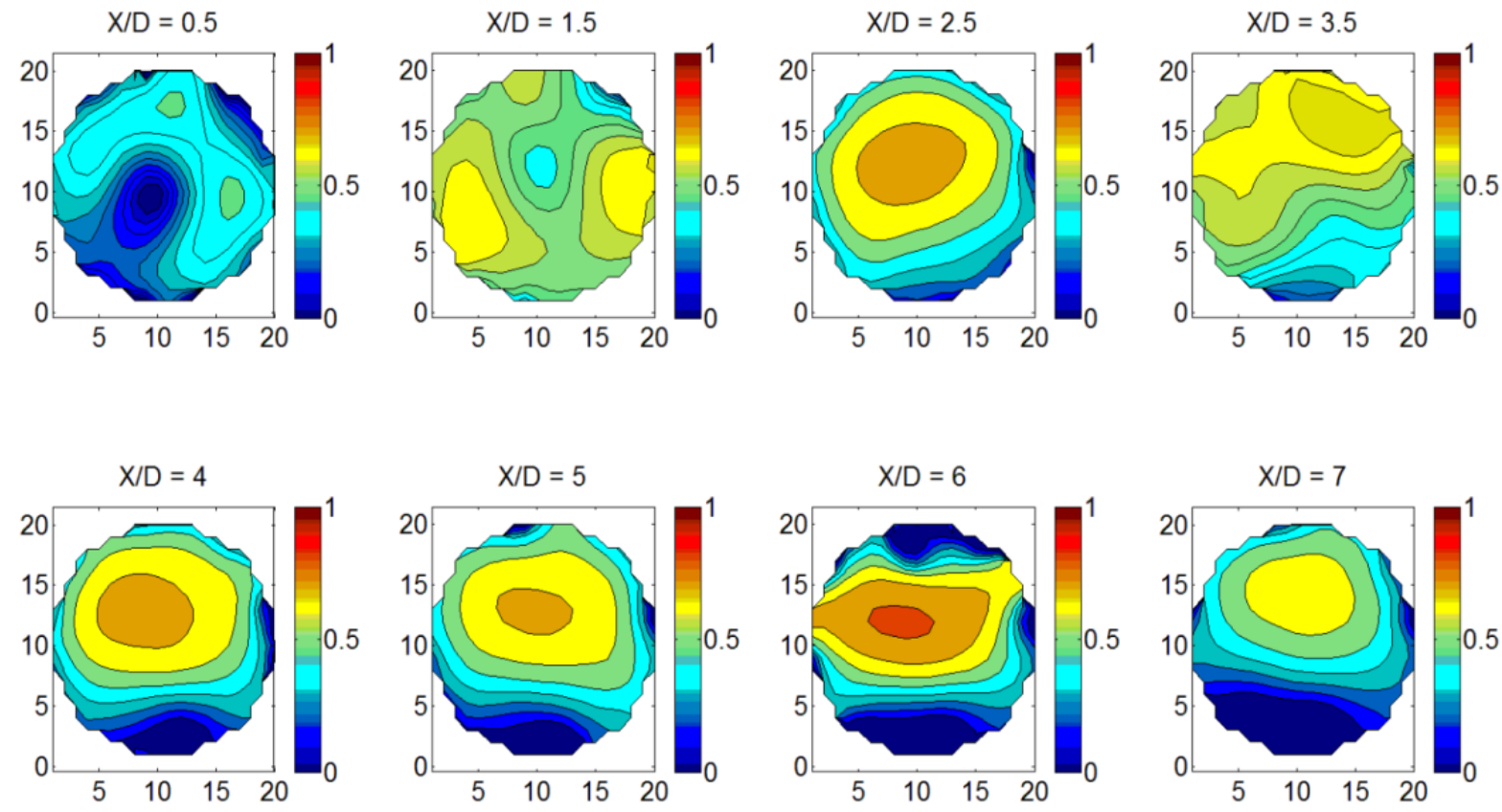

Figure 147: Concentration Tomogram at 40 psig_20 GPM_70\% GVF 

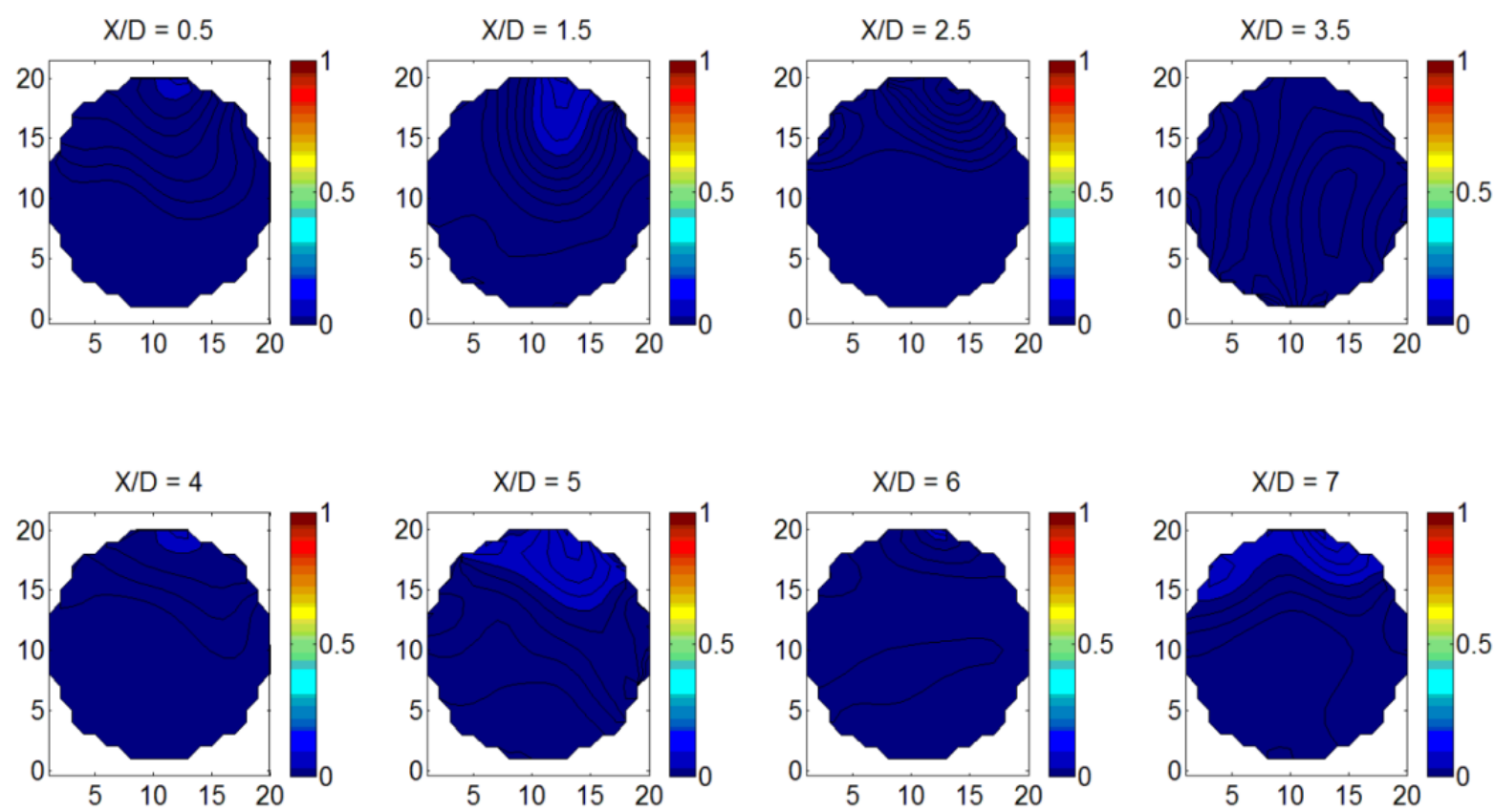

Figure 148: Concentration Tomogram at 40 psig_40 GPM_5\% GVF
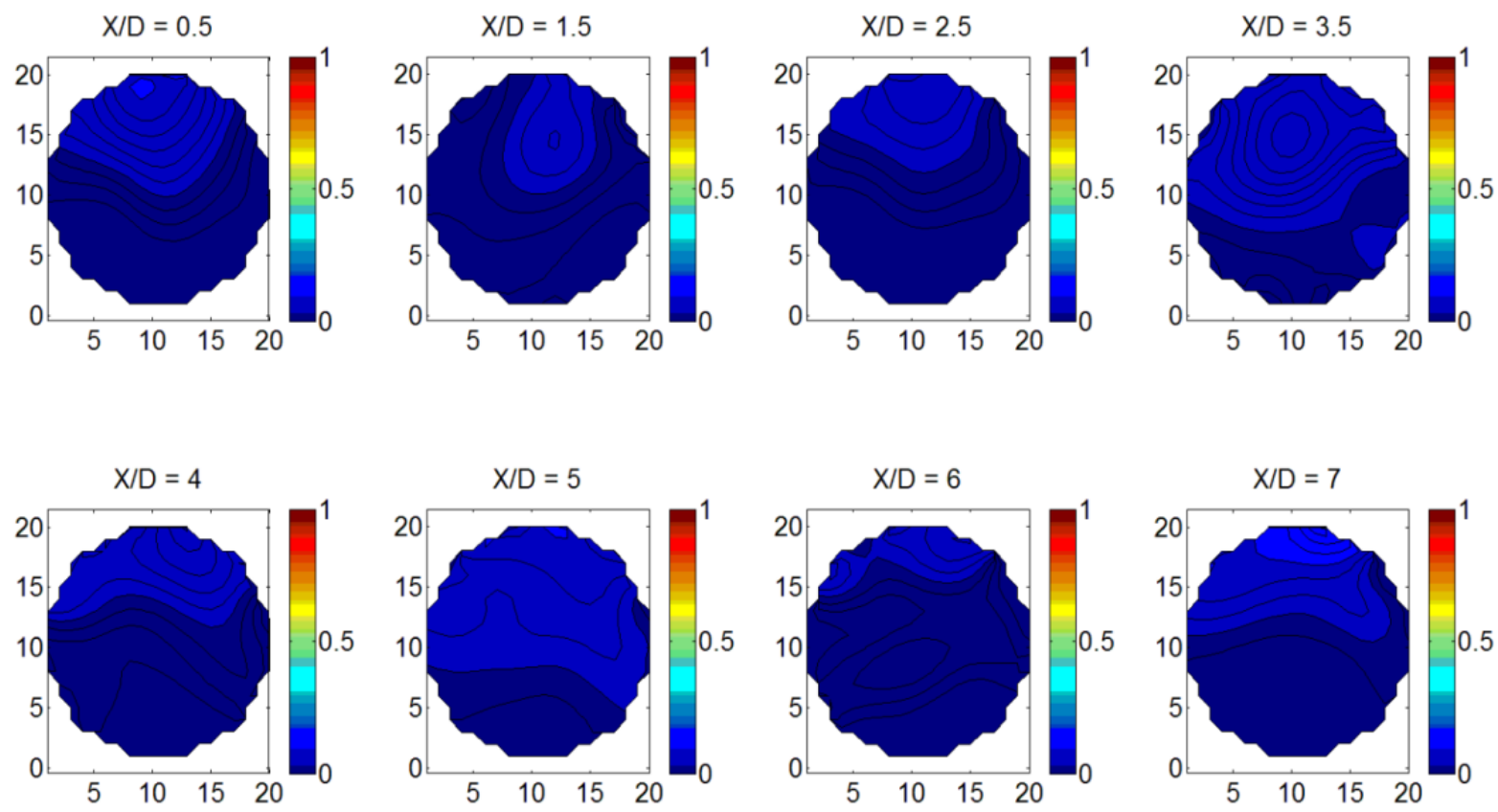

Figure 149: Concentration Tomogram at 40 psig_40 GPM_10\% GVF 

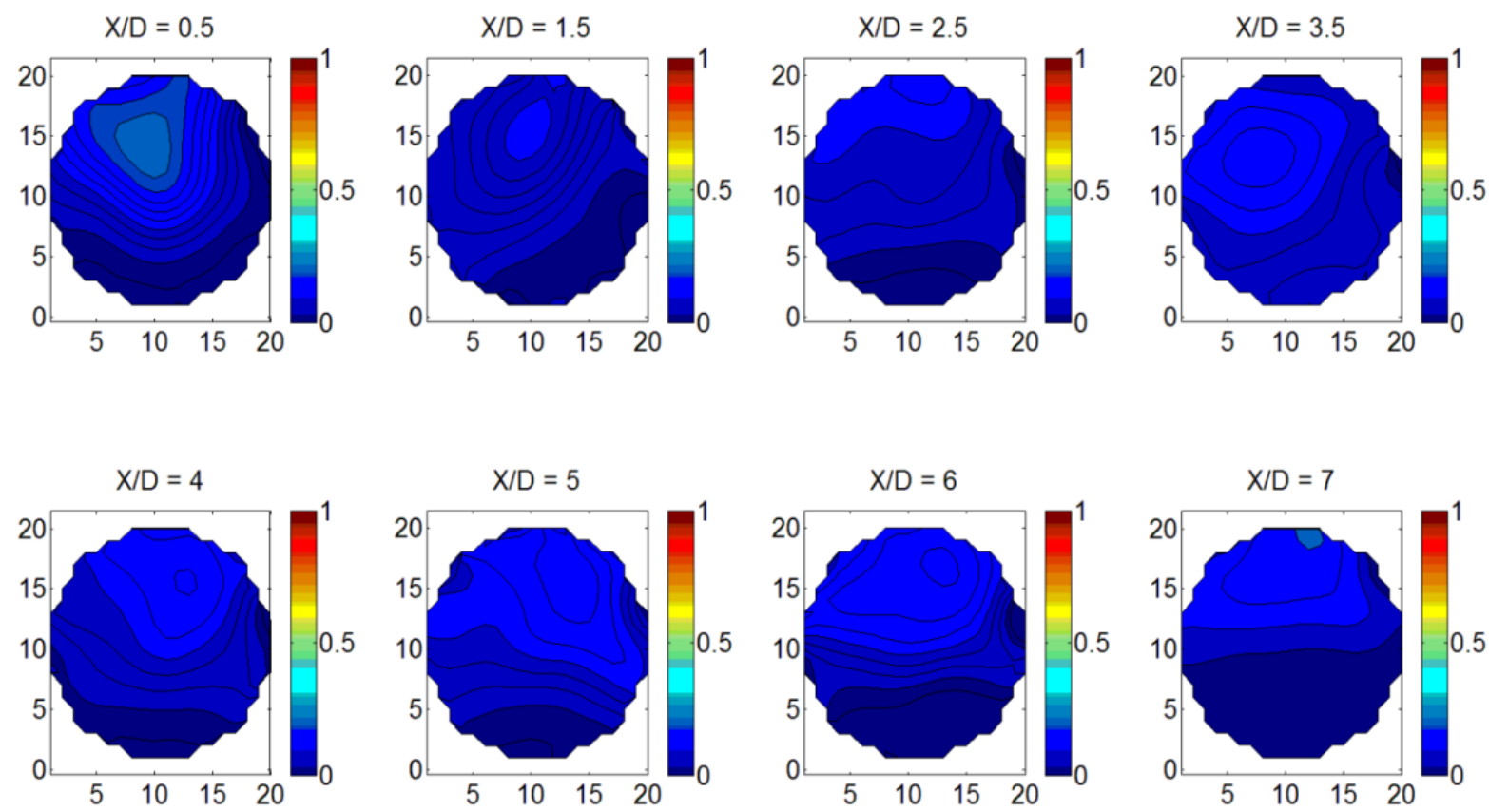

Figure 150: Concentration Tomogram at 40 psig_40 GPM_20\% GVF
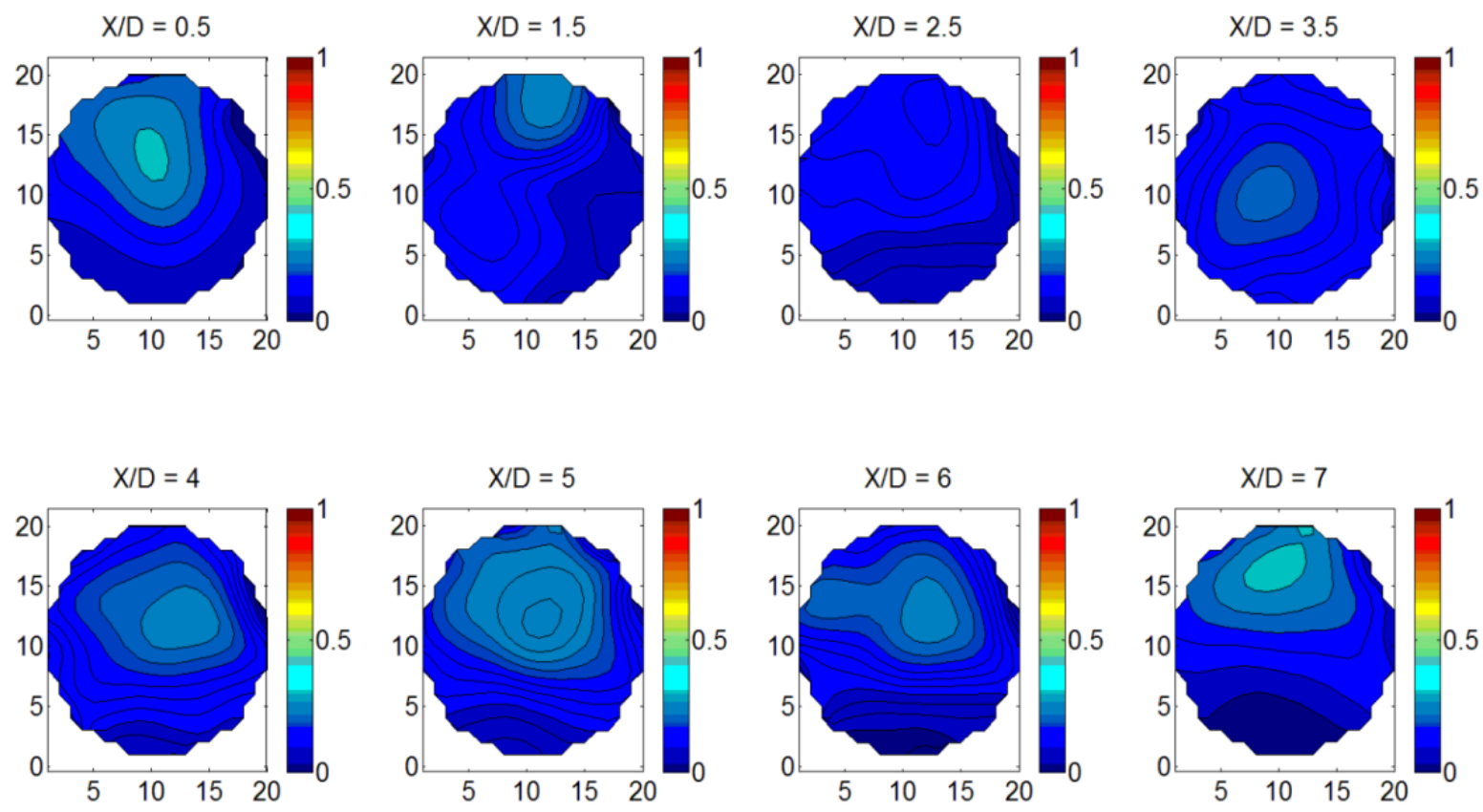

Figure 151: Concentration Tomogram at 40 psig_40 GPM_30\% GVF 

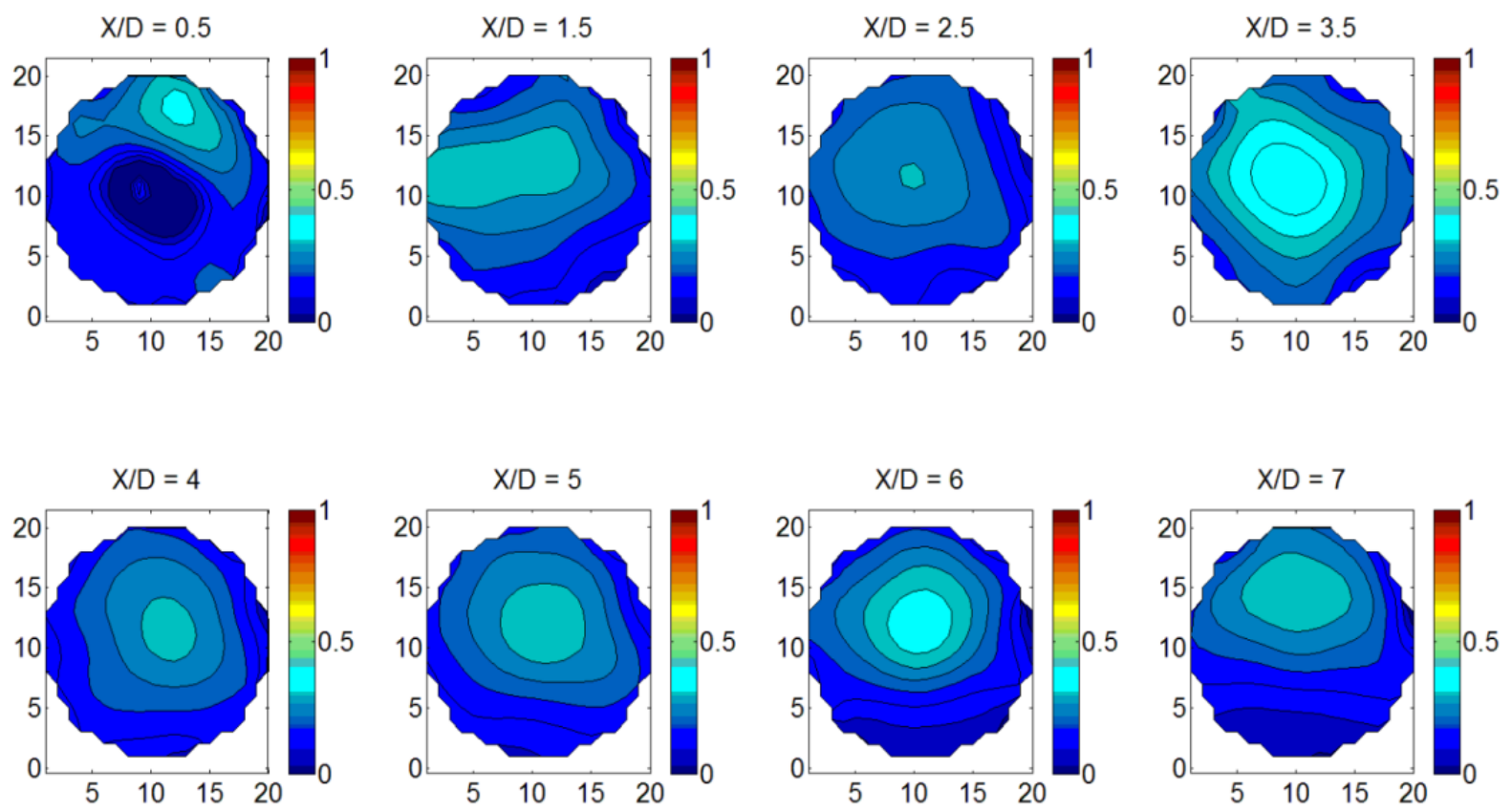

Figure 152: Concentration Tomogram at 40 psig_40 GPM_40\% GVF
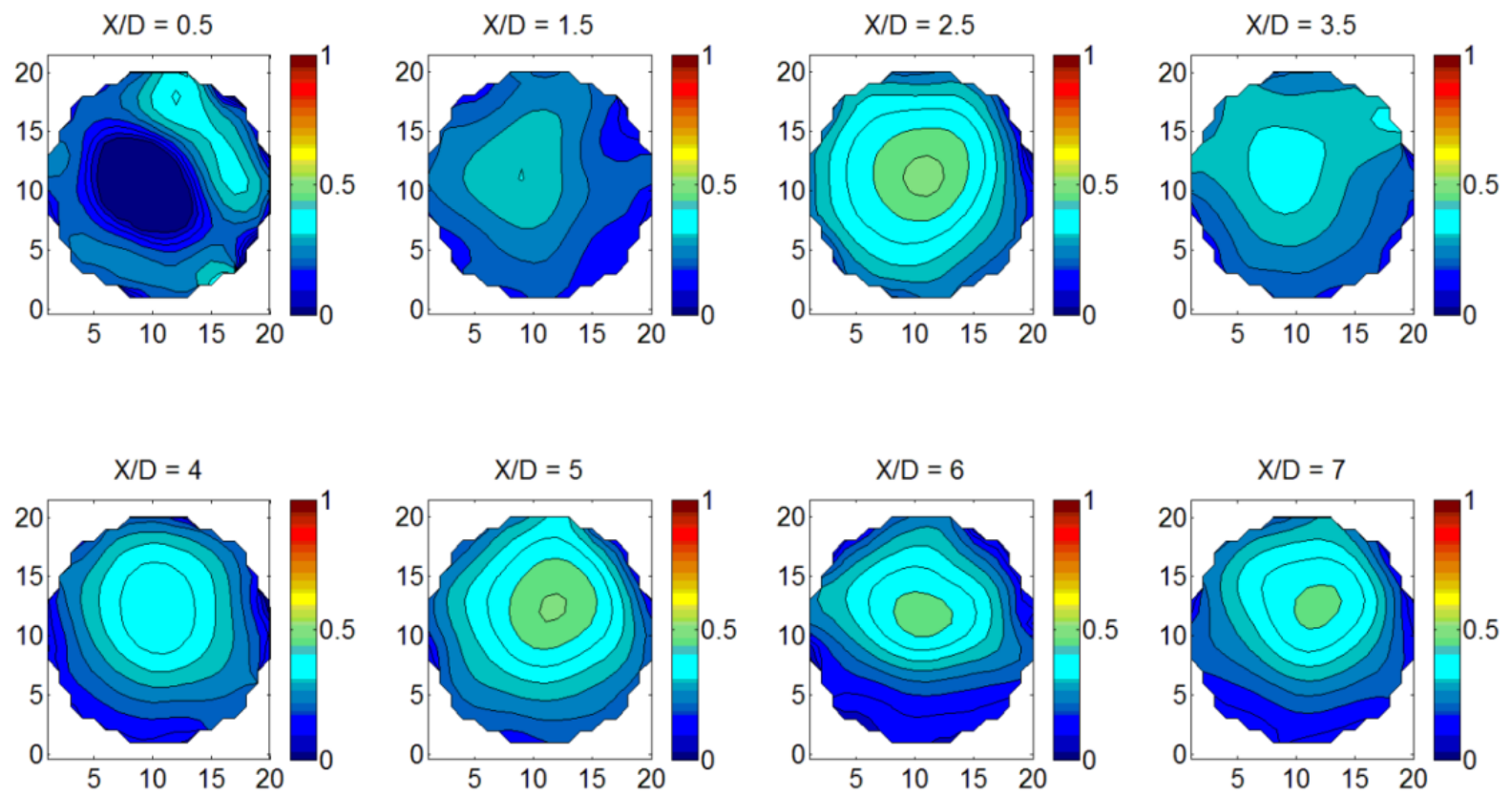

Figure 153: Concentration Tomogram at 40 psig_40 GPM_50\% GVF 

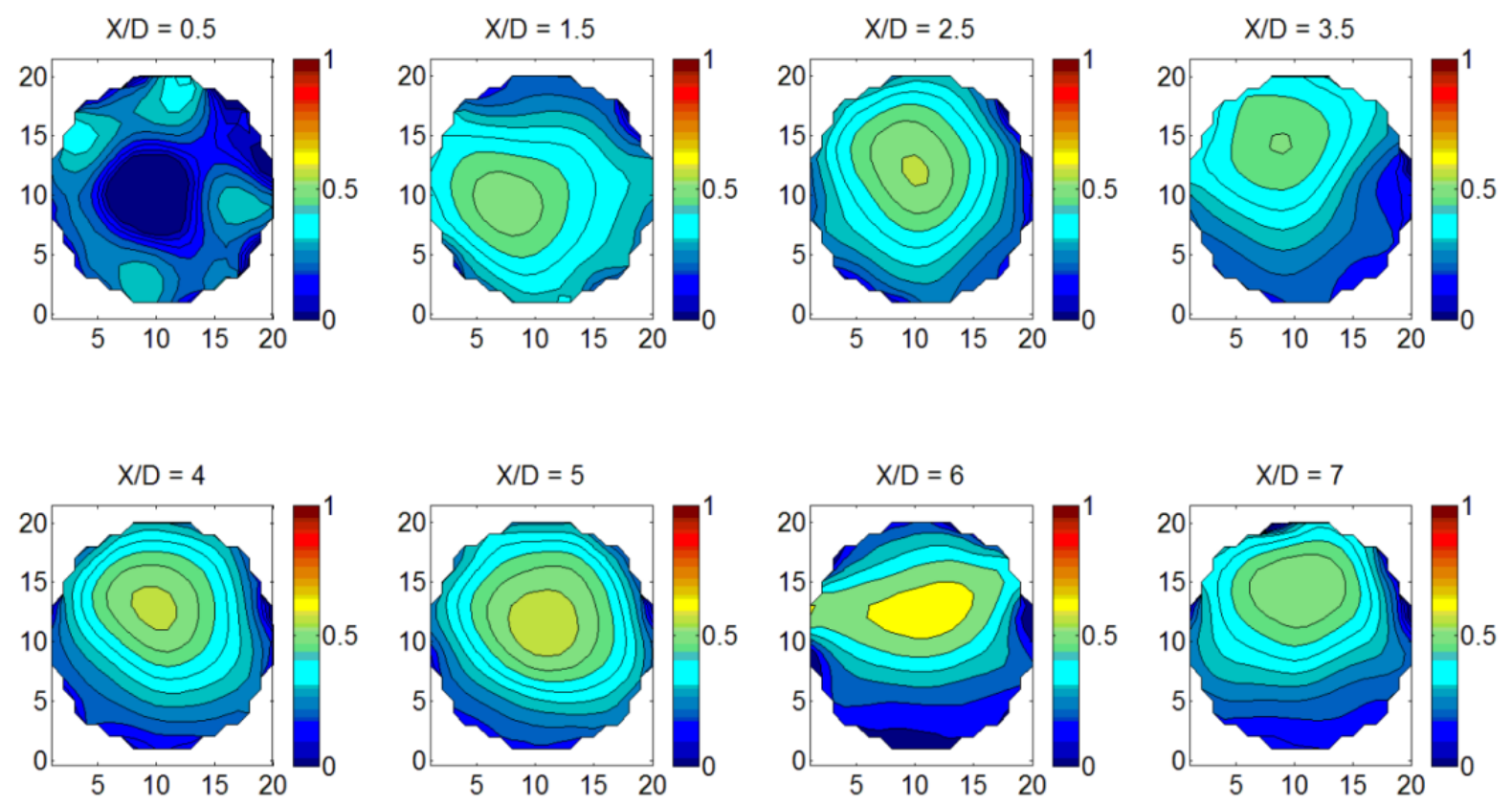

Figure 154: Concentration Tomogram at 40 psig_40 GPM_60\% GVF
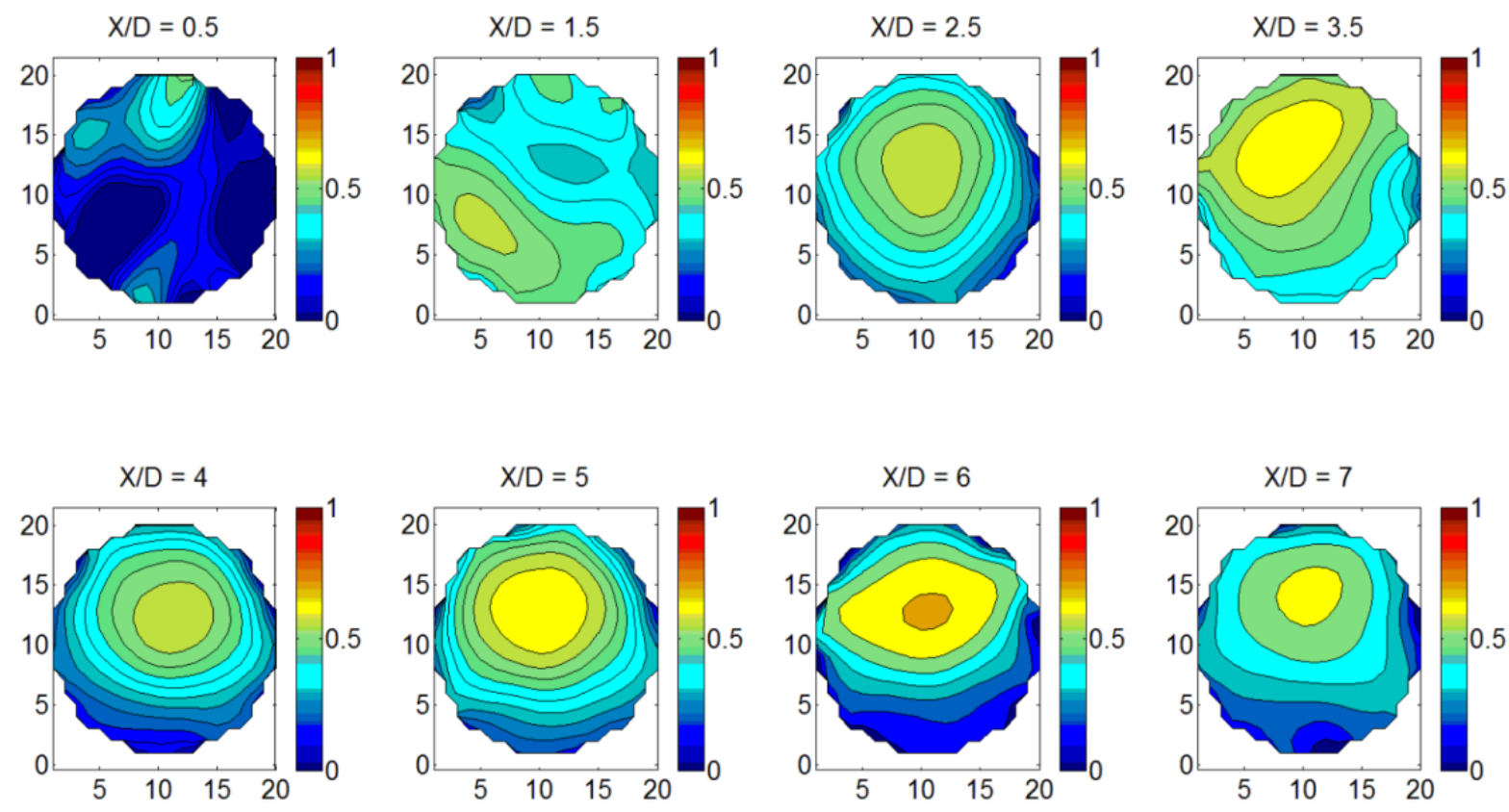

Figure 155: Concentration Tomogram at 40 psig_40 GPM_70\% GVF 

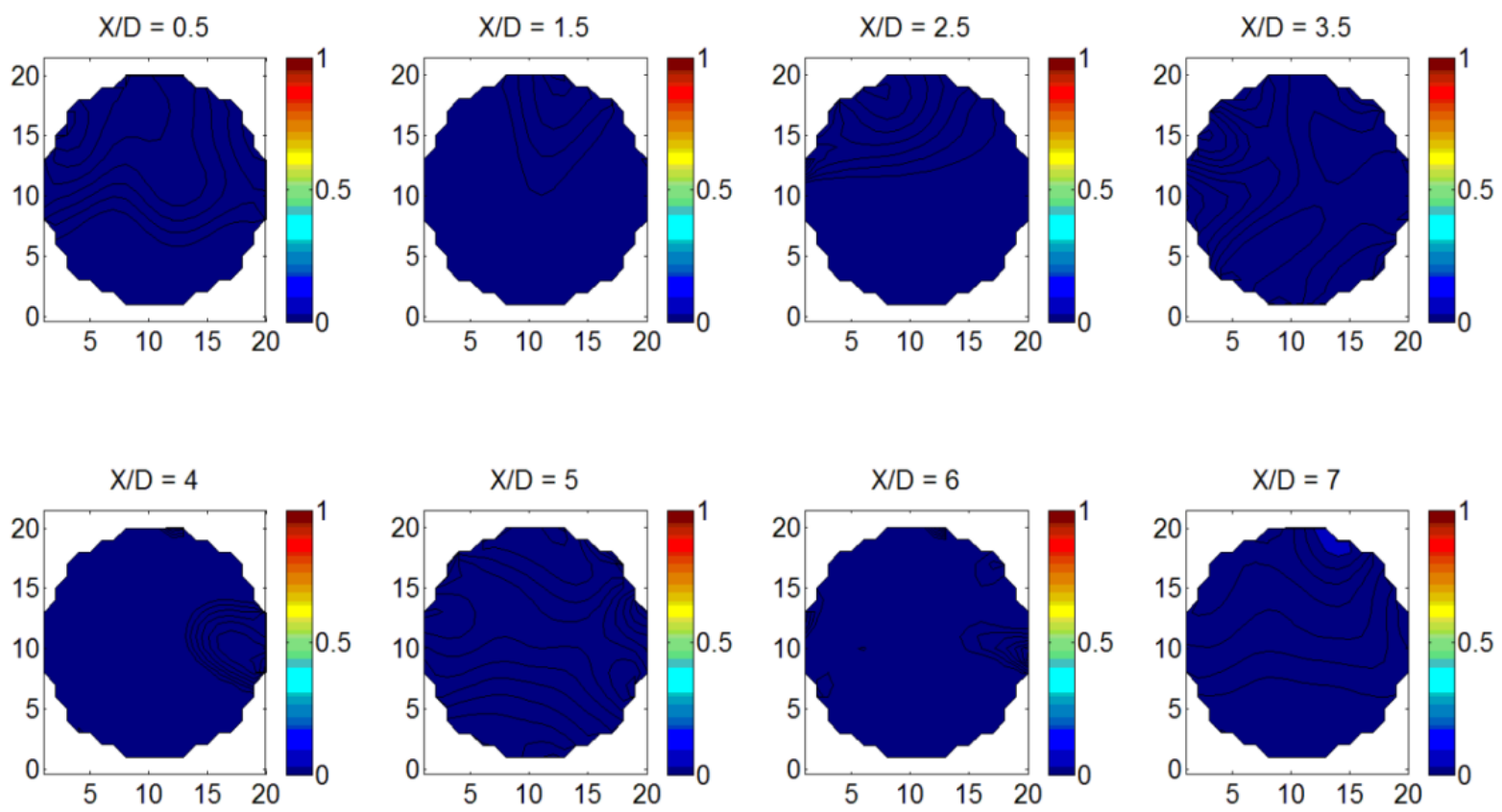

Figure 156: Concentration Tomogram at 40 psig_60 GPM_5\% GVF
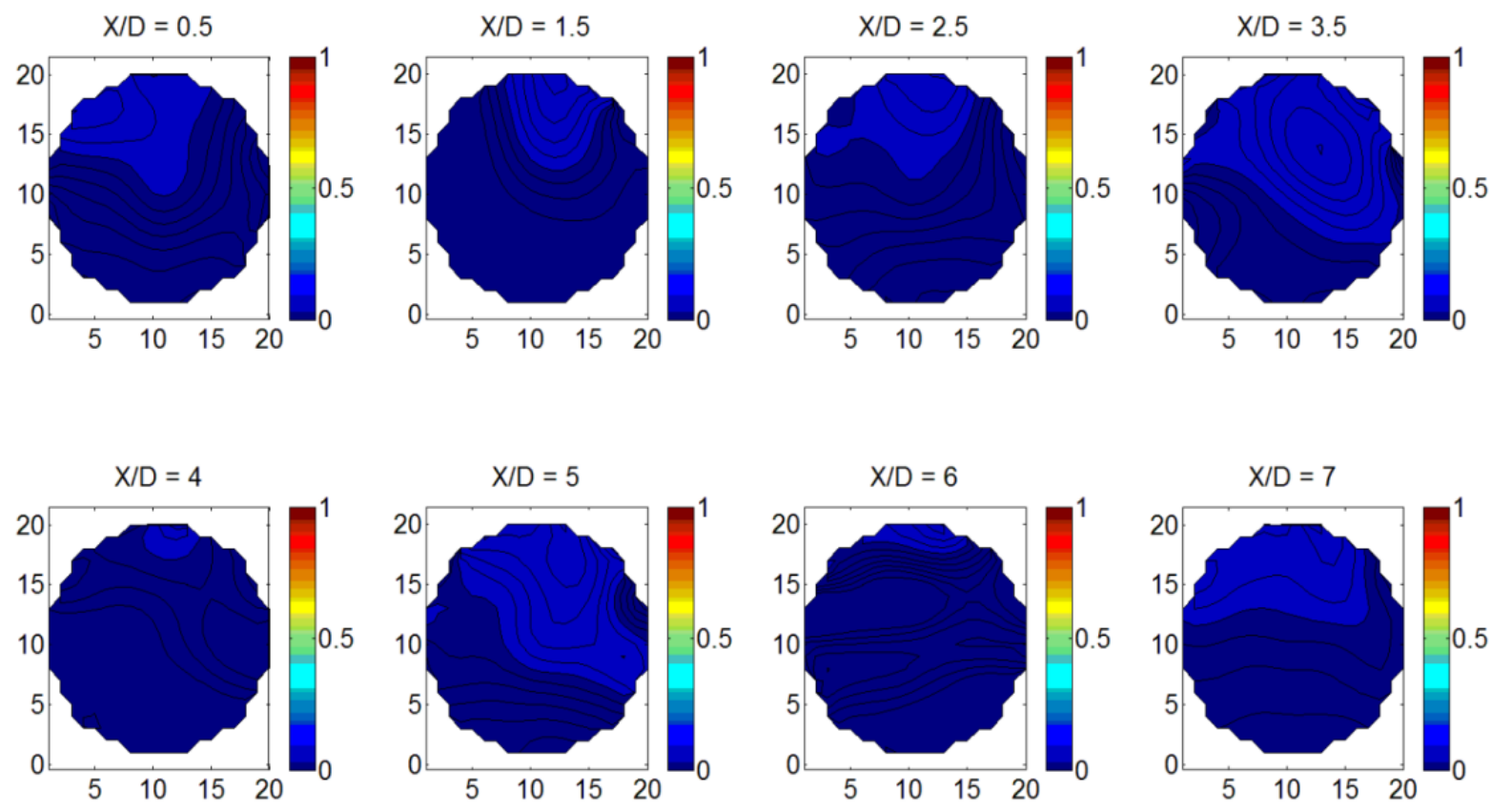

Figure 157: Concentration Tomogram at 40 psig_60 GPM_10\% GVF 

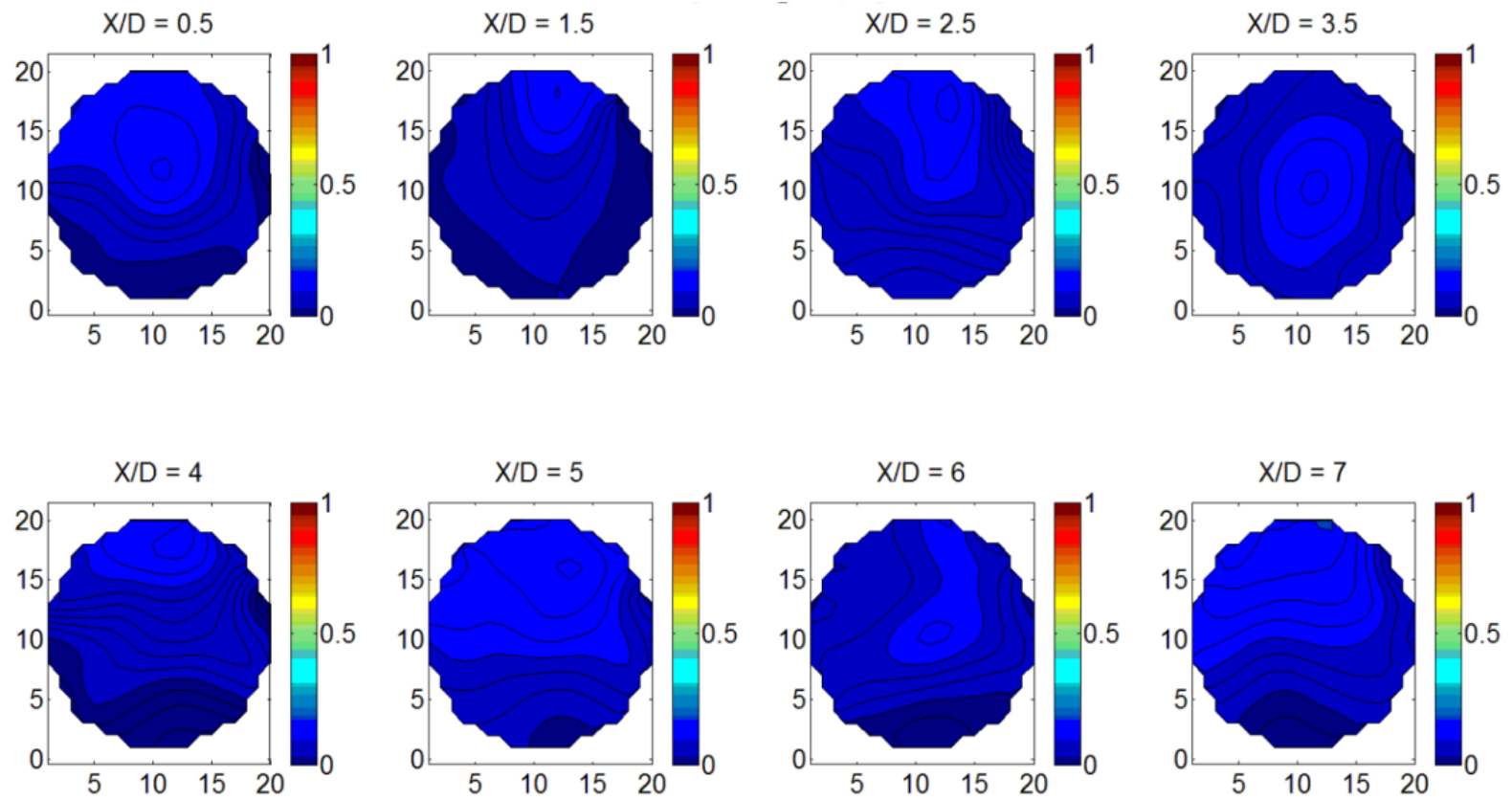

Figure 158: Concentration Tomogram at 40 psig_60 GPM_20\% GVF
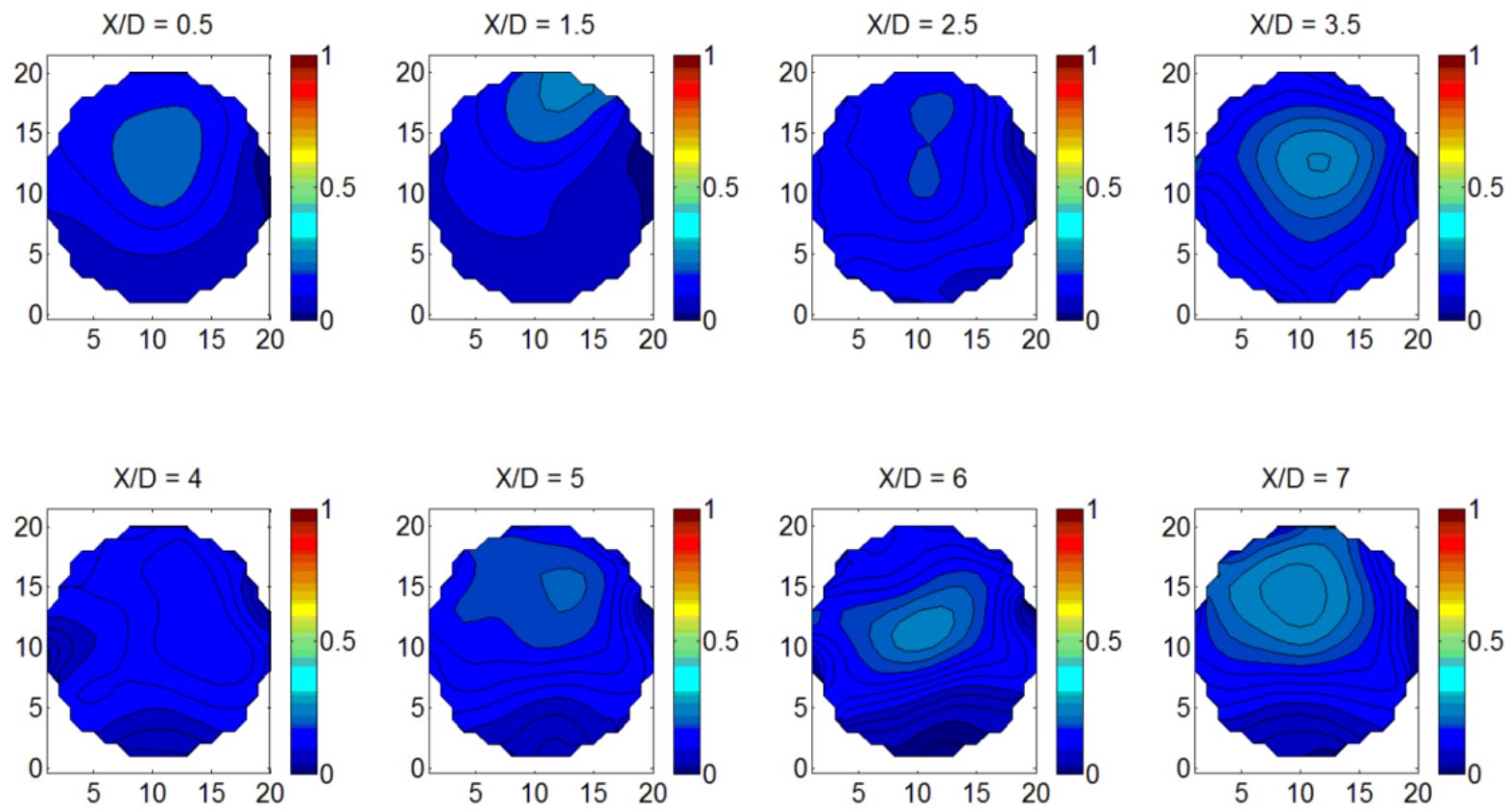

Figure 159: Concentration Tomogram at 40 psig_60 GPM_30\% GVF 

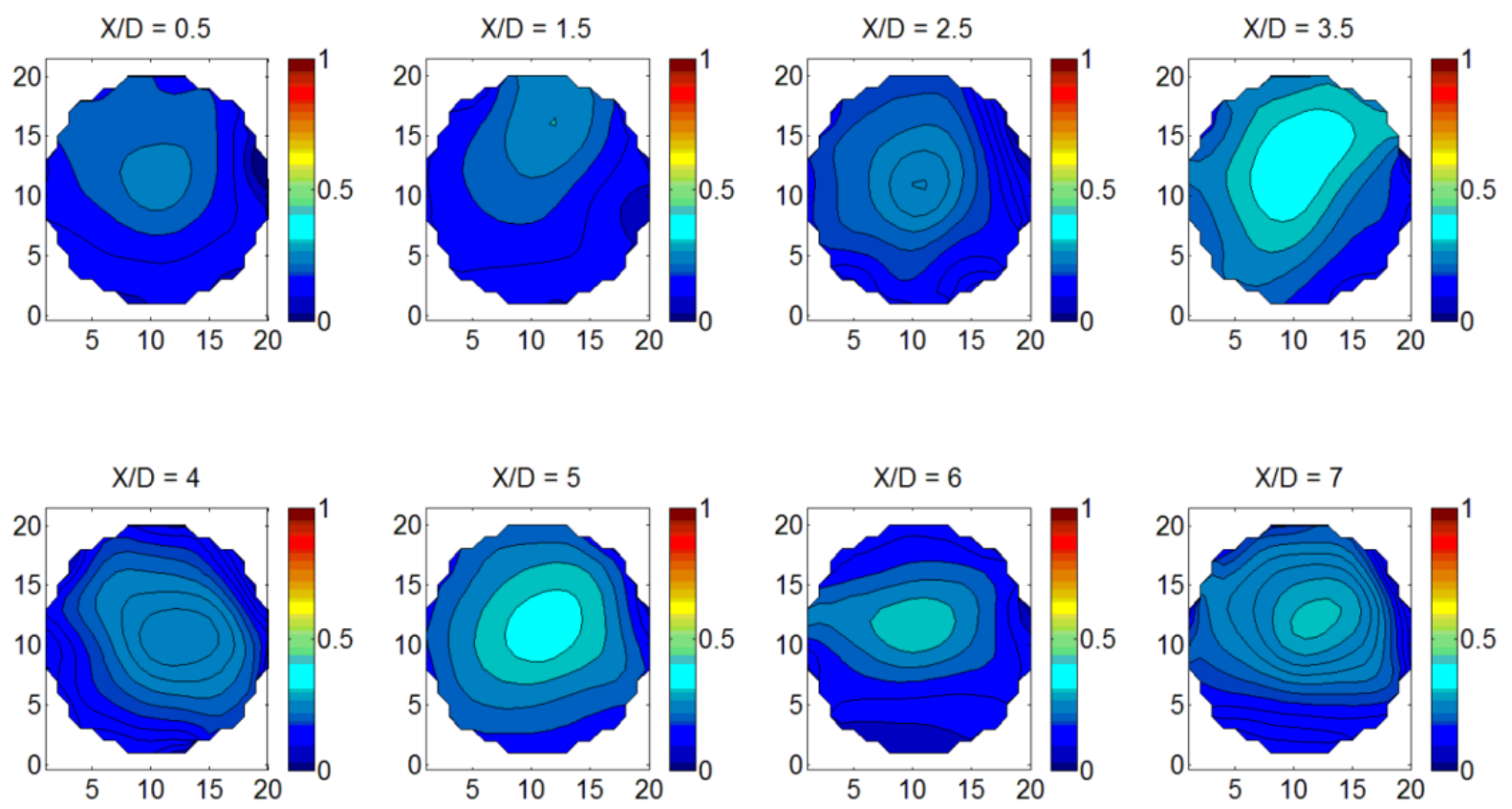

Figure 160: Concentration Tomogram at 40 psig_60 GPM_40\% GVF
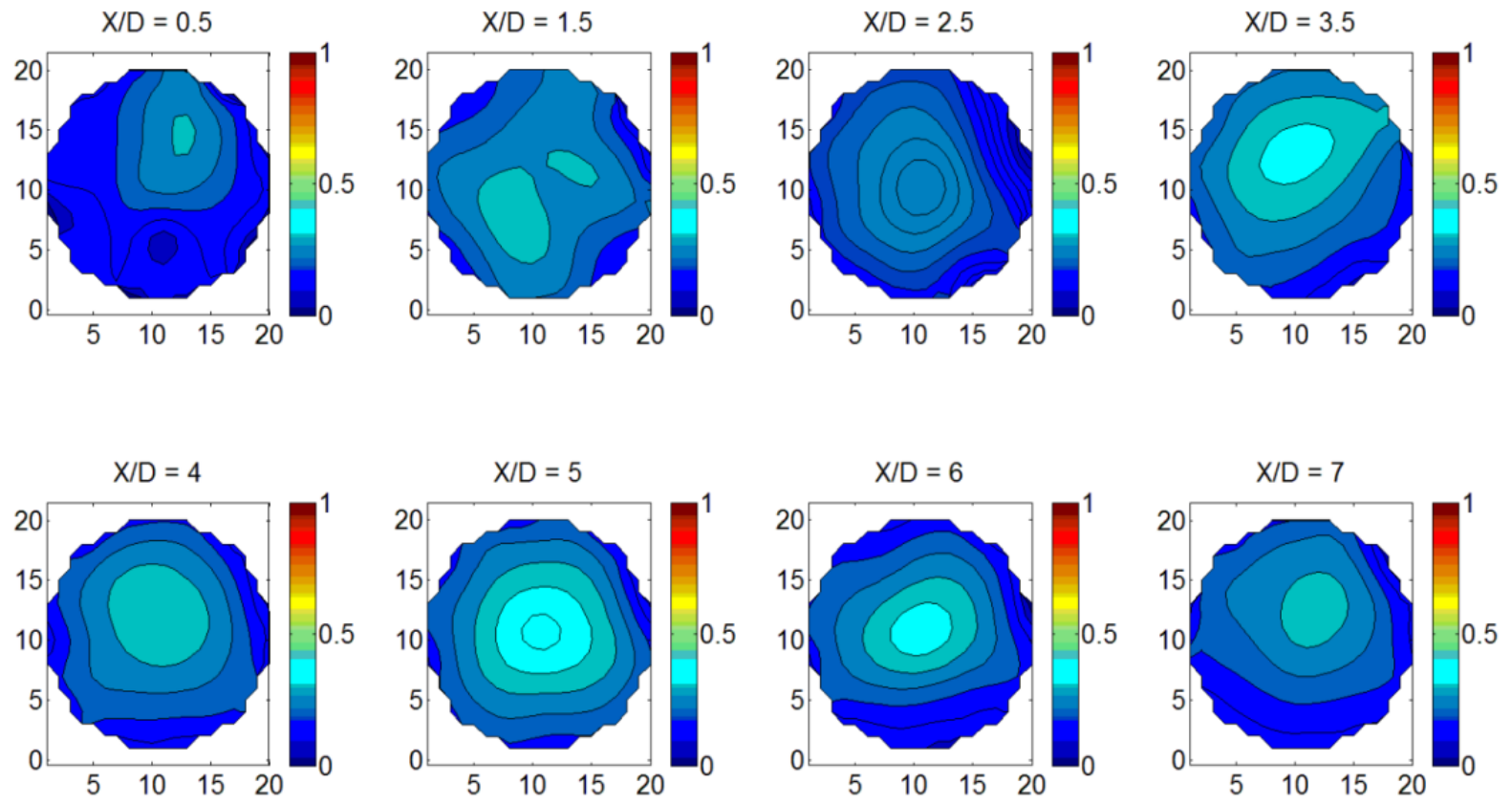

Figure 161: Concentration Tomogram at 40 psig_60 GPM_50\% GVF 

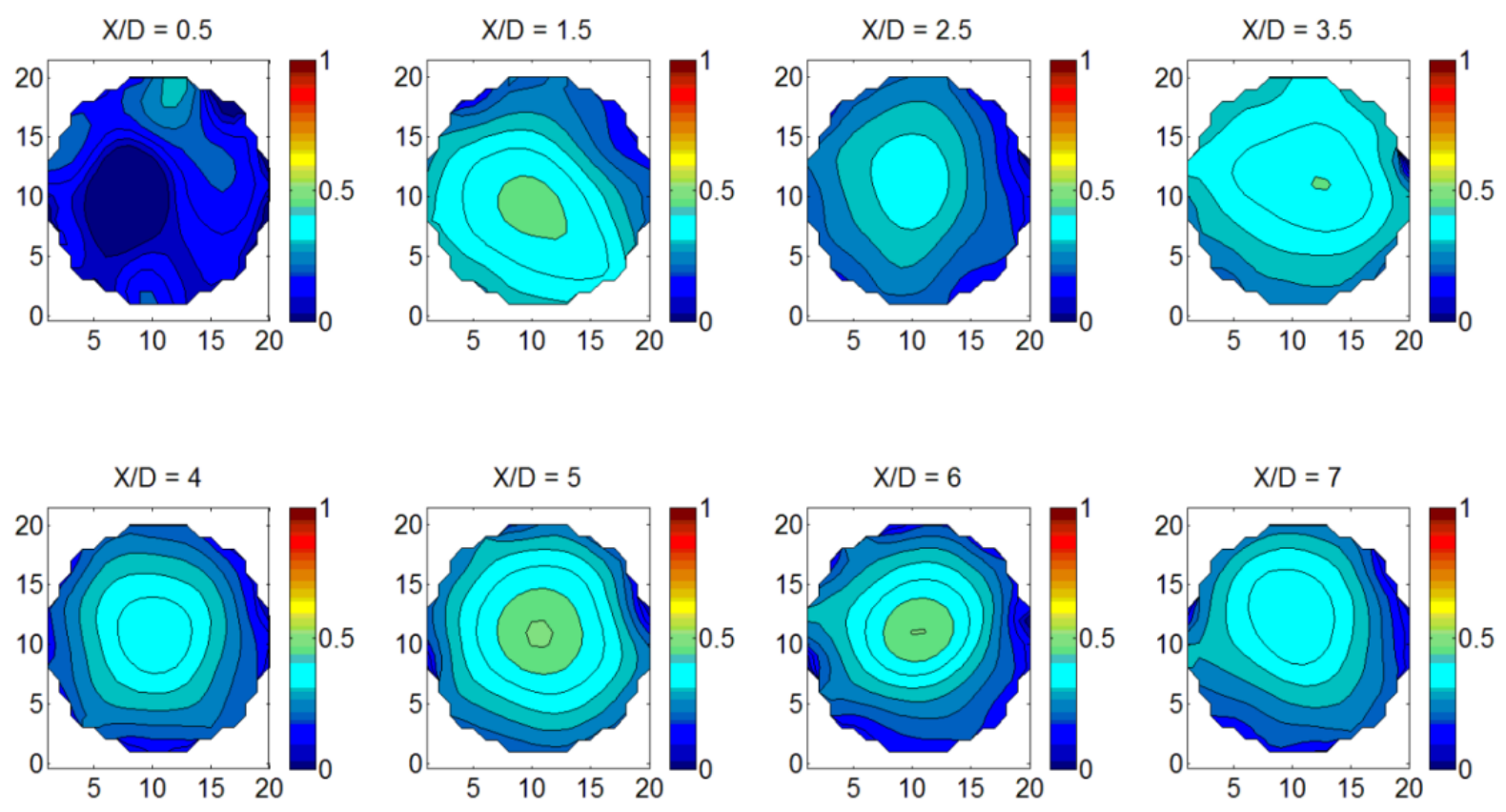

Figure 162: Concentration Tomogram at 40 psig_60 GPM_60\% GVF
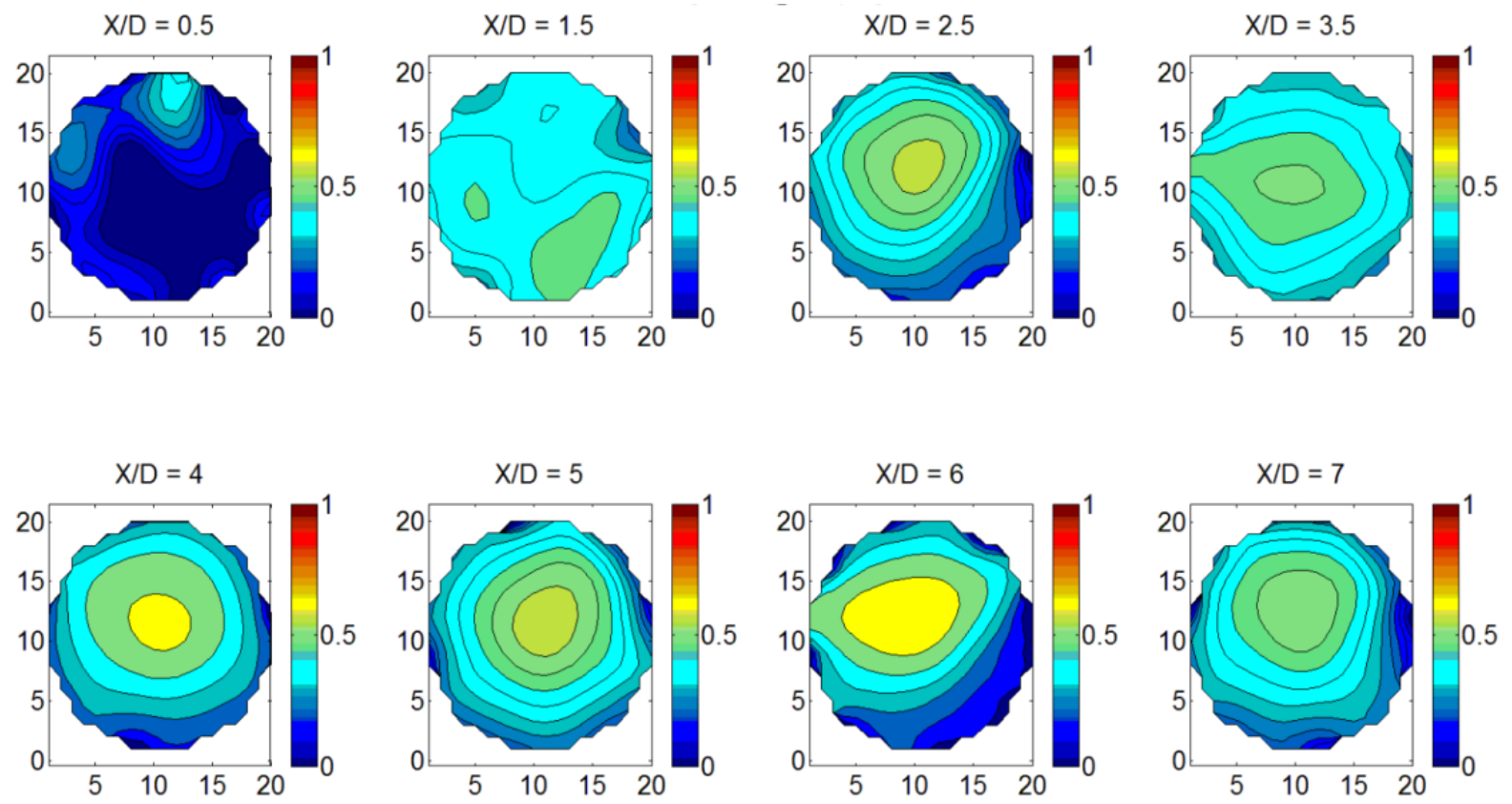

Figure 163: Concentration Tomogram at 40 psig_60 GPM_70\% GVF 

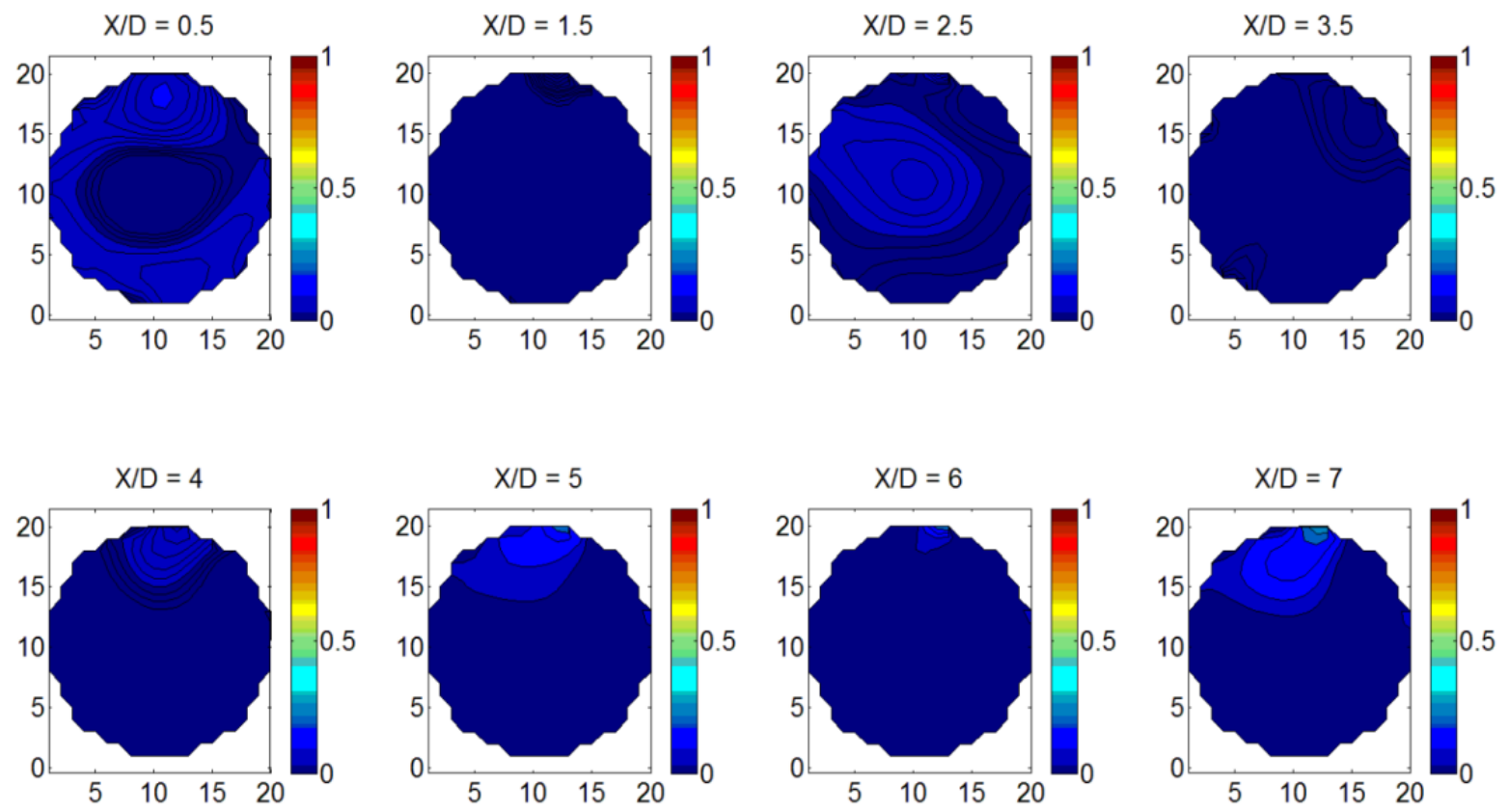

Figure 164: Concentration Tomogram at 60 psig_20 GPM_5\% GVF
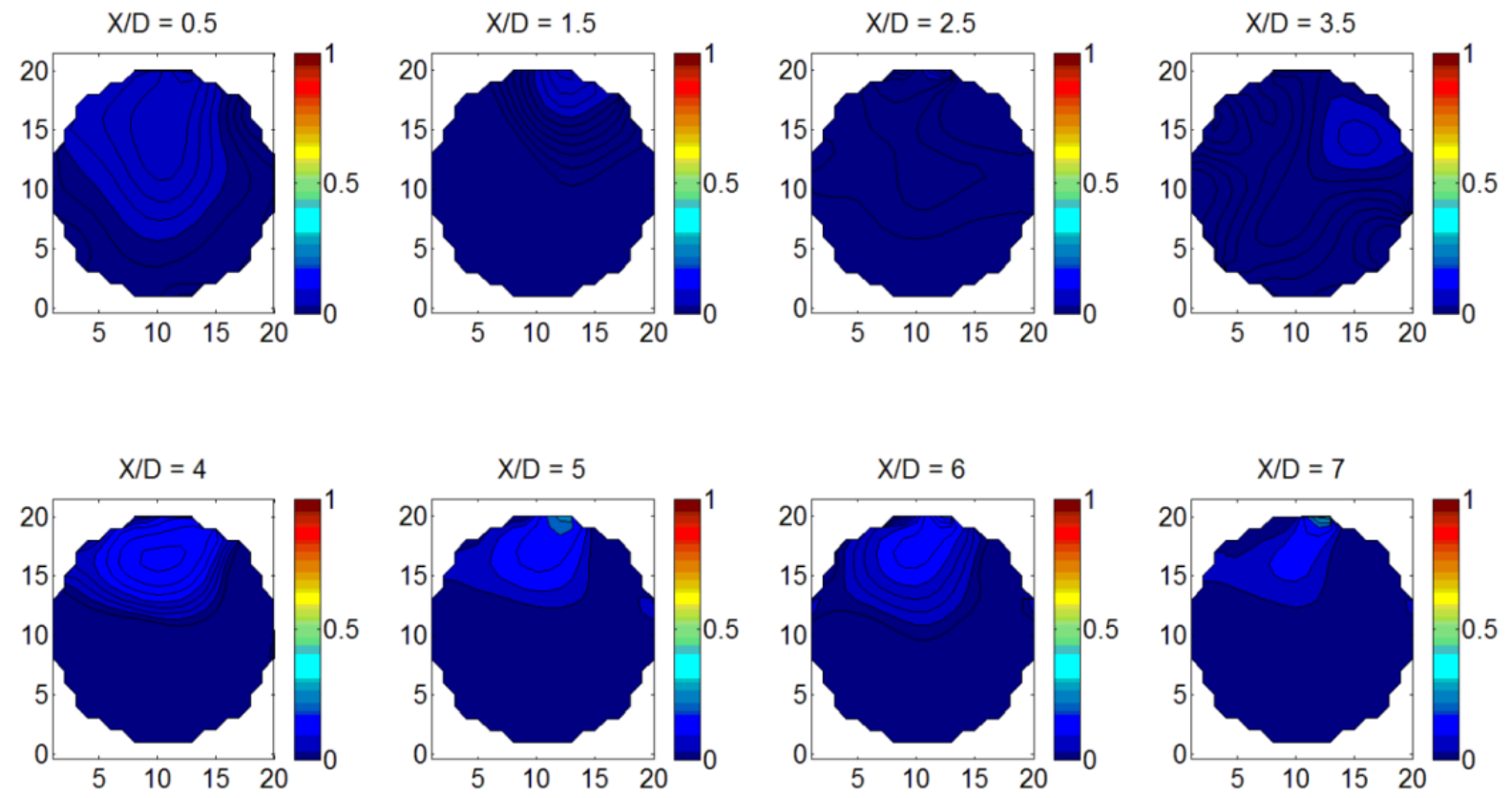

Figure 165: Concentration Tomogram at 60 psig_20 GPM_10\% GVF 

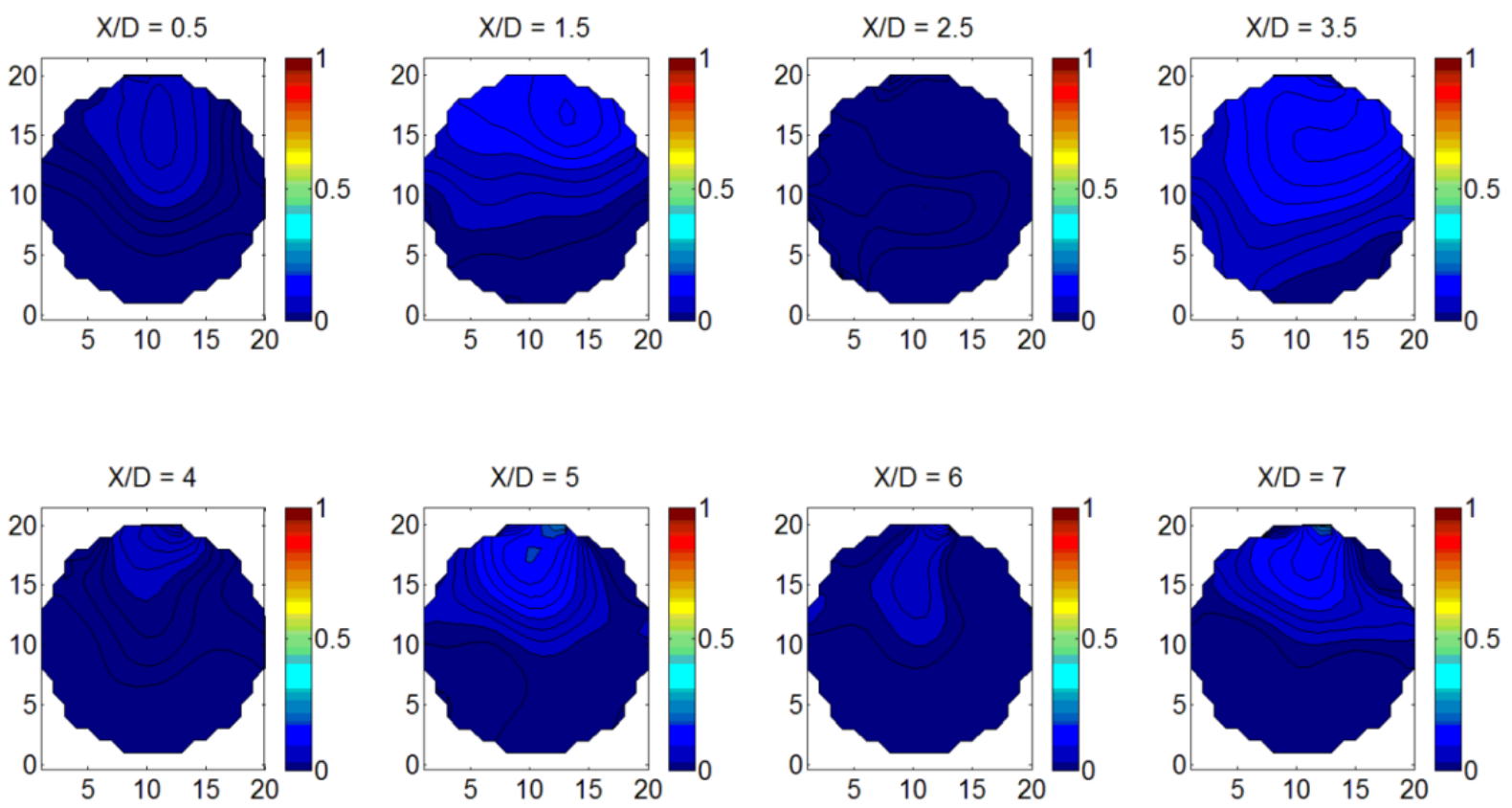

Figure 166: Concentration Tomogram at 60 psig_20 GPM_20\% GVF
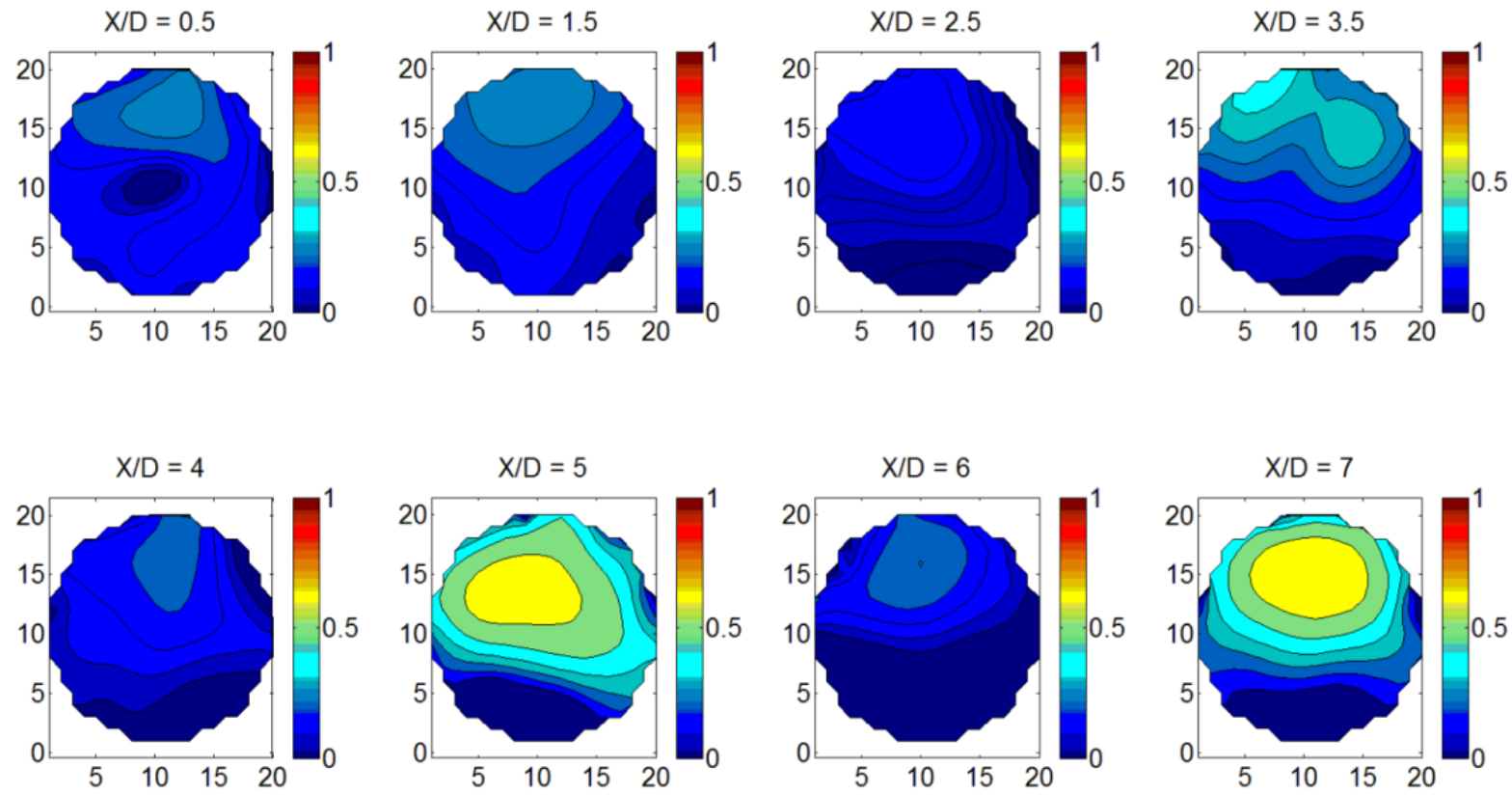

Figure 167: Concentration Tomogram at 60 psig_20 GPM_30\% GVF 

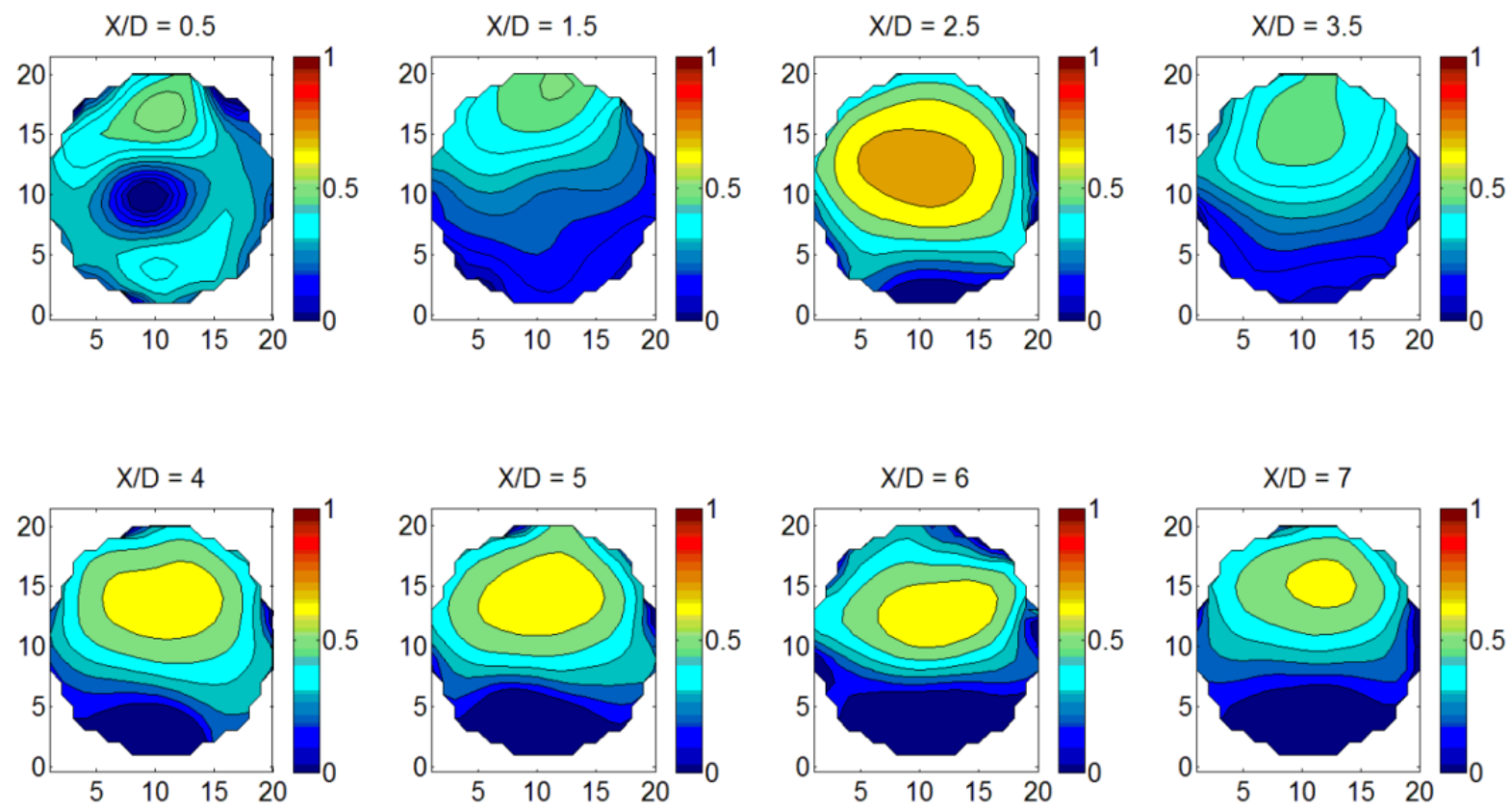

Figure 168: Concentration Tomogram at 60 psig_20 GPM_40\% GVF
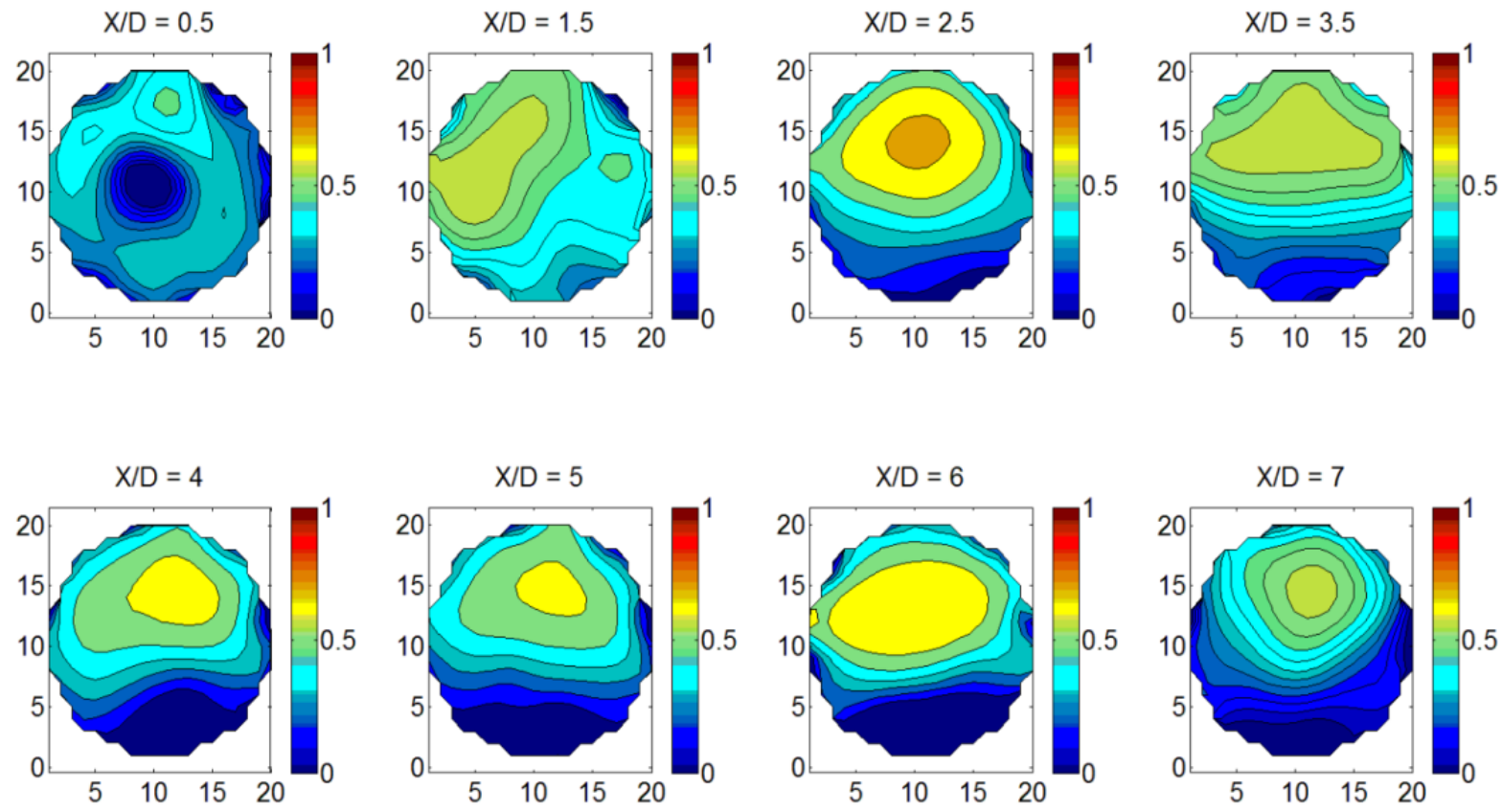

Figure 169: Concentration Tomogram at 60 psig_20 GPM_50\% GVF 

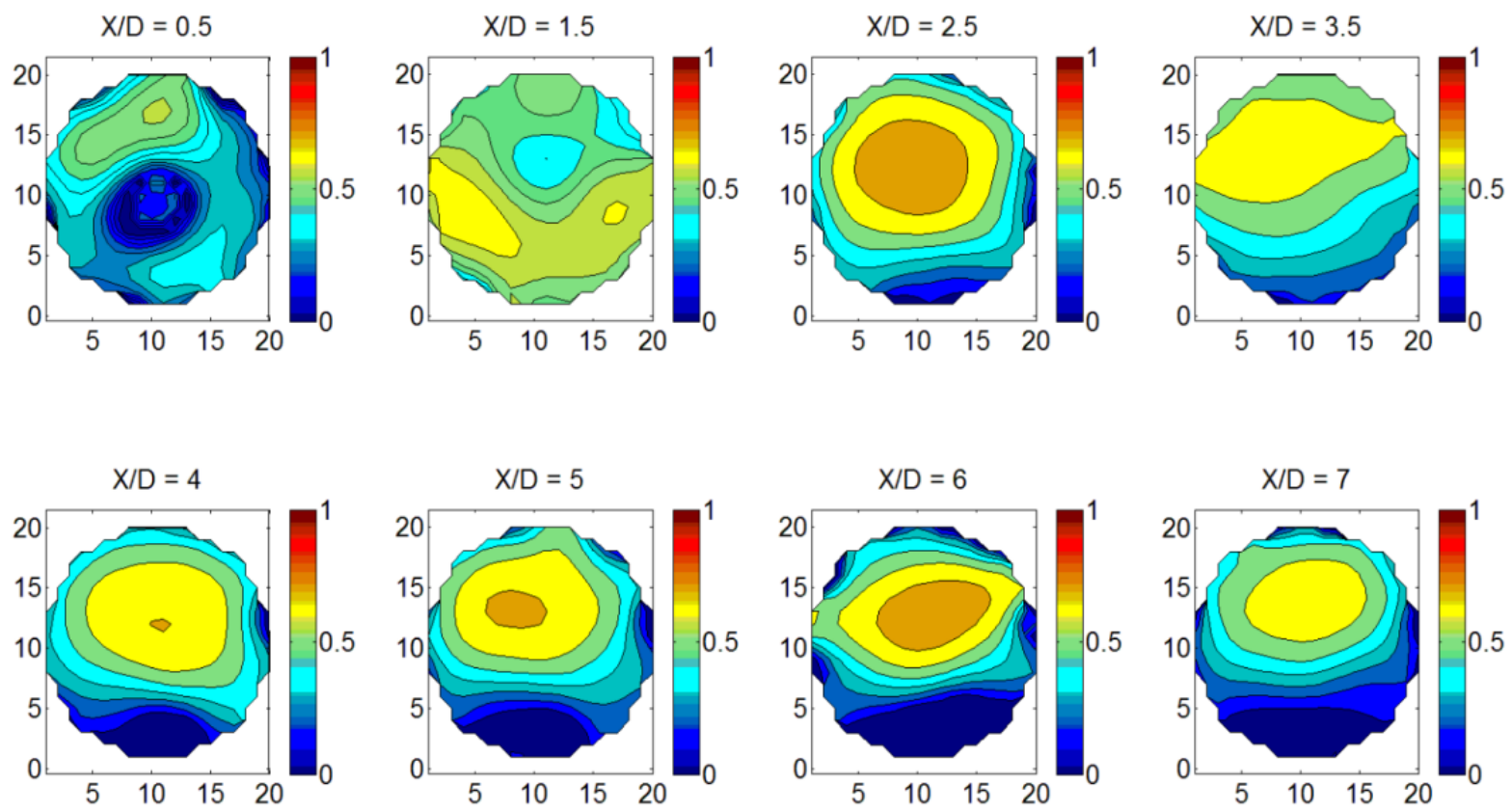

Figure 170: Concentration Tomogram at 60 psig_20 GPM_60\% GVF
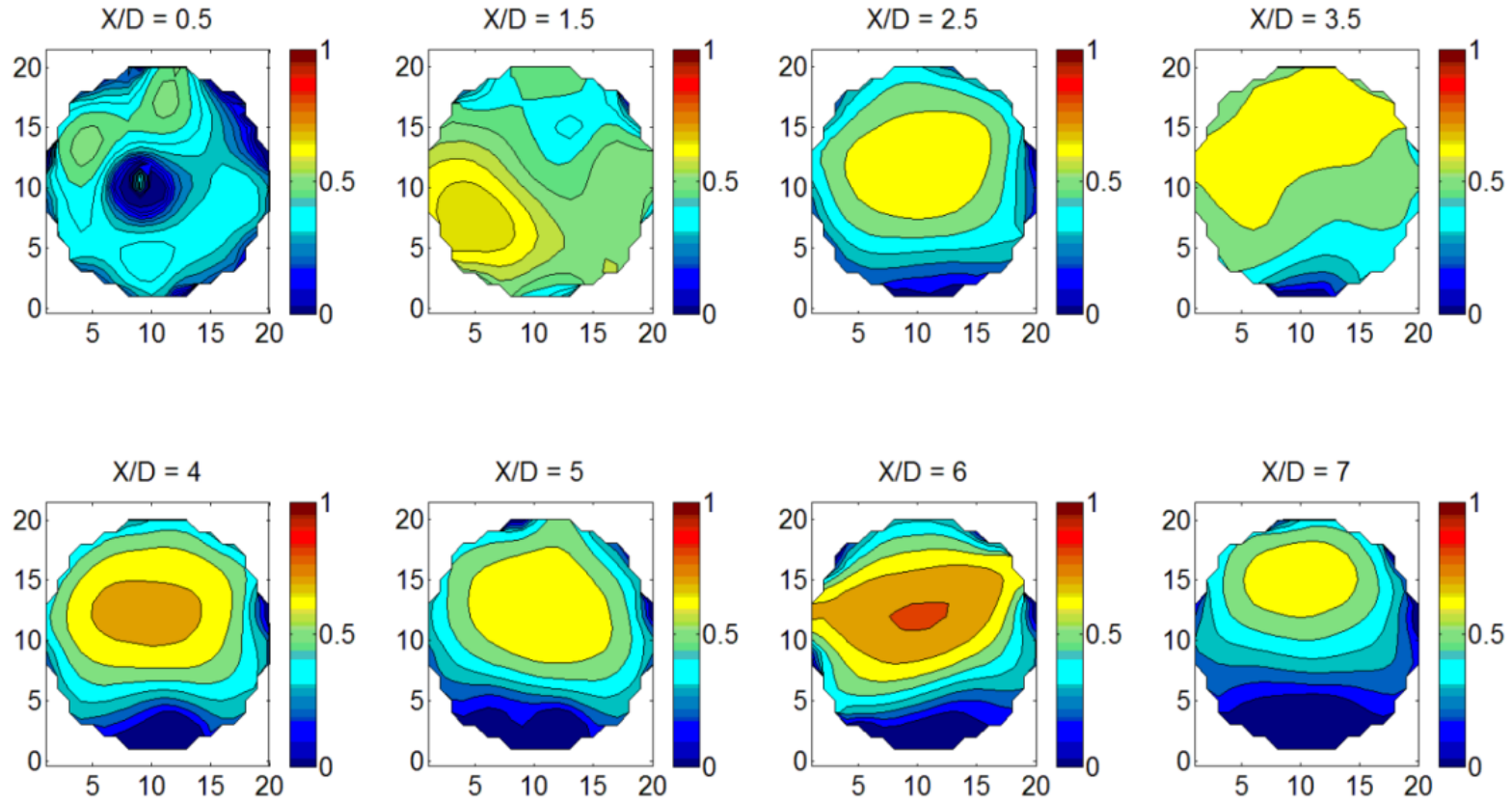

Figure 171: Concentration Tomogram at 60 psig_20 GPM_70\% GVF 

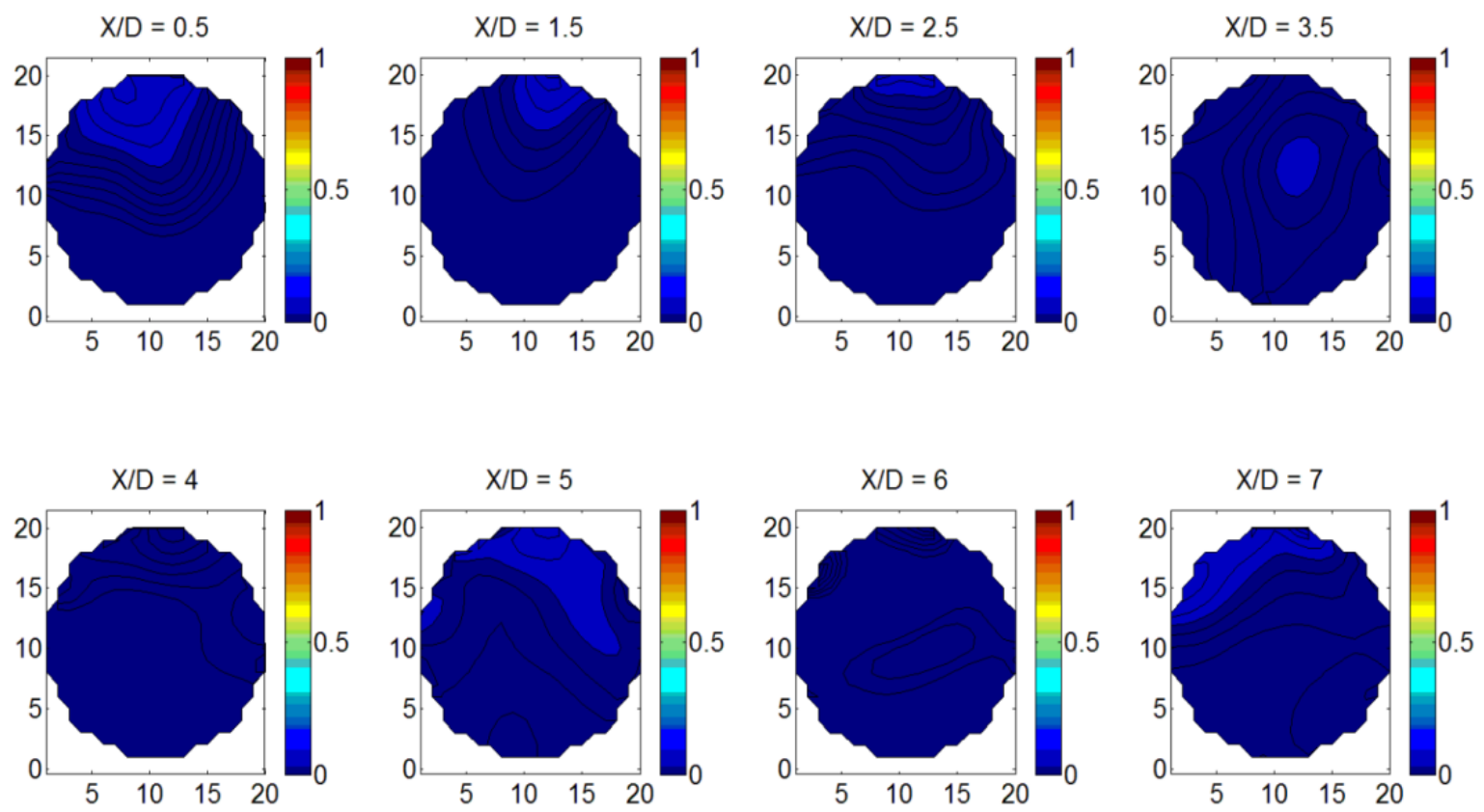

Figure 172: Concentration Tomogram at 60 psig_40 GPM_5\% GVF
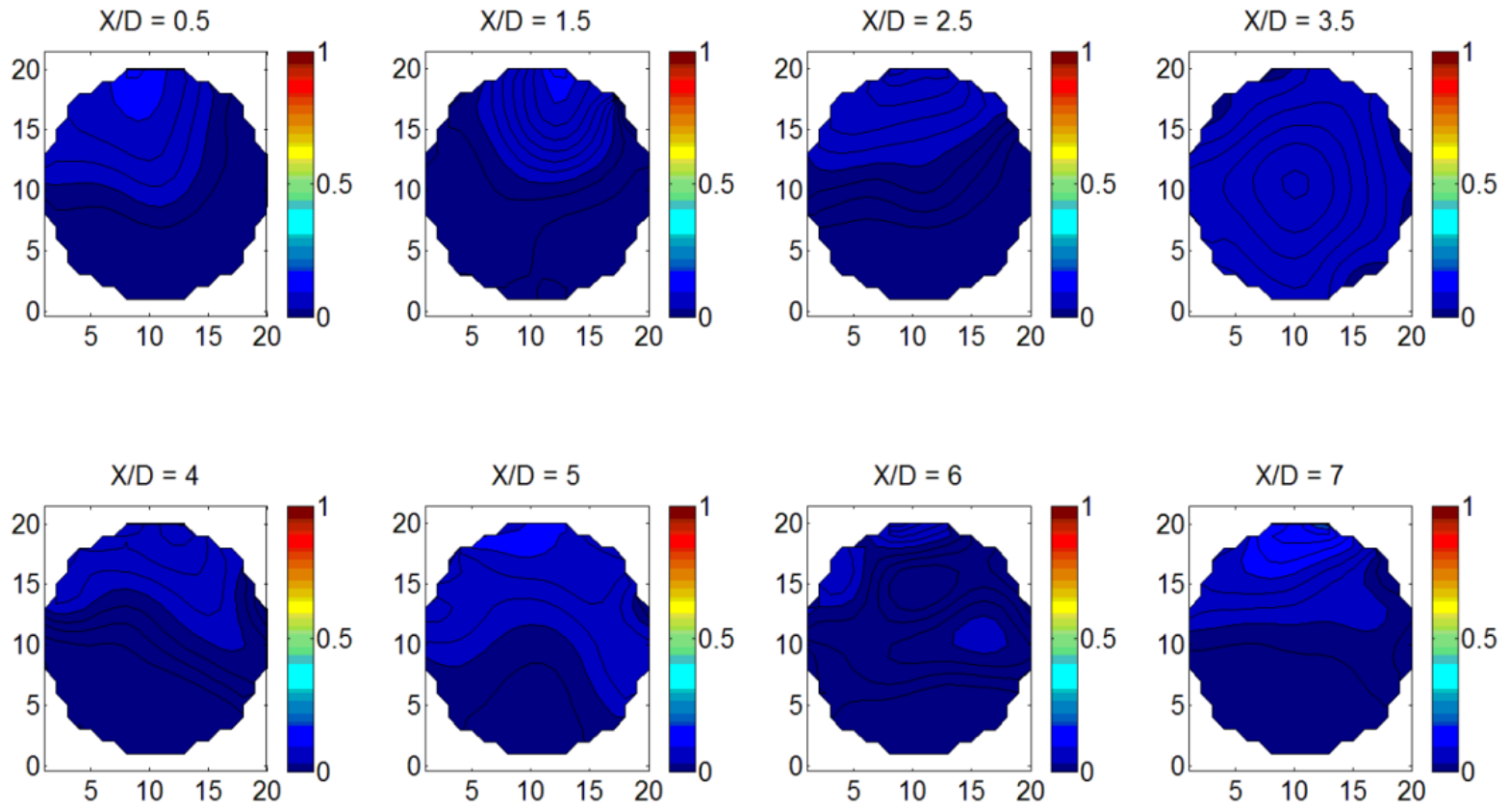

Figure 173: Concentration Tomogram at 60 psig_40 GPM_10\% GVF 

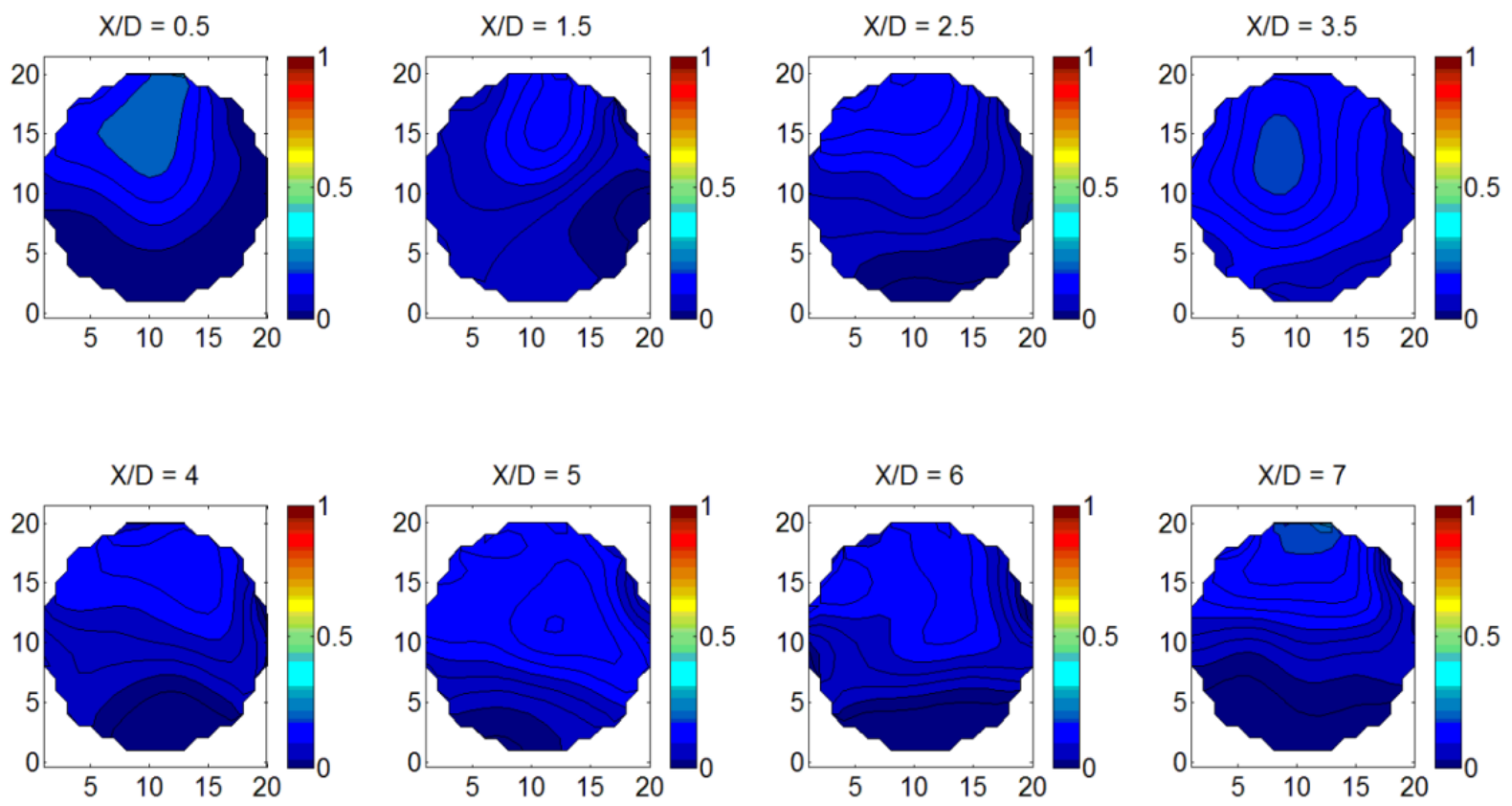

Figure 174: Concentration Tomogram at 60 psig_40 GPM_20\% GVF
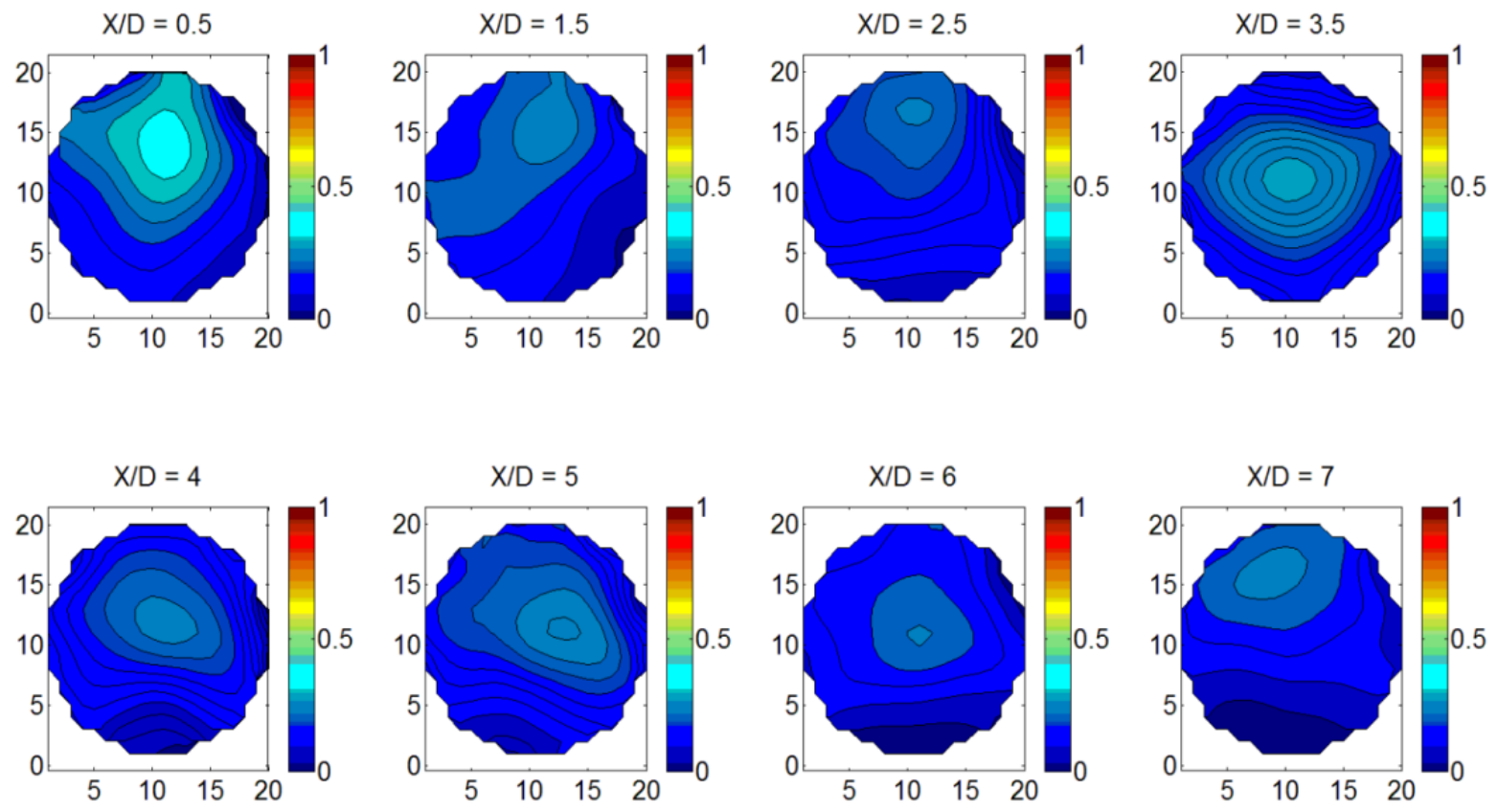

Figure 175: Concentration Tomogram at 60 psig_40 GPM_30\% GVF 

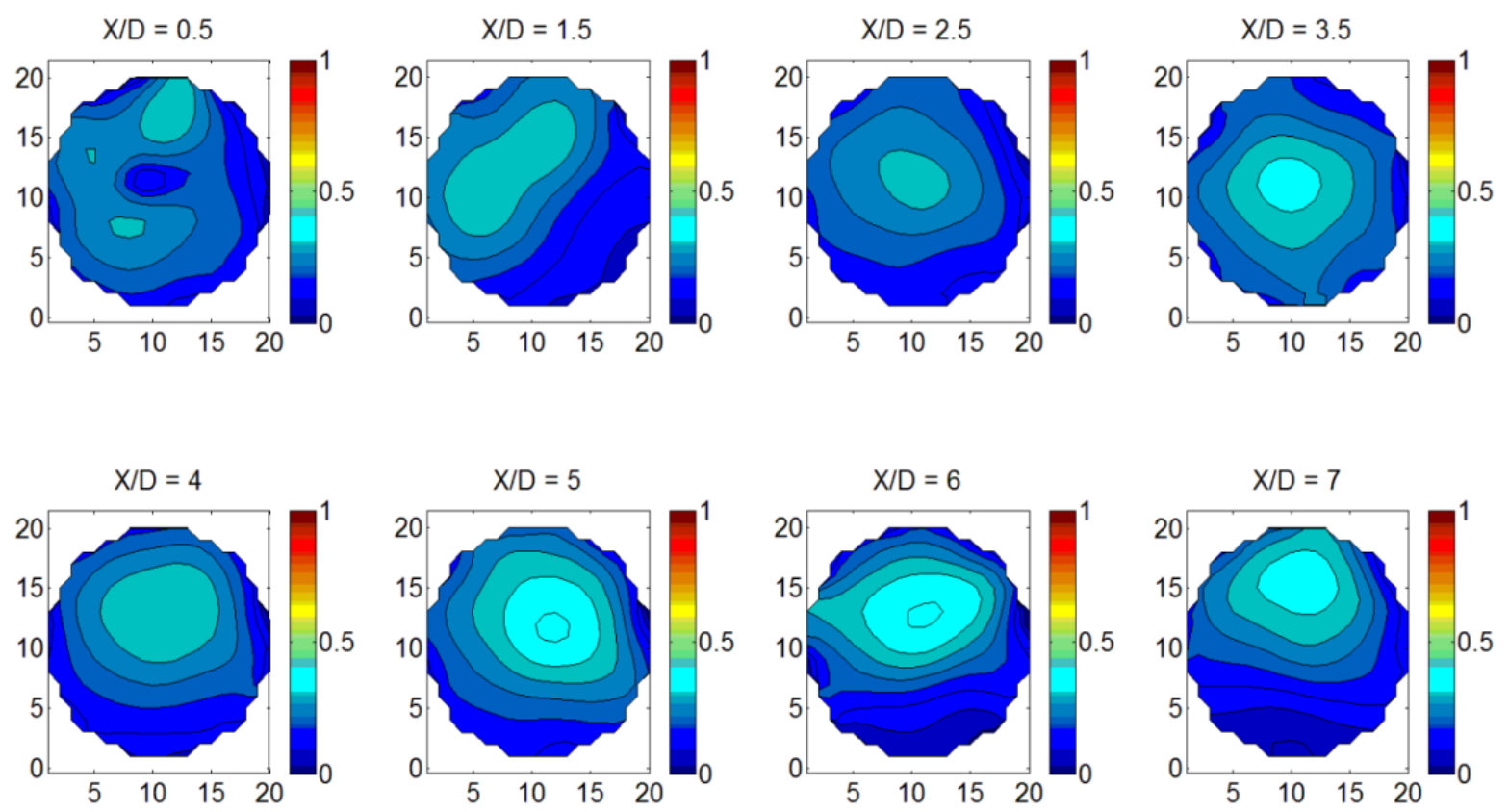

Figure 176: Concentration Tomogram at 60 psig_40 GPM_40\% GVF
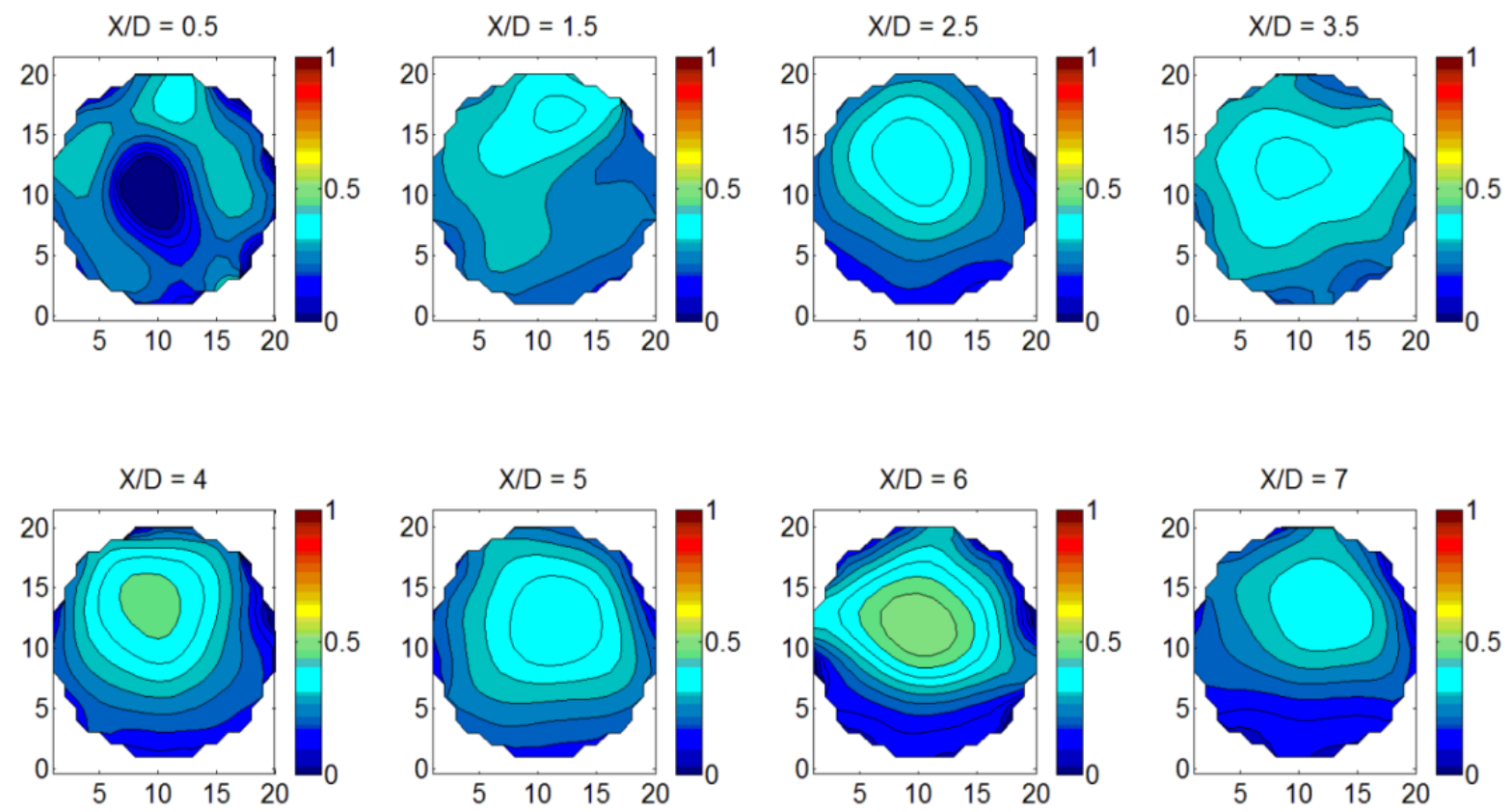

Figure 177: Concentration Tomogram at 60 psig_40 GPM_50\% GVF 

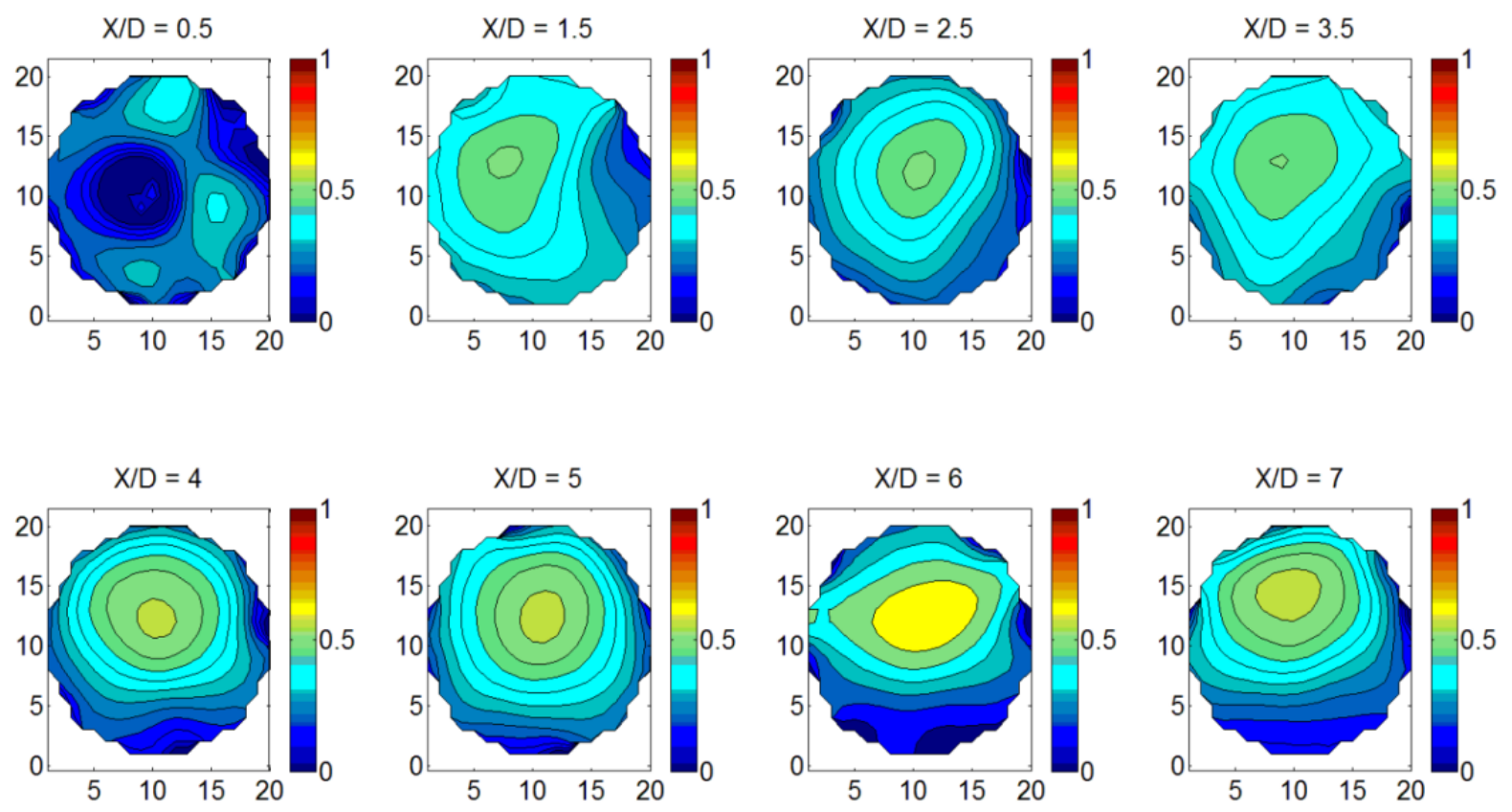

Figure 178: Concentration Tomogram at 60 psig_40 GPM_60\% GVF
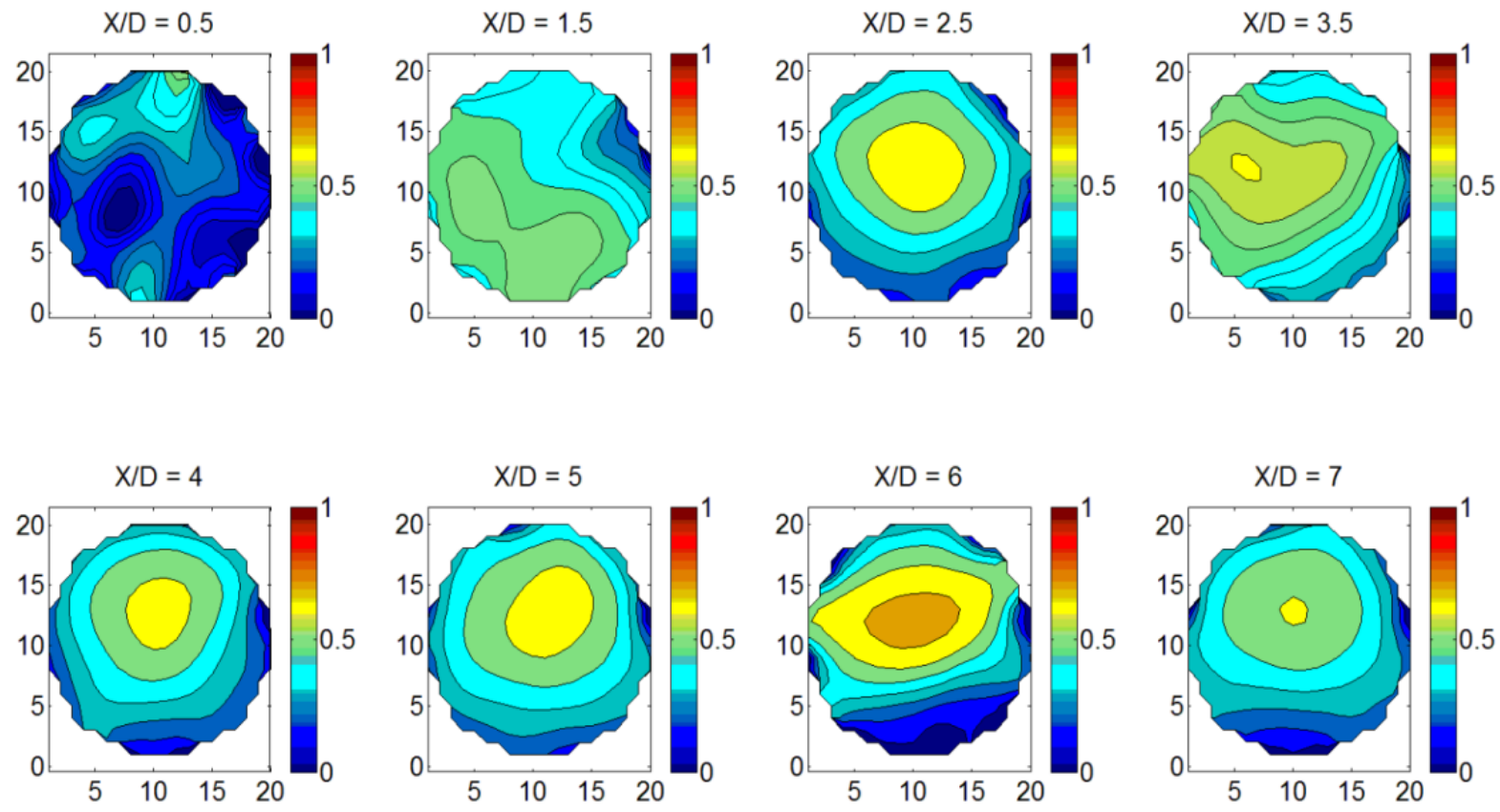

Figure 179: Concentration Tomogram at 60 psig_40 GPM_70\% GVF 

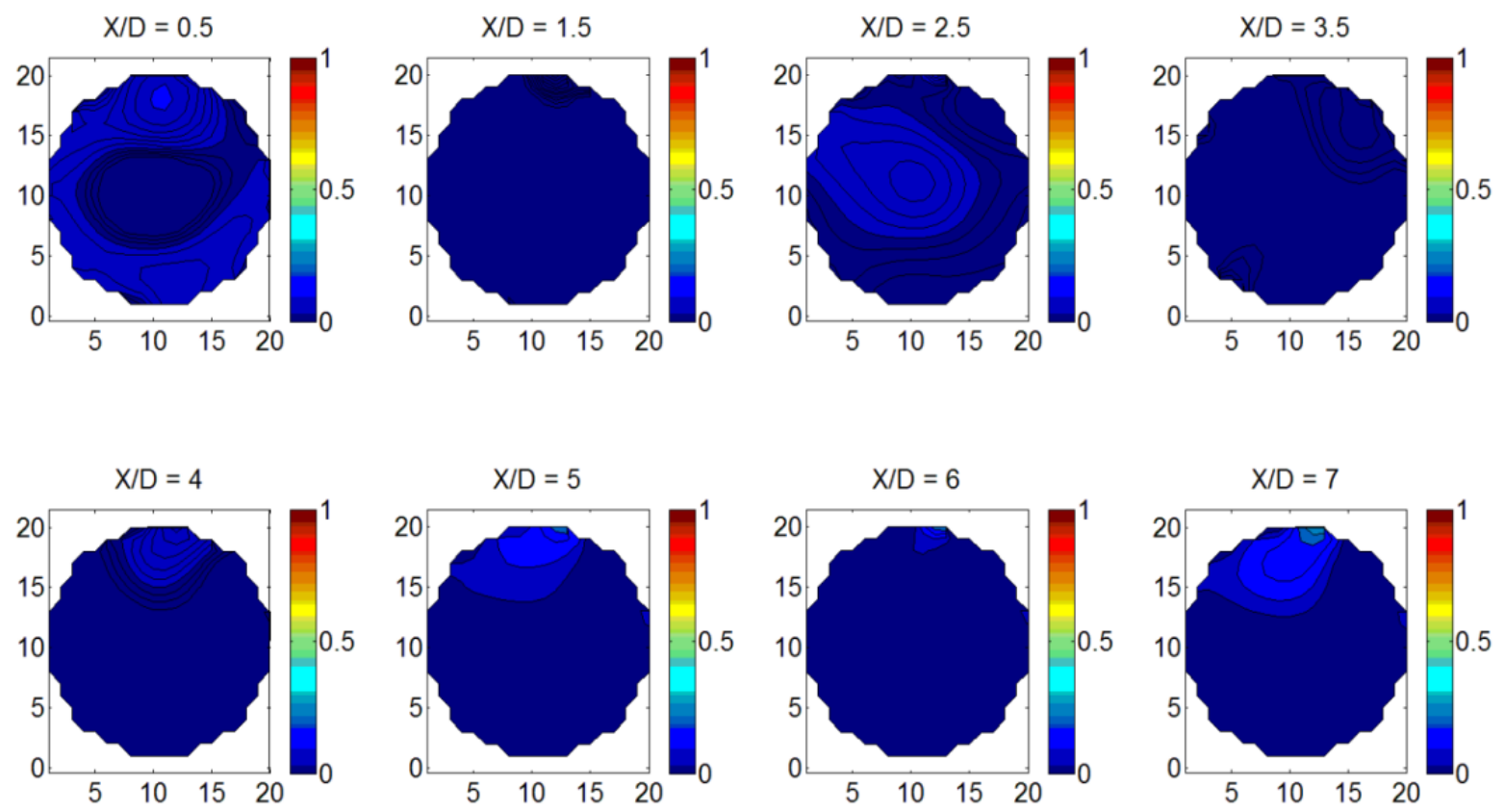

Figure 180: Concentration Tomogram at 60 psig_40GPM_5\%GVF
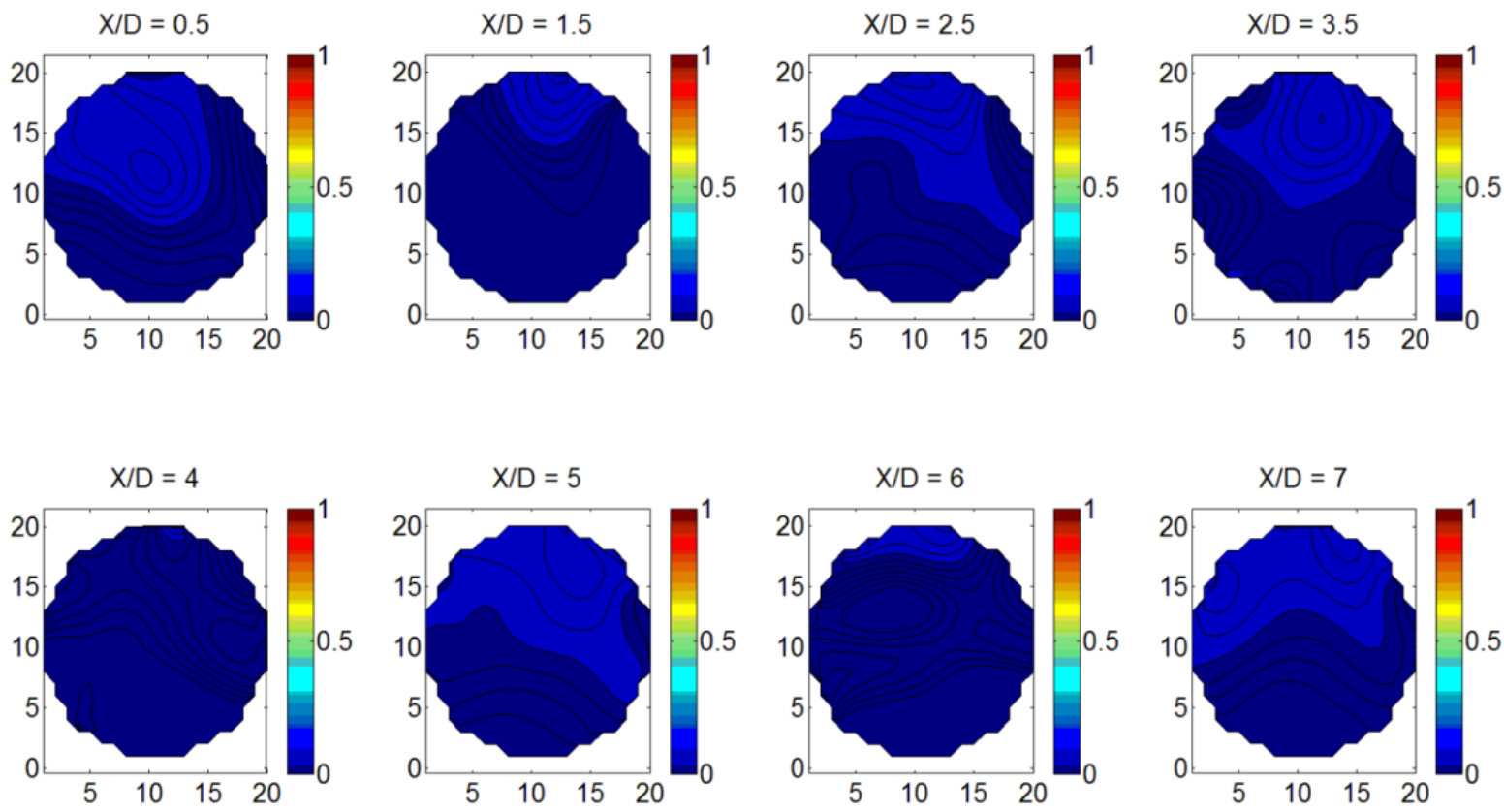

Figure 181: Concentration Tomogram at 60 psig_60 GPM_10\% GVF 

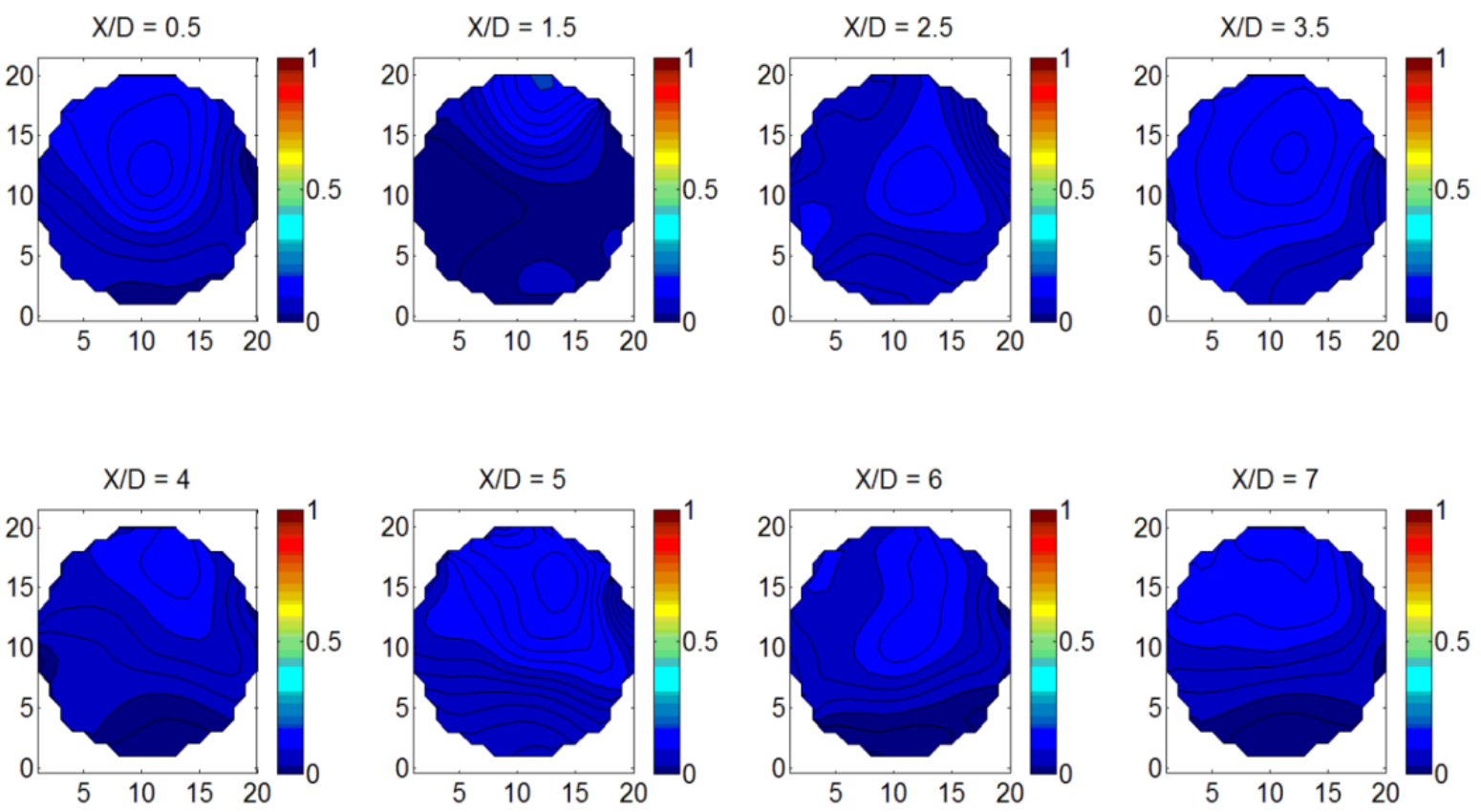

Figure 182: Concentration Tomogram at 60 psig_60 GPM_20\% GVF
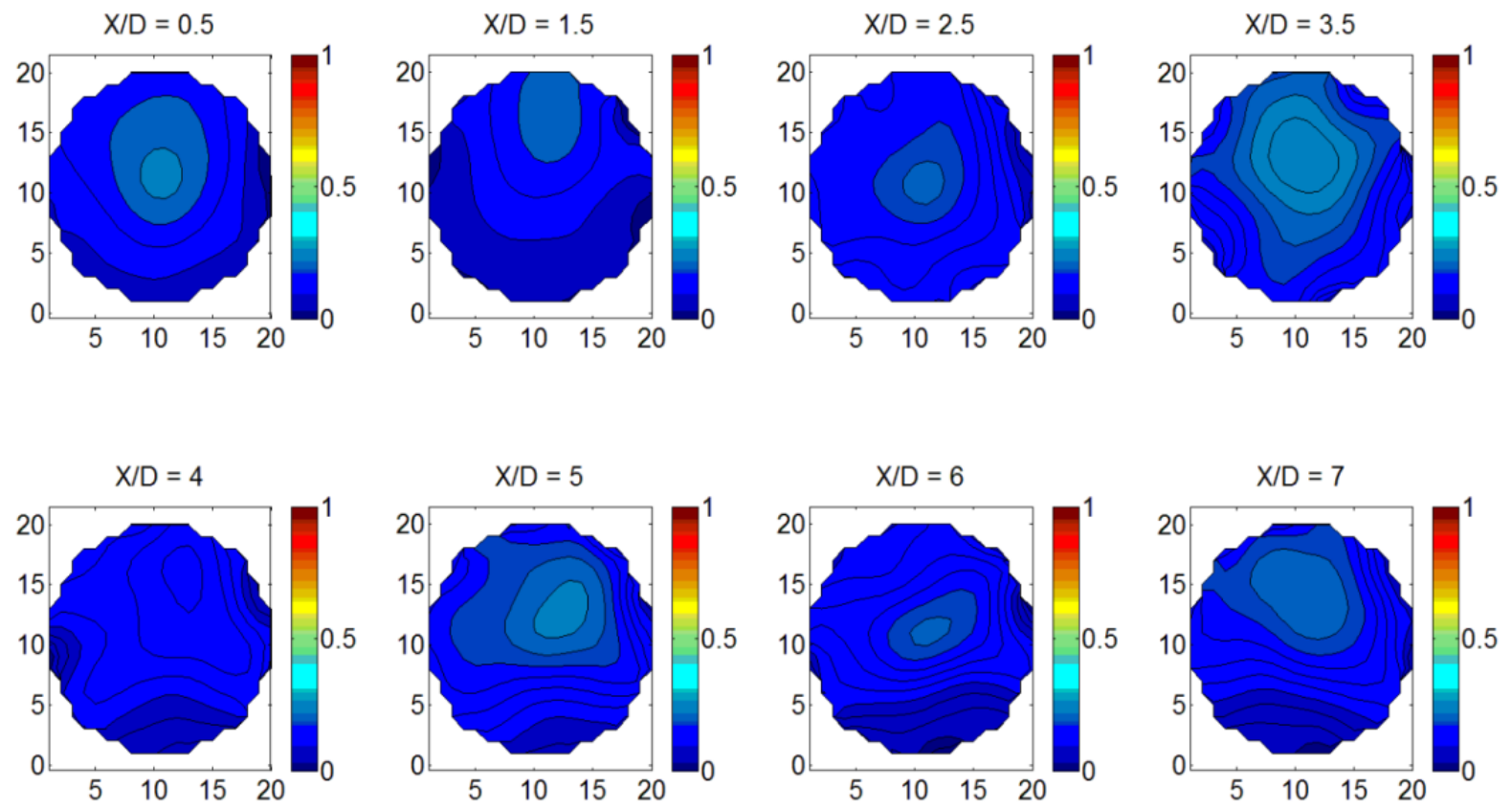

Figure 183: Concentration Tomogram at 60 psig_60 GPM_30\% GVF 

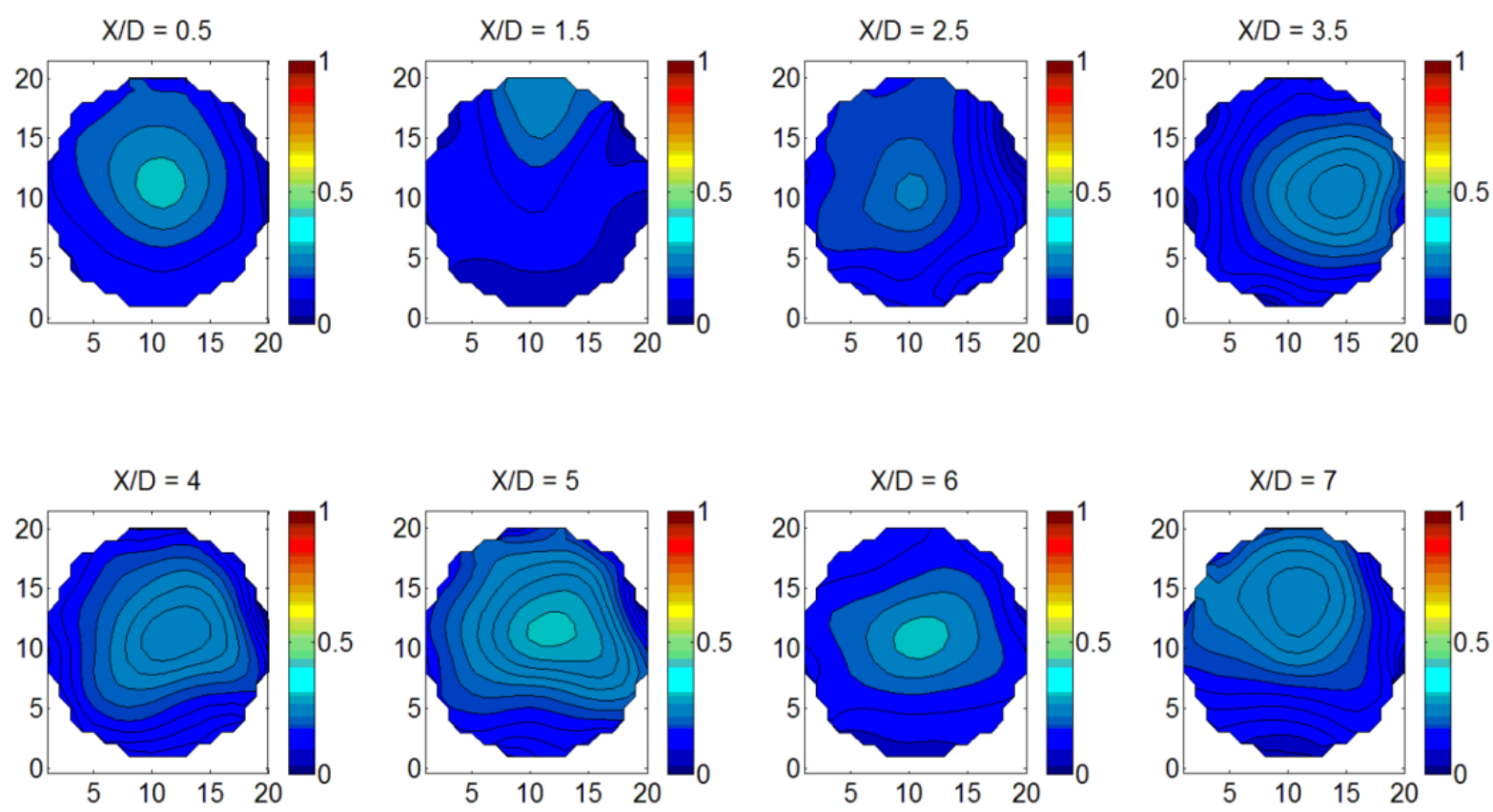

Figure 184: Concentration Tomogram at 60 psig_60 GPM_40\% GVF
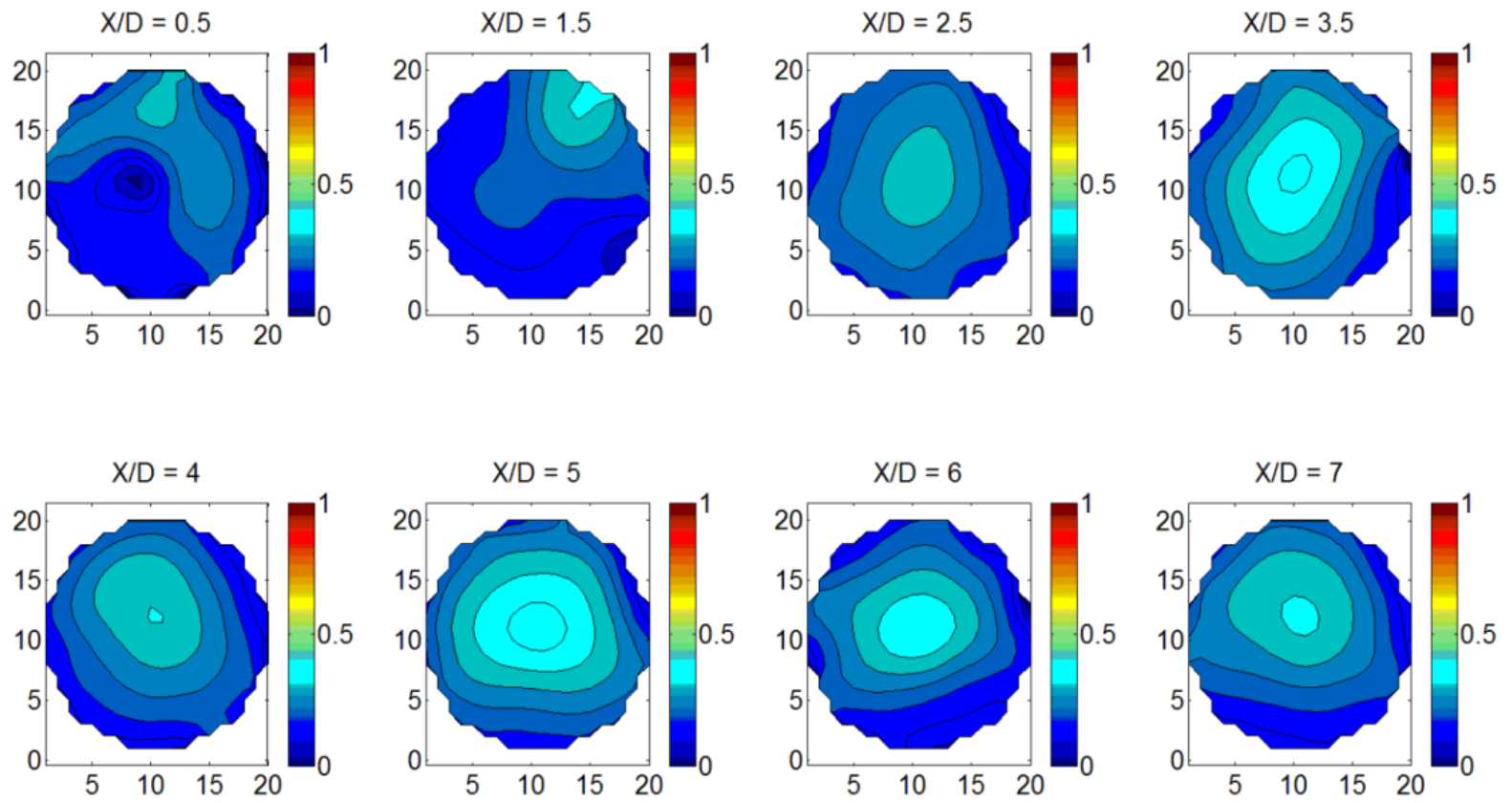

Figure 185: Concentration Tomogram at 60 psig_60 GPM_50\% GVF 

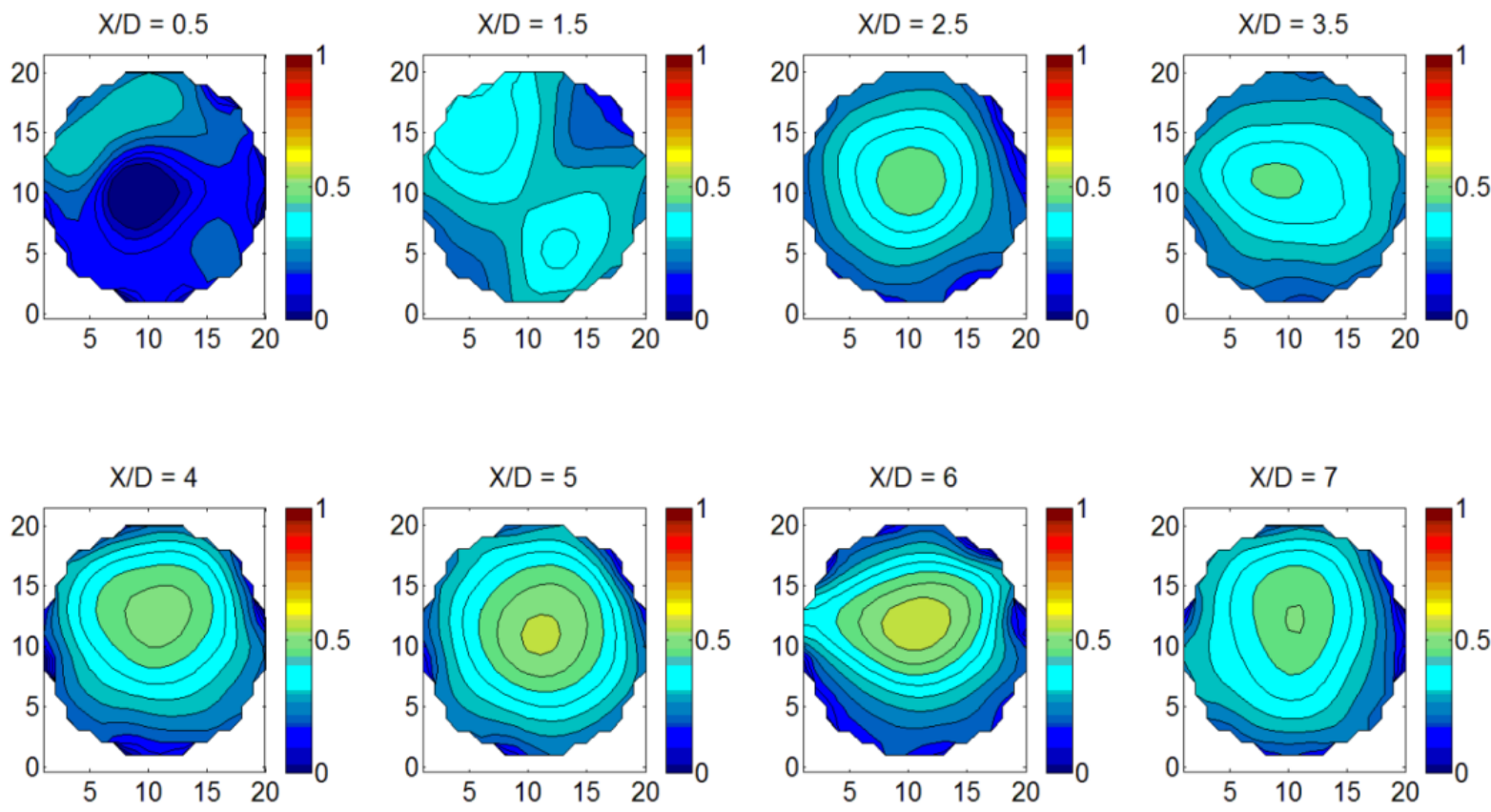

Figure 186: Concentration Tomogram at 60 psig_60 GPM_60\% GVF
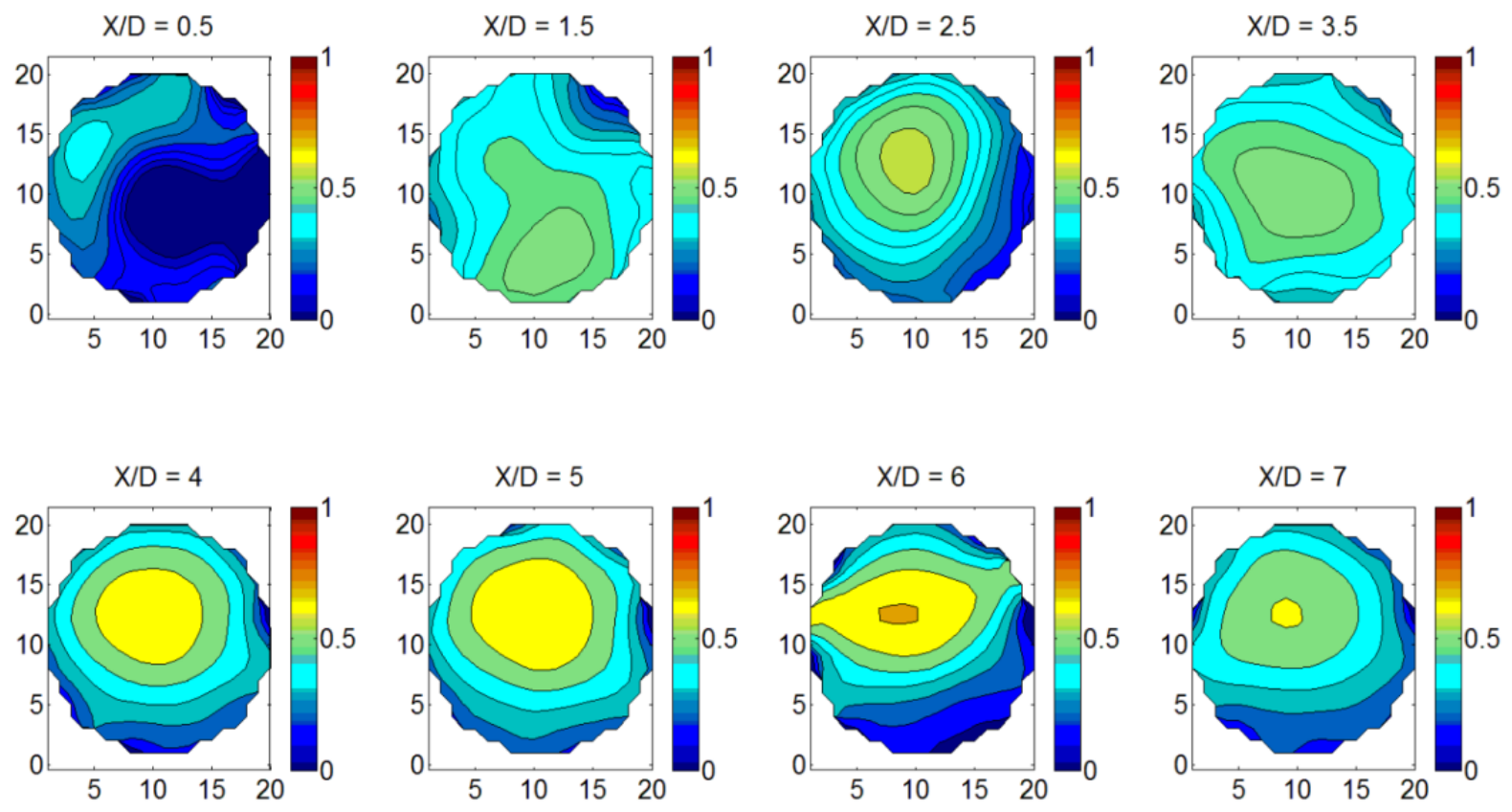

Figure 187: Concentration Tomogram at 60 psig_60 GPM_70\% GVF 


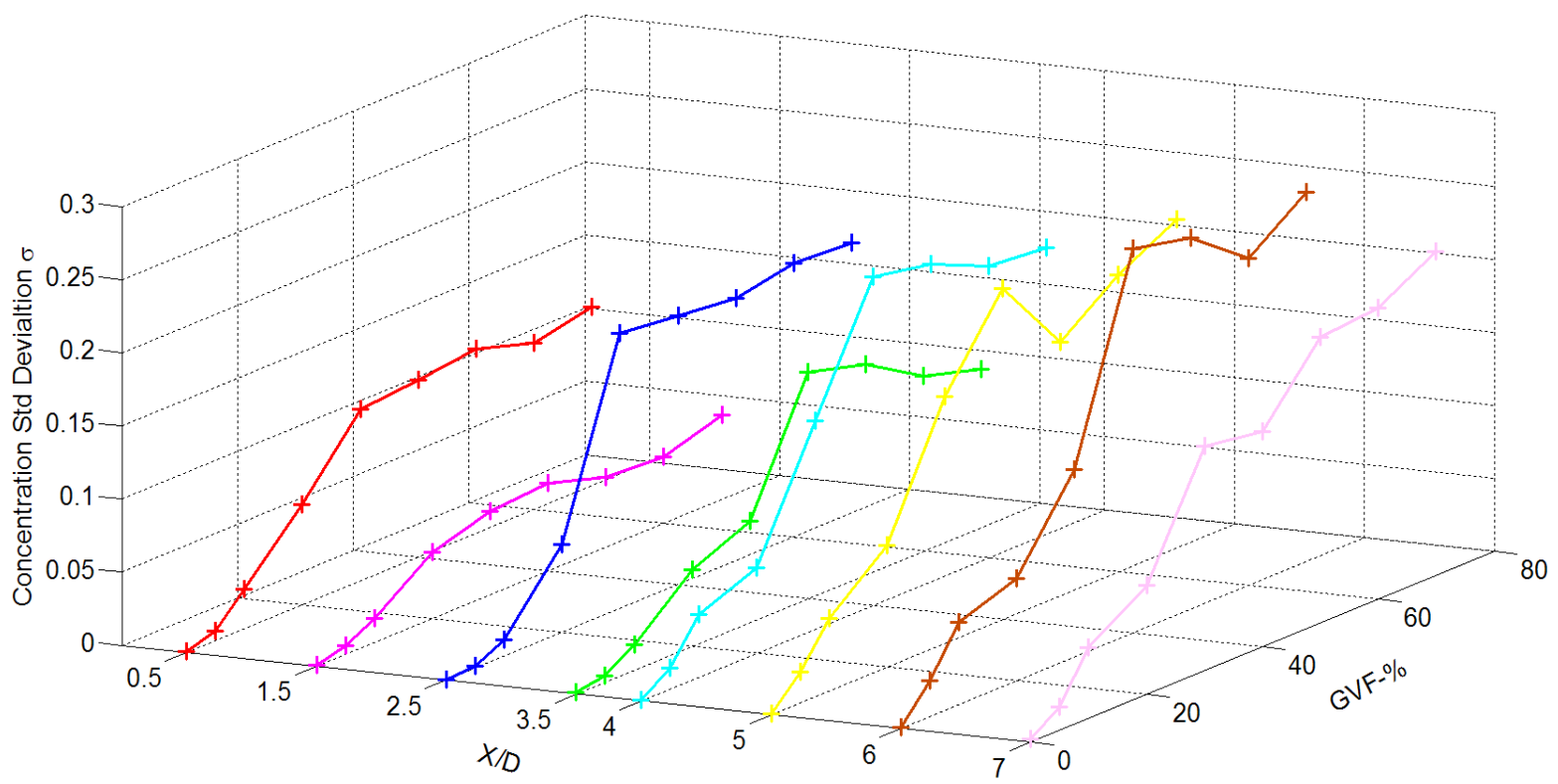

Figure 188: Standard deviation of the concentration tomogram pixel values at 20 psig \& 20 GPM

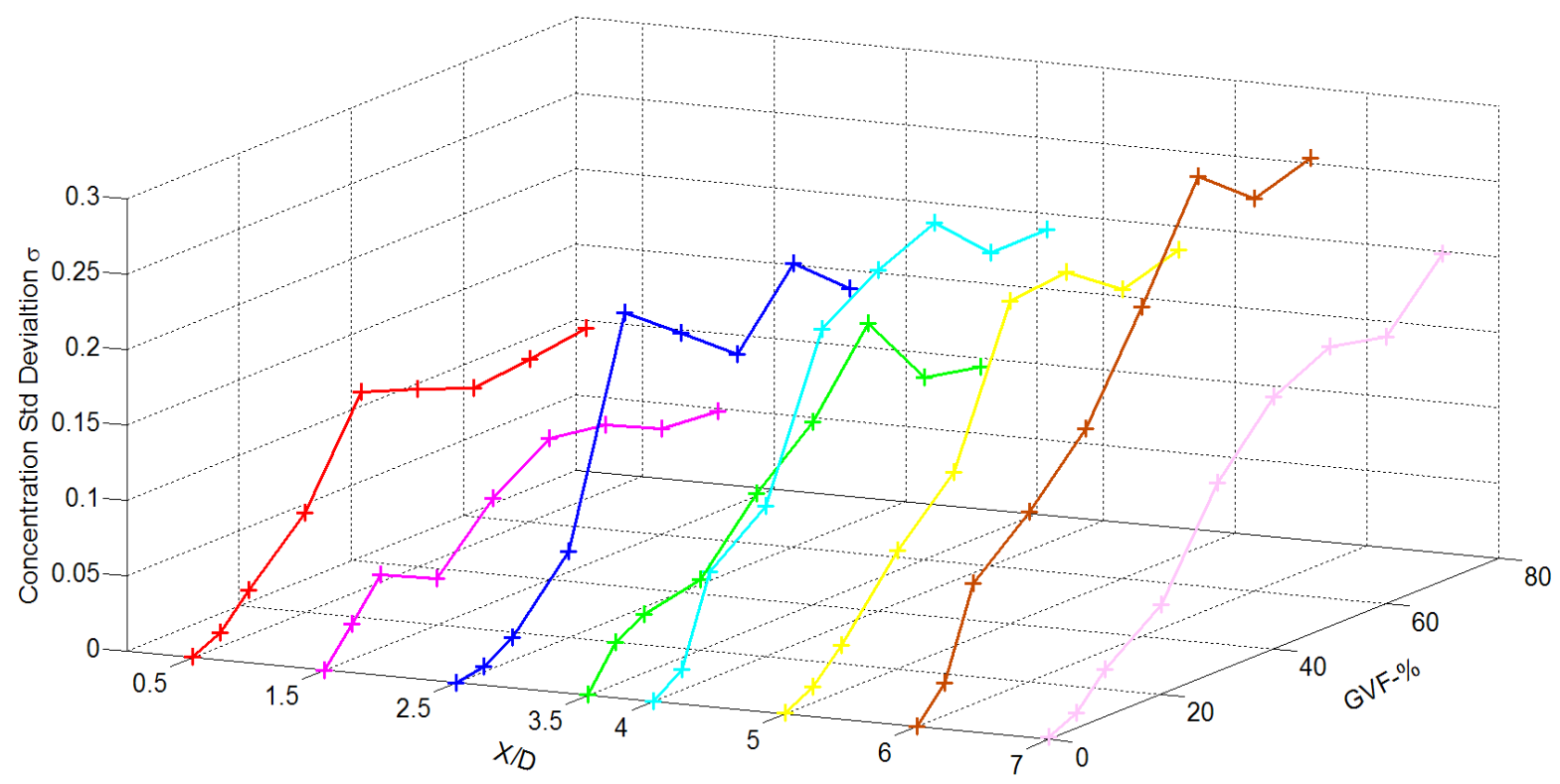

Figure 189: Standard deviation of the concentration tomogram pixel values at 40 psig \& 20 GPM 


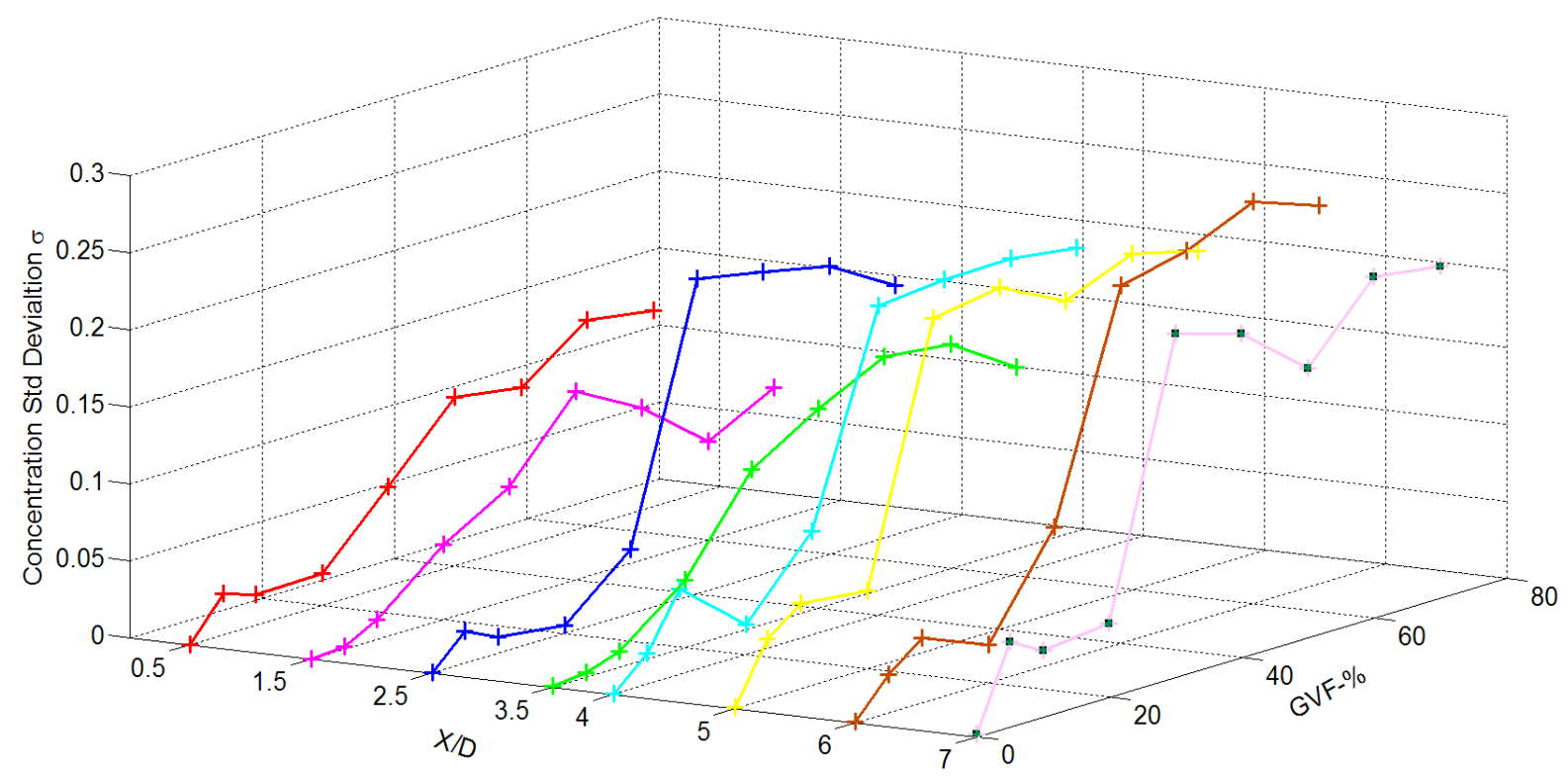

Figure 190: Standard deviation of the concentration tomogram pixel values at 60 psig \& 20 GPM 


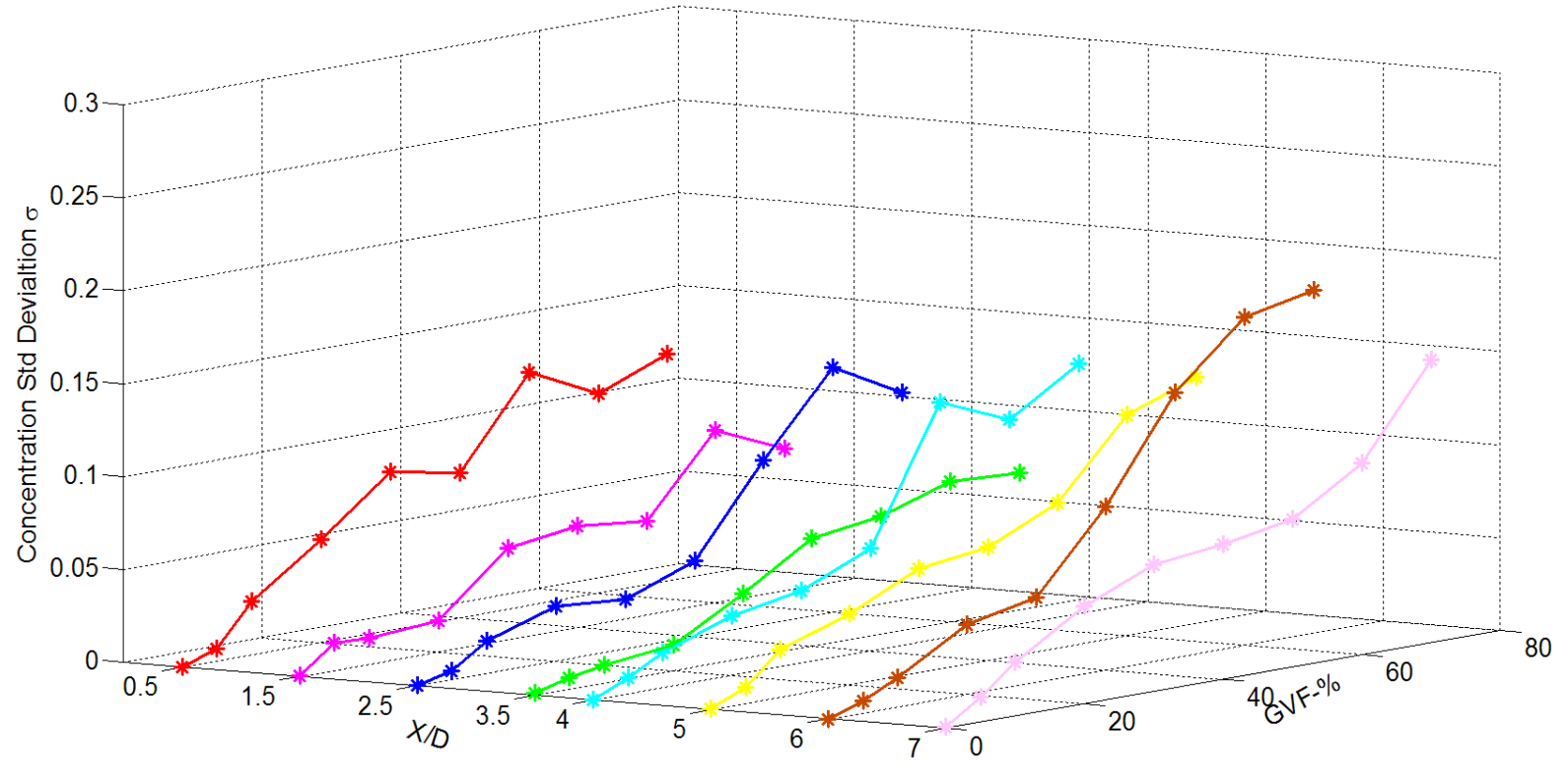

Figure 191: Standard deviation of the concentration tomogram pixel values at 20 psig \& 40 GPM

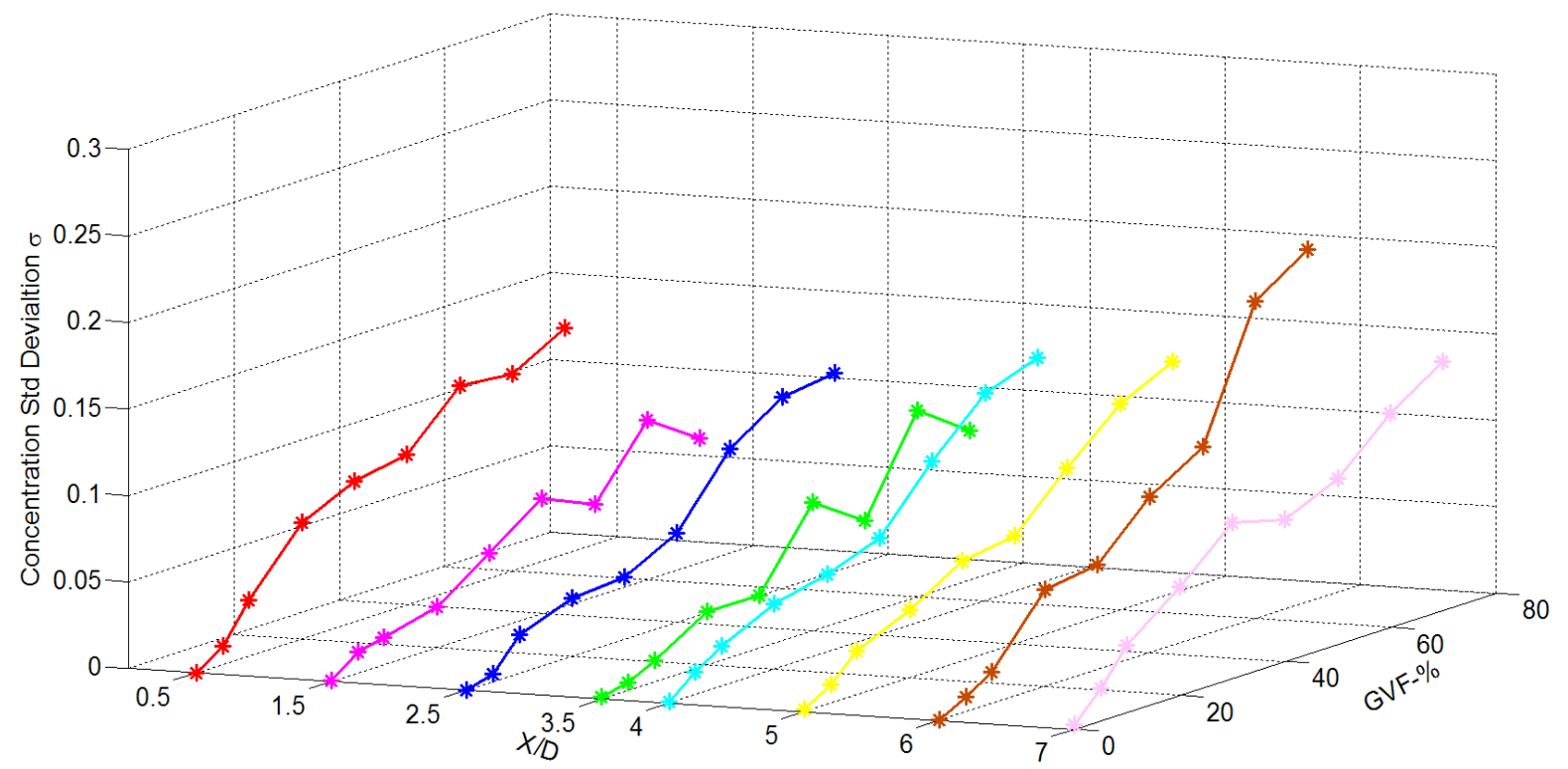

Figure 192: Standard deviation of the concentration tomogram pixel values at 40 psig \& 40 GPM 


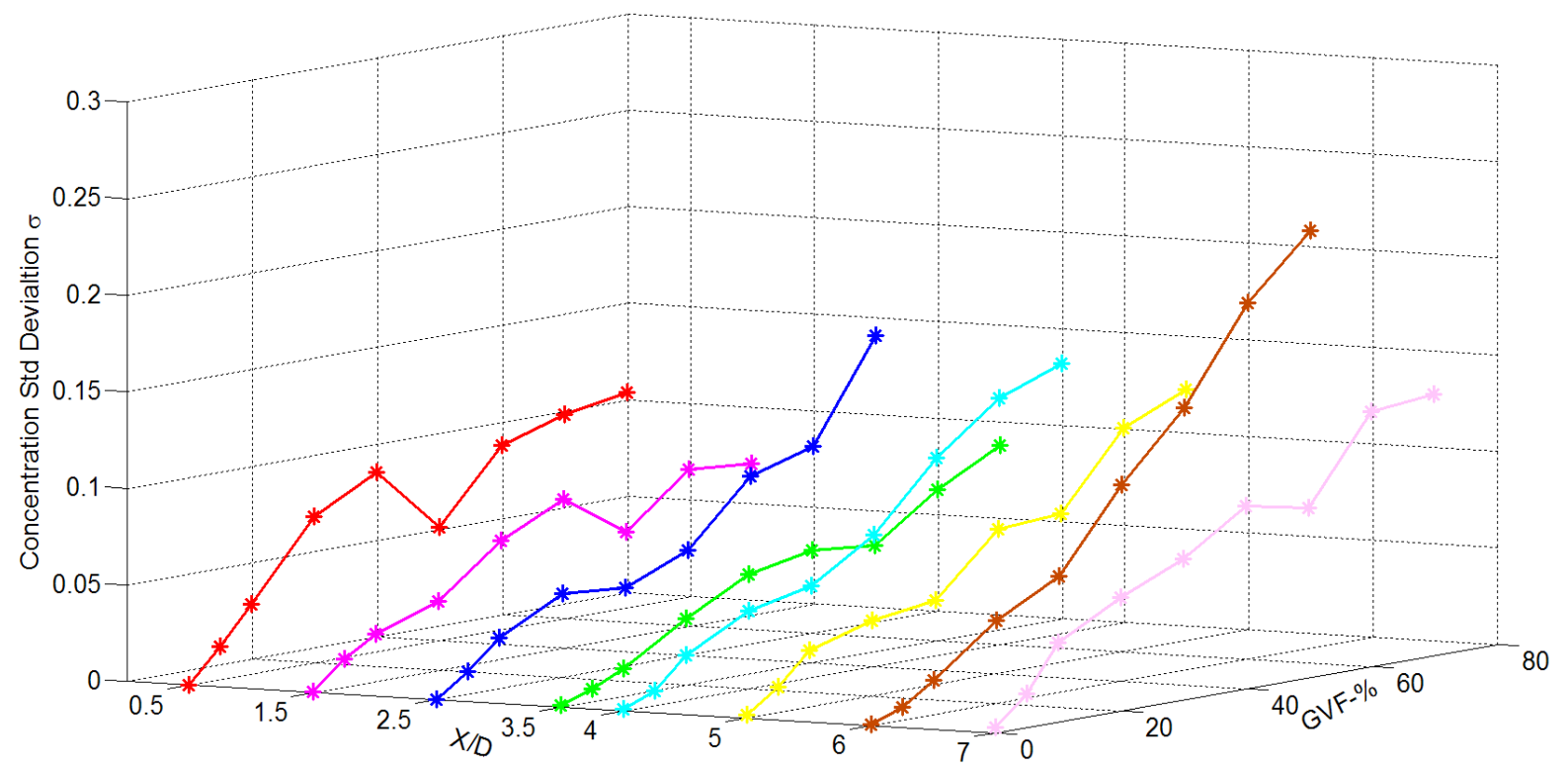

Figure 193: Standard deviation of the concentration tomogram pixel values at 60 psig \& 40 GPM 


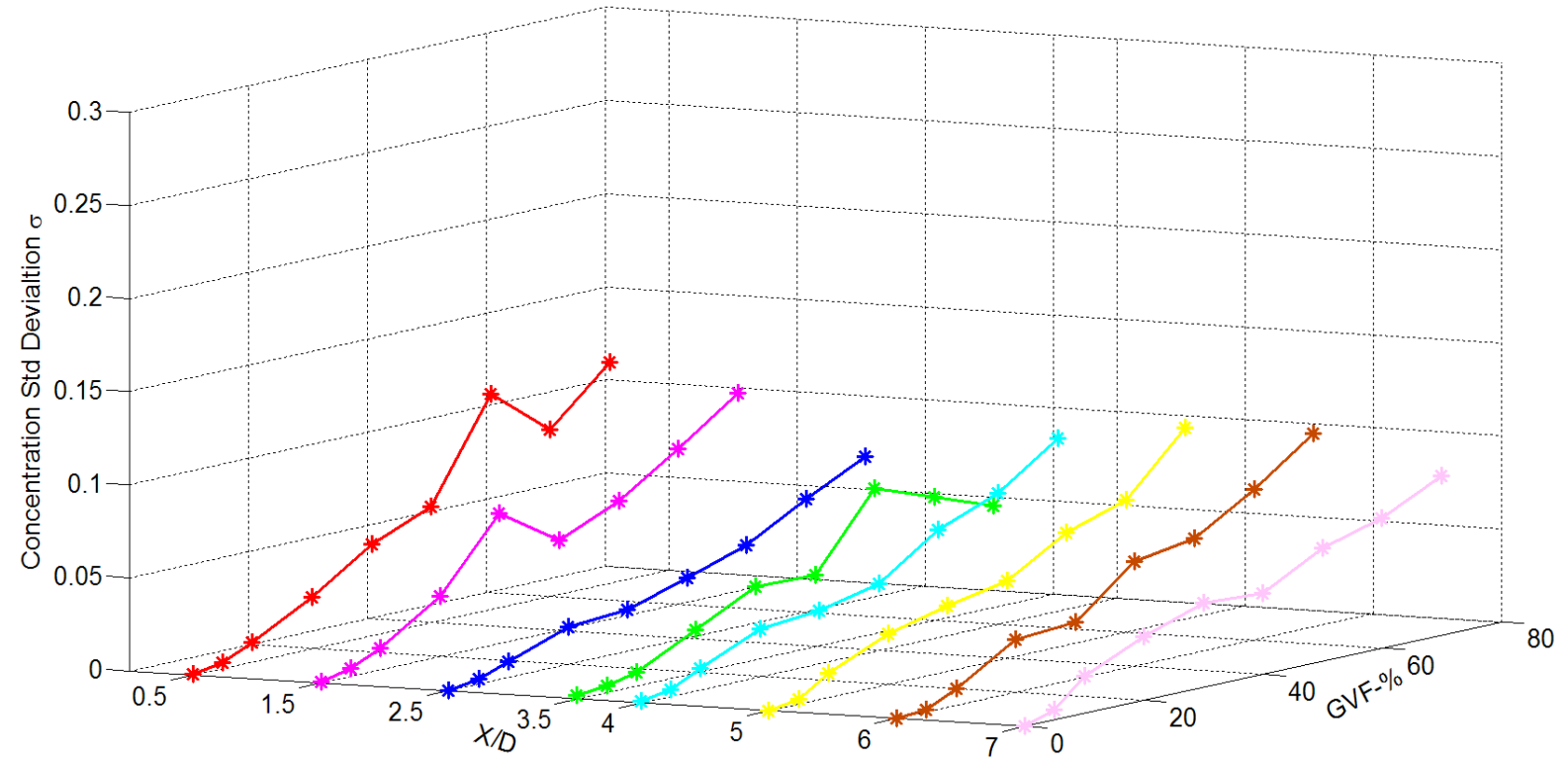

Figure 194: Standard deviation of the concentration tomogram pixel values at 20 psig \& 60 GPM

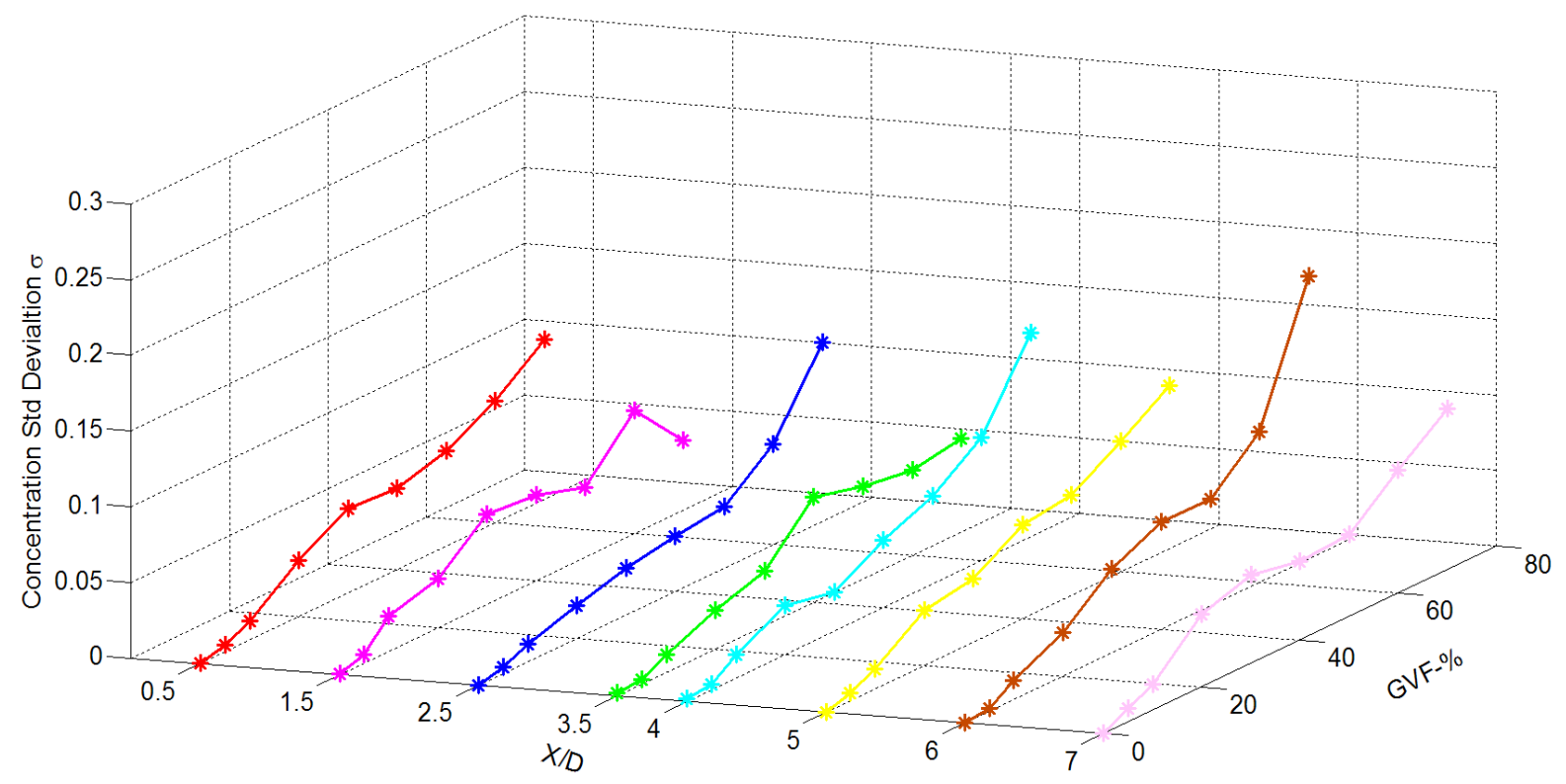

Figure 195: Standard deviation of the concentration tomogram pixel values at 40 psig \& 60 GPM 


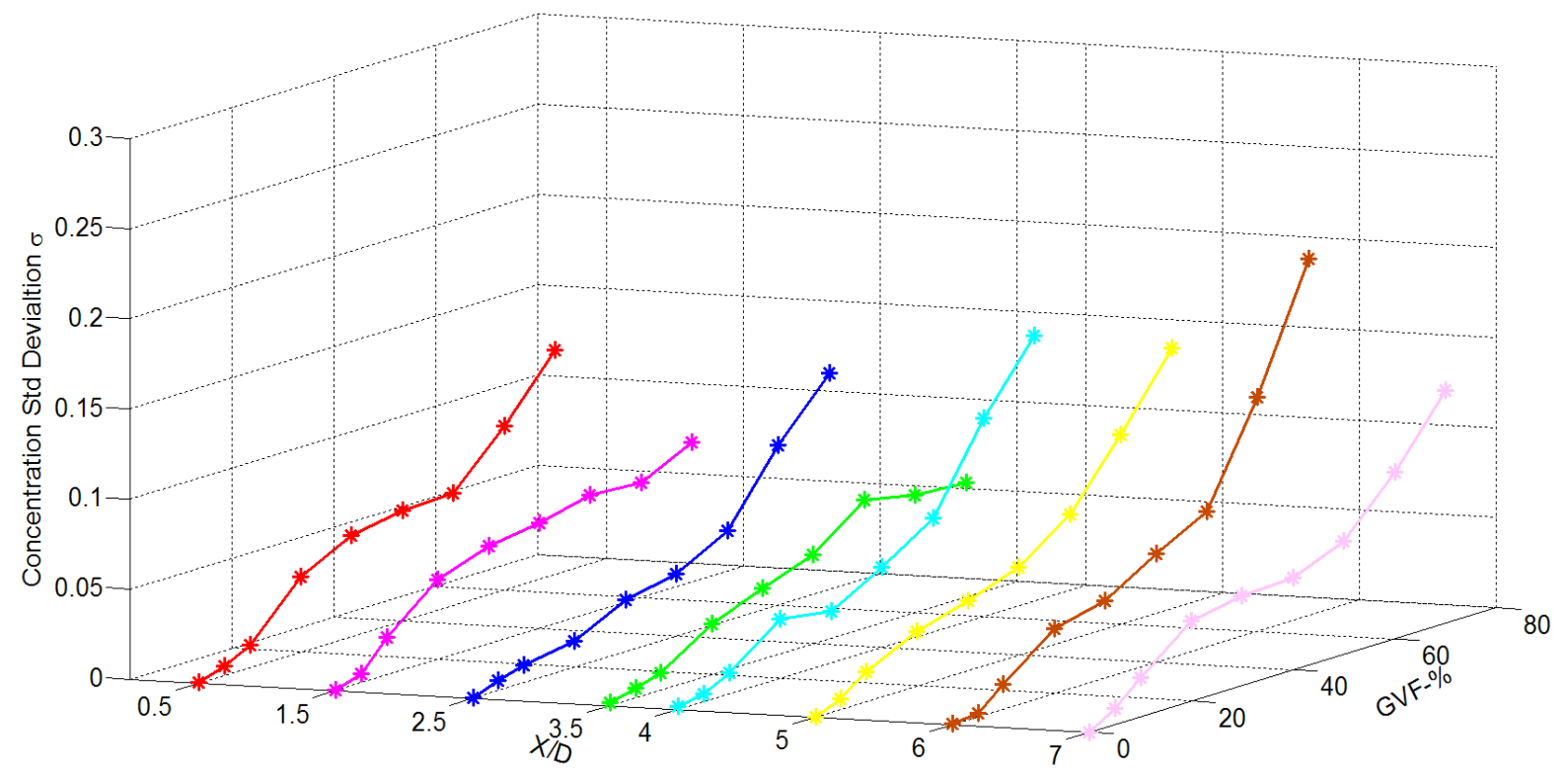

Figure 196: Standard deviation of the concentration tomogram pixel values at 60 psig \& 60 GPM

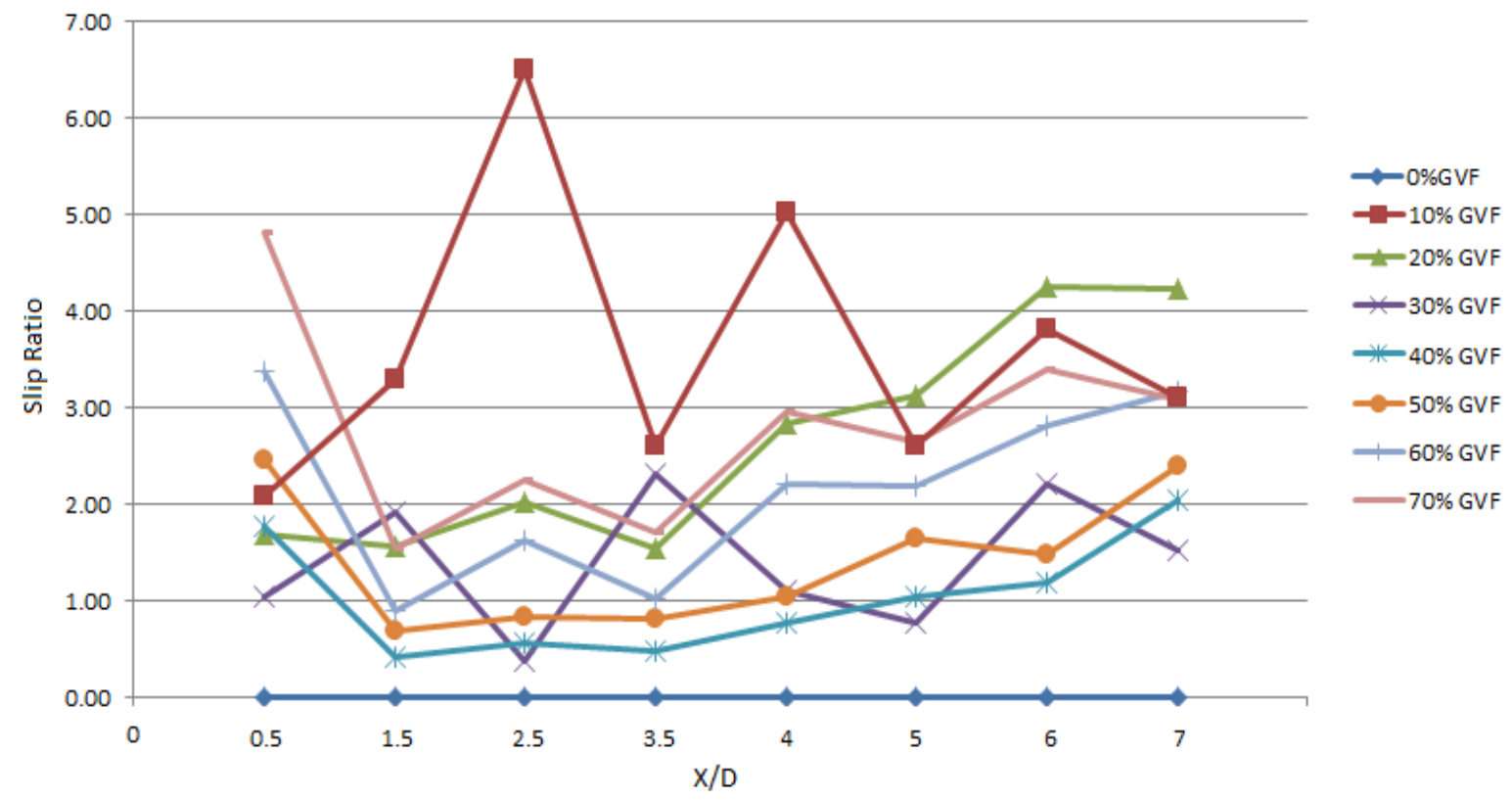

Figure 197: Slip Ratio at different downstream location for 20 GPM \& 20 psig 


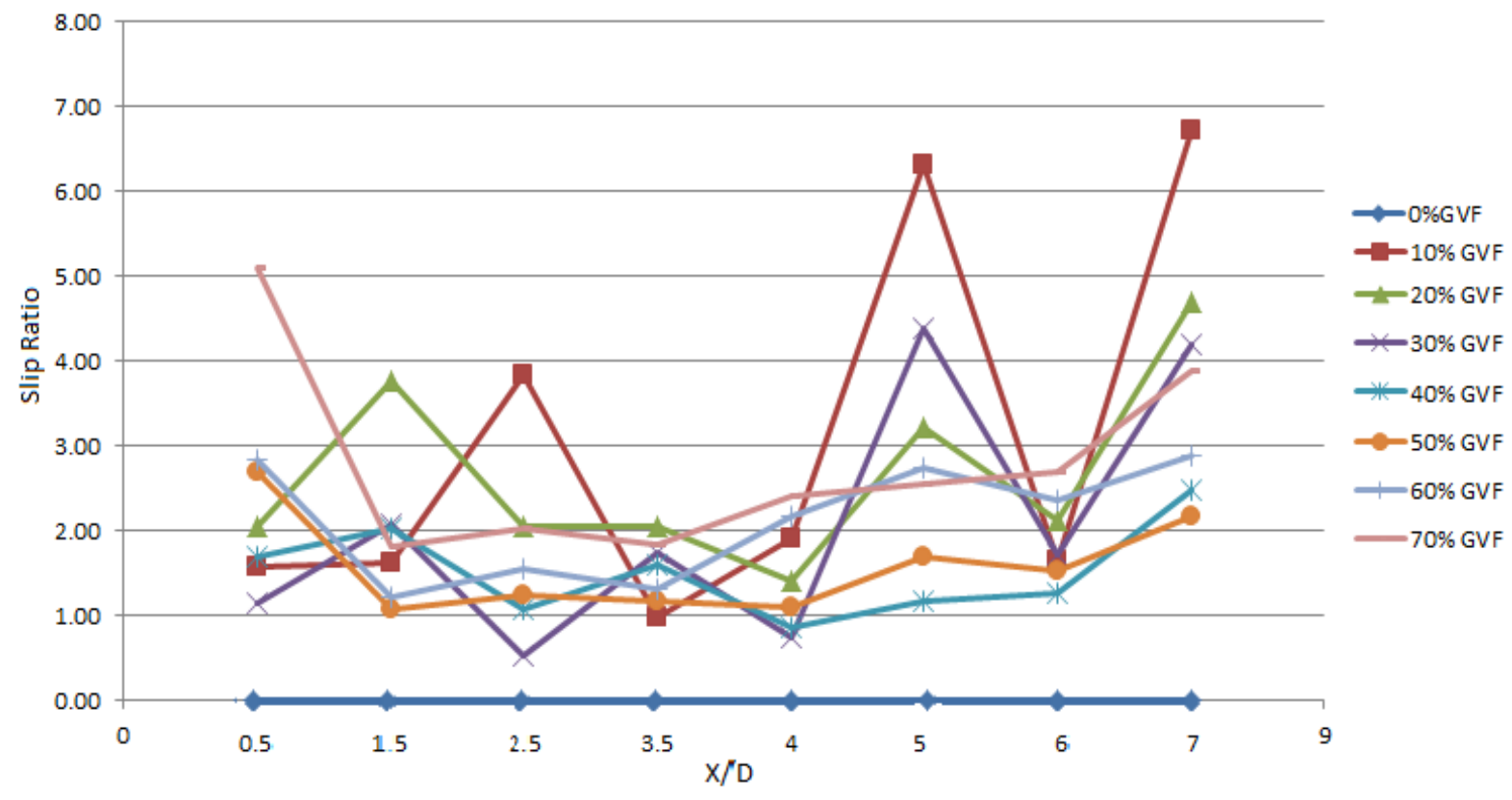

Figure 198: Slip Ratio at different downstream location for 20 GPM \& 40 psig

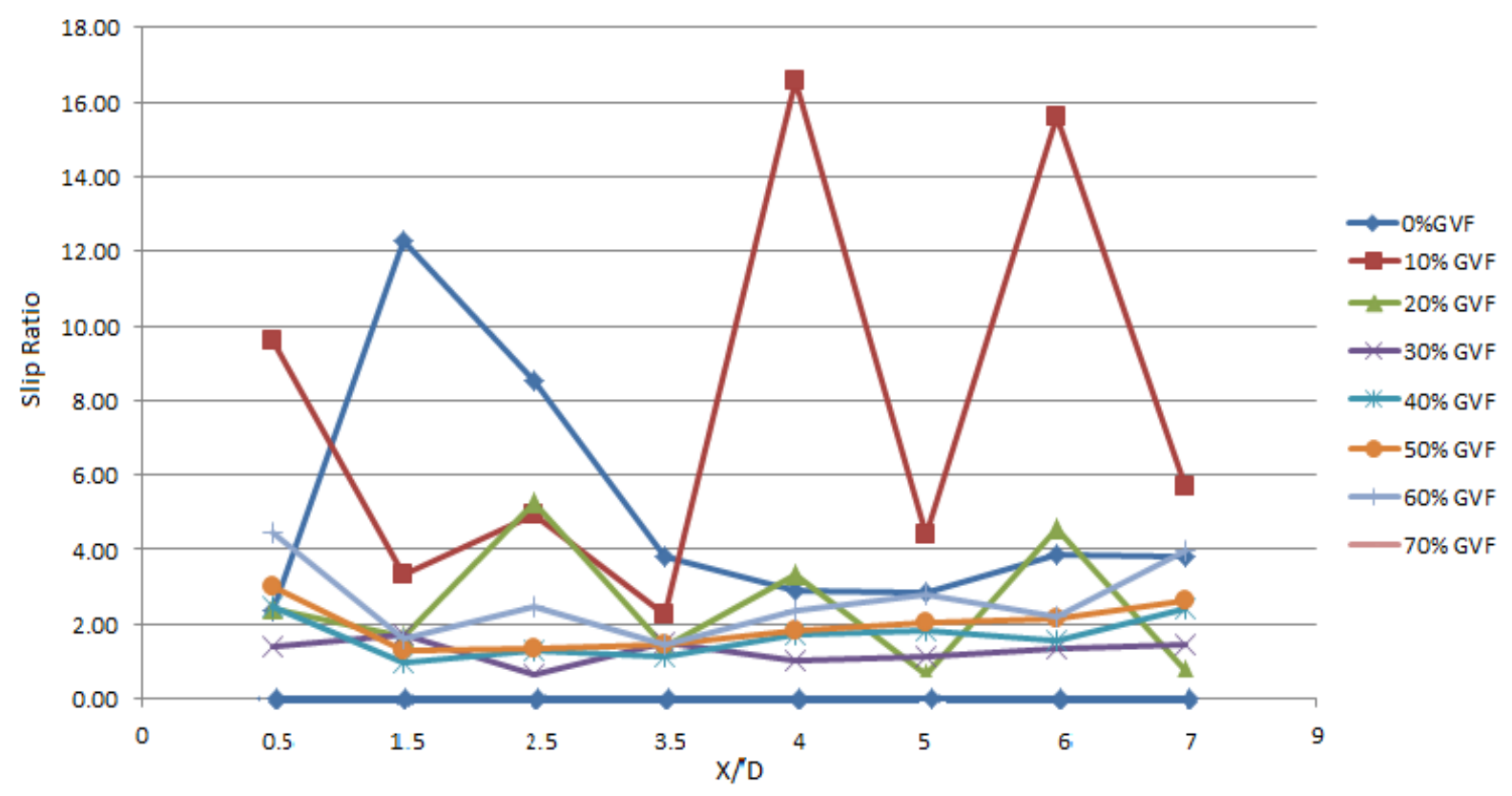

Figure 199: Slip Ratio at different downstream location for 20 GPM \& 60 psig 


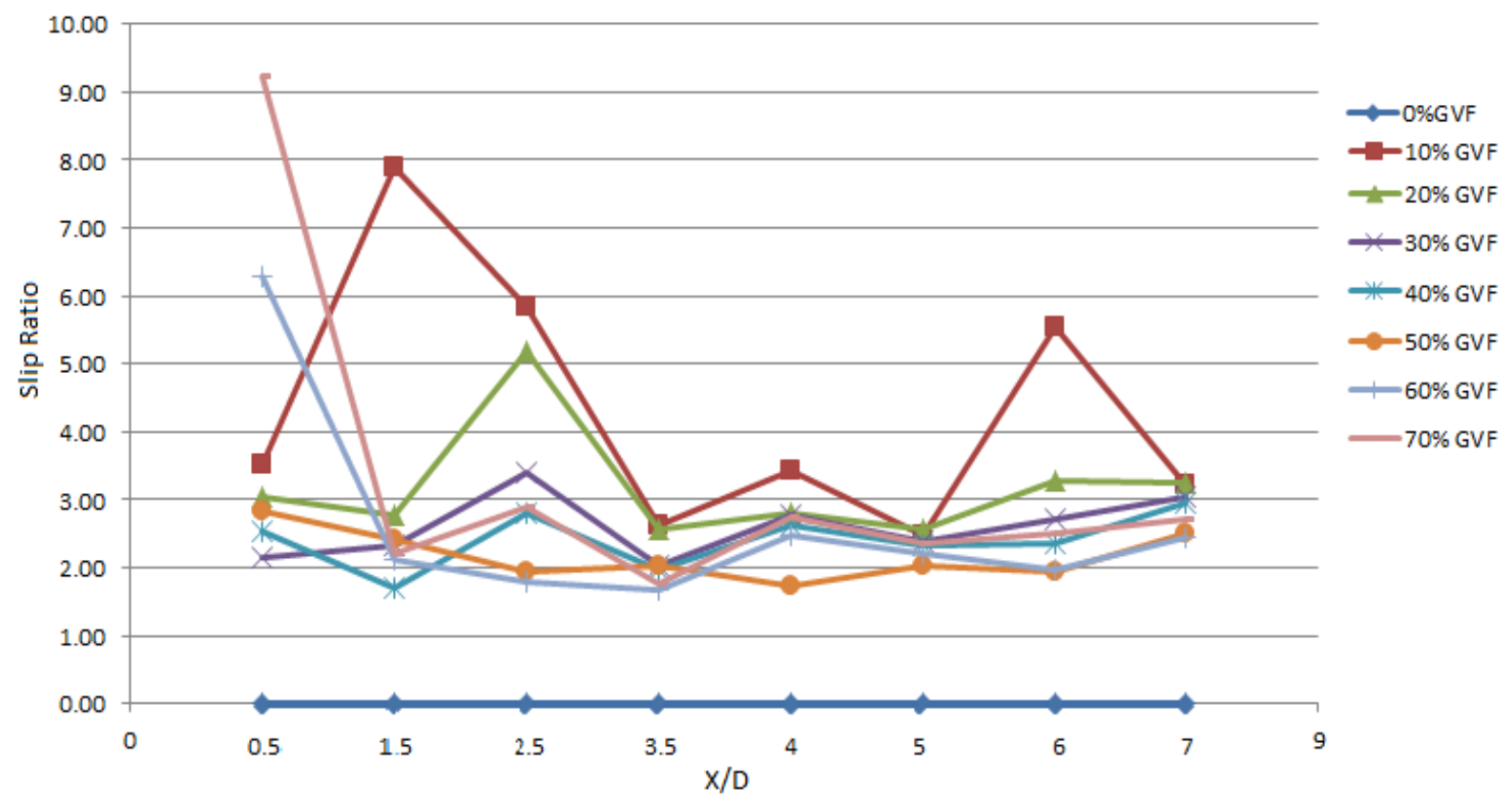

Figure 200: Slip Ratio at different downstream location for 40 GPM \& 20 psig

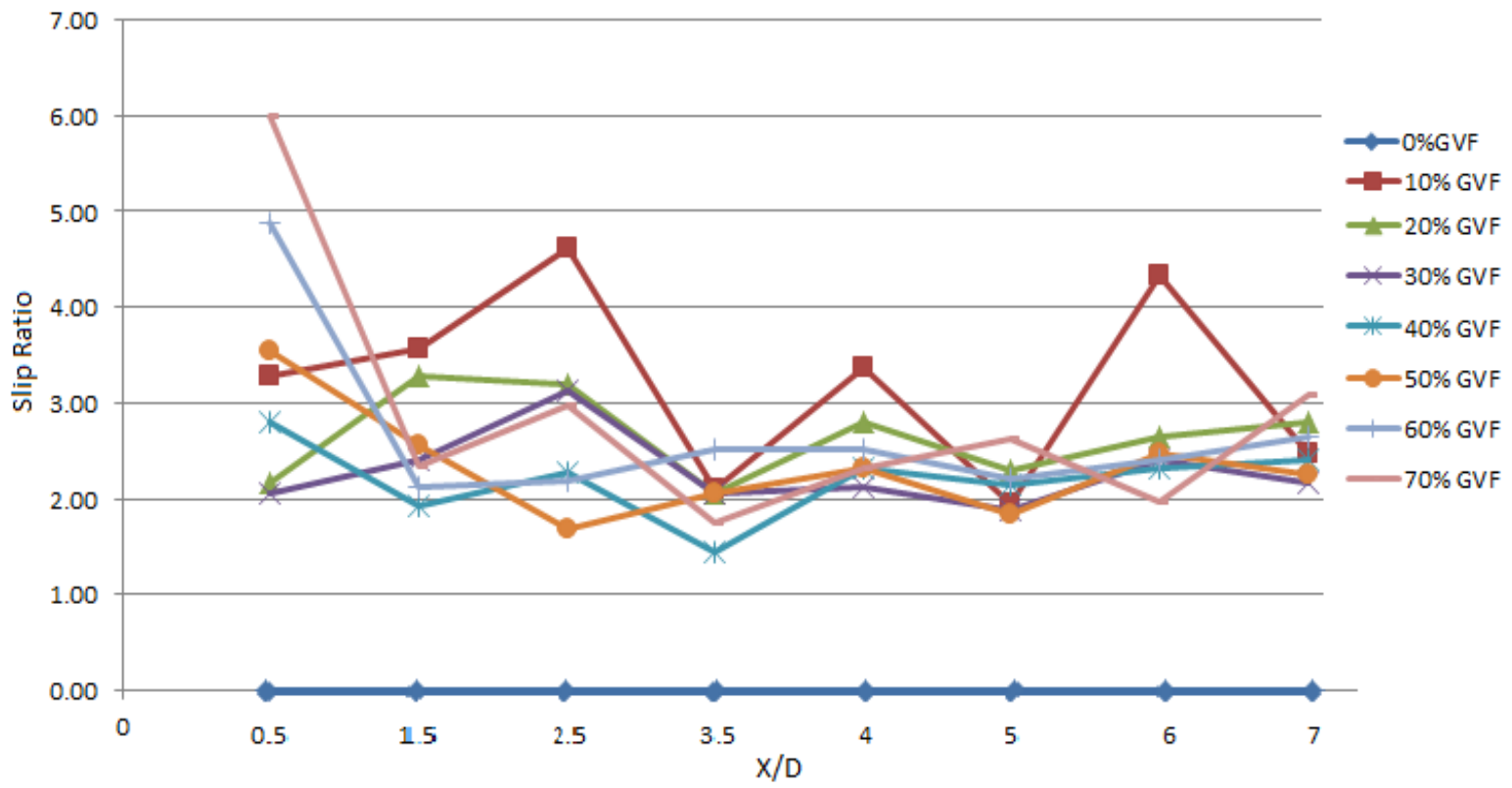

Figure 201: Slip Ratio at different downstream location for 40 GPM \& 40 psig 


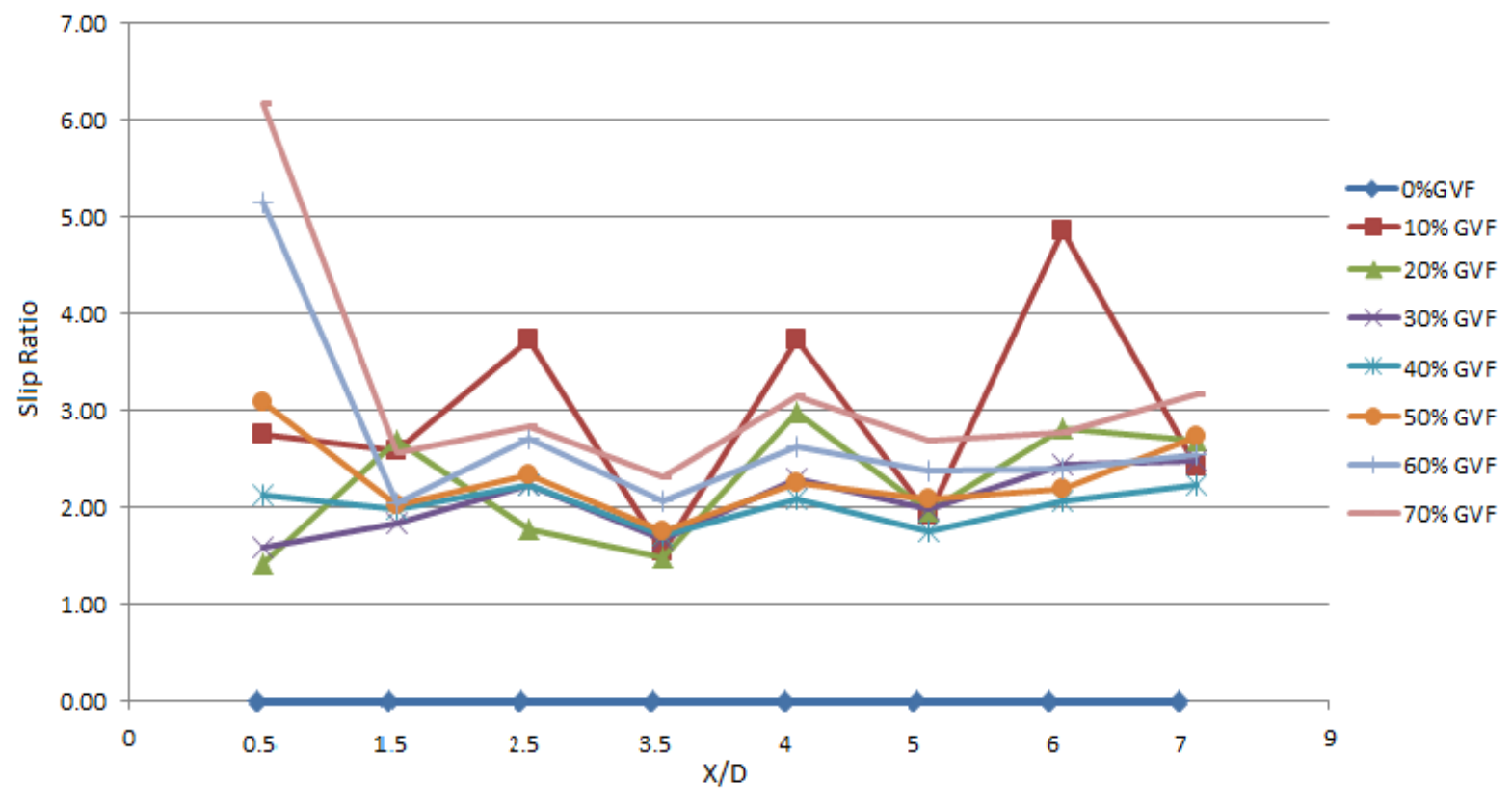

Figure 202: Slip Ratio at different downstream location for 40 GPM \& 60 psig

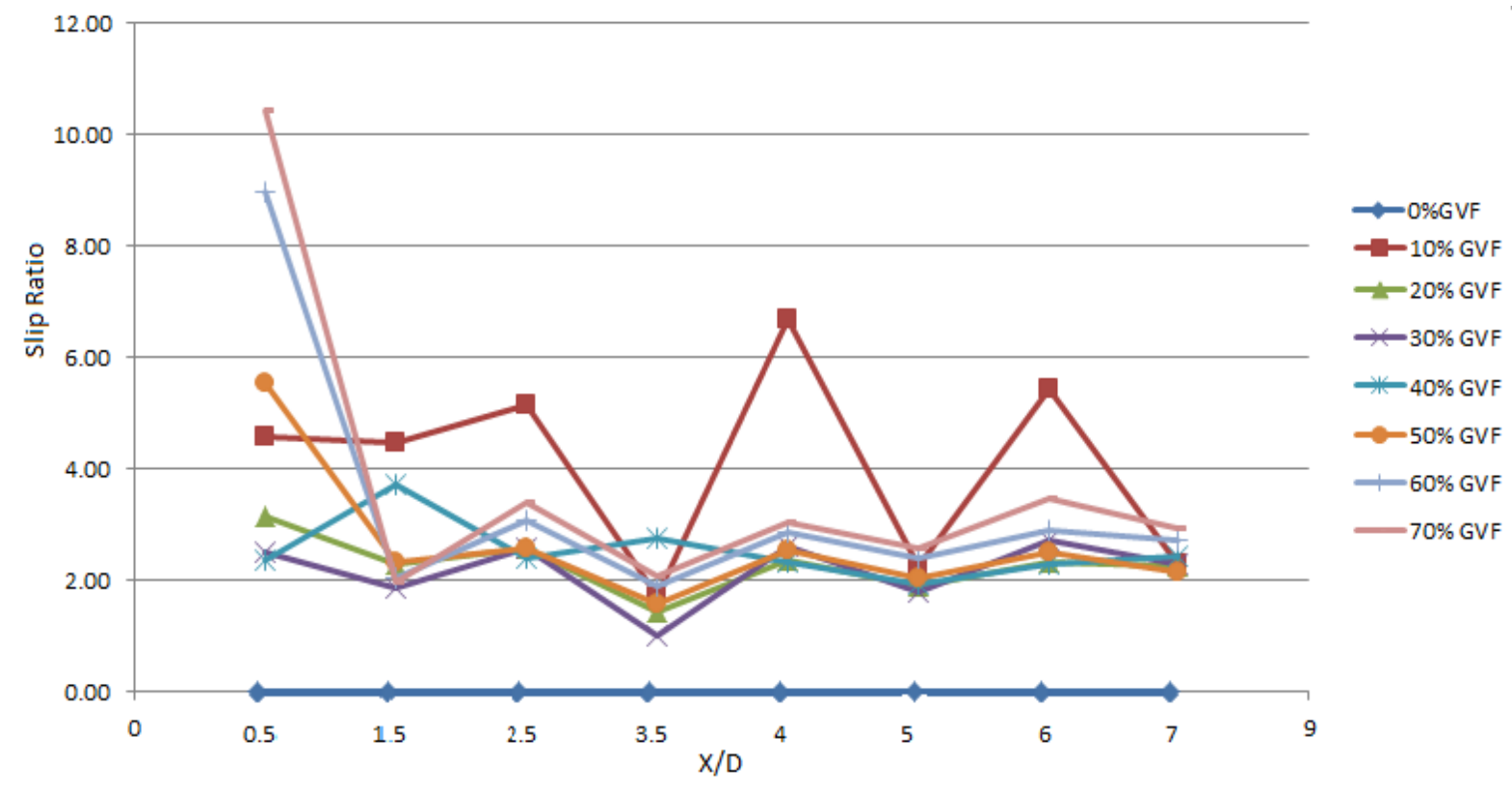

Figure 203: Slip Ratio at different downstream location for 60 GPM \& 20 psig 


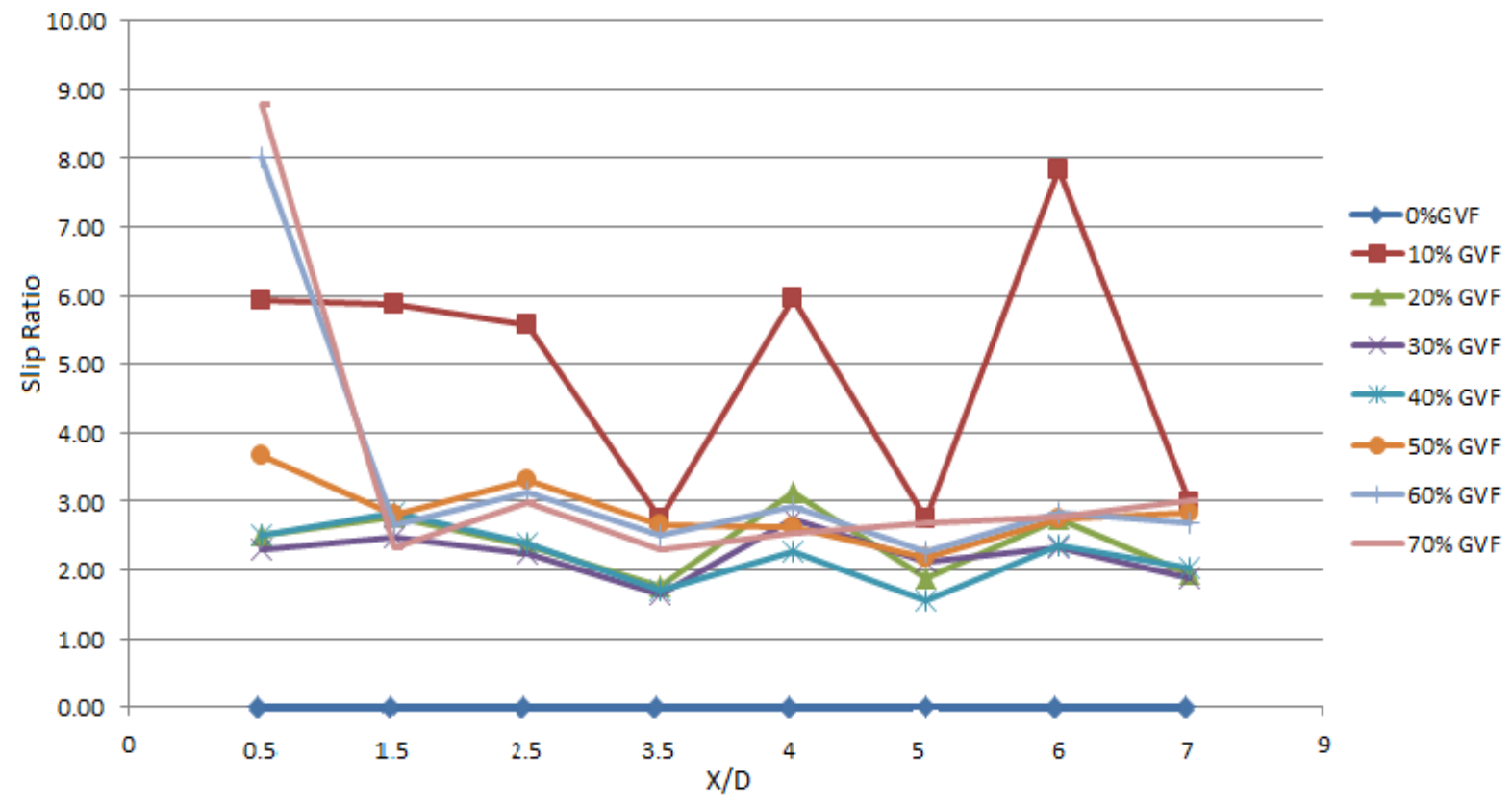

Figure 204: Slip Ratio at different downstream location for 60 GPM \& 40 psig

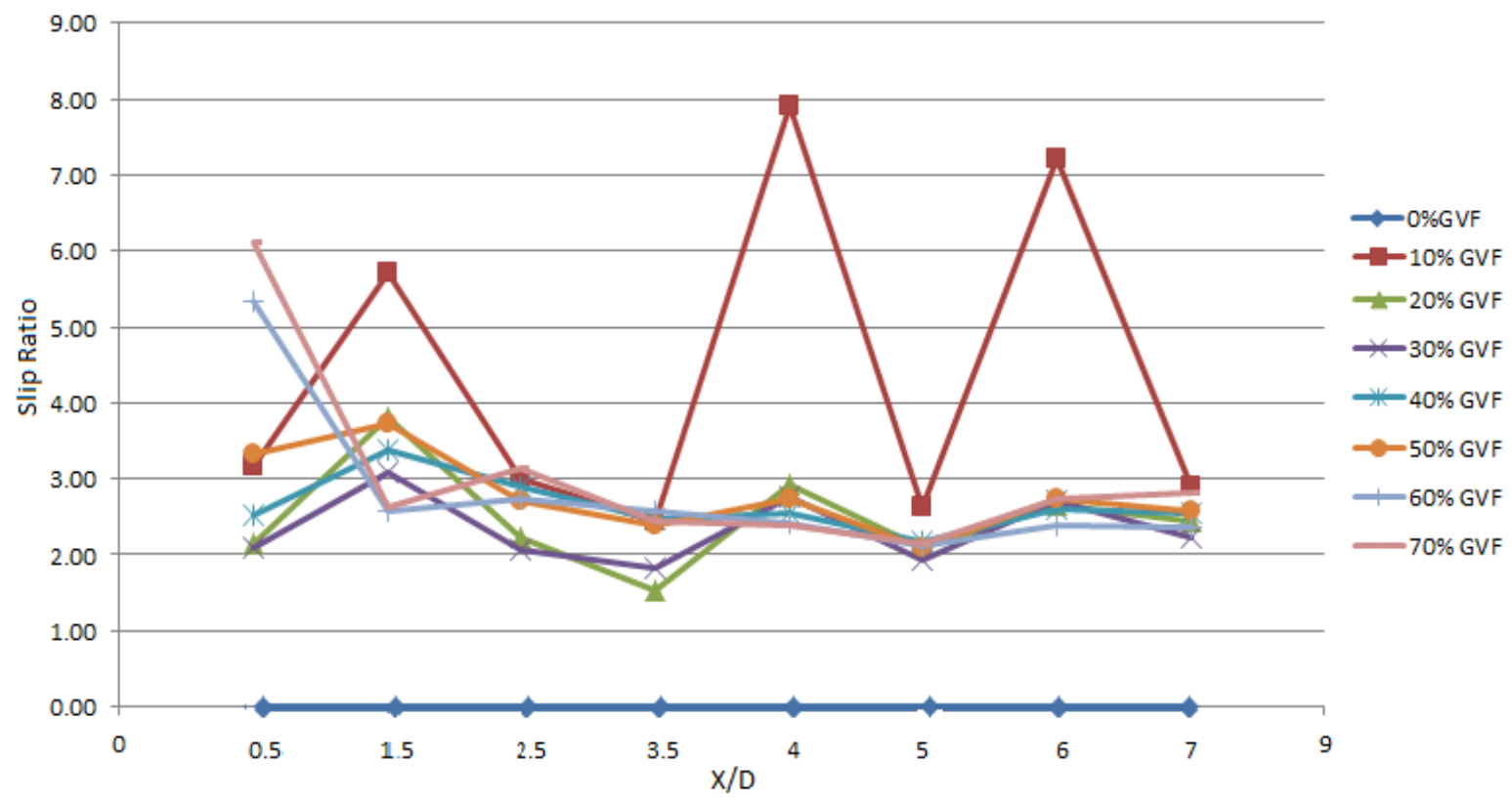

Figure 205: Slip Ratio at different downstream location for 60 GPM \& 60 psig 


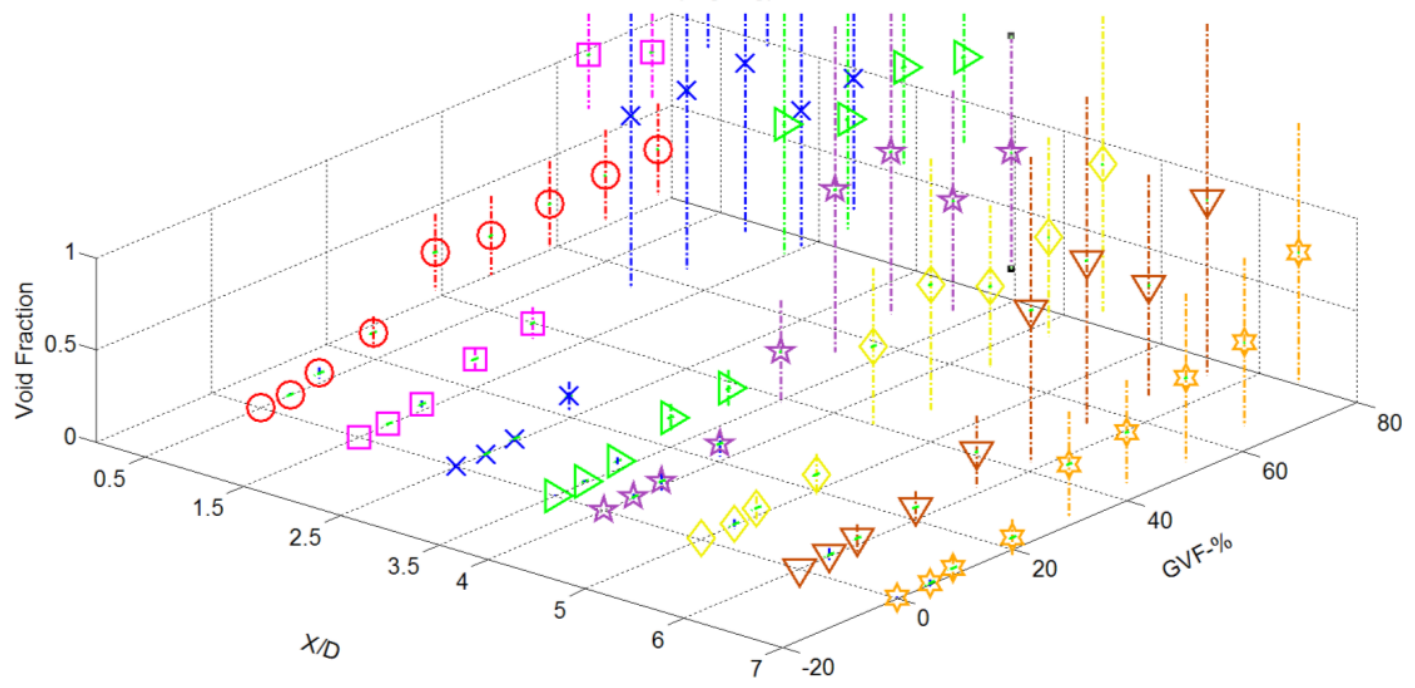

Figure 206: Standard deviation of Gas Void Fraction and GVF at 20 psig \& 20 GPM

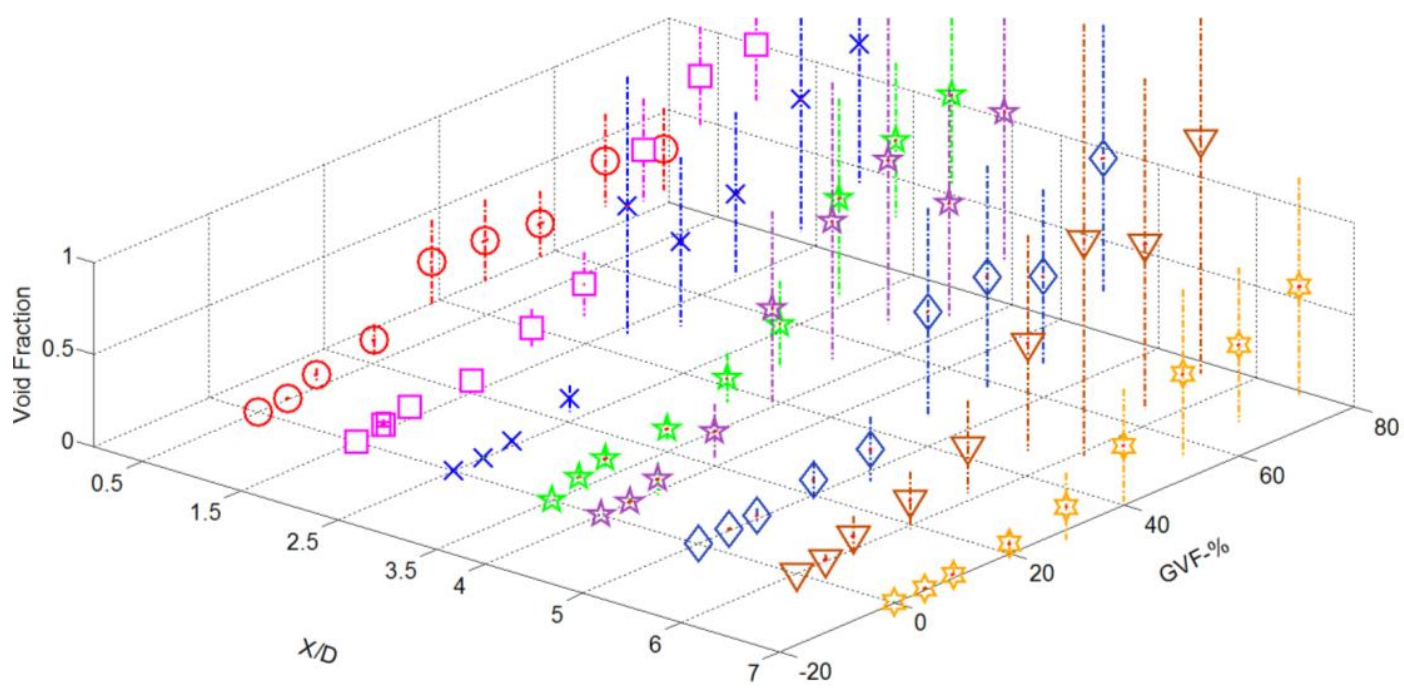

Figure 207: Standard deviation of Gas Void Fraction and GVF at 40 psig \& 20 GPM 


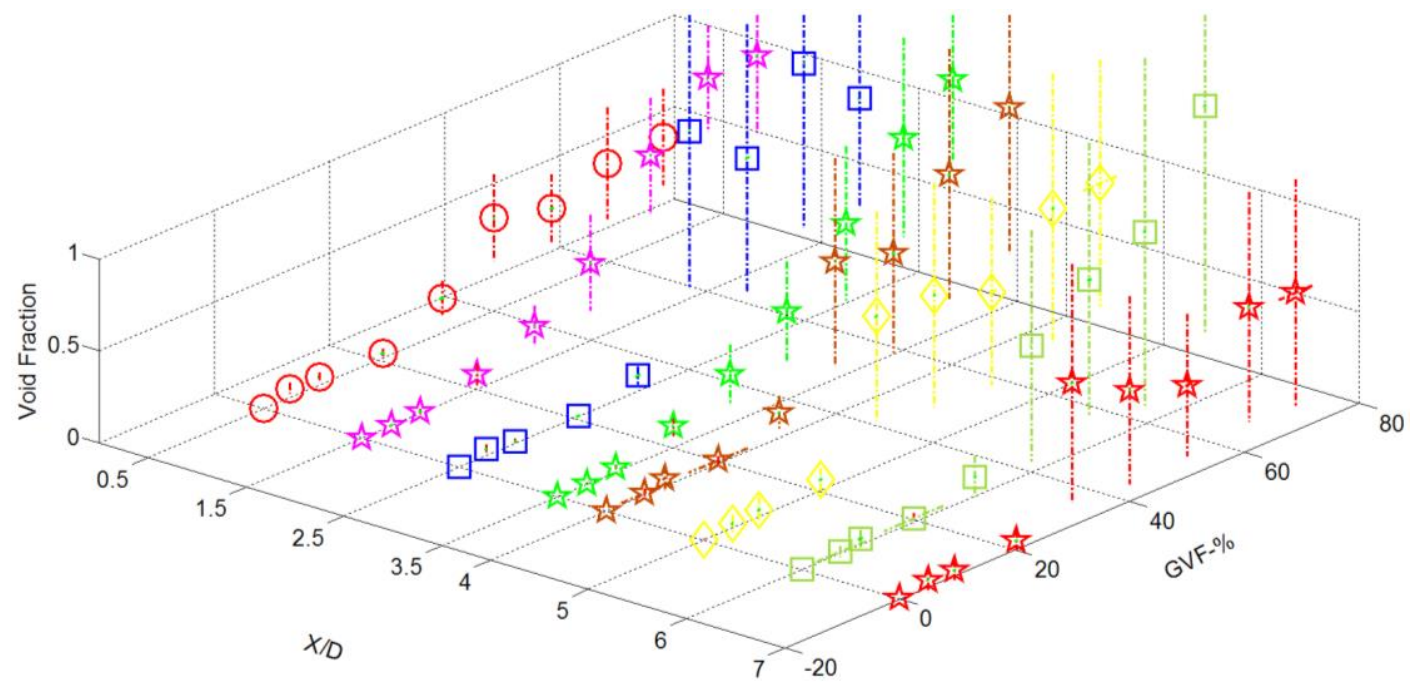

Figure 208: Standard deviation of Gas Void Fraction and GVF at 60 psig \& 20 GPM

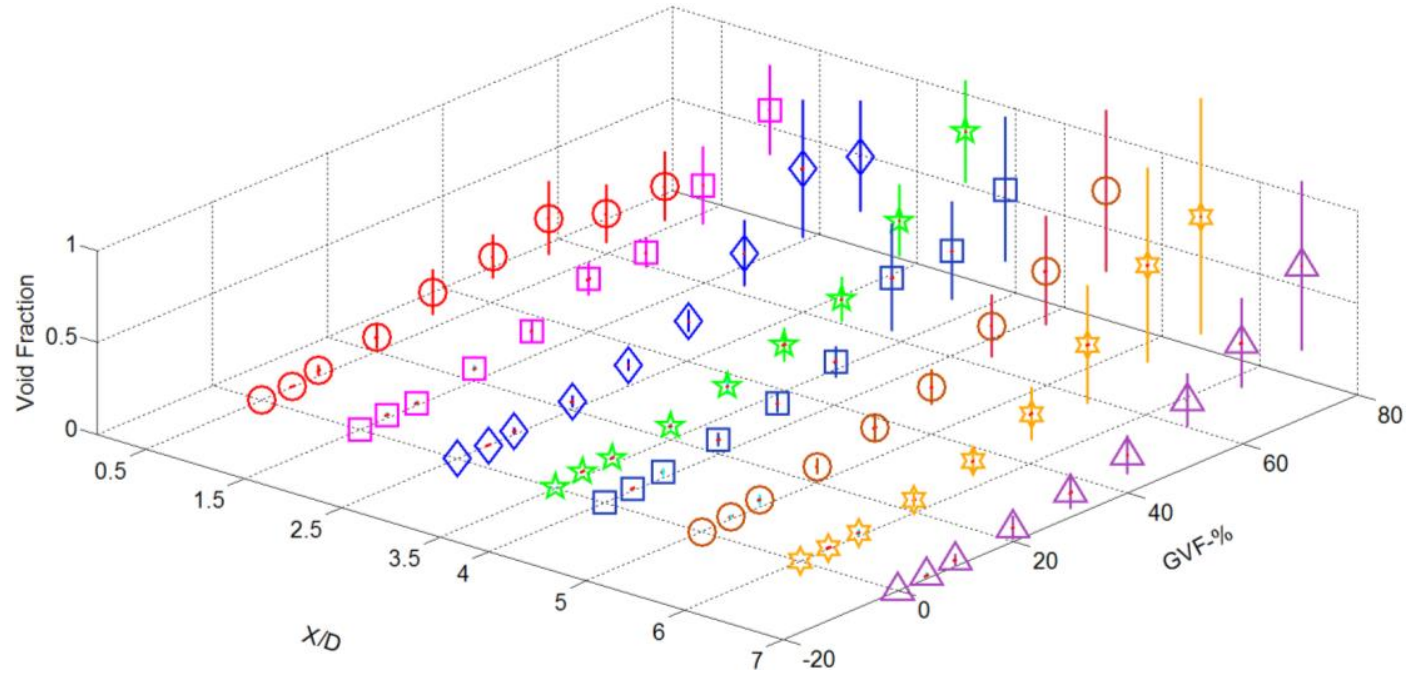

Figure 209: Standard deviation of Gas Void Fraction and GVF at 20 psig \& 40 GPM 


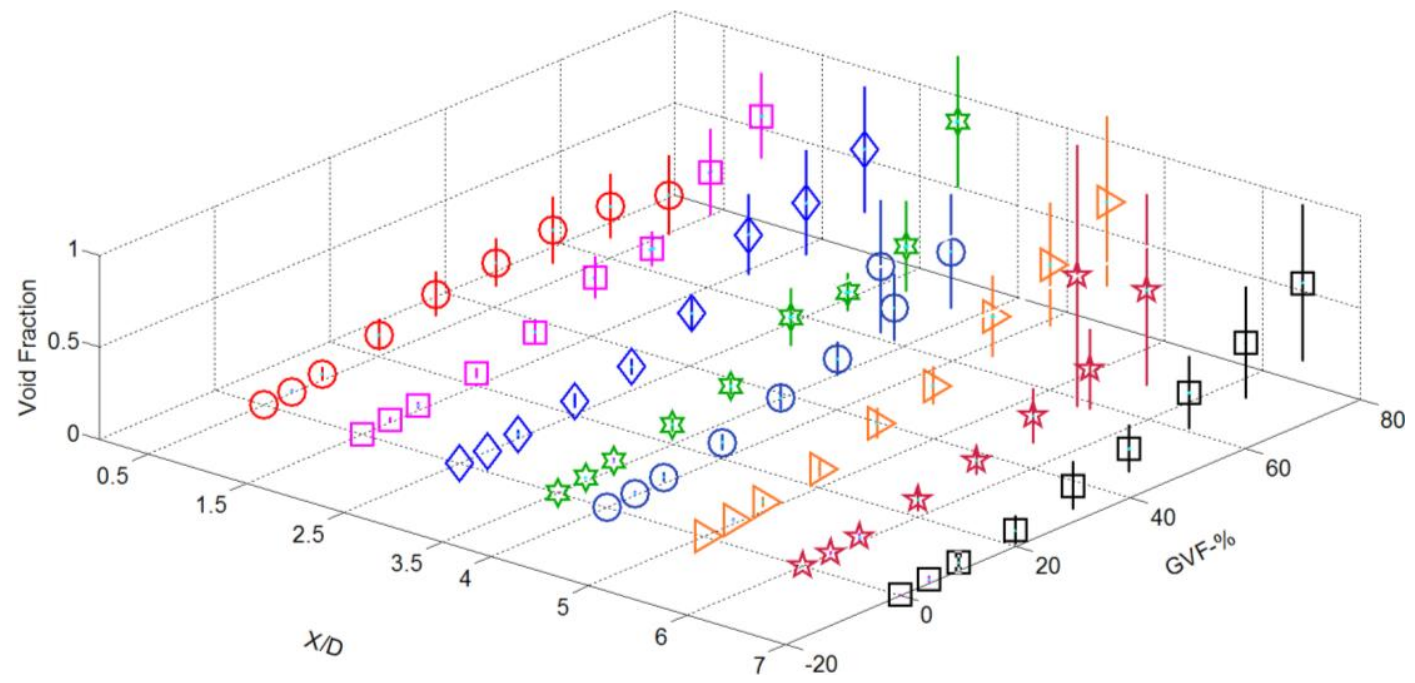

Figure 210: Standard deviation of Gas Void Fraction and GVF at 40 psig \& 40 GPM

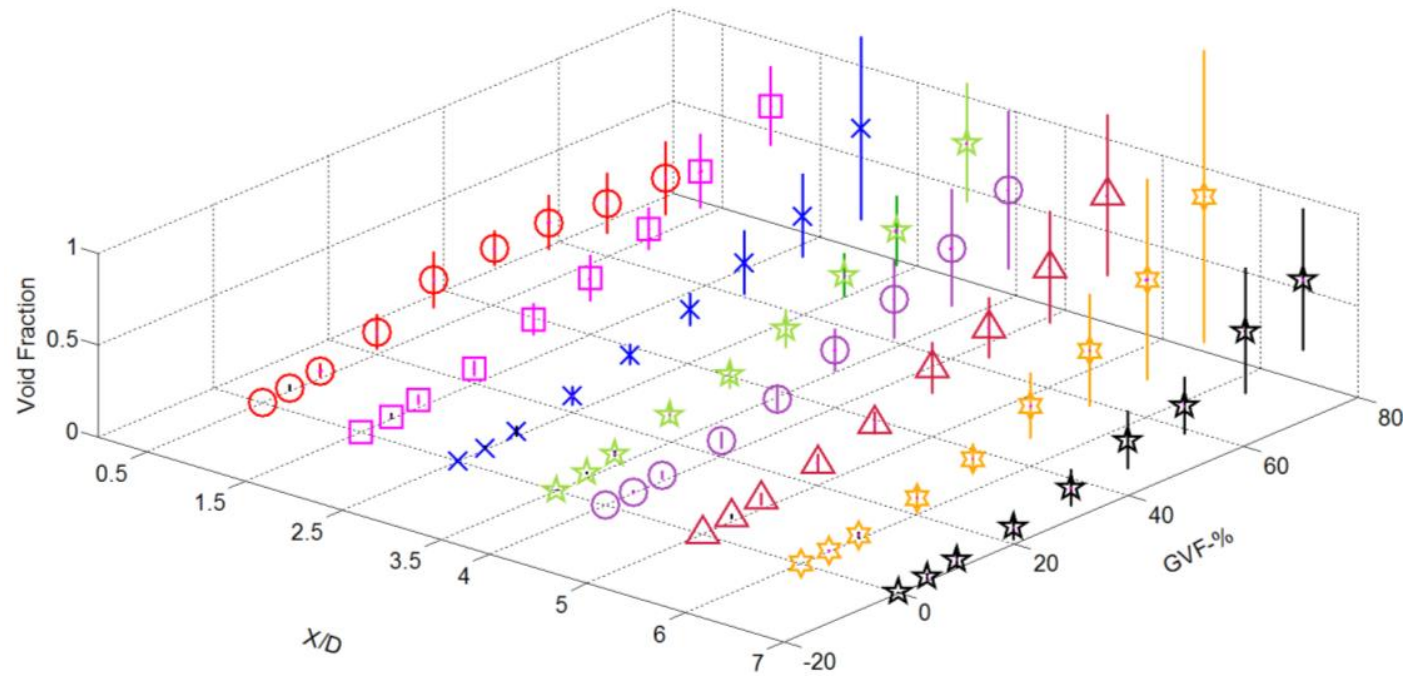

Figure 211: Standard deviation of Gas Void Fraction and GVF at 60 psig \& 40 GPM 


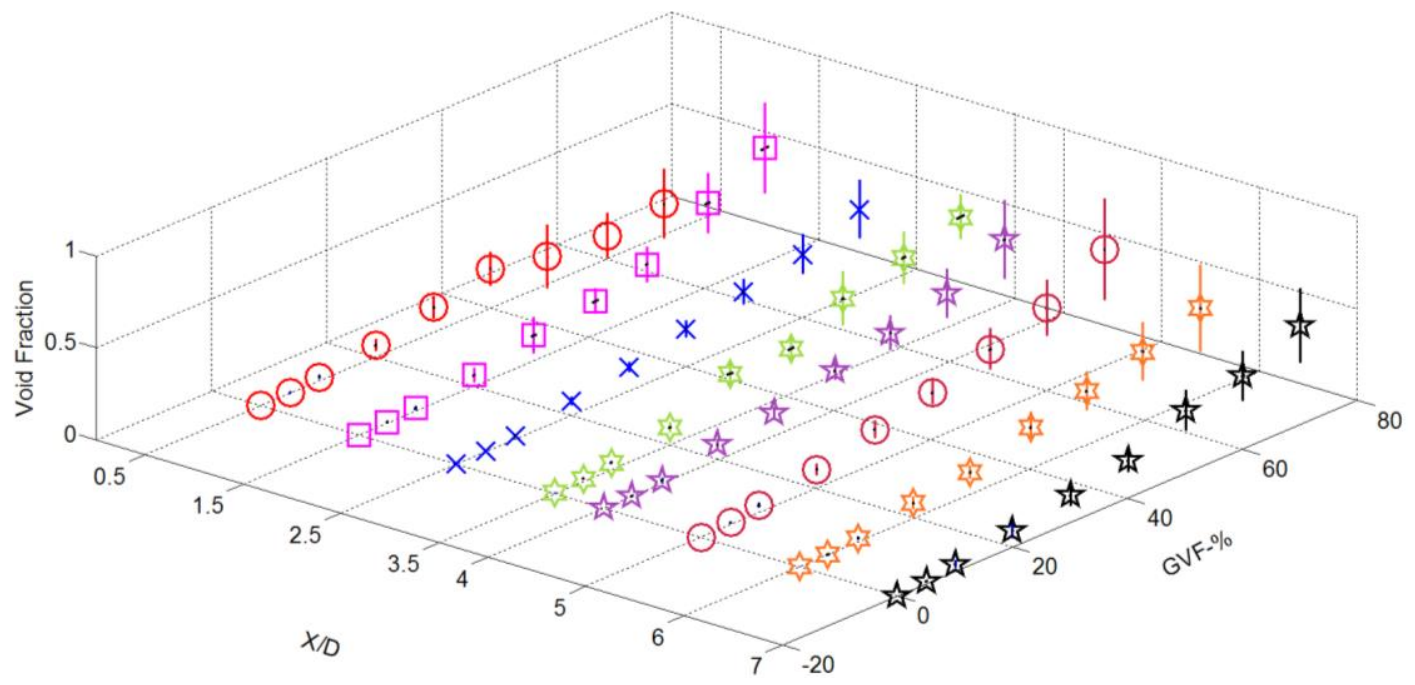

Figure 212: Standard deviation of Gas Void Fraction and GVF at 20 psig \& 60 GPM

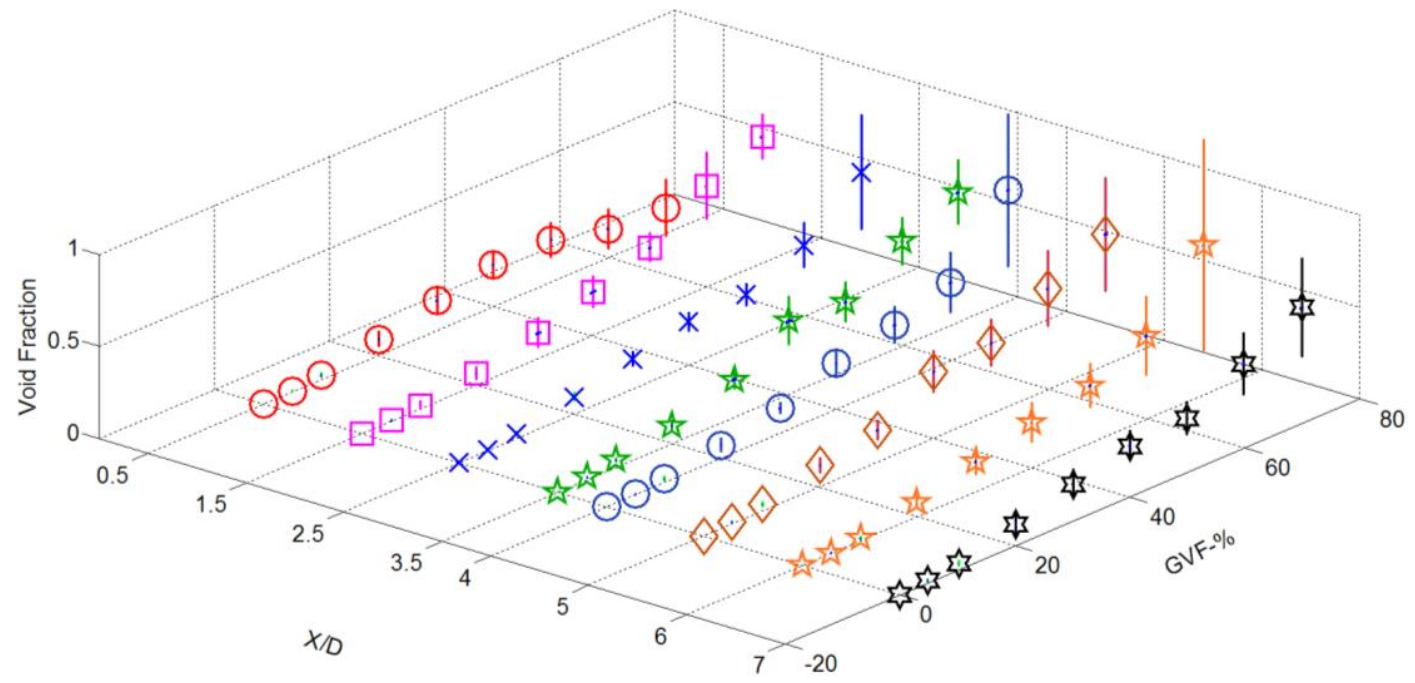

Figure 213: Standard deviation of Gas Void Fraction and GVF at 40 psig \& 60 GPM 


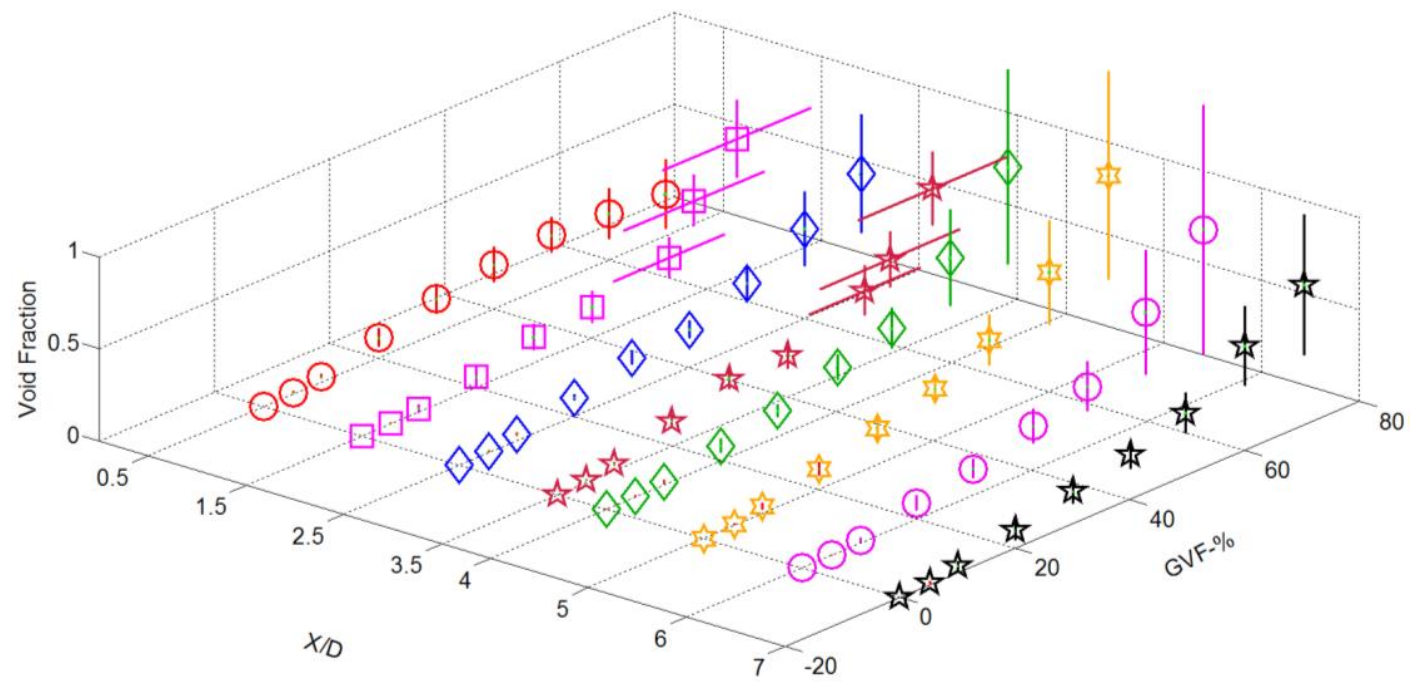

Figure 214: Standard deviation of Gas Void Fraction and GVF at 60 psig \& 60 GPM 